\title{
Progress towards the realization of an optical Far- Field Superlens
}

\author{
By
}

\author{
Farzaneh Fadakar Masouleh
}

\begin{abstract}
A thesis
submitted to the Victoria University of Wellington in fulfilment of the requirements for the degree of Doctor of Philosophy
\end{abstract}

Victoria University of Wellington 


\section{Abstract}

Conventional optics suffer from a fundamental resolution limit due to the nature of light. The near-field superlens concept was introduced two decades ago, and its theory for enabling high resolution imaging is well-established now. Initially, this superlens, which has a simple setup, became a hot topic given the proposition of overcoming the diffraction limit. It has been demonstrated that a near-field superlens can reconstruct images using evanescent waves emanating from small objects by means of resonant excitations on the surface of the superlens. A modified version of the superlens named the far-field superlens is theorized to be able to project the near-field subwavelength information to the far-field region. By design, the far-field superlens is a near-field superlens with nanostructures added on top of it. These nanostructures, referred to as diffraction gratings help couple object information available in the evanescent waves to the far-field.

Work reported in this thesis is divided to two major sections. The first describes the modelling technique that investigates the performance of a far-field superlens. This section focuses on evaluating the impact of the diffraction gratings geometry and the object size on the far-field superlens performance as well as the resulting far-field pattern.

It was shown that a far-field superlens with a nanograting having a duty cycle of $40 \%$ to $50 \%$ produces the maximum intensity and contrast in the far-field interactions. For periodic rectangular objects, an inverse-trapezoidal nanograting was shown to provide the best contrast and intensity for far-field interactions. The minimal simulation domain to model a symmetric far-field superlens design 
was determined both in $2 \mathrm{D}$ and $3 \mathrm{D}$. This input reduced the required modelling time and resources. Finally, a 3D far-field superlens model was proposed, and the effect of light polarization on the far-field pattern was studied.

The second section of this thesis contains the experimental study that explores a new material as a potential candidate for the construction of far-field superlens. The material conventionally used for superlens design is silver, as its plasmonic properties are well-established. However, scaling down silver features to the nanoscale introduces fundamental fabrication challenges. Furthermore, silver oxidizes due to its reactions with sulphur compounds at ambient conditions, which means that operating a silver far-field superlens is only possible in a wellcontrolled environment. This disagrees with our proposed concept of a low-cost and robust superlens imaging device. On the other hand, highly doped semiconductors are emerging candidates for plasmonic applications due to the possibility of tuning their optical and electrical properties during the fabrication process.

While the working principle of a superlens is independent of the plasmonic material of choice, every plasmonic material has a particular range of operating wavelengths. The pros and cons of each plasmonic material are usually identified once used experimentally.

In this work, aluminium-doped zinc oxide was the proposed material of choice for the far-field superlens design. The second part of this thesis details the characterization results of the optical, electrical and structural properties of this proposed alternative.

Our aluminium-doped zinc oxide samples were highly transparent for large parts of the spectrum. Their carrier concentration was of the order of $10^{+20} \mathrm{~cm}^{-3}$, 
and a resistivity of about $10^{-3} \Omega . c m$ was achieved. The modelled dielectric permittivity for the studied samples showed a cross-over frequency in the nearinfrared region, with the highest plasma frequency achieved in this study being $4710 \mathrm{~cm}^{-1}$. 


\section{Acknowledgement}

I have met many people along this winding yet enriching journey. This provided opportunities for me to grow as a person and hopefully have made me a better scientist. Many people have been there for me when I needed them and for that I am grateful.

First, I would like to thank my supervisors Dr. Bob Buckley and Dr. Eric Le Ru for having faith in me when I reached out. I will always be grateful for your patience, empathy and support, Bob.

My very special gratitude goes to Dr. Craig Watterson for his kind help and advice.

I would also like to acknowledge the generosity of Dr. John Kennedy, Dr. Peter Murmu, Dr. Simon Granville, and Sarah Spencer for kindly assisting me and granting me access to their facilities to carry my measurements.

To my officemates Eden Rafealov and Hamish Colenso, thank you for your support and friendship.

I would like to thank Dr. Murray Early for always being there to support me and raising my spirit when I needed it. I also like to thank Dr. Rod White for his advice and for encouraging me during the final stages of this work.

I dedicate this thesis to my parents and siblings for always believing in me and supporting me, regardless of the sacrifice.

Finally, I would like to thank my one and only, Ihab. Thank you for understanding me, supporting me, and helping me grow to be a better person. 
This page is intentionally left blank 



\section{Table of Contents}

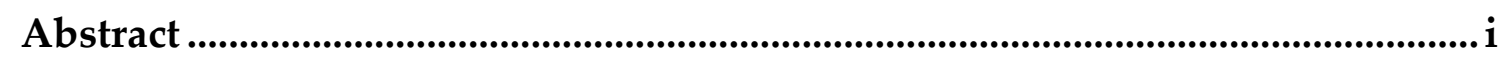

Acknowledgement............................................................................................................iv

Table of Contents.......................................................................................................... vii

Table of Figures.......................................................................................................................

Introduction ......................................................................................................... 1

Part one: Far-field superlens modelling .................................................................. 5

1 Electromagnetic wave theory …................................................................................ 7

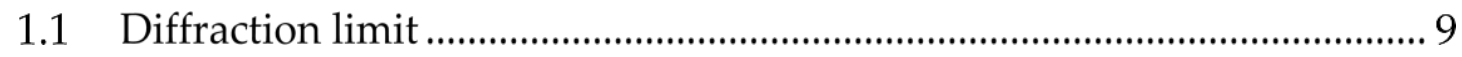

1.1.1 Resolution Limit ............................................................................. 10

1.2 Near-field and far-field optics ................................................................. 11

1.3 Devices to overcome the diffraction limit.................................................. 13

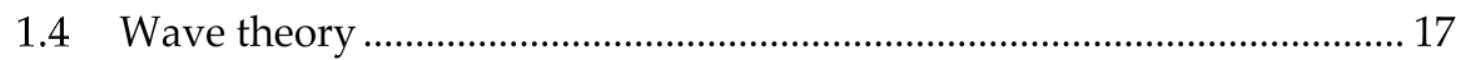

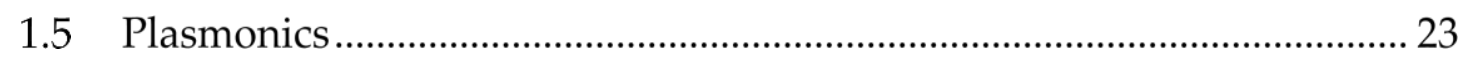

1.5.1 Basic Properties of Surface Plasmons................................................... 24

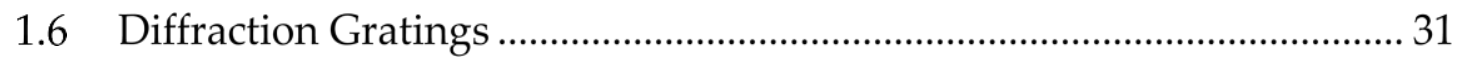

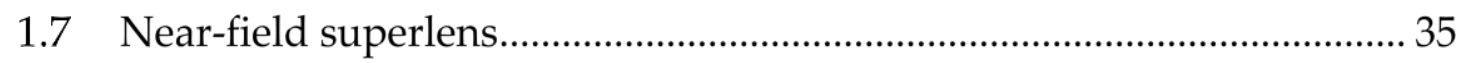

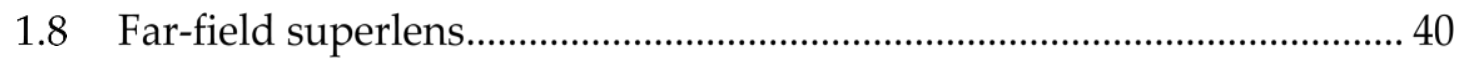

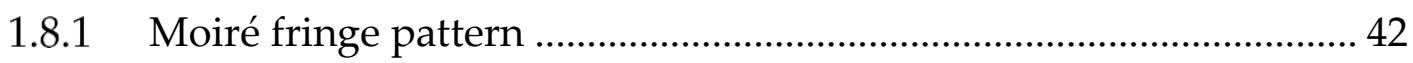

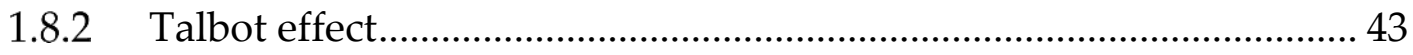

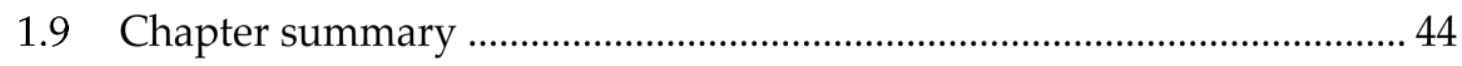




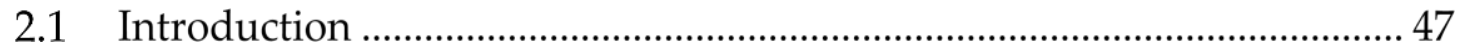

2.2 Meshing demands for modelling .......................................................... 50

2.3 Simulation Requirements and Available Resources ............................... 53

2.3.1 Available Computational Resources ................................................. 53

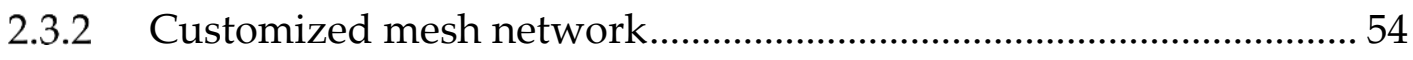

2.4 Modelling in COMSOL Multiphysics ${ }^{\circledR}$.................................................... 58

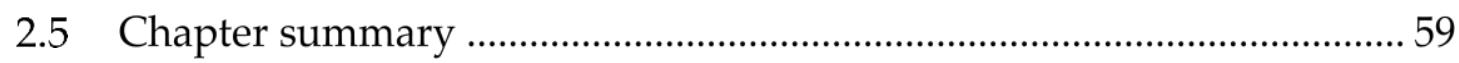

3 Modelling and Design of a Far-Field Superlens............................................ 61

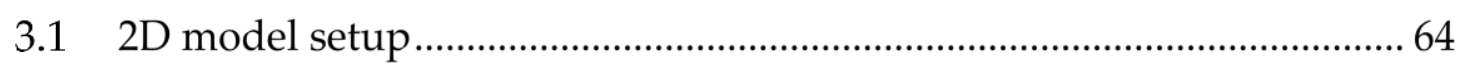

3.2 Near-field vs. Far-field superlens ......................................................... 73

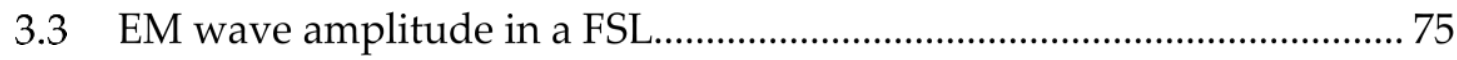

3.4 The effects of structures shape and size on the EM field pattern............ 78

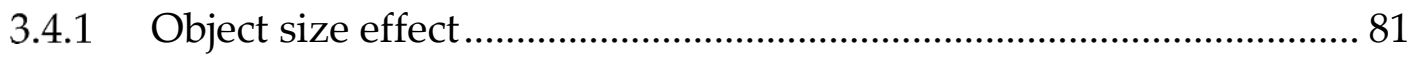

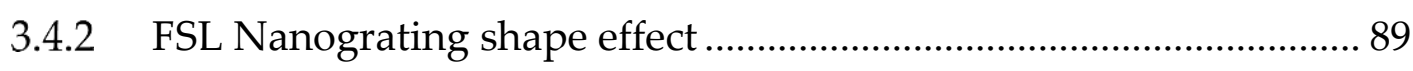

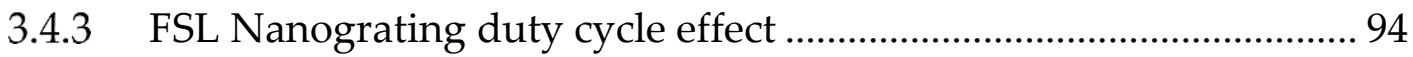

3.4.4 Anti-symmetric object positioning .............................................. 97

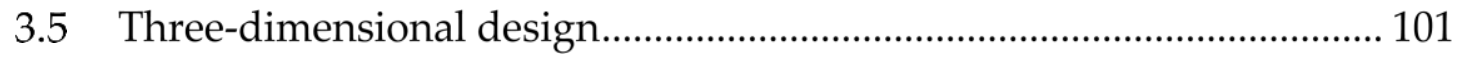

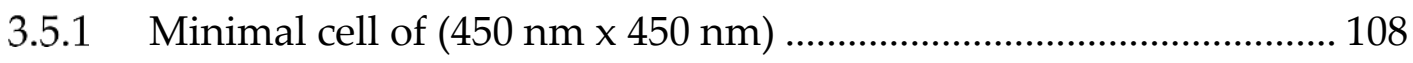

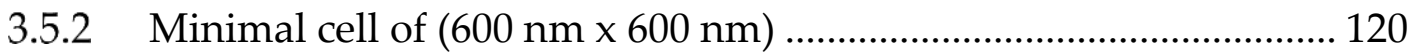

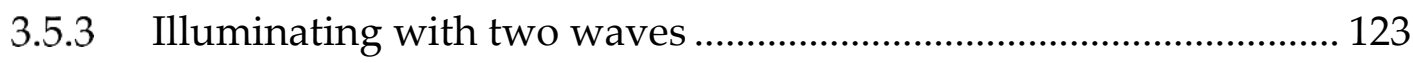

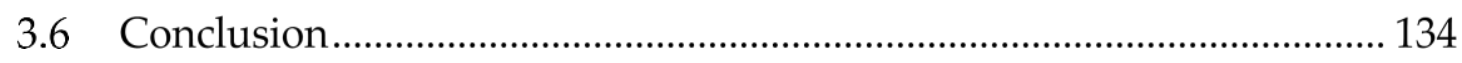

Part two: Novel Plasmonic Materials .................................................................. 139 


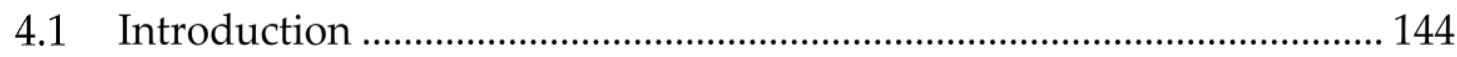

4.2 Potential Alternative Plasmonic Materials ................................................. 146

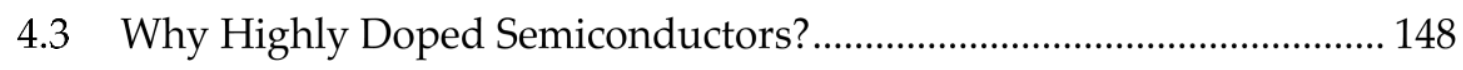

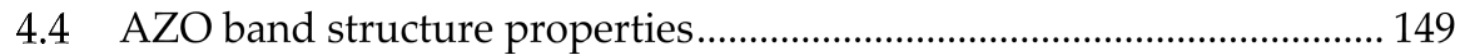

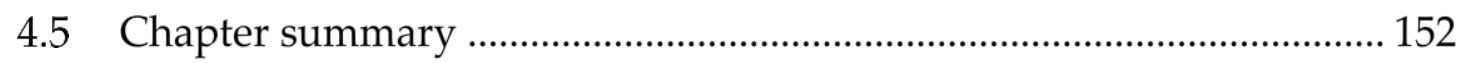

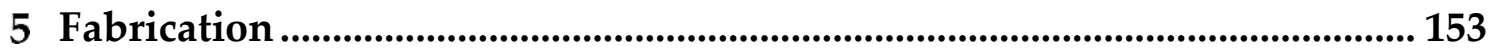

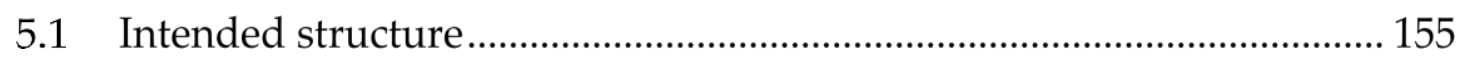

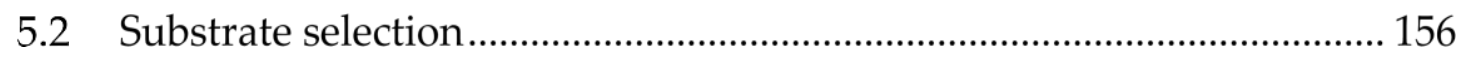

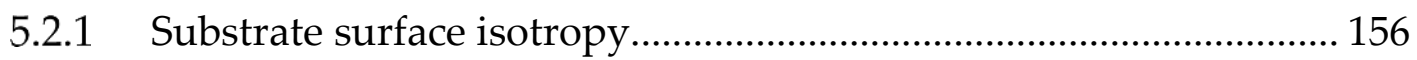

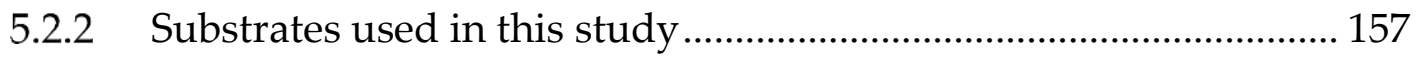

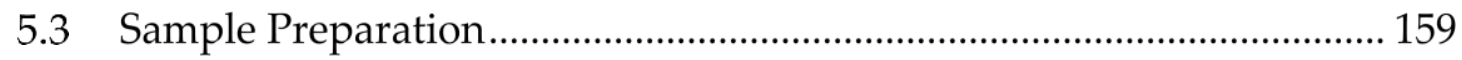

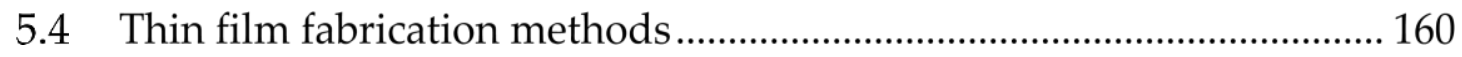

5.5 Sputtering techniques .......................................................................... 161

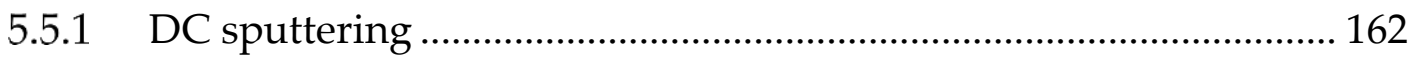

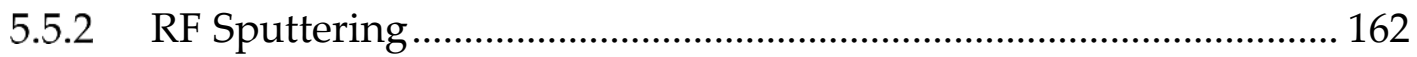

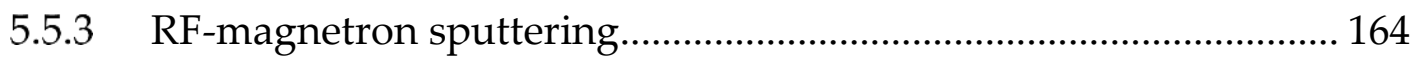

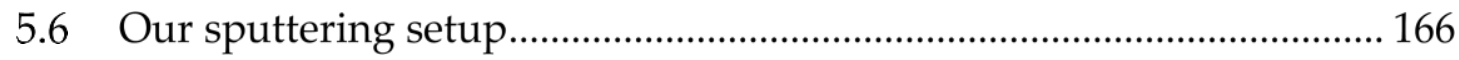

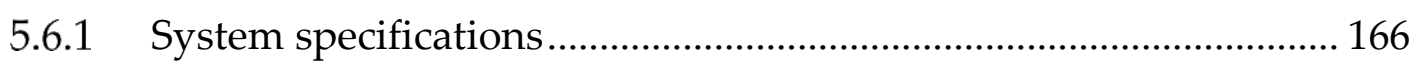

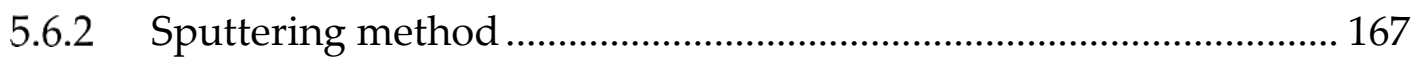

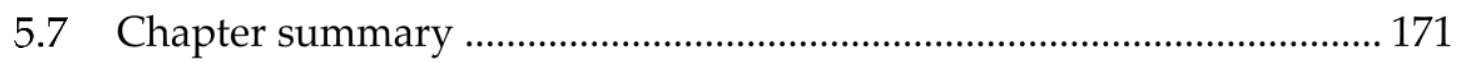

6 Characterization techniques................................................................................ 173

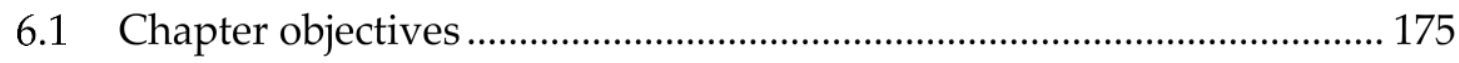




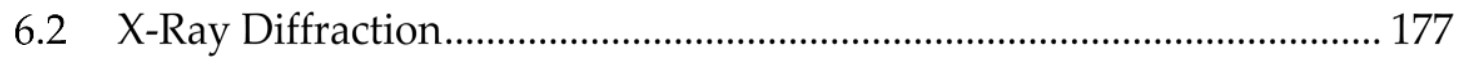

6.2.1 System specifications ...................................................................... 181

6.2.2 Properties studied using XRD ......................................................... 182

6.3 Thickness measurement ........................................................................ 184

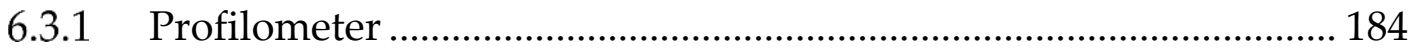

6.3.2 Rutherford backscattering spectrometry ………………………...... 185

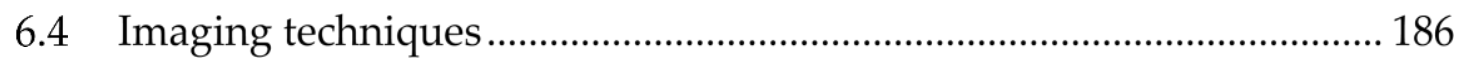

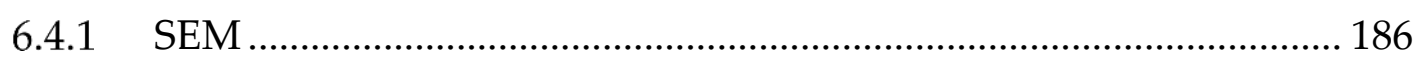

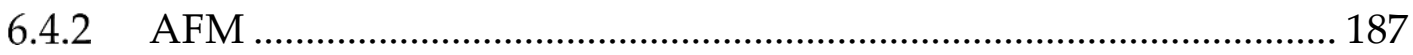

6.5 Hall Effect and PPMS measurements..................................................... 187

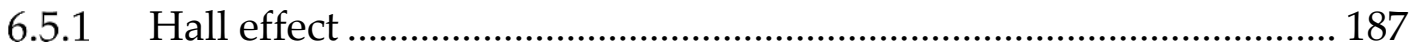

6.5.2 Physical property measurement system............................................. 193

6.6 Spectral Characterization ...................................................................... 195

6.6.1 Ultraviolet-Visible Spectroscopy.................................................... 197

6.6.2 Fourier transform infrared spectroscopy ………………………..... 199

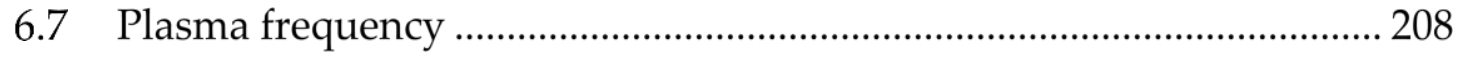

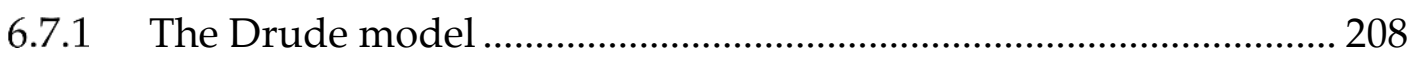

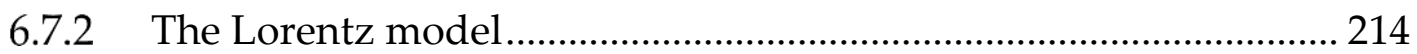

6.7.3 The Drude-Lorentz model .................................................................. 215

6.7.4 Calculating plasma frequency using the modelling software........ 215

6.7.5 Comparing methods ....................................................................... 220

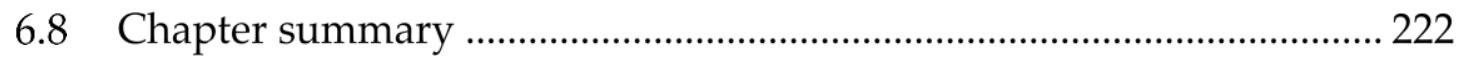

7 Results A: AZO thin films on Borofloat33 substrate ……………………....... 223 


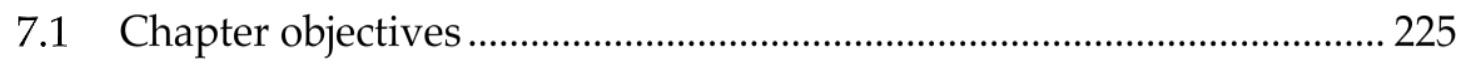

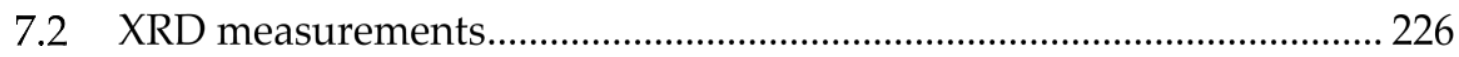

7.2.1 Dominant crystal orientation …………………………………….... 227

7.2.2 Mean crystallite domain size............................................................. 228

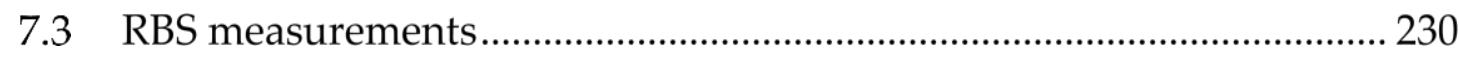

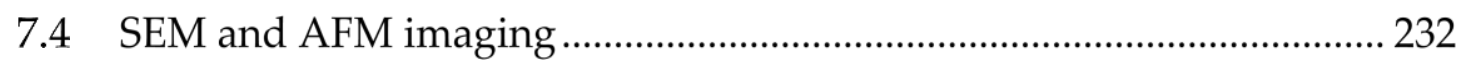

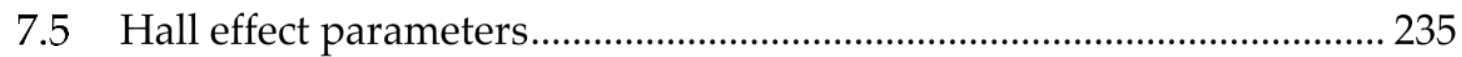

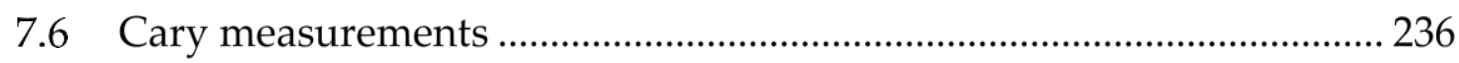

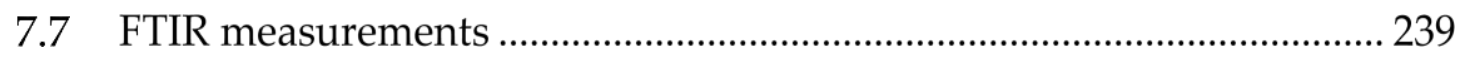

7.7.1 Transmission measurements............................................................. 239

7.8 Plasma frequency and dielectric permittivity of AZO........................... 249

7.8.1 Dielectric permittivity ……………………………………………. 255

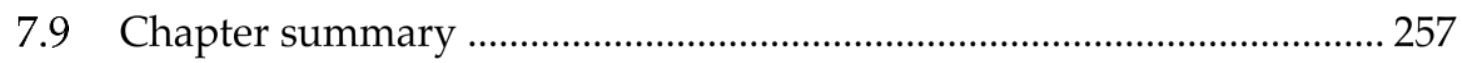

8 Results B: AZO thin films on Silicon, Sapphire and $\mathrm{CaF}_{2}$ substrates......... 259

8.1 Chapter objectives ................................................................................. 261

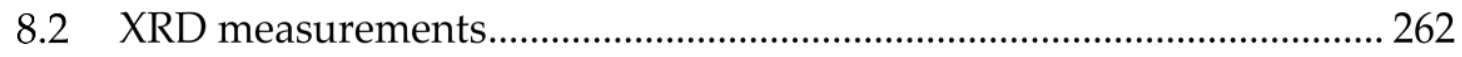

8.2.1 XRD analysis for AZO on sapphire.................................................. 262

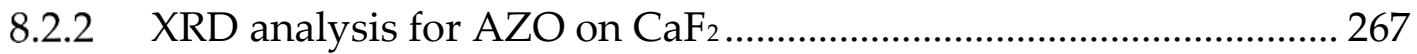

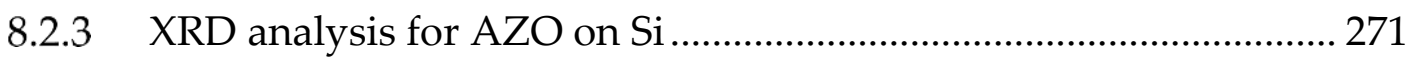

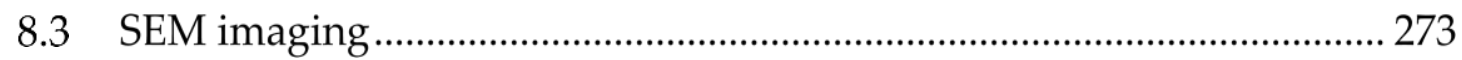

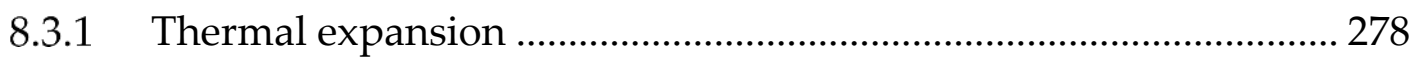

$8.4 \quad$ Hall effect parameters........................................................................... 284

8.4.1 Carrier concentration measurements............................................... 284 


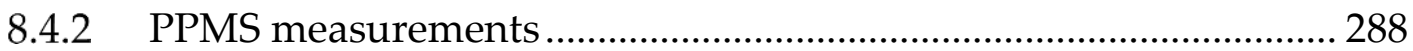

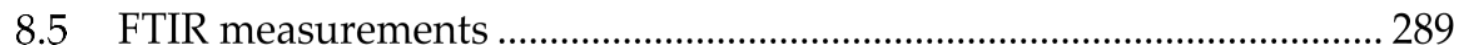

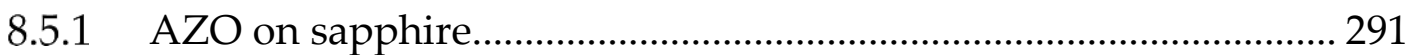

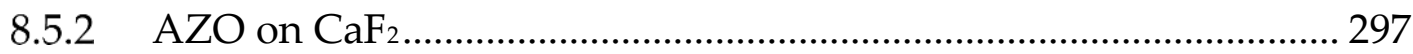

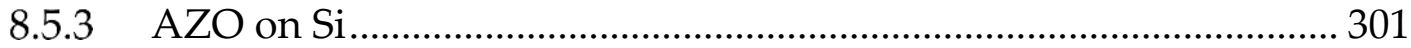

8.6 Plasma frequency and dielectric permittivity of AZO........................... 303

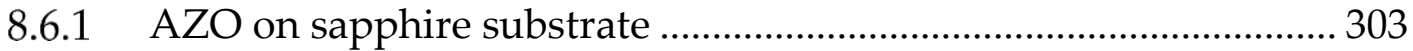

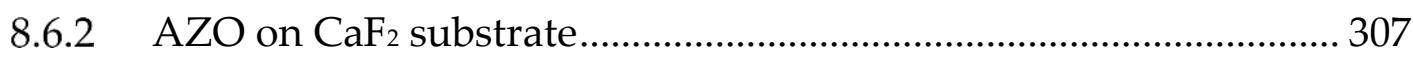

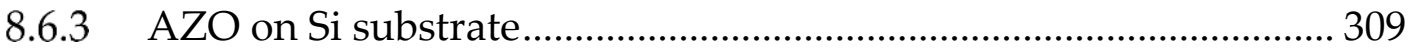

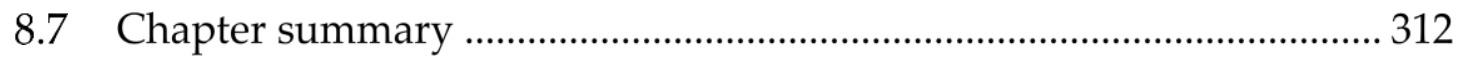

9 Results C: thermal annealing effect on AZO thin films ............................. 315

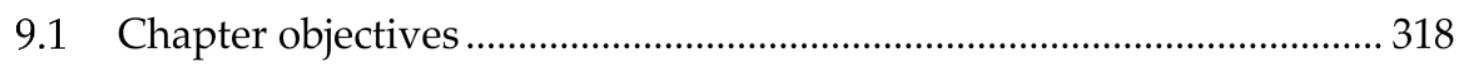

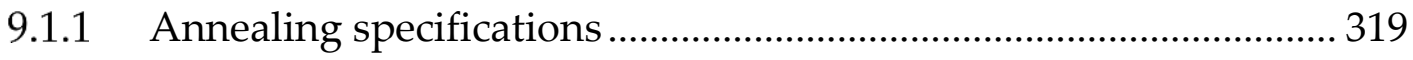

9.2 XRD results of annealed samples......................................................... 320

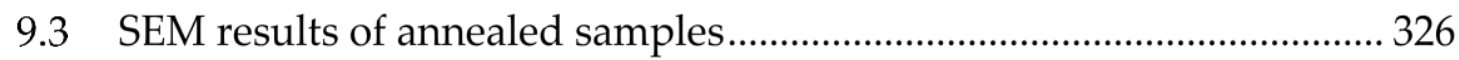

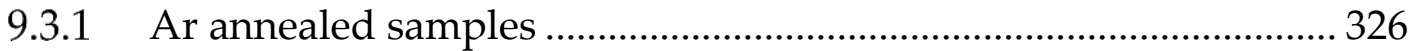

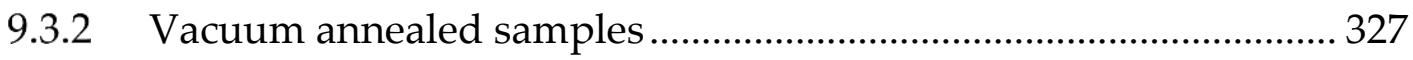

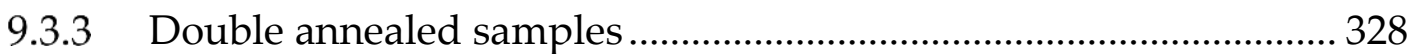

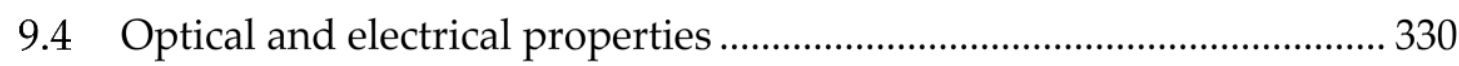

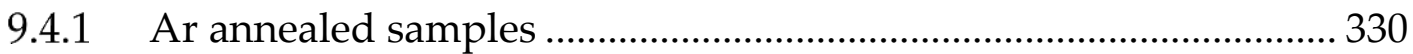

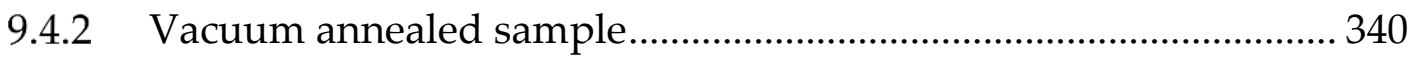

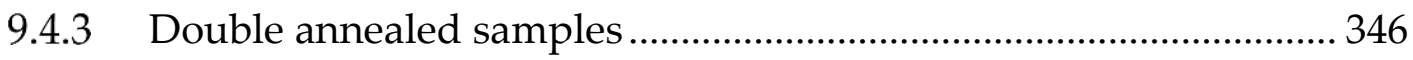

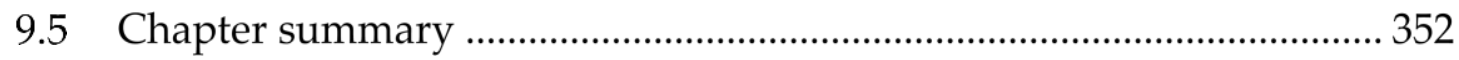




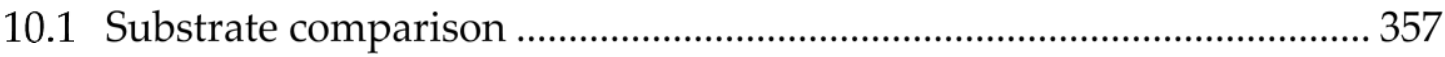

10.2 Thin film properties ............................................................................ 361

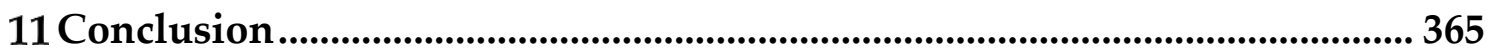

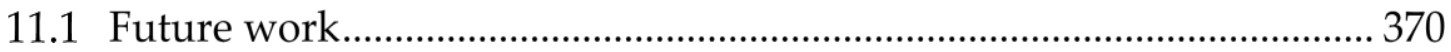

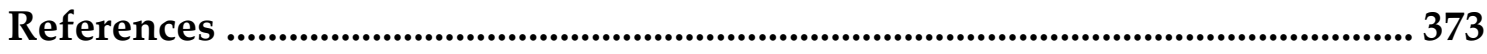

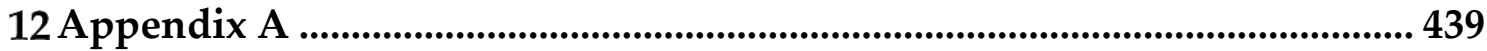

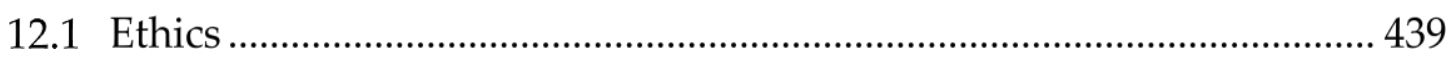

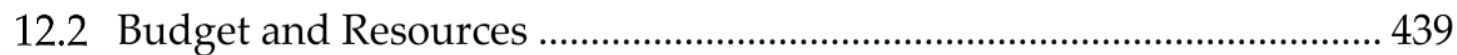

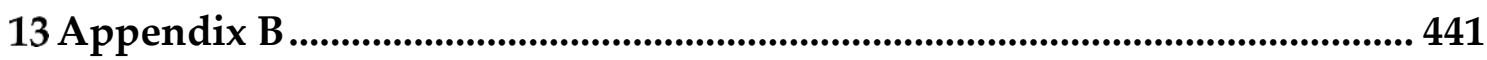

13.1 Challenges of FTIR measurements in our study .................................. 441

14 Appendix C ................................................................................................................... 447

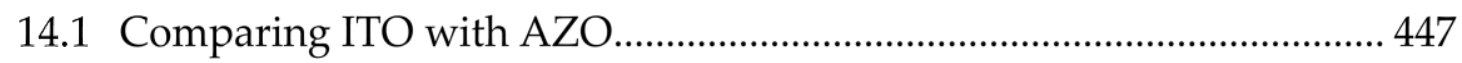




\section{Table of Figures}

Figure 1-1 (a) A flat NIM slab focuses light .......................................................... 17

Figure 1-2 Dispersion relation for bulk plasmons ……………………………...... 26

Figure 1-3 Refraction of light propagating.......................................................... 28

Figure 1-4 Diffraction gratings and the generated orders ..................................... 33

Figure 1-5 Refraction of light in positive and negative........................................... 36

Figure 1-6 Schematic presentation of focusing...................................................... 37

Figure 1-7 Amplitude of light at the output ........................................................... 39

Figure 2-1 1D mesh distribution for a line object.................................................. 55

Figure 2-2 A 2D mesh distribution for a FSL .......................................................... 56

Figure 2-3 Schematics of a 3D mesh distribution.................................................... 57

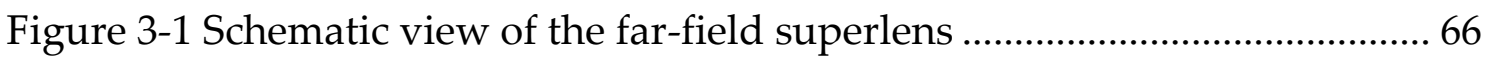

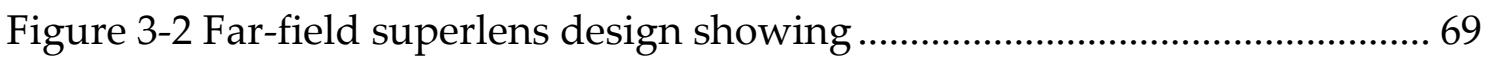

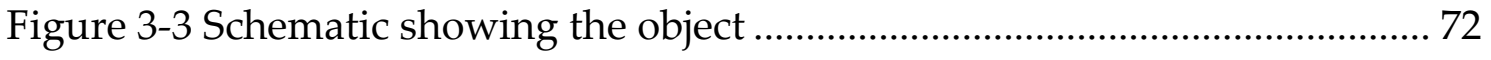

Figure 3-4 Electric field norm plots...................................................................... 74

Figure 3-5 Electric field norm of four models........................................................ 76

Figure 3-6 The magnitude of the electric field norm ............................................... 77

Figure 3-7 Different nanograting geometries as part .............................................. 80

Figure 3-8 Electric field norm for an object period of $96 \mathrm{~nm}$.................................. 83

Figure 3-9 Electric field norm density plot for ....................................................... 84

Figure 3-10 The electric field norm at the object ..................................................... 85

Figure 3-11 Fourier transforms of the electric field norm ....................................... 86

Figure 3-12 The magnitude transfer function for the ............................................. 88

Figure 3-13 Electric field norm for (a) the rectangular ........................................... 90 
Figure 3-14 (a) The contrast plots for three different FSL nanograting 91

Figure 3-15 (a) The amplitude-ratio plots for three different.............................. 93

Figure 3-16 The far-field pattern for similar models .......................................... 95

Figure 3-17 Variations of the amplitude of the electric field ...............................96

Figure 3-18 Schematic design of the arrangement of the object .........................97

Figure 3-19 Amplitude plots of the electric field norm ....................................... 98

Figure 3-20 The electric field norm plot at the .................................................. 99

Figure 3-21 Amplitude plots of the electric field norm.................................... 100

Figure 3-22 The electric field norm plot at the .................................................. 101

Figure 3-23 Top view arrangement of the object and FSL ................................. 103

Figure 3-24 Electric field norm of a 3D model with a 1D ................................. 110

Figure 3-25 Electric field norm of a 3D model with a 1D ................................. 112

Figure 3-26 Electric field norm of a 3D model with a 2D ................................ 114

Figure 3-27 Electric field norm of a 3D model with 2D .................................... 116

Figure 3-28 Electric field norm line plots at the Talbot ..................................... 118

Figure 3-29 The electric field norm amplitude plot ........................................... 122

Figure 3-30 Electric field norm amplitude plot ................................................. 125

Figure 3-31 Electric field norm amplitude plot for a model.............................. 126

Figure 3-32 Electric field norm amplitude plot for a model............................. 128

Figure 3-33 Electric field norm amplitude plot for a model.............................. 130

Figure 3-34 Electric field norm amplitude plot for a model............................. 131

Figure 3-35 Electric field norm amplitude plot for a model.............................. 132

Figure 3-36 Electric field norm amplitude plots in the $x-y$ plane ....................... 133

Figure 3-37 Schematics model combining the amplitude ................................. 135

Figure 4-1 Simple schematics showing the density of states in ........................ 151

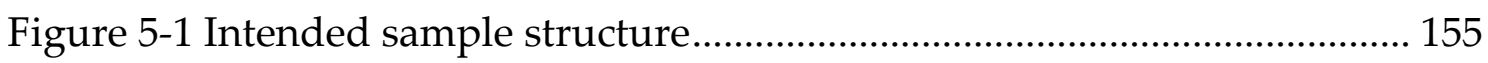

Figure 5-2 Schematics of a RF magnetron sputtering system ............................ 165 
Figure 5-3: The vacuum system diagram on the left and the HHV 167

Figure 5-4 Schematics of our RF magnetron sputtering setup. 169

Figure 6-1 characteristic radiation lines in a x-ray ............................................ 178

Figure 6-2 X-ray diffraction setup that fulfils Bragg's Law ............................... 180

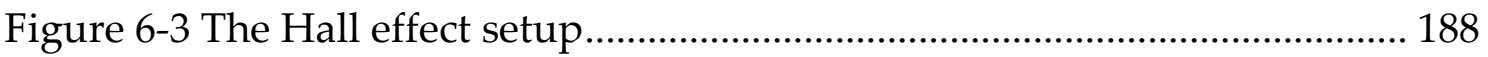

Figure 6-4 Illustration of the van der Pauw configuration ................................. 189

Figure 6-5 A PPMS setup with the sample ready for ........................................ 194

Figure 6-6 A diagram showing the UV/Vis spectrophotometer ........................ 198

Figure 6-7 A diagram of the electromagnetic spectrum ................................... 200

Figure 6-8 A simple Fourier transform spectrometer........................................ 201

Figure 6-9 Interferograms for the MIR and visible .......................................... 202

Figure 6-10 coherent transmission and reflection of light ................................ 218

Figure 7-1 AZO thin film diffractogram on a Borofloat33 substrate................. 228

Figure 7-2 SEM images of the AZO sample's surface ........................................ 232

Figure 7-3 SEM images of an AZO on Borofloat33 sample ................................ 233

Figure 7-4 AFM morphology of the AZO sample on Borofloat33 substrate .... 234

Figure 7-5 Transmission (T) plots of S1-S9 measured in the Cary ..................... 237

Figure 7-6 Transmission plots of S1-S9 measured in the Bomem FTIR............. 240

Figure 7-7 Reflectance plots of S1-S9 measured in the Bomem FTIR................. 241

Figure 7-8 Absorption plots of S1-S9 calculated from the reflectance .............. 242

Figure 7-9 Measured FTIR spectra for S4, S6, S9, and the blank....................... 244

Figure 7-10 (a) Bruker transmission plots for S4, S6 and S9 ............................. 246

Figure 7-11 Corrected reflection spectra measured using ................................ 247

Figure 7-12 Corrected absorption spectra measured using ............................... 248

Figure 7-13 Blank Borofloat33 substrate's measured FTIR................................ 250

Figure 7-14 Modelled transmission spectrum for AZO .................................... 251

Figure 7-15 FTIR measured transmission and reflection .................................. 253 
Figure 7-16 Real (solid lines) and imaginary .................................................. 256

Figure 8-1 Aligned diffractograms for AZO-on-sapphire …............................... 263

Figure 8-2 Image of the damaged AZO on $\mathrm{CaF}_{2}$ sample ....................................268

Figure 8-3 XRD diffractogram of AZO on $\mathrm{CaF}_{2}$ samples.................................. 269

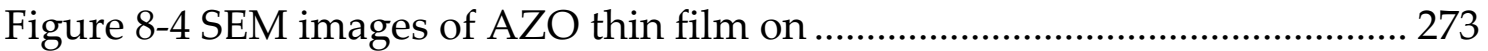

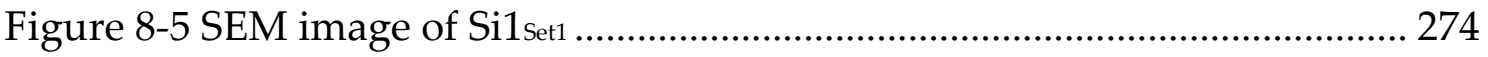

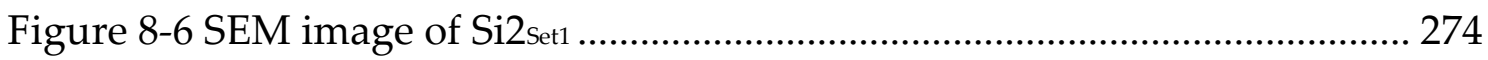

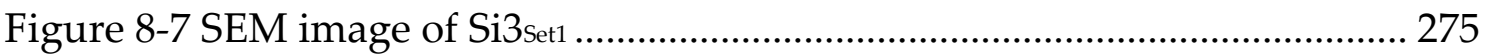

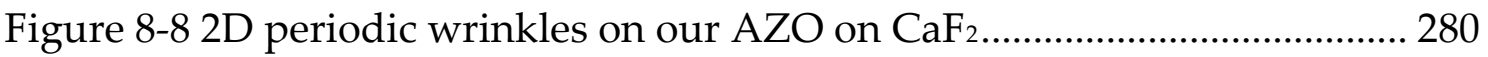

Figure 8-9 Cracks covering a large portion of the surface in ............................. 281

Figure 8-10 SEM image of AZO on $\mathrm{CaF}_{2}$ showing scratch ............................... 282

Figure 8-11 EDS analysis of AZO on $\mathrm{CaF}_{2}$ over Area......................................... 283

Figure 8-12 EDS analysis of AZO on $\mathrm{CaF}_{2}$ over Area......................................... 283

Figure 8-13 Temperature dependence conductivity of AZO ............................ 288

Figure 8-14 Transmission spectra for $\mathrm{CaF}_{2}$, Sapphire and Si............................. 290

Figure 8-15 Transmission spectra for AZO thin films on .................................. 291

Figure 8-16 Tauc plots for the AZO on sapphire samples ................................. 293

Figure 8-17 Transmission spectra for AZO thin films on sapphire.................... 294

Figure 8-18 Tauc plots for AZO on sapphire samples from Set2 ........................ 296

Figure 8-19 Transmission spectra for the four AZO on $\mathrm{CaF}_{2}$............................ 297

Figure 8-20 Tauc plots for the four AZO on $\mathrm{CaF}_{2}$ samples................................ 299

Figure 8-21 Transmission plots for the AZO thin films on Si ............................ 302

Figure 8-22 FTIR measured transmission spectra and Reffit ............................. 304

Figure 8-23 Real and imaginary parts of the dielectric permittivity .................. 306

Figure 8-24 Real and imaginary parts of the dielectric permittivity .................. 307

Figure 8-25 Real and imaginary parts of the dielectric permittivity ................. 309

Figure 8-26 Real and imaginary parts of the dielectric permittivity .................. 311 
Figure 9-1 SEM image of an Ar annealed AZO on sapphire sample. 326

Figure 9-2 SEM image of an AZO on sapphire sample 327

Figure 9-3 SEM image of the vacuum annealed AZO on Si.............................. 328

Figure 9-4 AZO on sapphire sample after the double annealing step.............. 329

Figure 9-5 Transmission spectra for S9 before and............................................. 331

Figure 9-6 Transmission spectra for $\mathrm{CaF}_{2}-\mathrm{A}$ and $\mathrm{CaF}_{2}-\mathrm{B}$ before ......................... 334

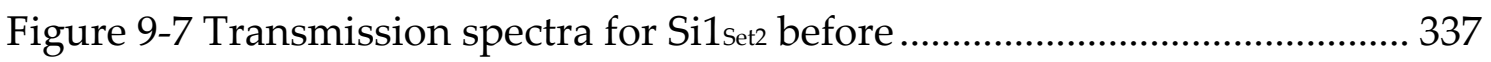

Figure 9-8 Transmission spectra for S7 before .................................................... 340

Figure 9-9 Transmission spectra for Sap2set2 before ........................................... 341

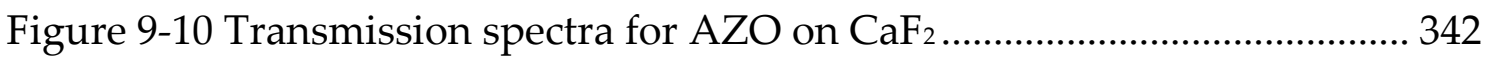

Figure 9-11 Transmission spectra for Si2set1 before ........................................... 343

Figure 9-12 Transmission spectra for two Ar and vacuum annealed ................ 347

Figure 9-13 Tauc plots to estimate the optical bandgap .................................... 348

Figure 9-14 Transmission plots for the non-transparent ................................... 350

Figure 9-15 Real (E1) and imaginary (E2) dielectric permittivity ....................... 352

Figure 10-1 Plot of the measured carrier concentration versus .......................... 360

Figure 10-2 Plot of the calculated carrier concentration versus ......................... 361

Figure 10-3 A comparison of FWHM $\left(^{\circ}\right)$ between the reported ........................... 364

Figure 13-1 Transmission discontinuity at the end ........................................... 441

Figure 13-2 Reference spectrum (RSC) and sample....................................... 442

Figure 13-3 FTIR spectrum for AZO sample with ................................................ 443

Figure 13-4 Transmission anomaly from the Si surface ..................................... 445

Figure 14-1 Transmission spectra included in our AZO - ITO comparison. .... 448 


\section{Introduction}

Common imaging tools typically rely on glass lenses to focus light and construct an image. These lenses provide a resolution about half the wavelength of the illuminating light due to the diffraction limit. As a result, smaller object features cannot be resolved using conventional imaging techniques. To produce images of such small objects, alternative solutions were proposed. Among these solutions lies a simple design, first suggested by Pendry in his paper in 2000, which was a miniaturized device concept called a superlens [1].

Following this theoretical paper, several experiments attempted to verify the super-resolution imaging theory $[2,3,4,5,6]$. Nonetheless, to the best of our knowledge, a low-cost, efficient and robust subwavelength imaging tool that can provide reliable and reproducible images has not yet been realized. Meanwhile, there remains a continuous interest in developing solutions that can image subwavelength sized objects using optical techniques $[7,8,9,10,11]$. This is partly driven by the growing research in the fields of nanotechnology and material science $[12,13,14,15]$. In addition, recent developments in nanophotonics, near-field optics and plasmonics amplified this interest $[16,17,18,19]$.

\section{Motivation}

Near-field superlens imaging is a promising technique that forms images using a combination of propagating waves and evanescent waves through a thin slab of metal. In such a technology, super-resolution optical imaging can be achieved by recovering the near-field spatial information from the evanescent 
waves. Therefore, a conventional superlens performance is limited to the near field given the inherent decay property of plasmonic waves [20]. A valuable property for a practical subwavelength imaging device is the ability to project the recovered object information to a distance beyond the near-field region. The work presented in this thesis aimed to progress the development of such a device, namely a far-field superlens, through both modelling and material studies.

\section{Scope and Contribution of this Work}

In the first part of this thesis, we present an extensive modelling study of a farfield superlens design made of silver. We perform both 2D and 3D simulations and propose ways to optimize the design to allow practical operation. We also investigate the effects of using polarized light on the superlens performance, as illumination is provided from different directions. Results from our modelling work can potentially contribute to the development of a superlens made of any plasmonic material as the working principle remains unchanged.

Choosing silver as a conventional material to build a superlens entails several shortcomings, such as the expected high surface roughness of deposited thin films when using non-epitaxial processes. This results in optical losses via scattering, which contribute to the high absorption loss reported in silver [21]. The other main drawback is surface oxidation at ambient conditions, which degrades with time, hence, is not ideal for an easy to use and low-cost subwavelength imaging tool.

Aluminium-doped zinc oxide has a lower absorption loss and is stable at ambient conditions; however, its plasmonic response range should be obtained for assessing its suitability for plasmonic applications. Therefore, in the second part 
of this thesis, we present a study that details the plasmonic properties of $\mathrm{Al}$ doped zinc oxide through optical and electrical characterization. This enables us to find the suitable electromagnetic range of $\mathrm{Al}$ doped zinc oxide for plasmonic applications. To the best of our knowledge, such a comprehensive study of $\mathrm{Al}$ doped zinc oxide over a broad electromagnetic range has not been reported elsewhere. These results provide a base for future studies intending to investigate the suitability of Al doped zinc oxide to fabricate a superlens.

Regarding contribution, all simulation and experimental work presented in this thesis was carried by the author (Farzaneh Fadakar Masouleh), except for RBS measurements, which were carried by Dr Peter Murmu at GNS Science. 

Part one: Far-field superlens

\section{modelling}



1 Electromagnetic wave theory 

In this chapter, the main limitation to the attainable resolution of conventional optical microscopes will be discussed. Alternative technologies, which rely on the working principles of near- and far-field optics will be introduced.

\subsection{Diffraction limit}

The attainable optical resolution of conventional microscopes is tied to the diffraction limit, which defines the smallest distance that two objects can be unambiguously distinguished through an aperture [22, 23, 24]. This limit was initially defined and experimentally proved by Ernest Abbe. The first ever lens with a good compatibility with the optical theory and the laws of physics was also built by Abbe. He published a paper in 1873 and described that the resolution of an image captured by a conventional microscope is limited to about half of the operating wavelength [25]. For instance, this implies the ability to detect a feature as small as $250 \mathrm{~nm}$ given an effective incoming wavelength of around $550 \mathrm{~nm}$. This wavelength is in the visible range with a size almost as big as the smallest bacterium. The first mathematical formulation for diffraction limit was presented by von Helmholtz in 1874, one year after Abbe's paper. Apart from presenting the mathematical formulation, von Helmholtz's experimental setup was different from that of Abbe's, as his study of diffraction considered the phase relationship of light at different points of the object [26].

Light illuminating an object is divided into two fractions after interaction, i.e., the absorbed and the scattered light. An observer sees everything through the scattered light. In a homogeneous medium, it can be assumed that light travels 
in a straight line without bending; however, in the presence of objects or inside narrow apertures diffraction occurs. The image of an object through a microscope is also formed by the scattered light.

\subsubsection{Resolution Limit}

An ideal microscope with high precision optics produces a point image of a point object. However, this ideal behaviour is not achieved given that a lens suffers from optical losses, diffraction and aberration effects. Rayleigh's criterion defines the minimum distance at which two point-objects can be still distinguished. This is a well-established definition of the resolution limit that relates to the operation of telescopes, microscopes, gratings, prisms and many other optical devices $[27,28]$.

While the Rayleigh criterion is a reliable rule of thumb, Abbe also proposed another well-known model to quantify the possible resolution through an objective lens [29]. Diffraction and scattering of light are inevitable sources of imaging problems and are related to the specific sizes of apertures in optical systems [30]. Accordingly, an image of an object will be inherently blurred regardless of the precision of the measurement system. In a typical imaging system, optical broadening occurs as light passes through each lens [31]. This effect originates from the inability of conventional lenses to collect parallel rays of light into a single focal point, as collection power is strongest when light is incident at a lens's axis of symmetry. Given the aforementioned non-idealities, there is a lower limit below which an imaging system cannot resolve two pointobjects. Based on the Abbe criterion, this minimal distance is close to half of the illuminating wavelength for a good numerical aperture (NA) [32]. The numerical aperture concept was first introduced by Abbe and defines the amount of light 
that is collected through a lens. In other words, it is a dimensionless value that relates to the light gathering power of an objective lens [33], which is given by [30]:

$$
N A=n \sin \theta \sim n \frac{\text { Radius of lens }}{\text { Focal length of lens }} .
$$

\section{Equation 1-1}

Here $n$ is the refractive index of the medium in which the lens is located, and $\theta$ is half of the angular aperture through which light is collected by the objective lens, often referred to as objective's collection angle [34].

In Abbe's description, resolution or the smallest object that an optical device can resolve, $d_{\min }$ in only dependant on the wavelength of light and the objective's NA, regardless of the quality of the used components $[35,36,37]$.

$$
d_{\min }=\lambda /(2 \mathrm{NA})
$$

\section{Equation 1-2}

Nowadays, there is a need to develop powerful imaging systems that can cater to the needs of emergent niches in various fields, such as semiconductor and electronics manufacturing, sensor and transducer development, molecular and biological characterization and material science $[38,39,40]$.

\subsection{Near-field and far-field optics}

Any energy transfer modality that can be described using wave equations, whether it is mechanical [41, 42], thermal [43, 44], electromagnetic [45, 46], or other, has developed concepts regarding the near and far -field zones. Adopting this two-zone concept helps clarify the effects of diffraction and scattering on the 
relevant propagating waves, despite the loose definition of the boundary that separates these zones. In optics, once light impinges on an object, the behaviour of the diffracted or scattered light varies with the propagating distance. Using the near and far -field zone concept, this behaviour can be simply modelled, as light is assumed to conform to defined characteristics in each zone [47]. In the near field, non-propagating evanescent waves dominate, while the nature of the wave depends on the source characteristics [48]. On the other hand, behaviour in the far-field is largely concerned with propagating waves, hence, the wave nature largely depends on the properties of the propagation medium. Regardless of the source, waves in the far-field can be considered to be plane waves as the distance travelled in the propagation medium is large enough to allow such an approximation.

Near-field interactions between light and matter are localized within the vicinity of the object, in a range comparable to that of the light's wavelength. One established definition for the near-field region in the multipole basis is given by [49]:

$\mathrm{kR} \ll 1$

\section{Equation 1-3}

where $R$ is the distance from the electromagnetic source and $k$ is the wavenumber. The far-field zone is located where [49]:

$\mathbf{k R} \gg 1$

\section{Equation 1-4}

A conventional optical microscope cannot image subwavelength details of an object as it operates on propagating waves in the far-field, where the high spatial frequency information that is carried by the evanescent waves in the near field is 
already lost [50,51]. Evanescent waves are lost during this imaging process since they rapidly decay when passing through a medium.

Near-field imaging techniques are developed to overcome the diffraction limit resolution problem. The first proposal of a near-field imaging device can be traced back to Edward Hutchinson Synge in 1928, where he speculated the use of a small gold particle to realize subwavelength imaging of a biological sample [49].

\subsection{Devices to overcome the diffraction limit}

Conventional microscopes were optical in nature, hence the optical diffraction limit was the threshold for possible resolution of the lens. However, non-optical microscopes using shorter wavelengths, such as electron microscopes can produce images with a magnifying ability for objects thousands of times better than what is possible in the diffraction limited optical microscopes [52]. Electron microscopy is one of several techniques that enables such levels of magnification.

Near-field scanning optical microscopy (NSOM, SNOM), stimulated emission depletion (STED) microscopy and near-field cathodoluminescence microscopy are among the optical techniques that enable us to resolve features smaller than the diffraction limit.

The first technique described here is known by two names, i.e., NSOM or SNOM [53]. The abbreviation, NSOM, focuses on near-field optics while SNOM emphasis is on scanning characteristic of the instrument. Therefore, using either of the abbreviations is correct based on the user's intention [54]. A NSOM relies 
on utilizing a sharp probe that interacts with the surface of the sample and collects the near-field evanescent signals from that surface [55]. Typically, the sharp optical probe has a nanometre sized aperture that scans the surface of the sample point-by-point in a raster like fashion, within a distance comparable to a fraction of the incident light's wavelength, thereby producing two dimensional subwavelength images of the sample's surface. Accordingly, resolution in a NSOM is independent of light's wavelength, and is solely defined by the probe aperture size and shape. However, there is a trade-off between image resolution and attainable contrast, according to the probe characteristics [56].

Stimulated emission depletion (STED) is a fluorescence microscopy technique that can overcome the diffraction limited resolution of confocal microscopes. This technique is based on the nonlinear optical effects that require the application of multiple high-intensity pulsed lasers with specialized modulation filters to control the excitation beam geometry. In STED, the scanning spot size can be significantly reduced by spatially-controlled stimulated quenching of the excited fluorescent molecules [57].

Near-field cathodoluminescence microscopy is a high-resolution imaging technique that analyses the luminescence (emitted light) from a material when stimulated by the electron beam of an electron microscope [58]. The luminescence may be in the ultraviolet (UV), visible or infrared wavelength ranges.

One downside of the aforementioned subwavelength imaging techniques is resorting to slow raster scanning rather than forming a shot image in real-time [59].

Another super-resolution imaging method is achieved by utilizing an optical medium or "metamaterial" that can amplify and transport the decaying nearfield evanescent waves then recover the contained high spatial frequency 
information in the far-field [60]. Earlier approaches within the optical region mostly rely on metallic thin films or metal-dielectric multilayers to amplify the evanescent waves but are restricted to layers that are thinner than the wavelength of illumination. However, due to optical losses in the thin film material, a short object-to-image distance is required [61].

Metamaterials are artificially arranged periodic structures and they could be made to have a negative index of refraction. This means that the reflected or transmitted light travels a nonconventional path, as it is bent in a reverse direction when compared to the conventional case of positive index materials; hence exotic propagation characteristics are made possible [62].

Metamaterials are custom designed with variable sizes, shapes and orientations, to interact with specific wavefronts at given wavelengths that depend on the intended application [63]. Early metamaterials had dimensions larger than those of visible wavelengths; however, recent efforts have been made to realize these metamaterials in the visible region. Optical subwavelength imaging is one of many applications that could be enabled by negative index metamaterials in the visible region [64].

Materials with negative index of refraction were theorized to enable subwavelength imaging [62], however, due to inability of creating such materials, this idea was sidelined until Pendry introduced the concept of a perfect lens [1]. Based on Pendry's theory, the evanescent waves containing details about the object, which cannot be resolved using conventional lenses, can be amplified while preserving the contained high spatial frequency information [65]. Pendry's lens consists of a slab of left-handed material, with a negative refractive index, magnetic permeability and dielectric permittivity [1]. The term "left-handed material" was coined by Veselago in 1968 given that a plane electromagnetic wave propagating inside such a material would have its Poynting vector 
opposite to its wavevector [62]. Energy loss in Pendry's lens is expected to be large given the lack of a way to reconstitute the evanescent waves emitted by the object, and losses caused by the negative permittivity [66]. This lens was only presented in a theoretical study, which suggested a way for super-resolution imaging without experimental validation. Hence, sceptical debates arose regarding the possible performance of his proposed lens [67, 68, 69, 70, 71, 72].

Early experimental research relating to such super-resolution imaging took a couple of years before fruition, where in 2003 Parimi et al. presented a superlens made of photonic crystalline material for the RF/microwave region [73]. Then by 2005, Melville et al. and Fang et al. described superlens designs that were able to produce super-resolution images on the scale of a few tens of nanometres $[2,74]$. However, to reduce optical losses in these setups, the object and image planes needed to be very close to one another. Later, there has been an interest to modify the superlens setup to acquire super-resolution image reconstruction far from the object plane or in the far-field. The hyperlens and far-field superlens were among these proposed solutions [75, 76, 77]. These concepts were developed for the microwave [78] and optical regions [59]. However, the working mechanism and the image quality in the optical frequency range has yet to be improved to acquire a better resolution for subwavelength objects.

A superlens provides an opportunity to generate real-time subwavelength images using plasmonic effects. Surface plasmon polaritons (SPP) occur at the metal interface when the real part of the dielectric permittivity is equal to the dielectric permittivity of the surrounding medium $[79,80]$.

Figure 1-1 illustrates the idea behind perfect image construction using a negative-index material (NIM) slab, which brings both the propagating and evanescent waves to a perfect focus [2]. Given that a superlens amplifies the evanescent mode, it allows real-time sub-wavelength imaging. Therefore, it is 
more efficient when compared with near-field scanning optical microscopy (NSOM), which relies on a slower raster scan process [81].

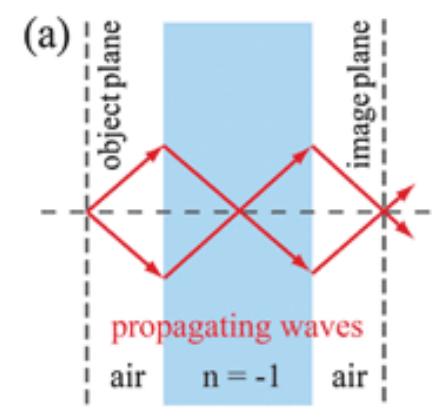

(b)

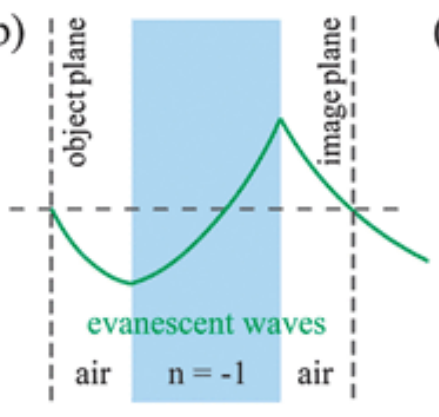

(c)

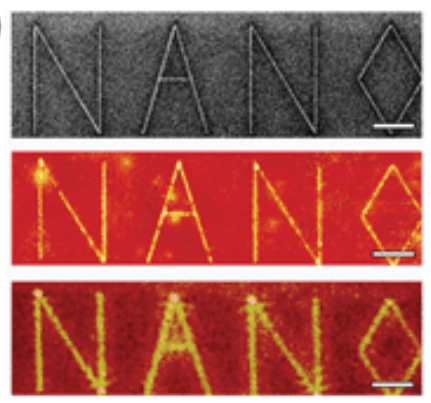

Figure 1-1 (a) A flat NIM slab focuses light from a point source. (b) The same flat NIM slab amplifies the evanescent waves which contain the high spatial frequency information, resulting in a perfect image. (c)

Experimental demonstration of an optical superlens: the top image shows a mask that was patterned inside a FIB system, the middle image was recorded using a AFM and shows a developed photoresist layer that utilized the silver superlens during exposure, the bottom AFM image shows the developed photoresist layer without using the superlens, all scale bars are $2 \mu \mathrm{m}$ long [2].

In the next section, we introduce the mathematical formulation for Maxwell's equations, which describe the electromagnetic wave propagation in a superlens [82].

\subsection{Wave theory}

Relations between magnetic and electric fields should be carefully considered when attempting to understand and model light propagation, and more generally electromagnetism. Maxwell's equations describe a time-varying 
relationship between electric and magnetic fields. Maxwell's differential equations are given by:

Faraday's law

$$
\nabla \times \vec{E}=-\frac{\partial}{\partial t} \vec{B}
$$

\section{Equation 1-5}

Ampere's law

$$
\nabla \times \overrightarrow{\mathbf{H}}=\frac{\partial}{\partial \mathbf{t}} \overrightarrow{\mathbf{D}}+\overrightarrow{\mathbf{J}}
$$

\section{Equation 1-6}

Gauss's law

$$
\nabla \cdot \vec{D}=\rho
$$

\section{Equation 1-7}

Gauss's magnetic law

$$
\boldsymbol{\nabla} \cdot \overrightarrow{\mathbf{B}}=\mathbf{0}
$$

Equation 1-8

where $E(\mathrm{~V} / \mathrm{m})$ is the electric field. $\mathrm{B}\left(\mathrm{Wb} / \mathrm{m}^{3}\right)$ is the magnetic flux. $H(\mathrm{~A} / \mathrm{m})$ is the magnetic field. $D\left(\mathrm{C} / \mathrm{m}^{2}\right)$ is the electric displacement vector. $\mathrm{J}\left(\mathrm{A} / \mathrm{m}^{2}\right)$ is the current density and $\rho\left(\mathrm{C} / \mathrm{m}^{3}\right)$ is the charge density.

In electromagnetism, the two fundamental parameters responsible for the characteristics of a medium are electric permittivity $(\varepsilon)$ and magnetic permeability $(\mu)$. Electric permittivity is measured by Farads per meter, and describes the electric field properties in a dielectric medium. Free space for 
instance has a permittivity of $\varepsilon_{0}$. Magnetic permeability has a unit of Henrys per meter [83] and describes magnetic field effects in a material. Free space has a permeability of $\mu_{0}$. Relative permittivity $\left(\varepsilon_{\mathrm{r}}\right)$ and relative permeability $\left(\mu_{\mathrm{r}}\right)$ of a material are given by:

$$
\begin{aligned}
& \varepsilon_{r}=\text { Electric permittivity }(\varepsilon) / \text { free space permittivity }\left(\varepsilon_{0}\right) \\
& \mu_{r}=\text { Magnetic permeability }(\mu) / \text { free space permeability }\left(\mu_{0}\right)
\end{aligned}
$$

Rewriting $D$ and $B$ to integrate permittivity and permeability results in:

$$
\overrightarrow{\mathbf{D}}=\varepsilon_{0} \overrightarrow{\mathbf{E}}
$$

\section{Equation 1-9}

$$
\overrightarrow{\mathrm{B}}=\mu_{0} \overrightarrow{\mathrm{H}}
$$

Equation 1-10

In the case of light waves, and to get a wave like solution from Maxwell's equations, Helmholtz wave equation is introduced [84]. In this case, the medium is considered to be non-conductive, hence current density is null, $J=0$. The modified Faraday's law is given by:

$$
\nabla \times \nabla \times \overrightarrow{\mathrm{E}}=-\frac{\partial(\nabla \times \overrightarrow{\mathrm{B}})}{\partial \mathrm{t}}
$$

\section{Equation 1-11}

$$
\nabla \times \overrightarrow{\mathrm{B}}=\varepsilon_{0} \mu_{0} \frac{\partial \overrightarrow{\mathrm{E}}}{\partial \mathrm{t}}
$$

Equation 1-12 
Therefore, Helmholtz's equation is written as:

$$
\nabla \times \nabla \times \overrightarrow{\mathrm{E}}=-\varepsilon_{0} \mu_{0} \frac{\partial^{2} \overrightarrow{\mathrm{E}}}{\partial^{2} \mathrm{t}}
$$

\section{Equation 1-13}

In order to simplify this equation, we assume a charge free medium, $\rho=0$, and therefore,

$$
\nabla \times \nabla \times \overrightarrow{\mathrm{E}}=\nabla(\nabla \cdot \overrightarrow{\mathrm{E}})-\nabla^{2} \overrightarrow{\mathrm{E}}
$$

Equation 1-14

which narrows down to:

$$
\nabla \times \nabla \times \overrightarrow{\mathrm{E}}=-\nabla^{2} \overrightarrow{\mathrm{E}}
$$

\section{Equation 1-15}

Helmholtz's equation is finally rearranged as follows:

$$
\nabla^{2} \mathrm{E}-\varepsilon_{0} \mu_{0} \frac{\partial^{2} \mathrm{E}}{\partial \mathrm{t}^{2}}=0
$$

Equation 1-16

Solving the same equations for magnetic flux (B) provides a similar form:

$$
\nabla^{2} \mathrm{~B}-\varepsilon_{0} \mu_{0} \frac{\partial^{2} \mathrm{~B}}{\partial \mathrm{t}^{2}}=0
$$

Equation 1-17 
Equation 1-16 and 1-17 are vector equations. Using Equation 1-16, the electric field can be expressed in the following form:

$$
\frac{\partial^{2} \mathrm{E}_{\mathrm{i}}}{\partial \mathrm{t}^{2}}=\frac{1}{\varepsilon_{0} \mu_{0}}\left(\frac{\partial^{2} \mathrm{E}_{\mathrm{i}}}{\partial \mathrm{x}^{2}}+\frac{\partial^{2} \mathrm{E}_{\mathrm{i}}}{\partial \mathrm{y}^{2}}+\frac{\partial^{2} \mathrm{E}_{\mathrm{i}}}{\partial \mathrm{z}^{2}}\right)
$$

Equation 1-18

This equation is valid for all components of the field, where $i$ refers to $x, y$, and z. Fourier decomposition of Helmholtz's equation is presented as a plane wave with the following form [85]:

$$
\overrightarrow{\mathrm{E}}=\operatorname{Re}\left[\overrightarrow{\mathrm{E}_{0}} \mathrm{e}^{\mathrm{i}(\overrightarrow{\mathrm{k}} \cdot \overrightarrow{\mathrm{r}}-\omega \mathrm{t})}\right]
$$

\section{Equation 1-19}

where $\overrightarrow{E_{0}}$ is the polarization vector for the electric field, and $\vec{k}$ is the wavevector that shows the direction in which the wave is moving. The electric field vector for any plane wave is constantly orthogonal to the direction of propagation given by $\vec{k}$, i.e., $\overrightarrow{\mathrm{E}_{0}} \cdot \overrightarrow{\mathrm{k}}=0$. Furthermore, for a plane electromagnetic wave, the wavevector for both the magnetic and electric fields is [86, 85]:

$$
\vec{k}=\overrightarrow{k_{x}} i+\overrightarrow{k_{y}} j+\overrightarrow{k_{z}} k
$$

\section{Equation 1-20}


The magnitude of the wavevector is the wavenumber, which can be derived using Maxwell's equations in the following form [87, 88]:

$$
\mathrm{k}_{0} \cdot \mathrm{k}_{0}=\mathrm{k}_{\mathrm{x}}^{2}+\mathrm{k}_{\mathrm{y}}^{2}+\mathrm{k}_{\mathrm{z}}^{2}=\frac{\omega^{2}}{\mathrm{c}^{2}}
$$

\section{Equation 1-21}

where $\omega$ is the angular frequency, and $c$ is the speed of light.

A plane wave propagating at a planar interface can be either evanescent or propagating. As mentioned previously, the decaying nature of evanescent waves results in imperfect images, as the information that evanescent waves carry are usually lost before reaching the detector. This information has a high spatial frequency, or large k vectors, and contributes to resolving the small details of an object. Considering a wave propagating along the $\mathrm{z}$ direction, the plane wave equation with an evanescent $k_{z}$ can be written as [86]:

$$
A(r) e^{i\left(k_{x} X+k_{y} Y-\omega t\right)}=\operatorname{Re}\left[E_{0} e^{ \pm i\left(k_{x} x+k_{y} y\right)-i \omega t}\right] e^{\mp\left(\sqrt{k_{0}^{2}-k_{x}^{2}-k_{y}^{2}}\right) z} .
$$

\section{Equation 1-22}

And a plane wave equation with a propagating $k_{z}$ component is given by [86]:

$$
A(r) e^{i\left(k_{x} X+k_{y} Y-\omega t\right)}=\operatorname{Re}\left[E_{0} e^{ \pm i\left(k_{x} x+k_{y} y\right)-i \omega t}\right] e^{\mp\left|k_{z}\right| z} e^{ \pm i\left(\sqrt{k_{x}^{2}+k_{y}^{2}-k_{0}^{2}}\right) z}
$$

Equation 1-23 
The time dependent part of the wave is usually shown with a complex term, $e^{-i \omega t}$, containing the wave frequency, $\omega . \mathrm{A}(\mathrm{r})$ is either $\mathrm{E}(\mathrm{r})$ or $\mathrm{B}(\mathrm{r}) . k_{x}$, $k_{y}$ and $k_{z}$ are the components of the wavevector in the corresponding directions, showing the direction of propagation, with the positive $(+)$ sign indicating propagation along the $\mathrm{k}$ direction, while a negative $(-)$ sign indicates propagation in a direction opposite to that of $\mathrm{k}$.

In the following section, we provide a brief introduction to plasmonics, which is needed to better understand the superlens performance.

\subsection{Plasmonics}

Despite the recent surge of interest, the realization of surface plasmon resonances on metallic interfaces has been sought after for the last 150 years [89]. Surface plasmons are useful for many fields of science where propagation and manipulation of light plays a critical role. One of these fields is high resolution imaging. During recent years, the better utilization of plasmonic properties and the synergy of the fields of metrology and microfabrication have paved the way to attain subwavelength imaging.

The electrical properties of semiconductor materials enable nanoscale computation and information storage, however the speed in its performance is limited by interconnect delay-time. Photonics, on the other hand, is limited by the fundamental laws of diffraction [90, 91]. Plasmonics merges the high bandwidth offered by photonics with the nanoscale integration offered by nanoscale electronics via coupling a photon's energy with the free-electron gas, 
resulting in a subwavelength oscillating mode, known as a plasmon [92]. This pretext provides the reasoning for seeking to develop plasmonic devices.

\subsubsection{Basic Properties of Surface Plasmons}

Materials that conventionally dominated the field of plasmonics are noble metals [93]. At a plane interface between noble metals and a dielectric (e.g. air), quantized oscillations of conductive electrons can interact with light. These enhanced interactions occur at the interface of metals with high levels of free electrons, such as noble metals. These interactions allow field enhancement in areas close to the surface, enabling surface plasmons (collective longitudinal oscillations of electrons, abbreviated SP) to be excited. Waves created by plasmonic effects are bound to the metal surface, and exponentially decay in both directions away from the interface [94]. Therefore, in this context, the metal plays a more important role than the surrounding dielectric medium, given its electrical and optical characteristics which arise from its electronic properties.

Noble metals exhibit strong absorption of electromagnetic waves with frequencies within the visible and ultraviolet regions, where free electrons can flow through the noble metal by electron transitions due to available vacancies [95].

Initially, plasmonic applications were developed in the visible range, where a well-known example for an early implementation of plasmonics is the roman Lycurgus Cup, dating back to the $4^{\text {th }}$ century [96]. This cup changes colour to red or green when it is illuminated from the inside or outside, respectively. This effect is attributed to plasmonic resonances within the visible spectrum, and it was suggested that silver nanoparticles are responsible for the green colour while gold nanoparticles are responsible for the red. This can be explained by the fact 
that changing the size of nanoparticles affects their resonance frequency [97]. Currently, plasmonic materials cover an expanded range of the electromagnetic spectrum, targeting various applications [98, 99, 100, 101].

It should be noted that plasmonic materials in their bulk form cannot be used for plasmonic applications, as free electrons exist near the surface. Therefore, dimensions of these materials have to be restricted in a given direction to allow plasmonic interactions to occur [102]. Examples include nanoparticles, thin films and nanostructured surfaces.

In 1908, Mie explained the notion of surface plasmon resonance (SPR) using a solution of Maxwell's equation for spherical particles interacting with plane electromagnetic waves [103]. This work covered the interaction of light with gold colloids having sizes smaller than or close to that of light's wavelength. These interactions included absorption, scattering, polarization and the colour change of the particles [104]. However, with the lack of proper tools, studying the optical properties of metal nanoparticles was not a topic of interest even 50 years after the publication of Mie's work [105]. Currently, Mie's paper has been cited more than 5000 times, as plasmonic effects have found applications in chemistry, biology, optics and electronics.

Having materials with low optical losses is generally desirable in plasmonic applications [106]. Dispersion is another important aspect when considering such materials, as the refractive index of dispersive materials changes with the wavelength, which is the case for most dielectric materials [107]. A material's dispersion is directly related to its dielectric loss [108].

Surface plasmon polaritons are electromagnetic excitations that propagate at the interface between a metal and a dielectric with decaying nature in both interfaces [109]. The dispersion relation of surface plasmon polaritons is shown 
in Figure 1-2, where it can be seen that SPPs always lie outside the propagating light cone, which is the area inside the blue solid curve [110,97]. To circumvent this problem, there are several available techniques to excite the SP waves, such as prism coupling and grating coupling. For instance, metallic nanogratings act as a coupling medium to excite surface plasmons. The interference of SPPs at the output side of these nanogratings can redistribute the illuminated light into subwavelength scaled regions along the metallic surface, with strong intensity that results in electric field enhancement $[111,94]$.

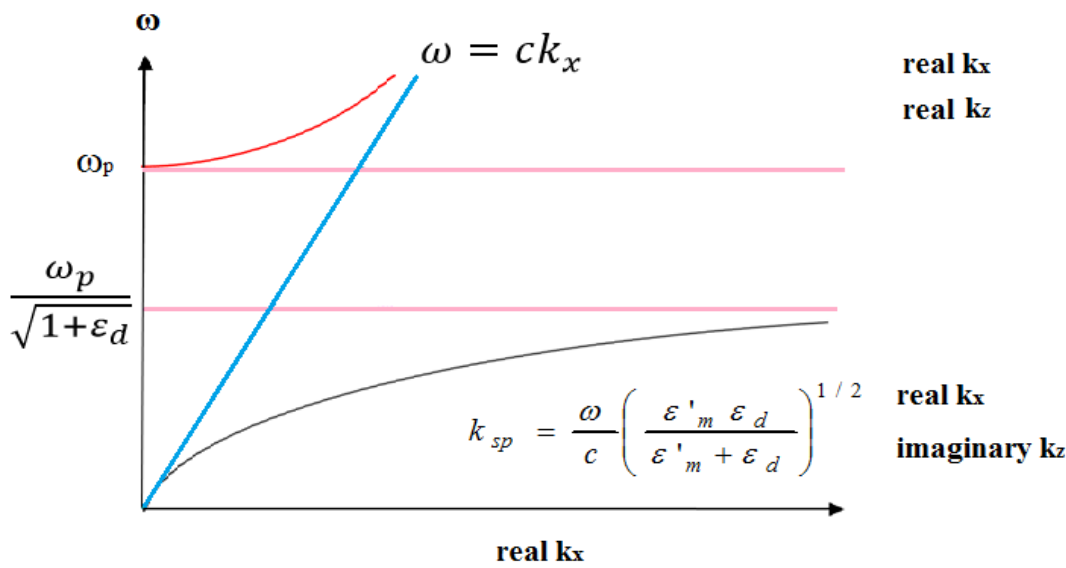

Figure 1-2 Dispersion relation for bulk plasmons (red curve), free-space electromagnetic waves (blue line), and surface plasmons (black curve).

The requirement of a coupling medium, such as a prism or nanograting to excite surface plasmon can be explained by describing the surface plasmon dispersion relation since not all light wavelengths are suitable to excited surface plasmons independently. Dispersion relation shows the relation between wavevector and angular frequency. As shown in Figure 1-2, the dispersion relation of surface plasmon resonance (black solid curve) and the dispersion 
relation of normal light (blue solid line) intersect only at the origin. For simplicity, by choosing a wavevector with two components in the $\mathrm{x}$ and $\mathrm{z}$ directions the problem can be defined as two dimensional. Given that the real part of the wavevector in the $z$-direction exceeds that of free light $(\omega=\mathrm{ck})$, a coupling mechanism is required.

To have propagating bound SPs, the wavevector component must be real along the interface $\left(k_{x}\right)$ and evanescent in the vertical direction $\left(k_{z}\right)$. In the presence of a transverse magnetic (TM, also commonly referred to as $\mathrm{P}$ ) polarized light, the SPs exist along a metal-dielectric interface $[112,97]$.

The complex reflection coefficient $\left(\mathrm{r}_{\mathrm{p}}\right)$ for a TM polarized wave at the metaldielectric interface is given by [102]:

$$
r_{p}=\frac{E_{i}}{E_{r}}=\left|r_{p}\right| e^{i \varphi}=\left|\frac{\tan (\alpha-\beta)}{\tan (\alpha+\beta)}\right| e^{i \varphi}
$$

\section{Equation 1-24}

where $E_{i}$ and $E_{r}$ are the incident $(i)$ and reflected $(r)$ electric fields. The angle $\alpha$ is the incident angle and $\beta$ is the transmitted angle from the Snell's law, as shown in Figure 1-3. $\varphi$ is the phase angle for the reflected light with respect to the incident light. 


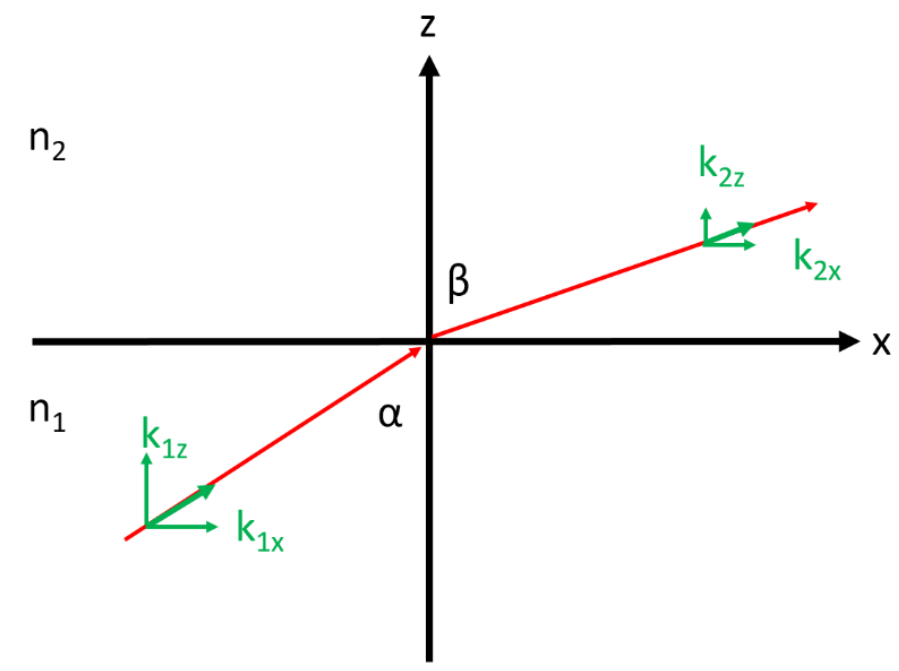

Figure 1-3 Refraction of light propagating from medium 1 to medium 2

Consequently, reflectance at the metal-dielectric interface $\left(R_{p}\right)$ is given by:

$$
\mathrm{R}_{\mathrm{p}}=\left|\mathrm{r}_{\mathrm{p}}\right|^{2}
$$

Equation 1-25

There are two special cases that follow from Equation 1-24; the first is when $\alpha+\beta=\pi / 2$ which satisfies the condition for the Brewster angle when reflection is zero $\left(R_{p}=0\right)$ for the TM polarized light, the other case is when $\alpha-\beta=\pi / 2$ leading to an infinite reflectance.

This latter case indicates that the electric field component of the reflected wave is much larger than that of the incident wave, i.e., resonance occurs. As $\alpha$ and $\beta$ are complementary angles:

$$
\cos (\alpha)=-\sin (\beta)
$$

\section{Equation 1-26}


Snell's law can be rewritten as:

$$
\tan (\alpha)=-\frac{n_{2}}{n_{1}}=\frac{k_{1 x}}{k_{1 z}}
$$

\section{Equation 1-27}

Where $n_{1}$ and $n_{2}$ are the refractive indices of the metal and dielectric medium, respectively, while $\tan (\alpha)$ is expressed in terms of the wavevectors in the incident region (region 1 ). Note that we assumed that light is propagating in the $x-z$ plane, which doesn't affect the generality of this solution.

Given the resonance condition, the dispersion relation for the two-dimensional wavevector $k_{1}=\left(k_{1 x}, k_{1 z}\right)$ can be calculated according to the following:

$$
\mathrm{k}_{1}^{2}=\mathrm{k}_{1 \mathrm{x}}^{2}+\mathrm{k}_{1 \mathrm{z}}^{2}
$$

\section{Equation 1-28}

Rearranging:

$$
\Rightarrow \mathrm{k}_{1 \mathrm{x}}^{2}=\mathrm{k}_{1}^{2}-\mathrm{k}_{1 \mathrm{z}}^{2}
$$

\section{Equation 1-29}

Substituting Equation 1-27 in Equation 1-29 gives:

$$
\mathrm{k}_{1 \mathrm{x}}^{2}=\mathrm{k}_{1}^{2}-\frac{\mathrm{n}_{1}^{2}}{\mathrm{n}_{2}^{2}} \mathrm{k}_{1 \mathrm{x}}^{2}
$$

\section{Equation 1-30}


Considering that the wavevector equation in medium 1 (metal) is $\overrightarrow{k_{1}}=\varepsilon_{1} \frac{\vec{\omega}}{c}$, and given that the relation between the refractive index and the dielectric permittivity for a non-magnetic medium is $n^{2}=\varepsilon$, we can substitute in Equation 1-30 to yield the SPR dispersion relations:

$$
k_{1 x}=\frac{\omega}{c} \sqrt{\frac{\varepsilon_{1} \varepsilon_{2}}{\varepsilon_{1}+\varepsilon_{2}}}=k_{x}
$$

\section{Equation 1-31}

$$
k_{i z}=\frac{\omega}{c} \sqrt{\frac{\varepsilon_{i}}{\varepsilon_{1}+\varepsilon_{2}}}, i=1 \& 2
$$

\section{Equation 1-32}

Considering the part of the electromagnetic spectrum below the plasma frequency of the metal, the dielectric permittivity of the metallic and dielectric media should hold the following relations to allow SPRs to exist at their interface $[94,97]:$

$$
\operatorname{Re}\left(\varepsilon_{\text {metal }}\right)<\mathbf{0} \text { and }\left|\varepsilon_{\text {metal }}\right|>\varepsilon_{\text {dielectric }}
$$

\section{Equation 1-33}

In this case, an electromagnetic wave propagates along the interface with evanescent side-lobes extending into both media. If the wavevector components parallel to the interface $\left(\mathrm{k}_{\mathrm{x}}\right)$ are semi-directional at the interface, then the conditions for excitations of plasmonic interactions are satisfied [113]. Consequently, the normal components of the wavevector across the interface are 
imaginary and have opposite directions. This indicates wave decay in both media, above and below the interface $[114,115]$.

\subsection{Diffraction Gratings}

Gratings are carefully arranged periodic features. They have numerous applications in optics and are prevalent in many optoelectronic and plasmonic devices [116, 117]. Different types of gratings are used for diffraction, reflection, transmission, and phase-control applications. A typical optical grating has a structure composed of ordered corrugations on a substrate and interacts with light having a frequency comparable to its dimensions. Gratings are most efficient if their design is symmetrical and periodic [118]. Gratings are said to be continuous when the base is constructed from the same material as the periodic features, and discontinuous if the base material is different.

Diffraction gratings diffract light after it passes through the small spacings in between the periodic features of the grating. Consequently, light is split into different directions. The resulting effect is analogous to that of a prism, which separates a beam of light to its composing frequencies.

A diffraction grating that is illuminated with an electromagnetic wave introduces different orders of diffractions denoted by $\mathrm{m}$ at the grating output. The number of available diffraction orders is in direct relation with the period of the grating and the incident light's wavelength [119]. The diffraction orders are integer numbers, with zero being the order without any diffraction. Figure 1-4 (a) shows the 0 and \pm 1 diffraction orders generated based on the localization of different beams from all openings of the grating at one point. Figure 1-4 (b) shows a different sketch that illustrates the definition of diffraction orders based on the 
propagation of the wavefronts, as well as the direction of the wavevectors at each opening. These figures give a clear idea of how diffraction orders are created in a diffraction grating. The zero-order gratings, in which the nanograting period $(\Lambda)$ is too small compared to the incident light wavelength, $\Lambda \ll \lambda$, are the gratings that only produce the zero order, $\mathrm{m}=0$. In this case, diffraction is non-existent, and the effect of the grating structure is studied according to the laws of reflection and refraction. Diffraction gratings can be designed to only generate certain orders of diffraction with respect to the illuminating wavelength, and have useful applications in sensing, filtering, imaging [120, 121, 122]. 


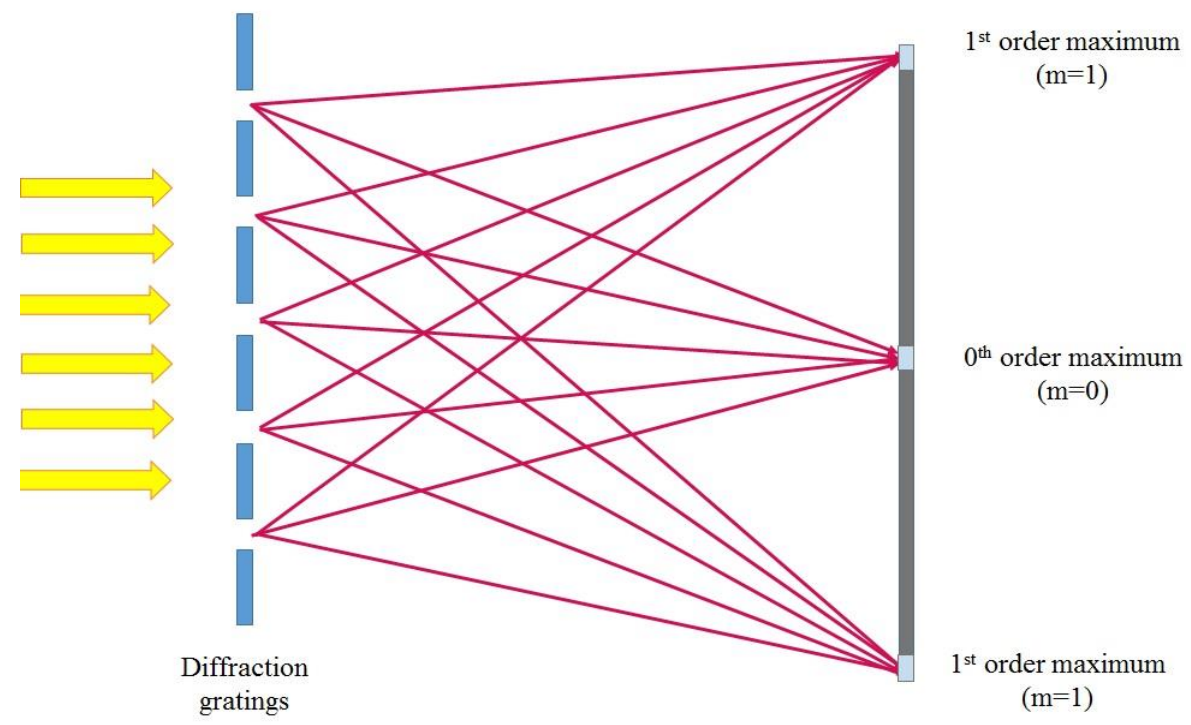

(a)

$\lambda \downarrow$

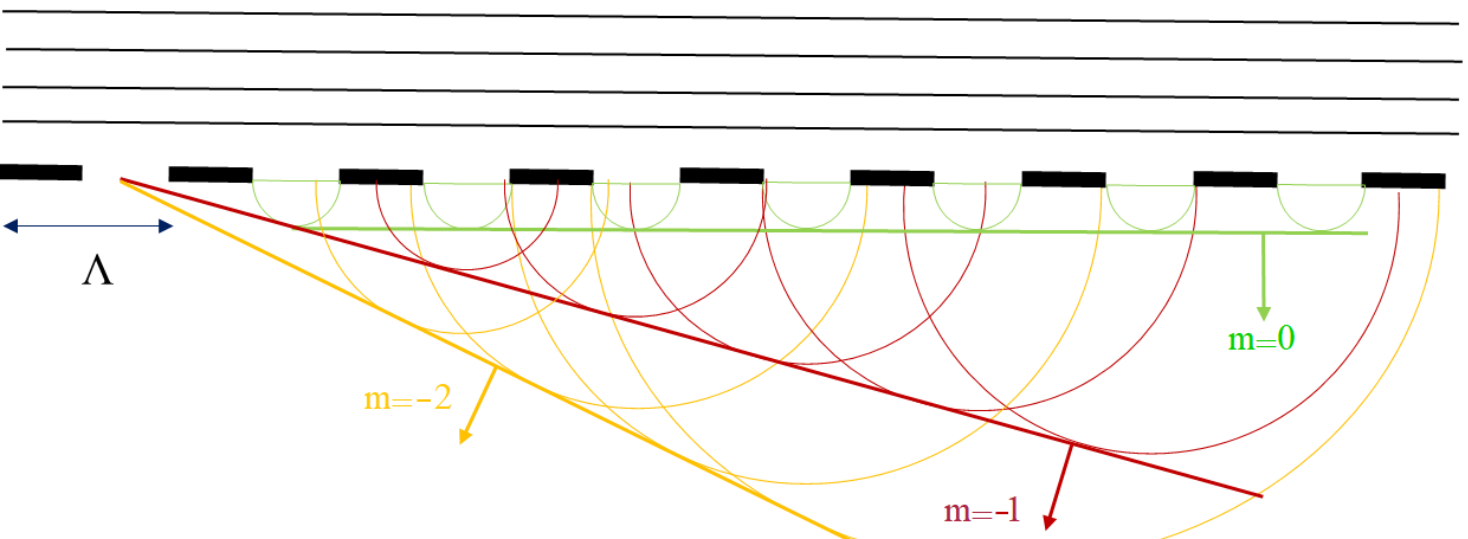

(b)

Figure 1-4 Diffraction gratings and the generated orders of diffraction shown in two different configurations.

A classic single ray diffraction on a plane surface results in a transmitted and reflected ray [118]. For monochromatic light illumination, each spacing acts as a light source which diffracts light into discrete directions that depend on the light 
wavelength [123]. Diffraction gratings can be reflective or transmissive, but the most important function of these gratings is the creation of diffraction orders which appears in both types.

The interaction of a monochromatic light with grating features that are comparable to its wavelength results in light bending as it passes through the small openings or near the grating's edges. This results in diffraction. According to Huygens-Fresnel principle, each point on the wavefront is perceived as a source point, accordingly, the traveling wavefront generates new wavefronts propagating in every direction. These wavefronts interfere with one another, resulting in a diffraction pattern [124].

As previously mentioned, diffraction gratings are a common type of coupling medium for plasmonic applications. By careful design, a grating structure can maximize the interaction of a light beam having a given angular frequency, so that the incident beam and the resulting wavevector match the frequency of a medium's plasmonic excitation, hence surface plasmon polaritons are generated.

Given the popular scenario where a monochromatic light is impinging on a metal grating surrounded by a dielectric medium, the grating equation that provides the relation between the grating period, light wavelength and different orders of diffraction is written as follows [125]:

$$
k_{s p p}=\frac{\omega}{c} \sin (\theta) \pm \frac{2 \pi l}{\Lambda}=\frac{\omega}{c} \sqrt{\frac{\varepsilon_{m}^{\prime} \varepsilon_{d}}{\varepsilon_{m}^{\prime}+\varepsilon_{d}}}
$$

Equation 1-34 
where, $\Lambda$ is the metal nanograting period, $\omega$ is the angular frequency of the incident light wave, $\mathrm{c}$ is the speed of light in vacuum, $\theta$ is the angle between the incident light and the device normal, and 1 is an integer number. The dielectric permittivity is shown as $\varepsilon_{d}$, while a metal's dielectric permittivity is complex, given by $\varepsilon_{m}=\varepsilon_{m}^{\prime}+i \varepsilon_{m}^{\prime \prime} ; \varepsilon_{m}^{\prime}$ is the real part of the dielectric permittivity and $\varepsilon_{m}^{\prime \prime}$ is the imaginary part of it. As a result, the wavevector is also complex.

When light is propagating along a grating interface, SPP damping is determined by the imaginary part of the parallel component of the wavevector [126]. Therefore, to maximize a grating's plasmonic behaviour, the selected metal should have a large negative real part and a small imaginary part for its dielectric permittivity. Silver satisfies these conditions within the visible frequency range. Therefore, a high field confinement at the interface will be possible while losses are kept to a minimum. When first order plasmonic excitations occur, the left hand side of Equation 1-34 matches the wavevector of the excited SPP ( $\left.\mathrm{k}_{\mathrm{spp}}\right)$, which is equivalent of the incident radiation and $1^{\text {th }}$ diffracted order interaction with the wavevector of SP at the interface.

In the next sections of this chapter, we will introduce the theory of operation of near-field and far-field -superlenses.

\subsection{Near-field superlens}

The theoretical concept of a superlens was proposed by V. G. Veselago in 1968 [62]. In his paper, Veselago explained how a beam of light can be super-focused through a left-handed metamaterial slab without aberrations, however, he couldn't find any naturally occurring material with these properties. It was in year 1999 when Pendry realized that the electromagnetic properties of a material 
can be varied using small scaled structures on its surface [127, 128]. This theory predicted the generation of properties that do not normally occur in nature. For instance, metamaterial structures would provide infinite spatial resolution if their refractive index, dielectric permittivity and magnetic permeability are all equal to -1 , i.e., $n=\varepsilon=\mu=-1$ [129]. Typical refraction paths of light in a metamaterial, which has a negative refractive index, and a normal material with a positive refractive index are shown in Figure 1-5. Thus, energy flow direction in a negative index metamaterial is opposite to that of the wavevector [130].

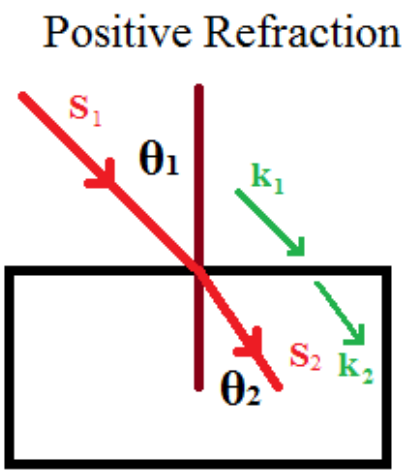

(a)
Negative Refraction

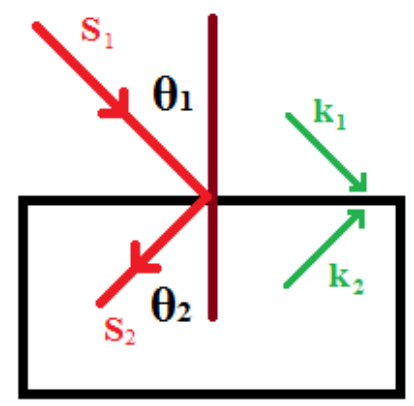

(b)

Figure 1-5 Refraction of light in positive and negative index materials, $S$ being the Poynting vector while $\mathrm{k}$ is the wave vector [131].

An imaging device designed using a negative index metamaterial is able to amplify the evanescent waves and beat the diffraction limit. Pendry termed such a device a "perfect lens" [1], where an image is formed by both propagating wave and evanescent waves.

Figure 1-6 illustrates the performance differences between a conventional glass lens (right-handed material) with a perfect lens (left-handed material). 
While the glass lens has a resolution dictated by the diffraction limit, the perfect lens is able to recover information about the subwavelength features of an object in the generated image. Diverging beams passing through a slab with negative refractive index can theoretically converge back to a point to achieve perfect imaging. This characteristic is known as the double focusing ability of the lens. Passing through the perfect lens slab, light undergoes two bending stages, then converges for the second time [132], as shown in Figure 1-6.

Conventional lens

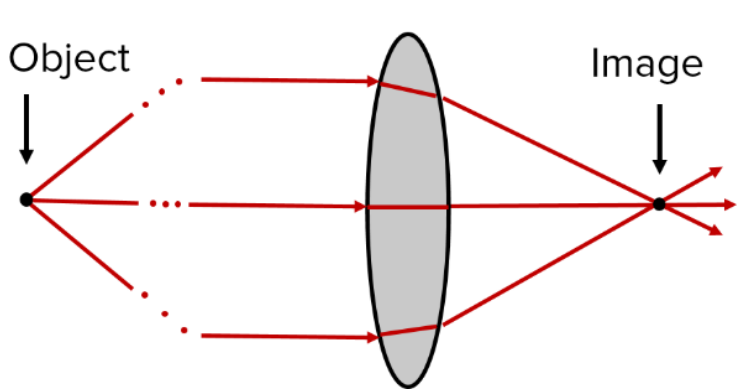

Perfect lens

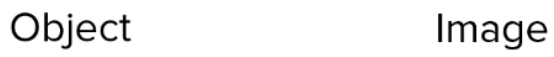

Figure 1-6 Schematic presentation of focusing through (a) a glass lens and (b) Pendry's perfect lens.

In search of a naturally occurring material as an alternative to metamaterials, noble metals were suggested as they partly fulfilled design conditions given their high free carriers [1]. Therefore, the possibility to fabricate a perfect lens using naturally occurring materials was proposed, where such a lens was termed a superlens [127]. To form this theory about the suitability of noble metals, certain assumptions were made. The crucial condition to be fulfilled by such a material is having a negative permittivity in the region close to the operating wavelength [133]. However, considering the electrostatic condition in subwavelength dimensions, it is not necessary to have negative values for both the dielectric 
permittivity and the magnetic permeability, as the electric field and magnetic field components can be decoupled [134]. Under this circumstance, the electric and magnetic response of the material, can be decoupled [1,135]. Therefore, by using light with a TM polarization or a TE polarization, the electrostatic or the magnetostatic response governs, respectively; thus, having a negative dielectric permittivity value suffices. Given the electrostatic response assumption of the material, a TM-polarized light is required to illuminate subwavelength features [136], where this specific light polarization is one of the important conditions for exciting plasmon resonance [137].

Following from Equation 1-32, the impedance matching equation is given by [2]:

$$
\frac{k_{z 1}}{\varepsilon_{1}}+\frac{k_{z 2}}{\varepsilon_{2}}=0
$$

Equation 1-35

Based on the wavevector equations for plasmonic excitations, it is wellestablished that the existence of plasmonic excitations across an interface necessitates that the dielectric permittivity values of the two adjacent media meet the following relation:

$$
\left|\varepsilon_{m}\right|>\varepsilon_{d}
$$

Equation 1-36

This equation regarding the condition for exciting surface plasmons is in agreement with the assumed electrostatic condition; however, the range of $k_{x}$ 
values that could meet this excitation condition is very narrow. There is another permittivity relation for metal-dielectric interface that satisfies the excitation condition for surface plasmons while allowing a broader range of $k_{x}$ values to be coupled $[2,1]$ :

$$
\left|\varepsilon_{m}\right| \sim \varepsilon_{d}
$$

Equation 1-37

Meeting this condition is the main reason behind the superlensing effect of such structures. An example of a thin metal slab superlens is shown in Figure 1-7.
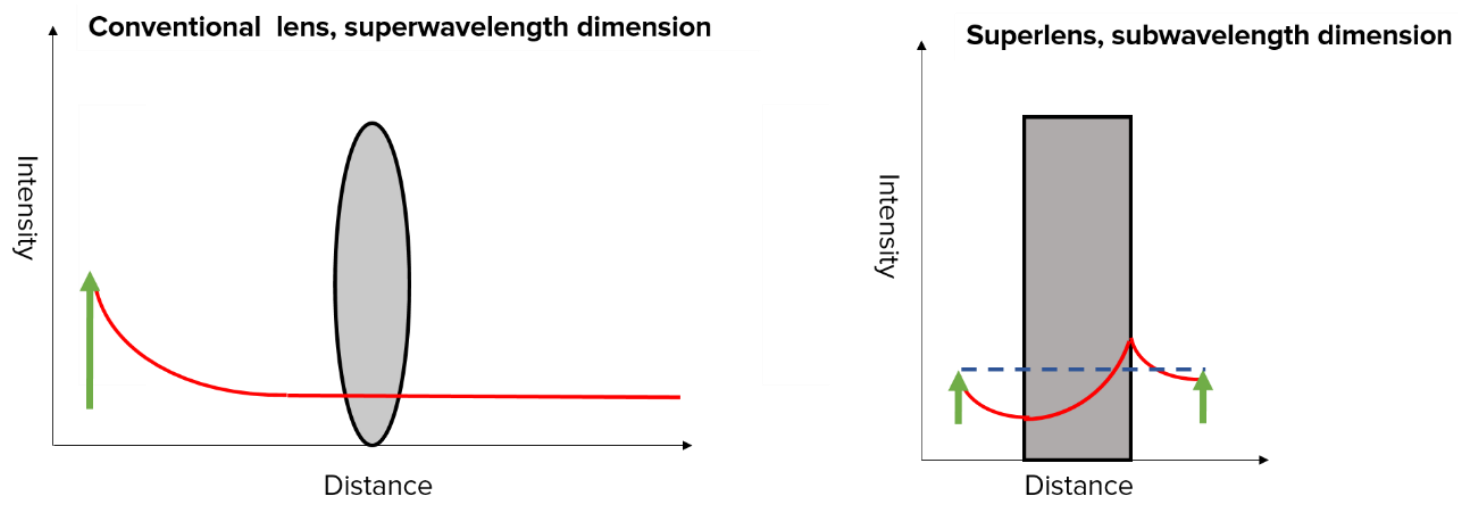

Figure 1-7 Amplitude of light at the output for (a) a glass lens, (b) a superlens where exponentially decaying evanescent modes are amplified.

Given the aforementioned conditions, silver is one of the best suggested materials for designing a superlens. In the visible optical region, silver meets all these conditions, hence the dependency on permeability is eliminated. As a result, the only influential factor is the dielectric function, and a silver superlens can recover more information from the near-field range to beat the resolution of conventional lenses [47]. 
There are several conditions that need to be satisfied in order for a silver superlens to perform properly.

First, the dielectric permittivity of silver is complex and depends on the illuminating wavelength. However, optical losses in such a material has a direct relationship with the imaginary part of the dielectric permittivity. Therefore, the range of proper wavelengths in which the optical loss or the imaginary part of the dielectric permittivity has a low value should be identified. Meanwhile, the real part of dielectric permittivity of the material must be negative in that range.

Second, there is a need to identify a neighbouring material that has the real part of dielectric permittivity close to that of the superlens material, i.e., dielectric matching. This matching will satisfy the conditions for SP band excitation [2].

Once the above requirements are satisfied, a smooth silver thin film can be used as a superlens. The operating wavelength range for a silver superlens is from the UV band to beginning of the visible range [74]. Other materials could potentially fulfil the requirements for operation in different ranges of electromagnetic spectrum, such as the microwave, infrared and optical frequencies $[138,2,139,140,141]$.

Following the emergence of the near-field superlens, the far-field superlens concept was proposed, with the purpose of enabling practical operation by carrying the high spatial frequency information to the far-field region.

\subsection{Far-field superlens}

A far-field superlens (FSL) is proposed to direct the high-resolution information produced by a near-field superlens to the far-field region through 
conversion of the evanescent waves into propagating waves, thereby projecting a perfect image. Accordingly, super-resolution optical imaging would not be confined to the near-field region of the lens [142].

The far-field superlens is a complex lens that builds on the principles of a nearfield superlens. Such a lens relies on the addition of a diffraction grating on top of the near-field superlens, where this diffraction grating is made of the same lefthand material of the near-field superlens. Typically, this left-handed material is a noble metal such as silver [142].

The production of high-resolution imaging in a FSL is possible due to coupling the evanescent waves from the object to surface excitations on the metallic thin film of the superlens. The strong field enhancement of such plasmonic excitations by the FSL grating enables them to propagate to the far-field, as a shift in their wavevectors towards the propagating region occurs. This wavevector shift is determined by size of the applied diffraction grating of the FSL [133, 142]. The grating size is defined according to the wavelength of the illuminating light, where the chosen subwavelength size ensures the enhancement of the plasmonic excitations at such a wavelength.

The higher spatial frequency wavevectors of the coupled evanescent waves are translated into lower spatial frequency wavevectors through the grating's diffraction orders [142], according to the following equation:

$$
k^{\prime}=k \pm \Lambda l,
$$

Equation 1-38 
where $k^{\prime}$ is the propagating wavevector, $k$ is the evanescent wavevector that contains the object information, $\Lambda$ is the diffraction grating wavevector, and $l$ is an integer number that corresponds to the diffraction order.

It is suggested that for a far-field superlens, only the 0 and \pm 1 orders are significant with regards to their contribution to image formation, while higher orders, such as \pm 2 only contribute to an improved definition of object edges [143]. Furthermore, higher orders generated by a grating are lossy, hence they are not significant for image reconstruction in a far-field superlens.

Next, we describe two visual effects that help explain the electromagnetic field interactions at the output of our far-field superlens, namely the Moiré and the Talbot effect. These effects are usually superimposed when illumination is applied at periodic structures like those of our FSL-object models [144].

\subsubsection{Moiré fringe pattern}

Once a far-field superlens is placed on top of a periodic object, Moiré fringe patterns are created. This effect arises due to the superimposition of the two periodic patterns with comparable dimensions. The theoretical formula that specifies the resulting period of a Moiré pattern is given by [145]:

$$
P_{M}=\frac{P_{A} P_{B}}{\left|P_{A}-P_{B}\right|}
$$

\section{Equation 1-39}

where $P_{A}$ and $P_{B}$ are the periods of the two superimposed patterns, and $P_{M}$ is the resulting Moiré period. The Moiré effect is observed in the simulated electromagnetic field patterns of our models in Chapter 3. 


\subsubsection{Talbot effect}

A second effect that is observed in our simulation results of the FSL's electromagnetic field is the Talbot effect. This effect is referred to as the selfimaging characteristic of periodic structures at fixed lengths [146]. When a coherent (plane) wave impinges on to structures with periodic geometry, the outcome is composed of hot spots that replicate a certain shape within fixed distances along the propagation direction after transmission through the periodic structures. This effect was first described by Henry Fox Talbot in 1836. Back then, light properties were not clearly understood [147]. In 1881, Lord Rayleigh found potential applications for the Talbot effect in various areas such as acoustics and optics [148]. The Talbot effect is commonly observed in conventional optics; however, its importance is recurrent in the emerging fields of optics [149].

Diffraction patterns generated at the output of diffraction grating can be periodic in one or two dimensions. Rayleigh believed that the Talbot effect is a consequence of the Fresnel diffraction arising from a grating as a function of propagating distance [149]. Therefore, he introduced a formula that estimates the period $\left(z_{T}\right)$ of a simple case Talbot effect:

$$
z_{T}=\frac{2 d^{2}}{\lambda}
$$

Equation 1-40

where $d$ is the period of the grating and $\lambda$ is the wavelength of the incident light. 


\subsection{Chapter summary}

In this chapter, limitations to the attainable resolution of conventional optical microscopes were discussed. Thereafter, a basic introduction of plasmonics was provided. Finally, the concepts of near-field and far-field superlenses were presented. In the next chapter, we will introduce the modelling requirements to develop our superlens design. 
2 Modelling method 

In this chapter, COMSOL Multiphysics ${ }^{\circledR}$, a common finite element modelling package is introduced. Modelling as a tool is used to inform the design and evaluate the performance of our superlens. Finally, the main settings and requirements of COMSOL Multiphysics® relevant to our study are discussed.

\subsection{Introduction}

Advances in nanophotonic and plasmonic devices have been accelerating during the last decades. This is associated with the substantial improvements of modelling and computational tools, experimental techniques and characterization methods [150]. Efficient and accurate modelling of complex plasmonic designs comprised of nanoscale features under electromagnetic illumination requires powerful and robust numerical modelling tools. Given the inherent shortcomings of analytical solutions, it is evident that developing a numerical model that accounts for realistic conditions and constraints is a challenging process [151]. In this chapter the modelling tool and computational requirements for designing our far-field superlens will be discussed.

Modelling electromagnetic problems usually requires solving fundamental physics equations in the time and space domains. These modelling techniques should be capable of providing accurate dynamic and steady-state information about the electromagnetic field interactions throughout the relevant modelling domain, from the near- to the far-field. Common modelling methods follow an iterative process to converge the approximated solutions of a model's partial 
differential equations, bringing them into acceptable agreement with real analytical solutions [152].

The two common methods for simulating electromagnetic waves in complex media are the finite difference time domain method (FDTD) and the finite element method (FEM).

FDTD is a simple method that relies on numerically solving the model's partial differential equations. In electromagnetic problems, this method solves for the central difference approximation to discretise the two Maxwell's curl equations, i.e., Faraday's and Ampere's equations, in both time and space domains. Then, it solves the resulting equations numerically to derive the electric and magnetic field distributions at each time step using an explicit leapfrog scheme [153].

On the other hand, the Finite element method constructs the solution to a problem using various discretizations and can improve and optimize the solution in real time [152]. Therefore, this method translates our physical structure into a mathematical model and solves it through a numerical procedure using the differential equations that describe the domain.

FDTD and FEM both use meshing to divide the domain into smaller sections, then carry solutions in each mesh element/cell $[154,155]$. However, the FEM is a more flexible given the ability to solve models with complex geometries and boundary conditions. Therefore, FEM requires a relatively longer computation time $[156,157]$.

In finite element or finite difference-based modelling, partial differential equations are solved on the points defined by the mesh distribution. High quality meshing in modelling enables accurate solution for the field components in each mesh element. However, the size of meshing elements should be carefully selected given that the whole computation domain needs to be covered with a 
mesh network. Thus, there is a direct relation between the accuracy of the numerical method, and the required computation time and cost, which rises in power law fashion with denser mesh coverage, and the complexity of physics defined in the study. Furthermore, efficient convergence of the computed solutions towards the real solution has a direct relation with the density, size and distribution of the mesh network in the modelling design. Failing to select proper meshing can result in incorrect solutions, while using denser meshing might exponentially increase computation time and resources.

Regardless of the selected numerical method for simulation, one fundamental requirement is to define suitable boundary conditions. Implementation of suitable boundary conditions can simplify the solution. Boundary conditions are usually responsible for refining the solution by removing unwanted effects arising from geometries, materials, and physical fields.

In this study, we have selected the finite element method for simulating the far-field superlens. The finite element method, first developed in 1940s by Richard Courant [158], is a mathematical and numerical technique widely used to analyse and model a broad range of physical problems. The current FEM approach has evolved from what was initially proposed to make use of the development of computational tools in the recent decades [159]. Nowadays, FEM modelling is an essential tool with applications in many fields of science and engineering such as aerospace, electromagnetism, mechanics, civil engineering, mining, chemistry, microfabrication, biomechanics and geology [160, 161, 162, $163]$.

Our design models in this thesis are either two dimensional (2D) or three dimensional (3D). In both cases, the computational domain is fully meshed, where mesh elements never overlap [164]. Driven by the requirements of this 
study, mesh elements can be fine/dense in one area, and coarse in other parts. However, a too coarse mesh network reduces the accuracy of the solution, and a too dense mesh requires high computation resources. Therefore, the user needs to be knowledgeable of the requirements and constraints of the model to optimize with respect to the underlying trade-offs. Each mesh element is surrounded by discrete point named nodes. These nodes connect different mesh elements. To solve the model, each mesh element hosts a specific set of governing equations, and any field variation in the modelling procedure is calculated at the corresponding nodes [165].

In this thesis, COMSOL Multiphysics ${ }^{\circledR}$ is the available FEM based modelling tool used to design and simulate our structure $[166,167]$. We use COMSOL Multiphysics ${ }^{\circledR}$ to simulate the transmitted field using the Wave Optics Module and the Radio Frequency (RF) Module, version 5.2-5.3.

\subsection{Meshing demands for modelling}

In this section, the meshing requirements for modelling in COMSOL Multiphysics ${ }^{\circledR}$ are described. As mentioned previously, improving the quality coverage of the meshing network results in optimization of the solution and fast convergence of accurate results. This is more important at the physical boundaries of the design components, where specific physics settings or boundary conditions are applied.

Throughout the modelling process, we defined the ideal mesh size based on the smallest features in our design and the operating wavelength. In the models presented in this study, most features were very close in size to the working 
wavelength, hence in order to observe the field interactions around the small features a fine and high density mesh network was required [168].

Generally, mesh grid network topology can be categorized into three groups: structured, unstructured (free) and block-structured (hybrid) [169]. For each problem, a specific topology or a combination of them can result in the arrangement of the meshing elements to conform the optimal shape and size that covers the geometry/domain. For instance, structured meshing is used in COMSOL Multiphysics® for isotropic geometries, while unstructured meshing is used for complex geometries with irregular shapes.

The structured mesh network can be applied for 1D, 2D, or 3D geometries to cover the simulation domain. Based on their shape, the structured mesh cells are categorized into groups of triangular, tetrahedral, quadrilateral, hexagonal, prism and pyramid mesh elements $[170,171,172]$. 1D meshing is suitable for the scenarios where mesh points are distributed along a line. In a 2D or 3D structured grid, cell edges are connected to the neighbouring cells, which stretch out to the domain boundaries. Structured meshing is suitable for simple scenarios as it offers a limited mesh-density control over the entire domain area. To satisfy the desired coverage of the geometry, the mesh network is composed of quadrilateral cells in a 2D structured grid, while coverage in 3D is achieved by using hexahedral cells. This offers simplicity, a good coverage, and acceptable resolution for a broad range of designs.

Unstructured grids, however, are usually defined using a triangular mesh in 2D, and a tetrahedral mesh in 3D. Unstructured meshing offers convenient adaptability for complicated geometries, with supple mesh density control [173]. Compared with other FEM modelling techniques, COMSOL Multiphysics® uses discretization polynomials and standard meshing elements to improve tetrahedral meshing efficiency of given geometries [174, 175, 176]. 
The hybrid mesh grid topology is versatile as it incorporates a variety of meshing geometries and is able to reasonably cater for the modelling constraints that require either structured or unstructured mesh topologies. By using a hybrid grid, mesh transitions between adjacent regions/groups in a domain can be smooth. This is applicable to any two boundaries or different parts of one object. A smooth mesh transition provides an easy union for the final compilation of the mesh grid from the different compartments [177].

COMSOL Multiphysics® allows broad customization of mesh settings. Thus, 2D and 3D designs can be divided into specific partitions, where different meshing styles/topologies can be defined based on the specific role of the partition domain in the model [178]. In most cases, the software's "automatic mesh" setting generates a mesh grid with a good coverage over the selected geometry/area.

Throughout this study, our approach was to define the minimal viable mesh structure that satisfies a given problem, then solve for that mesh to evaluate the quality of the resulting solution. Thereafter, progressive refinements were made before attempting to solve the final design. During the refinement process, meshing parameters, such as the maximum and minimum mesh sizes were optimized to achieve smooth wave solutions. Once the computation of the model with a coarser mesh and a reasonable run time gave solutions that are visibly comparable to those of a finer mesh setting, the efficiency and accuracy of the lighter mesh setting could be verified. 


\subsection{Simulation Requirements and Available Resources}

\subsubsection{Available Computational Resources}

Although, COMSOL Multiphysics ${ }^{\circledR}$ automatically chooses the most robust solver based on the physics settings of the model [179], generation of a solution and data assembly can demand vast computation resources. In some extensive 2D or 3D designs, our desktop computers were unable to process the computation and converge to a solution. Therefore, it was advised to use a high performing computation facility to solve these models. In our study, we used Victoria University of Wellington's high-performance computing facility (Sic-Fac HPC). This HPC facility of the Science faculty provides a 512 GB and a 1TB large memory nodes, along with further 34 processing nodes each having 64 GB of memory; yielding an effective 880 processing cores with a nominal distribution of 2.6 GB of memory per core. The facility is equipped with InfiniBand networking allowing for high throughput and low latency while servicing parallel computation programs [180]. A bash script is used to submit requests based on the estimated resources needed to solve the model. The Local Resource Manager then approves/rejects a given request, then queues the approved submitted jobs in a waiting list. It should be noted that this protocol for submitting jobs was often challenging given the unfamiliarity of the Local Resource Manager with the requirements of a FEM software like COMSOL Multiphysics ${ }^{\circledR}$, which often caused delays and required vast amounts of patience to resolve. 
Using the HPC facility provided access to more powerful computational resources thereby, requiring shorter times when solving our models.

\subsubsection{Customized mesh network}

After building the geometry in COMSOL Multiphysics ${ }^{\circledR}$, the components of geometry should be categorized to groups based on the design requirements. Accordingly, different mesh settings could be applied to each group. The predefined mesh settings include mesh size and shape associated with given geometry specifications. The meshing transition between different groups could be continuous or discontinuous based on the model requirements. Finally, different mesh groups are integrated to form the entire geometry [155].

It is important to specify the minimum mesh element size for each group of components in the design. In electromagnetic simulations, it is a rule of thumb to consider that the biggest mesh size should be smaller than $\lambda / 10$, where $\lambda$ is the wavelength of the utilized electromagnetic wave [181].

For a $1 \mathrm{D}$ structure, the mesh is composed of linear elements. The element displacements are given by:

$$
x_{i} \leq x \leq x_{i+1} ; \Delta x_{i} \equiv x_{i+1}-x_{i}
$$

Equation 2-1

where, $\Delta x_{i}$ is the displacement between two consecutive linear elements. Using COMSOL Multiphysics $®$, a simple line object with its mesh nodes are shown in Figure 2-1 [182]. 


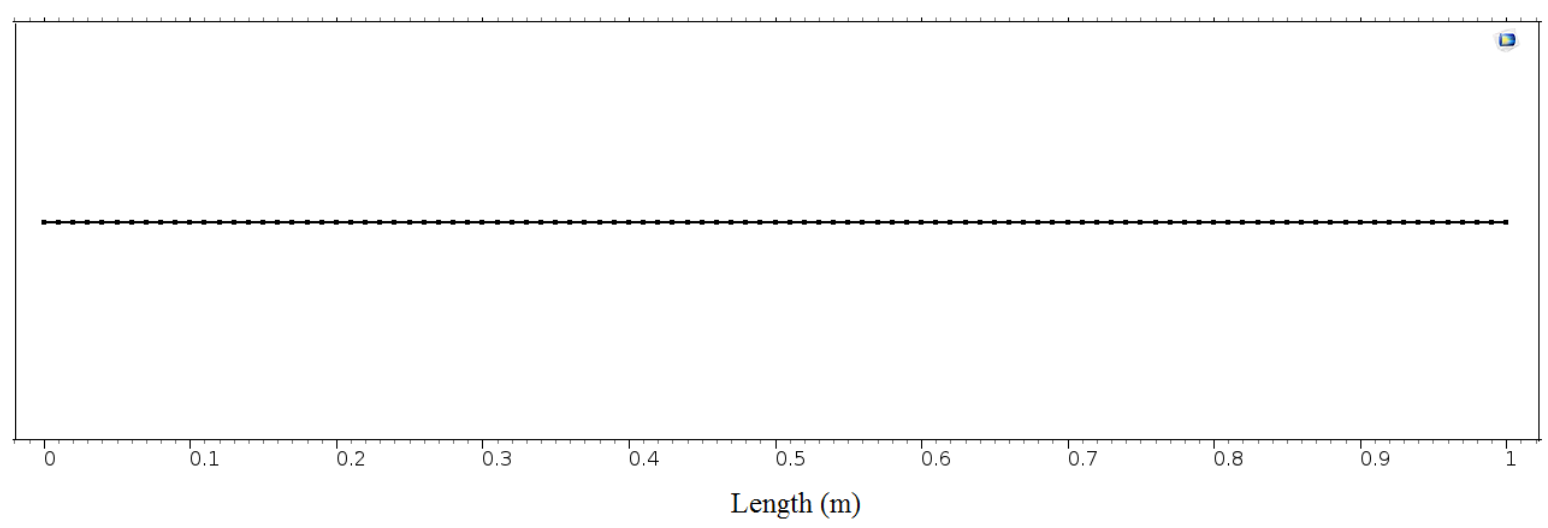

Figure 2-1 1D mesh distribution for a line object in COMSOL Multiphysics ${ }^{\circledR}$.

In case of a 2D simulation model, the entire domain is inside a polygon. In our 2D case, we used a rectangular plane, where all the geometry components are defined within. The default meshing process in COMSOL Multiphysics ${ }^{\circledR}$ is "physics-controlled meshing", which automatically adapts the mesh size and shape to fit the existing physical settings of a given model. The mesh size can be broadly tuned, ranging from extremely coarse to extremely fine. COMSOL Multiphysics ${ }^{\circledR}$ also allows a manual meshing process referred to as the "usercontrolled mesh". In this mode, the user defines the "primary size node" that contains the initial size constraints, which include minimum and maximum element sizes, element growth rate, the curvature factor, and the resolution of narrow regions. Extra "size nodes" can be added to different domains or boundaries, while size constraints are adjustable for each specific node. A selected sample of our 2D FSL design, which contains a free triangular mesh for the geometry, and a mapped mesh for the side boundaries is shown in Figure 2-2. Given the importance of accurately analysing light interactions near the nanoscale grating features, the mesh size constraint was set to small over this 
area. The mesh configuration was coarser when moving away from this area as a lower density mesh distribution was sufficient to yield accurate results for these areas.
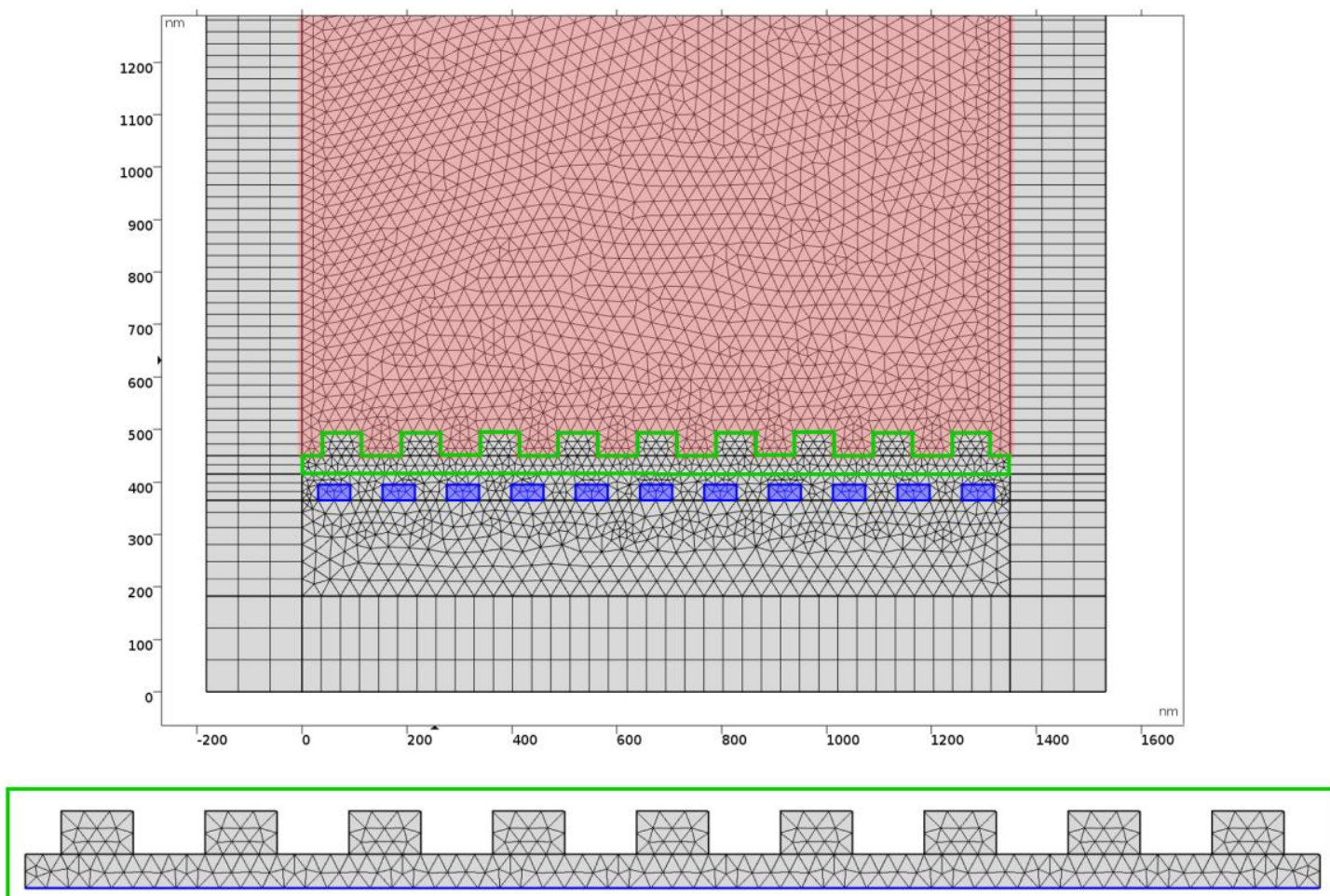

Figure 2-2 A 2D mesh distribution for a FSL design studied in this thesis using COMSOL Multiphysics ${ }^{\circledR}$.

In 3D simulations, the mesh design process is similar to that of 2D simulations. However, COMSOL Multiphysics® allows a wide range of mesh element geometries when designing the mesh network. In our case, we used free tetrahedral and free triangular mesh cells, which create an unstructured mesh grid. 3D simulations are usually resource intensive, given that the meshing and computation processes expand in three dimensions. 


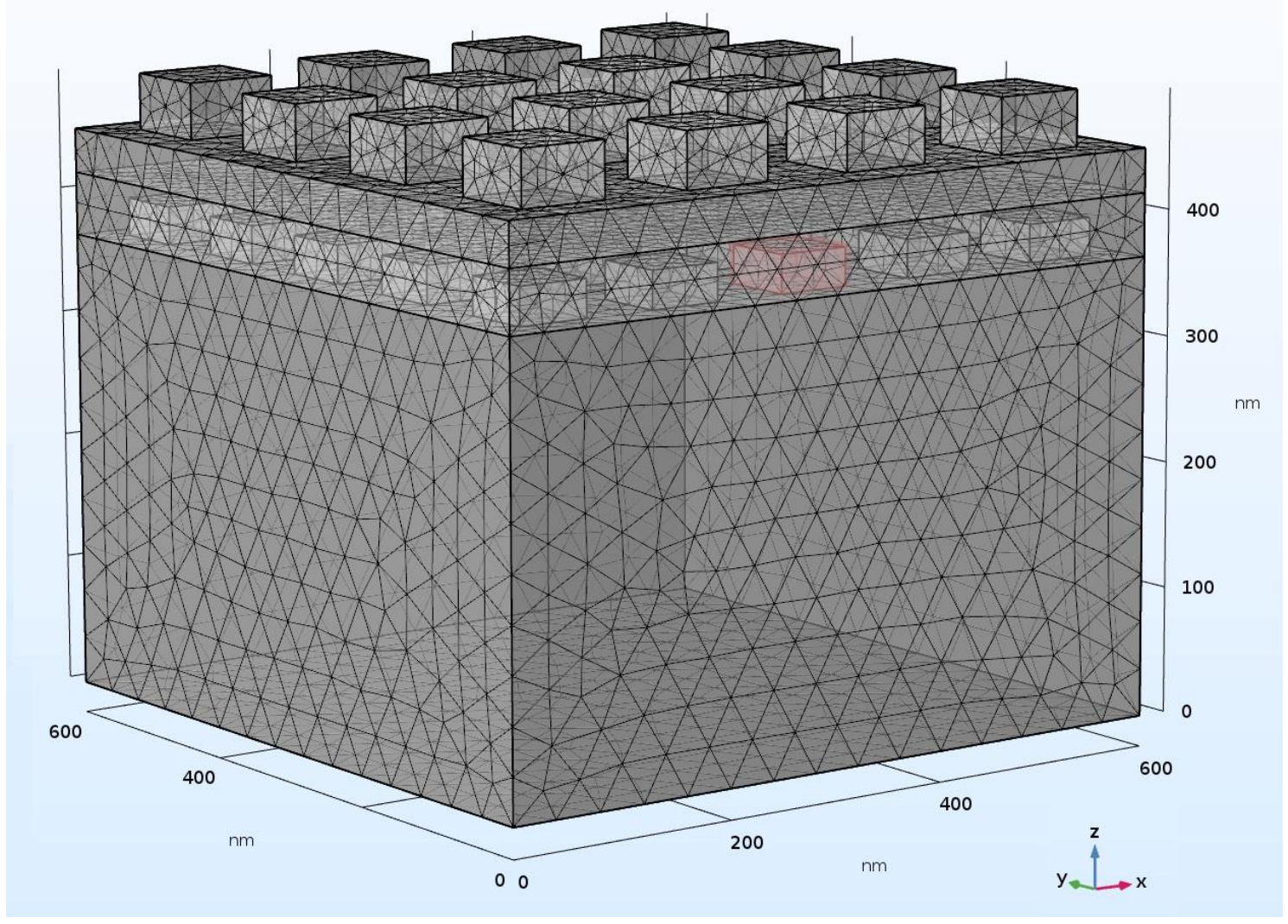

Figure 2-3 Schematics of a 3D mesh distribution based on a FSL design studied in this thesis. The part in red shows one of the objects.

Each mesh node in the computational domain can be assigned to one or more equations, which is defined based on the physical settings of the problem. A square matrix is then created based on the selected physics, material properties, boundary condition, element type, and element shape. Next, the solution is computed using a finite element approach. The number of degrees of freedom (DOF) defines the size of this solution matrix. Finer mesh results in a higher number of degrees of freedom. The DOF is a function of the number of elements, the discretization order used in each governing physics, and the number of 
variables solved for [183]. Therefore, the number of degrees of freedom is directly related to the memory and time requirements for solving a model [184].

\subsection{Modelling in COMSOL Multiphysics ${ }^{\circledR}$}

After designing the geometry and a suitable mesh coverage over the computation domain, the next steps to carry a simulation are:

- Materials and their properties need to be defined for each group in the domain. COMSOL Multiphysics ${ }^{\circledR}$ has a comprehensive material library where different data sets can be easily selected and applied [185]. In most cases, the magnetic and electric properties for each material are predefined in the material's property tables. However, any desired material can be manually added by the user. Given the constraints of the simulated model, only the relevant properties of materials need to be defined. Finally, each material is then manually assigned to the desired geometries/sections.

- The physics "setting nodes" should be added, where precise settings definition is the most important step when using COMSOL Multiphysics ${ }^{\circledR}$ modelling; however, specific physics modules can only be added if extra license keys are available [186]. The selection of a domain or a boundary for application of a specific physical boundary condition needs to be done using the relevant physical nodes [187].

- The "results node" and "post-processing nodes" could be added or modified both after and before a computation is completed [188].

- $\quad$ The model is computed. 
- The "post-processing nodes" are used to evaluate the model.

\subsection{Chapter summary}

In this chapter, our modelling software package of choice, COMSOL Multiphysics ${ }^{\circledR}$ was introduced. The main settings and requirements of COMSOL Multiphysics ${ }^{\circledR}$ relevant to our study were discussed. Additional properties of the specific physics involved in our design will be discussed in Chapter 3. 

3 Modelling and Design of a Far-

Field Superlens 

In this chapter, we discuss the results of our COMSOL Multiphysics ${ }^{\circledR}$ modelling study. State of the art for development of a far-field superlens before this research thesis was confined to identification of Moiré patterns using a CCD camera for a one dimensional rectangular object grating, with minor investigation in the far-field electromagnetic pattern produced from such a device $[189,142]$. Judging based on a review of the existing literature at the time, the author concluded that the literature was not comprehensive for practical operation of a FSL. Hence, several parameters such as different FSL nanograting shapes, object size, ideal domain size for memory use consideration and 3D structures were investigated. In Section 3.1 the design parameters of a 2D model are described. In Sections 3.2 and 3.4 modelling results for the designs in section 3.1 are presented. As part of this section, various object arrangements and geometries are considered, and their performance modelled. Furthermore, the shape of the nanograting as part of the FSL structure and its effects on performance are discussed. These results are then compared to that of a conventional near-field superlens.

Given that the required computation resources vary depending on the settings and requirements of our models, we studied -through 2D simulations- whether minimizing the domain size affects the performance of a given design. This provided the necessary input for designing our minimal cells in the 3D simulation section.

Section 3.5 includes the three-dimensional simulations of our FSL structure, with various arrangement of objects being considered. To the best of our knowledge, this is the first time where the three-dimensional performance of a 
FSL is investigated in the present form. 3D simulation allows us to evaluate the performance of various combinations of objects and FSL nanograting arrangements. We can also examine the effects of using two illuminating waves on the far-field patterns of a FSL.

Given that the electric field norm $|E|$ provides a good indication of power flow within our model [190], we have adopted this parameter to describe the amplitude plots throughout the analysis of our models, where wave intensity and energy are proportional to the square for the amplitude.

It is worth noting that to the best of author's knowledge, the parameters investigated in this chapter are all novel and studied for the first time with respect to the FSL design and performance.

\section{$3.12 \mathrm{D}$ model setup}

To model our structures in 2D, we designed the cross-section of our device in the $x-y$ plane. In this case, we assumed that all components extend to infinity in the perpendicular direction to the modelling plane.

To create our 2D model, we start by adding a base or a substrate. Next, a set of periodic objects is created on top of the substrate. As we only studied periodic objects in this thesis, all mentions of "object" are equivalent to "a periodic set of objects".

The next step is to add the FSL on top of the object. The FSL is composed of two parts. The first part is a metallic thin layer, i.e., the near-field superlens, and the second part is a series of parallel periodic subwavelength nanograting on top of the metallic thin layer. In our design, both parts are made of the same material. 
Given the nanometre scale of the setup, it was not realistic to assume that the FSL could immediately be placed on top of the object, therefore, we included a supporting spacer layer between them, as shown in Figure 3-1. The spacer layer's thickness was carefully selected to minimize optical losses through the structure, otherwise, the evanescent waves from the object would decay before interacting with the FSL. In a real experimental situation, adding this spacer layer is crucial to ensure practical handling of the nanoscale objects/FSL arrangement in ambient conditions. At this stage, the geometry design of our model is complete.

The next step is material selection as all parts in the modelling domain must be assigned a material. In our model, the substrate is made of borosilicate glass, the object is made of chromium, the FSL is made of silver and the spacer layer is made of poly (methyl methacrylate) (PMMA), which is a photosensitive material that reacts with deep UV and x-ray wavelengths. This active range is far from that of the operating wavelength in this simulation, thus does not affect the performance of our FSL [191, 192].

The following step is to set the excitation illumination in the form of an optical wave. In this study a transverse magnetic polarized light with a wavelength of $365 \mathrm{~nm}$ as the operating wavelength illuminates the whole setup from the bottom, and passes along the normal direction through the substrate and the object. We apply the illuminating optical wave through a port boundary condition adjacent to the lower face of the substrate. This required a "complementary port boundary condition" in the far-field region at the domain's top edge to receive the final field interaction.

The metallic thin film in a far-field superlens, independently known as a nearfield superlens, should be thin enough to interact with the evanescent waves before they decay. The material used to construct the superlens has a negative permittivity at the operating wavelength which is desirable for super-resolution 
imaging applications. Silver has this property in the UV and visible ranges [193]. This metallic layer in the FSL attenuates the small wavevector of the propagating waves while enhancing the large wavevector of the evanescent waves. This helps to improve the quality of the image by resolving more information related to the small features, which are carried by the evanescent waves. Addition of the nanograting as part of a far-field superlens provides the opportunity to transfer the near-field information to the far-field, as discussed in section 1.8. The electromagnetic interaction of the incident light with the object and the FSL creates a distinct electromagnetic field at the output.

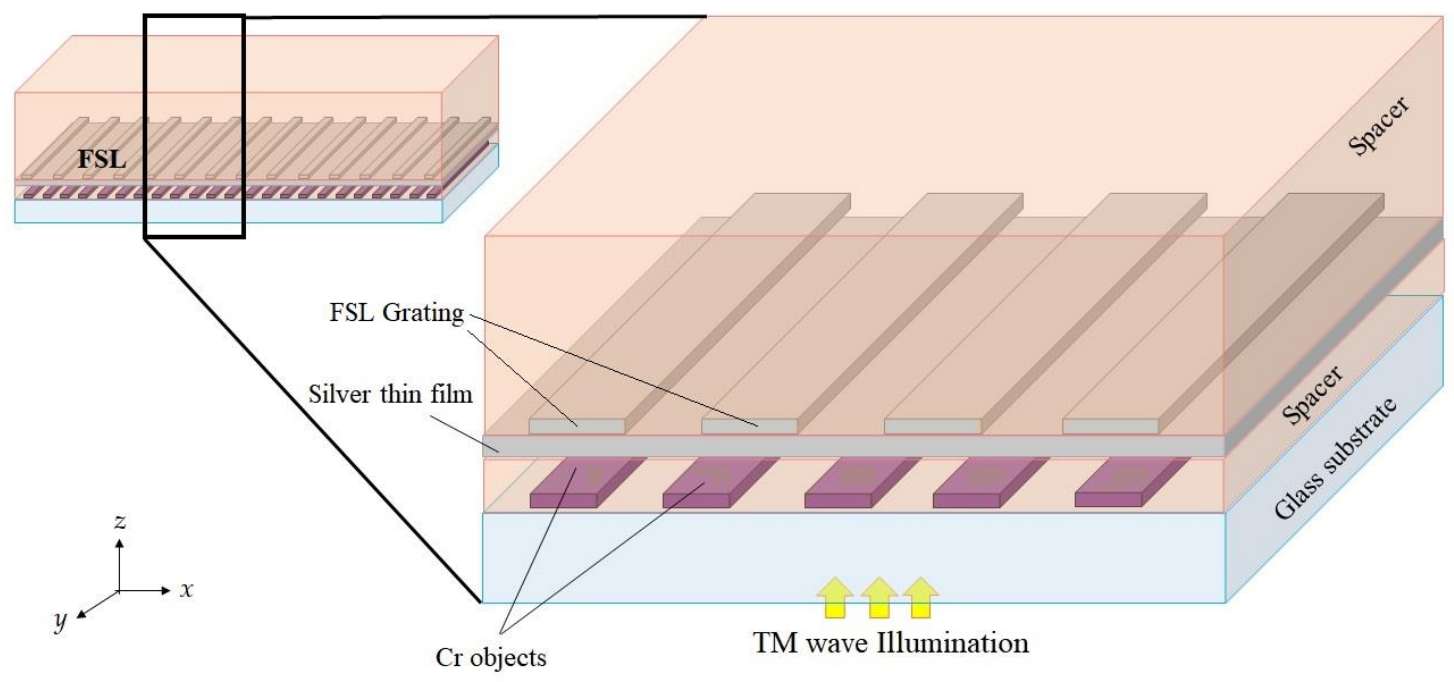

Figure 3-1 Schematic view of the far-field superlens under illumination. The object is shown just on top of the glass substrate.

Selecting a suitable surrounding medium in our FSL design is important because it needs to satisfy the permittivity (and index of refraction) matching (optical coupling) condition for adjacent media. The refractive index of this material should be approximately identical in magnitude and opposite in sign to that of the FSL material. In optical applications, it is essential to know the 
refractive index of adjacent materials if index matching constraints are to be satisfied [2]. Traditionally, the value of the refractive index for optical materials has been reported at the Sodium $D$ line, with a wavelength of $589 \mathrm{~nm}$ [194]. Given that refractive index is a wavelength dependent parameter, it is desirable to evaluate index matching for our design at the wavelength of interest, as the imaginary part of the refractive index (loss) might become too large.

PMMA is used in two places within our design as a structural material. The first being the spacer layer between the object and the FSL, while the second is a matching layer that infiltrates and covers the FSL nanograting. This satisfies the condition for permittivity matching, which is important in the superlens concept [195]. For dispersive materials, this condition is only satisfied within a small wavelength range. In the case of a FSL, the metal permittivity is set to be close to that of the surrounding medium's, i.e. , $\left|\varepsilon_{\mathrm{m}}\right| \approx \varepsilon_{\mathrm{d}}$ to satisfy impedance matching between them and to allow superlensing to exist [2], as mentioned in section 1.7. This condition dictates the superlens's operational wavelength [196]. Table 3-1 shows the list of materials used in our modelling and their corresponding refractive index and dielectric permittivity values. Other than silver and PMMA, all materials are assumed to have a null imaginary part, i.e., no optical loss. This loss is substantial for silver and should be considered. Therefore, the refractive index and dielectric permittivity values for our materials are presented at the operational wavelength, $\lambda=365 \mathrm{~nm}$ [197]. 
Table 3-1 The material properties used in our models throughout this simulation study.

\begin{tabular}{|c|c|c|c|c|}
\hline \multirow{2}{*}{ Material } & \multicolumn{2}{|c|}{ Refractive index } & \multicolumn{2}{c|}{ Dielectric Permittivity } \\
\cline { 2 - 5 } & Real & Imaginary & Real & Dielectric loss \\
\hline Air & 1 & 0 & 1 & 0 \\
\hline Silver (Ag) & 0.067 & 1.71 & -2.7 & 0.23 \\
\hline PMMA & 1.64 & 0 & 2.3 & 0.0014 \\
\hline Chromium & 3.05 & 0 & 9.3 & 0 \\
\hline Glass & 1.5 & 0 & 2.25 & 0 \\
\hline
\end{tabular}

The next step is to define the boundary conditions used in our model. In this study, we designed the object-FSL structure inside a perfectly matched layer (PML) boundary, which is a reflection-less boundary condition useful for shrinking the computational domain. For correct mesh distribution and results computation, the PML's boundary width should be larger than the operating wavelength. Using the PML helps to reduce the complexity of computation in our model and allows for simulating an unbound domain [198]. For the same reasons, periodic boundary conditions (PBC) were applied at both vertical boundaries of the modelling domain. The PBC is usually applied at both sides of a symmetrically arranged structure to provide an estimation of an infinite domain (an infinite horizontal domain in this case) [199]. Generally, having symmetry in the simulation model results in a high quality mesh coverage for the entire geometry and shorter computation time to solve the model.

Based on the settings described above, Figure 3-2 shows a typical model with the resulting field interactions using COMSOL Multiphysics®. The inset shows a close up of the area of the far-field superlens design with rectangular nanograting 
with a period of $\Lambda_{\mathrm{G}}=150 \mathrm{~nm}$, and the rectangular objects with a period of $\Lambda$ o $\approx 122.7 \mathrm{~nm}$.

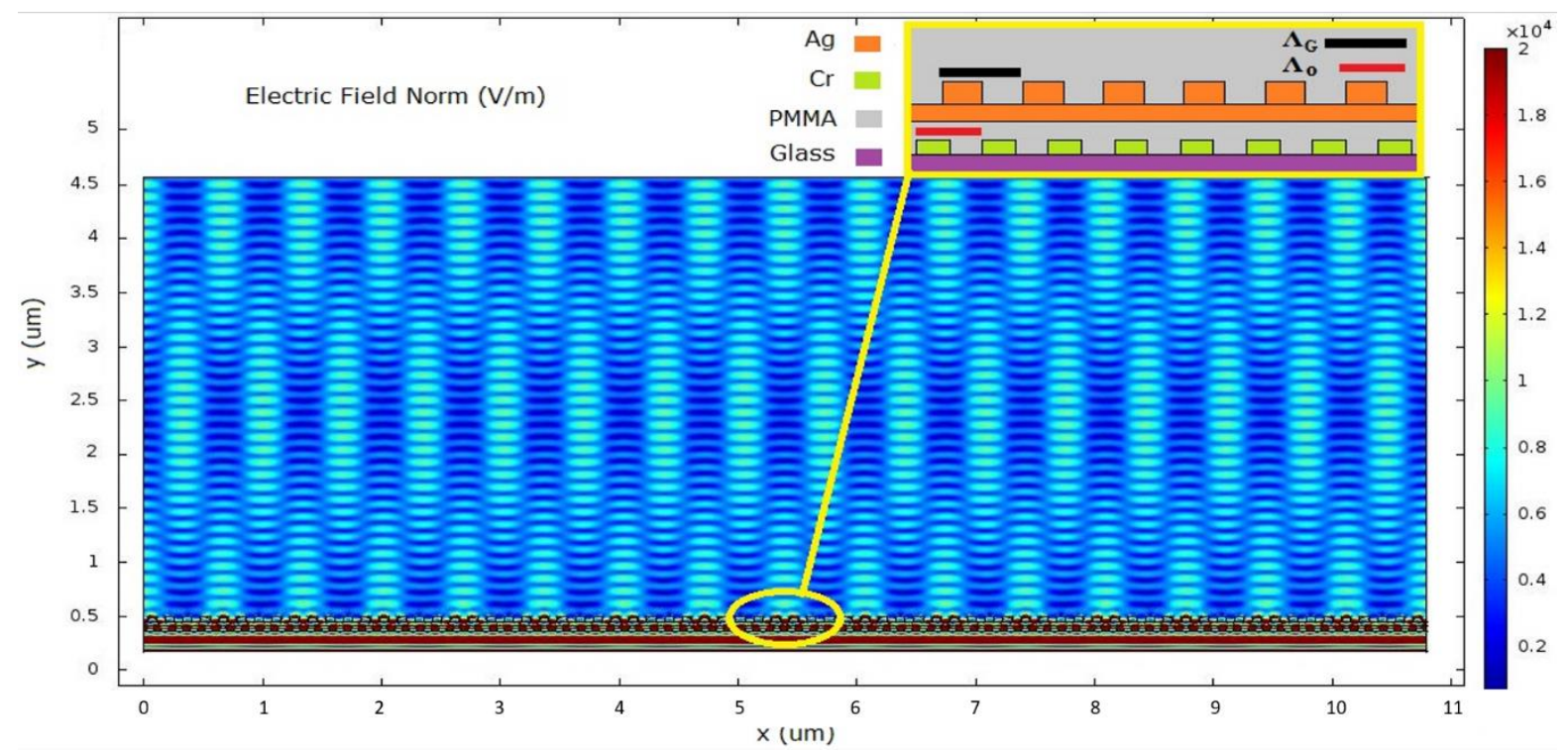

Figure 3-2 Far-field superlens design showing a far-field pattern over a $10,8 \mu \mathrm{m}$ wide domain with 88 object features and 72 FSL nanograting features.

This electromagnetic field pattern shown in Figure 3-2 is a typical recurrent result in our study. Analysing such patterns enabled us to compare the performance of different FSL designs and aided our optimization for the conditions in each setup.

Two effects are mainly responsible for production of these far-field patterns in our results, namely the Moiré effect and the Talbot effect, presented previously in sections 1.8.1 and 1.8.2, respectively.

The Moiré effect is a well-known phenomenon in optics and photography [200]. This effect explains the horizontal periodic pattern in the far-field. Based on the Moiré principle, when two sets of dots, lines, 2D grids [172] or any other periodic structures are superimposed within an illuminating field, a new pattern 
with a predictable size and period can be generated. The most common Moiré type is known as the linear Moiré pattern, which is created when two linear grating structures with different periods are superimposed. In our simulations, light passes through two sets of periodic components, i.e., objects and FSL's nanograting, and interacts with them. Constructive and destructive interference of light with these features creates Moiré patterns. The Moiré pattern size is proportional to the periods of the superimposed features [201]. Accordingly, the resulting electromagnetic field in our simulations should give us information about the size of the periodic object. Re-writing Equation 1-39 for our object and FSL gives the following formula for the Moiré pattern period $\left(\Lambda_{M}\right)$ [145]:

$$
\Lambda_{M}=\frac{\Lambda_{O} \Lambda_{G}}{\left|\Lambda_{O}-\Lambda_{G}\right|}
$$

\section{Equation 3-1}

where, $\Lambda_{\mathrm{o}}$ represents the period of the object and $\Lambda_{\mathrm{G}}$ is the period of the FSL nanograting. It is clear that the resultant Moiré pattern has a larger period than either of the superimposed structures. Considering the model presented in Figure 3-2, the calculated Moiré pattern from Equation 3-1 has a period of:

$$
\Lambda_{M}=\frac{122.7 \times 150}{|122.7-150|} \approx 675 \mathrm{~nm}
$$

The number of periods for the far-field Moiré pattern within the width of the domain of this simulation is given by the difference of the number of periods for the object and the FSL nanograting: 


$$
n_{M}=\left|n_{\text {periods of the object }}-n_{\text {periods of FSL }}\right|
$$

Equation 3-2

This agrees with the number of observed horizontal patterns in Figure 3-2:

$$
n_{M}=88-72=16
$$

The second periodic pattern observed in our models is along the vertical direction. This periodicity can be explained by the Talbot effect. The period for this electromagnetic field pattern is roughly estimated by [202, 203], as provided in section 1.8.2:

$$
Z_{T}=\frac{2 d^{2}}{\lambda}
$$

\section{Equation 3-3}

where $Z_{T}$ is the Talbot distance, $d$ is the horizontal period of a single pattern or the Moiré pattern period for superimposed periodic structures, and $\lambda$ is the wavelength of the illuminating light. The $Z_{T}$ value observed in our simulation in Figure 3-2 is close to the calculated $Z_{T} \approx 2497 \mathrm{~nm}$ with $\Lambda_{M}=675 \mathrm{~nm}$.

Another method that was used to compare the performance of different designs in this study is the magnitude transfer function, which we defined to provide an estimate of the amplitude of the far-field electromagnetic excitations 
with respect to the field amplitude at the object. The following steps are used to calculate the magnitude transfer function for our models:

- The object cutline is selected to be on the top edge of the object as shown in Figure 3-3. For instance, if the object height is not changed in different models, the object cutline stays at a fixed location.

- The image cutline may be placed at different vertical positions based on the Talbot length observed in the model. This is where the maximum amplitude of the far-field pattern is observed, which varies with the Talbot length in each design.

- The magnitude transfer function is given by the ratio of the corresponding amplitude peak in the Fourier transform of the electric field norm $|E|$ at the image cutline to that at the object cutline.

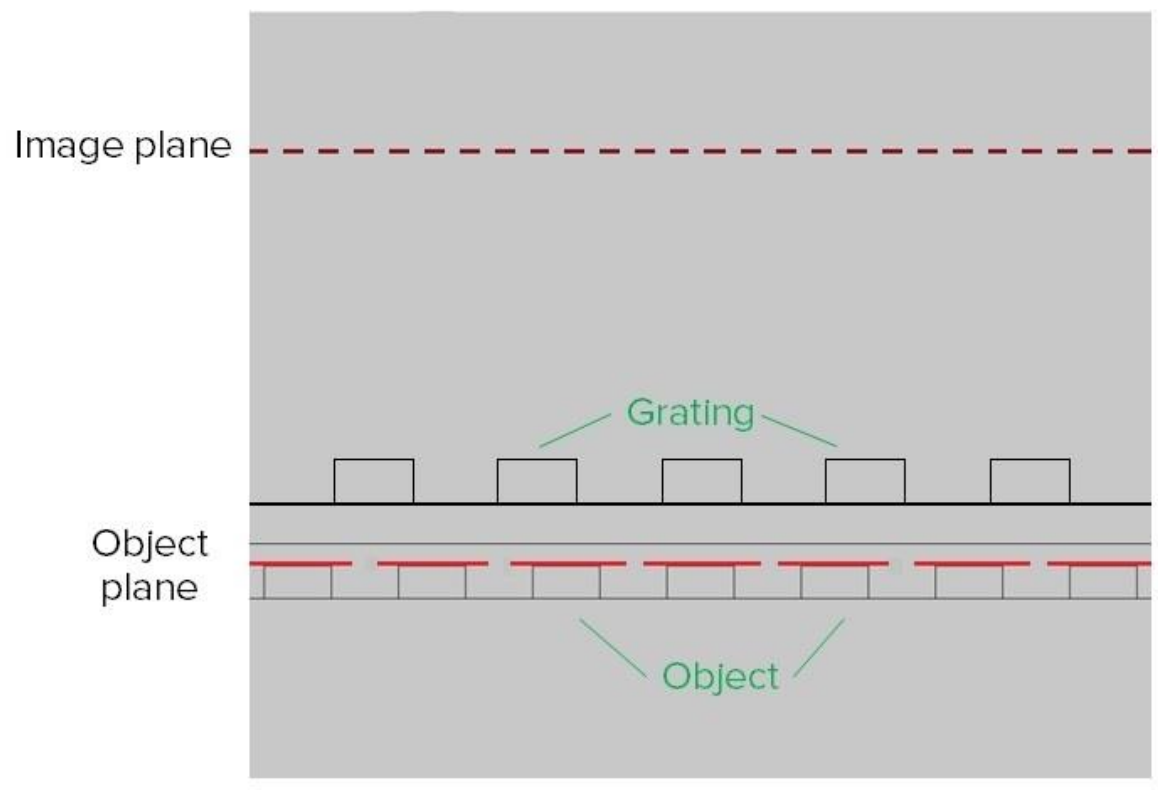

Figure 3-3 Schematic showing the object and the image cutlines. 


\subsection{Near-field vs. Far-field superlens}

In this section, we compare the performance of a far-field superlens with that of a near-field superlens using COMSOL Multiphysics®.

To illustrate this, we considered the scenario of having two identical simulation domains, geometries and settings, with the exception of having an added nanograting structure for the far-field superlens.

The electromagnetic field above a near-field superlens is shown in Figure 3-4(a), with a close-up on the lens structure in Figure 3-4(c). It is evident by these figures that the near-field superlens only operates in the near-field region [204], an outcome that is attributed to the decaying nature of the evanescent coupled plasmonic excitations emanating from the superlens structure.

On the other hand, Figure 3-4(b) shows the electromagnetic field for the farfield superlens. Comparing these two models indicates that replacing a near-field superlens with a far-field superlens expands the working area of the imaging device to the far-field. Given the geometric constraints, electromagnetic interactions that describe the object in the case of a near-field superlens are not easy to resolve [205]. The established method to capture the subwavelength features of the object using a near-field superlens is by including a photoresist layer directly on top of the superlens and analyse the resulting exposure pattern arising from the near-field [206]. On the other hand, a FSL creates electromagnetic patterns at the far-field region, where these patterns describe the properties of both the FSL and the objects. 


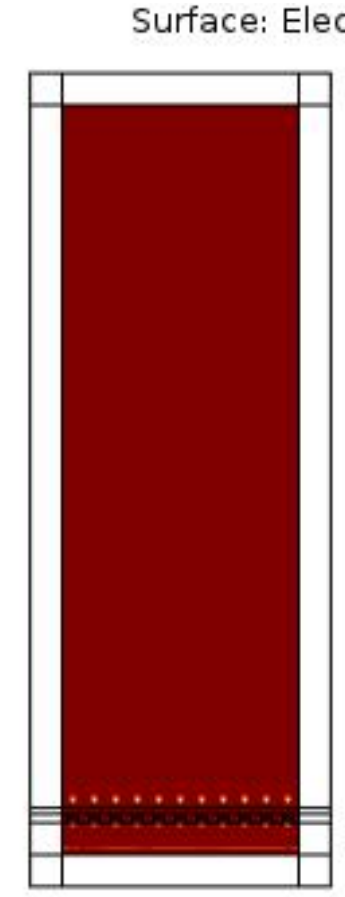

(a)

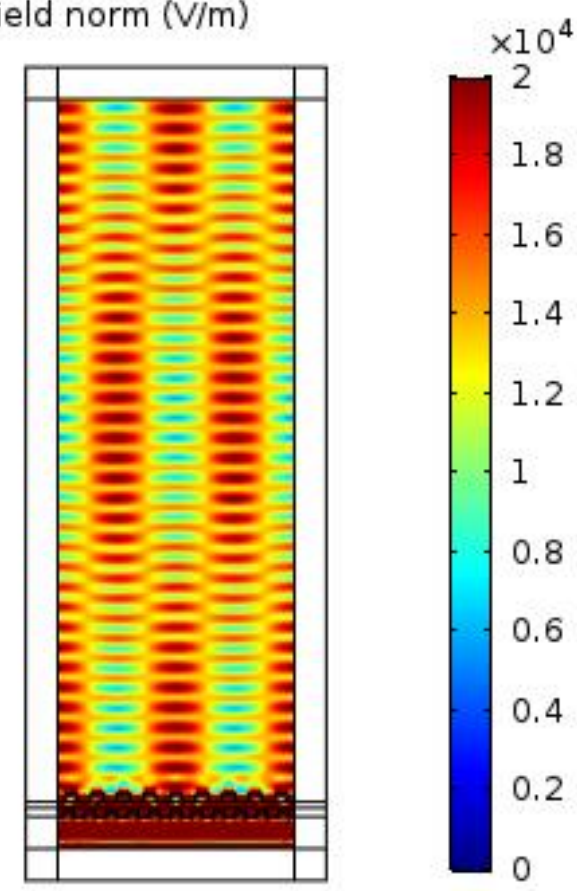

(b)

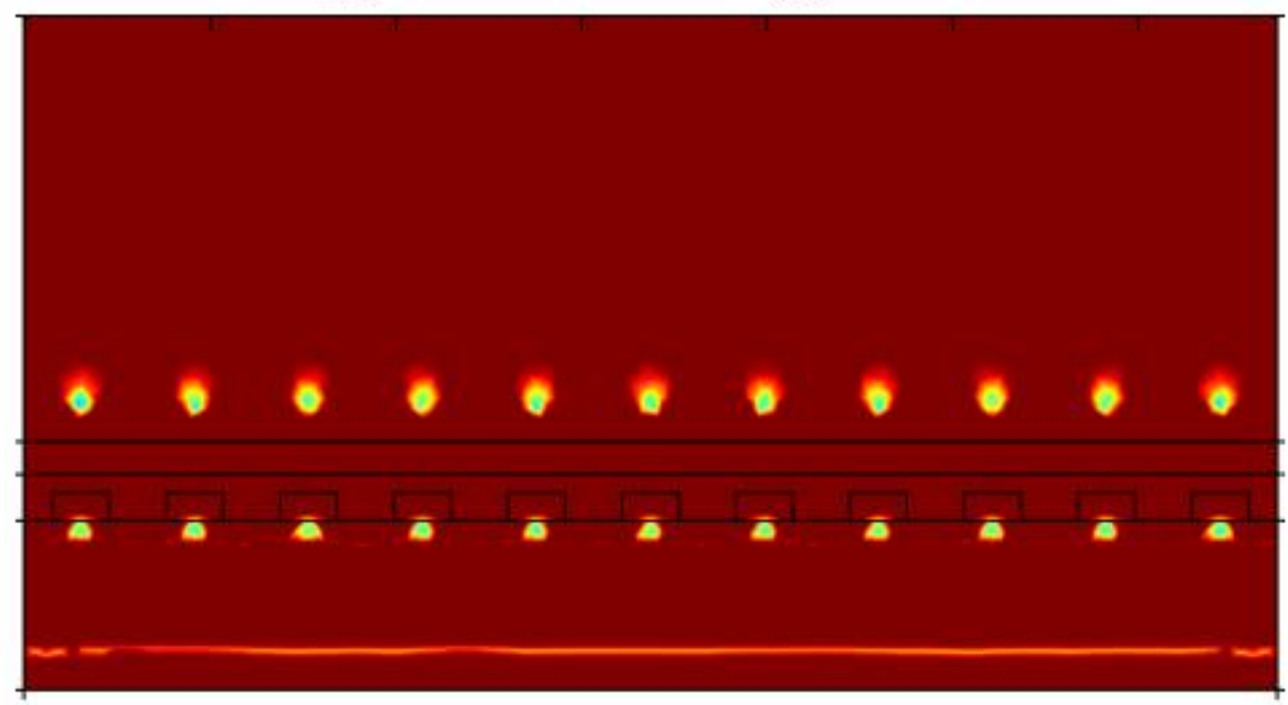

(c)

Figure 3-4 Electric field norm plots and the colour bar for (a) a near-field superlens, (b) a far-field superlens, (c) A close-up of the near-field region of (a). 
This example confirmed the expected difference in operation between a nearfield superlens and a FSL. The next sections of this chapter provide a detailed study regarding the performance of the far-field superlens.

\subsection{EM wave amplitude in a FSL}

In this section, we describe a study that aimed to evaluate the effect of domain length on the wave amplitude in the far-field of a FSL. Thus, the FSL design and model settings were kept similar for all four considered cases, where the only difference was the length of the far-field region. The horizontal width of the domains is solely dictated by the size constraint of a symmetric model to ensure the creation of a Moiré pattern. In this case, the domain width was set to $7800 \mathrm{~nm}$, where the object period was $120 \mathrm{~nm}$ and the FSL nanograting period was $150 \mathrm{~nm}$.

With regards to domain length variations, Figure 3-5(a) shows the first example where the domain length was set to $3630 \mathrm{~nm}$. In this example, one and a half vertical fringes are created within the far-field length. Deducing the height of all components immediately below the FSL nanograting, the peak amplitude of the first periodic pattern in the EM field (Talbot length/distance) occurs at the $1960 \mathrm{~nm}$ cutline from the top of the FSL nanograting. This location is shown with a red line in Figure 3-5(a).

In Figure 3-5(b), (c) and (d), as the length of the domain grows, more fringes appear in the far-field region. Similar to Figure 3-5(a), the first Talbot length occurs at a similar far-field distance for (b), (c) and (d). The second Talbot cutline is shown using a black line. In cases $(b)$ to $(d)$, the second Talbot pattern is similar to the first Talbot pattern with a horizontal shift equalling half of the Moiré period [207]. As the colour bar in Figure 3-5 suggests, the periodic pattern is 
repeated with almost the same amplitude for all four models, confirming the Talbot pattern characteristics and the low propagation loss in the selected farfield medium.

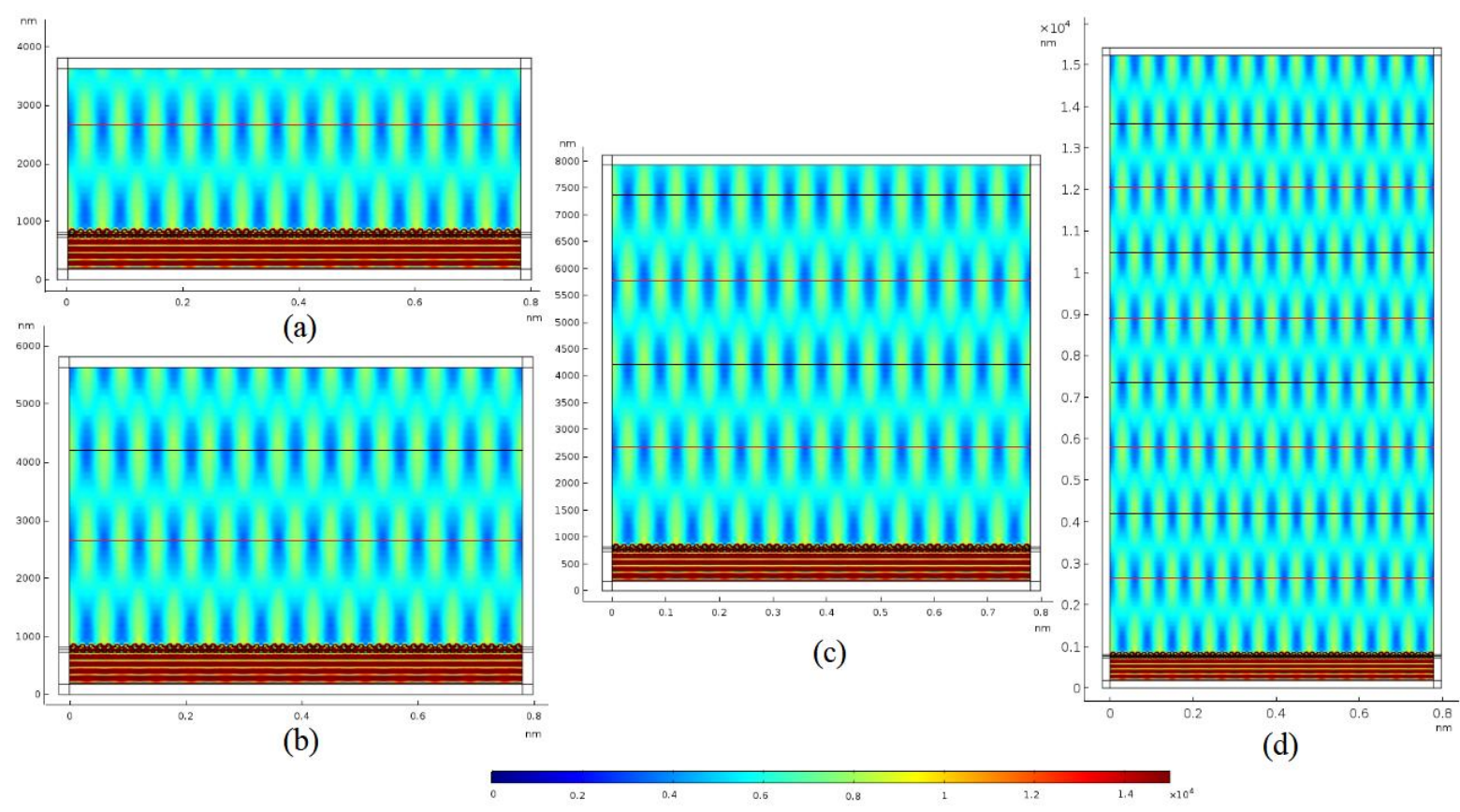

Figure 3-5 Electric field norm of four models with identical object and FSL but different domain sizes. The domain lengths are (a) $3630 \mathrm{~nm}$, (b) 5630 $\mathrm{nm}$, (c) $7930 \mathrm{~nm}$ and (d) $15230 \mathrm{~nm}$. The colour bar is identical for all plots. Red cutlines show the odd Talbot orders while the black cutlines show the even Talbot orders.

Figure 3-6 compares the magnitude of the electric field norm for odd and even orders of the Talbot length, which are represented with parallel red and black cutlines in Figure 3-5, respectively. 

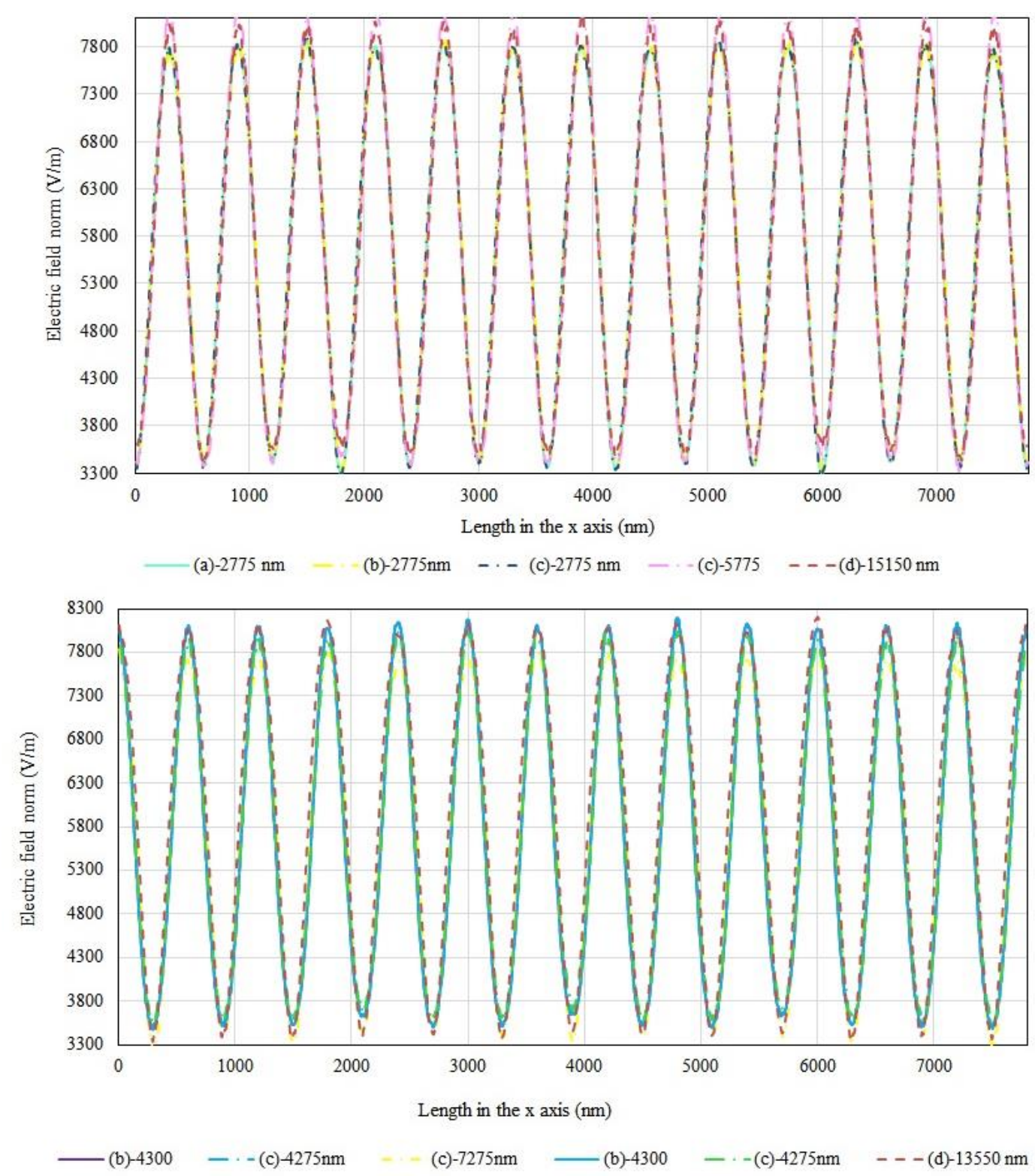

Figure 3-6 The magnitude of the electric field norm at Talbot cutlines placed at different lengths in Figure 3-5.

The plots shown in Figure 3-6 confirm that the electromagnetic pattern is reproducible in the far-field domain in a periodic manner. Given the implementation of the PML boundary condition, we were able to model a realistic scenario where the intensity of the field was preserved as light propagated to the far-field with minimal reflection losses at the boundaries [208]. 
Furthermore, judging by our modelling results, we confirm that models with the shortest and longest domain lengths produce identical results, thus, it is prudent to choose the shortest possible domain size to save on computation time and resources in our future models.

A main focus in this section was to investigate the performance of the proposed FSL design and observe its far-field electromagnetic interactions. Observing the Moiré and Talbot effects in our simulations confirmed that using a far-field superlens allows the light waves to propagate to the far-field.

\subsection{The effects of structures shape and size on the EM field pattern}

In this section, we evaluate the effects of changing the size and shape of the object and FSL nanograting on the overall performance of our FSL. To keep things simple, we started the experiment by changing either the shape of the size of the features at a time. This allowed us to identify their individual effects on the performance of our FSL. However, as the experiment developed, more complex permutations were considered.

In a real-world scenario, controlling the shape and size of nano-structures in the micro- and nano-fabrication processes is challenging. For instance, heat, moisture, exposure profile, and other variables relating to photoresist development and thin film deposition makes attaining the desired shapes possible within limits. As a result, the final products could have curved corners and rough side walls instead of the desired sharp corners aimed to form the rectangular nanograting. Therefore, it is imperative to study the effect of different 
nanograting shapes and non-idealities to understand the realistic performance of the FSL.

Based on our initial nanofabrication experiments in the cleanroom to construct silver nanograting, we identified the general shape profiles that resulted from this fabrication process using our laser lithography technique. Next, we incorporated these nanograting geometries in our COMSOL Multiphysics ${ }^{\circledR}$ models.

In Sections 3.4.1,3.4.2 and 3.4.3 of this thesis, the nano-structures shape effects are studied using two different models, the object-based and the FSL-based models.

The object-based model concerns investigating a range of periodic objects with varied sizes and evaluating their effects on the resulting EM field of the FSL.

On the other hand, the FSL-based model concerns investigating the effects of changing the shapes of the features of the FSL nanograting, and varying the grating's duty cycle, on the performance of the FSL. We define the duty cycle as the ratio or percentage of the width of the large base of the nanostructure to the value of the specified period. Figure 3-7 shows different geometries as part of the FSL design modelled in this thesis, where (a) shows a rectangular, (b) a roundedrectangular, (c) trapezoidal, (d) inverse-trapezoidal, and (e) a half-circle set of nanogratings. The FSL nanograting sets (a) to (d) have two bases, where the smaller base has a width of $x^{\prime \prime}$ and the larger base has a width of $2 x^{\prime}+x^{\prime \prime}$. The dependence of the FSL's performance on $x^{\prime}$ is reported in section 3.4.2. Although we use the same $x^{\prime}$ and $x^{\prime \prime}$ parameters to define the bottom base in Figure 3-7(e), this represents a special case where the geometry of the nanograting is composed of a single base. 


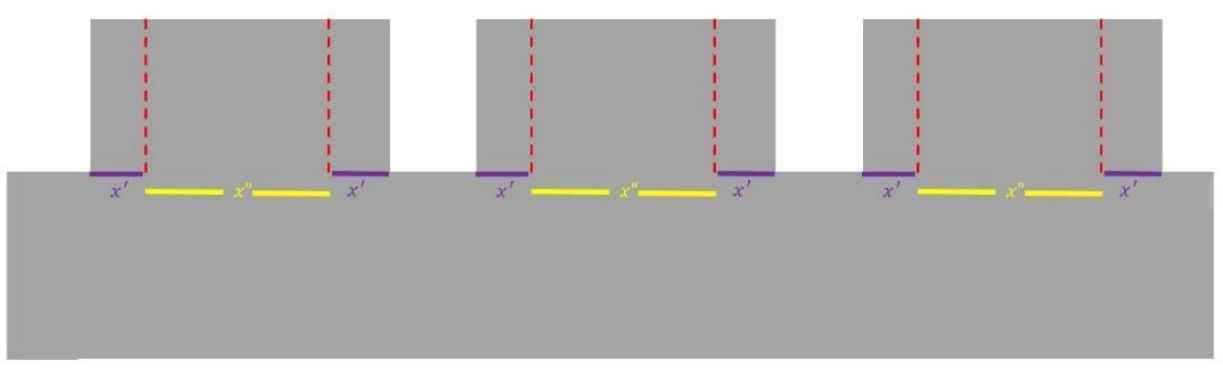

(a)

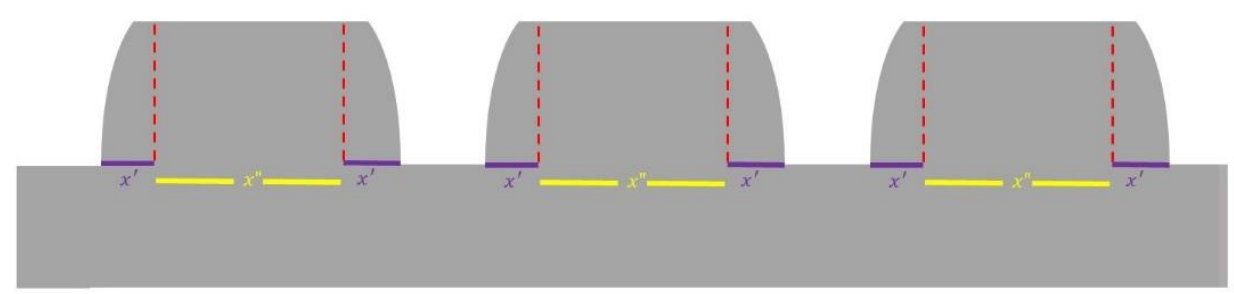

(b)

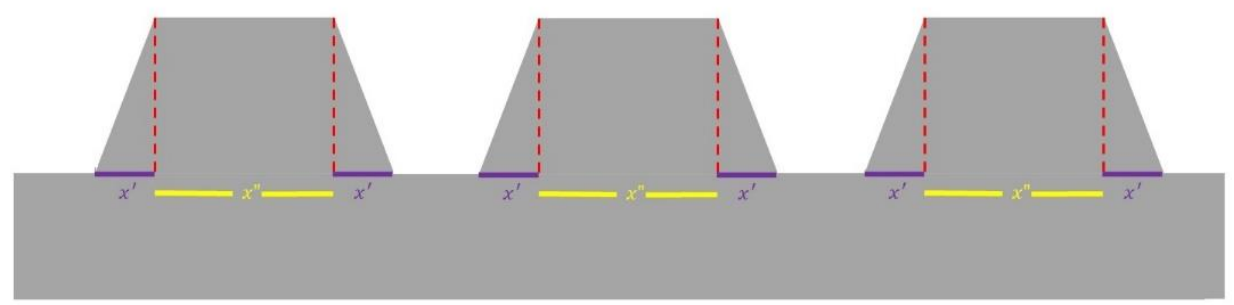

(c)

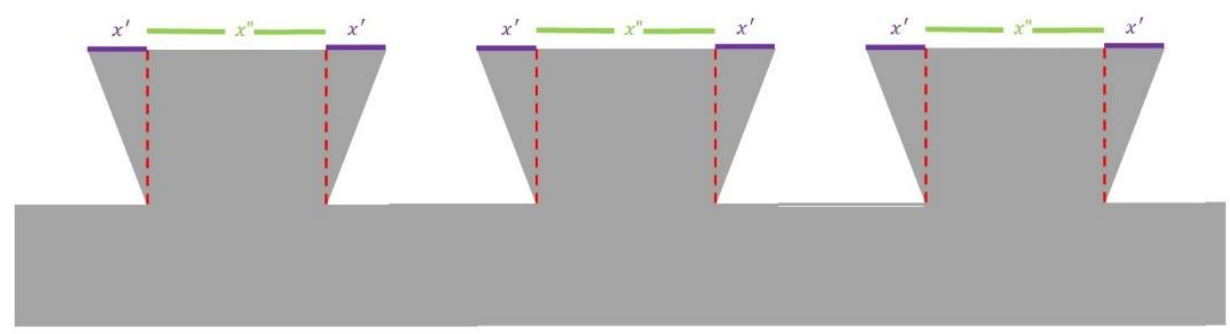

(d)

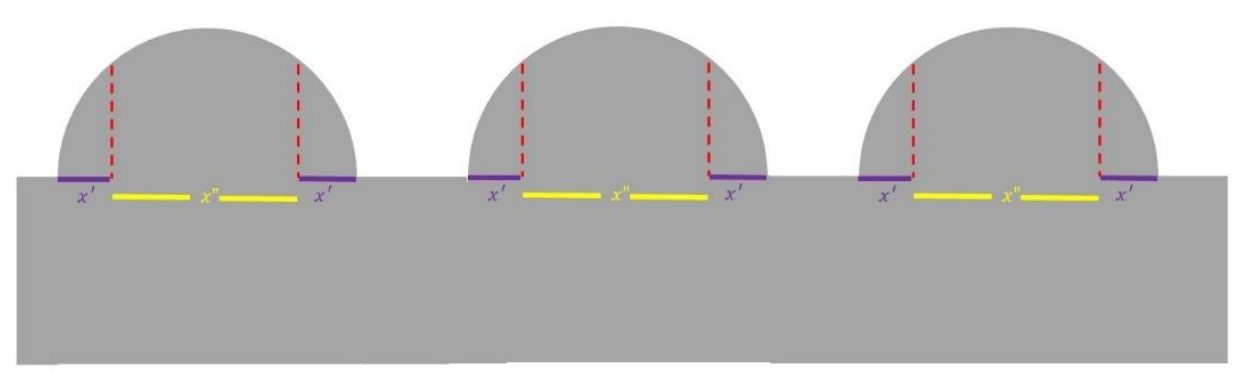

(e)

Figure 3-7 Different nanograting geometries as part of the FSL design investigated in the $2 \mathrm{D}$ modelling study. 


\subsubsection{Object size effect}

The central aim of the study reported in this section was to evaluate the impact of varying the object (periodic) size on the FSL's far-field pattern. In the first part of this study, the FSL structure and the model settings were kept fixed. Our ultimate aim was to determine the range of the object spatial frequencies that can be resolved using this FSL. Simulation results were analysed based on their magnitude transfer function [118], as defined in section 3.1. The next parts of this study incorporated a second variable, as the shape of the features of the FSL nanograting were varied to include the inverse trapezoidal and half-circular cases according to Figure 3-7.

In all considered models, objects had a rectangular cross-section, and a height of $30 \mathrm{~nm}$. The duty cycle was kept at 50\% for both the FSL nanograting and the objects. The main variable was the object width/period, where 11 different scenarios were considered. The secondary variable was the FSL nanograting shape, where the grating height was similarly kept constant.

It should be noted that horizontal reflectional symmetry had to be conserved throughout this study, to avoid the complications arising from an asymmetric model. Accordingly, the object pattern was kept symmetric with respect to the midline dividing the width of the modelling domain.

Given the symmetric condition in the geometry of the model, the width of the computation domain did not affect the far-field pattern as the correct boundary conditions were employed. Accordingly, the domain width was set to $1350 \mathrm{~nm}$. On the other hand, based on the results of Section 3.2, the length of the domain was selected to be $\sim 4500 \mathrm{~nm}$, to keep the required computation resources to a minimum. 
To aid in analysing the resulting field patterns, it should be noted that the FSL in this section had a total of 9 nanograting features, with a period of $150 \mathrm{~nm}$. Table 3-2 shows the considered object sizes and number of objects per domain width for this study, where the object period varied from $96 \mathrm{~nm}$ to $450 \mathrm{~nm}$. The case when the object period was $150 \mathrm{~nm}$ was omitted given the expectations by the Moiré and Talbot equations $\left(Z_{T}=\Lambda_{M}=\infty\right)$.

Table 3-2 Object period and number used to achieve a symmetric arrangement with respect to the domain width.

\begin{tabular}{|c|c|}
\hline $\begin{array}{c}\text { Object period } \\
\text { (nm) }\end{array}$ & $\begin{array}{c}\text { Object number } \\
\text { per } \\
\text { Domain width }\end{array}$ \\
\hline 96 & 14 \\
103 & 13 \\
112 & 12 \\
122 & 11 \\
135 & 10 \\
150 (omitted) & 9 \\
\hline
\end{tabular}

\begin{tabular}{|c|c|}
\hline $\begin{array}{c}\text { Object period } \\
\text { (nm) }\end{array}$ & $\begin{array}{c}\text { Object number } \\
\text { per } \\
\text { Domain width }\end{array}$ \\
\hline 168 & 8 \\
192 & 7 \\
225 & 6 \\
270 & 5 \\
337 & 4 \\
450 & 3 \\
\hline
\end{tabular}

Considering the smallest object scenario where the object period was $96 \mathrm{~nm}$ (object width of $48 \mathrm{~nm}$ given the $50 \%$ duty cycle), the resulting electric field norm is shown in Figure 3-8. This plot of the far-field shows a periodic pattern as expected. 


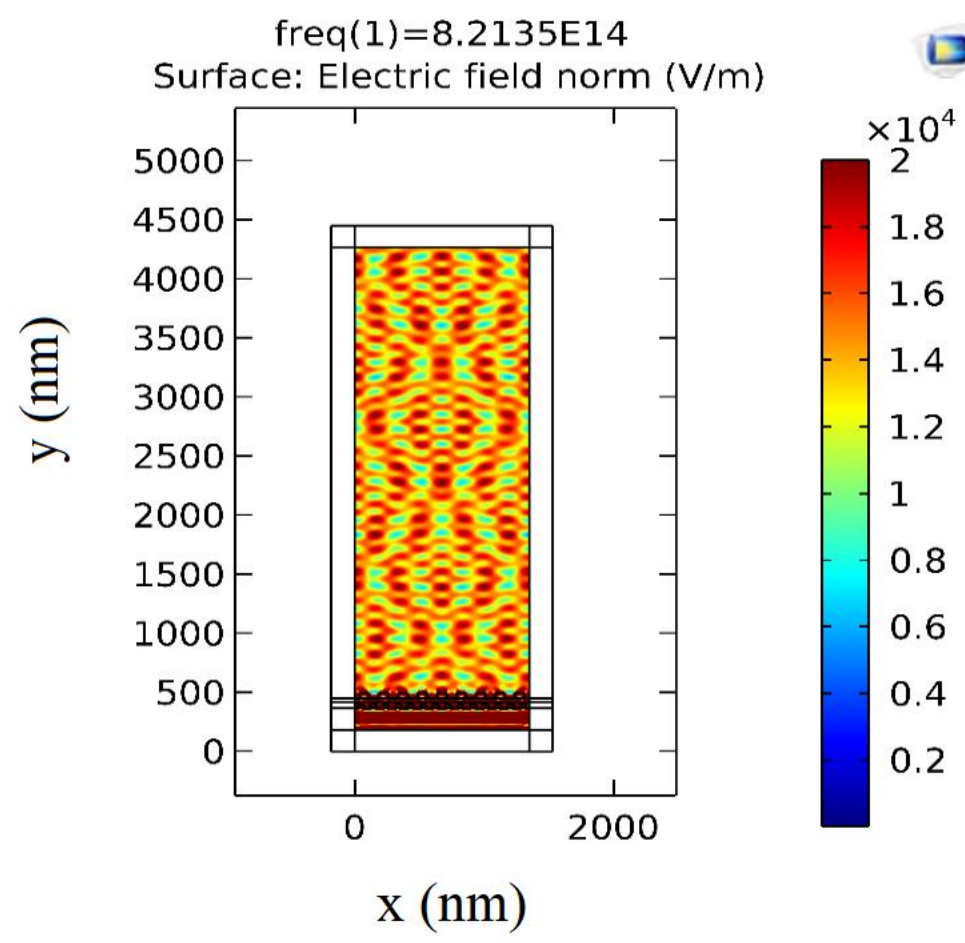

Figure 3-8 Electric field norm for an object period of $96 \mathrm{~nm}$. The far-field pattern is repeated according to Talbot effect, however the fringes are distorted when compared to the far-field patterns of larger objects.

Given the reflectional horizontal symmetry of the model, a horizontally symmetric yet distorted Moiré pattern was generated. The Moiré period was larger than that of both the object and FSL nanograting periods and agrees with the Moiré equation. It can also be seen that the self-repeating Talbot pattern exists along the direction of light propagation.

The resulting distortion in the field pattern might indicate that the selected illumination wavelength and FSL nanograting size were not suitable for directly imaging an object with this size. Therefore, resolving the object features in this case might require complicated image processing. 
Next, the remaining object sizes/periods were modelled. The resulting far-field for all of these cases showed non-distorted Moiré and Talbot patterns that agreed with the corresponding dimensions. As an example, the field interactions for objects with periods of $112 \mathrm{~nm}$, and $122 \mathrm{~nm}$ are shown in Figure 3-9.
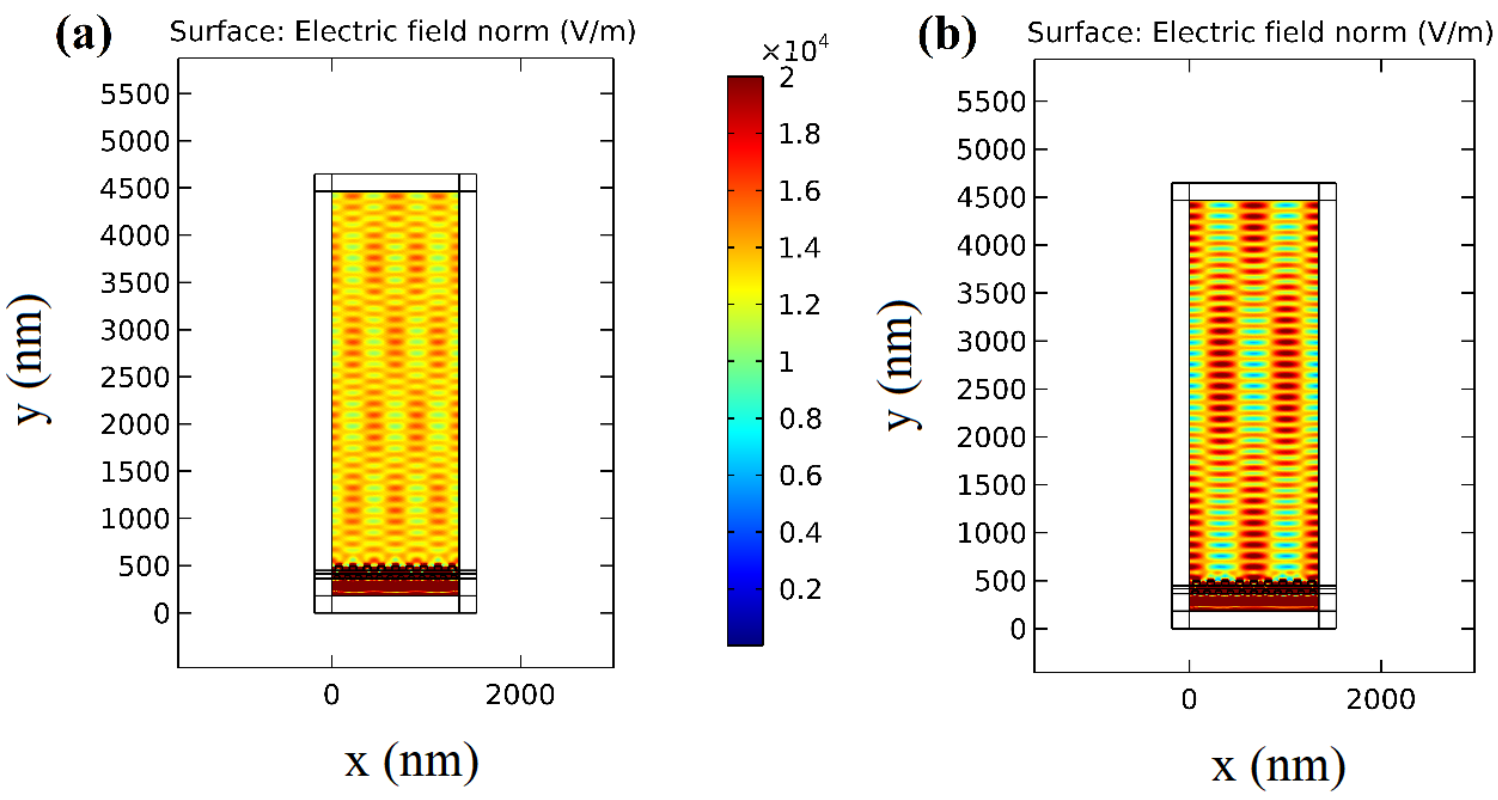

Figure 3-9 Electric field norm density plot for (a) $112 \mathrm{~nm}$, and (b) $122 \mathrm{~nm}$ object periods.

The same modelling study that was presented so far in this section was performed for the cases of inverse trapezoidal ( $\mathrm{x}^{\prime}$ was set to $30 \mathrm{~nm}$ ) and halfcircular FSL nanogratings. Thus, for each FSL nanograting shape, 11 different object periods, varying from $96 \mathrm{~nm}$ to $450 \mathrm{~nm}$ were simulated. The resulting electric field norm plots were used to calculate the magnitude transfer function of each permutation. 
To calculate the magnitude transfer function, we followed the steps highlighted in section 3.1. Thus, we start by extracting the electric field norm amplitude functions at the object cutline (at the top object boundary) and the image cutline (at the first Talbot distance).

An example is shown in Figure 3-10, where the electric field amplitude at the object cutline is shown in (a), while (b) shows that at the image cutline.

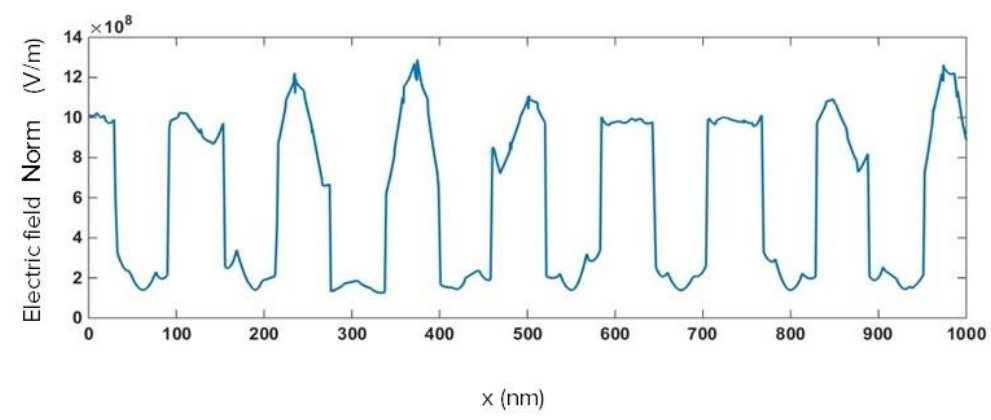

(a)

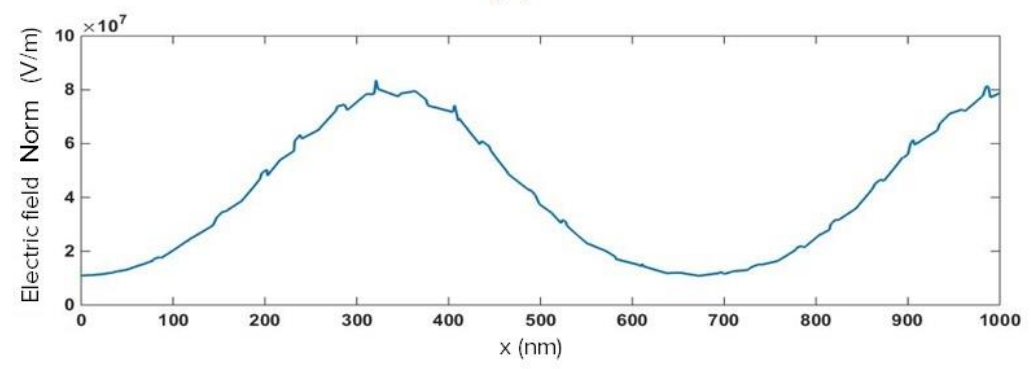

(b)

Figure 3-10 The electric field norm at the object (a) and image (b) cutlines

It is noted that in our study, the resulting electric field norm suffers from spatial irregularities due to the selected mesh resolution. This trade-off between mesh resolution and results' quality was accepted given that such irregularities are almost negligible (visually) in the 2D and 3D field plots. 
However, these irregularities become more prominent once viewed at a cutline, as highlighted by the plots of Figure 3-10. Fortunately, these irregularities are filtered out when calculating the magnitude transfer function, as the next step in this calculation involve performing Fourier transformation on both sets of data. The resulting plots are presented in Figure 3-11, where (a) and (b) show the frequency plots for the object and image cutlines, respectively.

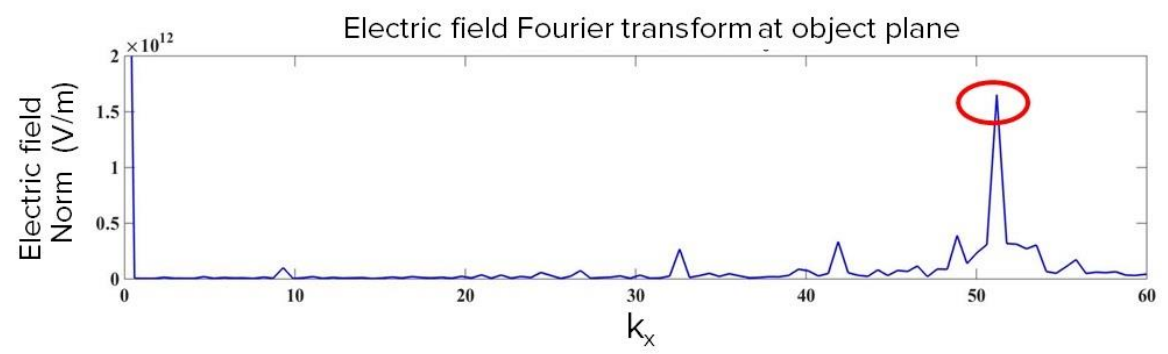

(a)

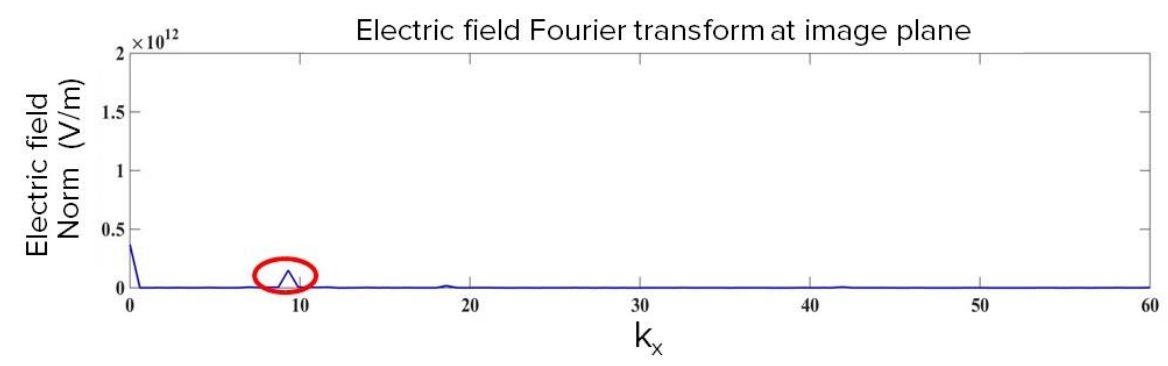

(b)

Figure 3-11 Fourier transforms of the electric field norm at the object (a) and image (b) cutlines.

According to the number of cycles observed at the object cutline and the image cutline, and given the sampling rate, we can estimate the frequencies that correspond to each period of interest, i.e., relating to the number of objects at the object cutline, and relating to the Moiré period at the image cutline. The specific 
amplitude values for each of the relevant peaks (highlighted in red in Figure 3-11) are shown in Table 3-3.

Table 3-3 Image and object peak amplitudes in Figure 3-11.

\begin{tabular}{|l|l|}
\cline { 2 - 2 } \multicolumn{1}{c|}{} & Peak amplitude \\
\hline Image cutline & $0.2 \times 10^{12}$ \\
Object cutline & $1.7 \times 10^{12}$ \\
\hline
\end{tabular}

Finally, the magnitude transfer function $(M T F)$ is given by the ratio between the image cutline peak amplitude $\left(\operatorname{FFT}\left(A_{I m g}\right)_{\max }\right)$ and the object cutline peak amplitude $\left(\operatorname{FFT}\left(A_{O b j}\right)_{\max }\right)$ :

$$
M T F=\frac{\operatorname{FFT}\left(A_{I m g}\right)_{\max }}{\operatorname{FFT}\left(A_{O b j}\right)_{\max }}
$$

\section{Equation 3-4}

This process was repeated for all modelled cases in this study, i.e., 11 object sizes for each of the three FSL nanograting shapes. The final result comparing the magnitude transfer function in all these cases is shown in Figure 3-12. 


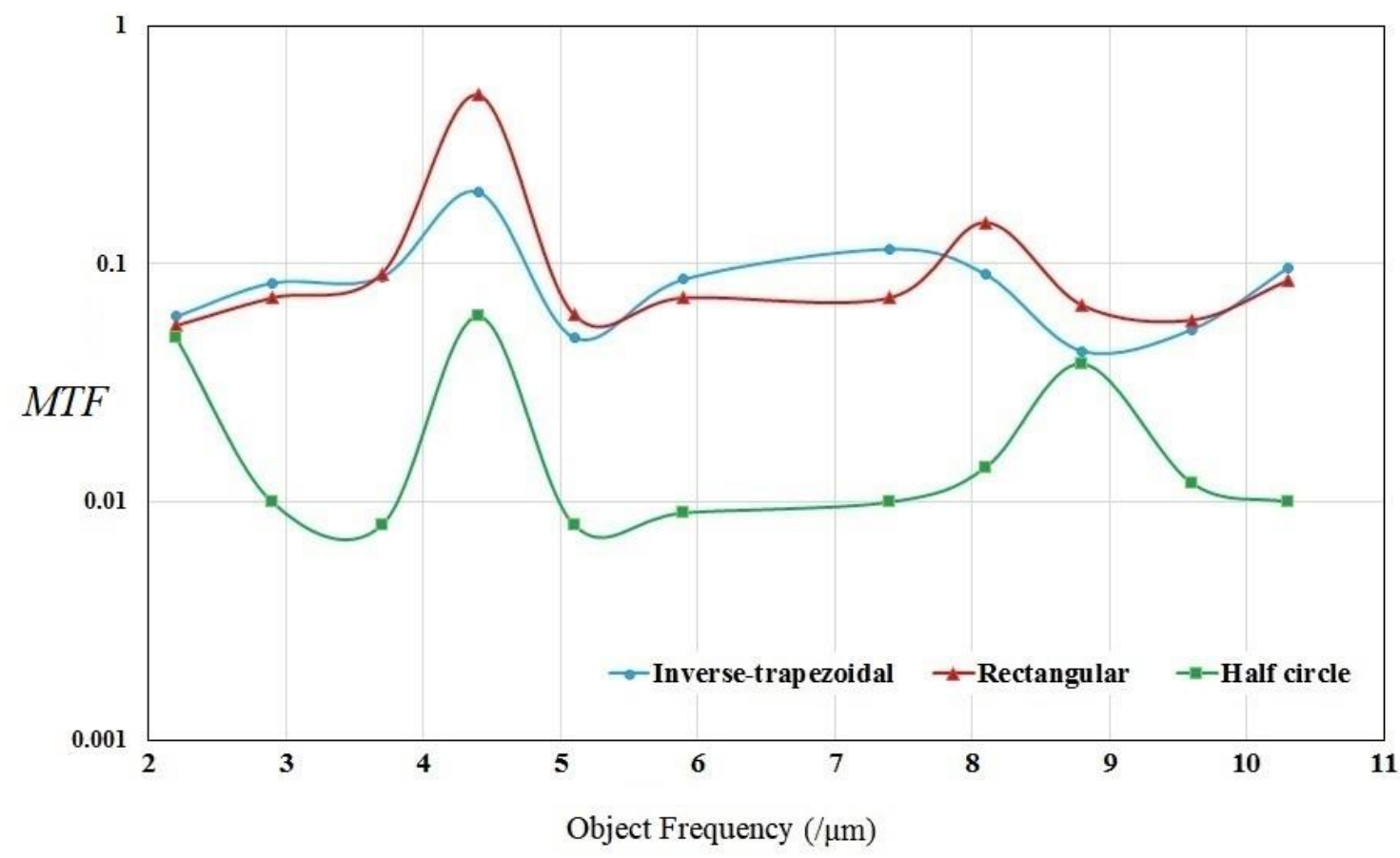

Figure 3-12 The magnitude transfer function for the inverse-trapezoidal, rectangular and half-circular FSL nanogratings with 11 different object periods, varying from $96 \mathrm{~nm}$ to $450 \mathrm{~nm}$.

The period of the object on the horizontal axis is represented in terms of its inverse, i.e., object frequency having a unit of $(/ \mu \mathrm{m})$. Here, the object period ranges from $96 \mathrm{~nm}<->(10.4 / \mu \mathrm{m})$ to $450 \mathrm{~nm}<->(2.2 / \mu \mathrm{m})$. Based on the simulated results, Figure 3-12 highlights the performance (in terms of MTF) for each FSL nanograting with respect to the object period/width.

The FSL designs with rectangular and inverse trapezoidal nanogratings have a higher magnitude transfer function over the whole range, when compared to the FSL with half circular nanograting.

It can also be seen that the maximum magnitude transfer function occurs for an object with a period of $225 \mathrm{~nm}(4.4 / \mu \mathrm{m})$ when using the rectangular FSL nanograting. A second high intensity area occurs for higher object frequencies (smaller objects) in the $8 / \mu \mathrm{m}$ to $9 / \mu \mathrm{m}$ range, and for all three FSL nanogratings. 
Given the latter observation, studies presented in section 3.4 .2 will focus on evaluating the effects of the FSL nanograting shape on the lens performance when the object frequency was in this region of interest, i.e., $8 / \mu \mathrm{m}$ to $9 / \mu \mathrm{m}$.

\subsubsection{FSL Nanograting shape effect}

In this section, different FSL nanograting geometries are introduced and their effect on the performance of the FSL is studied. All objects in this study are periodic with rectangular features and a duty cycle of $50 \%$. The period of the object is specified for each model, however, it is kept in the 8-9/ $\mu \mathrm{m}$ region of interest as defined by the study reported at the end of section 3.4.1. The different periodic geometries investigated in this section relate to the FSL nanograting design and should not be confused with the object. Figure 3-7 shows the range of different nanograting geometries studied in here.

We start this study by modelling the electric field norm for two different FSL nanograting geometries, where both designs have the same object with a period of $122.7 \mathrm{~nm}$. The FSL in Figure 3-13(a) has a rectangular nanograting feature, while the one in Figure 3-13(b) has a half-circle nanograting feature. 
(a)

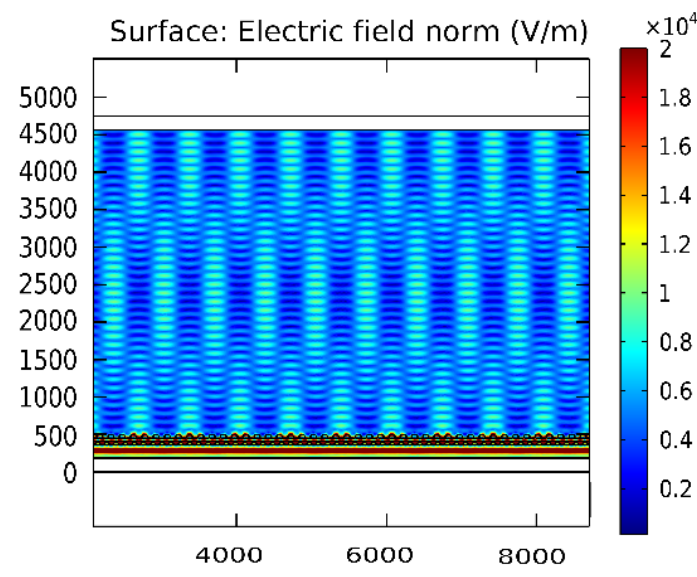

(b)

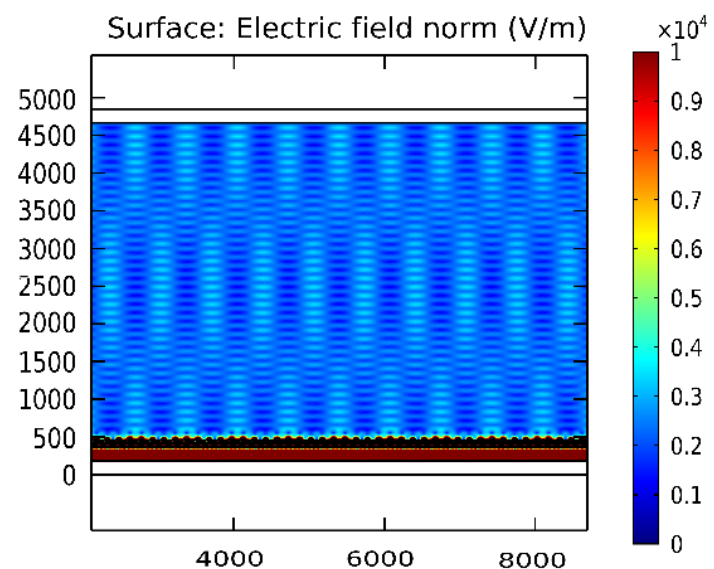

Figure 3-13 Electric field norm for (a) the rectangular nanograting, and (b) the half-circle nanograting.

It can be observed that far-field patterns are almost identical for both cases; however, the maximum amplitude for the half-circle nanograting is lower, noting that the colour bar in Figure 3-13(b) is half of that in (a). This can be explained by the fact that plasmonic excitations are stronger around sharp edges than curved walls due to electric field enhancement.

Given these results, it became evident that we needed to provide a measure for contrast achieved in our models. Therefore, we adopted the Michelson contrast equation to compare different results [209], where contrast is given by:

$$
\text { Contrast }=\frac{A_{\max }-A_{\min }}{A_{\max }+A_{\min }}
$$

Equation 3-5

Here, $A_{\max }$ and $A_{\min }$ are the highest and lowest amplitudes of the electric field norm at the image cutline (Talbot distance), respectively. 
Thus, the following steps were taken to calculate the contrast for our models:

- An image cutline was applied at the Talbot distance, where the maximum amplitude in the electric field norm plot was observed.

- Using MATLAB, all maximum and minimum peaks in this data set were identified and their averages produced, i.e., $\bar{A}_{\text {max }}$ and $\bar{A}_{\text {min }}$.

- Contrast was calculated using Equation 3-5.

Next, we modelled the performance in terms of the achieved contrast for three FSL nanograting shapes as a function of $x^{\prime}$. The three considered shapes were the inverse-trapezoidal, trapezoidal and rounded-rectangular geometries. The selected object in this case had a period of $112.5 \mathrm{~nm}$. The corresponding results are shown in Figure 3-14.

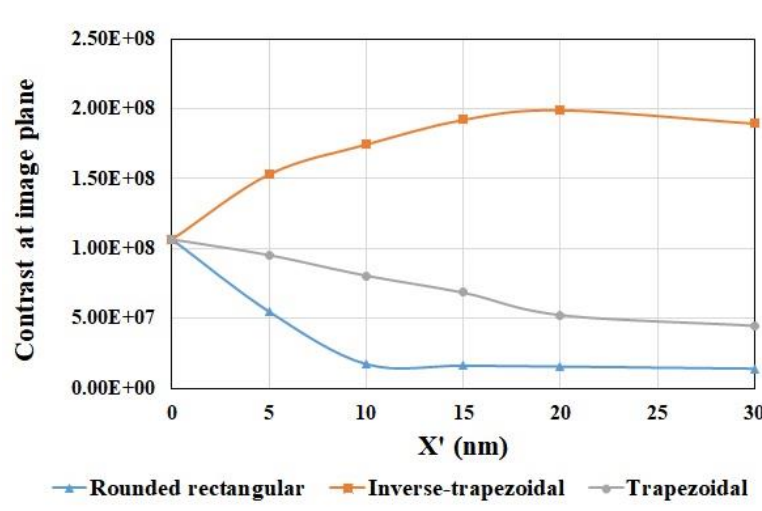

(a)

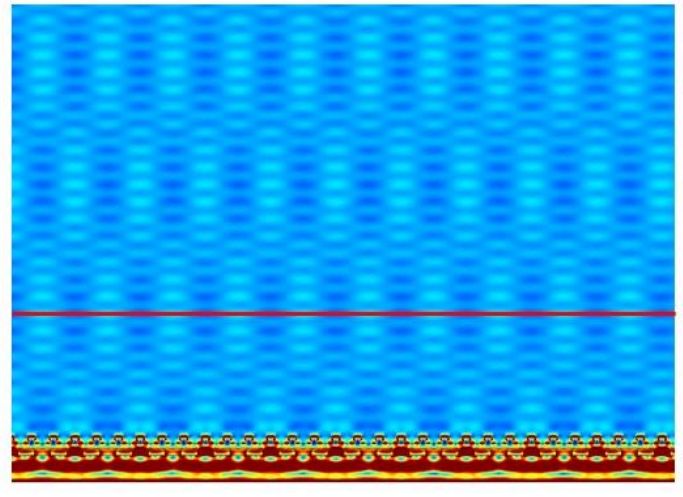

(b)

Figure 3-14 (a) The contrast plots for three different FSL nanograting shapes as a function of $x^{\prime},(b)$ a sample far-field plot for the inversetrapezoidal FSL nanograting with $x^{\prime}=30 \mathrm{~nm}$. 
Here, we evaluated the contrast at the image cutline for the three different FSL nanograting shapes while $x^{\prime}$ varied, where each data point in these plots was extracted from an individual model.

It is observed that the highest contrast was achieved using the inversetrapezoidal nanograting, which was twice as high as the rectangular nanograting case $\left(x^{\prime}=0 \mathrm{~nm}\right)$. Increasing $x^{\prime}$ seemed to result in enhanced contrast for the inverse-trapezoidal nanograting, however, we acknowledge that creating this scenario poses numerous microfabrication challenges. As expected, the worst contrast was for the rounded-rectangular FSL nanograting possibly due to lower electric field enhancement.

The next study featured in this section modelled the performance of three FSL nanograting shapes as a function of $x^{\prime}$. Performance in this case was judged based on comparing the maximum amplitude of the electric field norm at the object and the image cutlines, which we refer to as the amplitude-ratio parameter. The three considered shapes were the inverse-trapezoidal, trapezoidal and roundedrectangular geometries. The selected object in this case had a period of $122.7 \mathrm{~nm}$. The corresponding results are shown in Figure 3-15. 


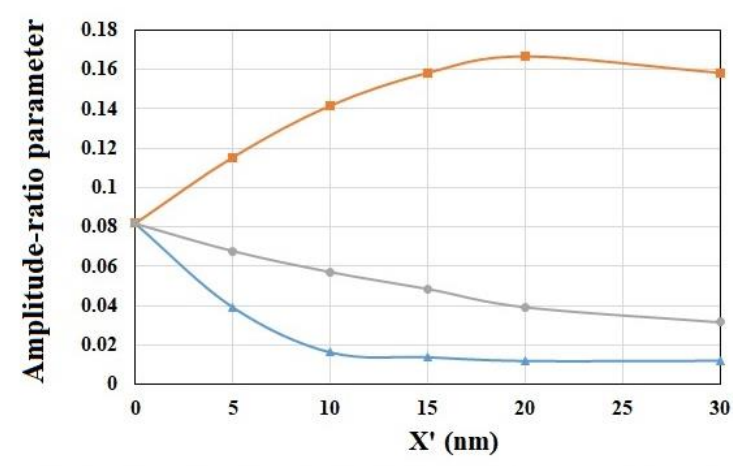

$\leadsto$-Rounded rectangular $\rightarrow-$ Inverse-trapezoidal $\rightarrow$ Trapezoidal

(a)

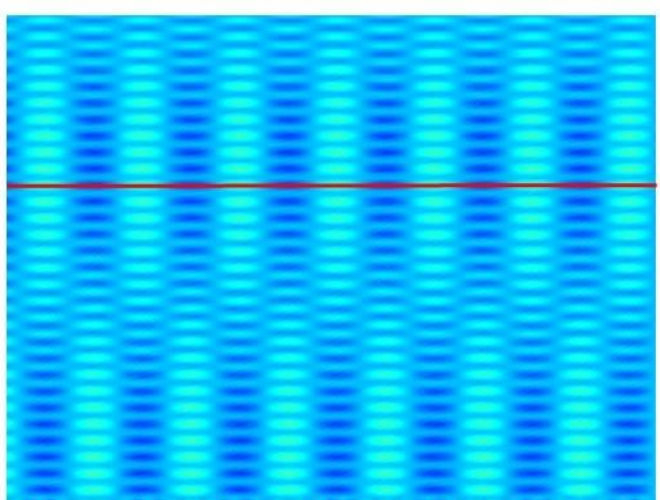

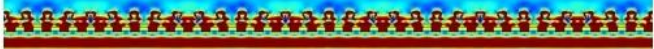

(b)

Figure 3-15 (a) The amplitude-ratio plots for three different FSL nanograting shapes as a function of $x^{\prime},(b)$ a sample far-field plot for the inverse-trapezoidal FSL nanograting with $x^{\prime}=30 \mathrm{~nm}$.

For each model considered in this study, the amplitude-ratio parameter (dimensionless) was calculated according to the following steps:

- An object cutline was applied at the top boundary of the object.

- An image cutline was applied at the Talbot distance, where the maximum amplitude in the electric field norm plot was observed.

- Using MATLAB, all maximum peaks in both data sets were identified and their averages produced, i.e., $\bar{A}_{\text {obj max }}$ and $\bar{A}_{\text {Img max }}$.

- The amplitude ratio parameter was calculated using $\bar{A}_{\text {Img } \max } / \bar{A}_{\text {Obj max }}$

The observed results in the amplitude-ratio plots of Figure 3-15 are similar to our findings in the contrast plots of Figure 3-14, where the inverse-trapezoidal FSL nanograting outperformed other considered geometries. Based on these results, the FSL performance is sensitive to both the slope and edge shape of the nanograting profiles. 
It is noted that both contrast and amplitude-ratio plots show a similar trend for different FSL designs, despite the minor variation in the object size. The main difference between these two plots is the inclusion of the object cutline information in the amplitude-ratio parameter. However, given that the illuminating wave was a fixed parameter, the contrast and amplitude-ratio parameters are found to be mostly proportional regardless of the FSL geometry variations.

\subsubsection{FSL Nanograting duty cycle effect}

In this section, we evaluate the effects of the FSL nanograting's duty cycle $\left(\Lambda_{G}\right)$ on the resulting far-field pattern. Throughout this study, the FSL nanograting had a rectangular profile with a period of $150 \mathrm{~nm}$, while the considered object had a rectangular profile with a period of $120 \mathrm{~nm}$.

Figure 3-16 shows the electric field norm plots for a range of FSL nanograting with a duty cycle between $30 \%$ and $70 \%$. 


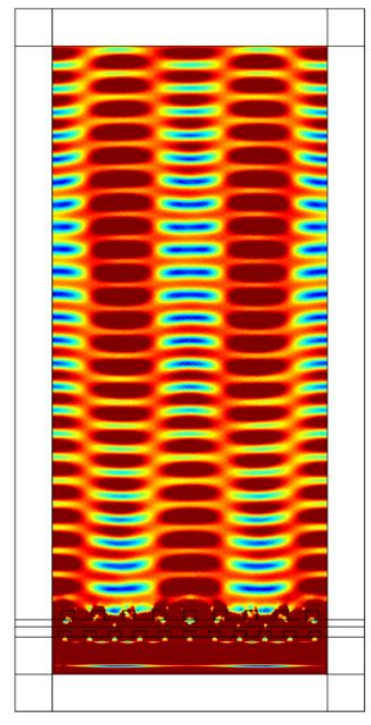

(a)

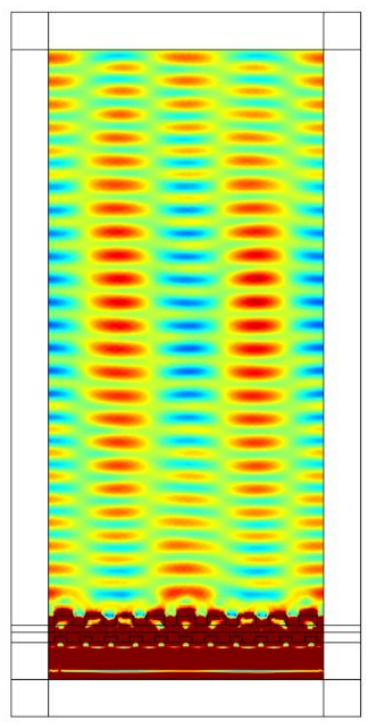

(c)

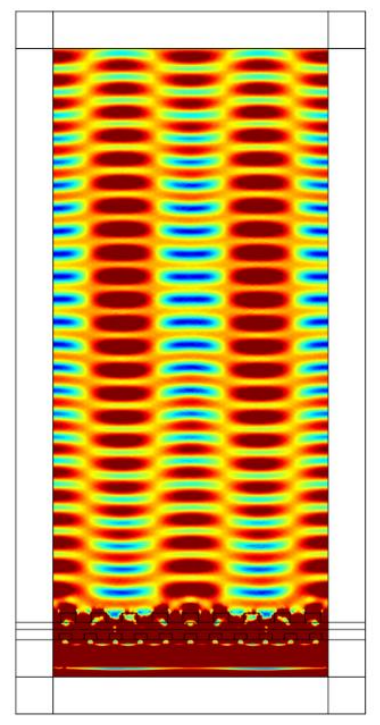

(b)

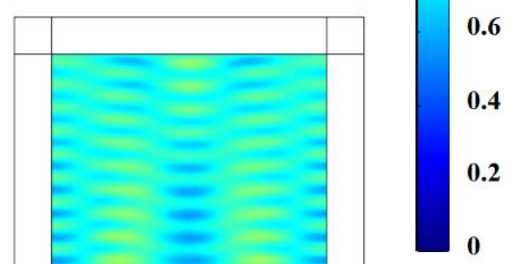

0.2
0

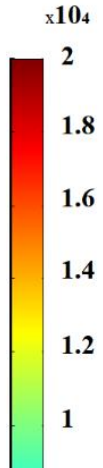

0.8

0.6

4

.


object and the FSL nanograting remain constant. For duty cycles higher than 70\% and lower than $40 \%$, the far-field patterns are laterally distorted, i.e., the resolution of the pattern is degraded, therefore we omitted their results from this study.

Figure 3-17 shows the amplitude of the electric field norm at the image cutlines (Talbot distance) for the models shown in Figure 3-16.

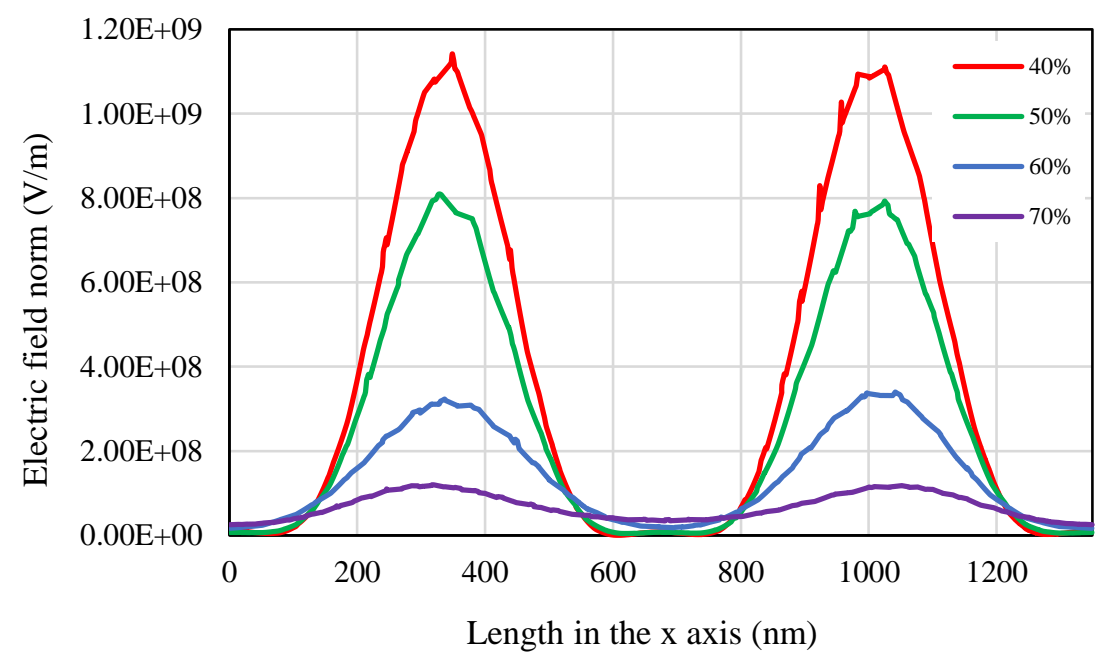

Figure 3-17 Variations of the amplitude of the electric field norm with respect to the FSL nanograting's duty cycle.

Even though the amplitude and contrast were highest for the model with a $40 \%$ duty cycle, we resorted to adopting a 50\% duty cycle for the rest of the studies in this chapter, given that fabricating smaller features is generally more challenging. 


\subsubsection{Anti-symmetric object positioning}

Thus far, our models had reflectional symmetry with respect to the vertical line in the middle of the modelling domain. In reality, given the small size of the objects, it is not reasonable to assume an accurate symmetry while arranging objects under the FSL.

To investigate the effects of potential asymmetries in the object location (horizontal) on the FSL's far-field pattern, we designed the following experiment. Four identical models were created, where the single difference was object placement. Accordingly, the horizontal position of the object was changed in 3 steps, where each step moved the object by a quarter of its period $\left(\Lambda_{o b j} / 4\right)$. These arrangements are shown below in Figure 3-18.

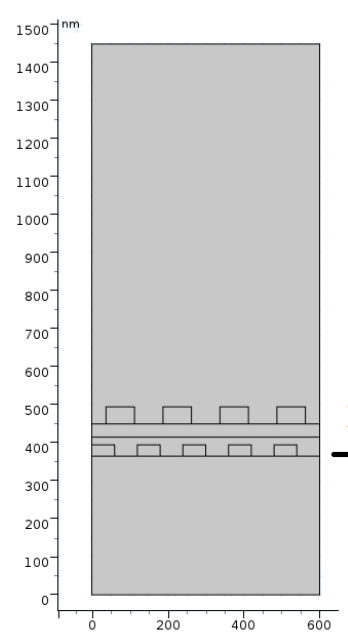

(a)

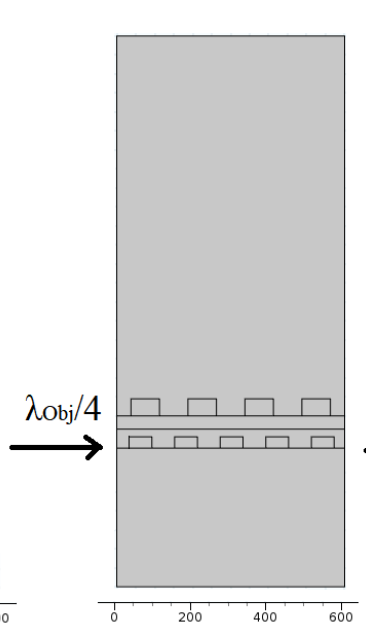

(b)

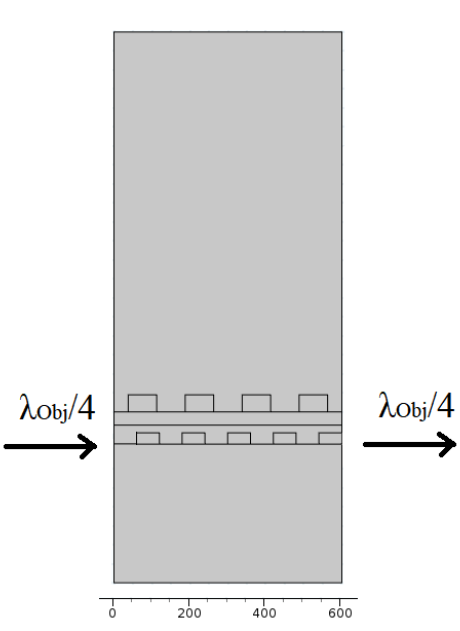

(c)

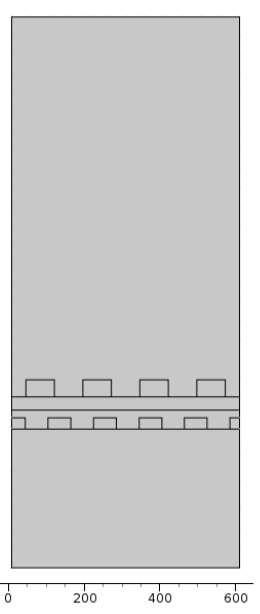

(d)

Figure 3-18 Schematic design of the arrangement of the object under the FSL. Each step shifted the object location by $\Lambda_{o b j} / 4$.

Prior to this study, our models necessitated reflectional symmetry for both the periodic object and the FSL nanograting. However, given the asymmetry 
consideration in this study, the boundary conditions and model settings needed to be changed. In order to simulate asymmetric designs, we set up a model using a "fixed periodic cell" as opposed to a domain with semi-infinite width. Thereafter, new settings were applied, which included a different definition for the illuminating wave and different boundary conditions. A Floquet boundary condition made it possible to treat the whole cell as a periodic problem that repeats itself to infinity [210]. The number of repetitions for the cell could be manually defined, however, our results indicated that using two repetitions gives the same outcome as higher orders.

Given our familiarity with the far-field interactions of a symmetric FSL-object design, we started our comparison by modelling the two symmetric arrangements in Figure 3-18, namely scenarios (b) and (d). The results showing the electric field norm of these simulations are shown in Figure 3-19.
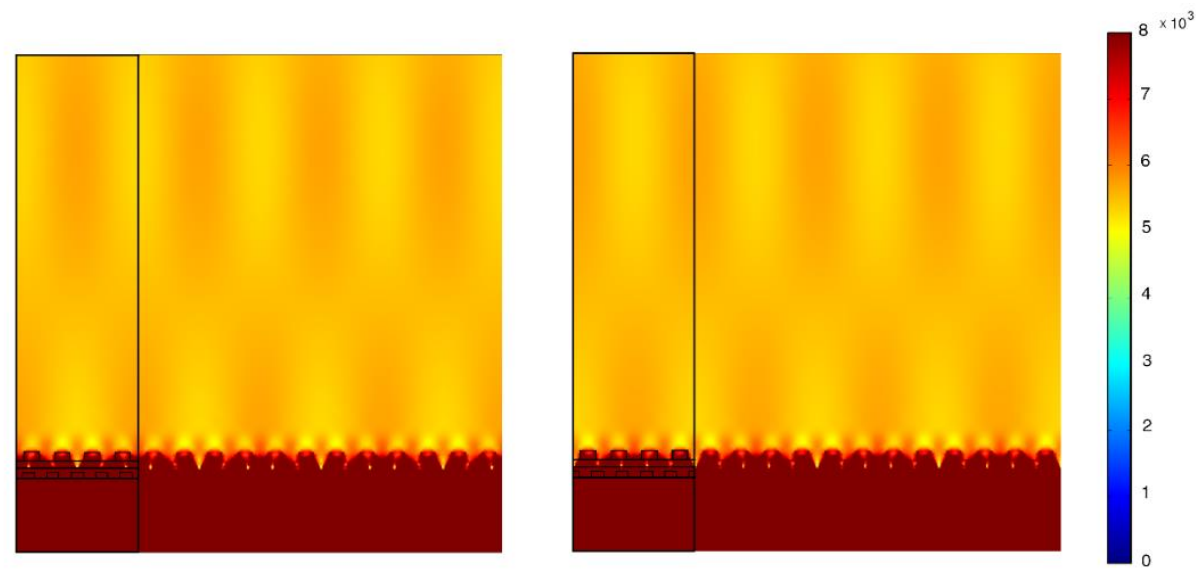

Figure 3-19 Amplitude plots of the electric field norm for models (b) on the left and (d) on the right from Figure 3-18. These models have symmetric object arrangement. The FSL nanograting and object period are $150 \mathrm{~nm}$ and $120 \mathrm{~nm}$, respectively. 
It can be observed that the familiar far-field pattern with correct Moiré and Talbot periods were achieved. This similar outcome was promising given the drastic changes in the new model settings.

In addition, the results shown in Figure 3-19 indicated that changing the position of the object resulted in a phase shift in the Moiré pattern in the far-field. Figure 3-20 shows the magnitude of the electric field norm at the Talbot length for both scenarios.

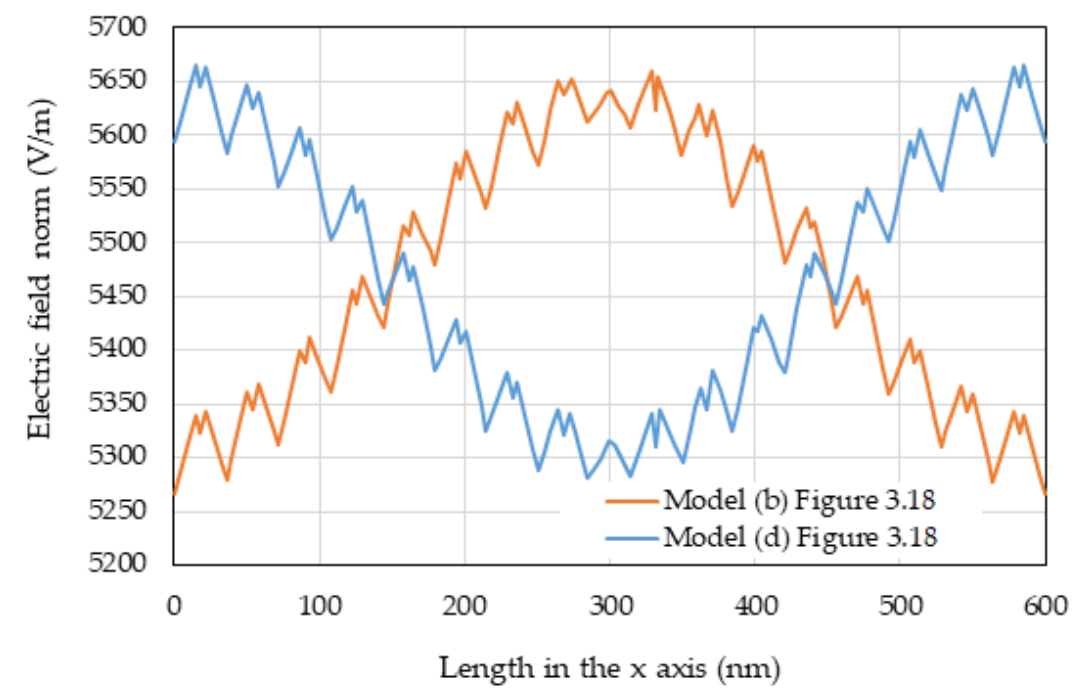

Figure 3-20 The electric field norm plot at the Talbot length for the models in Figure 3-19.

Accordingly, in these symmetric arrangements, we observed that changing the object location by half the object period, $\Lambda_{o b j} / 2$, shifts the far-field pattern by half the Moiré period $\left(\Lambda_{M} / 2\right)$.

Next, the asymmetric objects arrangement scenarios represented in Figure 3-18 (a) and (c) were modelled. The resulting electric field norm plots are shown in Figure 3-21. 


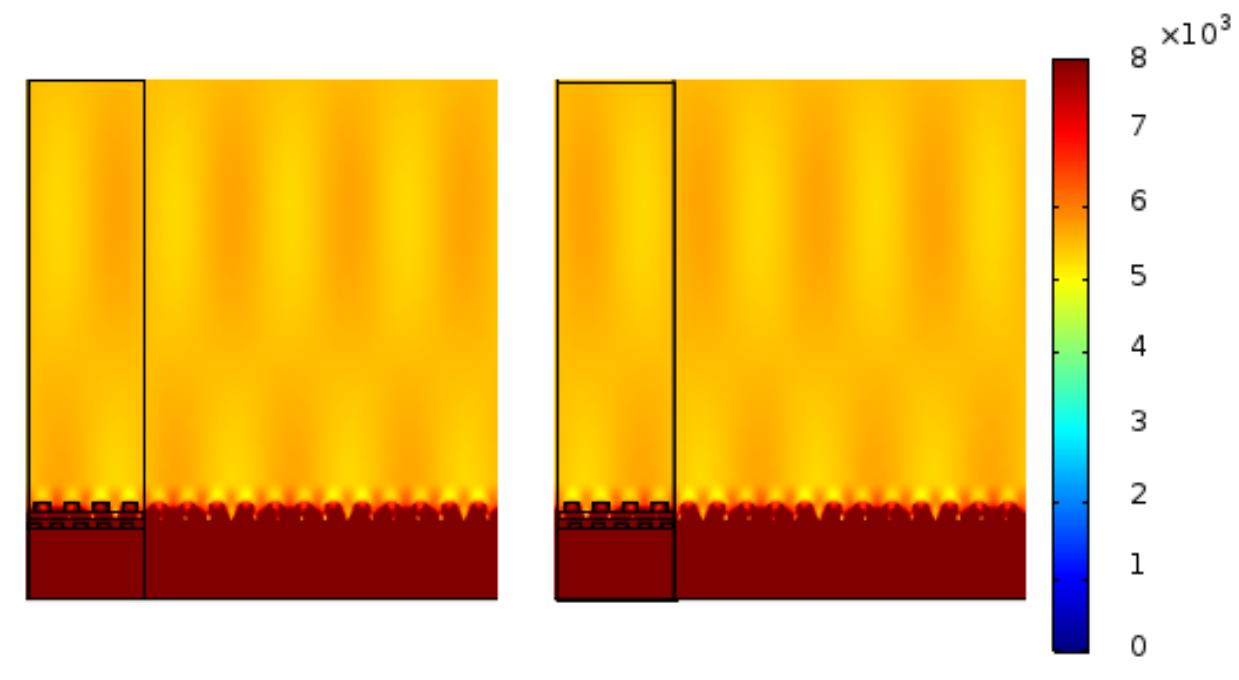

Figure 3-21 Amplitude plots of the electric field norm for models (a) on the left and (c) on the right from Figure 3-18. These models have asymmetric object arrangement. The FSL nanograting and object period are $150 \mathrm{~nm}$ and $120 \mathrm{~nm}$, respectively.

Based on our results, the far-field patterns in the asymmetric cases were similar to those of the symmetric design. Figure 3-22 shows the magnitude of the electric field norm at the Talbot length for both asymmetric scenarios. 


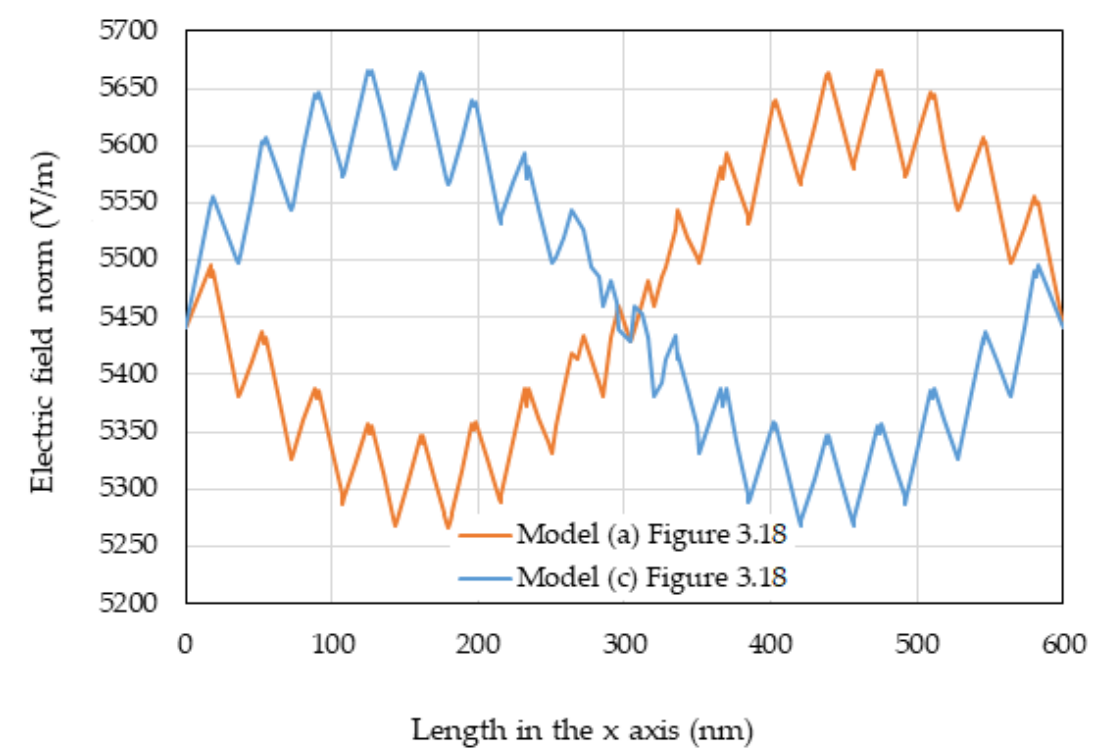

Figure 3-22 The electric field norm plot at the Talbot length for the models in Figure 3-21.

A general conclusion from Figure 3-20 and Figure 3-22 is that with each $\Lambda_{o b j} / 4$ shift in the location of the object provides a shift of $\Lambda_{M} / 4$ to the far-field pattern.

\subsection{Three-dimensional design}

In Section 3.5, we modelled our object-FSL designs in three-dimensions. This allowed us to improve our understanding of the FSL's far-field interactions, and to experiment with complex illumination conditions that are not possible to implement in 2D.

Solving 3D models with domain sizes as large as those used in our 2D models is impractical, given that simulation time and memory consumption increase drastically. Therefore, we identified the minimal cell that satisfied the constraints of our 3D model. This minimal cell had the minimum surface area in the $x-y$ plane 
that could accommodate the periodic object and the FSL nanograting while preserving the reflectional and translational symmetries in the design. In short, a correctly designed minimal cell for a given model should perform in a similar manner to the full-sized design.

Figure 3-23 shows the two groups of minimal cells that we used in this section. These two cells had specified areas of $(600 \mathrm{~nm} \times 600 \mathrm{~nm})$ and $(450 \mathrm{~nm} \times 450 \mathrm{~nm})$. The arrangement and combination of the objects and nanogratings that were studied using this setup are:

- 1D objects and 1D gratings,

- 1D objects and 2D gratings,

- 2D objects and 2D gratings,

- 2D objects and 1D gratings. 


\section{Top view}
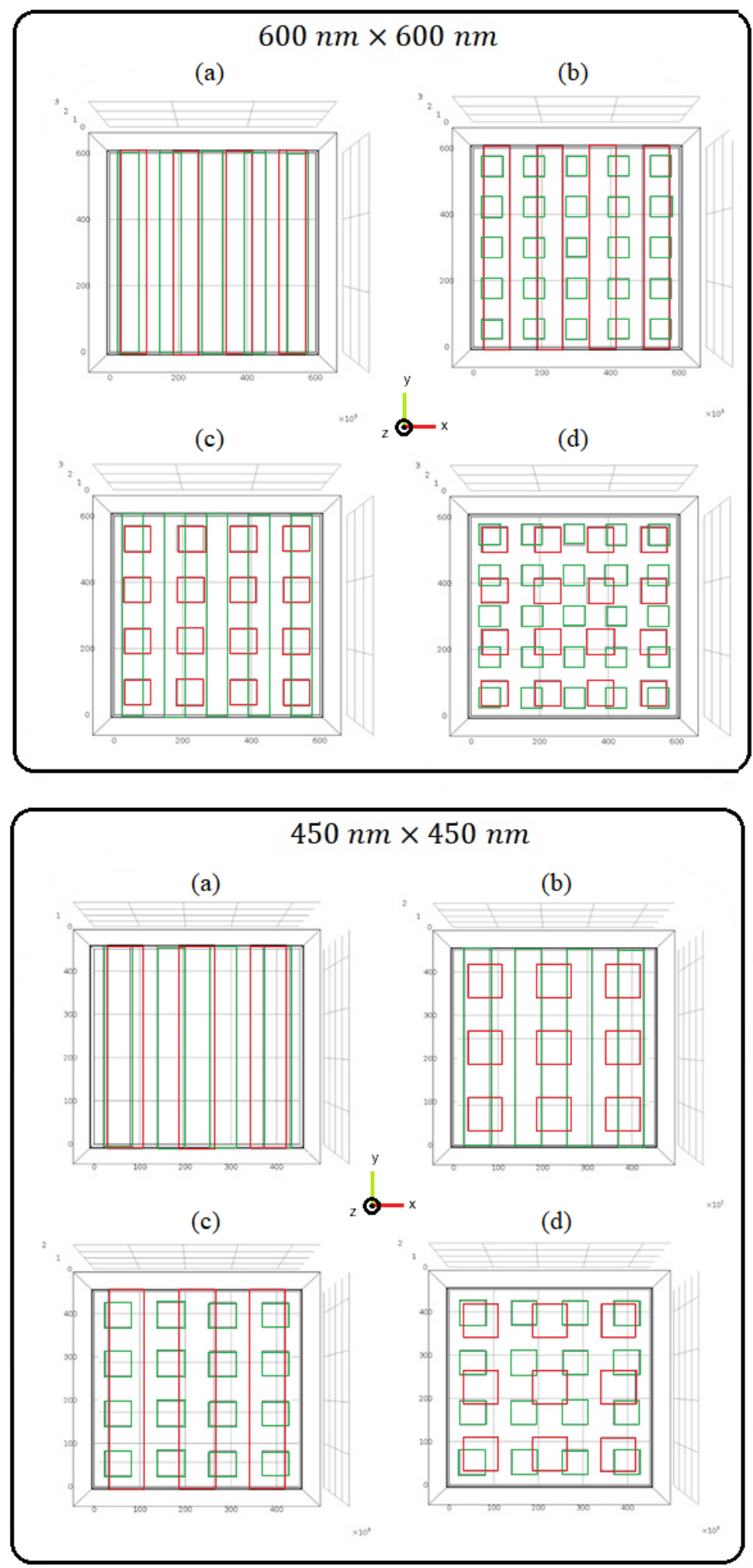

Figure 3-23 Top view arrangement of the object and FSL nanogratings in a 3D model. The nanograting features are shown in red while the object is shown in green. 
Here, the term "1D" specifies an object or nanograting with one dimension extending to infinity, and "2D" object or nanograting describes a structure with known length of feature in all 3 dimensions. The duty cycle was $50 \%$ for both the 1D and 2D features.

After designing the model geometry, we needed to define the elements of our incident wave in 3D. Accordingly, two periodic ports were assigned to our models. The first port was located at the bottom of the domain where incident light was introduced, while the second port was located at the top where it exited the domain. We chose to start with a simple transverse magnetic electromagnetic wave at the first port. The plane of incidence included definitions for the surface normal, $n$, and the wavevector, $k[211]$.

In order to define the electromagnetic wave input to our models, we recall that the magnetic $(H)$ and electric $(E)$ fields are perpendicular to one another, where the following relation is satisfied for the direction of propagation [212]:

$$
\vec{E} \times \vec{H}=\vec{k},
$$

\section{Equation 3-6}

Accordingly, linear polarization in our models was defined using the following steps:

- First, we defined the input and output ports in planes parallel to the $x-y$ plane at the bottom and top planes of the computation domain, respectively. 
- Next, the electromagnetic wave was defined at the input port. Based on Equation 3-6, a TM wave could be represented in terms of either its electric field or magnetic field components. Given the selected wavevector direction in our model, we opted to use the equivalent single magnetic field component instead of the more complex electric field component to define the TM polarized wave.

- Then, it was necessary to define the surface normal vector, $n$, at each port to navigate the modelling space, e.g., knowing the direction of $n$ allowed us to understand whether the domain extended in $+z$ or $-z$ direction, as this affected the set up used to define the periodic boundary condition of the 3D model.

- Next, we defined the incident wavevector, $k$, which is a property of the illuminating wave that shows the direction of propagation. To define the wavevector in a 3D domain, it was necessary to know the angle between the wavevector and the $z$ axis, denoted by $\alpha$, and the angle $\alpha^{\prime}$ between the $x$-axis and the projection of the wavevector on the $x y$-plane. Using these two angles, $\alpha$ and $\alpha^{\prime}$, the components of the wavevector in the Cartesian system are:

$$
\begin{aligned}
& k_{x}=k_{0} \times \cos \left(\alpha^{\prime}\right) \sin (\alpha) \\
& k_{y}=k_{0} \times \sin \left(\alpha^{\prime}\right) \sin (\alpha) \\
& k_{z}=k_{0} \times \cos (\alpha)
\end{aligned}
$$

\section{Equation 3-7}

These components were then applied in the port definition of our 3D models.

- Based on the possible directions for a TM polarized wave in such setup, the wave was incident along the $x$ or $y$ direction, to simplify 
the model's solution without affecting its generality. The suitability of either wavevector directions depended on the alignment of the FSL nanograting.

- The wavevector component in the $z$ direction, $k$, was defined to be evanescent, i.e., not propagating [213].

On that note, plasmonic excitations are created in the form of evanescent surface waves that propagate in the FSL nanograting's plane, which was the $x-y$ plane in our models. The propagation direction for plasmonic excitations was set to be orthogonal to the longest (infinite) dimension of the FSL nanograting [214, 215]. These excitations were set to decay along the vertical direction, $z$.

In such a scenario, having a TM polarized wave with a wavevector component parallel to that of the surface plasmons on the surface of the FSL generates plasmonic excitations that can propagate to the far-field domain [142].

Consequently, we identified the need to evaluate the effects of three different TM polarized wave illuminations on the performance of the FSL. The first TM wave was defined by a magnetic field component along the $x$ axis, $(H \hat{\imath}, 0,0)$, the second TM wave had a magnetic field component along the y axis, $(0, H \hat{\jmath}, 0)$ and the third TM wave was constructed by superimposing these two polarizations, i.e., $(H \hat{\imath}, H \hat{\jmath}, 0)$; where $(\hat{\imath}, \hat{\jmath})$ are the unity vectors along the $x$ and $y$ directions.

The two orthogonal TM waves described above are given by:

The wavevector of a TM polarized wave along the $y$-axis $(0, H \hat{\jmath}, 0)$ has both the $x$ and $z$ components of the electric field, and a $y$ component for the magnetic field. This wave has two components of the wavevector in the $x-z$ plane. Based on Equation 3-6 and Equation 3-7, the wavevector components are derived for the specific case of $\alpha^{\prime}=0$, 


$$
\begin{gathered}
k_{a x}=k_{a} \sin (\alpha) \\
k_{a y}=0 \\
k_{a z}=k_{a} \cos (\alpha)
\end{gathered}
$$

\section{Equation 3-8}

The wavevector of a TM polarized wave along the $x$-axis $(H \hat{\imath}, 0,0)$ has both the $y$ and $z$ components of the electric field, and the $x$ component of the magnetic field. This wave has two components of the wavevector in the $y-z$ plane. For the specific case of $\alpha^{\prime}=90$,

$$
\begin{gathered}
k_{a^{\prime} x}=0 \\
k_{a^{\prime} y}=k_{a} \sin (\alpha) \\
k_{a^{\prime} z}=k_{a} \cos (\alpha)
\end{gathered}
$$

Equation 3-9

Finally, we needed to define the wave equation at the output port. In our models, materials at the input and output ports were different, where we had glass at the input port, and PMMA at the output port, as mentioned in section 3.1. This should be taken into account when defining the wavevector, to adjust for the refractive index of the respective medium.

Fresnel's equation was applied to the wave at the output port where we calculated the transmitted plane wave through the planar interface [216]. In this study, the incident wavevector at the first medium was defined as $k_{a}$ and the transmitted wavevector in the last medium was defined as $k_{b}$. 
Moreover, according to Snell's law, passing from one medium to the other changes the angle of propagation, which in the new medium we name $\beta$ [217]:

$$
\beta=\arcsin \left(\frac{n_{g} \sin (\alpha)}{n_{p m m a}}\right)
$$

Equation 3-10

where $\alpha$ is the angle of incidence with respect to the surface normal and $\beta$ is the angle of refraction. The refractive index of the glass substrate or the first medium in which light enters is $n_{g}$, and the refractive index of PMMA which is used as a spacer and is the last medium before the output port is $n_{P M M A}$. Material properties used in this 3D models are identical to those used in the 2D models.

In the next subsections of this chapter, we present our 3D modelling results for two defined minimal domain sizes, where we consider the effects of geometry and illumination settings, then consider the scenario when the incident light has two superimposed orthogonal polarizations.

\subsubsection{Minimal cell of $(450 \mathrm{~nm} \times 450 \mathrm{~nm})$}

In this section, the 3D domain for the considered models had a minimal cell size of $450 \mathrm{~nm} \times 450 \mathrm{~nm}$. To preserve the reflectional symmetry of each model, the object had a period of $112.5 \mathrm{~nm}$ while the FSL nanograting had a period of $150 \mathrm{~nm}$. We start with a simple TM polarized wave having a non-oscillatory component in the $z$ direction [97]. 
The magnetic field component of this wave is given by:

$$
H_{y}=\exp i\left(k_{a x} x\right) \exp \left(-k_{a z} z\right)
$$

Equation 3-11

Using this equation, we solve the four models that include all possible combinations of the 1D/2D object and nanogratings (as shown in Figure 3-23).

In the first arrangement, the object and the FSL nanograting were both one dimensional features that lied on planes parallel to the $x-y$ plane. The long features of the one-dimensional object and nanograting were extended along the $y$ direction. Figure 3-24 shows a 3D representation of the resulting electric field norm. 

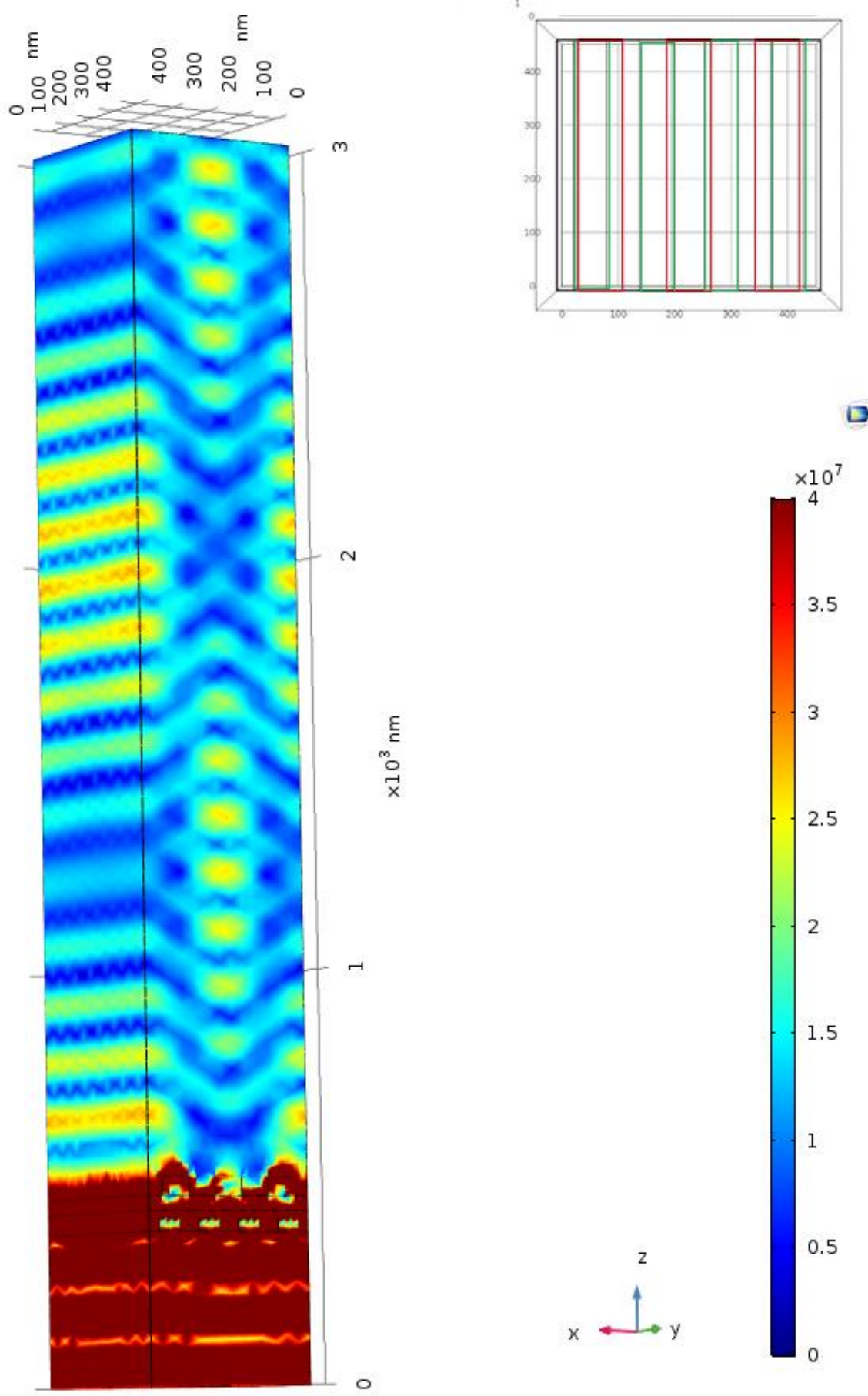

Figure 3-24 Electric field norm of a 3D model with a 1D object and a 1D

FSL nanograting. The top right image illustrates the simulated arrangement, where the nanograting features are shown in red while the object is shown in green. 
It can be seen that the obtained results for the 3D model were similar to the familiar results obtained for our 2D models. This was a promising outcome given the different settings and definitions these models used. Thus, the Moiré and Talbot effects are observed in this plot, where the relevant fringes showed the expected periodicity given the model's parameters.

Given that the propagating wavevector was along the $x$ direction, the Moiré pattern was created in the $x-z$ plane, while the $y-z$ plane did not show any specific fringes except those associated with the cross-section of the pattern in the $x-z$ plane. This is related to the placement of the nanogratings with their longer dimension extending along the $y$ axis, while the incident propagating wavevector was perpendicular to them. Therefore, object features are resolvable as they periodically extended along the $x-z$ plane.

The next arrangement studied in this section was the case of a 1D object and a 2D FSL nanograting, as shown in Figure 3-23(b). In this setup the nanogratings cannot be seen as they do not intersect with the minimal cell boundaries.

Using the TM polarized wave described in Equation 3-11 along with the 1D object - 2D FSL nanograting model, the resulting electric field norm amplitude plot is shown in Figure 3-25. The single change in the design of this model compared to that of Figure 3-24 was that the FSL nanogratings had a 2D arrangement instead of 1D. 

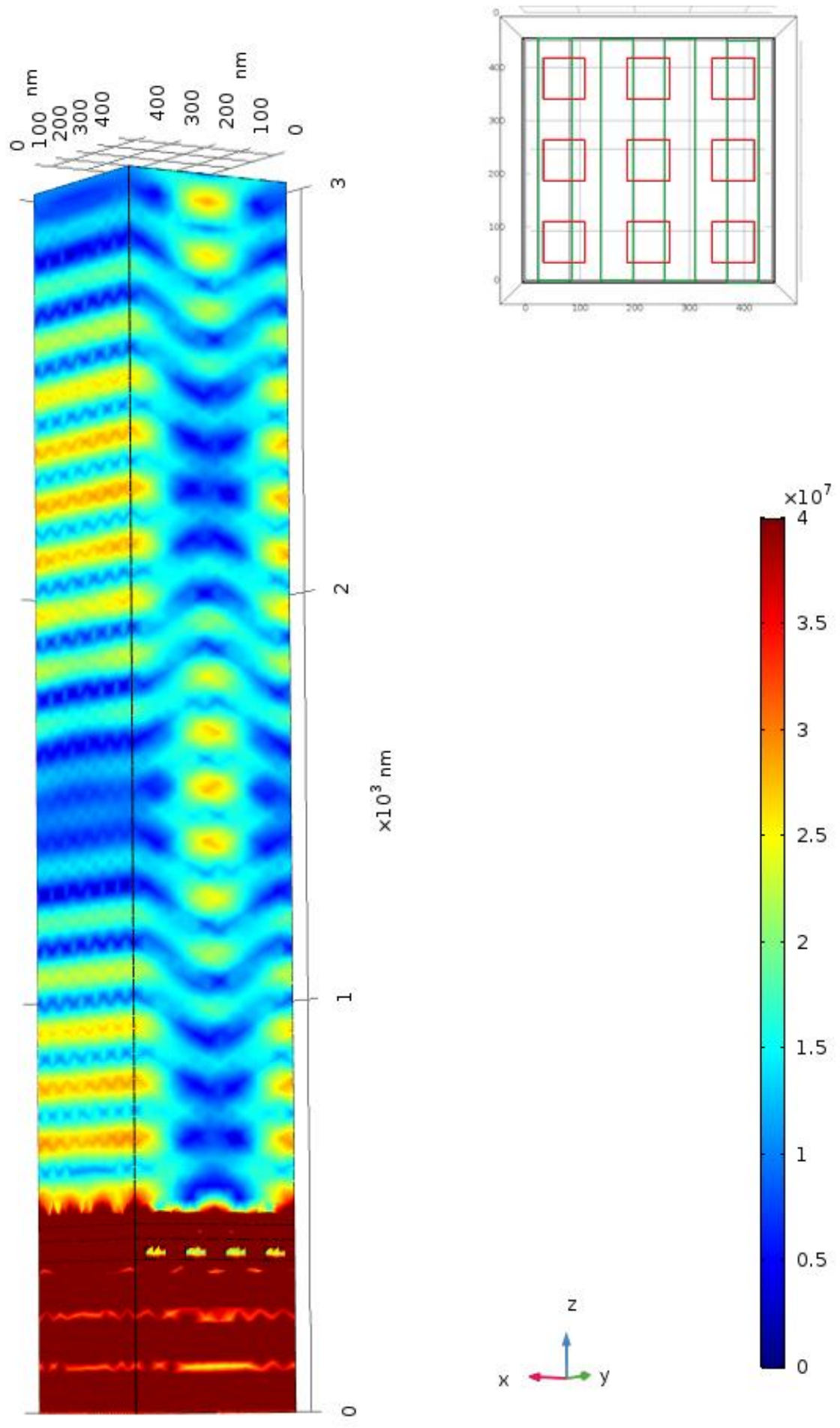

Figure 3-25 Electric field norm of a 3D model with a 1D object and a 2D

FSL nanograting. The top right image illustrates the simulated arrangement, where the nanograting features are shown in red while the object is shown in green. 
The resulting far-field interaction was similar to that of the 1D object - 1D FSL nanograting scenario. However, a higher field amplitude in $y$-z plane was observed when compared to Figure 3-24. This could be attributed to the larger field enhancement effect resulting from the higher number of corners in the $2 \mathrm{D}$ FSL nanograting case.

To our knowledge, this is the first time the far-field pattern of a FSL with 2D nanograting has been simulated in a 3D model. The properties of the far-field pattern created in the $x-z$ plane of Figure 3-25, in terms of the pattern shape and Moiré period, were identical to Figure 3-24 where a 1D FSL nanograting were used. This similarity is caused by the selected direction of the wavevector of the incident wave where no perpendicular component of the wavevector in the $y$ direction existed. Therefore, although the FSL nanograting was $2 \mathrm{D}$ and had the potential to enhance plasmonic excitations in both the $x$ and $y$ directions, the farfield Moiré pattern was only created in the $x$-z plane because the incident wavevector did not have a $y$ direction component. It should be noted that the observed Talbot distance was longer than that for the 1D object-1D FSL nanograting scenario, which suggests that the plasmonic excitations propagated further into the far-field region, likely due to electric field enhancement at the edges of the 2D FSL nanograting features.

The third arrangement studied in this section had a 2D object and 1D FSL nanograting. Similarly, this model utilized the TM polarized wave from Equation 3-11. The resulting electric field norm amplitude plot is shown in Figure 3-26. 


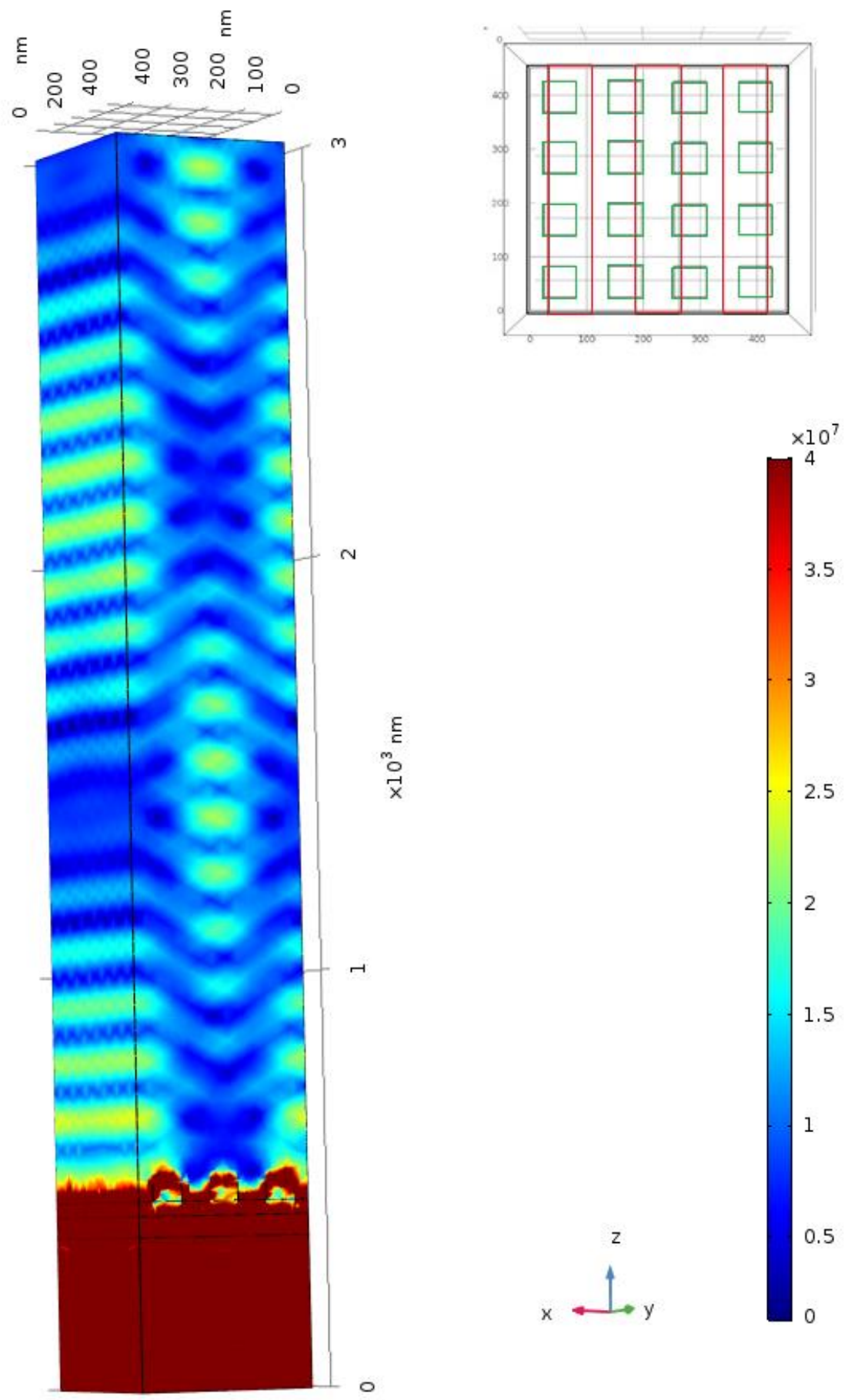

Figure 3-26 Electric field norm of a 3D model with a 2D object and a 1D

FSL nanograting. The top right image illustrates the simulated arrangement, where the nanograting features are shown in red while the object is shown in green. 
The far-field Moiré and Talbot patterns were created in the $x$-z plane, similar to previous arrangement. The Talbot distance in this case was less extended to the far-field when compared to the second arrangement, however, it still was farther than that of the first arrangement, possibly due to field enhancement of the evanescent waves by the edges of the $2 \mathrm{D}$ object. The creation of these patterns in the $x-z$ plane is again attributed to the direction of the propagating wavevector of the light and the alignment of the 1D FSL nanograting in the $y$ direction. Plasmonic excitations within our models did not seem to depend on the number of dimensions of the object structure (1D or $2 \mathrm{D}$ objects) as the incident wavelength did not match the plasmon resonance wavelength for chromium. However, a 2D object structure resulted in higher optical loss, probably due to light scattering and diffraction at the edges when compared to a 1D object.

The final arrangement studied in this section had both the object and the FSL nanograting composed of 2D features. Similarly, this model utilized the TM polarized wave from Equation 3-11. The resulting electric field norm amplitude plot is shown in Figure 3-27. Based on the arrangement of the object and FSL nanograting in the minimal cell, no cross-section of the object or FSL features was observed in the electric field norm plot as all constituents lied within the cell boundaries.

Despite having 2D features for both the object and FSL nanograting, the farfield showed the Moiré and Talbot patterns only in the $x-z$ plane, which again is attributed to the direction of the propagating wavevector of the light. This signifies the importance of evaluating the effects of illumination on the FSL farfield in 3D. 


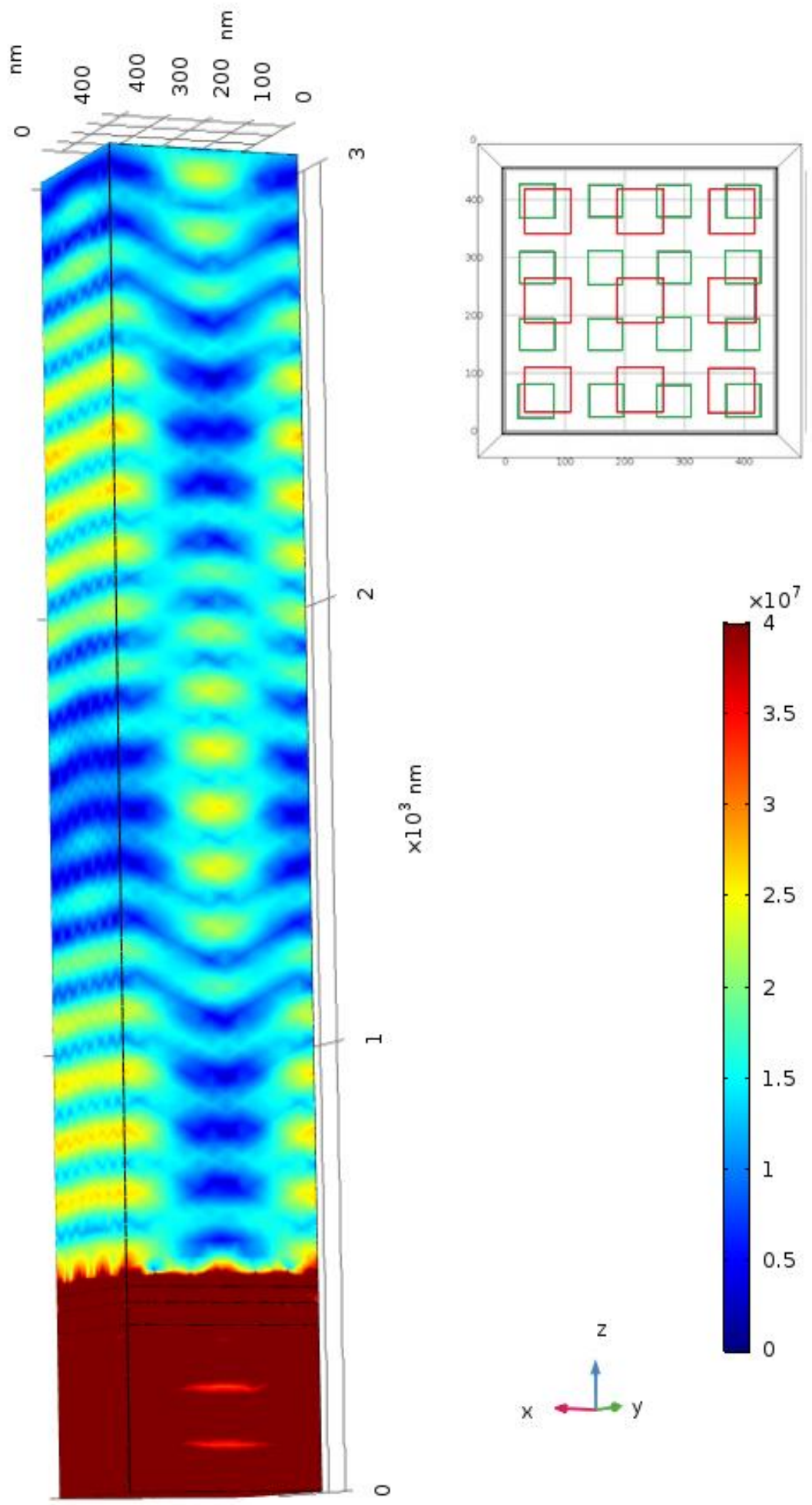

Figure 3-27 Electric field norm of a 3D model with 2D object and 2D FSL nanograting. The top right image illustrates the simulated arrangement, where the nanograting features are shown in red while the object is shown in green. 
It should be noted that the Talbot distance was the farthest of all arrangements in this scenario, probably due to the duel field enhancement effect by the edges of the 2D object and 2D FSL nanograting.

To summarize the aforementioned results, all four models presented from Figure 3-24 to Figure 3-27 had visual similarities in terms of the far-field patterns. This indicates that given a symmetric design, any combination (1D and 2D) of our object and FSL nanograting would be suitable to obtain a Moiré pattern with the correct size in the far-field. Therefore, by knowing the properties of the incident light and the FSL used for imaging, estimation of the object period is possible. Talbot distance on the other hand seemed to depend on the dimensions of the underlying features, where 2D features seemed to make the distance longer when compared to $1 \mathrm{D}$ features. It should be noted that there was a difference in the contrast obtained in the far-field patterns of each plot. Figure 3-28 shows the electric field norm over the image cutline at the Talbot length in the $x$ - $z$ plane for all four arrangements. These were extracted from Figure 3-24 to Figure 3-27. 


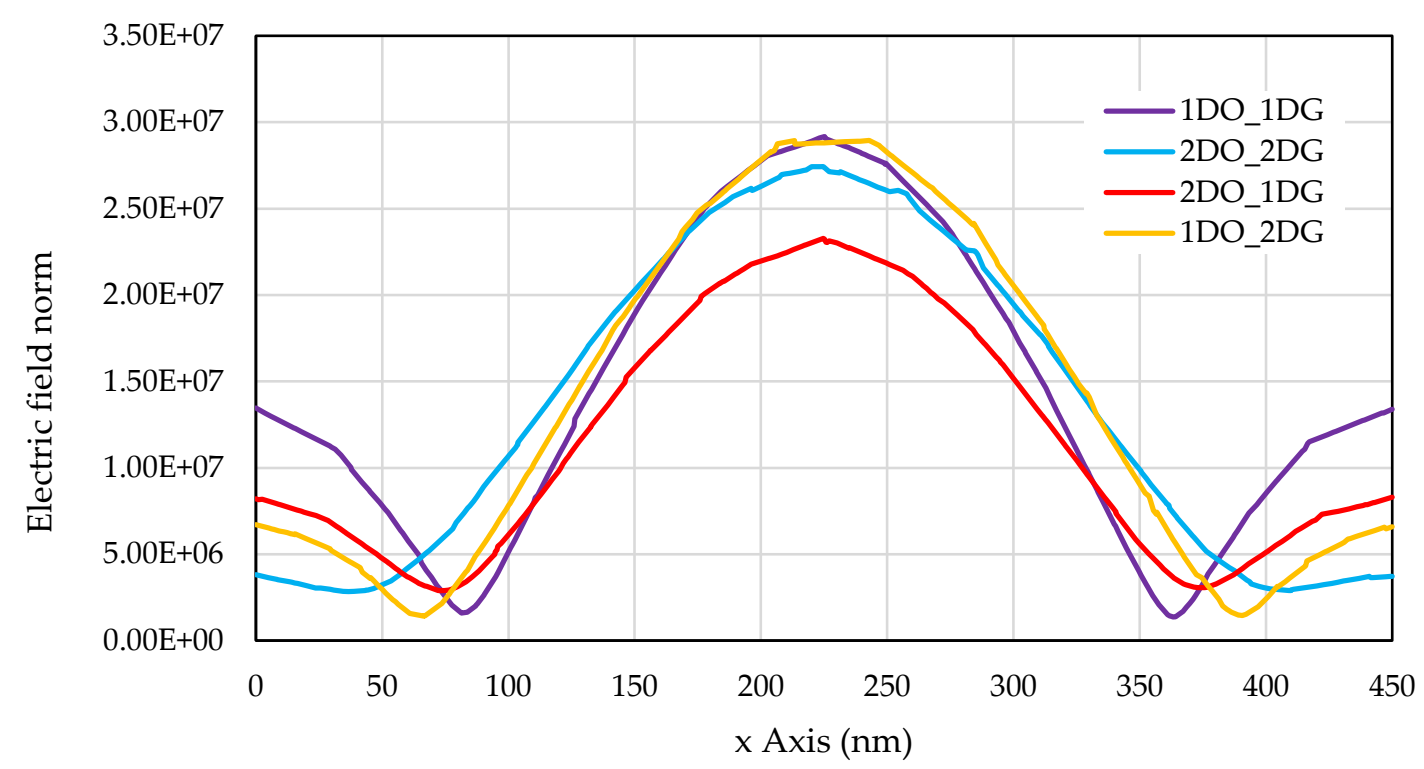

Figure 3-28 Electric field norm line plots at the Talbot length of the models in Figure 3-24 to Figure 3-27.

The red curve represents the lowest peak amplitude of the electric field norm from Figure 3-26 with a 2D object and 1D nanograting design. This is followed by the blue curve, which represents the 2D object and 2D nanogratings. The plots representing 1D objects and 1D nanogratings and 1D objects and 2D nanogratings have the maximum amplitude peaks.

In the case of the red curve, having a 2D object arrangement in the design implies more edges and a larger surface area; hence higher scattering and diffraction of light occurs as it passes through the object before reaching the FSL. This could be the reason for the red curve having the least amplitude.

The blue curve also represents an object geometry comprised of a 2D nanofeatures, however, although the scattering and diffraction effects of the 2D object should decrease the far-field amplitude, using 2D FSL nanograting contributed to the local field enhancement that justify an increased peak amplitude $[218,219]$. 
The yellow curve with 1D object and 2D nanogratings has slightly larger full width at the half maximum when compared to the violet curve for $1 \mathrm{D}$ object and 1D nanogratings. This could be related to local field enhancement at 2D objects.

Based on the curves from Figure 3-28, we calculate the contrast for each model according to Equation 3-5. These results are presented in Table 3-4 along with the observed Talbot lengths for these models.

Table 3-4 Calculated contrast for the four different models.

\begin{tabular}{|c|c|c|c|c|}
\hline Model name & 1DO_1DG & 1DO_2DG & 2DO_1DG & 2DO_2DG \\
\hline Contrast & 0.91 & 0.91 & 0.78 & 0.81 \\
\hline Talbot length (nm) & 785 & 1085 & 940 & 1145 \\
\hline
\end{tabular}

The Talbot lengths represented in Table 3-4 are of the same order of magnitude to that of the simple theoretical length given by Equation 1-40, which was 1560 $\mathrm{nm}$ for this model. However, the Talbot length equation represents a theoretical estimation for the periodic optical interactions of a simple case of two sets of 1D periodic features and not a combination of $1 \mathrm{D}$ and $2 \mathrm{D}$ periodic features as in our study [203]. 


\subsubsection{Minimal cell of $(600 \mathrm{~nm} \times 600 \mathrm{~nm})$}

A second minimal cell size was simulated using the same model settings. To preserve reflectional symmetry in these designs, selection of proper object and FSL nanograting periods had to done. Accordingly, the object was set to have a $120 \mathrm{~nm}$ period while the FSL nanograting had a period of $150 \mathrm{~nm}$. Duty cycle remained $50 \%$.

A main aim for the studies in this section was to examine the effects of illumination on the far-field region of the FSL. Thus, we assumed a 1D object 1D FSL nanograting setup, with the extended dimension of these periodic features being along the $y$ axis. Then, in a first scenario, we defined a TM polarized wave where the magnetic field was given by:

$$
H_{x}=\exp \left(i k_{a y} y\right) \exp \left(-k_{a z} z\right)
$$

Equation 3-12

Based on the wave polarization, the magnetic field has only one component in the $x$ direction. This TM polarized wave had a $90^{\circ}$ shift when compared with Equation 3-11 that was used in the previous section. The propagating wavevector in Equation 3-12 is $k_{a y}$ meaning that it travels in the $y$ direction along the length of the $1 \mathrm{D}$ objects (rather than the transverse direction used in the previous section). The decaying wavevector was kept similar to Equation 3-11, i.e., $k_{a z}$, hence the wave decayed along the thickness of the features and the length of the domain, in the $z$ direction.

In a second scenario, we defined a TM polarized wave with the magnetic field along the $z$ direction: 


$$
H_{z}=\exp \left(i k_{a y} y\right) \exp \left(-k_{a x} x\right) .
$$

Equation 3-13

Thus, propagation of this wave was along the $y$ direction, while the wave decayed along the $x$ direction.

As expected, simulation results for models with illumination defined by Equation 3-12 and Equation 3-13 showed no far-field pattern after the FSL. An example of the resulting electric field norm amplitude plot is shown in Figure 3-29 where the model's illumination was set according to Equation 3-12, i.e., light propagates along the $y$ axis and decays along the $z$ axis. As can be seen, the amplitude is zero everywhere in the far-field region.

This verified the requirements for enabling imaging through a FSL [142, 220], where correct selection of light polarization and propagation direction with respect to the alignment of the FSL nanograting is crucial. 


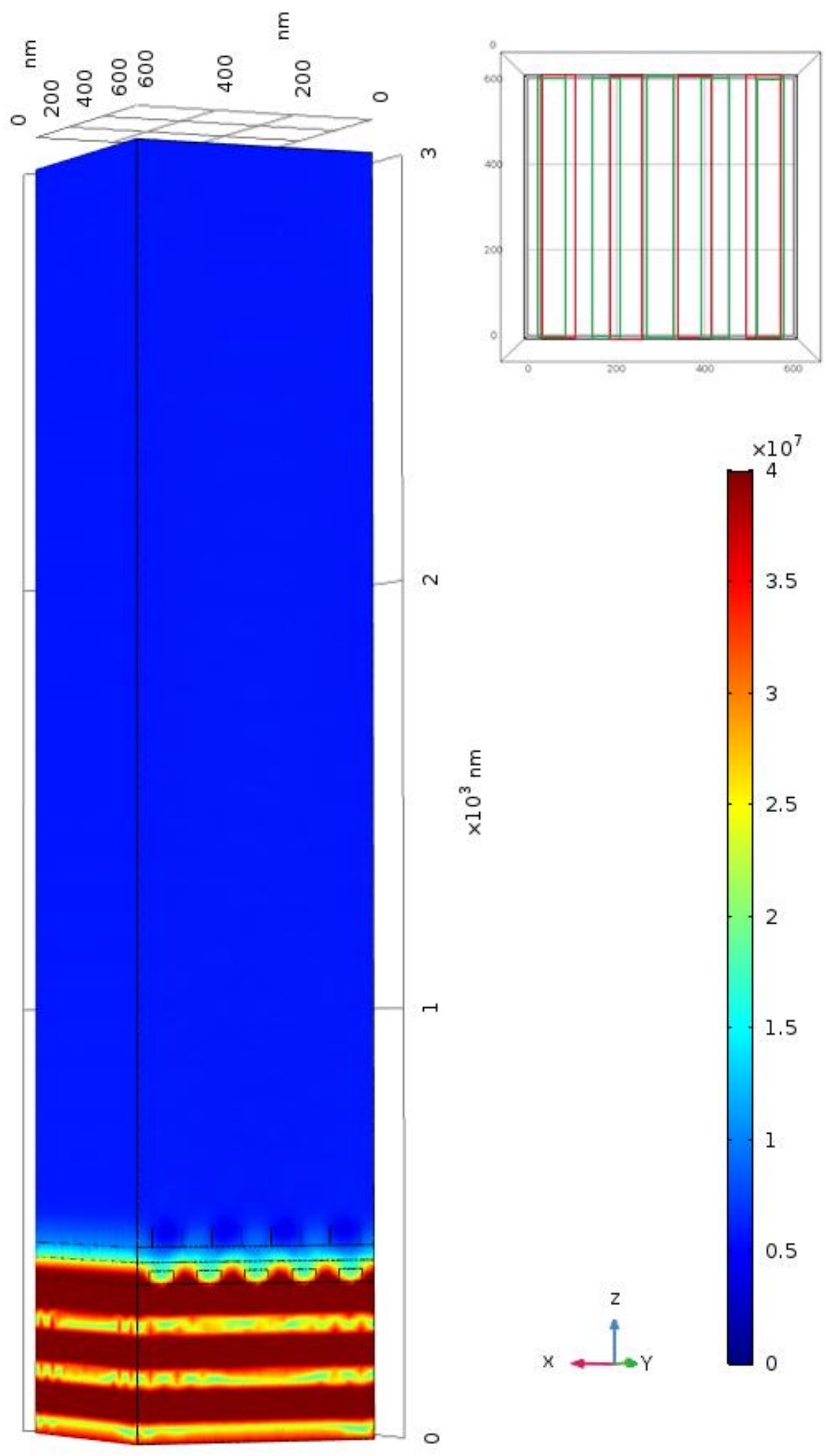

Figure 3-29 The electric field norm amplitude plot for a design with a 1D object and a 1D FSL nanograting, where the illuminating wavevector was propagating along the $y$ axis. The top right image illustrates the simulated arrangement, where the nanograting features are shown in red while the object is shown in green. 


\subsubsection{Illuminating with two waves}

In this section, we model more complex settings of illumination, in specific, wave packets that are generated when linearly polarized light sources are superimposed [217]. The first TM polarized wave that we considered for this study is given by:

$$
\left(H_{x}, H_{y}, H_{z}\right)=\left(0, e^{-i\left(k_{x} x+k_{z} z\right)}, 0\right)
$$

Equation 3-14

This equation represents a TM polarized wave with propagating wavevectors in the $x$ and $z$ directions. We also considered a similar illumination where the TM polarized wave was propagating along the $y$ and $z$ directions:

$$
\left(H_{x}, H_{y}, H_{z}\right)=\left(e^{-i\left(k_{y} y+k_{z} z\right)}, 0,0\right)
$$

Equation 3-15

Although all wavevector components in Equation 3-14 and Equation 3-15 have a propagating nature, only the in-plane wavevectors are able to excite plasmonic excitations.

In order to study the effects of the illuminating wavevector direction with respect to the FSL and object, we modelled a design comprised of $2 \mathrm{D}$ objects and 2D nanogratings. The waves were applied at the input ports of each model. The 
domain size was set to be $600 \mathrm{~nm} \times 600 \mathrm{~nm}$, the 2D object had a period of $120 \mathrm{~nm}$ and the 2D FSL nanograting had a period of $150 \mathrm{~nm}$.

Figure 3-30 and Figure 3-31 show the electric field norm amplitude plots for models illuminated according to Equation 3-14 and Equation 3-15, respectively. 


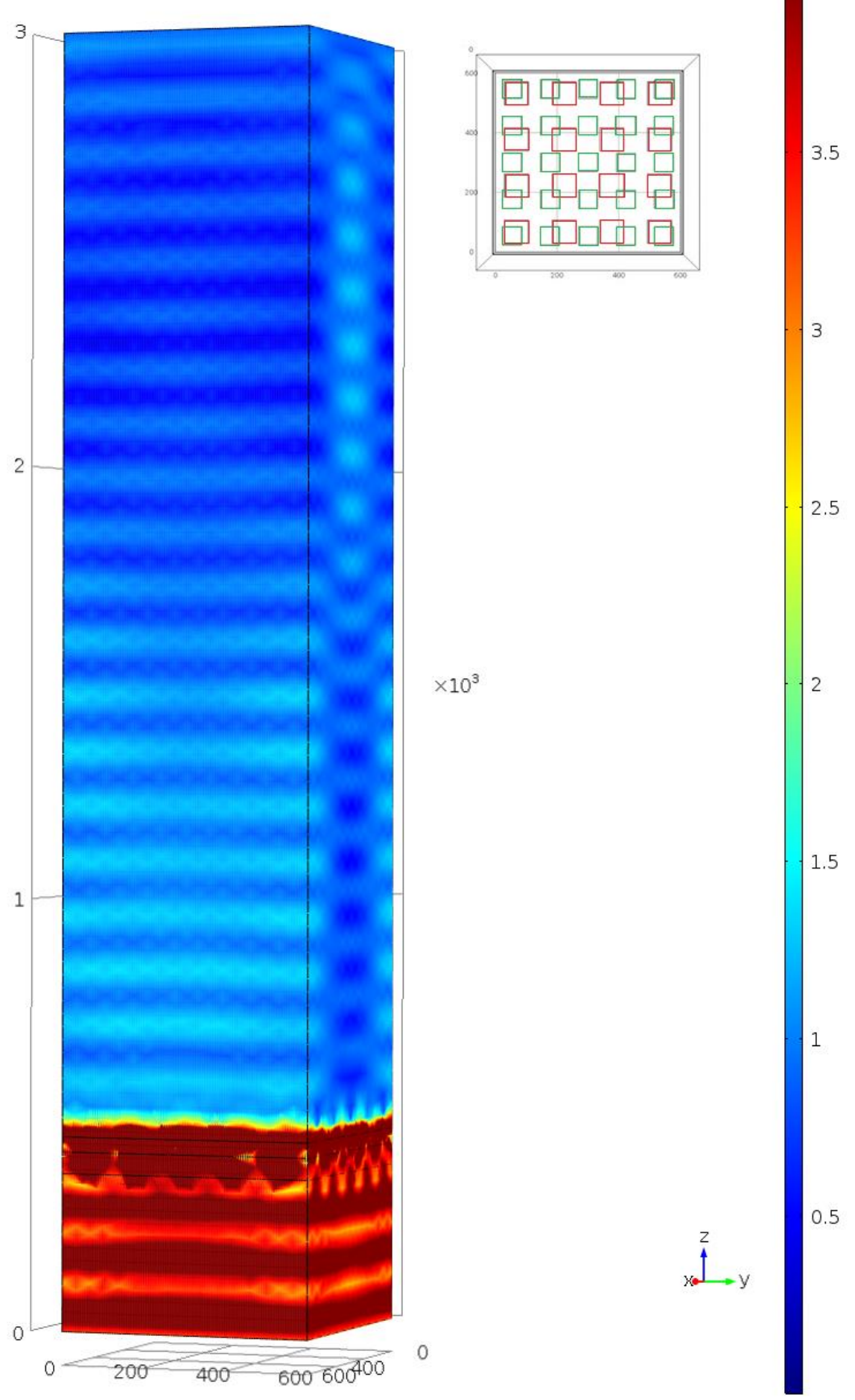

Figure 3-30 Electric field norm amplitude plot for a model with a 2D object and a 2D FSL nanograting, where the illuminating wave was set according to Equation 3-14. The top right image illustrates the simulated arrangement, where the nanograting features are shown in red while the object is shown in green. 


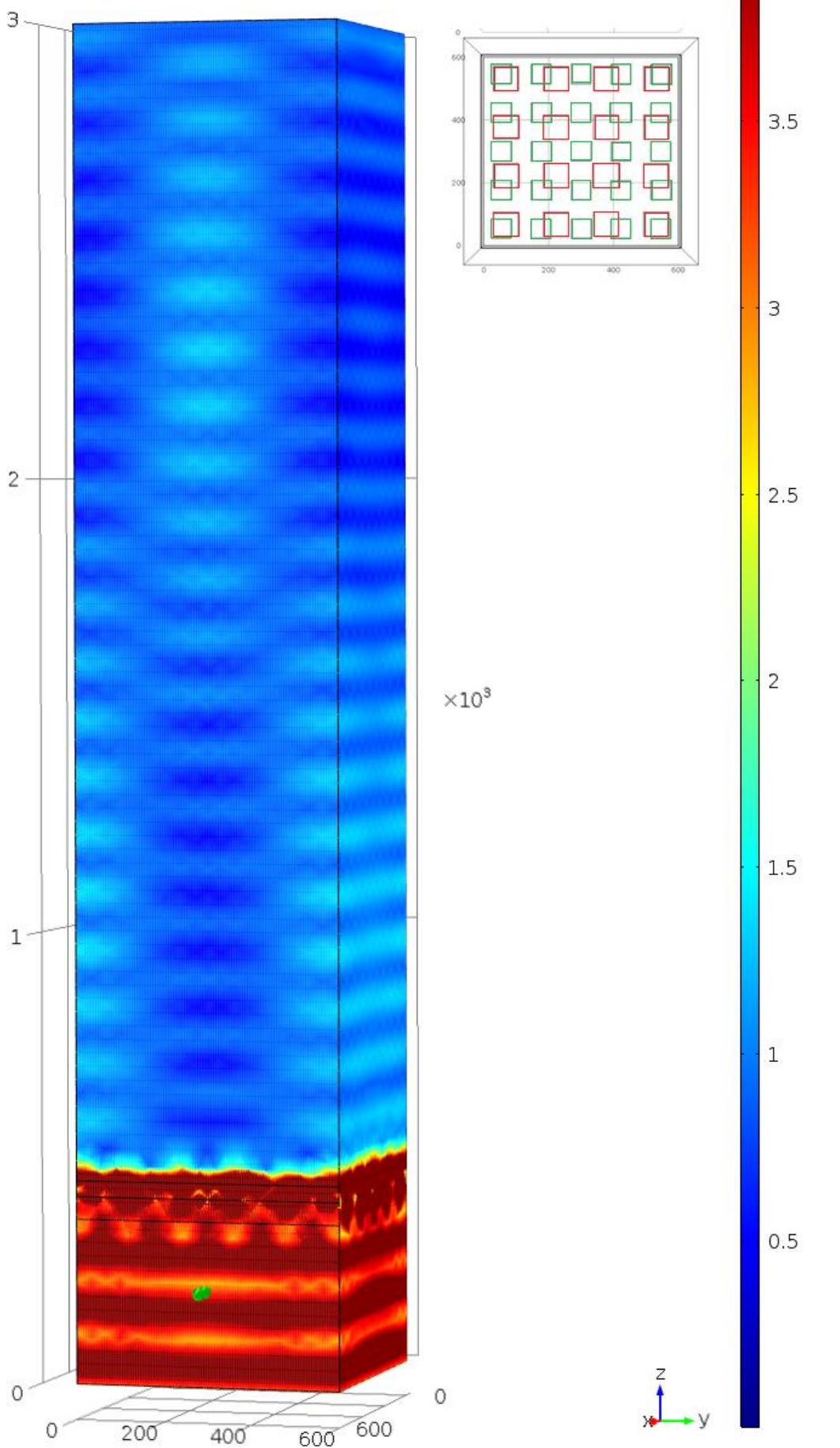

Figure 3-31 Electric field norm amplitude plot for a model with a 2D object and a 2D FSL nanograting, where the illuminating wave was set according

to Equation 3-15. The top right image illustrates the simulated arrangement, where the nanograting features are shown in red while the object is shown in green. 
It can be seen that the plane in which the far-field Moire pattern was created was the $x-z$ plane in Figure 3-30 and the $y-z$ plane in Figure 3-31. These far-field patterns observed in both models were identical, except for the $90^{\circ}$ rotation around the $z$-axis that stemmed from the illuminating wavevector directions. Therefore, the far-field created in each case provided unique information about one specific face of the object, which was perpendicular to the incident propagating wavevector.

Finally, we considered a third illumination scenario were the wave equation is given by:

$$
\left(H_{x}, H_{y}, H_{z}\right)=\left(e^{-i\left(k_{y} y+k_{z} z\right)}, e^{-i\left(k_{x} x+k_{z} z\right)}, 0\right)
$$

Equation 3-16

This wave has two magnetic field components in the $x$ and $y$ directions, i.e., $H_{x}$ and $H_{y}$, and propagating wavevector components in the $x, y$, and $z$ directions.

Using the same object and FSL grating arrangement as in the previous models of this section, the resulting electric field norm amplitude plot is shown in

Figure 3-32. 

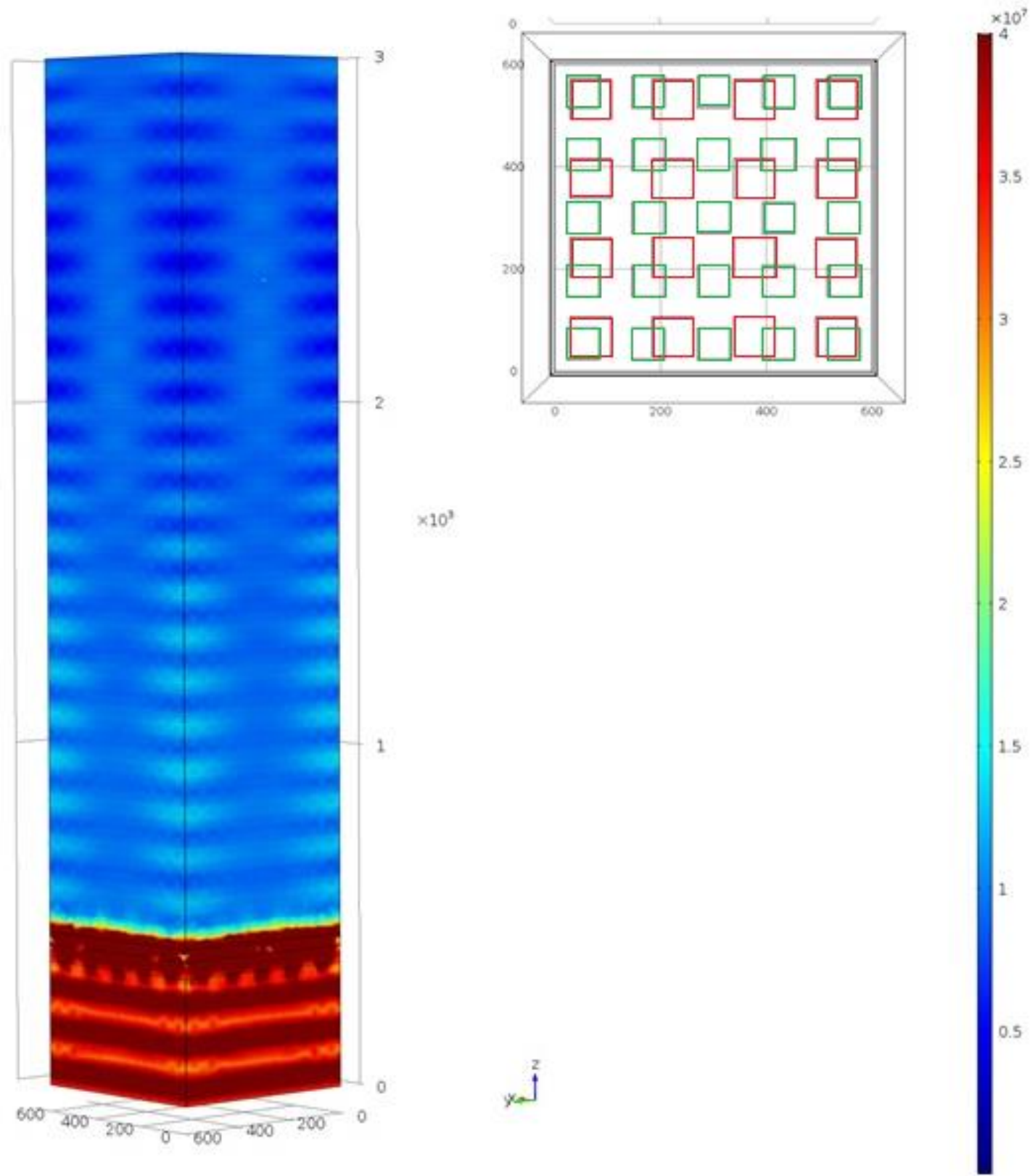

Figure 3-32 Electric field norm amplitude plot for a model with a 2D object and a 2D FSL nanograting, where the illuminating wave was set according

to Equation 3-16. The top right image illustrates the simulated arrangement, where the nanograting features are shown in red while the object is shown in green. 
Results in this case confirmed that by using the correct FSL geometry and incident illumination, far-field patterns containing information about 2D objects can be seen in both $x-z$ and $y-z$ planes. Therefore, illuminating the design with two linearly polarized waves (parallel propagation to object-FSL plane) provides the opportunity to resolve more information about 2D objects using a FSL with 2D nanogratings.

In order to identify the information that could possibly be lost when a $1 D$ FSL nanograting is employed, we modelled a 2D object under a 1D FSL nanograting. The 1D FSL nanograting was aligned in the $y$ direction. The domain size was kept to $600 \mathrm{~nm} \times 600 \mathrm{~nm}$, with an object period of $120 \mathrm{~nm}$ and a 2D FSL nanograting period of $150 \mathrm{~nm}$. For such settings, we considered all three illumination cases described in Equation 3-14 to Equation 3-16. The resulting electric field norm amplitude plots are shown in Figure 3-33 to Figure 3-35, respectively. 


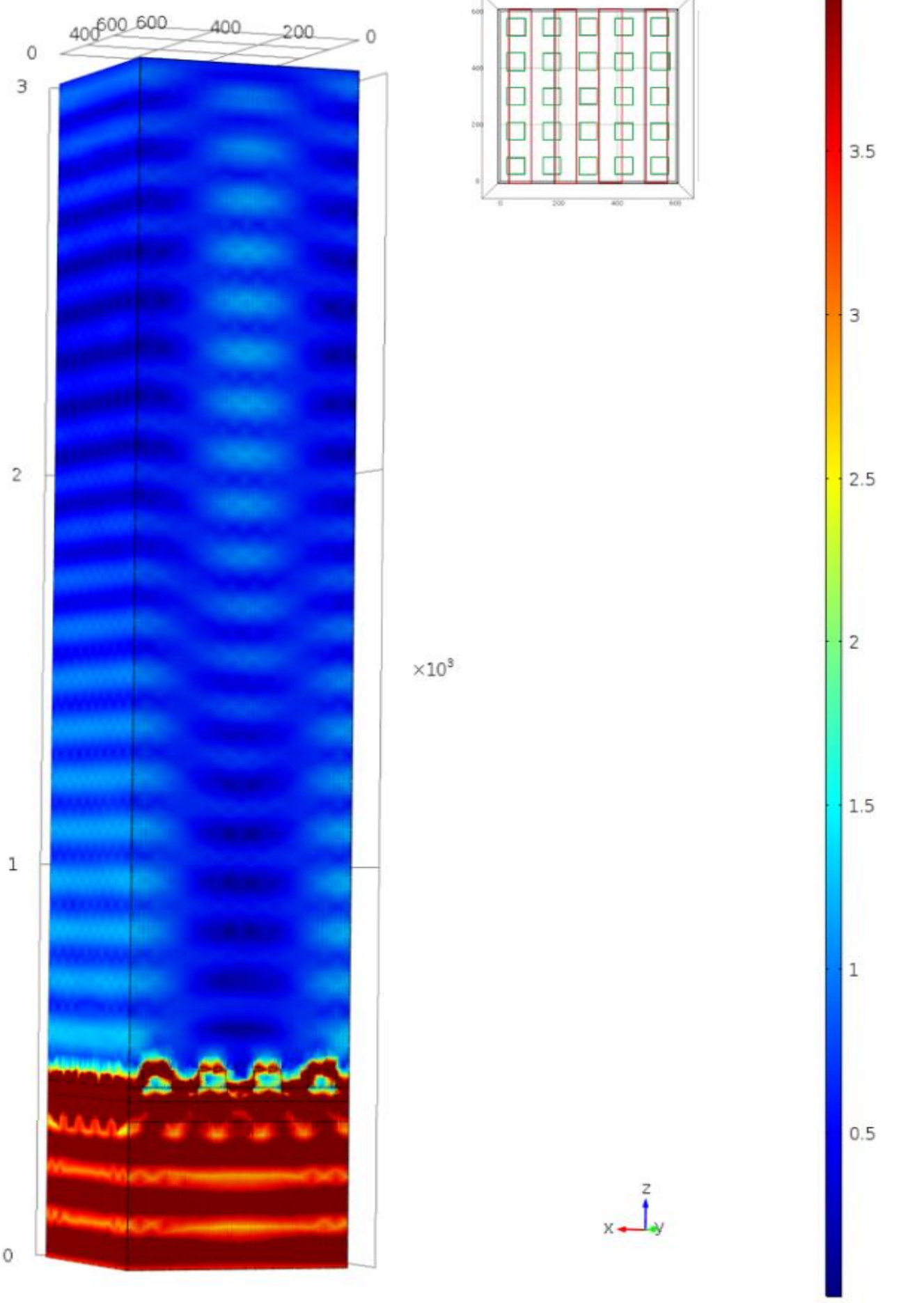

Figure 3-33 Electric field norm amplitude plot for a model with a 2D object and a 1D FSL nanograting, where the illuminating wave was set according

to Equation 3-14. The top right image illustrates the simulated arrangement, where the nanograting features are shown in red while the object is shown in green. 

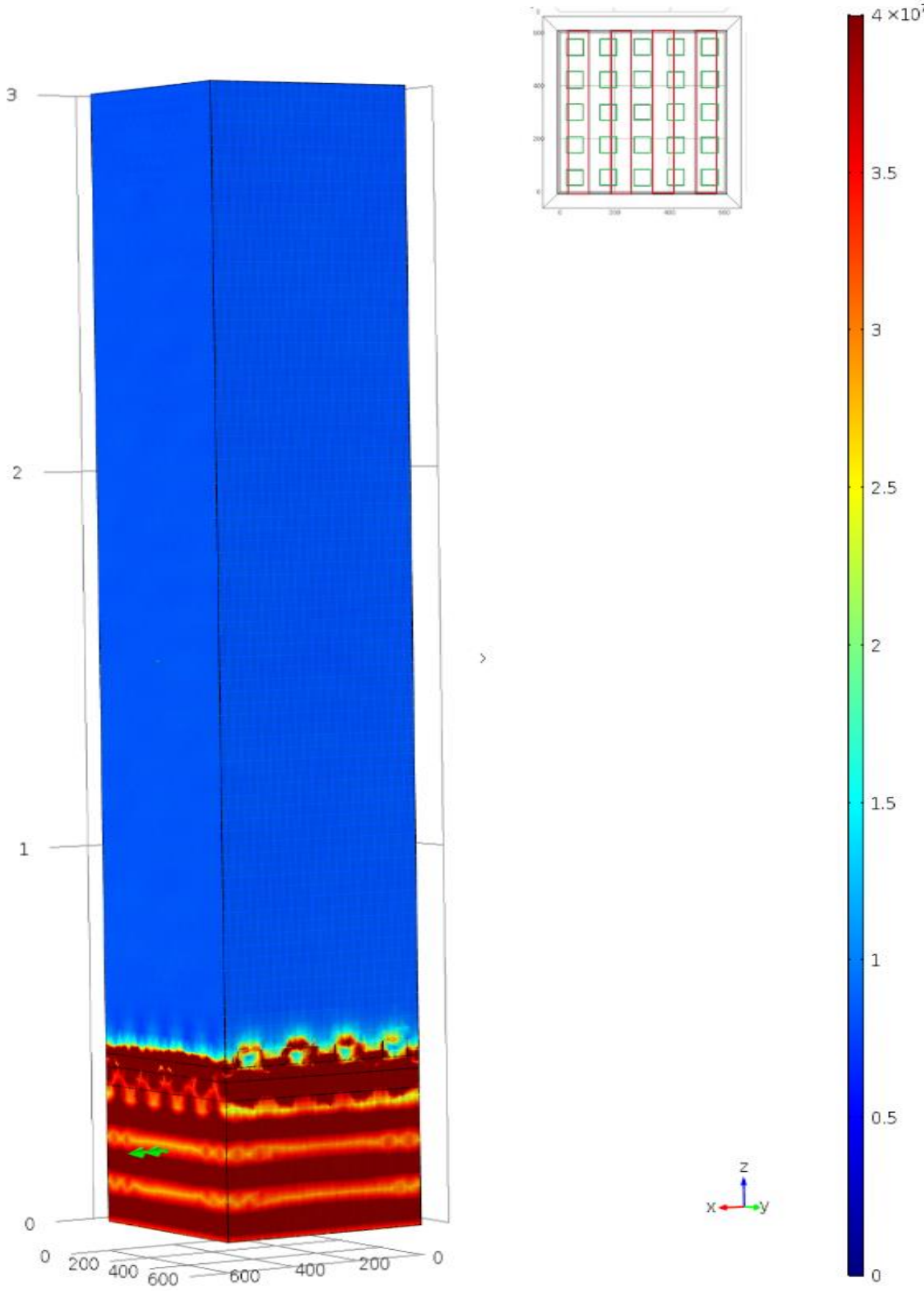

Figure 3-34 Electric field norm amplitude plot for a model with a 2D object and a 1D FSL nanograting, where the illuminating wave was set according

to Equation 3-15. The top right image illustrates the simulated arrangement, where the nanograting features are shown in red while the object is shown in green. 

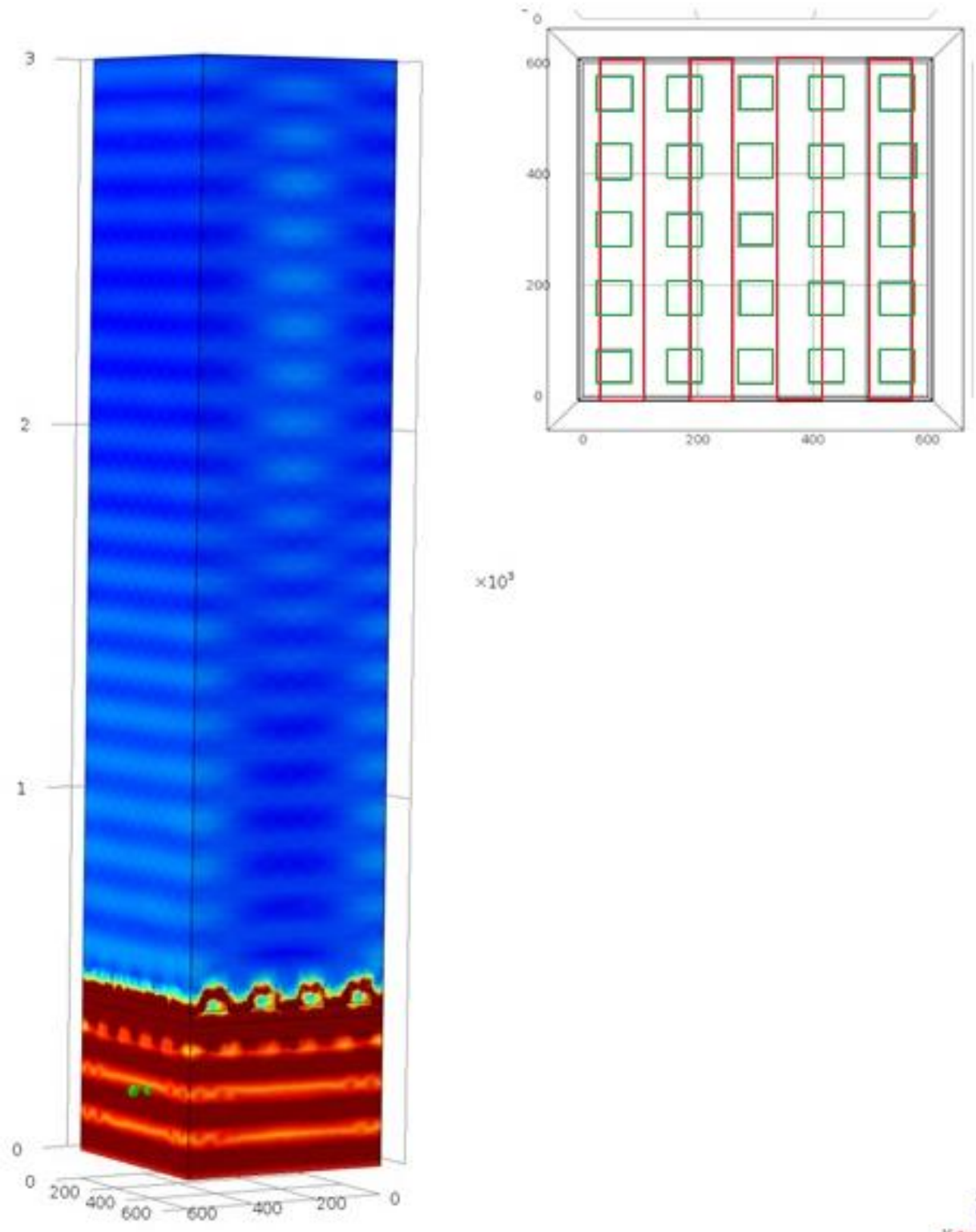

Figure 3-35 Electric field norm amplitude plot for a model with a 2D object and a 1D FSL nanograting, where the illuminating wave was set according

to Equation 3-16. The top right image illustrates the simulated arrangement, where the nanograting features are shown in red while the object is shown in green. 
In the amplitude plot of Figure 3-33 alignment of the FSL nanograting was perpendicular to the $k_{x}$ component of the illuminating wave, thus, the far-field Moiré pattern was created in the $x-z$ plane.

Considering Figure 3-34, the amplitude plot showed no far-field Moiré patterns due the lack of correct illumination (no $k_{x}$ ) with respect to the FSL nanograting.

Finally, the far-field pattern in

Figure 3-35 is a superimposition of Figure 3-33 and Figure 3-34. Accordingly, the result is similar to that of Figure 3-33 given that only the $k_{x}$ illumination contributed to the plasmonic excitations along the $x$-z plane.

Figure 3-36 shows amplitude plots at the image cross-section of our models of

Figure 3-32 and

Figure 3-35. These plots represent the in-plane electric field norm amplitude at the observed Talbot lengths for these models.
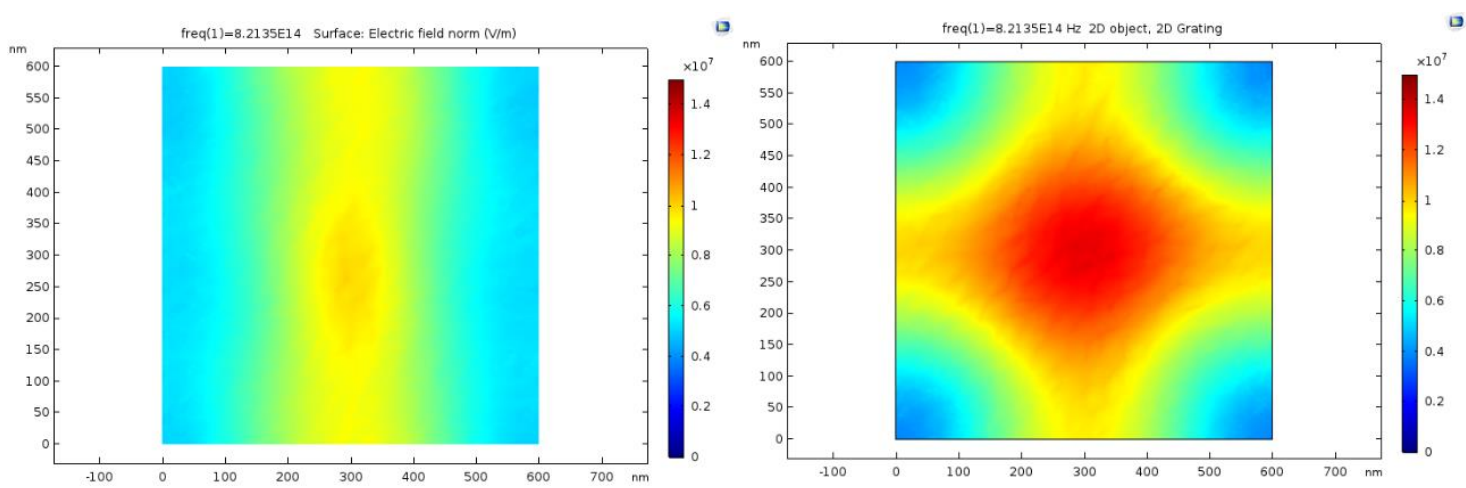

Figure 3-36 Electric field norm amplitude plots in the x-y plane crosssection of our models at the Talbot length. The (left) plot shows the result using the 1D FSL nanograting while (right) shows the resulting far-field using a 2D FSL nanograting, both gratings had a period of $150 \mathrm{~nm}$. The object was 2D with a period of $120 \mathrm{~nm}$ for both cases. 
The electric field amplitude at the Talbot length created a feature in two dimensions for the model in

Figure 3-32 versus the one dimensional feature for the model in

Figure 3-35. While the object and illuminating wave were similar in both designs, the 2D FSL nanograting lens resulted in a higher amplitude, better contrast and more resolved information about the object. This is due to the ability of such a FSL grating to achieve plasmonic excitations along two orthogonal directions, one of which is the excitation captured in the 1D FSL nanograting scenario.

\subsection{Conclusion}

To the best of our knowledge, reported literature that discusses the far-field pattern of a far-field superlens is not elaborative and the performance of a farfield superlens is not widely understood. In this study, we described the relationship between object geometry and the electromagnetic field in the farfield region of a FSL. The far-field patterns presented in this study are in the form of electric field norm amplitude plots. These plots follow the coherent approach in optical imaging where all beams at the output have the same phase relationship as time varies (time independent) [29]. 


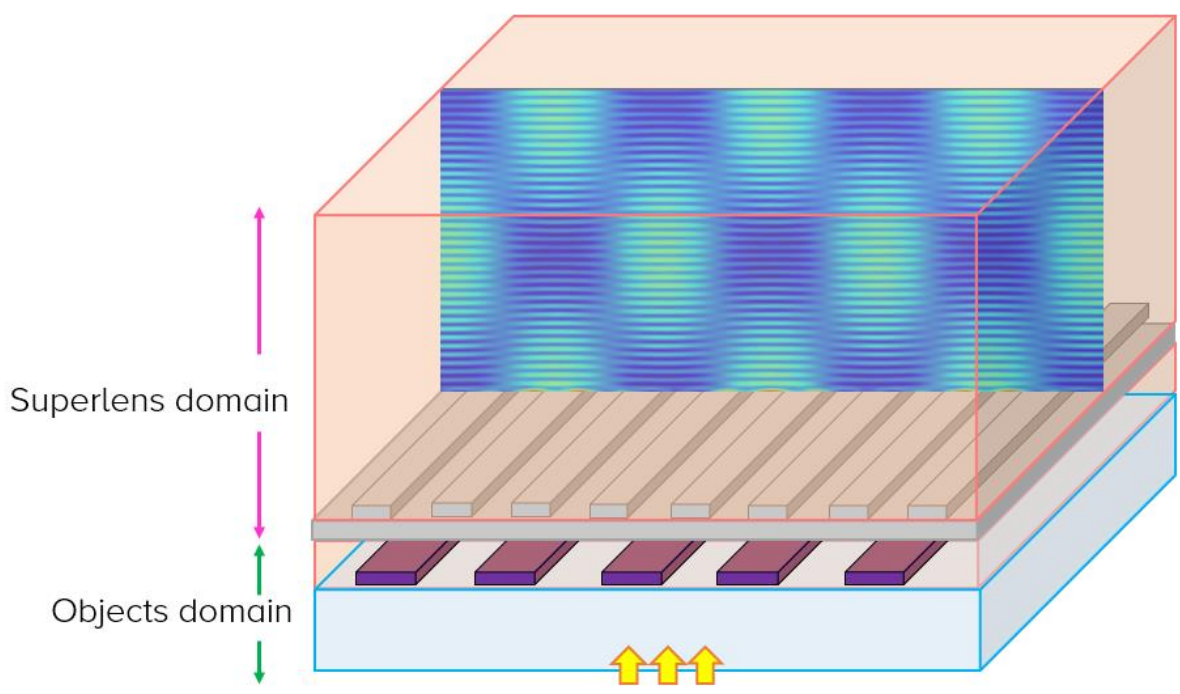

Figure 3-37 Schematics model combining the amplitude plot from simulations with the true geometry of respective case, i.e., a 1D object and 1D FSL nanograting combination.

Our 2D simulated models showed the far-field dependence on the object and FSL nanograting geometries.

An important test to verify the performance of a FSL is confirming whether changing a diffraction limited object size distorts the far-field pattern [135]. This was verified in our results where an example is provided in Figure 3-37, which shows that the FSL produces far-field patterns that correlate with the physical attributes of a subwavelength object.

In addition, we simulated different FSL nanograting shapes and compared their far-field patterns with the generalized rectangular nanogratings. This helped to explore two important aspects:

- How potential nanofabrication problems could affect the performance of our FSL. 
- How the FSL design could be optimized to improve performance.

One of the main outputs of the available studies that demonstrate the working principle of a FSL was focused on the creation of the far-field Moiré pattern. In these studies, images were produced using image processing techniques. In this work, we showed that Moiré patterns are created for all proposed designs, where there was a correlation between the size of the created Moiré pattern, the object and FSL nanogratings. This is shown to be the case regardless of the breath of the investigated designs. Furthermore, our 3D simulations showed Moiré patterns in two orthogonal direction of the imaging domain, which implies that higher dimensions offer more object information to resolve using image processing. This is a significant result, where by using a simple rotation of the object and correct light polarization more information could be produced in a simple setup.

Furthermore, it was found that other than a proper Moiré pattern, all nanograting shapes generate a vertical pattern, known as the Talbot pattern. Studying the properties of this vertical pattern could also help in image reconstruction if propagation decay was forgiving. The Talbot pattern has never been studied in a superlens context before, however, it reinforces the concept of projecting images to the far-field using a FSL.

Based on the differences in our designs, the amplitude and contrast varied for the generated Moiré and Talbot patterns. For instance, the inverse-trapezoidal nanogratings and the conventional rectangular nanogratings resulted in a comparable magnitude transfer function. However, the half circular nanogratings resulted in a weaker magnitude transfer function, hence this geometry was deemed less suitable for plasmonic excitations of a FSL. These 
results indicated that the amplitude and/or contrast of the far-field interactions are directly related to the geometry of the FSL nanogratings.

Furthermore, for the sake of simulation resource management, the simulation domain size and its effect on the observed far-field patterns was studied. While the far-field pattern is self-repeating by nature (Talbot effect), this study allowed us to evaluate whether changing the domain size imposes any artefacts on the far-field pattern. The results confirmed the accuracy of the selected minimal domain size, which was later used for designing of our 3D models, where resource management becomes critical.

In Section 3.5, three dimensional models were studied. Our simulated models showed the far-field patterns for several object sizes and shapes, and were used to evaluate the effects of FSL-object placement on these patterns. It was found that using a FSL with 2D nanogratings with bi-directional wave illumination produces more information about the object features given that the far-field pattern is generated in two directions. This could be attributed to the interaction of this wave with specific features of the object and the FSL nanograting. Such illumination approach allows collecting more information about the object, hence acquiring a better object resolution [221].

On this note, we conclude part one of this thesis, which concerned the electromagnetic modelling of the far-field superlens. In the second part of this thesis, we propose a novel plasmonic material to fabricate the far-field superlens. This decision is based on the previously mentioned challenges that restrict the realization of a far-field superlens made of silver, both from a microfabrication and a testing standpoint. 



\section{Part two:}

Novel Plasmonic Materials 



\section{Aluminium-doped Zinc Oxide for plasmonic applications}



In the second part of this thesis, we propose using a novel plasmonic material as a potential candidate to our intended FSL device. Most plasmonic devices are designed using well-known metals as the plasmonic material, for instance silver has been the proposed material to build a superlens. After doing initial thin film deposition using silver and characterizing its properties, in particular surface roughness, optical transparency, and photolithography resolution, we concluded that given the existing equipment at our cleanroom, doing further work on silver would not be conducive to this thesis. Therefore, we steered to focus on characterizing the plasmonic properties of a transparent conductive oxide. This selected material is $\mathrm{Al}$ doped zinc oxide [222]. The plasmonic active range of $\mathrm{Al}$ doped zinc oxide is expected to happen at larger wavelengths when compared to silver. Therefore, a far-field superlens made of Al doped zinc oxide can accommodate bigger grating structures for its design. This translates to less stringent fabrication challenges when trying to realize such a structure, which could minimize performance defects of such a device within its intended operating spectrum. The first step for developing this device required us to confirm the suitability of Al doped zinc oxide as a plasmonic material within the intended operating spectrum. To achieve this, we needed to analyse the properties of $\mathrm{Al}$ doped zinc oxide thin films produced in our lab.

The main focus for the second part of this thesis was the characterization of $\mathrm{Al}$ doped zinc oxide thin films, from the UV to the infrared range to estimate its plasmonic range. 
In this chapter, we discuss the reasons behind our consideration of $\mathrm{Al}$ doped zinc oxide as a suitable candidate for plasmonic applications, such as the proposed FSL.

\subsection{Introduction}

Conventional optical devices are fabricated from dielectric materials, such as glass or plastic [223]. However, regardless of how perfect the manufacturing

process is and high quality materials are, conventional optical devices have a resolution limit due to diffraction according to the Huygens-Fresnel principle. On the other hand, recent developments of subwavelength imaging devices have been ramping up, with several established techniques that are becoming more popular in the lab.

Plasmonic effects enable efficient light-matter interaction and manipulation at the nanoscale. Thus, plasmonics has been receiving a lot of research attention, paving the way for the construction of various novel instruments, significantly imaging related concepts. A plasmonic material has an abundance of free electrons that can couple with electromagnetic fields to produce collective oscillations. These oscillations are on a smaller scale than the optical diffraction limit, hence they can be used to resolve subwavelength information about nearby objects [224]. Common plasmonic materials include noble metals, such as silver and gold, which by nature are highly conductive, inert and clean [1].

The far-field superlens is a promising concept that can overcome the diffraction limit while providing real-time imaging with no dependence on raster scanning [225]. Silver is the material of choice for fabricating an optical superlens due to its plasma frequency, which occurs at $377 \mathrm{~nm}$ [226], and its lower optical 
loss than gold in the relevant part of the electromagnetic spectrum, i.e., ultraviolet and visible range [227]. Nonetheless, silver has significant shortcomings in terms of microfabrication control and oxidation in ambient conditions, which make the construction of a silver FSL highly challenging and requiring expensive resources, which are prohibiting to the development of the concept.

A simple near-field superlens is typically comprised of a thin silver film with a negative dielectric permittivity at the operating wavelength. Fabrication of a defect-free, smooth and inert surface is needed when building a near-field superlens. Ohmic losses due to non-radiative surface plasmon excitations are directly related to absorption losses in metals [228]. To keep these losses low, the thickness of the silver thin film should be kept of the order of 10s of nanometres, otherwise the device performance is quickly degraded [229, 230]. In the case of silver, formation of nanoscale islands during early stages of thin film growth hinders the creation of smooth thin films and results in light scattering [231].

A far-field superlens in comparison has a more complex design given that periodic nanostructures need to be created on top of the metallic thin film [232, 220]. These nanostructures are typically made from the same material of the thin film, and are used to radiate the coupled evanescent waves to the far-field region, hence enabling practical subwavelength imaging [233].

However, the fabrication of nanoscale structures is expensive and demanding especially when these structures are required to cover areas many orders of magnitude larger than them, such as in the case of a far-field superlens. Consequences of not achieving good quality nanostructures is the loss of subwavelength information and malfunction of the far-field superlens [234]. In short, using today's technology, the construction of a silver based superlens is very challenging. 
Given the aforementioned challenges for silver as the plasmonic material in a far-field superlens, and the relative shortage of experimental results on far-field superlenses, it became evident to us that there is a need to focus on enabling this technology by finding an alternative material with "better properties". It is worth noting that good plasmonic properties are not exclusive to metals; in fact, doped semiconductors can also exhibit properties similar to metals, having negative real permittivity in a specific part of the electromagnetic spectrum [92]. The term "better properties" mentioned in this paragraph is a relative term that refers to properties that maximize the prospects of realizing a FSL given the trade-off between plasmonic performance and microfabrication challenges. For instance, by selecting a material with a relatively high operating plasmonic wavelength compared to silver, the size of the features in such a far-field superlens does not have to be so small, hence, relaxed fabrication constraints would be expected, which allow robust development of this device. The downside of this trade-off would be the inability to resolve features as small as what is possible in a silver superlens. Nevertheless, as we go on, we explain that the candidate material selected in this study has tuneable dielectric properties. This means that its plasmonic operating wavelength could potentially be closer to the visible range of the spectrum [235].

\subsection{Potential Alternative Plasmonic Materials}

Suitable alternative materials for superlens imaging applications in the NIR, visible and near-UV include doped semiconductors and metallic compounds, such as transparent conductive oxides (TCOs), for example indium tin oxide and 
doped zinc oxide, and transition metal nitrides (TMN), for example titanium nitride [236, 237, 238, 239].

Transparent conductive oxides have been mass-produced and commercially used in various applications that require transparent electrodes and interconnects such as in the field of photovoltaics, where indium tin oxide is standardly used in solar cells. Recently, transparent conductive oxides have been proposed as potential plasmonic materials given their ability to accommodate high levels of doping, which can be varied allowing for tuneable dielectric properties [235, 240, 241, 242]. Literature also suggests that TCOs can have four times lower optical losses than silver in the near infrared region [243, 244]. Nonetheless, tuning the properties of such materials is not trivial, and often depends on specific deposition conditions and methodology.

By changing the plasmonic operating wavelength of a material, the scope of application varies. For instance, an infrared superlens might provide the opportunity to study biological systems, biomedical imaging, thermal microscopy, circuit elements characterization, while a visible light superlens should be able to capture images of sub-wavelength elements under visible illumination. Conventional gold- and silver-based plasmonic devices with their high carrier concentration of $\sim 1 \mathrm{E}+23 \mathrm{~cm}^{-3}$ mainly operate at wavelengths in the visible and UV range [66]. In contrast, TCOs have a carrier concentration of 1E+20 $\mathrm{cm}^{-3}$, promising plasmonic properties in the near-infrared region of the spectrum, which cannot be addressed by plasmonic devices made out of metals [245]. Aluminium doped zinc oxide (AZO) in particular seems to offer many advantages over other TCOs for optical and electrical applications [246]. These advantages include the relative abundance of its elements and the ability to produce this safe material without the need for toxic chemical precursors [247], such as those used for indium tin oxide that can lead to indium lung [248]. In 
addition, AZO offers good chemical and mechanical stability as well as relative ease of fabrication and a broad range of doping [249, 250, 251]. Therefore, choosing $\mathrm{AZO}$ as the candidate material for our FSL study seemed logical and straightforward [252], as our research group had already developed expertise in AZO thin film deposition [253].

\subsection{Why Highly Doped Semiconductors?}

Semiconductors can become more metallic by introducing dopant impurities [254]. Doping directly affects the concentration of free electrons in the crystal structure, hence the dielectric permittivity of the material changes. This ability to tune the dielectric properties of semiconductors is highly favourable to plasmonic applications as a broad range of the electromagnetic spectrum could be targeted. A key focus for our experimental study was to find the "crossover frequency" for our AZO thin films deposited by RF magnetron sputtering. The crossover frequency is where the real part of the dielectric permittivity equals zero [255]. Once this frequency is identified, the range where the material behaves like a metal can be recognized. Below the plasma wavelength, semiconductors can be transparent to electromagnetic fields. Therefore, the range of the operating wavelength for a superlens exists below the plasma frequency of the given material [133].

Optical loss is inevitable in plasmonic devices due to the ohmic loss and electron-core interactions in the process of plasmonic excitation. This loss is associated with the high imaginary part of the dielectric permittivity and is usually detrimental to most plasmonic applications [256]. However, recent literature suggests that a range of wide bandgap TCOs possess good optical 
properties with low loss interband transitions and low resistive losses [238, 239, 245]. An ideal transparent conducting oxide is transparent over a wide range of wavelengths [257]. Materials, such as gallium zinc oxide (GZO), indium tin oxide (ITO), and Al doped zinc oxide (AZO) show lower optical loss and have the potential to create tuneable plasmonic materials [258, 243]. AZO has a wide bandgap and could be made to have high carrier concentration in the conduction band [259]. Furthermore, AZO promises plasmonic properties for applications in the near-infrared range due to the small imaginary part of its dielectric permittivity [260].

\subsection{AZO band structure properties}

Zinc oxide $(\mathrm{ZnO})$ is a wide bandgap semiconductor, with a bandgap $\left(E_{g}\right)$ of $\sim 3.44 \mathrm{eV}$ at low temperatures and $\sim 3.34 \mathrm{eV}$ at room temperature $[261,262,263]$. $\mathrm{ZnO}$ thin films deposited at ambient temperatures usually have a wurtzite crystal structure [262]. The optical band edge for a material is defined as the point on the electromagnetic spectrum where higher energy (frequency) photons are absorbed, and lower energy photons are transmitted. Due to its wide bandgap, $\mathrm{ZnO}$ is optically transparent in the visible region.

The properties of intrinsic $\mathrm{ZnO}$ can be changed by introducing impurities. Some studies suggest the possibility of p-type doping in $\mathrm{ZnO}[264,265]$, however, this is often dismissed because of the tendency in zinc oxide to have native donors due to oxygen deficiency [266]. Therefore, $\mathrm{ZnO}$ is inclined towards n-type conductivity, and it is possible to increase its conductivity by extra doping [267, $268,269]$. This n-type doping occurs via substitution of zinc atoms with group III 
ions, such as $\mathrm{Al}^{3+}$. This ability to dope zinc oxide provides a means to tune the optical, electrical and thermal properties of $\mathrm{ZnO}$ [270].

However, in order to deposit highly conductive AZO thin films, $\mathrm{ZnO}$ should be doped beyond the degenerate limit [271]. This means that the number of electrons becomes large, often above $1 \mathrm{E}+20 \mathrm{~cm}^{-3}$ [272], which is comparable to the effective density of states in the conduction band. Increasing the number of electrons moves the fermi level closer to the conduction band in $\mathrm{n}$-type semiconductors. This Fermi level, Ef, rise can continue until it becomes within the conduction band, Ec. A common criterion to identify the degenerate doping limit of a semiconductor is when the fermi level is within $3 \mathrm{kT}$ of either band edges; i.e., conduction band for n-type doping and valence band for $\mathrm{p}$-type doping [273, 274]; k being Boltzmann's constant and $\mathrm{T}$ is the temperature.

For degenerate doping, band filling can be described by the Burstein-Moss (BM) effect $[275,276]$. This model accounts for the apparent optical band gap widening in heavily doped semiconductors, where energy states are filled in the lower part of the conduction band as the Fermi level rises to the conduction band, i.e., interband transitions from the valence band to available conduction band states become larger [277]. Optical bandgap widening $\left(\Delta E_{g}\right)$ according to the BM model is given by:

$$
\Delta E_{g}=\frac{h^{2}}{8 m_{e}^{*}} \times\left(\frac{3 n}{\pi}\right)^{\frac{2}{3}}
$$

\section{Equation 4-1}

where $h$ is Planck's constant, $m_{e}^{*}$ is the electron effective mass in the AZO and $n$ is the carrier concentration. 
Therefore, for degenerate doping the fermi level lies within an unfilled band, which might be an impurity band, i.e., donor band in n-type doping, or even be pushed into higher energy levels in the conduction band of the host material [278, 279]. Accordingly, photons having exactly the bandgap energy cannot be absorbed due to the occupied energy states at the lower part of the conduction band. Consequently, a larger photon energy is needed to excite valence electrons into vacant states within the conduction band, which is manifested in a wider bandgap in optical absorption measurements of such materials [275].

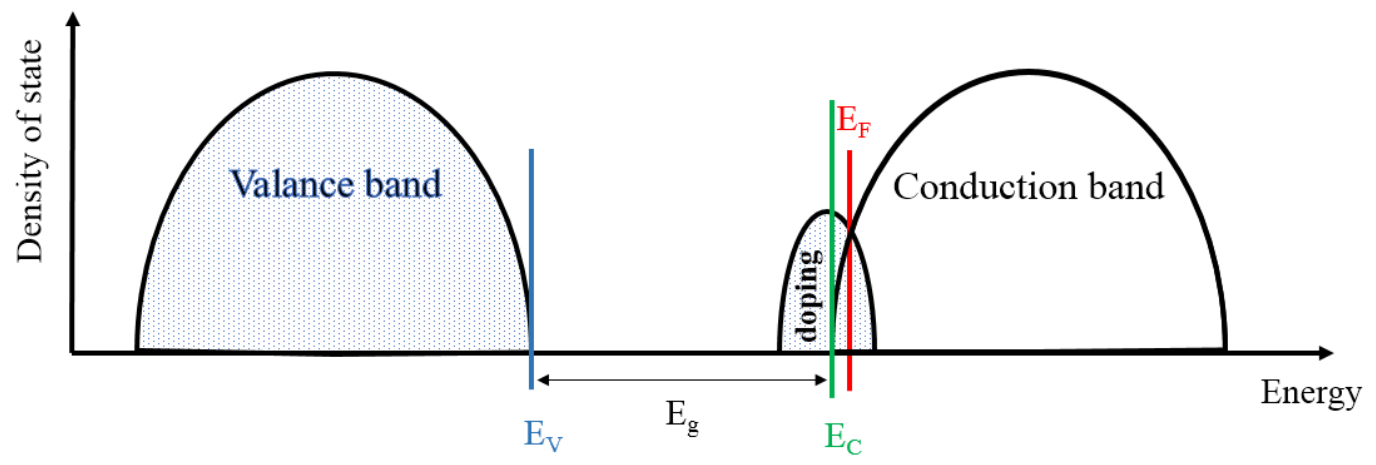

Figure 4-1 Simple schematics showing the density of states in degenerate doped n-type semiconductor. 
In the result chapters, we calculate the bandgap for selected AZO samples using Tauc plots [280]. In a Tauc plot $(\alpha E)^{2}$ is plotted against $E$ near the absorption edge. Here, E represents energy ( $E=h v, v$ being the frequency) and $\alpha$ is the absorption coefficient approximately represented in the form of [281]:

$$
\alpha=\frac{\ln \left(\frac{1}{T}\right)}{d}
$$

Equation 4-2

where, $T$ is wavelength dependant transmission and $d$ is the film thickness.

\subsection{Chapter summary}

In this chapter $\mathrm{AZO}$ was suggested as an alternative material for plasmonic applications. This necessitates that the selected material has an abundance of free electrons, while possessing a negative real permittivity below the plasma frequency $[282,283,284]$. Having a high optical transparency and electrical conductivity are also required for AZO thin films to enable the operation of a farfield superlens. Therefore, the main focus in the next few chapters is characterizing our AZO thin films to estimate their dielectric properties and confirm their suitability to the proposed FSL application. 


\section{Fabrication}



This chapter provides details regarding the fabrication process of our thin films. This includes a presentation regarding substrate selection criteria, an overview of the used thin film deposition technique, a description of our deposition system and a summary of the method used to create our thin films.

\subsection{Intended structure}

In the experimental part of this study we aimed to develop a recipe for creating a thin film sample with plasma frequency in the near-infrared region. This thin film should have a smooth surface and high transparency. Optical losses with the substrate-thin film structure should be minimal. Therefore, it is desirable to use flat substrates that have high transparency over the spectral region of interest.

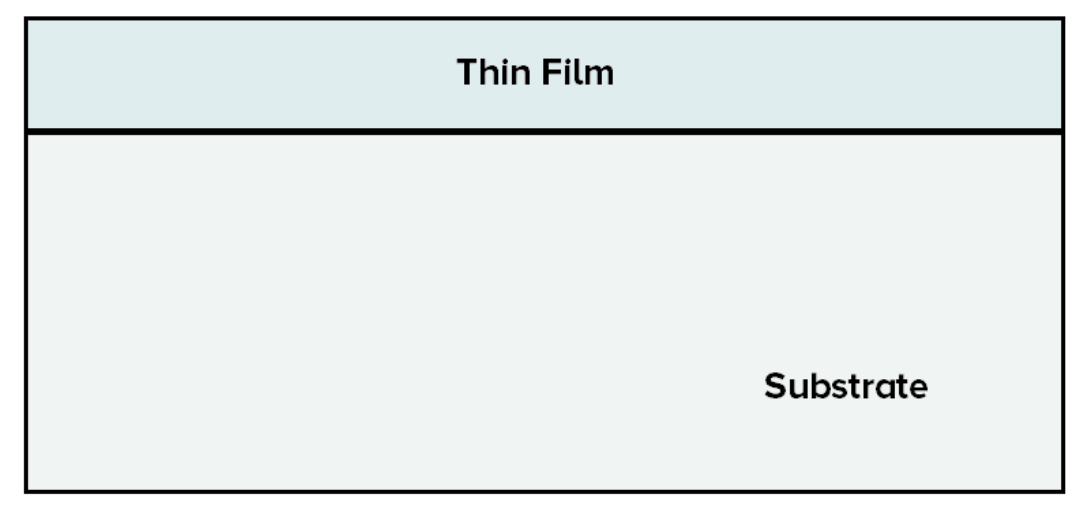

Figure 5-1 Intended sample structure 


\subsection{Substrate selection}

Selection of suitable substrates was a crucial task in this study. Although AZO is a transparent conductive oxide, it is not fully transparent in all regions of the electromagnetic spectrum. Furthermore, substrate thickness and its optical matching with the AZO film affects the overall response of the sample. Therefore, selecting a substrate with a limited transparency and compounding this with the thin film absorption and reflection properties might result in a low transmission for the whole sample, which might lead to a wrong or incomplete assessment of the optical properties of the thin film. Furthermore, the blank substrate should have a smooth and uniform surface to allow smooth thin film deposition to maximize lateral uniformity and minimize light scattering. The substrate should also be resilient to allow plasma aided deposition, dicing and handling during measurements. Furthermore, the substrate should have suitable thermal properties to allow heating during deposition and thermal annealing if required. Thermal expansion is a particularly important parameter during thin film deposition given that large thermal mismatches between the substrate and the thin film material can lead to stress related damage such as cracking and peeling.

\subsubsection{Substrate surface isotropy}

Substrates used in this thesis had either crystal or amorphous forms. Amorphous solids are isotropic in nature. Crystals on the other hand can be either isotropic or anisotropic depending on the dimensions of the crystallographic axes [285]. Anisotropy in this sense is manifested by the spatial differences of given physical properties within the material, such as complex refractive index, density, etc. when measured along the different axes [286]. 
Many transparent crystals are optically isotropic with constant refractive index in all directions of their lattice. In most optical characterization tools, having an isotropic material is desirable, as it allows light illumination from different angles or polarizations to behave in a simple constant way upon interacting with the sample [287].

In comparison, birefringence in many crystal material is an anisotropy that results in having different effective refractive indices for the material depending on the illuminating polarization and direction of propagation [288]. In this work, we aimed to have substrates with minimal birefringence values.

\subsubsection{Substrates used in this study}

In this thesis, $\mathrm{Al}$ doped zinc oxide thin films were deposited on Borofloat33 [289], Silicon [290], Sapphire [291] and Calcium Fluoride [292] substrates.

\subsubsection{Borofloat33 substrate}

Borofloat33 supplied by University Wafers ${ }^{\circledR}$ was initially selected as the substrate to deposit our AZO thin films. Borofloat33 is the industrial name for a high quality borosilicate glass manufactured by SCHOTT AG®. This substrate is highly transparent in the visible region. Furthermore, it has low thermal expansion that allows it to be resistant to thermal shock with a good thermal stability up to $450{ }^{\circ} \mathrm{C}$. This substrate can be easily diced with a diamond blade without shattering or cracking. However, Borofloat33 transparency in the infrared region is low with some absorption bands, hence it did not allow for full understanding of the optical properties of the deposited thin film in this region. 


\subsubsection{Sapphire substrate}

Another substrate used in this study was a two-side polished $\mathrm{Al}_{2} \mathrm{O}_{3}$ (sapphire) (006) c-oriented substrate supplied by University Wafers ${ }^{\circledR}$. Sapphire has a high temperature resistance and transmission in the near-infrared region. However, sapphire is slightly birefringent, but to mitigate this disadvantage, we restricted the optical characterization of our samples to a single direction, which was normal to the surface.

\subsubsection{Calcium fluoride}

The third selected substrate was calcium fluoride $\left(\mathrm{CaF}_{2}\right)$, which was supplied by Eksma Optics ${ }^{\circledR}$. Substrate specifications indicated a cubic crystal structure [292]. Compared to all other substrates used in this study, calcium fluoride has the highest transmission and a low absorption over a broad range of wavelengths from $0.2 \mu \mathrm{m}$ to $8 \mu \mathrm{m}$. $\mathrm{CaF}_{2}$ substrates are typically used as spectroscopic windows due to their high transmission properties. Thermally, calcium fluoride had the largest thermal expansion of the selected substrate materials, which was a disadvantage during the sputtering and annealing processes given the large expected thermal stresses in the deposited film [293]. Nonetheless, given its broad transmission spectra, $\mathrm{CaF}_{2}$ enabled us to isolate the deposited thin film transmission properties in our spectroscopic study.

\subsubsection{Silicon substrate}

The last substrate used for AZO deposition was boron doped silicon with a crystal orientation of (100) from MTI Corporation. Silicon wafers are not optically transparent. However, in a crystalline silicon wafer, atoms have covalent bonds, 
which results in good optical transmission through the material in the farinfrared range (unlike the other substrates) as photons do not have enough energy to interact with the covalent bonds [294]. Therefore, the transmission properties of our AZO thin films deposited on silicon could be measured in this spectral region. Silicon has a face-centred cubic lattice structure [295], hence is weakly anisotropic, where the low birefringence level could be neglected [296].

Table 5-1 shows the refractive indices $\left(n_{d}\right)$ and thermal expansion coefficients (a) for the substrates used in this study [297, 298]. Given that the refractive index varies with the illuminating wavelength, this index is typically shown at the wavelength of $587.6 \mathrm{~nm}$.

Table 5-1 Refractive indices $\left(n_{d}\right)$ and thermal expansion coefficients (a) for all substrates used in this study

\begin{tabular}{|c|c|c|c|c|}
\hline & Borofloate33 & Silicon & Sapphire & $\mathrm{CaF}_{2}$ \\
\hline $\boldsymbol{n}_{\boldsymbol{d}}$ & 1.4714 & 3.9766 & 1.7682 & 1.4338 \\
$(\lambda=587.6 \mathbf{n m})$ & {$[289]$} & {$[299]$} & {$[300]$} & {$[301]$} \\
\hline $\boldsymbol{a}, \mathbf{k}^{-\mathbf{1}}$ & $3.25 \times 10^{-6}$ & $2.60 \times 10^{-6}$ & $5.00 \times 10^{-6}$ & $18.85 \times 10^{-6}$ \\
& {$[289]$} & {$[302]$} & {$[303]$} & {$[293]$} \\
\hline
\end{tabular}

\subsection{Sample Preparation}

Sample preparation is a basic step to ensure reproducibility in microfabrication processes. Substrates were cleaned to reduce possible contamination during deposition step and improve adhesion and uniformity of 
our deposited AZO thin films. After dicing the substrates to the desired size, which was $10 \times 10 \mathrm{~mm}^{2}$ in our case, cleaning was done in preparation for deposition. The substrates were first rinsed with deionized water, then sonicated in an acetone bath for three minutes. This was followed by a three-minute sonication in instrument grade isopropanol (IPA) bath. Then, the samples were dried under a nitrogen flow, and placed on the relevant sample holder in the sputtering chamber.

\subsection{Thin film fabrication methods}

Thin films are primarily formed using wet processes, such as plating, chemical solvent deposition, and chemical melt deposition, or dry processes, such as physical vapour deposition and chemical vapour deposition [304]. Physical vapour deposition (PVD) of thin films is mainly done by thermal evaporation, electron beam evaporation, and sputtering techniques. To transfer atoms in a controlled manner from a purified source to a substrate and form the film, the atoms should possess a suitable energy level. Typically, the whole process occurs in a vacuum chamber, in which the source atoms are brought in to vapour state through physical mechanisms, such as Joule heating or collision [304].

Vapour atoms or molecules then condense onto the surface of the substrate to complete the deposition process. A uniform supply of incident atomic vapour results in uniformly distributed nucleation islands. These islands are initially small but grow until they coalesce as the voids between them are filled. Full thin film coverage on the surface of the substrate is obtained once the film thickness reaches a specific limit, typically in the order of several nanometres, and varies with the given material and deposition parameters. As long as the processing 
conditions are at a steady state, the crystallographic orientations and configuration are preserved within an island.

Sputtering is a well-established method for depositing TCOs due to the ability of controlling the deposition parameters for a broad range of materials, which results in consistent optical characteristics once a processing recipe is developed. $[305,306,307]$. Furthermore, plenty of literature relating to sputtering doped and undoped $\mathrm{ZnO}$ is already available $[308,309,310,311]$. Accordingly, RF magnetron sputtering was our method of choice to create our AZO thin films.

\subsection{Sputtering techniques}

Sputtering is a physical vapour deposition technique. The process simply involves the collision of energetic ions of the controlled plasma with a target material, thereby transferring the kinetic energy of the ions to the target surface through momentum exchange, which results in target atoms or molecules to be sputtered. The sputtered particles then condense on the substrate in a high vacuum environment $[312,307]$. Generally, small scale sputtering systems have the substrate and the target facing each other. The target acts as the cathode and is connected to the negative terminal of the system's power supply, while the rest of the chamber acts as the anode.

Different sputtering methods were established to allow certain deposition characteristic, where the main methods include the direct current (DC), radio frequency $(\mathrm{RF})$, magnetron and reactive sputtering [304]. Compound sputtering techniques are also available, for example, RF magnetron sputtering, which is used in this study. 


\subsubsection{DC sputtering}

DC sputtering is an easy and economical PVD method for large sample sizes. It uses a direct current (DC) power supply and is typically used for the deposition of highly conductive films, such as metals. In order to start a DC sputtering process, the sputtering chamber is brought into high vacuum, then back filled with a low pressured process gas. The process gas materials are chosen based on the deposited material, where argon and oxygen are commonly used. DC sputtering employs two electrodes to carry the deposition process; the negative DC potential is applied to the target making it a cathode, while the ground is applied to the chamber including the substrate holder making it the anode. As a result of the applied DC electric field, free electrons within the chamber are accelerated towards the anode. When these energetic electrons collide with the atoms of the process gas, they can turn them into positively charged ions, and knocking out extra electrons in the process. This cascaded process will accelerate until plasma is ignited and sustained. In this plasma, positively charged ions are accelerated towards the target, and with enough kinetic energy, they can cause the target atoms or molecules to be sputtered. This "vapour" of sputtered atoms then condenses on the substrate to form the deposited film [313]. The inability to deposit highly resistive and insulating material, the low deposition rates due to low plasma density, and the higher discharge voltage and gas densities are among the disadvantages of DC sputtering [314].

\subsubsection{RF Sputtering}

Non-conductive materials can be sputtered using an AC-drive signal applied to the electrodes. This process is referred to as RF sputtering and is usually used to deposit insulating materials and semiconductors onto the substrate. Plasma 
dynamics during a RF sputtering process are very different from that of a DC sputtering process [315]. In a DC sputtering system, charge builds up on each electrode, however, in a RF sputtering system, the electrical polarity at the electrodes alternates, therefore, there is no permanent cathode and anode. This minimizes charge build-up at the target surface [316].

The RF power supply can be in the order of kilohertz $(\mathrm{kHz})$ to megahertz $(\mathrm{MHz})$, however, $13.56 \mathrm{MHz}$ is a standard frequency for plasma processing defined by Federal Communication Commission (FCC) [317]. At the standard frequency, which is typical of most sputtering systems, the path length of heavy ions is much slower than that of the electrons [304]. As a result, the target is selfbiased to a negative potential. In this situation, positive ions sputter away ions from the target and deposition happens. The application of a RF signal to the target results in a larger electron flow in the positive half-cycle, and a smaller $\left(\mathrm{Ar}^{+}\right)$ions displacement in the negative half cycle. Accordingly, the net charge accumulation during a RF cycle is not zero.

To understand how efficient directional sputtering occurs in a RF sputtering system, it is important to consider the equivalent electrical model of the system. This model is composed of one capacitor at the substrate, with the second capacitor at the target, where both capacitors are connected in series. These capacitors have different areas, hence different values. This implies that the net voltage drop in each electrical cycle is far from unity. Given a small target contact and a larger substrate contact this voltage drop is given by:

$$
\frac{V_{C}}{V_{d}}=\left(\frac{A_{d}}{A_{C}}\right)^{4}
$$

\section{Equation 5-1}


where $V_{C}$ is the voltage drop for the target cover capacitance with the area of $A_{C}$, and $V_{d}$ is the voltage drop at the substrate capacitance with the area of $A_{d}$.

It is worth noting that the substrate area is not limited to the substrate surface that we deposit on, but any surface within the sputtering chamber. In comparison, the surface of the sputtering target is small. This raises the potential of the target surface while minimizing sputtering of chamber surfaces. Due to this area difference, a net electrical polarity exists in a RF sputtering system.

There are some disadvantages associated with RF sputtering, most prominently, the degradation of the deposited film quality due to potential plasma arcing, which imposes high surface roughness and can distinguish the plasma [318].

\subsubsection{RF-magnetron sputtering}

An RF magnetron sputtering system is a compound form of a RF sputtering system, where a permanent magnet is placed close to the target. Figure 5-2 shows different components of a RF magnetron sputtering system. 


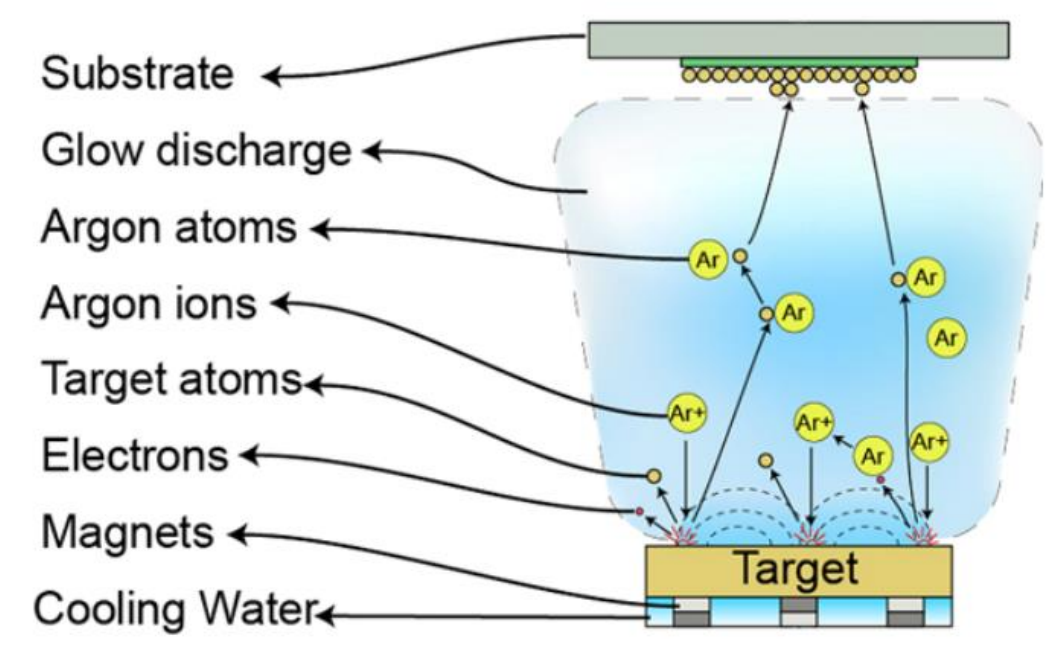

Figure 5-2 Schematics of a RF magnetron sputtering system [319].

Given the magnetic field presence, Lorentz force traps the free electrons near the target, which results in more collisions between the sputtering gas atoms and the electrons in that area. Lorentz force is given by [320]:

$$
\vec{F}=q(\vec{E}+\vec{v} \times \vec{B}),
$$

Equation 5-2

where $\mathrm{q}$ is the free charged carrier, $\mathrm{E}$ is the electric field, $\mathrm{v}$ is the velocity and $\mathrm{B}$ is the magnetic field.

Employing the magnetic field in such a setup enhances the efficiency of the initial ionization process and allows for plasma to be generated at lower pressure levels [321]. Consequently, the energy loss in the sputtering atoms through gas collisions and background gas involvement in the film growth is reduced. 


\subsection{Our sputtering setup}

\subsubsection{System specifications}

An Auto500 RF magnetron sputtering system from HHV® was used to deposit our AZO thin films. Figure 5-4 shows the schematics and a photo of our sputtering system.

This system has a $600 \mathrm{~W}$ adjustable RF power supply that operates at a frequency of $13.56 \mathrm{MHz}$. Processing gases supplied to the system include both argon and oxygen, where gas flow-rate sensors provide flow-rate readings during processing.

The system could be configured to allow substrate heating using a single quartz lamp, where a type-K thermocouple is used to sense the temperature while a proportional integral derivative (PID) controller is used to control the temperature during operation. In our case, a custom sample holder was built to accommodate the heater to be placed at a close distance from the sample to attain higher substrate temperatures than what is possible in the standard setup. 

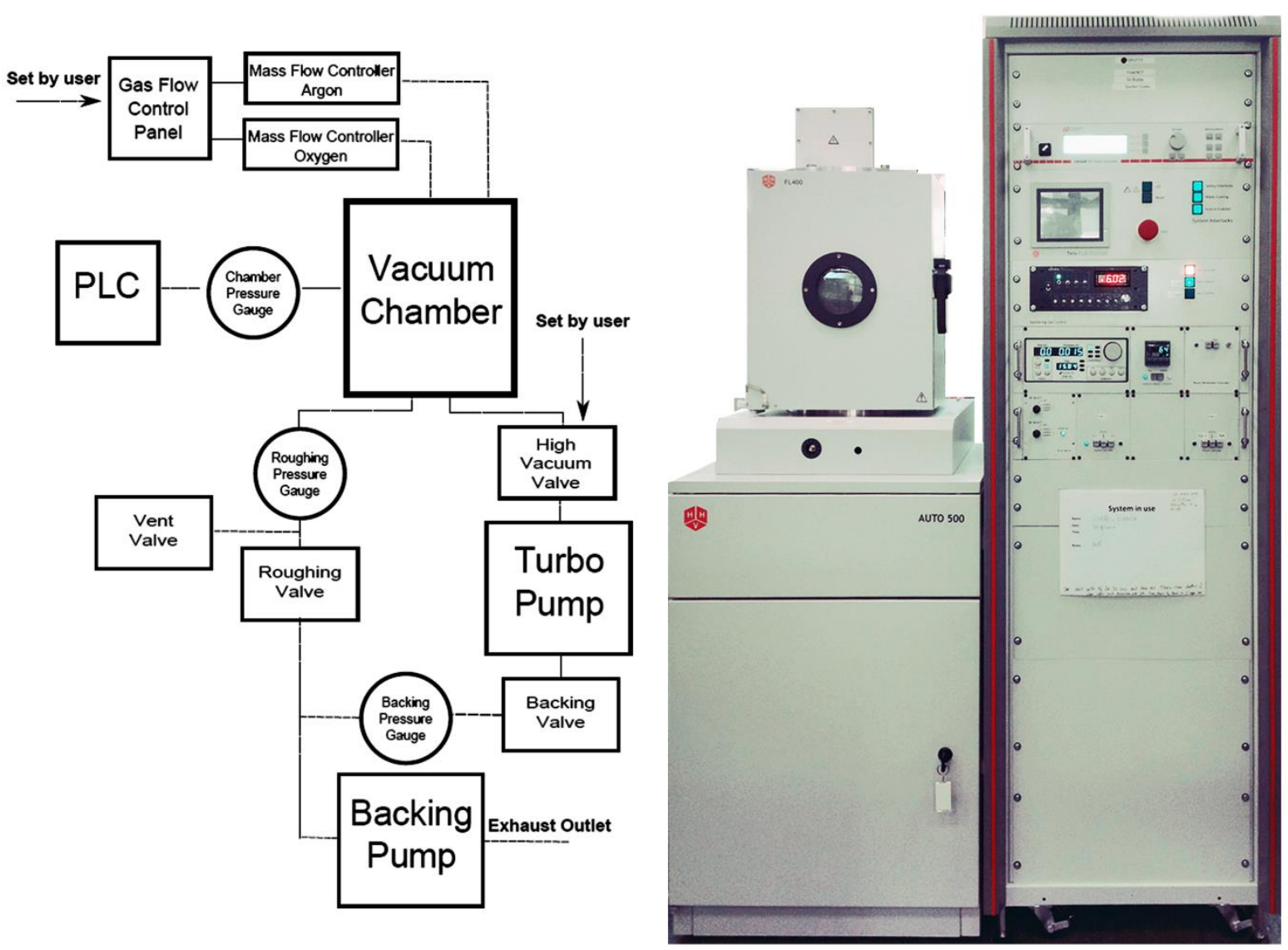

Figure 5-3: The vacuum system diagram on the left and the HHV Auto500 sputter coater system on the right. This system can support three different targets and has a single RF magnetron sputtering source [253].

It should be noted that in this system, the thermocouple is directly inserted into the sample holder, which means that the user has to sacrifice sample rotation if substrate heating is required.

\subsubsection{Sputtering method}

Our AZO thin films were deposited using RF magnetron sputtering. Parameters, such as the substrate material, deposition pressure, substrate temperature, target material and many others directly affect the resulting thin 
film properties. In most cases it is not trivial to understand the individual effects of these parameters unless an expansive study is undertaken. Given the small scale of our sputtering system, we were also limited by several constraints that made uniform deposition of suitable films over large surface areas challenging. The fact that we did not use rotation during deposition and the short distance between the target and substrate was expected to result in a lower film thickness uniformity and high levels of inherent film stress. Fortunately, a previous sputtering study relating to $\mathrm{ZnO}$ and $\mathrm{AZO}$ thin film deposition was recently done at our group at Victoria University of Wellington (VUW) [253], and learnings from that study were largely implemented in our deposition process.

The target used for sputtering our AZO thin films was supplied by Kurt J. Lesker ${ }^{\circledR}$ and had a purity of $99.999 \%$ with a $98 \% \mathrm{ZnO}: 2 \%$ Al composition.

Our sputtering run was started by pumping the system down to a level of $<$ $2 \times 10^{-6}$ mbar. This was usually done overnight as the backing and turbomolecular pump needed 8-9 hours to bring the chamber to this pressure. Upon reaching this base pressure, the processing gas was introduced to the chamber; this gas was instrument grade argon in our case. Gas flow rate was regulated to our desired set point of $6 \mathrm{sccm}$, then the high vacuum valve was adjusted to the required processing pressure. The chamber was then brought up to the desired temperature of $345{ }^{\circ} \mathrm{C}$ before plasma was ignited.

When igniting the plasma, it was crucial to keep the argon pressure close to $1 \times 10^{-2}$ mbar, as a low sputtering gas pressure reduces the chance of energetic gas atoms colliding with the available free electrons, while a high sputtering gas pressure results in a large number of collision events which reduces the mean free path of ions; both of these scenarios prevent the ignition of plasma $[322,323]$. 
Once plasma was ignited, the RF power was ramped up to the desired set point, which was $150 \mathrm{~W}$ in our case. The sputtering gas pressure was then lowered to reach our target of $3 \times 10^{-3} \mathrm{mbar}$.

Similar to any thin film deposition process, the growth quality of our AZO thin films highly depended on the substrate-film interface and the first deposited layer of the material, which acts as a seed layer to facilitate the growth $[324,325]$. Having a well-defined crystal orientation within the seed layer is critical for controlling the optical properties of the deposited AZO thin films, given that defects directly affects the visual appearance of the thin film; hence rapid changes in the sputtering conditions are not desirable at the early stages of deposition.

For each of our deposited sets, sputtering was performed for about one hour, with the aim to deposit an AZO film with a nominal thickness of $100 \mathrm{~nm}$. An illustration of our sputtering setup is shown in Figure 5-4.

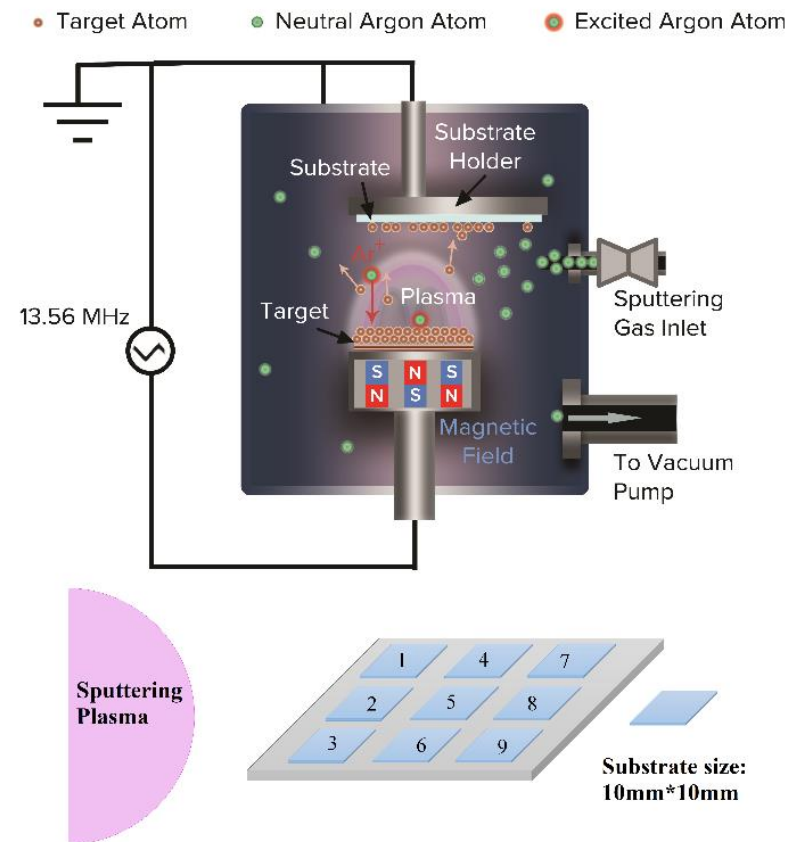

Figure 5-4 Schematics of our RF magnetron sputtering setup detailing the samples location with respect to the sputtering target [326]. 
Given that our sample holder was not allowed to rotate, we had to precisely align the samples with respect to the sputtering target, both laterally and vertically. The two main reasons that necessitate this precise alignment were:

- Thin film uniformity, which relates to thickness uniformity and crystal orientation and grain size uniformity across the deposition area. Both factors vary with the deposition angle and distance from the plasma centre [327].

- Avoiding film damage due to bombardment by highly energetic negative plasma species, oxygen in this case, which is consistent with other observations of deposition directly on top of the target in small sputtering systems. In literature, film damage is observed at substrate positions facing the racetrack eroded target area [328, 329, 330]. This damage pattern is assumed to correlate with the lateral distribution of the different species of negative ions within the RF magnetron sputtering plasma [331], which were chiefly $\mathrm{O}^{-1}$ and $\mathrm{O}^{-2}$ in our AZO target case, as investigated in the previous VUW study [253].

In this setup, samples were mounted on an off-centre sample holder with respect to the plasma axis [332]. We designed the sample holder to allow placing the samples in a 2D array arrangement, as shown in Figure 5-4, thus, registration of our samples with respect to the plasma centre was consistent in all runs. The sample holder was raised to the maximum working distance from the target, which was $92 \mathrm{~mm}$. The off-centre radial distance was approximately $40 \mathrm{~mm}$, where the target had a 3 inch diameter. 
This sputtering arrangement was found to minimize the size of the damaged region on the samples and resulted in thin films with more uniform optical and electrical properties.

\subsection{Chapter summary}

In this chapter, physical vapour deposition techniques were introduced with a focus on RF magnetron sputtering, which was employed to create our thin films.

In order to meet our requirements of high electrical conductivity and low optical loss in the NIR range, and given our small-scale sputtering system, the specific deposition parameters were discussed. Substrate selection criteria were also explained. The individual substrate effects on the overall properties of our samples will be discovered through a non-trivial exploratory process in the following results chapters. 



\section{Characterization techniques}



This chapter introduces the different material characterization techniques that were used in the study. These techniques were used to measure the thickness, carrier concentration, resistivity, surface morphology and topography and the crystallographic properties of our deposited thin films. Furthermore, we discuss the working principles of spectroscopic methods that were used to measure the spectral response of our samples. Finally, we describe the models that were used to calculate the plasma frequency of our thin film material.

\subsection{Chapter objectives}

This chapter describes the techniques and equipment used to characterize our deposited AZO thin film samples.

- In section 6.2 , the working principle of $x$-ray diffraction (XRD) is introduced. In this study, the XRD was used to examine the crystal structure and orientation of the samples.

- Section 6.3 introduces the used thickness measurement methods. Initially, a Dektak profilometer was used to provide an estimation of the thin film thickness. However, given the importance of accurate thickness measurement in this study, we used Rutherford backscattering spectroscopy to measure the thickness of our thin films with an accuracy of a few nanometres.

- In section 6.4, imaging techniques used to identify surface morphology of our samples are introduced. In our study, we used scanning electron 
microscopy and atomic force microscopy to produce surface images of the deposited samples.

- Section 6.5 introduces the specifications of the utilized Hall effect system. Hall effect measurements were used to estimate the carrier concentration of our deposited thin films. The basic operation principles of a physical property measurement system (PPMS) are also presented in this section. A PPMS system was mainly used to study the effect of temperature on the electrical conductivity of our deposited thin films.

- In section 6.6, the spectroscopy systems used to collect optical -mainly transmission- spectra of our samples are introduced, and their working principles explained. Spectroscopy was a cornerstone characterisation technique in this study, where an ultraviolet-visible (UV-Vis) spectrophotometer and two Fourier transform infrared spectroscopy (FTIR) systems were employed. The used UV-Vis spectrometer was a Cary 100 while the FTIR spectrometer systems included a Bomem DA8 and a Bruker Vertex $80 \mathrm{~V}$. These FTIR spectrometers have a wider scanning range that covers the infrared region.

- Section 6.7 describes the analytical and modelling methods used to calculate the plasma frequency of our samples. The first method, which we refer to as "FTIR-Reffit modelling" was used to calculate the Lorentzian oscillation terms of the thin films, by fitting the FTIR measured transmission data. The second method is a Drude-based estimation that we refer to as the "Hall effect-Drude formula". 


\subsection{X-Ray Diffraction}

X-rays are highly energetic electromagnetic waves that were discovered by Wilhelm Conard Röntgen while studying cathode rays of discharge tubes. Röntgen realized that mysterious rays were emitted near discharge tubes, affecting photographic paper in a similar fashion to light [333]. Cathode rays are accelerated beams of electrons that produce x-rays upon hitting a metal surface. Röntgen called this beam a x-ray, because of its unknown nature. This name was befitting the nature of the beam given that only a few fundamental facts were discovered about these rays over the next two decades [334].

Generally, x-rays can be categorised into hard and soft x-rays based on their energy, where hard x-rays have an energy range between $10 \mathrm{keV}$ to $200 \mathrm{keV}$ [335] while soft x-rays have a lower energy between $0.250 \mathrm{keV}$ to $4 \mathrm{keV} \mathrm{nm} \mathrm{[336].} \mathrm{Hard}$ $\mathrm{x}$-rays are usually used for medical radiography and airport security, and soft $\mathrm{x}$ rays are used for microscopy of small particles [337].

Nowadays, x-rays are usually produced in vacuum x-ray tubes. Under an applied voltage, electrons are accelerated from a cathode to an anode target. The target anode should be metallic to absorb electrons and have a high atomic number to increase the intensity of the generated breaking radiation, known as bremsstrahlung [338]. Bremsstrahlung is polychromatic with characteristic radiation lines that arise due to the electrons being decelerated by the nuclei of target atoms [339]. Transitions between two adjacent orbitals creates an $\alpha$ transition, while transitions across two orbitals creates a $\beta$ transition. The orbitals are named according to the principal quantum level with predefined letters, such as $\mathrm{K}, \mathrm{L}, \mathrm{M}$, and so on. Each atomic orbital can accommodate a specific number of 
electrons. Examples of electron transitions and the naming of orbitals are shown in Figure 6-1.

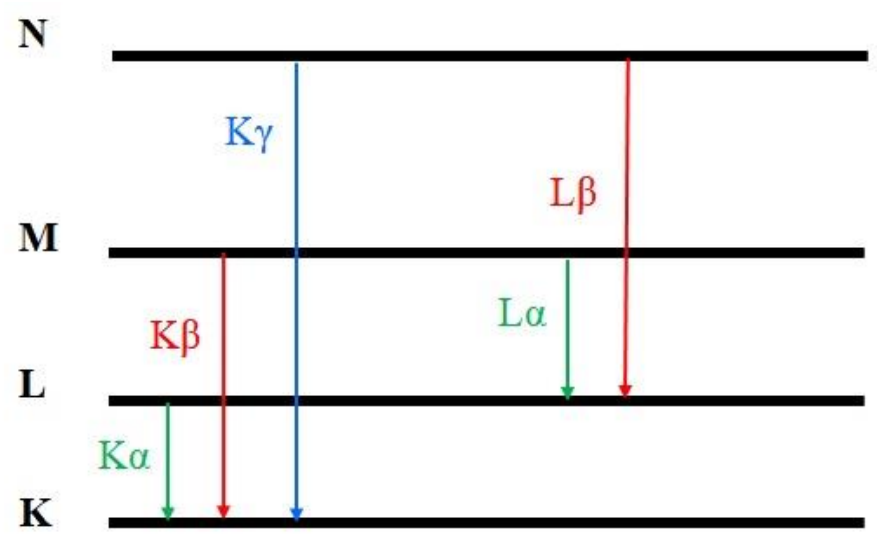

Figure 6-1 characteristic radiation lines in a x-ray

An x-ray anode should have a good heat dissipation ability and a reasonably high melting point. This is because most of the kinetic energy transferred during electron collision with the target turns into heat, where only $\sim 1 \%$ of that energy produces a x-ray [340]. The most commonly used anode target materials for xray production are chromium $(\mathrm{Cr})$, iron $(\mathrm{Fe})$, cobalt $(\mathrm{Co})$, copper $(\mathrm{Cu})$, molybdenum (Mo), silver (Ag), nickel (Ni), and tungsten (W) [341, 342]. For a copper target, which was used in our system, the wavelength of three main characteristic lines are:

$$
\begin{aligned}
& \lambda_{K_{\alpha 1}}=0.154056 \mathrm{~nm} \\
& \lambda_{K_{\alpha 2}}=0.154439 \mathrm{~nm} \\
& \lambda_{K_{\beta}}=0.139222 \mathrm{~nm}
\end{aligned}
$$

Equation 6-1 
Diffractometers usually have a filter to eliminate $\lambda_{K_{\alpha 2}}$ and $\lambda_{K_{\beta}}$.

Given that diffraction is maximized when an electromagnetic wave has a wavelength comparable to that of the object size [343], x-rays with wavelengths similar to those in Equation 6-1 can be used to provide information on the atomic and molecular composition in a crystal structure. The ordered arrangement of atoms in a crystalline material can be also studied using x-ray diffraction techniques. This characterization method was first proposed in 1912 [344].

In specific, x-ray scattering from a crystal structure occurs when Bragg's law conditions are satisfied. Consider $n+1$ parallel equidistant atomic planes with a spacing $d$ in between, and a highly monochromatic x-ray beam with an angle $\theta$ incident on the sample surface as shown in Figure 6-2; this results in x-ray scattering due to electrons in the atoms of the crystal planes. The condition for constructive interference as a result of multiple reflections from a crystal's lattice plane is given using Bragg's law [345]:

$$
n \lambda=2 d_{h k l} \sin \theta
$$

\section{Equation 6-2}

where $d_{h k l}$ is the lattice distance between the parallel crystal planes, $(h k l)$ are Miller' indices of the specific crystal plane [346, 347, 348], $n$ is an integer that shows the order of diffraction and $\lambda$ is the wavelength of the photons. 


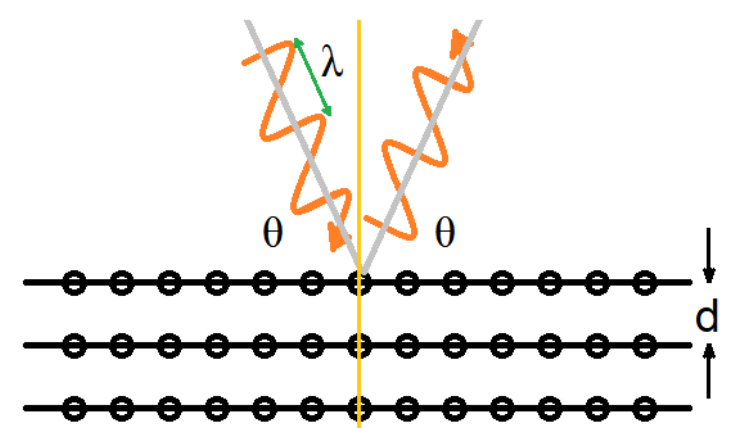

Figure 6-2 X-ray diffraction setup that fulfils Bragg's Law.

By varying the angle $\theta$ for a monochromatic $x$-ray beam with a wavelength $\lambda$, the crystal lattice spacing $d$ can be determined. The x-ray detector is always placed at angle $(\pi-\theta)$, hence intensity peaks in the diffractogram occur only at Bragg's angle. Thus, each material will have a characteristic x-ray diffraction pattern (diffractogram) with specific peaks at unique angles [349].

Accordingly, lattice constants can be calculated for each sample using the corresponding $2 \theta$ angle in the diffractogram. For instance, a c-axis grown $\mathrm{ZnO}$ (wurtzite) film will show a dominant (002) orientation in its diffractogram; thus, a high peak will be observed when the angle $\theta$ satisfies Bragg's equation for the (002) lattice distance. For the (002) orientation in wurtzite $\mathrm{ZnO}, d$ is given by [350]:

$$
\frac{1}{d_{h k l}^{2}}=\frac{4}{3}\left(\frac{h^{2}+h k+k^{2}}{a^{2}}\right)+\frac{l^{2}}{c^{2}}
$$

Equation 6-3

Thus, the $2 \theta$ and $d$ values for such a geometry are presented below [351]: 
Table 6-1 (002) oriented peak for hexagonal lattice geometry in $\mathrm{ZnO}$.

\begin{tabular}{|l|l|l|l|l|}
\hline $2 \theta\left(^{\circ}\right)$ & dhkl $(\AA)$ & $h$ & $k$ & 1 \\
\hline 34.447 & 2.60150 & 0 & 0 & 2 \\
\hline
\end{tabular}

\subsubsection{System specifications}

The used XRD system in our study was from the X'Pert Pro series by PANalytical [352]. When carrying our measurements, the thin film samples were mounted on the flat sample stage. The accelerating beam voltage was set to 45 $\mathrm{kV}$ and the current was set to $40 \mathrm{~mA}$. To control the incident beam width, a 10 mm divergence slit, and a $1^{\circ}$ anti-scattering slit were used.

The type of scan used in our study was a $(\theta-2 \theta)$ scan. During this scan, both the $x$-ray source and the detector move in order to preserve a symmetrically arranged angle $(\theta)$ with respect to the normal vector to the surface of the sample [353]. Before the start of each measurement session, a test measurement using a fluorescent sample was performed to confirm the spot size and location of the $x-$ ray beam.

Despite the care taken during our samples' placement, this process was manual and depended on our visual judgement, thus, alignment errors were assumed to be unpredictable. Fortunately, minimizing the effects of such errors was possible for samples deposited on crystalline substrates, as the well-defined substrate peaks were used as a reference. Accordingly, diffractograms of such samples could be shifted along the $\theta$-axis to maintain a substrate's reference peak 
at a fixed abscissa. Understandably, this step was not applicable for amorphous substrates.

\subsubsection{Properties studied using XRD}

The three main outcomes of our XRD study were the assessment of dominant crystal orientations or orientation selectivity in our thin films, the estimation of the crystallite domain size (CDS) of a given orientation [354], and the estimation of in-plane residual stress.

The dominant orientation was identified based on the normalized peak intensities in a diffractogram. Orientation selectivity was the ratio of the dominant orientation's peak intensity to that of a competing orientation.

Imperfections are unavoidable in any thin film deposition processes. Grain dislocations in a crystal for instance, results in diffraction peak broadening [350]. By measuring peak broadening in a XRD diffraction pattern, an estimation of the crystallite domain size can be made according to Scherrer's equation. This equation provides a rough estimate to the mean crystallite domain size (CDS) for a specific crystal orientation. The estimated CDS is less than or equal to the grain size and is given by $[354,355]$ :

$$
C D S=\frac{K \lambda}{B \times \cos (\theta)}
$$

\section{Equation 6-4}

$K$ is a dimensionless shape factor close to unity and is usually assumed to be 0.9 but can vary based on the sample type [356]. $\lambda$ is wavelength of the $x$-ray, $B$ is the 
full width at half maximum of the diffractogram peak in radians, and $\theta$ is the Bragg angle.

The in-plane residual stress $(\zeta)$ can be evaluated using Hooke's law. This was further derived in [253] for the specific case of c-axis grown $\mathrm{ZnO}$, which is given by:

$$
\zeta(\mathrm{GPa})=-232.812 \frac{d-d_{0}}{d_{0}}
$$

Equation 6-5

where $d$ is the measured lattice distance along the (002) orientation of $\mathrm{ZnO}$, while $d_{0}$ is the theoretical unstrained value for this lattice distance, given in Table 6-1 to be $2.60150 \AA$. Given that our AZO thin films are $98 \% \mathrm{ZnO}$ by composition, we assume these relations to be valid for AZO. Thus, the in-plane stress for c-axis grown AZO is estimated by:

$$
\begin{gathered}
\zeta(\mathrm{GPa})=-232.812 \frac{d(\AA)-2.6015}{2.6015}=-89.4914(d(\AA)-2.6015) \\
\text { Equation 6-6 }
\end{gathered}
$$

In this case, negative stress values indicate compressive stress, while positive stress values indicate tensile stress. 


\subsection{Thickness measurement}

In this section, we introduce the methods used to measure the thickness of the deposited thin films. In a typical sputtering system, thin film thickness can be monitored using a quartz crystal, which also provides an estimate to the deposition rate. However, based on our experience, this estimation was not reliable given the location of the thickness monitor and the high temperatures used during our deposition process. Furthermore, we visually observed a thickness gradient at different places of the deposited thin films, which seemed to depend on the location of the sample with respect to the sputtering plasma.

In this work, accurate thickness measurement was necessary to obtain correct calculations for our Hall effect and resistivity measurements. Thin film thickness was also a required input for our fitting software used to estimate the plasma frequency of an AZO sample.

\subsubsection{Profilometer}

After our AZO deposition runs, we used a Veeco Dektak 150 profilometer to provide a rough measurement of the thin film thickness over the deposited surface. The Dektak profilometer raster scans a defined length on the sample with a stylus having a sharp diamond tip. In our system, the measured thickness accuracy was of the order of several hundred nanometres, which was too rough for our thin films that were supposed to be about $100 \mathrm{~nm}$ thick. As a result, there was a need to use other methods for measuring thickness in a reliable and accurate manner, while the profilometer was simply reserved for quick assessment after each sputtering run. Given that the atomic force microscope at 
VUW was down for repairs for a significant period during this study, we relied on a Rutherford backscattering spectrometer (RBS) to provide an accurate estimate to our thin films thickness.

\subsubsection{Rutherford backscattering spectrometry}

Rutherford backscattering spectrometry is a non-destructive ion beam analysis technique used for surface analysis of thin film samples. RBS uses charged atomic particles to reveal information about the structure and stoichiometric composition of most types of thin film materials, such as oxides, nitrides, metallic films, and compound semiconductors. It can also be used to determine the thickness of thin films [357].

The main parts of a RBS system include the ion source, a particle accelerator to accelerate ions and a detector. The penetration depth of a near-surface analysis using RBS is determined by the ion source energy. In this setup, a ${ }^{4} \mathrm{He}^{+}$ion beam with an energy of $2.5 \mathrm{MeV}$ from a $3 \mathrm{MeV}$ Van de Graaff accelerator was directed to the sample surface at normal incidence. Therefore, high energy particles from the ${ }^{4} \mathrm{He}^{+}$beam can collide with the atoms of the sample, where collisions are mostly elastic. The energy spectrum of the backscattered ions is measured using a detector. Changes in the energy of the backscattered particles correlate with the mass of the atoms in the measured sample [358, 359].

RBS measurements were performed inside a vacuum chamber, where both compositional and depth profiling analysis was carried for the deposited thin films. The RBS system provided spectral plots regarding the number of backscattered particles picked by the detector as a function of particle energy [360]. The measured spectrum for each thin film sample included the individual substrate and thin film peaks. A data deconvolution program named 
"Rutherford Universal Manipulation Program" (RUMP) [361] was used to fit models to the RBS spectra to estimate the thin film stoichiometry and thickness. The statistical and common errors in our RBS measurements were estimated to be $5-10 \%$.

\subsection{Imaging techniques}

\subsubsection{SEM}

In a scanning electron microscope, a well collimated high energy electron beam is accelerated towards the surface of the specimen [362]. The electron interaction with the specimen is received by the detectors in the system [363] and specific types of images, including topographical and compositional images are produced. The imaging process is performed in vacuum otherwise the electron beam interacts with the particles in the environment degrading the image quality [364]. Usually, beam properties, lenses and apertures should be adjusted before imaging to ensure the electron beam is focused on the surface of the specimen [363]. For conductive samples no sample preparation is required as they can easily dissipate the electron beam. However, non-conductive samples are coated with a thin layer of a conductive material, such as carbon. This additional layer conducts electrons from the surface of the samples and minimizes surface charging effects, thus increasing the image quality [365].

In this study, SEM images were produced using a FEI Nova NanoSEM 450. Furthermore, for the purpose of analysing the elemental composition of the deposited thin film, this system was used to acquire energy dispersive $x$-ray spectrographs (EDS) for selected samples, where spectral analysis is carried out for the detected beam to identify the atomic composition [366]. 


\subsubsection{AFM}

An atomic force microscope is used to produce three dimensional images of a sample's surface. The specified area on the sample's surface is raster scanned using a sharp tip mounted on a cantilever to provide an image. There is no sample preparation required before imaging with an AFM, however, the sample should be reasonably clean with a relatively small surface area to fit within our system.

\subsection{Hall Effect and PPMS measurements}

\subsubsection{Hall effect}

The Hall effect system was used to measure the carrier concentration and resistivity of each sample. The resistivity value confirmed how conductive a sample was. The measured carrier concentration was employed in the Drude formula to help calculate the plasma frequency value of a given sample.

Hall effect was first discovered by Edwin Hall in 1879 [367]. Nowadays, the Hall effect measurement systems have numerous applications in semiconductor related industries and in basic material research [368, 369]. They can be used to measure several parameters, which include the Hall voltage/Hall coefficient, resistivity, magnetoresistance, carrier concentration, carrier mobility, carrier conductivity and doping type [370, 274].

The fundamental phenomena behind the Hall effect are the Coulomb and Lorentz forces. In a Hall effect setup, an electric field is applied across two opposite faces of a semiconducting sample, which will mobilize charged carriers 
within the sample by Coulomb (electrostatic) force. When an orthogonal magnetic field is simultaneously applied to the sample surface, as per

Figure 6-3, an electric potential gradient is induced by Lorentz force according to the right-hand rule. The Lorentz force equation combines the magnetic and electric forces [371] as shown in Equation 5-2. This force pushes the carriers to one side of the sample. Localization of the charge carriers at the sides of the sample produces a voltage across, which balances the magnetic force. The resulting potential difference across the sample is the Hall voltage [372]. The Hall voltage sign can be either positive or negative, depending on the sign of the charge carriers. By inputting the thickness (d) of the measured sample, we can estimate the bulk p- or n-type carrier densities.

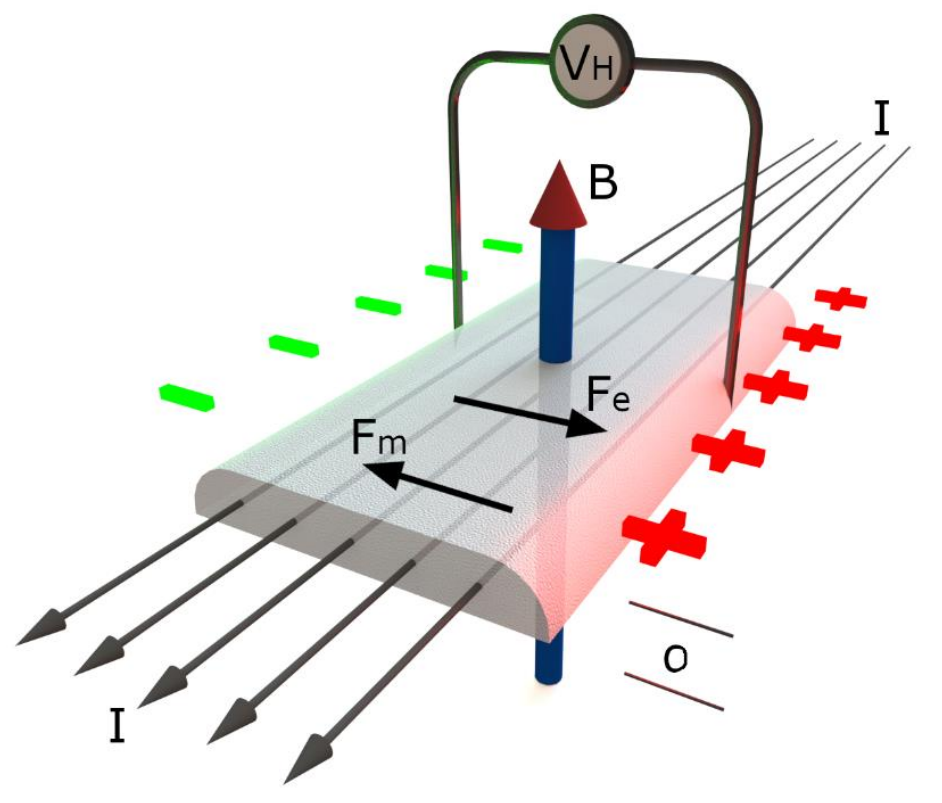

Figure 6-3 The Hall effect setup. 
In our Hall effect system, resistivity measurement was done using a four point probe setup (van der Pauw) [373], where $I_{n m}, n=m=[1, \ldots, 4]$ were recorded; $I_{n m}$ being the DC current applied at contact $n$ and sourced through contact $m$. Likewise, DC voltages between each two contacts were measured in the absence of the permanent magnet, denoted by $V_{n m}$. This van der Pauw arrangement is illustrated in Figure 6-4.

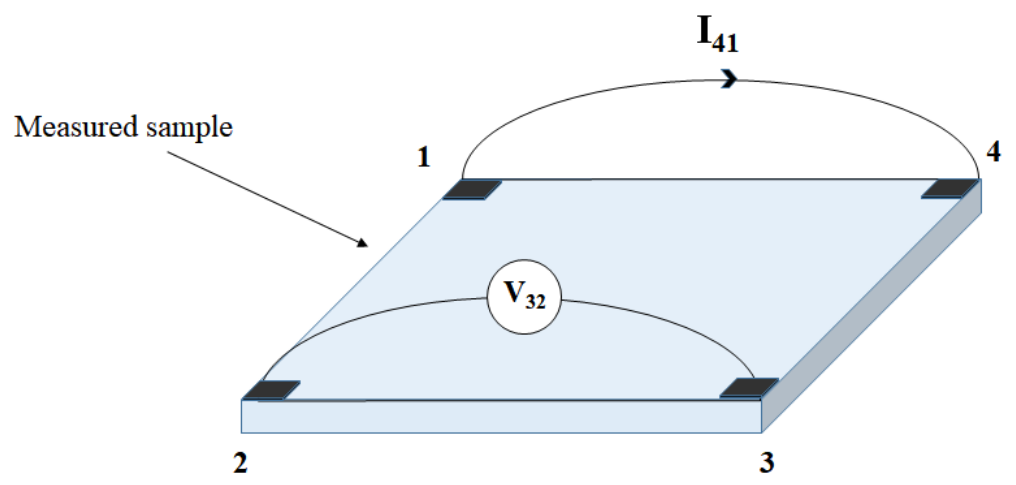

Figure 6-4 Illustration of the van der Pauw configuration described in this section.

Consequently, the positive values of resistance across the sample could be given by [374]:

$$
\begin{aligned}
& R_{21,34}=\frac{V_{34}}{I_{21}}, R_{12,43}=\frac{V_{43}}{I_{12}} \\
& R_{32,41}=\frac{V_{41}}{I_{32}}, R_{23,14}=\frac{V_{14}}{I_{23}} \\
& R_{43,12}=\frac{V_{12}}{I_{43}}, R_{34,21}=\frac{V_{21}}{I_{34}} \\
& R_{14,23}=\frac{V_{23}}{I_{14}}, R_{41,32}=\frac{V_{32}}{I_{41}}
\end{aligned}
$$

Equation 6-7 
Based on measurements' consistency and current reversal properties, the following equations should be satisfied:

$$
\begin{aligned}
& R_{21,34}=R_{12,43} \\
& R_{32,41}=R_{23,14} \\
& R_{43,12}=R_{34,21} \\
& R_{14,23}=R_{41,32}
\end{aligned}
$$

\section{Equation 6-8}

According to the reciprocity theorem the following is true for the combinations of $R_{m n}$ :

$$
\begin{aligned}
& R_{21,34}+R_{12,43}=R_{43,12}+R_{34,21} \\
& R_{32,41}+R_{23,14}=R_{14,23}+R_{41,32}
\end{aligned}
$$

Equation 6-9

The sheet resistance $\left(R_{S}\right)$ was calculated from the following equations:

$$
\begin{aligned}
& R_{A}=\left(R_{21,34}+R_{12,43}+R_{43,12}+R_{34,21}\right) / 4 \\
& R_{B}=\left(R_{32,41}+R_{23,14}+R_{14,23}+R_{41,32}\right) / 4
\end{aligned}
$$

Equation 6-10

Solving the van der Pauw equation for $R_{S}[375]$ : 


$$
\exp \left(-\frac{\pi R_{A}}{R_{S}}\right)+\exp \left(-\frac{\pi R_{B}}{R_{S}}\right)=1
$$

Equation 6-11

Knowing the film thickness, the bulk resistivity $(\rho)$ could be calculated:

$$
\rho=R_{S} O
$$

\section{Equation 6-12}

where $o$ is the film thickness.

For each of our deposited thin film samples, the ohmic contact quality and the thin film uniformity were verified. Then, to carry the hall voltage measurements, we placed the permanent magnet around the sample. In order to change the polarity and the applied current across the sample, this action was repeated for both magnetic polarities. In each case, the voltage and current values were measured for all sample contacts diagonally, in a manner similar to that of Figure 6-3. To calculate the carrier concentration from the measured Hall voltage, the following equation was used [376]:

$$
n=\frac{B I}{V_{H} e o}
$$

Equation 6-13 
where, $B$ is the magnetic field, $I$ is the current, $V_{H}$ is the Hall voltage and $e$ is the electron charge. The sign of the Hall voltage indicates type of the majority charge carriers in the sample.

The carrier mobility for n-type materials is given by:

$$
\mu_{n}=-\frac{\sigma_{n} V_{H} o}{I B}
$$

Equation 6-14

where, $\sigma_{\mathrm{n}}$ is the resistance calculated using the resistivity value.

\subsubsection{System specifications}

We used an Ecopia HMS-3000 Hall Effect system from Bridge Technology to measure the resistivity and Hall parameters of our deposited AZO thin films. The following equipment were also part of our Hall effect measurement setup:

A permanent magnet with a power of 0.55 Tesla, a current source with a range of $1 \mathrm{nA}-20 \mathrm{~mA}$, and a high input impedance voltmeter with $\pm 12 \mathrm{~V}$ range. The HMS3000 software provided an easy access to two interfaces to measure the I-V curves and the Hall parameters for each sample.

\subsubsection{Measurement procedure}

Initially a test measurement was performed where a test sample with known properties was measured. After confirming correct system operation, we carried our samples' measurements. 
For improved accuracy, we repeated each measurement four time, rotating the sample by $90^{\circ}$ for each measurement; the measured results were then averaged to provide the final result.

\subsubsection{Physical property measurement system}

As mentioned in section 4.4, AZO is a n-type doped semiconductor, where a high doping level is required to have metal-like AZO thin films. This property is correlated with low thin film resistivity, which depends on the doping level and factors that affect carrier mobility such as grain size and other crystal properties [377].

The physical property measurement system (PPMS) is an analytical instrument designed to carry a variety of automated measurements. The PPMS used in our study was from Quantum Design Inc., and it enabled us to measure the films' resistance versus temperature. Measurement results depended on the inherent characteristics of the deposited thin films, which could vary between sputtering runs.

Resistance dependence on temperature has established models for both metals and semiconductors. In metals for instance, resistivity increases with temperature in a mostly linear fashion for temperatures higher than $100 \mathrm{~K}$, whereas semiconductors become more resistive with temperature decrease due to the drop in the fermi level as carriers fall into lower energy states [378].

The PPMS used in this study has a chamber that is kept under vacuum, except when loading and unloading the samples, where helium gas is used as a backfill to prevent moister and other contaminants from getting into the chamber. The sample holder has a delicate temperature sensor at its end where the sample is 
placed. This sensor measures temperature accurately near the sample's surface. Another temperature sensor is located at the bottom of the chamber, hence the temperature difference between the bottom of the chamber and at the sample's surface can be monitored.

Before a sample was loaded into the PPMS, its resistance was measured using a precision digital multimeter (Agilent 34410A) to use as a reference. This value was later compared with the resistance measured by the PPMS, where both readings should conform.

Thus, after the reference measurement, the sample was loaded into the PPMS. Figure 6-5 shows an illustration of the components at the bottom of the PPMS chamber.

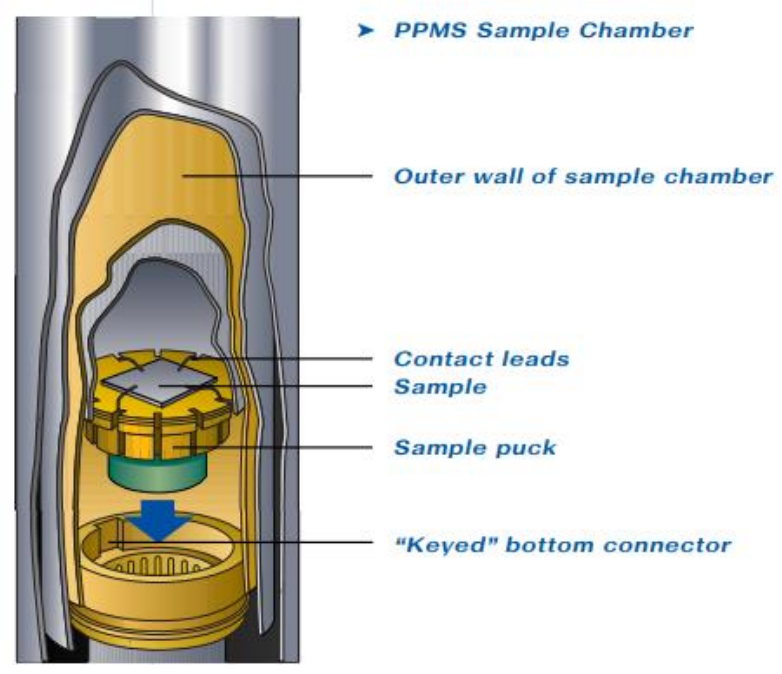

Figure 6-5 A PPMS setup with the sample ready for measurement [379].

Thereafter, resistance was measured as a function of temperature, which provided important information about variations between our samples' electrical properties. For our measurements, resistance was converted into resistivity based 
on NIST's algorithm for sheet resistance estimation using van der Pauw's equation [375].

\subsection{Spectral Characterization}

Spectroscopy is a technique that studies the spectral interaction of electromagnetic waves with a given sample [380]. This technique could also be applied using other types of waves, such as acoustic and gravitational waves $[381,382,383]$. Historically, spectroscopy was used to study samples under the illumination of visible light. In 1672, Isaac Newton published his findings about shining the visible light on a prism, which provided the first understanding of the rainbow [384].

The output of a spectrometer is a spectrum, which could either be quantized or continuous. For instance, atomic emission spectra are quantized, as atoms selectively absorb certain quanta of energy (photons) for transitions between their different energy states. Thus, when a polychromatic light beam passes through a hydrogen gas tube, electrons in the hydrogen atoms selectively absorb photons that possess the required energy to transit to higher energy levels, i.e., energy matching the discrete absorption spectrum of hydrogen. Similarly, once an electron drops to a lower energy level, a photon with a specific energy, compatible with the difference between the two energy levels is emitted, which can be seen in the discrete emission spectrum of a hydrogen atom. Meanwhile, a prism separates a beam of white light to all its constituent colours, which resemble a continuous spectral output. 
Spectroscopy techniques can be used for both qualitative and quantitative analysis [385]:

- In qualitative analysis, certain qualities of the inspected sample are identified. This often relates to detecting the elemental or molecular composition of a material, where specific spectral signatures are associated with each "ingredient".

- In quantitative analysis, certain quantities are estimated in each sample, such as stoichiometry and concentration.

In this thesis, UV-Vis spectroscopy, Fourier transform infrared spectroscopy and Rutherford backscattering spectroscopy were used to characterize the deposited samples, both qualitatively and quantitatively. Further analysis of the UV-Vis and FTIR data enabled us to estimate the samples' physical properties, such as dielectric permittivity and plasma frequency, and some qualitative properties such as absorption. Rutherford backscattering spectrometry is a quantitative technique which was used to provide information about the atomic composition and thin film thickness of our samples.

In UV-Vis and FTIR spectrometers monochromatic light is transmitted through or reflected by a sample then measured by a detector, as the frequency of the light is changed within a given range of the electromagnetic spectrum. Thus, a transmission, reflection, absorption or photoluminescence spectrum could be generated from these measurements. In some systems, several light sources and detectors can be integrated to allow measurements over a broad range of the EM spectrum.

UV-Vis and FTIR spectrometers can provide information about the characterized samples based on the measured wavelength range, where different 
interactions between the molecules or atoms can be excited and probed. For instance, the UV and visible light ranges have relatively shorter wavelengths, i.e., higher energies, which can result in electronic transitions at the atomic or molecular scale. On the other hand, infrared wavelengths are longer, i.e., lower energy, which can excite the vibrational transitions of molecules through phonon coupling in the far infra-red region (FIR).

The transmission, reflection and absorption spectra of a sample can be quantitatively known through the measured spectra. Depending on the sample's composition, specific spectral bands can be absorbed, which can excite electrons in the sample. The remaining portion of the light is either transmitted or reflected from the sample.

In the following section, we describe how a UV-Vis spectrometer works.

\subsubsection{Ultraviolet-Visible Spectroscopy}

The major parts of a photospectrometer include the light source, the diffraction grating and the detector.

In such a system, the light beam impinges on a dispersive grating before it hits the sample. This is a necessary step as the dispersive grating acts like a prism that deconstructs light to its constituent wavelengths [386]. Furthermore, to confirm that the specified measurement band is accurate, the deconstructed beam passes through a monochromator or a band-pass filter before hitting the sample. After the light interacts with the sample, it reaches a light sensitive detector. As a result, the amount of light transmitted through or reflected from the sample can be measured. In a case where the reflection is assumed to be negligible, the 
absorbance of the light beam within the sample can also be directly measured using the transmission spectrum.

An illustration of the Cary 100 spectrometer used in this study is shown in Figure 6-6. Light sources in this photospectrometer include a deuterium lamp for the UV region and a tungsten halogen lamp for the visible/near-infrared region. The deuterium light source needed to be turned on for at least 15 minutes before starting the measurements to reach a stable intensity level. The system operates at ambient conditions.

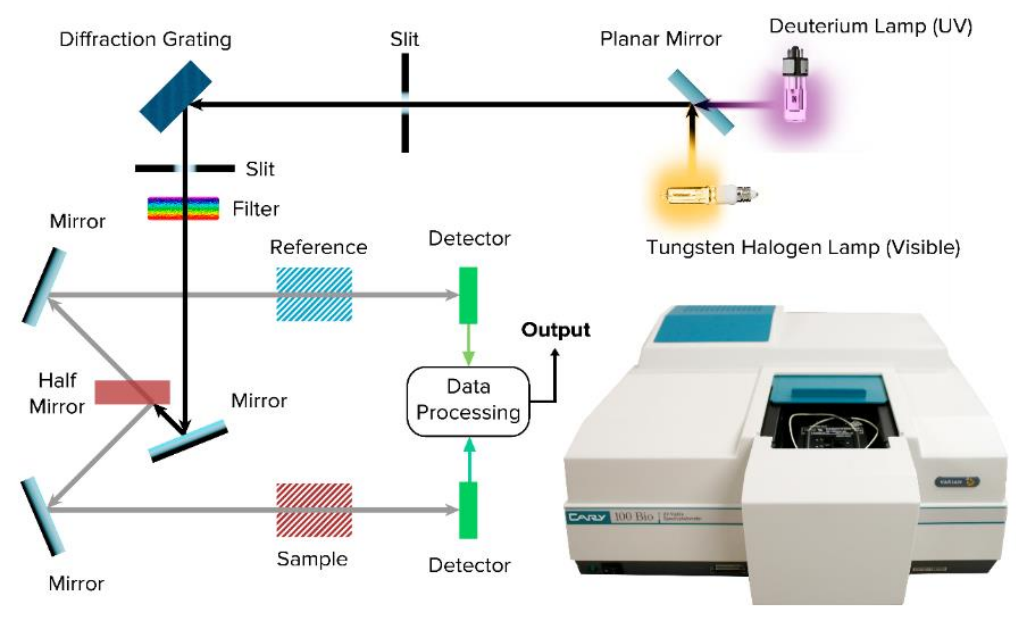

Figure 6-6 A diagram showing the UV/Vis spectrophotometer working steps, and the Cary 100 photospectrometer used in this work [253].

There are two compartments inside the chamber of a Cary 100, one for the sample while the other is for a reference. Input light is divided into two rays by means of a beam splitter or half mirror. These rays then pass through the sample and reference compartments. Finally, identical photodetectors are used to measure the output intensities of both compartments. Before starting the 
transmission measurement, the detected background level should be adjusted for by running the measurements using just the reference, then assigning $100 \%$ to the result over the measured spectrum.

After mounting the sample, the system is ready for the measurement. Thus, when a sample is measured, the resulting transmission spectrum would be normalized with respect to the adjusted reference response.

In our study, we used the Cary 100 to measure the transmission response of the AZO thin film samples in the range of $190 \mathrm{~nm}$ to $900 \mathrm{~nm}$.

According to literature [245, 235], AZO has plasmonic properties in the near infrared region (NIR). Therefore, UV-Vis spectroscopy did not have the suitable range to characterize our $\mathrm{AZO}$ thin films in the region of interest (NIR), nonetheless, it provided a quick sanity check regarding the visible properties of these samples.

\subsubsection{Fourier transform infrared spectroscopy}

Unlike the UV-Vis spectrometer that uses a dispersive grating to generate the monochromatic light, Fourier transform infrared spectroscopy (FTIR) uses an interferometer to generate the spectra of a sample, then performs a Fourier transform on the resulting interferogram to calculate the final spectrum. By using different detectors and light sources, a FTIR system can characterize samples over a broad range of the EM spectrum. In our study, we used two FTIR systems, namely the Bomem DA8 FTIR spectrometer and the Vertex V80v Bruker FTIR spectrometer, where both systems operate under vacuum.

Typically, in a FTIR spectrometer, light is emitted from a source, and is directed to an interferometer that modulates it. Passing through the 
interferometer, light is directed to the relevant sample compartment for a transmission or reflection measurement. Then, light exiting the compartment is focused onto a suitable detector.

Figure 6-7 shows the electromagnetic spectrum with specifications regarding the different energy ranges starting from $\gamma$-rays to radio broadcast waves.

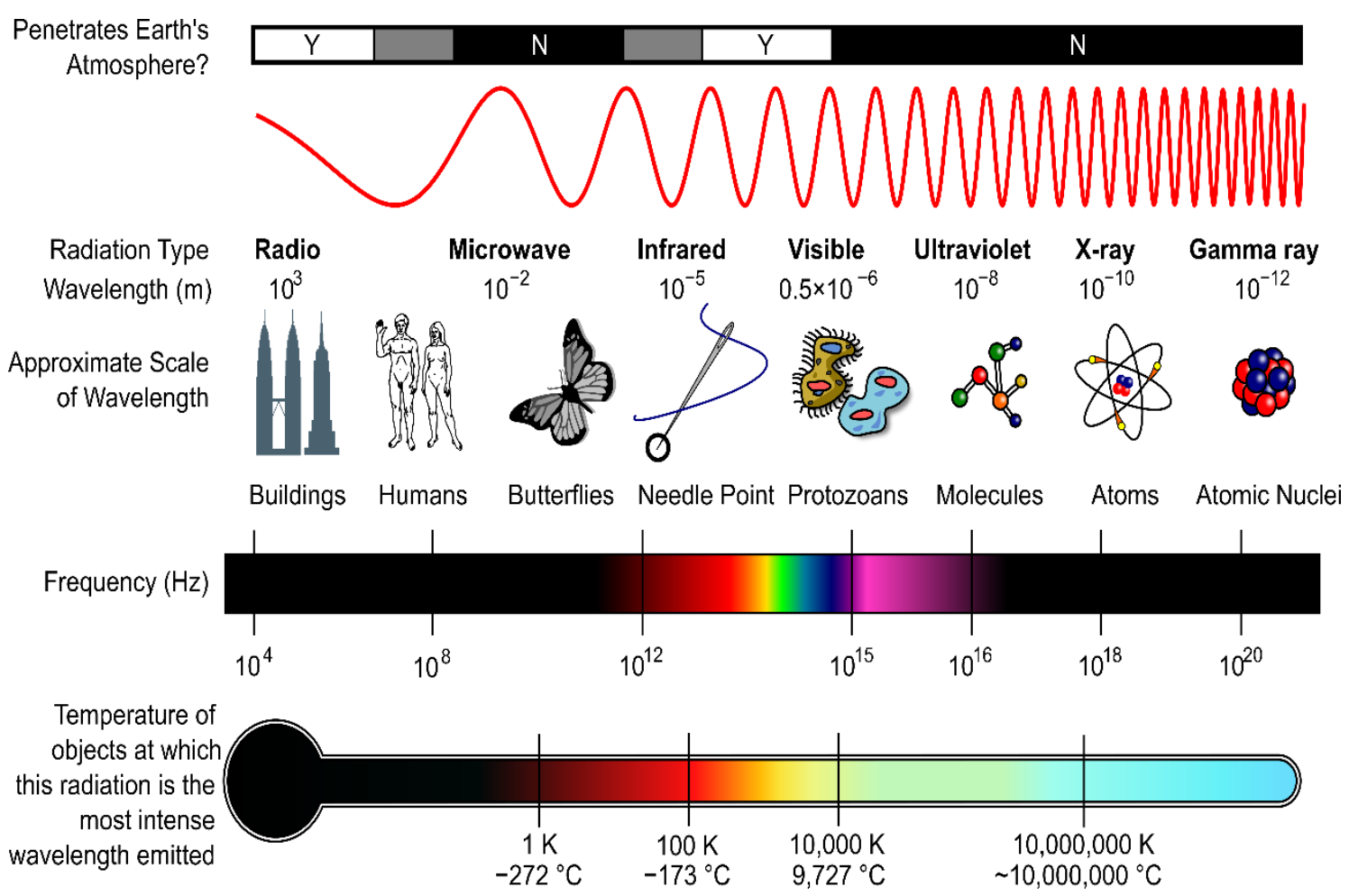

Figure 6-7 A diagram of the electromagnetic spectrum [387]

For a better understanding, some terms relevant to FTIR spectrometry are defined in the subsections below. 


\subsubsection{The interferogram}

A simple Fourier transform spectrometer consists of a Michelson interferometer with a beam splitter, plus one fixed mirror and one moving mirror. Figure 6-8 shows a simple Fourier transform spectrometer. As seen in the figure, a beam of light from a given source is divided into two rays by means of the beam splitter. These rays then hit the two mirrors, where a right angle exists between the fixed mirror and the moving mirror. The recombined beam's intensity at the detector is shown in the diagram as a function of the moving mirror position, starting from the zero-path difference position [388]. At zero path difference, the rays form a constructive interference and the recombined signal intensity is at the maximum value; hence the primary peak of the interferogram is created [389].

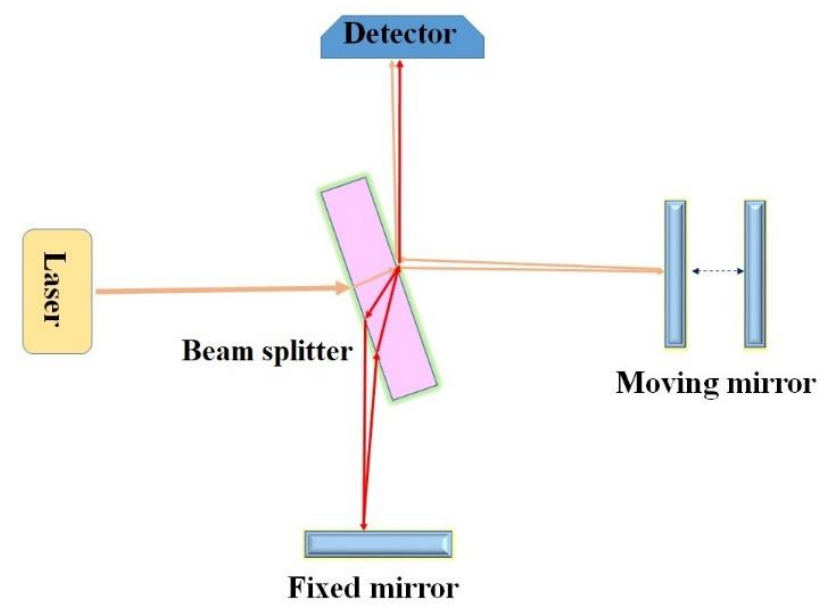

Figure 6-8 A simple Fourier transform spectrometer consists of a laser beam, a beam splitter, a detector, one fixed mirror and one moving mirror.

Once the optical path difference increases, the recombined beam shows a series of destructive and constructive interference regions. The resulting 
interferogram displays the recombined light intensity that corresponds to the spectral range of interest. This range should be within the spectral range of the active detector. Fourier transform is used to convert the spatial interferogram into a spectral beam.

Two typical FTIR interferograms captured in real time in our measurements are shown in Figure 6-9. These interferograms belong to the mid-infrared (MIR) and visible detectors with their primary peak positions specified in each plot.
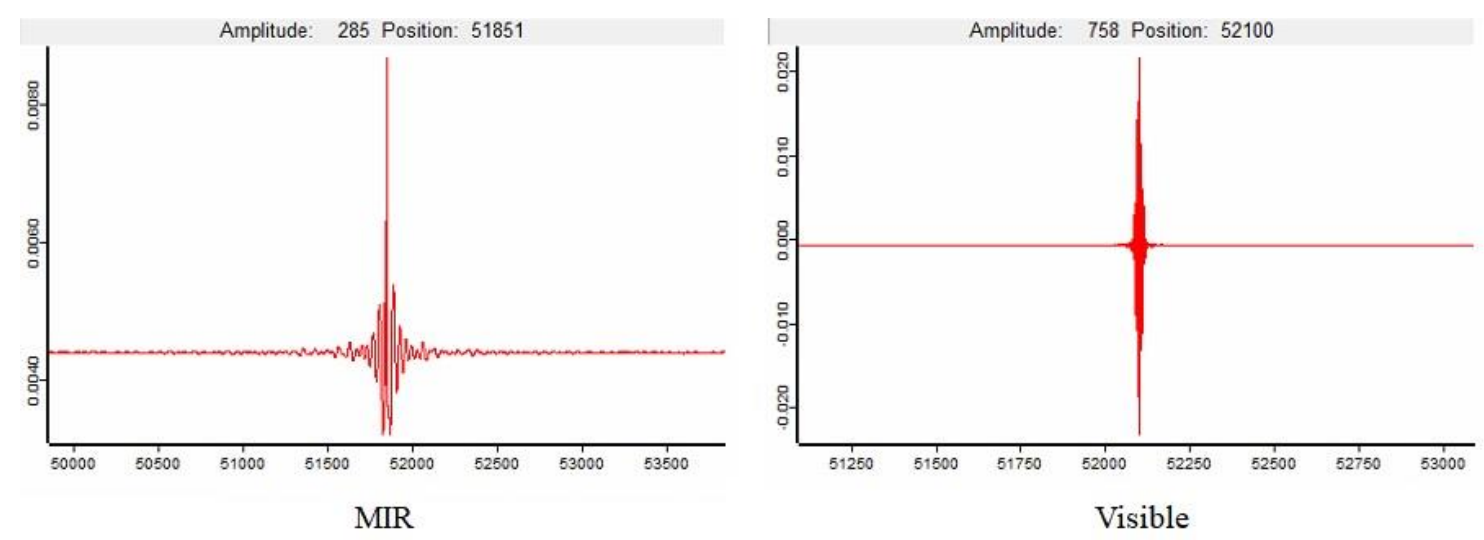

Figure 6-9 Interferograms for the MIR and visible detectors in the Bruker FTIR

\subsubsection{Fourier transform}

Fourier transformation is the theory behind the performance of a FTIR spectrometer where an interferogram generated by a spectrometer is transformed into the spectral domain using the relevant mathematical algorithm. The general Fourier transform function is a continuous signal based on an integral function. The Bruker FTIR spectrometer uses discrete furrier transformation (DFT) because it uses digitized interferogram signals [389]. Using 
this function, a wavefront from the incoming beam can be decomposed to its constituent frequencies using sinusoidal terms. Each sinusoidal term is identified by its frequency and amplitude [390].

$$
S(k . \nabla v)=\sum I(n . \nabla x) e^{-i 2 \pi n k / N}
$$

Equation 6-15

As can be seen, in DFT, the continuous values of $v$ and $x$ are replaced with the discrete interferogram points of $k . \nabla v$ and $n . \nabla x$ [391].

\subsubsection{The background spectrum}

The background spectrum is generated when the beam passes through the sample holder while the sample hole is empty. The background is collected in most FTIR setups in order to cancel out the instrument contribution to the overall measured spectrum. For consistency in measurements, all measured spectra from our thin film samples were normalize over the background spectrum, similar to the carried approach in our UV-Vis measurements. However, in this instance, the background collection process is not a one-off and must be repeated before measuring each thin film sample.

Thus, to collect a transmission spectrum using our Bruker FTIR spectrometer, we first measured the interferogram of the background, i.e., sample holder without any sample, then the sample of interest are measured. Fourier transforms of both interferograms were calculated by the Bruker's user-interface, where the final transmission spectrum was presented according to the following equation: 


$$
T(v)=\frac{S(v)}{R(v)^{\prime}}
$$

Equation 6-16

where, $v$ is the frequency, $S$ is the sample's spectrum and $R$ represents the reference or background spectrum

\subsubsection{Wavenumber}

The output data from a FTIR spectrometer is either transmission or reflection data expressed versus frequency, where frequency has a wavenumber unit $\left(\mathrm{cm}^{-}\right.$ ${ }^{1}$ ), which corresponds to radians per distance. Frequency with the wavenumber unit is a direct inverse of wavelength without the use of a $2 \pi$ factor. For example, a frequency value with the unit of wavenumber has the following conversion equation:

$$
\text { Frequency }\left(\mathrm{cm}^{-1}\right)=\frac{10^{7}}{\text { Wavelength }(\mathrm{nm})}
$$

Equation 6-17

\subsubsection{Beamsplitters}

To achieve reliable measurements, specific beamsplitters for different spectral ranges were required. In our Bruker v80 spectrometer, the beamsplitters can be changed manually or automatically with the help of a software. The advantage 
of an automatic operation is the elimination of the need to break the vacuum of the system.

Beamsplitters shown in Table 6-2 are available in our system to cover different spectral ranges. $\mathrm{KBr}$ is the default beamsplitter, which covers a spectral range of $350\left(\mathrm{~cm}^{-1}\right)$ to $8000\left(\mathrm{~cm}^{-1}\right)$.

Table 6-2 Different beamsplitters used in the Bruker FTIR with their corresponding operating ranges [389]

\begin{tabular}{|l|c|}
\hline Beamsplitter & Range of operation $\mathbf{~ c m}^{-1} \mathbf{)}$ \\
\hline Mid-Infrared (KBr) & $350-8000$ \\
\hline Near-Infrared (CaF2) & $1200-15000$ \\
\hline Visible and UV (CaF2 NIR/Vis/UV) & $50-4000$ \\
\hline
\end{tabular}

\subsubsection{Sources}

Five sources are available in our spectrometer. Each source operates in a specific spectral range. The MIR source (Globar) is the default source installed inside the spectrometer. Globar is a resistively heated silicon carbide bar, which can produce radiation over a broad EM range of $1 \mu \mathrm{m}$ to $50 \mu \mathrm{m}$ [392].

Externally connected sources can be plugged in at any of the four inlet ports available on the optical bench, however, a preferred inlet port exists for each specific source to achieve better alignment. Table 6-3 shows the different available sources and their installation locations. 
Table 6-3 Different sources used in the Bruker FTIR with their locations.

\begin{tabular}{|l|l|}
\hline Type of source & Location \\
\hline High-power Mid-Infrared (Globar) & internal \\
\hline Vis/NIR (tungsten halogen lamp) & internal \\
\hline Ultra-Violet (Deuterium lamp) & external \\
\hline FIR (mercury lamp) & external \\
\hline
\end{tabular}

\subsubsection{Detectors}

In an ideal measurement, all photons originating from the light source that hit the sample are collected by the detector. These photons then generate an electrical current in the detector. The current intensities from the reference cell and the sample cell provide a ratio that defines the transmission of a given sample.

Detectors, however, have finite ranges of operation; hence, the measured EM spectrum is often divided into different sections that correspond detectors' ranges. Available detectors in the spectrometer used in this study are presented in Table 6-4. 
Table 6-4 Different detectors used in the Bruker FTIR with their estimated spectral range of operation.

\begin{tabular}{|l|l|}
\hline Detector name & Spectral range $\mathbf{( c m}^{-1} \mathbf{)}$ \\
\hline DLaTGS FIR with KBr window & Mid-Infrared, 600-5000 \\
\hline InGaAs diode & Near infrared, 3850-11500 \\
\hline RT-Si diode & Visible, 9500-20500 \\
\hline RT-GaP diode & $\mathrm{UV}, 20000-37000$ \\
\hline
\end{tabular}

All four detectors were located inside the external detector chamber and they operated at room temperature. The spectral range information provided in Table 6-4 shows the estimated range where each detector provides the best coverage of the spectrum while the signal to noise ratio is minimal, these ranges are usually assumed under ideal conditions.

To convert the analog signal from the detector to a digital one, an amplifier and a built in analog to digital converter (ADC) are used, which can be set automatically or manually. Therefore, both a high signal to noise ratio and an interference free transmission signal can be obtained.

When switching between detectors during a measurement, mirrors are automatically adjusted to create the best path for the light beam, from the source to the corresponding detector. It is noted that removal of obstacles along the optical path is essential to ensure correct measurements. We also note that a red alignment laser within the system is set to pass through the centre of the sample hole to ensure correct placement. Finally, our FTIR spectrometer features a robust 
computer interface (OPUS 7.5) that controls the system and records the measured data in real-time.

\subsection{Plasma frequency}

\subsubsection{The Drude model}

A well-known method to calculate the plasma frequency is based on the Drude model, which was the first of its kind to study the properties of metals [393]. The Drude model is semi-classical where electrons are treated as free solid spheres that can travel in all directions and undergo elastic collisions in random directions with the fixed heavy ions. An important constraint of this model is that the electron-electron and electron-ion interactions need to be ignored. The Drude model is suitable where there is abundance of free electrons in the material, for instance in metals. The elastic collision assumption in the Drude model is similar to that of the ideal gas theory where the dominant energy ruling the model's performance is the kinetic energy. Therefore, based on the kinetic molecular theory, we estimate the average speed of a gas particle to have a direct relation with its temperature [394].

$$
\begin{gathered}
\frac{1}{2} M \overline{v^{2}}=\frac{3}{2} R T \\
v_{r m s}=\sqrt{\frac{3 R T}{M}}
\end{gathered}
$$

Equation 6-18 
where, Vrms is the root mean square speed, $\mathrm{R}$ is the molar gas constant $(\mathrm{J} / \mathrm{mol} \times \mathrm{K})$, $T$ is the temperature in $(\mathrm{K})$, and $M$ is the molar mass $(\mathrm{kg} / \mathrm{mol})$. Similarly, based on our assumption for the Drude model, the average speed of an electron at room temperature can be written as:

$$
\bar{v}=\sqrt{\frac{3 k_{B} T}{m_{e}}}
$$

Equation 6-19

where $m_{e}$ is the electron mass $(\mathrm{kg}), \mathrm{k}_{\mathrm{B}}=\mathrm{R} / \mathrm{N}_{\mathrm{A}}, \mathrm{k}_{\mathrm{B}}$ is the Boltzmann constant $(\mathrm{J} / \mathrm{K})$, and $\mathrm{N}_{\mathrm{A}}$ is the Avogadro number.

In the Drude model, relaxation time $(\tau)$ is defined as the average time an electron travels before a collision happens. Scattering rate is the inverse of relaxation time, denoted as $G$ in our study, where $(G=1 / \tau)$. Therefore, the average distance travelled by a particle, i.e., the mean free path is given by:

$$
l=\tau \bar{v}
$$

\section{Equation 6-20}

Using these concepts in the Drude model, the dispersion relation for the dielectric permittivity is given by:

$$
\varepsilon^{f}(\omega)=\varepsilon_{\infty}+\frac{\omega_{p}^{2}}{-\omega^{2}+i \omega G}
$$

Equation 6-21 
where $\mathrm{f}$ denotes the electronic intraband transitions produced by free electrons, $\varepsilon_{\infty}$ is the high frequency dielectric constant, which usually is equal to or bigger than unity [395], $\omega_{p}$ is the plasma frequency that relates to the intraband transitions, $\omega$ is the frequency of the external electromagnetic radiation, and $G$ is the scattering rate, also known as the damping constant [396, 193, 397].

The following equation describes the electrical DC conductivity (when $\omega=0$ ) in the Drude model [398]:

$$
\sigma=\frac{n e^{2} \tau}{m_{e}^{*}}
$$

Equation 6-22

where $\sigma$ is the electrical conductivity, $n$ is the carrier concentration, $e$ is the charge of an electron, $m_{e}^{*}$ is the electron's effective mass and $\tau$ is the relaxation time [399].

The Drude model is most appropriately used to describe the properties of free carriers in metals and semiconductors [400]. It does not account for interband transitions in metals and semiconductors. Thus, this model will be applied to AZO for energies lower than the bandgap energies [395].

\subsubsection{Plasma frequency calculation using the Drude model}

Plasma oscillations are collective, meaning that they are described as a group as opposed to charged ionized particles. Accordingly, the centre of mass of the group's nuclei and the centre of mass of the group's electrons represent the collective nuclei and electrons while in motion. These centres coincide at equilibrium, and once their positions are shifted with respect to one another, the 
Coulomb force acts to restore equilibrium resulting in an oscillatory behaviour. Plasma frequency is the frequency at which these oscillations resonate, hence it's a characteristic material property [401].

Most metals are opaque and reflective within the visible region [402]. Based on the free electron theory, their reflectivity depends on their plasma frequency, where reflectivity reaches zero at the screened plasma frequency, $\omega_{p}$ [403]. Thus, when light with a frequency below $\omega_{p}$ is incident on a metal's surface, most of the light energy is reflected with the same incident wavelength, while any absorbed light is re-emitted by the surrounding free electrons. This explains why metals are highly reflective.

Nonetheless, metals are transparent to light with frequencies higher than that of the screened plasma frequency, where there is no absorption in the material. This is due to the inability of electrons to quickly interact with the applied electric field (with in the light) at these frequencies [404]. Once a perturbation field is introduced to the material, electrons require a certain amount of time to react to the field. Therefore, the screened plasma frequency defines the screened response time, $t_{p}$, as follows:

$$
t_{p}=\frac{2 \pi}{\omega_{p}}
$$

\section{Equation 6-23}

For a time varying field at frequencies below plasma frequency, the perturbation variation time, $t=2 \pi / \omega$, is longer than the screened response time. Hence, the electrons can react to the field, thereby screening it. However, for frequencies above the plasma frequency, the perturbation variation time is shorter than $t_{p}$, and there is not enough time to screen the field [405]. 
When electrons move within a plasma wave, the electron density is changed accordingly. The average charge density in one dimension is given by:

$$
\rho=\left(n-n_{a f t e r}\right) e=n e \frac{d s}{d x}
$$

Equation 6-24

where $e$ is the electrons charge, $n$ is the initial density, $n_{\text {after }}$ is the density after motion, $\mathrm{ds} / \mathrm{dx}$ is the ratio of displacement compared to the equilibrium location. By combining Gauss's law (Equation 1-7) and the charge density equation (Equation 6-24), we can derive the one dimensional electric field function $\left(E_{x}\right)$ due to the displacement of electrons as:

$$
\frac{\partial E_{x}}{\partial x}=\frac{n e}{\varepsilon_{0}} \frac{\partial s}{\partial x}
$$

Integrating with respect to $\mathrm{x}$ :

$$
E_{x}=\frac{n e}{\varepsilon_{0}} s+K
$$

Equation 6-25

The integration constant $\mathrm{K}$ is zero given that $E_{x}$ is null when $s=0$. Accordingly, moving electrons with an effective mass of $m_{e}^{*}$ will experience the following restoring force $\left(F_{x}\right)$ at the new position: 


$$
F_{x}=m_{e}^{*} \frac{d^{2} s}{d t^{2}}=-\frac{n e^{2}}{\varepsilon_{0}} s
$$

Equation 6-26

Based on the definition of the wave, we assume that displacement follows a harmonic path with a time variation of $e^{i \omega_{p} t} / \cos \omega_{p} t$ [406]. Therefore, the frequency of oscillation or the plasma frequency is given by:

$$
\omega_{p}=\sqrt{\frac{n e^{2}}{\varepsilon_{0} m_{e}^{*}}}
$$

\section{Equation 6-27}

where $\omega_{p}$ is the plasma frequency, $n$ is the carrier concentration, $e$ is the charge of an electron, $\varepsilon_{0}$ is the permittivity of free space, and $m_{e}^{*}$ is the electron's effective mass in the material [269]. In our study, the electron effective mass in zinc oxide was initially selected to be $m_{e}{ }^{*}=0.27 m_{e}$, as suggested by Noriega et al [407], a figure which was later revised to $0.65 m_{e}$ as revealed in our later sections.

An increase in the carrier concentration in a material will result in a higher plasma frequency. It is noted that charge tends to move to the surface of a good conductor, thus, no electric fields exist inside its bulk.

The plasma frequency directly affects the optical and electrical properties of a material [395]. For instance, assuming the Drude model, the real part of the dielectric permittivity is negative and the index of refraction is complex for frequencies below plasma frequency [395]. On the other hand, for frequencies 
higher than the plasma frequency the material loses its reflectivity and becomes transparent [408].

Despite its limitations, the Drude model could be applied to estimate the properties of highly doped semiconductors, such as our AZO thin film samples.

\subsubsection{The Lorentz model}

Once multiple resonant frequencies are compounded to the material's dispersion relation, the semi-quantum Lorentz model can be applied [409]. This model describes the electronic interband transitions, and assumes an atomic model where electrons are bound to the nuclei. In this instance, EM induced oscillations can be described by a damped mass on a spring model [410]. Each oscillator in this model corresponds to an absorption band in the material, or interband transition energies, referring to a bound electron effect [411].

$$
\begin{gathered}
\varepsilon^{b}(\omega)=\sum_{m=1}^{M} \frac{A_{i} f_{m} \omega_{p}^{2}}{\omega_{m}^{2}-\omega^{2}+i \omega G} \\
A_{i}=\frac{f_{m}}{\omega_{p}^{2}}, \sum_{1}^{M} f_{m}=1
\end{gathered}
$$

Equation 6-28

where, $m$ denotes the oscillator number, $\omega_{m}$ is the resonant frequency of the corresponding oscillator, $f_{m}$ is the oscillator strength, $\omega_{p}=\sqrt{G_{m}} \omega_{m}$ is the plasma frequency associated with the $m^{\text {th }}$ resonance [412]. 


\subsubsection{The Drude-Lorentz model}

The Drude-Lorentz model is a combined model that takes into account both the interband and intraband transmission effects, which offers a more realistic dielectric model for materials [413]. Accordingly, the dispersion relation for the dielectric permittivity is given by:

$$
\varepsilon_{r}(\omega)=\varepsilon^{f}(\omega)+\varepsilon^{b}(\omega)=\varepsilon_{\infty}+\sum_{m=0}^{M} \frac{f_{m} \omega_{m}^{2}}{\omega_{m}^{2}-\omega^{2}+i \omega G}
$$

Equation 6-29

In the case when $m=\omega_{0}=0$, this equation turns into the Drude model formula. In the next section, we introduce a software package that models the Drude and Lorentzian terms of our deposited thin film samples.

\subsubsection{Calculating plasma frequency using the modelling software}

In this work, plasma frequency was mostly estimated by means of fitting the measured transmission spectra in the FTIR system. This fitting provides one Drude term and, based on the complexity of the spectrum, one or more Lorentzian oscillator terms for the corresponding AZO thin film. The Drude term is then used to estimate the plasma frequency value. 
For this fitting process, we consider our sample to be multilayered, composed of a substrate and a thin film. This process also requires the knowledge of the substrate and thin film thicknesses with high precision. The spectral data from the FTIR spectrometer must be known for both the bare substrate, and the thin film on substrate, to be able to model their individual properties.

Once a transmission spectrum is fitted using the software, the resulting oscillator terms provide information about the properties of the material, such as the plasma frequency and the scattering rate [414]. This scattering rate $(G)$ should agree with the results from the PPMS measurement, and comply with the Drude DC conductivity model shown in Equation 6-22.

Information from the plasma frequency fitting model can then be used to provide an estimate for the material's dielectric permittivity [415].

Given the large number of samples that we produced, and the extensive effort required to fully characterize each sample, we resorted to nominating certain samples from each deposition run, thereby providing a representative analysis. The main criteria we used to select these nominal samples were:

- High transparency, samples with high transparency were selected as this is a desirable property for our FSL.

- High carrier concentration, which directly relates to the transmission spectrum of a given sample. In this case, it was desirable to have samples that are metal-like, i.e., having a high carrier concentration to yield a high plasma frequency.

- High film uniformity, both visually and thickness wise, which depend on the location of the sample in the sputtering chamber and could affect the transmission characteristics of the sample. 


\subsubsection{Coherent and Incoherent Fields}

In order to comply with the requirements of the Reffit model, coherency of the layers in each sample must be specified, as samples were assumed to have two layers, corresponding to the substrate and the thin film, respectively [416].

Coherency is a term referring to phase matching between the field components in time or space. Thus, in a coherent layer, orders of transmission (or reflection) are in-phase and interfere constructively; this is often the case in thin films. The following equation describes the phase difference relation during this interference [417]:

$2 n o \cos (\theta)=m \lambda$

Equation 6-30

where $\lambda$ is the input light wavelength, $m$ is an integer, $n$ is the refractive index of the medium and $o$ is the thickness. Figure 6-10 shows the orders of transmission from a thin film with coherent light transmission resulting in a Fabry-Perot effect. 


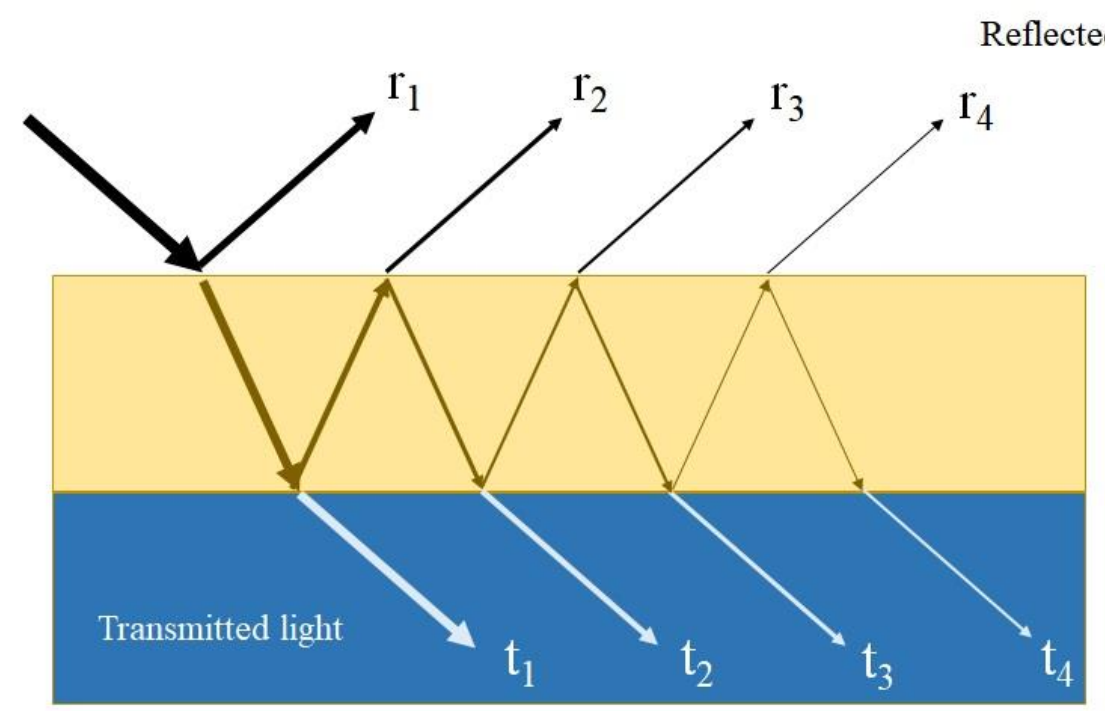

Figure 6-10 coherent transmission and reflection of light upon hitting a thin film sample's surface

For an unsupported weakly absorbing thin film, the coherent normal incidence reflection is given by [418]:

$$
R=\left|\frac{r_{1}+r_{2} \exp (-2 i \gamma o)}{1+r_{1} r_{2} \exp (-2 i \gamma o)}\right|^{2}
$$

\section{Equation 6-31}

and the coherent normal incidence transmission is given by [418]:

$$
T=\left|\frac{t_{1} t_{2} \exp (-i \gamma o)}{1+r_{1} r_{2} \exp (-2 i \gamma o)}\right|^{2}
$$

Equation 6-32

Both of which allow for phase interference in the thin film, where 1 and 2 refer to the front and back surfaces on the film, respectively. In these equation, $\mathrm{r}^{\prime} \mathrm{s}$ and $\mathrm{t}^{\prime} \mathrm{s}$ 
represent the normal incident Fresnel coefficients for the front and back surfaces of the thin film. $o$ is the thin film thickness and $\gamma=2 \pi N / \lambda$, where $N$ is the complex refractive index of the thin film.

For a thin film-on-substrate system, the reflectance $(\mathcal{R})$ and transmittance $(\mathcal{T})$ are given by:

$$
\mathcal{R}=R+\frac{T^{2} R_{0}}{1-R_{0} R^{\prime}}
$$

Equation 6-33

$$
\mathcal{T}=\frac{T\left(1-R_{0}\right)}{1-R_{0} R^{\prime}}
$$

Equation 6-34

where $\mathrm{R}$ and $\mathrm{T}$ are given by Equation 6-31 and Equation 6-32, respectively. $R_{0}$ is the reflectance of the back surface of the substrate, given by $\left(\left(1-n_{s}\right) /\left(1+n_{s}\right)\right)^{2}$, where $n_{s}$ is the refractive index of the substrate. $\mathrm{R}^{\prime}$ is light reflectance from the substrate boundary onto the back of the film, and is given by:

$$
R^{\prime}=\left|\frac{r_{2}+r_{1} \exp (-2 i \gamma o)}{1+r_{1} r_{2} \exp (-2 i \gamma o)}\right|^{2}
$$

Equation 6-35

In reality, for a given illumination wavelength, the thickness of an incoherent layer is much larger than that of a coherent layer. Thus, the constructive phase relationship does not apply for an incoherent layer scenario, where the 
incoherent multiple reflections inside the layer only include the intensity while the phase is lost. This phase loss is attributed to the reasons of incoherency, such as unparalleled layer boundaries or defects within the material, which is mostly the case in thicker layers.

\subsubsection{Comparing methods}

For selected samples, plasma frequency values estimated form the Hall effectDrude model method were compared to those estimated using the FTIR-Reffit method. This helped us to improve the accuracy of the estimated plasma frequency values, where an iterative approach was applied to minimize the differences between the results of both methods. This exercise was necessary given that a large range of plasma frequency values could fit the same FTIR curve using Reffit, which meant that relying solely on the fitting approach was not reliable.

Initially, a range of acceptable plasma frequency values from fitting agreed with the calculated values from the Hall effect-Drude model method. This was done while assuming that the effective mass of the carriers (electrons) was 0.27 of an electron mass, a value widely reported in literature for AZO films [419,407]. However, for some samples, this was not possible as plasma frequency values obtained from the Hall effect-Drude model method and the FTIR-Reffit method were too far to reconcile. Consequently, we analysed the main deficiencies in our methods while scouring literature. We identified that the effective mass value used in our calculations was inadequate, as effective mass can vary with the carrier concentration for degenerate doping levels [420]. It is also noted that providing an accurate model for the behaviour of the effective mass requires an extensive investigation, which falls outside the scope of this study. Therefore, we 
resorted to a compromise by selecting a value for the effective mass that reconciles both plasma frequency estimation approaches for all samples. This value for effective mass was about 0.65 of the electron mass. The following paragraphs describe the process that we carried to estimate this effective mass.

Firstly, the Reffit software was used to fit the FTIR transmission spectra to analytical models using the aforementioned multilayer approach [414]. For that, we opted to select the $\mathrm{AZO}$ on $\mathrm{CaF}_{2}$ samples given that the substrate was the most transparent across the measured spectrum. Therefore, we were able to isolate the AZO film's transmission properties most clearly, in particular in the infrared region where we suspected our plasma frequency to be, given the results of our Hall effect measurements.

Next, the fitted plasma frequency values from the Reffit model were incorporated into the Hall effect-Drude model to estimate the effective mass. It should be noted that for $\mathrm{AZO}$ on $\mathrm{CaF}_{2}$ samples, only a very limited range of plasma frequency values was suitable to fit the transmission curve as the substrate was highly transparent in that region. Accordingly, the effective mass value was estimated to be around 0.65 of an electron mass.

Using this estimated effective mass, plasma frequency values for both the FTIR-Reffit method and the classical Hall effect-Drude model method had good agreement with each other, for all samples included in this study. The full results will be presented in the following chapters. 


\subsection{Chapter summary}

Selecting suitable characterization techniques is an important step when evaluating the properties of thin films. In this chapter, a summary of the main characterization techniques and equipment used to study the structural, electrical and optical properties of our deposited thin films were presented. 
7 Results A: AZO thin films on

\author{
Borofloat33 substrate
}



This chapter describes the procedure to characterize the AZO thin films deposited on Borofloat33 substrate. This substrate was prioritized in this study given that the group at VUW had an established process for depositing $\mathrm{ZnO}$ on Borofloat33 and sapphire substrates. However, as sapphire is a crystal, its optical birefringence was perceived as a disadvantage for our plasmonic application, hence, was considered as a secondary option.

It is worth noting that this level of depth in investigation of the deposited AZO thin film on Borofloat33 using an off-centre magnetron sputtering setup in such wide electromagnetic range has never been reported to the best of author's knowledge and provides a much needed insight into the properties of this class of deposited AZO thin films.

\subsection{Chapter objectives}

In this chapter, nine AZO samples deposited on Borofloat33, namely S1-S9, are presented. These samples are from a single sputtering set that had the best transparency and carrier concentration values among our AZO on Borofloat33 samples.

The characterization methods can be divided into two groups; structural properties and optical-electrical properties.

Structural properties were measured using methods such as Rutherford backscattering spectroscopy, XRD, and SEM-EDS, where we investigated the stoichiometric ratios, thin film thickness and crystallography. 
When determining the optical-electrical properties of these samples, we used a Hall effect setup to measure the carrier concentration in our deposited thin films. The carrier concentration was then used to estimate the plasma frequency based on the Drude model. In addition, absorption in samples was calculated using transmission and reflection spectroscopy in the specified frequency range. Finally, based on the transmission spectra and the carrier concentration of the deposited thin films, a fitting procedure was performed for selected samples to calculate the plasma frequency and the dielectric permittivity.

The main objectives for the study presented in this chapter were:

- To measure the spectral properties of our samples using three different spectrometers. Each spectrometer had a limited wavelength range and by replacing the spectrometer, each time we were able to cover a broader wavelength range.

- To evaluate the transmission properties of Borofloat33 with and without the deposited thin film.

- To calculate the plasma frequency values using the FTIR-Reffit method and the Hall effect-Drude model method.

\subsection{XRD measurements}

XRD measurements were used to characterize the crystal properties of our $\mathrm{Al}$ doped zinc oxide thin films. Based on our pre-measurement checks, we confirmed that the x-ray beam covered a large area of the samples' surface, as mentioned in section 6.2. The studied parameters in this section are:

- The dominant crystal orientation for the deposited thin film. 
- The full width at half maximum of the diffraction peaks for the dominant crystal orientation, which provides an estimate for the mean crystal domain size.

- We note that we could not accurately evaluate the in-plane residual stress $(\zeta)$ as Borofloat33 is a polycrystalline material, hence alignment errors could be significant while measuring the lattice constant $(d)$.

XRD results of the selected samples from this chapter are presented below.

\subsubsection{Dominant crystal orientation}

Our deposited AZO on Borofloat33 samples produced diffractograms similar to that shown in Figure 7-1. The dominant peak in the diffractogram shows the preferred crystal orientation on the surface of the thin films [421]. For our films, the dominant intensity peak was the (002) orientation, which meant that our films grew along the c-axis direction. Based on literature, the (002) crystal orientation is the most thermodynamically stable growth direction for wurtzite zinc oxide [422], thus benefits from high energy deposition conditions [423]. This orientation has the lowest free surface energy as atoms are packed in a dense arrangement [424]. 


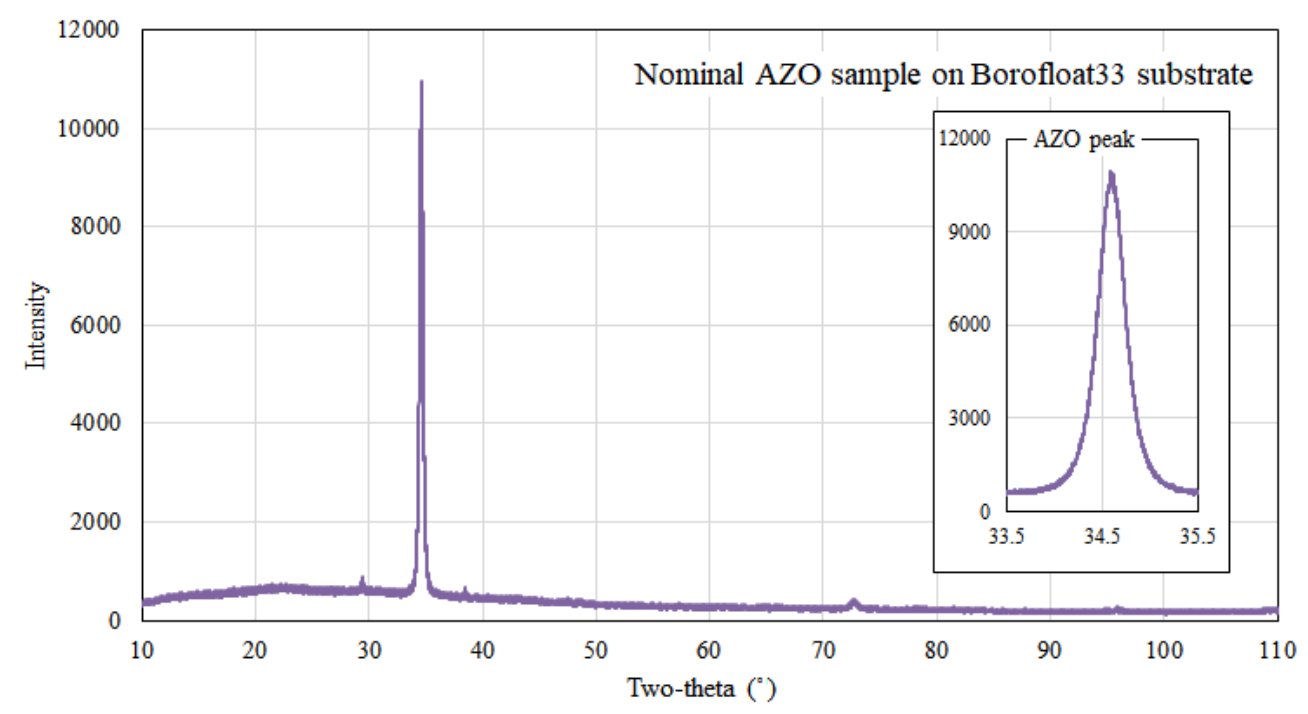

Figure 7-1 AZO thin film diffractogram on a Borofloat33 substrate.

In order to confirm the orientation selectivity and dominant AZO material's peak, the ratio of the dominant peak from the material's diffractogram can be compared with other competing peaks. Figure 7-1 shows the diffractogram of sample S9. The (002) crystal orientation is the only high intensity peak occurring at a $2 \theta$ of $\sim 34.6^{\circ}$ in this plot. The inset shows a close-up of this AZO intensity peak. As confirmed by the diffractogram, the Borofloat33 substrate does not show peaks given that it is amorphous.

\subsubsection{Mean crystallite domain size}

After recognizing the dominant crystal orientation, calculating the FWHM for each peak can be used to estimate the crystallite domain size for the corresponding orientations using Scherrer's formula [425, 354]. This formula is merely used to provide an estimate value, and its accuracy significantly degrades when the thin film thickness is larger than $100 \mathrm{~nm}$. Furthermore, Scherrer's formula assumes perfect conditions exist during the measurements. This is 
usually affected by imperfection in the XRD setup. The calculated FWHM and mean crystallite domain values are reported in Table 7-1 for selected AZO thin films on Borofloat33 substrates.

Table 7-1 FWHM and mean crystallite domain size for nominal AZO on Borofloat33 samples.

\begin{tabular}{|c|c|c|c|}
\hline Name & $S 4$ & $S 6$ & $S 9$ \\
\hline FWHM ( $\left.^{\circ}\right)$ & 0.31 & 0.33 & 0.30 \\
\hline CDS (nm) & 26.6 & 25.3 & 27.5 \\
\hline
\end{tabular}

Samples in Table 7-1 are selected from different locations on the sample holder during the same sputtering process. Differences between values of the mean crystallite domain size for these samples indicate a crystal domain size variation at the sputtered location. However, these values appear to be close to each other confirming the uniformity of our deposited thin films.

As the Borofloat33 substrate is amorphous, there is no substrate reference peak available to perform a stress analysis. These parameters will be studied in Chapter 8 where thin film deposition took place on crystalline substrates. 


\subsection{RBS measurements}

Using a Rutherford backscattering spectroscopy system, the atomic compositions of thin films could be quantitatively measured. This tool provided a means to confirm the stoichiometric homogeneity of the AZO thin film samples.

For each AZO on Borofloat33 sample, the atomic density composition was measured at 3 locations. The averages for these measurements are presented in Table 7-2, where 9 samples are included.

The measurement error is estimated to be $10 \%$. Averaging this density composition for all 9 samples gives $(77.0 \pm 8.0) \times 10^{+15}$ atoms per $\mathrm{cm}^{2}$ for both $\mathrm{Zn}$ and $\mathrm{O}$, and $(4.0 \pm 0.4) \times 10^{+15}$ atoms per $\mathrm{cm}^{2}$ for $\mathrm{Al}$.

The percentage of $\mathrm{Al}$ concentration shown in Table $7-2$ ranges from $2.26 \%$ to $2.74 \%$ while the sputtering target used to deposit these films had only $2 \% \mathrm{Al}$. This difference indicates that in the sputtering process, there are different profiles of sputtering where some elements sputter better than others. Controlling this outcome requires a complex process that is beyond the scope of our study. 
Table 7-2 Average atomic density composition (atoms per $\mathrm{cm}^{2}$ ) for our AZO films, along with the estimated thickness (nm) using RBS.

\begin{tabular}{|c|c|c|c|c|c|}
\hline Name & $\begin{array}{l}\text { Zn atoms } \\
\times 10^{+15} \mathrm{~cm}^{-2}\end{array}$ & $\begin{array}{l}\mathrm{O} \text { atoms } \\
\times 10^{+15} \mathrm{~cm}^{-2}\end{array}$ & $\begin{array}{l}\text { Al atoms } \\
\times 10^{+15} \mathrm{~cm}^{-2}\end{array}$ & $\begin{array}{c}\text { Al percentage } \\
\%\end{array}$ & $\begin{array}{c}\text { Thickness } \\
\text { nm }\end{array}$ \\
\hline S1 & 87.0 & 87.0 & 4.3 & 2.4 & $178 \pm 17$ \\
\hline S2 & 75.0 & 75.0 & 4.0 & 2.6 & $154 \pm 15$ \\
\hline S3 & 81.0 & 81.0 & 4.0 & 2.4 & $166 \pm 16$ \\
\hline S4 & 79.0 & 79.0 & 4.0 & 2.5 & $162 \pm 16$ \\
\hline S5 & 85.0 & 85.0 & 4.0 & 2.3 & $174 \pm 17$ \\
\hline S6 & 88.0 & 88.0 & 4.3 & 2.4 & $180 \pm 18$ \\
\hline S7 & 65.0 & 65.0 & 3.0 & 2.3 & $134 \pm 13$ \\
\hline S8 & 71.0 & 71.0 & 4.0 & 2.7 & $146 \pm 14$ \\
\hline S9 & 69.0 & 69.0 & 3.7 & 2.6 & $142 \pm 14$ \\
\hline
\end{tabular}

Thus, it is evident that the location of the sputtered film with respect to the sputtering plasma has an influence on the composition of the deposited AZO thin film, which is compounded by the fact that we did not rotate the samples during sputtering. Further characterization techniques will be applied to investigate the uniformity of our samples. 


\subsection{SEM and AFM imaging}

A scanning electron microscope (SEM) and atomic force microscope (AFM) were used to image the AZO samples studied in this chapter. These two techniques provided useful information regarding the surface properties of the deposited thin films. SEM images of the AZO sample (S6) in Figure 7-2 show a smooth surface with a grain size of about $60 \mathrm{~nm}$.

SEM images in Figure 7-3 show the surface of S9. Other samples showed similar properties, and by comparison, the uniformity and continuity of the thin film structure from this sputtering set was evident.

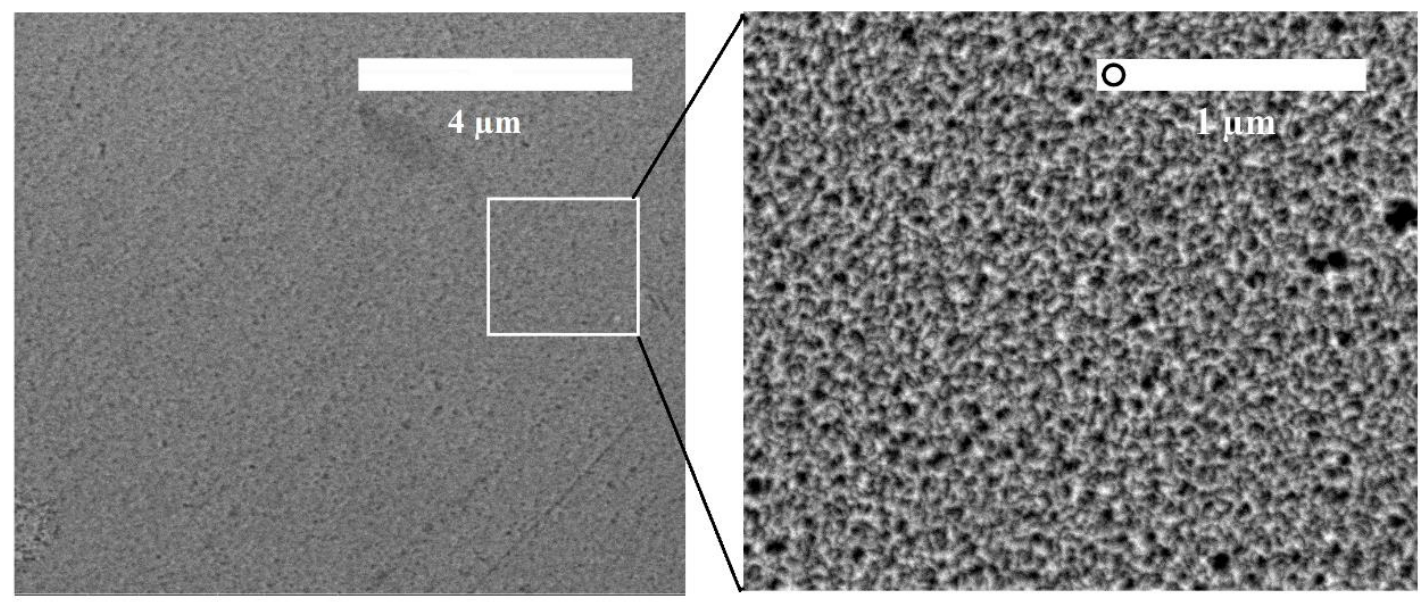

Figure 7-2 SEM images of the AZO sample's surface. As a reference to the grain size, a circle having a diameter of $60 \mathrm{~nm}$ is included in the scale bar of the magnified SEM image. 


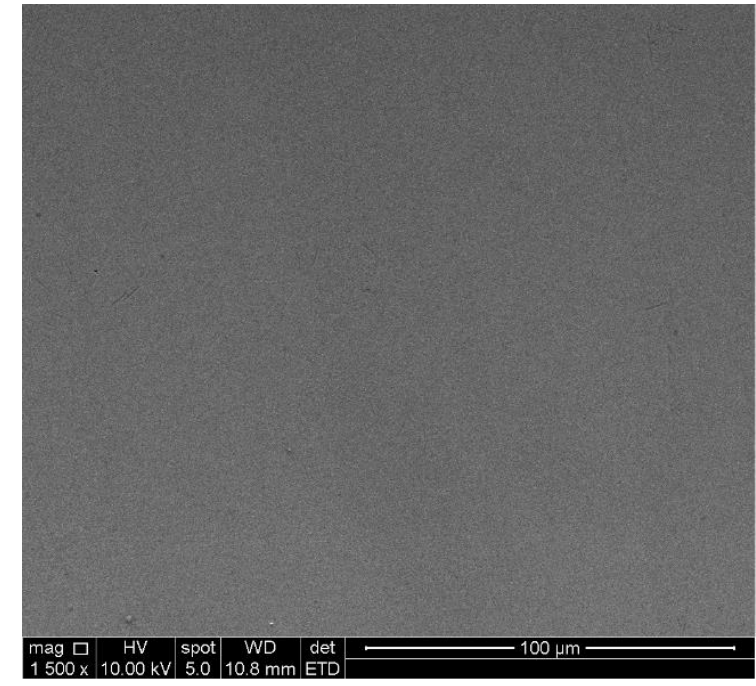

(a)

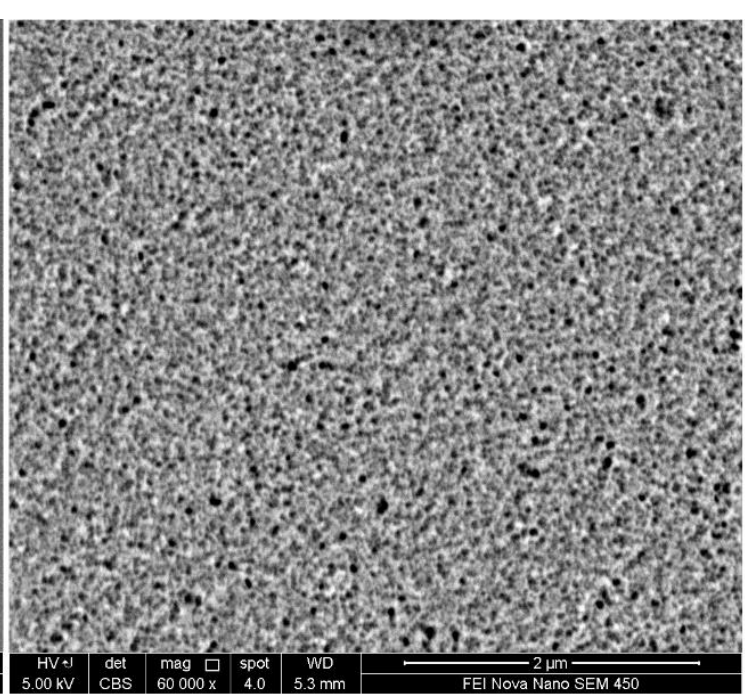

(b)

Figure 7-3 SEM images of an AZO on Borofloat33 sample at two different scales, (a) $100 \mu \mathrm{m}$, (b) $2 \mu \mathrm{m}$.

Three samples (S4, S6, and S9) were selected for an energy dispersive x-ray spectroscopy (EDS) analysis inside the SEM, where several spots were examined on each sample. These measurements were carried out using a $10 \mathrm{kV}$ tension, and the resulting mean atomic percentages are presented in Table 7-3.

Table 7-3 Mean atomic composition for S4, S6, and S9 using EDS.

\begin{tabular}{|c|c|c|c|}
\hline \multirow{2}{*}{ Element } & \multicolumn{3}{|c|}{ Atomic percentages } \\
\cline { 2 - 4 } & $S 4$ & $S 6$ & $S 9$ \\
\hline $\mathrm{O}$ & $53.1 \pm 4$ & $52.3 \pm 4$ & $54.1 \pm 4$ \\
\hline $\mathrm{Zn}$ & $31.8 \pm 1$ & $33.3 \pm 1$ & $30.7 \pm 1$ \\
\hline $\mathrm{Al}$ & $1.7 \pm 0.4$ & $1.6 \pm 0.3$ & $1.6 \pm 0.3$ \\
\hline $\mathrm{Si}$ & $12.0 \pm 0.7$ & $10.4 \pm 0.6$ & $11.2 \pm 0.6$ \\
\hline $\mathrm{Na}$ & $1.4 \pm 0.3$ & $2.4 \pm 0.4$ & $2.3 \pm 0.4$ \\
\hline
\end{tabular}


$\mathrm{Zn}$ is assumed to be exclusively detected from the thin film, while $\mathrm{Al}$ and $\mathrm{O}$ occur both in the film and the substrate. Si and $\mathrm{Na}$ are constituents of the substrate [289]. The results in Table 7-3 confirm the homogeneity of the grown thin films.

AFM images of our samples' surfaces showed that root mean square (RMS) roughness was around $1.5 \pm 0.6 \mathrm{~nm}$ for a sampled area of $100 \mu \mathrm{m}^{2}$, which indicate the smooth nature of our films regardless of thickness variations. A sample AFM image is shown in Figure 7-4.

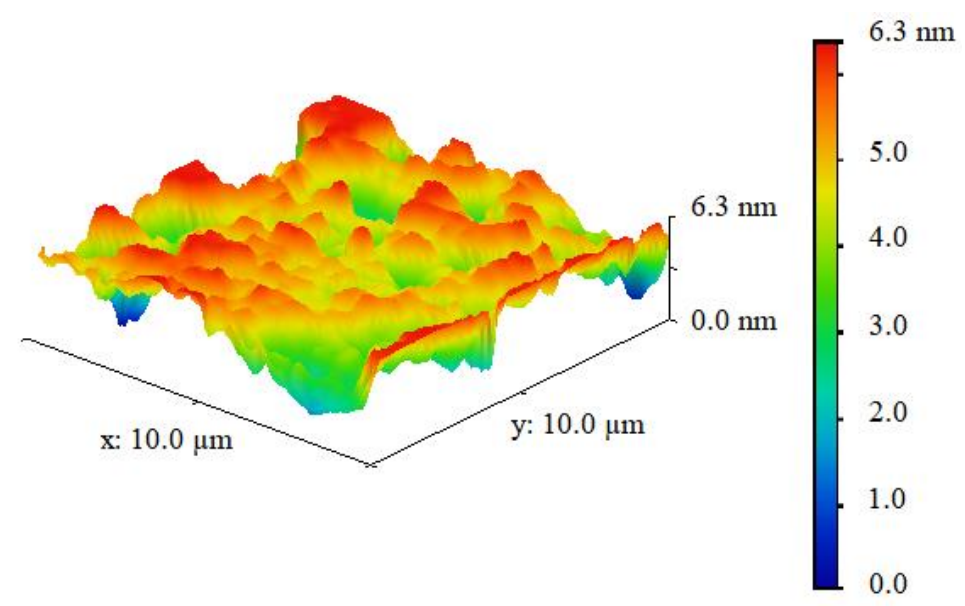

Figure 7-4 AFM morphology of the AZO sample on Borofloat33 substrate.

So far, the characterization methods used in this chapter reveal similarities among our AZO samples which are developed in one sputtering set. These films were found to be homogeneous with little variation in stoichiometry, and had a dominant (002) crystal orientation. The thickness was found to vary with location with respect to the sputtering target, however, surface roughness was found to 
be consistently low indicating smooth film topography. In the following sections, we describe the electrical and optical properties of these samples.

\subsection{Hall effect parameters}

Prior to the Hall effect measurements, we measured the resistance of our samples using a digital multimeter to verify their ohmic behaviour, and the resulting readings were used as a reference, as mentioned in section 6.5.1. For each sample, the Hall effect measurement was repeated four times to increase accuracy and adjust for variations, with the sample being rotated by $90^{\circ}$ in each measurement. The measured Hall effect parameters are presented in Table 7-4.

Table 7-4 Hall effect parameters measured for the nine samples represented in this chapter. The carriers are n-type.

\begin{tabular}{|c|c|c|}
\hline Name & Carrier concentration $\left.\mathbf{( c m}^{-3}\right)$ & $\rho(\mathbf{\Omega} . \mathbf{c m})$ \\
\hline$S 1$ & $(0.59 \pm 0.06) \mathrm{E}+20$ & $(1.74 \pm 0.05) \mathrm{E}-02$ \\
\hline$S 2$ & $(0.27 \pm 0.10) \mathrm{E}+20$ & $(3.92 \pm 0.12) \mathrm{E}-01$ \\
\hline$S 3$ & $(0.31 \pm 0.10) \mathrm{E}+20$ & $(2.30 \pm 0.10) \mathrm{E}-01$ \\
\hline$S 4$ & $(1.09 \pm 0.07) \mathrm{E}+20$ & $(6.95 \pm 0.07) \mathrm{E}-03$ \\
\hline$S 5$ & $(1.36 \pm 0.26) \mathrm{E}+20$ & $(8.23 \pm 0.08) \mathrm{E}-03$ \\
\hline$S 6$ & $(1.37 \pm 0.27) \mathrm{E}+20$ & $(6.97 \pm 0.06) \mathrm{E}-03$ \\
\hline$S 7$ & $(2.32 \pm 0.37) \mathrm{E}+20$ & $(3.60 \pm 0.05) \mathrm{E}-03$ \\
\hline$S 8$ & $(2.54 \pm 0.38) \mathrm{E}+20$ & $(2.83 \pm 0.04) \mathrm{E}-03$ \\
\hline$S 9$ & $(2.60 \pm 0.54) \mathrm{E}+20$ & $(2.75 \pm 0.01) \mathrm{E}-03$ \\
\hline
\end{tabular}


The represented parameters are averaged over several measurements. The carrier concentration gets as high as $2.6 \mathrm{E}+20 \mathrm{~cm}^{-3}$ for sample S9, while the resistivity for this sample is $2.75 \mathrm{E}-3 \Omega-\mathrm{cm}$, the lowest value among all nine samples.

Only high-quality samples are selected for the plasma frequency study at the end of this chapter. Thus, the selected samples had to have a carrier concentration value of the order of $1 \mathrm{E}+20 \mathrm{~cm}^{-3}$, which potentially can result in plasmonic properties in the near infrared region. Applying this condition omits the first three samples in Table 7-4. A second characterization method that helps to investigate the plasmonic properties of these samples is spectrometry which will be discussed in sections 7.6 and 7.7.

\subsection{Cary measurements}

Initially, we used a Cary photospectrometer to measure the transmission spectra in the range of $190 \mathrm{~nm}$ to $900 \mathrm{~nm}$. This range of wavelengths can be converted to a frequency range with the unit of wavenumber from $52631.58 \mathrm{~cm}^{-1}$ to $11111.11 \mathrm{~cm}^{-1}$. This frequency unit conversion was done to ensure consistency given that other spectrometers used in this study use the wavenumber convention.

The sample's (thin film + substrate) transmission in the Cary spectrometer is measured against a blank Borofloat33 substrate as the background. This means that the output measured spectrum of our sample using a Cary spectrometer is 
the ratio of transmission of the sample to that of the blank substrate. The transmission spectra for all 9 samples included in this study are presented in Figure 7-5.

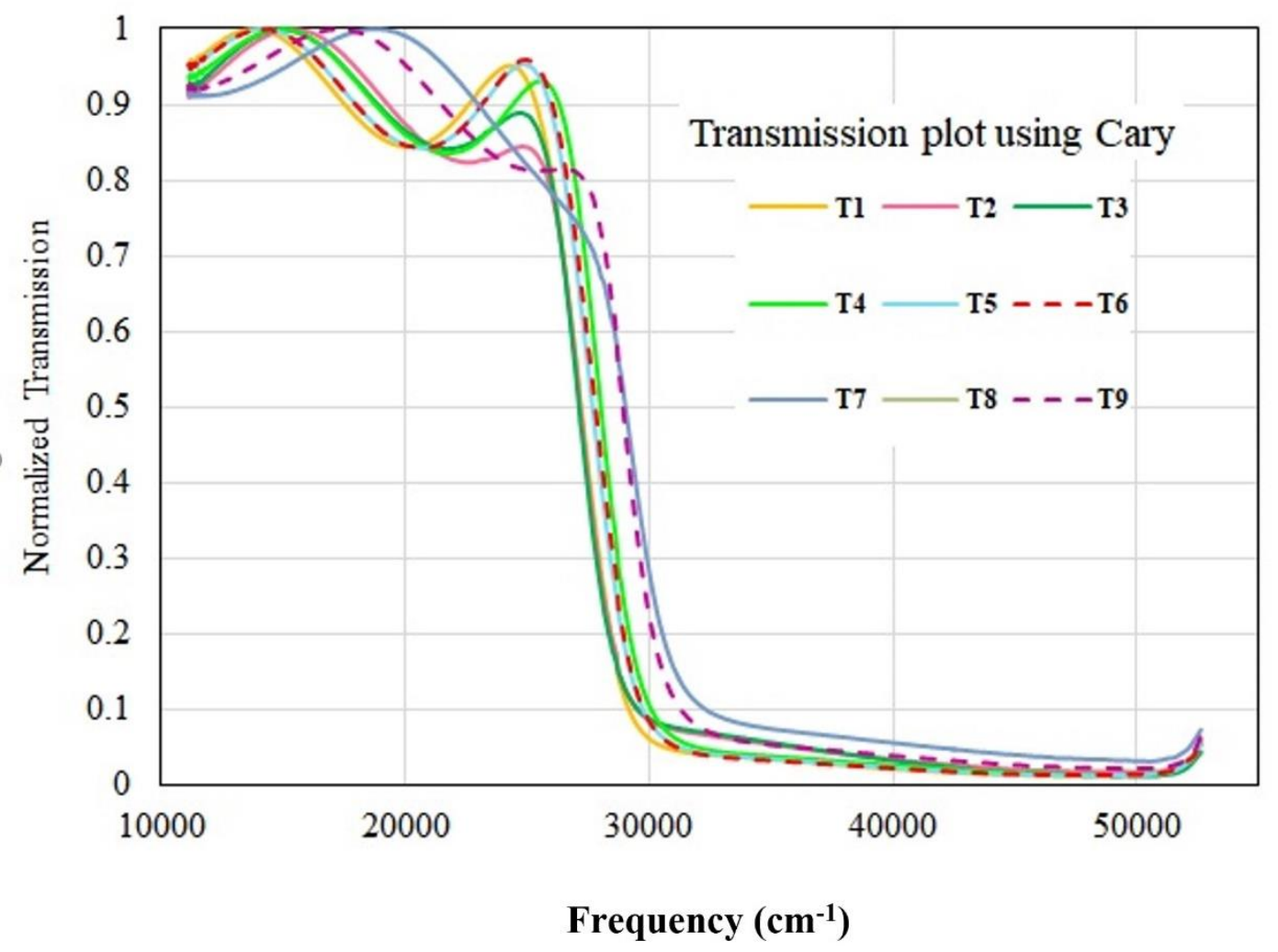

Figure 7-5 Transmission (T) plots of S1-S9 measured in the Cary spectrometer.

Results from the Cary photospectrometer indicate that our sputtered AZO samples are over $80 \%$ transparent in the visible range. In the following section, we present the FTIR spectrometer transmission results over a wider range of the electromagnetic spectrum. 
Using these measured transmission spectra, Tauc plots were used to estimate the bandgap for the AZO on Borofloat33 samples reported in this chapter.

Table 7-5 Estimated bandgap and measured carrier concentration for the AZO on Borofloat33 samples.

\begin{tabular}{|c|c|c|}
\hline Name & Bandgap (eV) & Carrier concentration $\left.\mathbf{~ c m}^{-3}\right)$ \\
\hline$S 1$ & 3.45 & $(5.89 \pm 0.65) \mathrm{E}+19$ \\
\hline$S 2$ & 3.40 & $(2.68 \pm 1.02) \mathrm{E}+19$ \\
\hline$S 3$ & 3.41 & $(3.11 \pm 1.01) \mathrm{E}+19$ \\
\hline$S 4$ & 3.52 & $(1.09 \pm 0.07) \mathrm{E}+20$ \\
\hline$S 5$ & 3.50 & $(1.36 \pm 0.26) \mathrm{E}+20$ \\
\hline$S 6$ & 3.51 & $(1.37 \pm 0.27) \mathrm{E}+20$ \\
\hline$S 7$ & 3.62 & $(2.32 \pm 0.37) \mathrm{E}+20$ \\
\hline$S 8$ & 3.62 & $(2.54 \pm 0.38) \mathrm{E}+20$ \\
\hline$S 9$ & 3.62 & $(2.60 \pm 0.54) \mathrm{E}+20$ \\
\hline
\end{tabular}

The estimated bandgap values are higher than what literature suggests for undoped $\mathrm{ZnO}$ at room temperature $[426,427]$, and it agrees with literature values for heavily doped $\mathrm{ZnO}[428,429,430]$. This difference could be attributed to the degenerate doping of a semiconductor, where doping $\mathrm{ZnO}$ with $\mathrm{Al}$ widens its optical bandgap due to the occupation of the lower part of the conduction band with dopants, as described by the Burstein-Moss (BM) effect [431] that was presented in section 4.4. Plotting the measured carrier concentration data against 
the estimated optical bandgap energies for the different samples of Table 7-5 is in a good fit with the BM model [428].

\subsection{FTIR measurements}

To study the transmission properties of our thin films in the infrared range, we used two different FTIR spectrometers which have different spectral ranges. These two systems are the Bomem DA8 FTIR spectrometer and the Vertex V80v Bruker FTIR spectrometer.

\subsubsection{Transmission measurements}

The first FTIR spectrometer used in this study is the Bomem DA8. To have reproducible measurements, this system should be kept under vacuum overnight. This reduces the amount of water vapour traces in the measured spectra. Furthermore, after changing each detector, a sufficient waiting time should be considered before any measurement is initiated to ensure system stability. The FTIR transmission plots were measured for all 9 samples over a range of $2500 \mathrm{~cm}^{-1}$ to $8500 \mathrm{~cm}^{-1}$ (4000 $\mathrm{nm}$ to $1176.47 \mathrm{~nm}$, respectively).

When using the Bomem FTIR system, the transmission spectrum of both the background and the blank substrate were separately collected just before or after measuring each thin film sample. Thus, the resulting transmission spectrum of each sample was normalized with respect to that of the blank substrate.

Transmission spectra for all 9 AZO on Borofloat33 thin film samples are shown in Figure 7-6. As these samples have been deposited in the same sputtering run, the main influence which directly affects the transmission spectrum of the thin 
film is the location of the sample in the chamber with respect to the centre of the sputtering plasma.

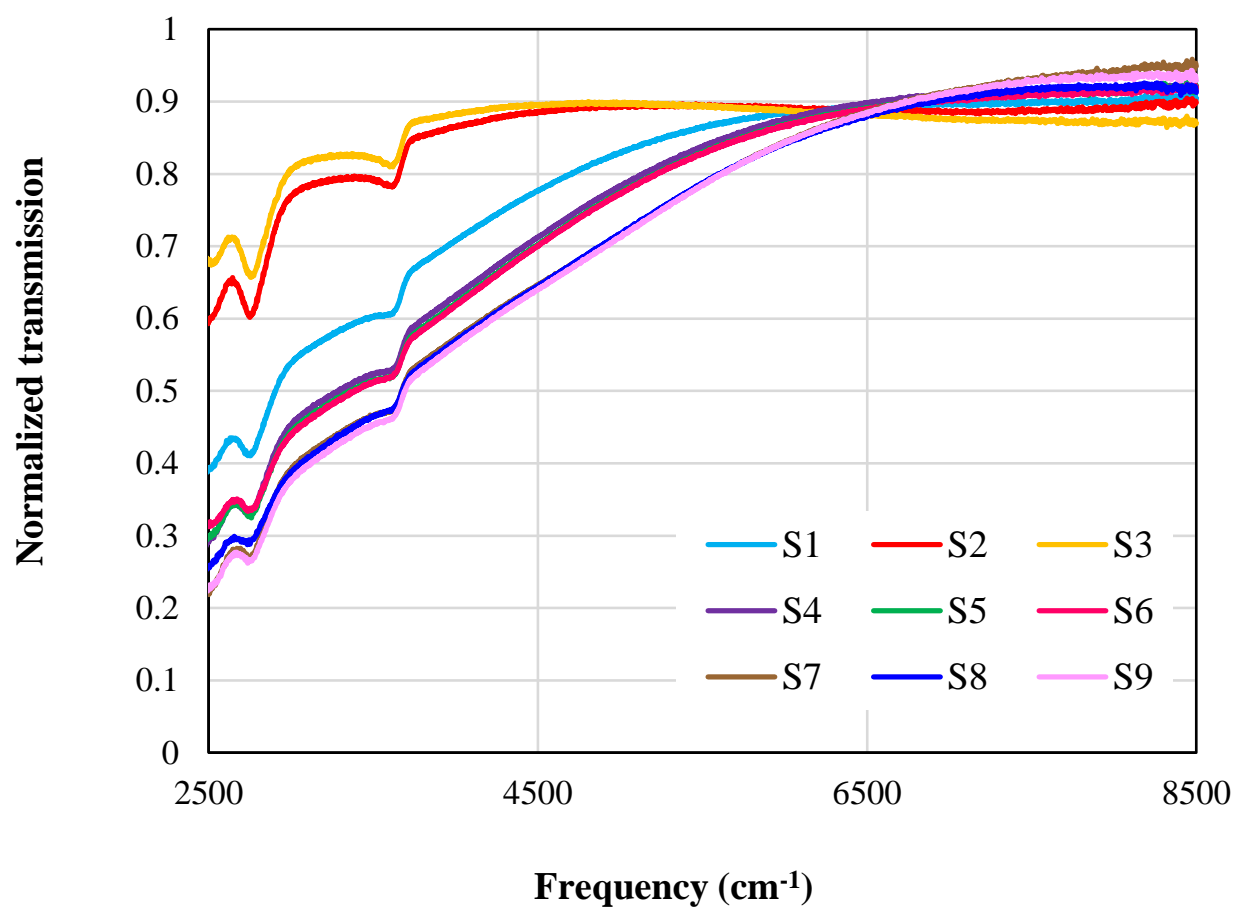

Figure 7-6 Transmission plots of S1-S9 measured in the Bomem FTIR.

As shown in Figure 7-6 below $6500 \mathrm{~cm}^{-1}, \mathrm{S1}, \mathrm{S} 2$, and S3, which are closest to the plasma centre show higher transparency, while S4, S5, and S6 have lower transparency. It is noted that S2 and S3 had significantly higher resistance values as measured in section 7.5, which might indicate that these samples suffered from peeling and oxygen ions bombardment damage during sputtering; i.e. higher transparency and lower electrical conductivity due to having less thin film coverage.

Finally, S7, S8, and S9 have the lowest overall transmission levels. Comparing transmission values of S4 to S6 with S7 to S9 shows a 5-7\% difference at the 4000 
$\mathrm{cm}^{-1}$, while all nine samples have similar transmission behaviour towards the visible region.

The Bomem spectrometer also allows for reasonably straightforward reflection (R) measurements. We measured R for S1-S9, and the results are shown in Figure 7-7.

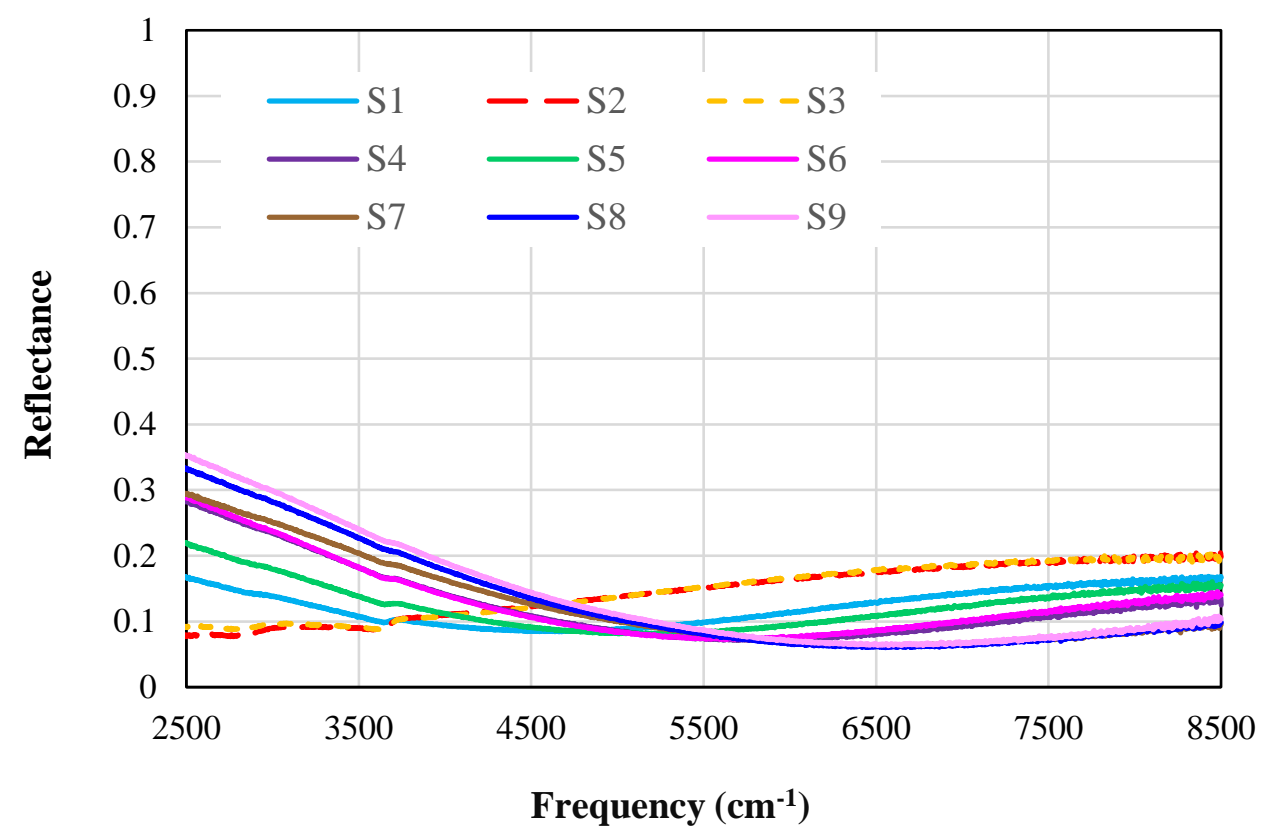

Figure 7-7 Reflectance plots of S1-S9 measured in the Bomem FTIR.

Comparing the results from Figure 7-6 and Figure 7-7 it can be seen that as the transmission increases the reflection decreases in the region below $4000 \mathrm{~cm}^{-1}$.

Based on the conservation of energy for a beam of light interacting with a thin film the following equation should apply:

$$
A+R+T=1
$$

\section{Equation 7-1}


where $R$ is the reflection, $T$ is the transmission and $A$ is the absorption for each measured sample. Figure 7-8 shows the calculated absorption for S1 to S9. The absorption plot covers the wavelength range starting from $4 \mu \mathrm{m}\left(2500 \mathrm{~cm}^{-1}\right)$ to $\sim 1.2 \mu \mathrm{m}\left(8500 \mathrm{~cm}^{-1}\right)$. Given the high absorption of the substrate, the absorption spectra have reasonably high values at lower frequencies, reducing towards the higher frequencies (shorter wavelengths).

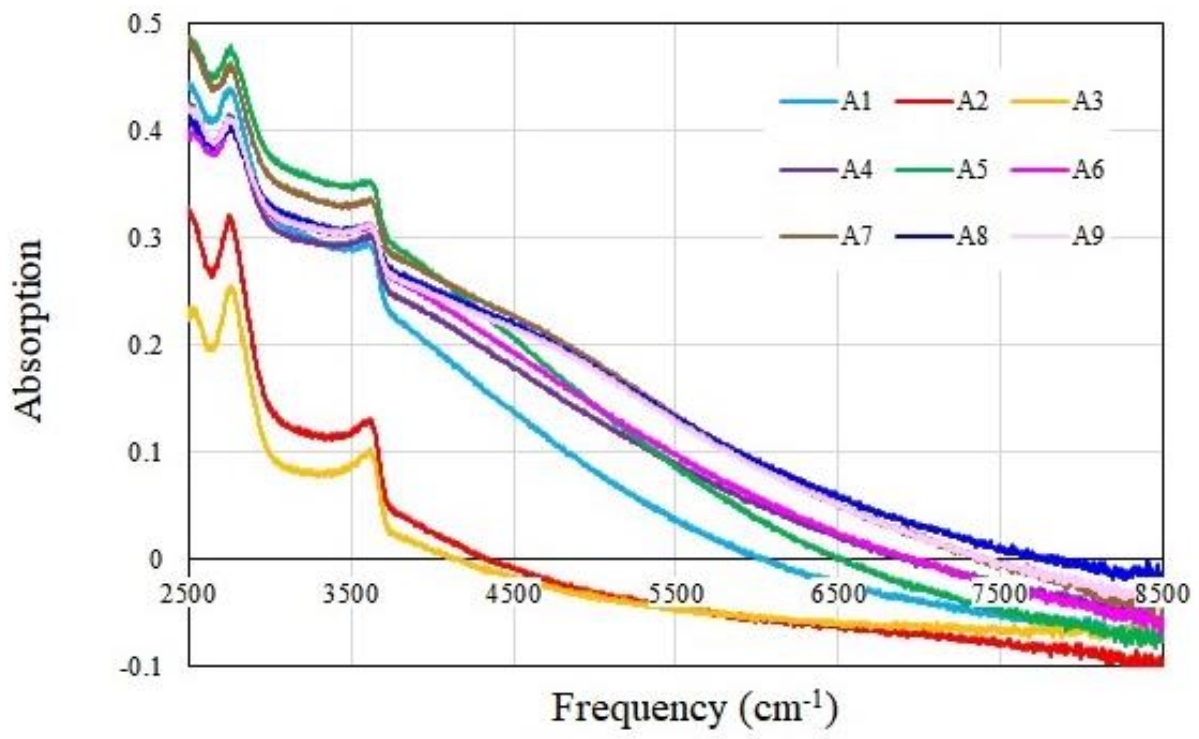

Figure 7-8 Absorption plots of S1-S9 calculated from the reflectance and transmission spectra using Bomem FTIR.

Figure 7-8 shows negative absorption values at higher wavenumbers present in almost all measured samples. While negative absorption does not have a physical meaning in this instance, the consistent observation of these values indicates an adjustment limitation in our Bomem FTIR system. Therefore, for a measured sample, the negative value for absorption can originate from either the transmission or reflection setup error. To investigate this, we can compare either 
T or R spectra from Bomem against another FTIR spectrometers and evaluate the accuracy of the Bomem-measured spectra.

At this stage, a newly commissioned Vertex V80v Bruker FTIR spectrometer was used as a second spectrometer to measure the transmission spectra, thereby providing a reference.

In the Bruker spectrometer, measurements were done in similar fashion our protocol while using the Bomem spectrometer, thus, both the blank substrate and the film-on-substrate were successively measured under vacuum; however, the resulting spectra in this instance were normalized with respect to the background. Compared to the Bomem spectrometer, the Bruker collects data over an extended range of $700 \mathrm{~cm}^{-1}$ to $30000 \mathrm{~cm}^{-1}$, which is desirable for a comprehensive investigation of our samples' properties.

We carried the Bruker FTIR measurements on three selected samples out of the nine samples included in this study. This selection was based on:

- The Hall effect results and the values of carrier concentration, as shown in Table 7-4. Samples S1 to S3 have a carrier concentration value of the order of $1 \mathrm{E}+19$ which is not desirable, hence only six samples were left to consider.

- The transmission plots of the samples S1-S9, measured using the Bomem FTIR. These plots shown in Figure 7-6 illustrate very similar behaviour for two groups of samples. These two groups are S4 to S6 and S7 to S9. Out of these six samples S4, S6 and S9 were selected for further investigation.

Figure 7-9 shows extended transmission spectra for S4, S6, S9, and the blank substrate using the Bruker spectrometer. 


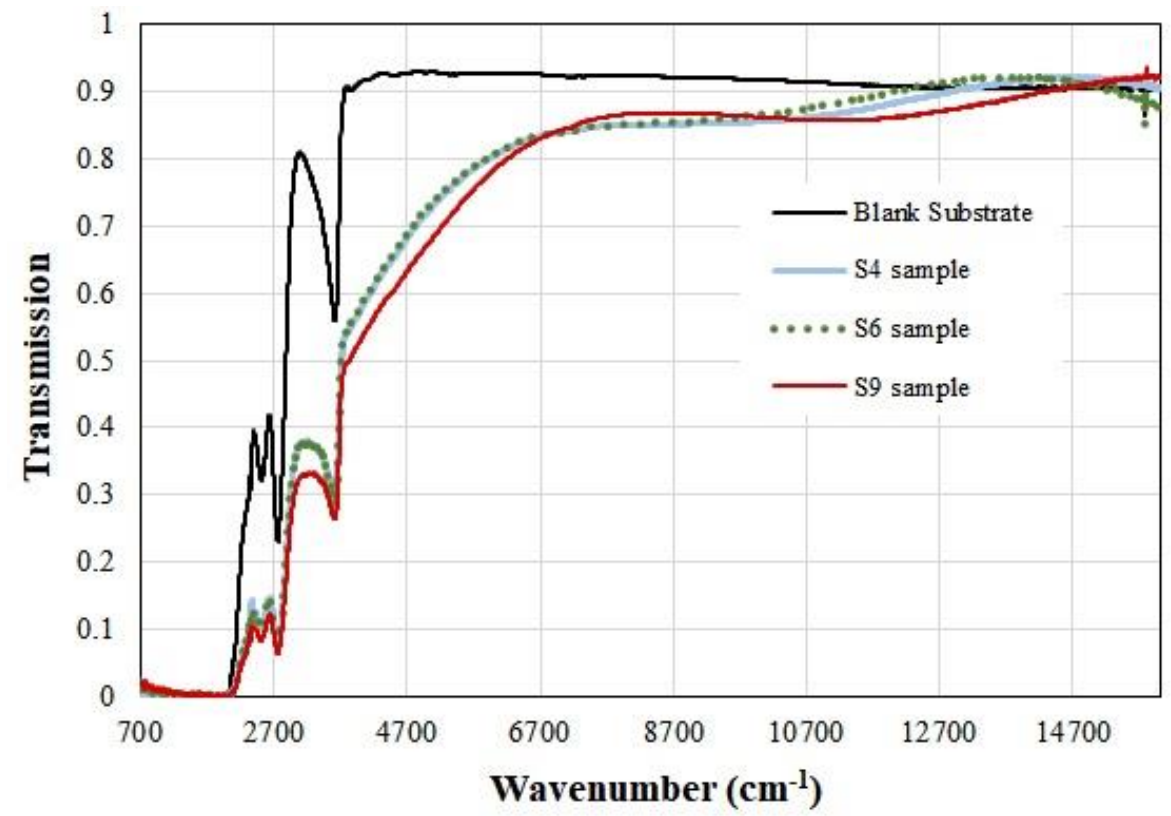

Figure 7-9 Measured FTIR spectra for S4, S6, S9, and the blank Borofloat33 substrate using the Bruker spectrometer.

Upon comparing the transmission plots of S4, S6 and S9 from the Bruker with that of the Bomem (Figure 7-6), it is apparent these results do not look similar. The reason is that the transmission spectra from the Bomem spectrometer shown in Figure 7-6 is composed of the sample normalized by the blank substrate spectrum, that is:

$$
\mathrm{T}_{\text {Bomem }}=\mathrm{T} \text { sample } / \mathrm{T} \text { blank substrate }
$$

Equation 7-2

However, the Bruker's transmission spectrum is normalized by the spectrum measured through a hole (background, i.e., power spectrum of the source), accordingly: 


$$
\mathrm{T}_{\text {Bruker }}=\mathrm{T} \text { sample } / \mathrm{T} \text { hole }
$$

\section{Equation 7-3}

Therefore, we needed to modify data from one spectrometer to allow proper comparison. We decided to modify our Bruker transmission data, shown in Figure 7-9, by dividing S4, S6 and S9 spectra by that of the inverse of the blank substrate, hence we get the following for data from the Bruker:

$$
\mathrm{T}_{\text {Bruker (new) }}=\mathrm{T} \text { sample } / \mathrm{T} \text { blank substrate }
$$

\section{Equation 7-4}

Figure 7-10 shows the modified Bruker data overlayed on the transmission data from the Bomem spectrometer for S4, S6 and S9. As can be seen from Figure 7-10(a) and (b), the two transmission spectra collected from the same sample using two different spectrometers are almost identical.

Measuring our AZO samples and the blank substrate in the Bruker spectrometer allowed us to examine the transmission behaviour over a broader frequency range. We note that the blank substrate showed a strong absorption behaviour in the lower frequency spectrum (infrared region) as seen in Figure 7-9.

The transmission spectra of our AZO samples followed a similar trend, with peaks and dips following that of the blank substrate. This meant that the overall transmission of our samples was affected by the substrate's transmission properties. However, these features have different intensities in the thin film 
sample, which could be attributed to the compound absorption effects in both the thin film and the blank substrate.

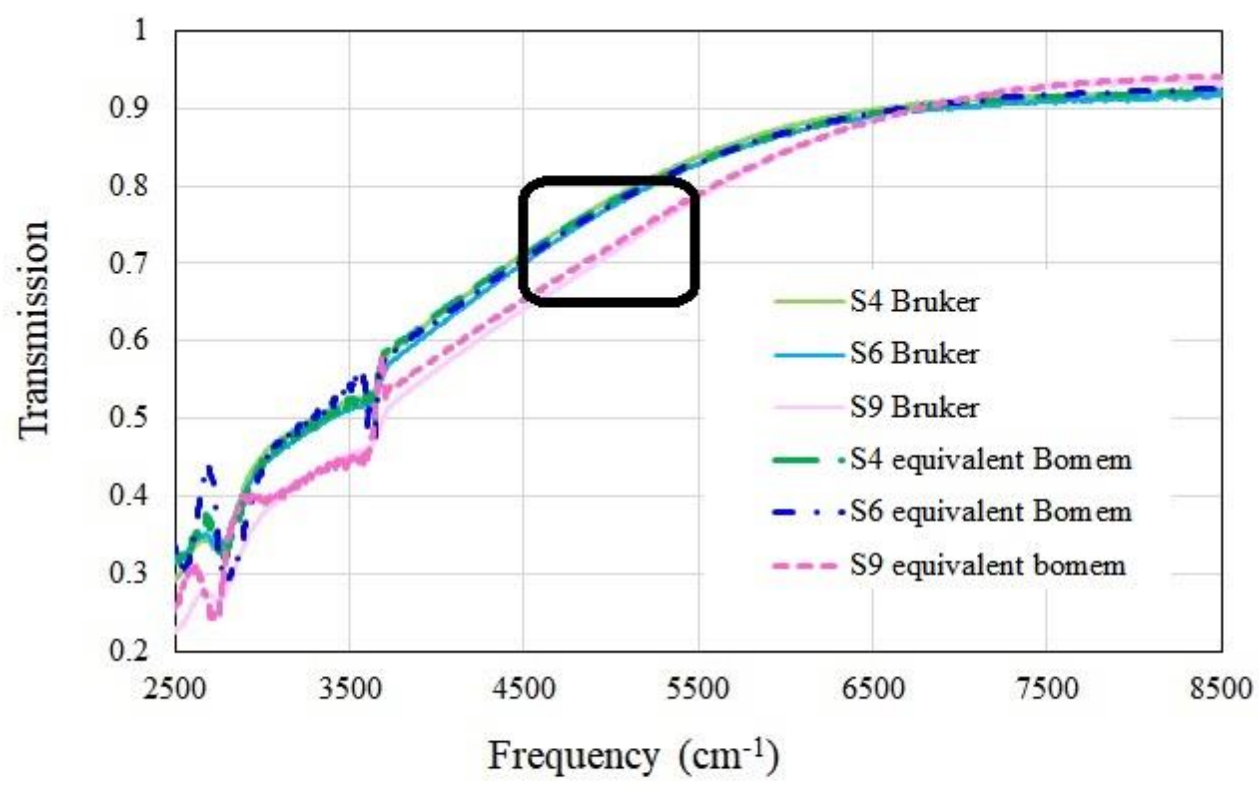

(a)

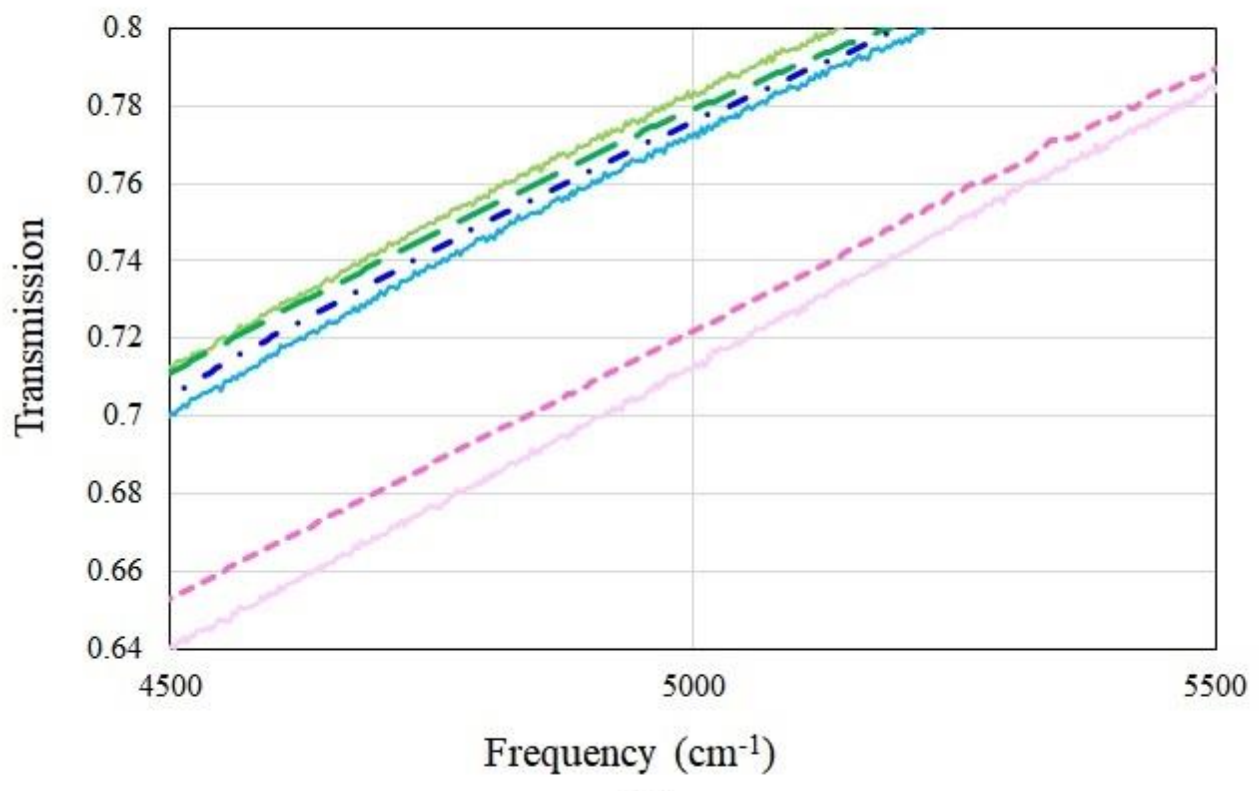

(b)

Figure 7-10 (a) Bruker transmission plots for S4, S6 and S9, and Bomem modified plots of the same samples for comparison. (b) Close up of the frame provided in (a). 
Given the normalized transmission spectra presented in Figure 7-10, we became confident that the transmission spectra from the Bomem were reasonably accurate, as a second FTIR system produced almost identical results. This indicated that the observed negative absorption in the spectra of Figure 7-8 was due to an adjustment (systematic) error in the Bomem spectrometer's reflection setup. Assuming this to be an offset in the results, as a first approximation, a simple correction of the reflection and absorption spectra could be achieved by shifting the absorption spectra so that the minimum value equals zero, then adding the difference to their respective reflection spectra. The corrected reflection spectra are shown in Figure 7-11.

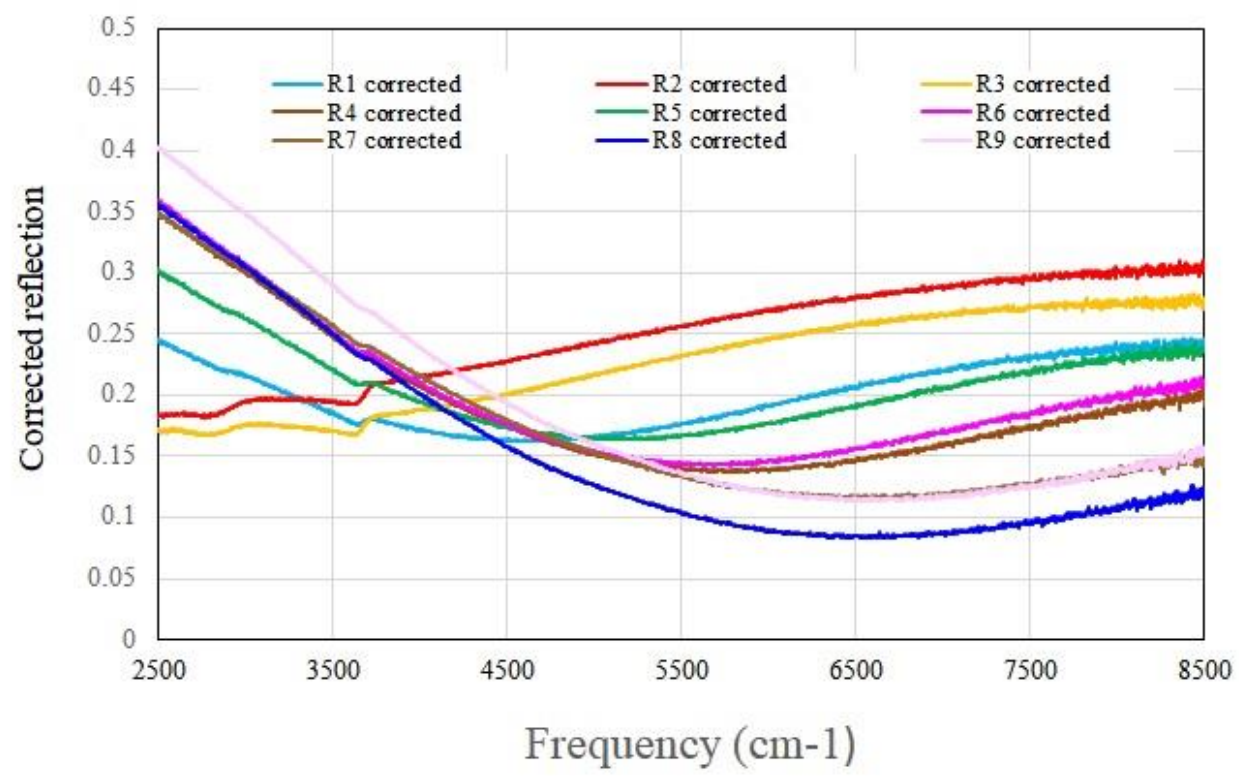

Figure 7-11 Corrected reflection spectra measured using the Bomem FTIR.

Similarly, the corrected absorption spectra are shown in Figure 7-12. 


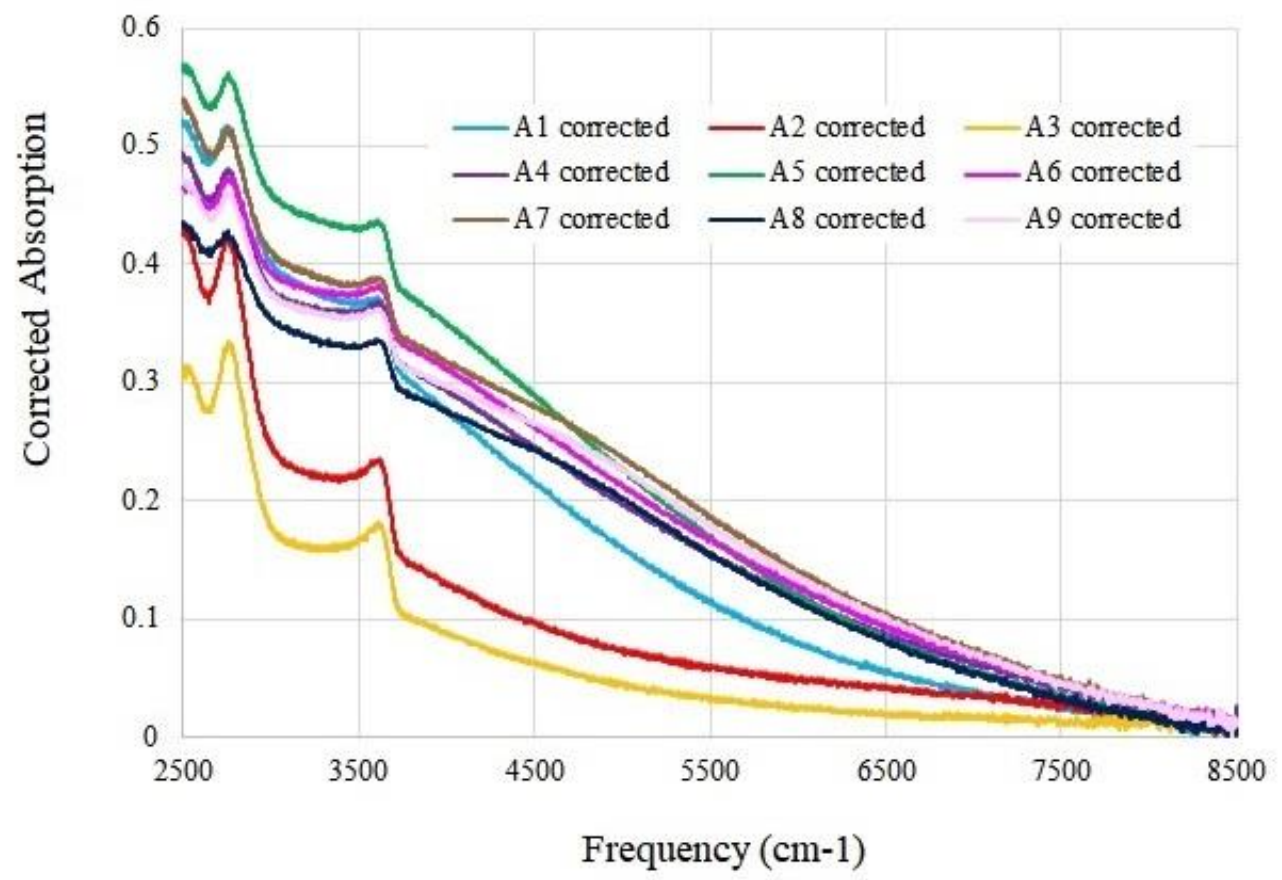

Figure 7-12 Corrected absorption spectra measured using the Bomem FTIR.

A general observation in the measured spectra using FTIR is high absorption in the infrared region for all AZO on Borofloat33 samples. These localized absorption regions could be attributed to the substrate's optical characteristics, as seen in Figure 7-9. Therefore, substrate absorption reduced our confidence in judging the properties of the AZO thin films in the region of interest to our plasmonic application.

Given the limited spectral coverage of the Bomem FTIR system and the high absorption loss in the substrate, the following two decisions were taken to enable us to study the properties of our AZO thin films:

- Concentrate on using the Vertex V80v Bruker FTIR spectrometer for future measurements to make use of the wider spectral range. 
- Depositing our samples on different substrates that have better transmission properties in the IR region (more transparent).

Using different substrates with higher transparency in the region of interest allows us to measure the properties of the deposited AZO thin films.

\subsection{Plasma frequency and dielectric permittivity of AZO}

Based on the characterization results from this chapter, S4, S6, and S9 are selected as representatives for plasma frequency calculation given that they showed desirable electrical and optical properties.

As mentioned in section 6.7.5, in the early stages of the modelling the AZO on Borofloat33 samples, we had to accept that plasma frequency estimation would yield a broad range, given the high absorption (or low transmission) of the Borofloat33 substrate in the region of interest, i.e., where plasma frequency is most likely to be. Nonetheless, we were able to narrow the estimated plasma frequency range down by comparing it with the results from the Drude model.

Our AZO samples were defined to have two layers (the substrate and the thin film) in the Reffit software, as mentioned in section 6.7.4. Having two widely different thicknesses for the thin film and the substrate layers, the coherency of the multiple reflected light rays was expected to vary in each layer. In this model, the substrate was treated as an incoherent layer and the thin film was treated as a coherent layer [416].

When trying to fit the transmission spectrum of a sample, the first step was to fit the transmission spectrum of the substrate. Knowing the correct thickness of 
the substrate is essential for this fitting. Once a good fit is established, i.e., the fitting line overlaps with the FTIR transmission spectrum of the substrate, the corresponding Lorentzian oscillator terms for the substrate are extracted. These terms are then employed in the second fitting step [415]. The number of the Lorentzian terms required to get a good fit critically depends on the material's properties.

Figure 7-13 shows the fitted curve for the Borofloat33 substrate's FTIR transmission spectrum.

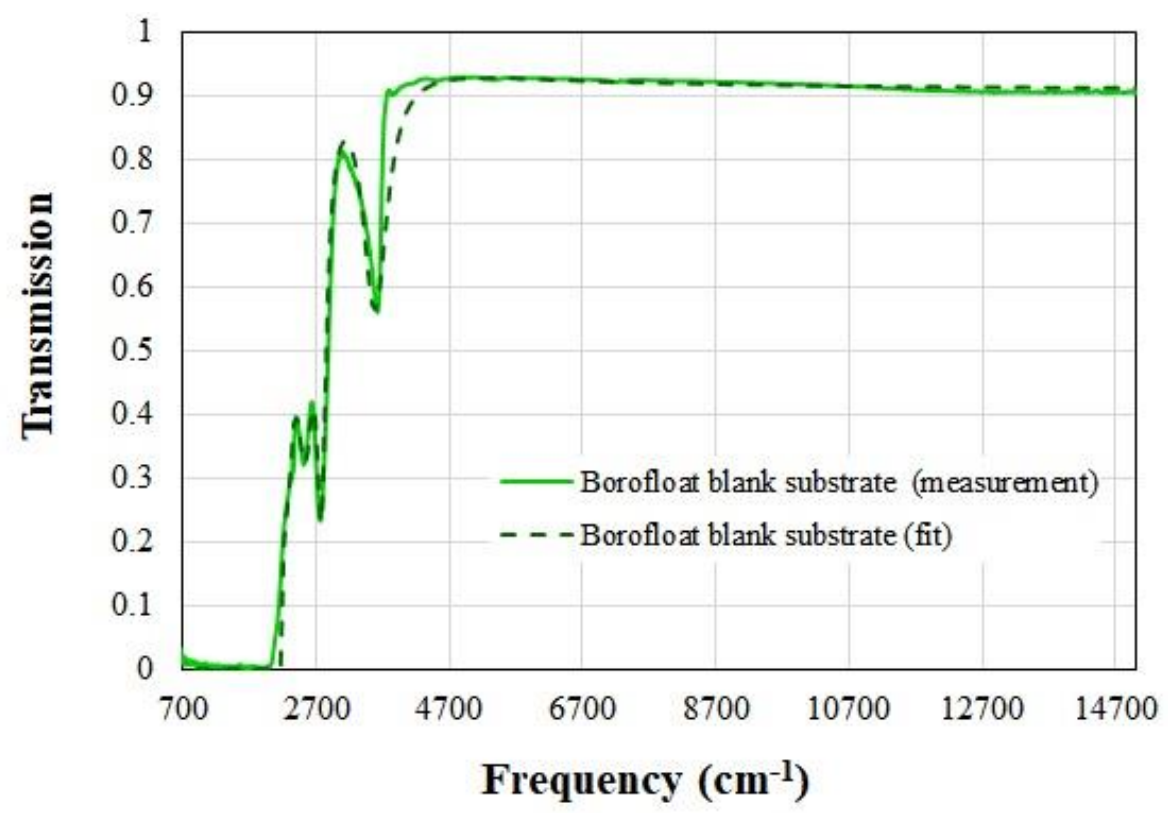

Figure 7-13 Blank Borofloat33 substrate's measured FTIR transmission spectrum and the fitted curve in the Reffit software.

The dashed line fit in Figure 7-13 was created using six Lorentzian terms, and without any Drude term. This is due to the fact that the substrate did not have free electrons to justify the use of a Drude term. The Drude oscillator is a special 
case of the Lorentz oscillator where electrons in the conduction band are free, therefore, the restoring force and the resonant frequency $\left(\omega_{0}\right)$ are set to zero [401].

For the second and final fitting step, the FTIR measured transmission spectrum for the AZO thin film-on-substrate was modelled using the two-layer approach. The first layer was the substrate, which already had defined Lorentz oscillator terms, while the second layer was the thin film. Thus, by adding the necessary Drude and Lorentz oscillator terms for the second layer, we could fit the measured FTIR spectrum. Thus, the extracted Drude and Lorentzian terms for the second layer would describe the properties of the AZO thin film. Figure 7-14 shows the measured FTIR transmission spectrum of S9 in a solid line, and the fitted curve of S9 (AZO film-on-substrate) in a dashed line.

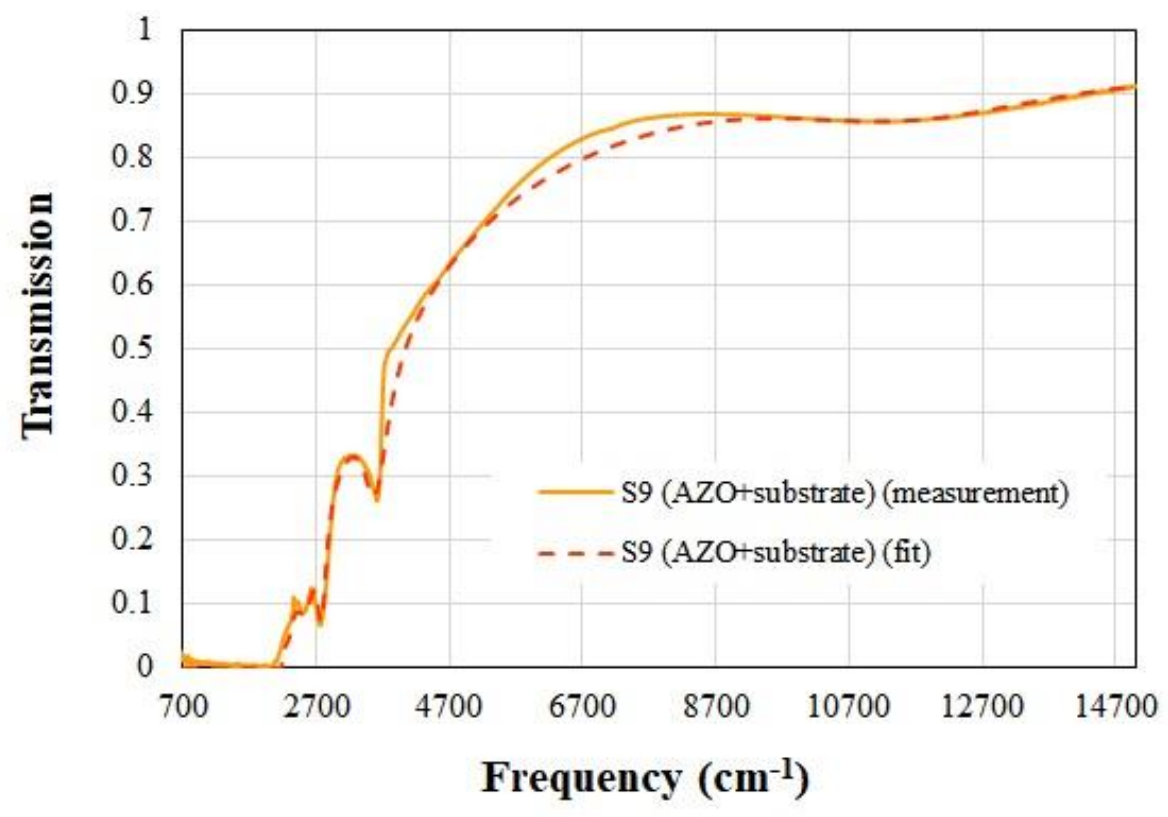

Figure 7-14 Modelled transmission spectrum for AZO thin film for S9 using one Lorentzian term. 
Table 7-6 shows the Drude term's plasma frequency $\left(\omega_{\mathrm{p}}\right)$ and scattering rate $(G)$, obtained from the fitting process for S9. As mentioned previously, the resonance frequency $\left(\omega_{0}\right)$ of the Drude model is set to zero, given that the Drude model assumes no dependencies between the electrons and the nuclei in the dispersion equation $[404,401]$.

\section{Table 7-6 The Drude term that was used to fit the FTIR transmission spectrum of sample S9.}

\begin{tabular}{|c|c|c|c|}
\hline \multicolumn{4}{|c|}{ S9 fitted Drude term } \\
\hline$\omega_{0}$ & $\omega_{p}\left(\mathrm{~cm}^{-1}\right)$ & $\mathrm{G}$ & $\varepsilon_{\infty}$ \\
\hline 0 & 5980 & 3000 & 3 \\
\hline
\end{tabular}

It is worth noting that the fit for the AZO thin film-on-substrate in Figure 7-14 was achieved by using a single Drude term for the AZO thin film layer, which is shown in Table 7-6.

Despite having a good fit for sample S9 using a single Drude term, it is noted that samples with complex transmission spectra might require the addition of extra Lorentzian terms in the thin film layer model to achieve improved fitting. Thus, the overall quality of a fitted model to a sample's FTIR spectrum has a direct impact on the estimated plasma frequency.

In this work, although we have solely relied on the FTIR transmission spectra to extract the Drude and Lorentzian terms from our fitted curve, this fitting process could have been carried using the reflection data instead. 
The simplicity of the fitted term for S9 offered an easy way to test the validity of our fitting. The testing method was to repeat the fitting procedure for the FTIR measured reflection spectrum of the same sample, i.e., S9. If the fitting parameters of the reflection spectrum match those of the transmission spectrum, then the validity of the fitting model is verified.

The measured and fitted transmission (T) and reflection (R) spectra for sample S9 are shown in Figure 7-15, where a single Drude term (reported in Table 7-6) was used for the fitting process.

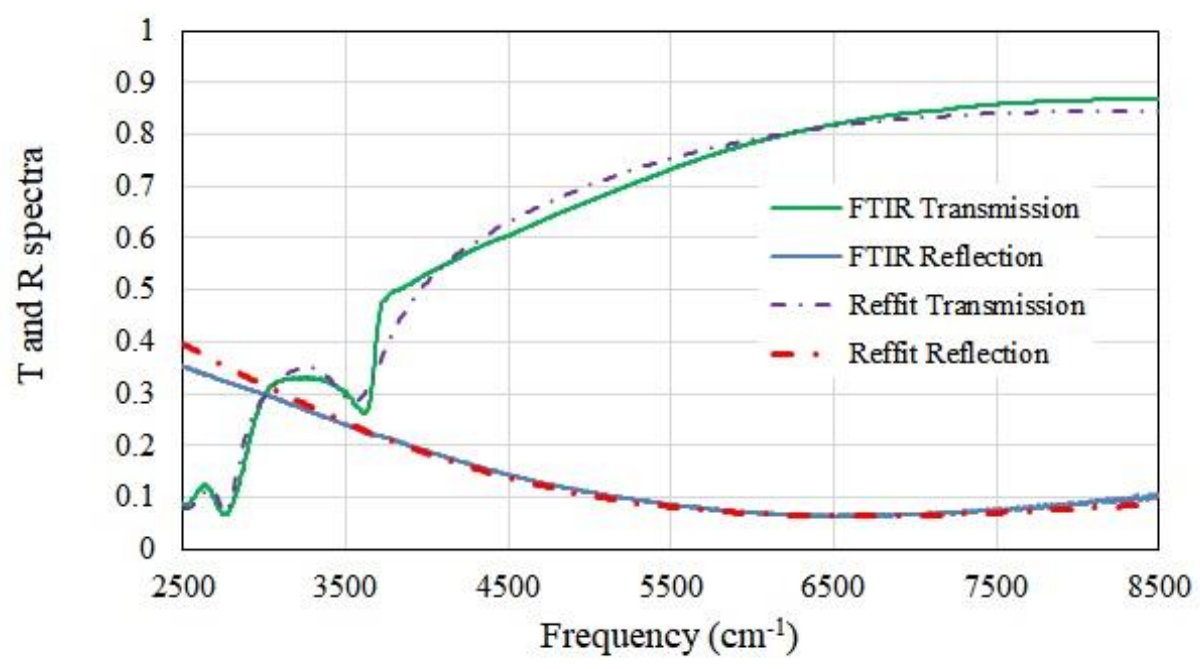

Figure 7-15 FTIR measured transmission and reflection spectra, with the Reffit modelled plots for sample S9.

As seen in this figure, an important result of this process was that a simple Reffit model comprising of only one Drude term provided good quality fitted models for both the transmission and reflection spectra of the sample. This gave us confidence about the results and the estimated plasma frequency value, as two 
independently measured $\mathrm{T}$ and $\mathrm{R}$ spectra were fitted in a Reffit model using the same Drude term.

Finally, the plasma frequency values for three samples from the FTIR-Reffit method are presented in Table 7-7, which fully agreed with values calculated using Equation 6-27 from the Hall effect-Drude method while assuming that $m_{e}^{*}=0.65 m_{e}$. The plasma frequency $\left(\mathrm{cm}^{-1}\right)$ and its wavelength equivalent are both reported using this method. As part of this fitting procedure, we also present the estimated values for scattering rate $(G)$ from each Drude term, which describes the response of free electrons, and dielectric permittivity at infinity $\left(\varepsilon_{\infty}\right)$.

S9 had the highest carrier concentration among the three samples, hence its plasma frequency value was higher, meaning that the plasma frequency value is closer to the visible region.

Table 7-7 Plasma frequency values calculated from the two different methods.

\begin{tabular}{|c|c|c|c|c|}
\hline \multirow{2}{*}{ Name } & \multicolumn{3}{|c|}{ FTIR-Reffit method } & Hall effect-Drude method \\
\cline { 2 - 5 } & $G$ & $\boldsymbol{\varepsilon}_{\infty}$ & $\begin{array}{c}\text { Plasma frequency } \\
\left(\mathbf{c m}^{-1}\right)\end{array}$ & $\begin{array}{c}\text { Plasma frequency } \\
\left(\mathbf{c m}^{-1}\right)\end{array}$ \\
\hline S4 & 3000 & 1.9 & 3882 & 3882 \\
\hline S6 & 3000 & 2 & 4340 & 4341 \\
\hline S9 & 3000 & 3 & 5980 & 5980 \\
\hline
\end{tabular}


Estimating the properties of a sample using optical data is more conclusive when the covered frequency range in the measurements is broad, which is a strength of our study. This important point is usually overlooked in literature studying AZO [432, 433, 434]. In this section, a broad spectrum from the UV to the infrared was used to produce the Drude model for each sample. Furthermore, the Hall effect-Drude formula was used to calculate the plasma frequency values using the carrier concentration values measured in the Hall effect setup. A main result in this section was the ability to calculate the plasma frequency for a given sample by two independent methods, provided the iterative approach described in section 6.7.5. The scattering rate estimated from our fitting procedure was also in good agreement with the scattering rate directly measured from the PPMS. This provided confidence in our measurement and calculation approaches, which were later applied in the following chapters.

\subsubsection{Dielectric permittivity}

Achieving a good fit to extract the plasma frequency allows us to estimate the dielectric function of our deposited thin films using Reffit. The Lorentzian terms that result in the fitted software model are used to plot the dielectric function, i.e., the real $\left(\varepsilon_{1}\right)$ and imaginary $\left(\varepsilon_{2}\right)$ parts of the dielectric permittivity of a sample. Being a function of frequency, the real part of the dielectric permittivity can be used to define the plasmon-active region for our AZO thin films. Figure 7-16 shows the modelled $\varepsilon_{1}$ and $\varepsilon_{2}$ plots for S4, S6 and S9. 


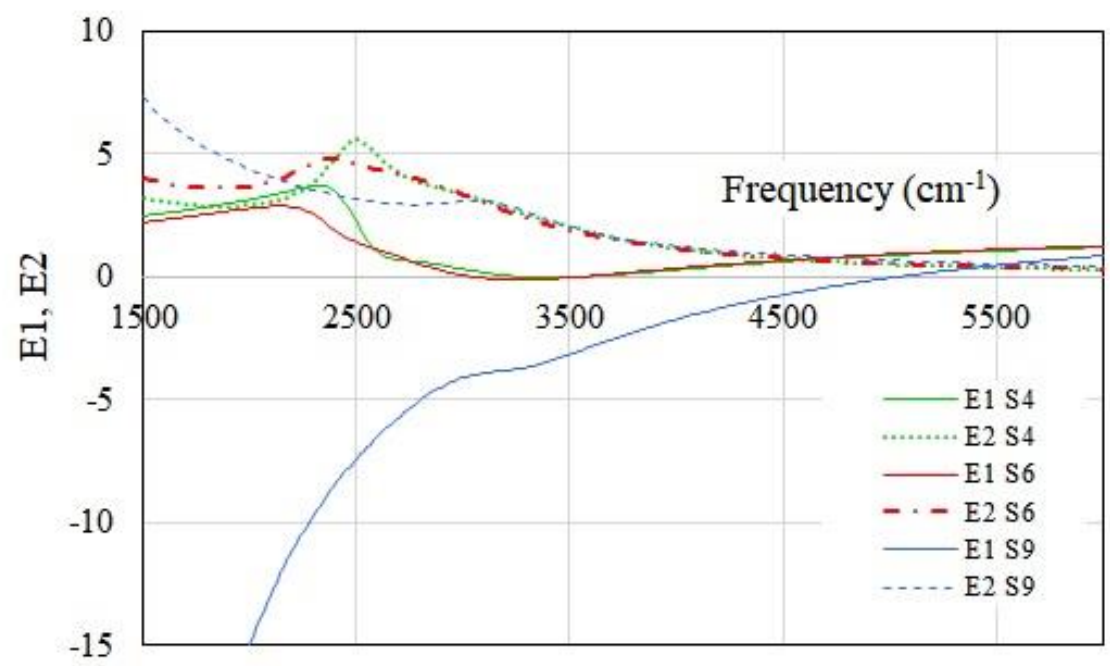

Figure 7-16 Real (solid lines) and imaginary (dashed and dotted lines) parts of the dielectric permittivity for three different samples within the near-infrared range.

It can be seen that only S9 shows a crossover frequency region where the real part of the dielectric permittivity goes negative. The imaginary part of the dielectric permittivity for $\mathrm{S} 9$ is also low around the same frequency region, which suggests a lower optical loss [435]. A high value of the imaginary part of the dielectric permittivity results in high optical absorption (i.e., ohmic losses of free electrons) and is detrimental to the efficient design of plasmonic devices. It is therefore desired to have values of $\varepsilon_{1}<0$ while $\varepsilon_{2}$ remains close to zero. It is worth noting that only S9 met the criteria for plasmonic applications as S4 and S6 did not exhibit a negative real permittivity. This could be related to the value of carrier concentration measured in each sample, where $\mathrm{S} 9$ had almost double the carrier concentration, which indicated that we needed to produce samples with carrier concentrations close to or higher than that of S9 on this substrate. This could also be related to the fact that we are close to the phonon absorption in the glass substrate and our models are not quite good enough to disentangle the substrate and the film properties. 


\subsection{Chapter summary}

In this chapter, the material composition and stoichiometry of the deposited samples were studied using RBS and SEM-EDS. Then, based on our XRD measurements, the preferred crystal orientation was observed, and the mean crystallite domain size was calculated. AFM and SEM images of the deposited samples surface were shown. This was followed by calculating the carrier concentration of the samples, based on the thickness measurements using RBS spectroscopy. UV-Vis and FTIR spectroscopy were then performed on the samples, where transmission and reflection spectra were measured over a broad range of $2500-8500 \mathrm{~cm}^{-1}$. Transmission larger than $80 \%$ was observed for our samples in the near-infrared, visible and UV regions. Next, Reffit modelling and plasma frequency calculations were performed on nominal samples with high carrier concentrations of the order of $1 \mathrm{E}+20 \mathrm{~cm}^{-3}$. Based on the plasma frequency calculations, the real and imaginary parts of the dielectric permittivity were estimated. A negative dielectric permittivity in the near infrared region was achieved for one of our samples, while the dielectric losses remained small. This was a promising result for our AZO material in terms of confirming its suitability for plasmonic applications in the near infrared region.

In this chapter, the spectral data from the FTIR system did not completely clarify the properties of our deposited AZO thin films. This was due to the observed absorption in the Borofloat33 substrate in the near-infrared region of the electromagnetic spectrum. This reduced the overall transmission range of our samples and made it hard to separate the substrate and the film properties. In 
order to avoid the absorption effects of this specific substrate, we decided to use more transparent substrates for the AZO deposition process. This will be investigated in the following chapter. 
8 Results B: AZO thin films on

\title{
Silicon, Sapphire and $\mathrm{CaF}_{2}$
}

\author{
substrates
}



This chapter discusses the characterization results of AZO thin films deposited on three substrates; sapphire, calcium fluoride, and silicon. The main reason to use new substrates for AZO deposition was to achieve higher transparency for our AZO samples as compared with the limited transparency of our samples of Chapter 7, which were deposited on Borofloat33 substrates.

It is worth noting that this level of depth in investigation of the deposited AZO thin film on aforementioned substrates using an off-centre magnetron sputtering setup in such wide electromagnetic range has never been reported to the best of author's knowledge and provides a much needed insight into the properties of this class of deposited AZO thin films.

\subsection{Chapter objectives}

The main objectives of this chapter are:

- To measure the carrier concentration and optical transmission properties for AZO samples deposited on three different substrates. The selected substrates for this study are (006) sapphire (Sap), (111) calcium fluoride $\left(\mathrm{CaF}_{2}\right)$ and $(100)$ silicon $(\mathrm{Si})$.

- To calculate the plasma frequency values based on the measured transmission data and estimate the real and imaginary parts of the dielectric permittivity for these samples. 
Deposition of the AZO thin films reported in this chapter relied on the established deposition recipe that was used for our Borofloat33 based samples. Once the samples were created using all three new substrates materials, we conducted the characterization process, which involved the same techniques that were used in the previous chapter. Thus, we characterized AZO on sapphire, $\mathrm{CaF}_{2}$ and $\mathrm{Si}$, highlighting the results of selected samples. These samples were selected to represent the full diversity of all deposited samples.

$\mathrm{XRD}$ is the first characterization method discussed in this chapter, and its results are presented in the following section.

\subsection{XRD measurements}

Firstly, XRD diffraction patterns were measured for AZO thin films deposited on sapphire, calcium fluoride, and silicon. Unlike Borofloat33, these substrates are crystalline. For a crystalline substrate, the XRD diffractogram shows defined peaks of the relevant crystal. Accordingly, the measured diffractograms were adjusted based on the location of the dominant peak of each substrate, thereby minimizing alignment errors as discussed in section 6.2.1.

\subsubsection{XRD analysis for AZO on sapphire}

Diffractograms of a total of seven AZO-on-sapphire samples from two different sets of sputtering, labelled Set1 and Set2, were measured using our XRD system. The diffractograms of five samples from Set1 are shown in Figure 8-1. The AZO peaks were aligned based on the substrate peak. All measured AZO thin films are (002) crystalline with their c-axis perpendicular to the substrate 
plane. As can be seen, there is a difference in the (002) peak intensity between these five samples. This difference is probably attributed to the only variable in a single sputtering run, i.e., samples' location with respect to the sputtering plasma/target.

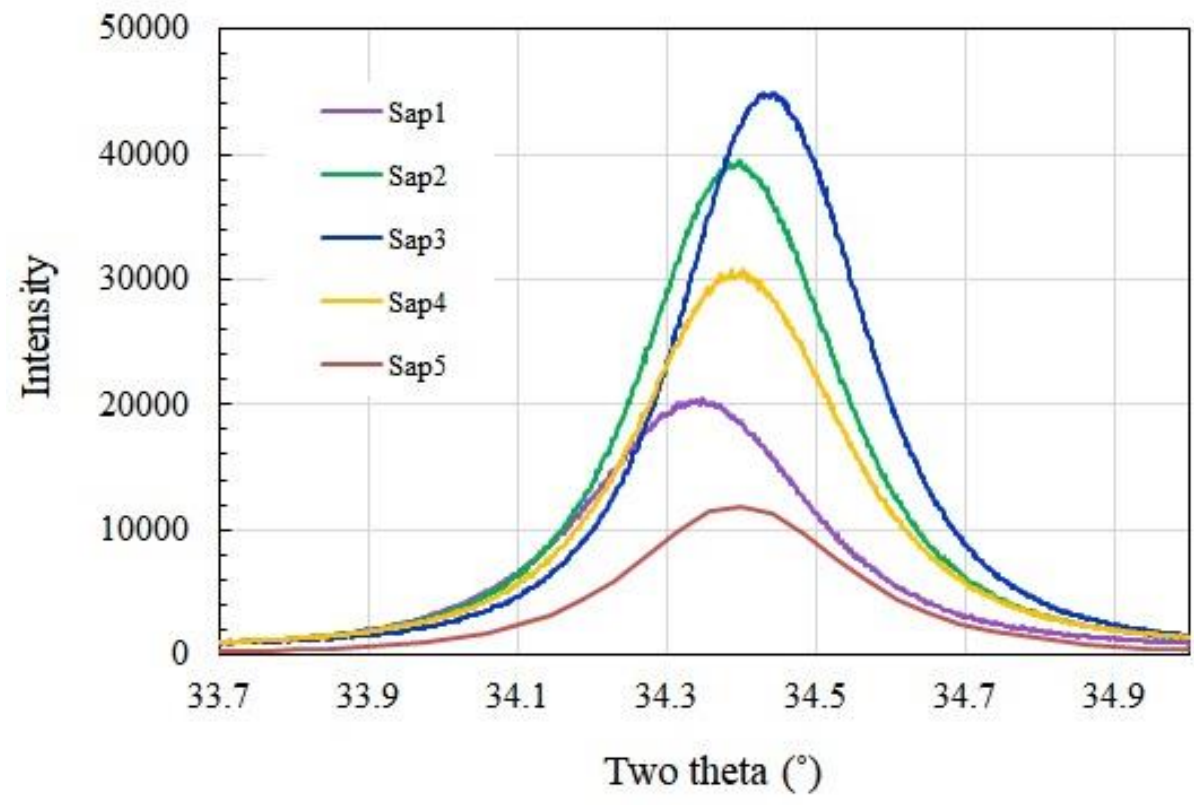

Figure 8-1 Aligned diffractograms for AZO-on-sapphire samples from Set1.

Table 8-1 shows the lattice constant $(d)$ and estimated in-plane residual stress $(\zeta)$ for the five AZO thin film on sapphire samples from Set1. The literature value for $d$ along the c-axis direction, i.e., (002), for pure wurtzite $\mathrm{ZnO}$ is $2.6015 \AA$ [253]. The in-plane residual stress for these samples was calculated using Equation 6-6. 
Table 8-1 Lattice constant for Set1 AZO samples.

\begin{tabular}{|c|c|c|}
\hline Name & d $(\AA)$ & $\zeta(\mathrm{GPa})$ \\
\hline Sap1 ${ }_{\text {set1 }}$ & 2.609 & -0.67 \\
\hline Sap2set1 & 2.605 & -0.31 \\
\hline Sap3set1 & 2.602 & -0.04 \\
\hline Sap4set1 & 2.605 & -0.31 \\
\hline Sap5set1 & 2.605 & -0.31 \\
\hline
\end{tabular}

The negative sign of the estimated stress values indicated that the stress was compressive in nature. The average lattice constant for Set1 was 2.605 with a standard deviation of $0.2 \%$ from the samples made over the whole sputtering area. This reported stress and difference in the average lattice constant from that of the theoretical value for wurtzite zinc oxide has been attributed to the sputtering process and doping effects as suggested in literature [436, 304, 253].

Next, the FWHM values corresponding to the (002) oriented peak for AZO samples from Set1 was estimated. Using the XRD diffractogram of samples and implementing Scherrer's formula, the mean crystallite domain size can be calculated for each sample. Table 8-2 presents the (002) oriented FWHM and mean crystallite domain size values for the samples of Set1. 
Table 8-2 XRD analysis showing the FWHM and the mean crystallite domain size for the AZO samples of Set1.

\begin{tabular}{|c|c|c|}
\hline Name & FWHM $\left.\mathbf{(}^{\circ}\right)$ & CDS (nm) \\
\hline Sap1set1 & 0.35 & 23.78 \\
\hline Sap2set1 & 0.30 & 27.46 \\
\hline Sap3set1 & 0.29 & 28.71 \\
\hline Sap4set1 & 0.32 & 26.12 \\
\hline Sap5set1 & 0.34 & 24.60 \\
\hline
\end{tabular}

It can be seen that sample Sap3 had the highest (002) orientation count and narrowest FWHM, hence a better crystal quality due to a larger mean crystallite domain size, as recommended for our plasmonic application.

Summarizing these results, the deposited AZO thin film on sapphire in Set1 had the same (002) crystal orientation selectivity despite the varied locations for the different samples with respect to the sputtering plasma and target. The mean crystallite domain size had an average of $26.13 \mathrm{~nm}$ with a standard deviation of $7.7 \%$ over the sputtering area.

The main incentive to carry the XRD measurements in this study was to make sure that our sputtering recipe was consistent and resulted in films that were comparable in their crystal properties. Accordingly, we performed XRD measurements on a different set of AZO on sapphire samples (labelled as Set2) to verify that Set2 samples had the same dominant crystal orientation, mean crystallite domain size and lattice constant. 
Checking the measured diffractograms for Set2, it was quickly confirmed that these samples were also (002) oriented. Table 8-3 shows the (002) peak intensity, lattice constant and in-plane residual stress for the samples from Set2.

Table 8-3 XRD analysis of AZO thin films on sapphire from Set2.

\begin{tabular}{|c|c|c|c|}
\hline Name & (002) Peak intensity & $\mathbf{d}(\mathbf{A})$ & $\zeta$ (GPa) \\
\hline Sap1set2 & 7412 & 2.608 & -0.58 \\
\hline Sap2set2 & 18316 & 2.611 & -0.85 \\
\hline
\end{tabular}

It can be seen from these results that the lattice constant and estimated stress for samples from Set2 were comparable to those of Set1 samples.

Table 8-4 shows the FWHM and the mean crystallite domain size for Set2 samples.

Table 8-4 XRD analysis showing the FWHM and the mean crystallite domain size for the AZO samples of Set2.

\begin{tabular}{|c|c|c|}
\hline Name & FWHM $\mathbf{(}^{\circ}$ ) & CDS (nm) \\
\hline Sap1set2 & 0.39 & 21.4 \\
\hline Sap2set2 & 0.37 & 22.4 \\
\hline
\end{tabular}


To summarize, we can confirm that for AZO on sapphire, samples from Set1 and Set2 had comparable crystal properties, which included the orientation selectivity and peak intensity, the crystallite domain size and the in-plane residual stress inherent in the films.

In the next section, similar $\mathrm{XRD}$ analysis for $\mathrm{AZO}$ thin films deposited on $\mathrm{CaF}_{2}$ are present.

\subsubsection{XRD analysis for $\mathrm{AZO}$ on $\mathrm{CaF}_{2}$}

In this section, we selected four samples, each representative of a different sputtering set to highlight any differences in properties between these sputtering runs. The selected samples are referred to as the "non-transparent" sample, the "damaged" sample, the "thickest" sample and the "thinnest" sample.

All of our sputtering sets resulted in thin film samples that were visually transparent, except for a single run that produced samples with a darker grey tint. During this particular sputtering process, the plasma became occasionally unstable and some sparks were observed, in particular at the beginning of the process. These sparks could have originated due to electric field enhancement by the sharp flakes that can cover the internal sputtering chamber's surface due to improper cleaning [437]. Samples from this run are referred to as "nontransparent" samples.

The next selected sample in this section was the "damaged" sample, which can be seen in Figure 8-2. This image was taken by an optical microscope and shows the damaged AZO thin film deposited on $\mathrm{CaF}_{2}$. This peeling effect was mildly increased with time. 


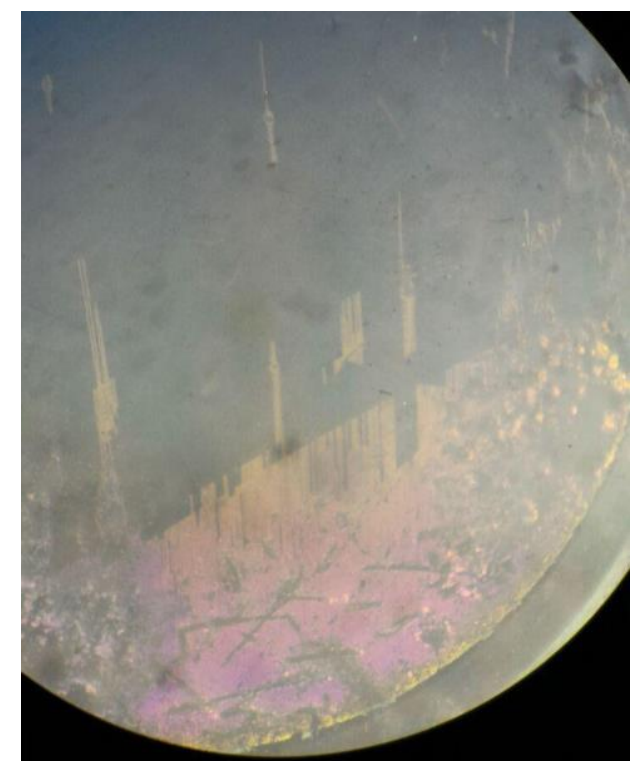

Figure 8-2 Image of the damaged $\mathrm{AZO}$ on $\mathrm{CaF}_{2}$ sample showing a peeled surface.

Samples with the thickest and thinnest (using Dektak) deposited thin film thickness amongst all our $\mathrm{AZO}$ on $\mathrm{CaF}_{2}$ samples were also selected for characterization and were simply labelled the "thickest" and "thinnest" samples.

When conducting the XRD measurements of these four samples, we soon found out that the substrate showed no peaks in the $(\theta-2 \theta)$ diffractogram, which was unexpected given the datasheets of these samples [438]. Nonetheless, we carried on with our characterization study, knowing that we would not be able to align the theta location of the AZO peaks by using the substrate's reference peaks. Consequently, the stress analysis was not performed for these samples, and we suspected that our $\mathrm{CaF}_{2}$ wafers might be amorphous. At a later stage of this work, we had temporary access to a XRD setup that accommodated rocking curve measurements. These measurements revealed that our $\mathrm{CaF}_{2}$ wafers were indeed crystalline in nature; however, there seemed to be a tilt between the (111) crystal plane direction and the surface of our wafer. This meant that the normal 
vector on the surface of the substrate was not aligned with the vector normal to the (111) $\mathrm{CaF}_{2}$ crystal orientation, hence it was not possible to observe a $\mathrm{CaF}_{2}$ peak in our theta-two theta XRD setup.

The XRD diffractograms presented in Figure 8-3 show the intensity of the dominant peak for all four samples included in this study.

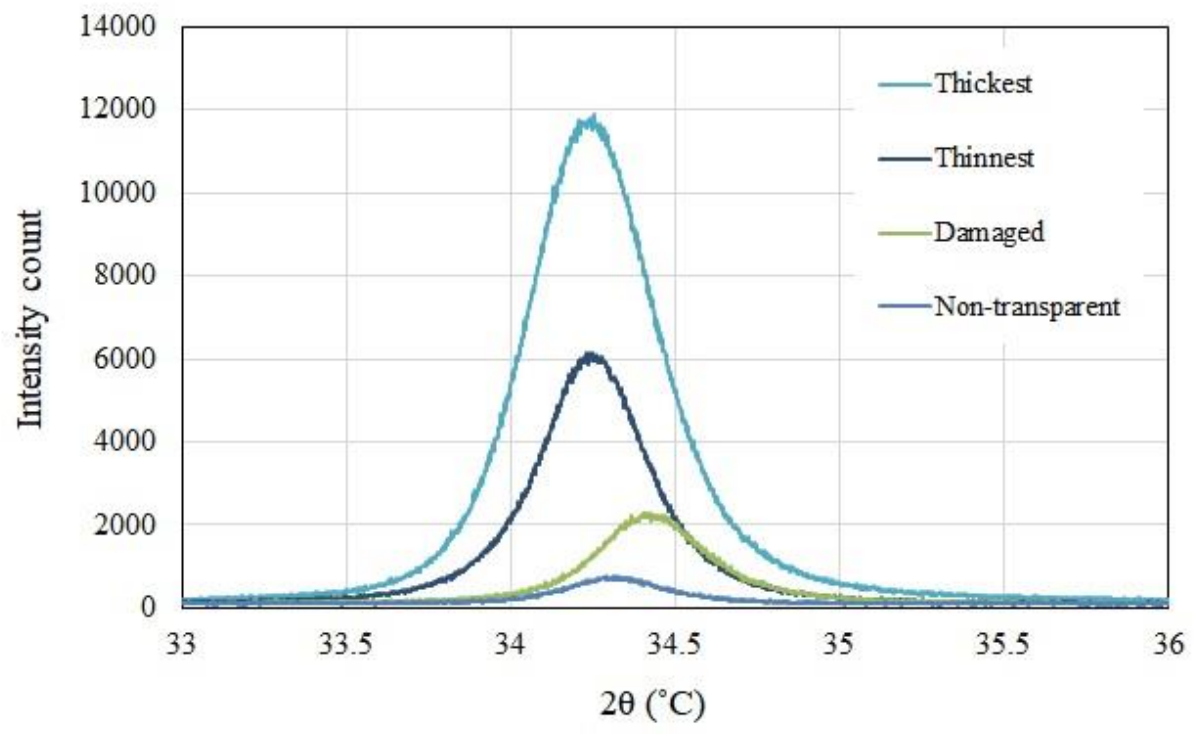

Figure 8-3 XRD diffractogram of $\mathrm{AZO}$ on $\mathrm{CaF}_{2}$ samples.

As shown in Figure 8-3, regardless of their visual and thickness characteristics, all four samples had the same dominant crystal orientation. The thickest sample showed the highest count for the dominant peak. However, the thinnest sample did not have the minimum peak intensity, as both the non-transparent and damaged samples displayed low peak intensities. The intensities of the dominant crystal orientation peaks for these samples are shown in Table 8-5. 
Table 8-5 XRD analysis of AZO thin films on $\mathrm{CaF}_{2}$ substrate.

\begin{tabular}{|c|c|}
\hline Name & (002) Peak intensity \\
\hline non-transparent & 767 \\
\hline damaged & 2297 \\
\hline thickest & 11913 \\
\hline thinnest & 6152 \\
\hline
\end{tabular}

The FWHM and mean crystallite domain size for the (002) orientation for all four samples are shown in Table 8-6.

Table 8-6 XRD analysis showing FWHM and the mean crystallite domain size for $\mathrm{AZO}$ on $\mathrm{CaF}_{2}$ samples.

\begin{tabular}{|c|c|c|}
\hline Name & FWHM $\left.\mathbf{(}^{\circ}\right)$ & CDS (nm) \\
\hline non-transparent & 0.38 & 21.6 \\
\hline damaged & 0.39 & 20.9 \\
\hline thickest & 0.45 & 18.3 \\
\hline thinnest & 0.38 & 21.7 \\
\hline
\end{tabular}


In summary, it was confirmed that samples from different sputtering sets had the same dominant (c-axis) crystal orientation. The thickest sample had the maximum (002) intensity count, however, it had the widest FWHM (smallest mean crystallite domain size), while the thinnest sample had the best crystal quality with the second highest (002) intensity and the largest mean crystallite domain size.

\subsubsection{XRD analysis for AZO on $\mathrm{Si}$}

The third substrate studied in this chapter is (100) silicon, where we carried two AZO sputtering runs on this substrate, denoted by Set1 and Set2. XRD diffractograms for our AZO on Si samples from Set1 and Set2 showed strong Si crystal intensity peaks. Therefore, we were able to shift and align the measured diffractograms and provide estimates to the (002) oriented lattice constant and the in-plane residual stress for the AZO thin films. These results for three samples from Set1 and one sample from Set2 are compiled in Table 8-7, which also shows the (002) peak intensities for these samples.

Table 8-7 XRD analysis of the AZO thin films on Si substrates.

\begin{tabular}{|c|c|c|c|}
\hline Name & (002) peak intensity & $\mathbf{d}(\mathbf{A})$ & $\boldsymbol{\zeta}(\mathbf{G P a})$ \\
\hline Si1 set1 & 11040 & 2.604 & -0.22 \\
\hline Si2 set1 & 28777 & 2.606 & -0.40 \\
\hline Si3 set1 & 20853 & 2.607 & -0.49 \\
\hline Si1set2 & 38950 & 2.610 & -0.76 \\
\hline
\end{tabular}


It can be seen that AZO thin films deposited on Si showed a comparable (002) peak intensity to what was observed on other substrates. In addition, the estimated lattice constant and in-plane residual stress values were similar to what was found for sapphire substrates, suggesting that our sputtering recipe yields thin films with compressive stresses.

Table 8-8 summarizes the FWHM and mean crystallite domain size values for our selected samples on Si.

Table 8-8 XRD analysis showing FWHM and the mean crystallite domain size for the AZO on Si samples.

\begin{tabular}{|c|c|c|}
\hline Name & FWHM $\mathbf{(}^{\circ}$ ) & CDS (nm) \\
\hline Si1 set1 & 0.36 & 23.4 \\
\hline Si2 set1 & 0.32 & 26.0 \\
\hline Si3 set1 & 0.34 & 24.2 \\
\hline Si1 set2 & 0.35 & 23.9 \\
\hline
\end{tabular}

All samples presented in Table 8-8 had comparable FWHM values, hence their mean crystallite domain sizes were similar.

To conclude the findings of this section, the XRD results confirmed that our AZO films had similar crystal properties, despite having very different substrates. This was a positive outcome for our plasmonic application, given that 
three different substrate materials were found to be compatible with our sputtering recipe, which expanded our design choices.

\subsection{SEM imaging}

In this section, we present SEM images of nominal AZO samples on sapphire, $\mathrm{Si}$ and $\mathrm{CaF}_{2}$ substrates. Judging from a selection of SEM images of various samples produced in this study, a similar surface morphology was observed for thin films deposited on the same substrate. Figure 8-4 shows two SEM images at the same scale for selected $\mathrm{AZO}$ samples on sapphire and $\mathrm{CaF}_{2}$ substrates.

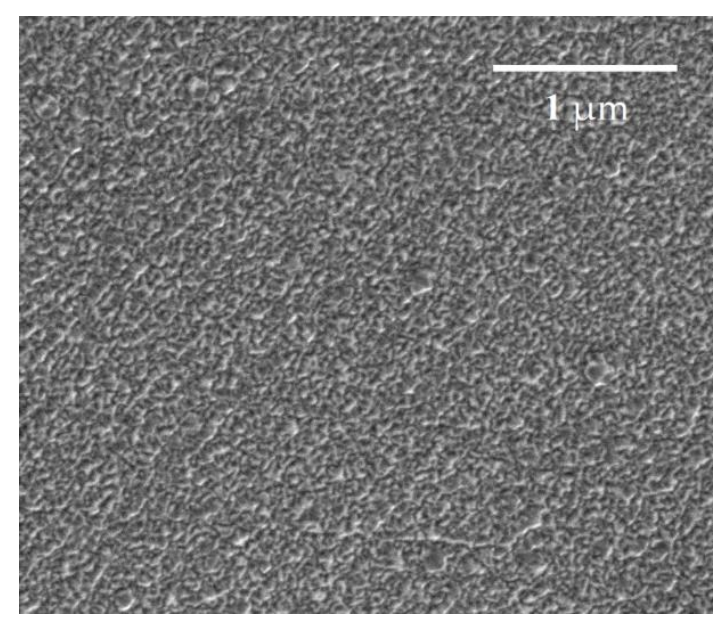

(a)

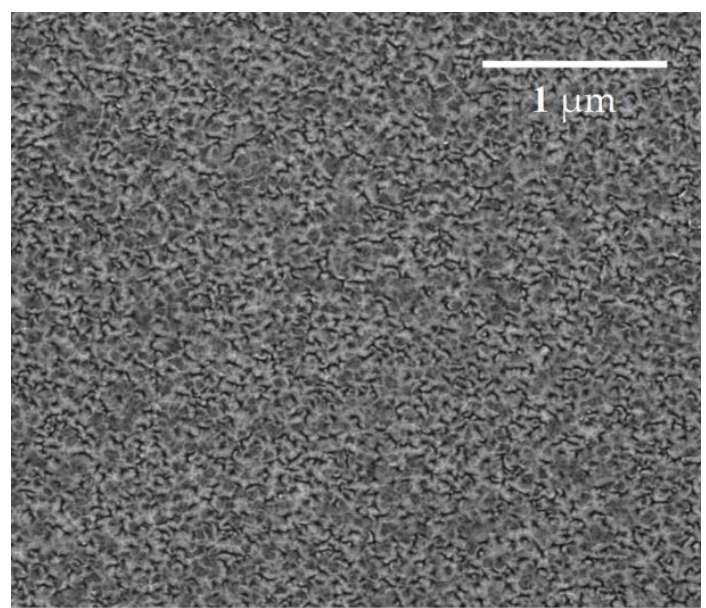

(b)

Figure 8-4 SEM images of $\mathrm{AZO}$ thin film on (a) $\mathrm{CaF}_{2}$ and (b) sapphire.

In each SEM image from Figure 8-4, the uniformity for the deposited thin films over the imaged area can be observed. In order to identify the differences between samples produced in one sputtering run, we provide SEM images and EDS analysis for three AZO samples deposited on Si in Set1: Si1set1, Si2set1 and 
Si3set1 as defined in section 8.2.3. These SEM images are shown in Figure 8-5 to Figure 8-7, while the results of the EDS analysis are presented in Table 8-9.

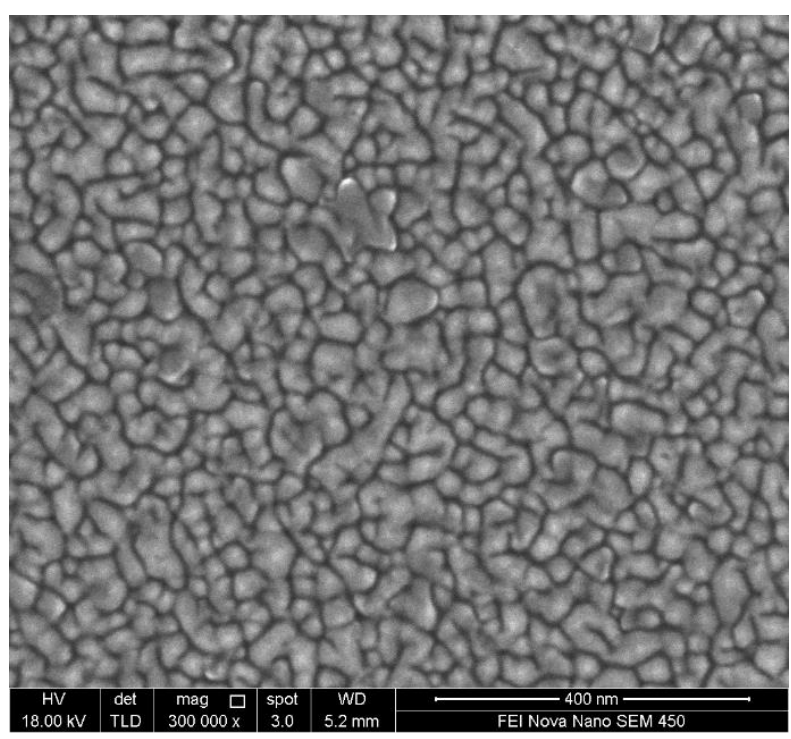

Figure 8-5 SEM image of Si1set1.

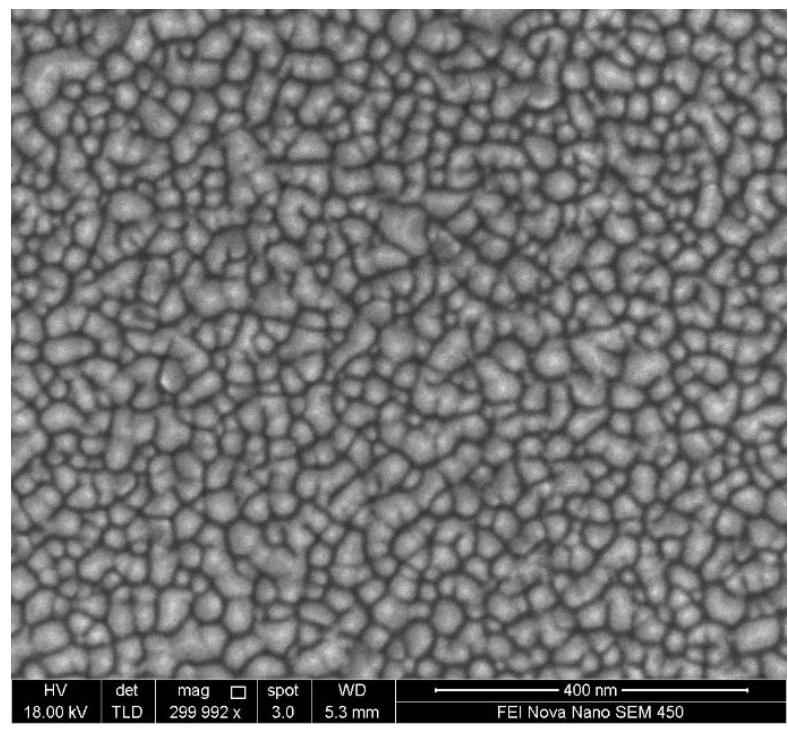

Figure 8-6 SEM image of Si2set1. 


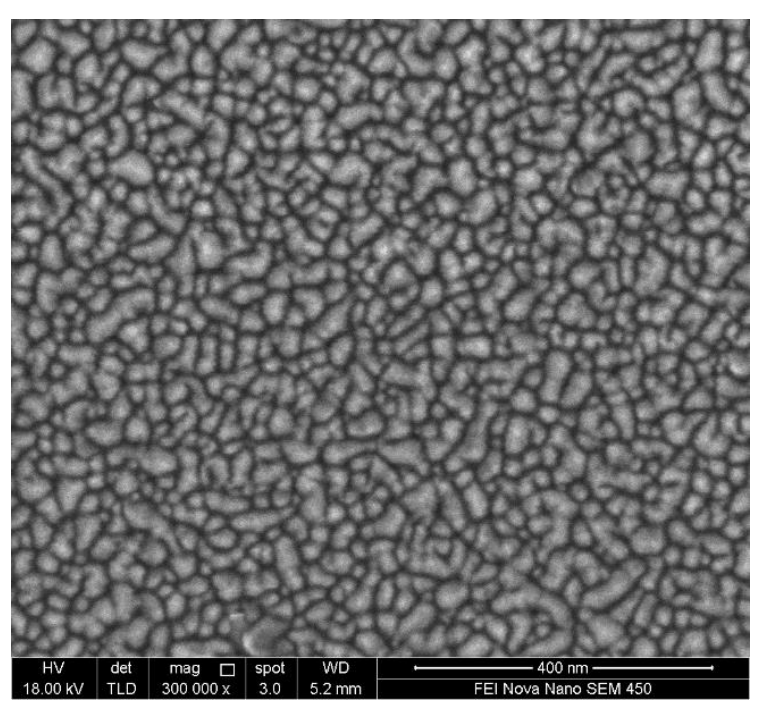

Figure 8-7 SEM image of Si3set1.

It can be seen that these three samples had similar grain shapes and sizes. It is noted that the location of these samples with respect to the centre of the sputtering plasma during the sputtering process was almost equidistant. Furthermore, these samples had similar properties during the characterization processes, which included the Hall effect, XRD and RBS measurements. Their thickness values were close with a difference of about $20 \mathrm{~nm}$.

Table 8-9 Mean atomic composition (\%) for Si1set1 to Si3set1 using EDS.

\begin{tabular}{|c|c|c|c|}
\hline \multirow{2}{*}{ Element } & \multicolumn{3}{|c|}{ Atomic percentages } \\
\cline { 2 - 4 } & Si1set1 & Si2 Set1 $_{1}$ & Si3set1 \\
\hline $\mathrm{O}$ & $48 \pm 4$ & $50 \pm 4$ & $50 \pm 4$ \\
\hline $\mathrm{Zn}$ & $42 \pm 2$ & $44 \pm 2$ & $43 \pm 2$ \\
\hline $\mathrm{Al}$ & $3 \pm 1$ & $2 \pm 1$ & $2 \pm 1$ \\
\hline $\mathrm{Si}$ & $7 \pm 1$ & $3 \pm 1$ & $6 \pm 1$ \\
\hline
\end{tabular}


Each EDS measurement was acquired over an area within the SEM image frames shown for Si1set1 to Si3set1. Zn, O, Al from the thin film, and Si from the substrate, were the detected elements in the EDS spectra. These results highlight the uniformity of our films in deposition process, where all samples showed similar composition within the margins of error. It should be noted that samples Si1set1 and Si3set1 are $110 \mathrm{~nm}$ thick while Si2set1 is $130 \mathrm{~nm}$ thick, which is consistent with obtaining a lower percentage for $\mathrm{Si}$ in the Si2set1 sample as the electron beam had to travel through a thicker thin film.

Table 8-10 shows the elemental composition percentages of the AZO thin films of these three samples, extracted from the same EDS data, where the Si contribution was excluded.

Table 8-10 Measured stoichiometry of samples Si1set1 to Si3set1 using EDS, with the stoichiometry provided for the AZO target material.

\begin{tabular}{|c|c|c|c|c|}
\hline \multirow{2}{*}{ Element } & \multicolumn{4}{|c|}{ Atomic percentages (\%) } \\
\cline { 2 - 5 } & Si1set1 & Si2set1 & Si3set1 & Target Stoichiometry \\
\hline $\mathrm{O}$ & $52 \pm 4$ & $52 \pm 4$ & $53 \pm 4$ & 50.5 \\
\hline $\mathrm{Zn}$ & $45 \pm 2$ & $46 \pm 3$ & $45 \pm 3$ & 47.6 \\
\hline $\mathrm{Al}$ & $3 \pm 1$ & $2 \pm 1$ & $2 \pm 1$ & 1.9 \\
\hline
\end{tabular}


As it can be seen, the atomic composition percentages agree with the stoichiometry of the target.

Using "ImageJ” [439], an image processing tool developed by the US National Institutes of Health $(\mathrm{NIH})$, we analysed the size of the grains (mean grain diameter) for the thin films presented in Figure 8-5 to Figure 8-7. These results are shown in Table 8-11.

Table 8-11 Mean grain diameter (nm) estimated using ImageJ with the corresponding error.

\begin{tabular}{|c|c|}
\hline Name & Mean grain diameter $\mathbf{( n m )}$ \\
\hline Si1 set 1 & $46.54 \pm 4.65$ \\
\hline Si2 set 1 & $44.75 \pm 4.47$ \\
\hline Si3 set 1 & $40.31 \pm 4.03$ \\
\hline
\end{tabular}

Based on these results, the mean grain diameter seemed to be comparable across the imaged samples.

In this section, $\mathrm{SEM}$ images of $\mathrm{AZO}$ samples on sapphire, $\mathrm{CaF}_{2}$ and $\mathrm{Si}$ were presented. These images confirmed the uniformity of our deposited films. We note that $\mathrm{AZO}$ films deposited on $\mathrm{CaF}_{2}$ substrates often suffered from peeling and cracking, which was visually evident as shown in Figure 8-2. In the next subsection, aided with SEM images, we explore the effects of thermal expansion on the expected residual thin film strain in our samples, and compare the suitability of all four used substrates with AZO from a thermal matching standpoint. 


\subsubsection{Thermal expansion}

The thermal expansion property describes the tendency of a material to expand or contract due to temperature variation [440]. This material characteristic is usually modelled using the thermal expansion coefficient, which denotes changes in the size of an object with respect to temperature variation at a constant pressure.

Depending on the application and dimensions of the sample, different forms and approximations of the coefficients of thermal expansion can be used. In this study, we are interested in evaluating the effects of thermal expansion at the substrate-thin film interface. Thus, assuming the linear thermal expansion equation is adequate as long as the assumption that the medium is isotropic is valid. Accordingly, in the linear form, a variation in the temperature $(\delta T)$ will cause thermal expansion or contraction, resulting in a net strain $(\Delta L / L)$. This is given by the following equation:

$$
\frac{\Delta L}{L}=a \delta T
$$

\section{Equation 8-1}

where $a$ is the coefficient of thermal expansion.

Applying this to our deposited thin films samples and given that we sputtered the $\mathrm{AZO}$ at $345^{\circ} \mathrm{C}$, then by the time they and the corresponding substrate cool to a room temperature of $20^{\circ} \mathrm{C}$, a significant net strain could be trapped in the thin film structure. 
Table 8-12 presents a summary of the coefficients of thermal expansion for our substrates and thin film material, and provides an estimate for the net strain in each material due a variation in temperature of $325{ }^{\circ} \mathrm{C}$. In this table, we also provided an estimate for the relative strain of each substrate with respect to the thin film material $\left(R_{\Delta L / L}\right)$, given by:

$$
R_{\Delta L / L}=\frac{(\Delta L / L)_{A Z O}-(\Delta L / L)_{\text {Substrate }}}{(\Delta L / L)_{A Z O}}
$$

Equation 8-2

Assuming that the thin film remains in intimate contact with the substrate, i.e., no peeling or delamination, the relative strain provides an indication of the mismatch between the two materials, which relates to the residual thermal stress in the film. A negative relative strain in this instance indicates that the substrate shrunk more than the thin film during the cooling process, which should result in compressive in-plane stress within the thin film, and vice versa.

Table 8-12 Net strain calculated for our substrates.

\begin{tabular}{|c|c|c|c|c|c|}
\cline { 2 - 6 } \multicolumn{1}{c|}{} & $\begin{array}{c}\text { Calcium } \\
\text { Fluoride }\end{array}$ & Silicon & Borofloat33 & Sapphire & AZO \\
\hline$a\left({ }^{\circ} \mathrm{C}^{-1}\right)$ & $\begin{array}{c}18.85 \mathrm{E}-6 \\
{[301]}\end{array}$ & $\begin{array}{c}2.60 \mathrm{E}-6 \\
{[299]}\end{array}$ & $\begin{array}{c}3.25 \mathrm{E}-6 \\
{[289]}\end{array}$ & $\begin{array}{c}5.00 \mathrm{E}-6 \\
{[300]}\end{array}$ & $\begin{array}{c}3.53 \mathrm{E}-6 \\
{[441]}\end{array}$ \\
\hline$\Delta L / L$ & $6.13 \mathrm{E}-03$ & $8.45 \mathrm{E}-04$ & $1.06 \mathrm{E}-03$ & $1.63 \mathrm{E}-03$ & $1.15 \mathrm{E}-03$ \\
\hline $\mathrm{R} \Delta L / L$ & $-4.34 \mathrm{E}+00$ & $2.63 \mathrm{E}-01$ & $7.93 \mathrm{E}-02$ & $-4.16 \mathrm{E}-01$ & $0.00 \mathrm{E}+00$ \\
\hline Stress type & Compressive & Tensile & Tensile & Compressive & Null \\
\hline
\end{tabular}


The calculated relative strain is significantly higher for the $\mathrm{CaF}_{2}$ substrate compared with other substrates. Observing the surface of our deposited thin films in the SEM images, the only samples that showed a sign of damage on the surface in the form of cracks and delamination were AZO samples deposited on the $\mathrm{CaF}_{2}$ substrate.

The damage on the surface of our $\mathrm{AZO}$ thin films deposited on the $\mathrm{CaF}_{2}$ substrate was observed within 24 hours after deposition and deteriorated with time. This damage is associated with the residual stress in these films, a significant part of which was suspected to originate from thermal effects. The short-term damage manifested in 2D periodic wrinkles in the form of buckledelamination, as shown in the SEM images of Figure 8-8 [442, 443].
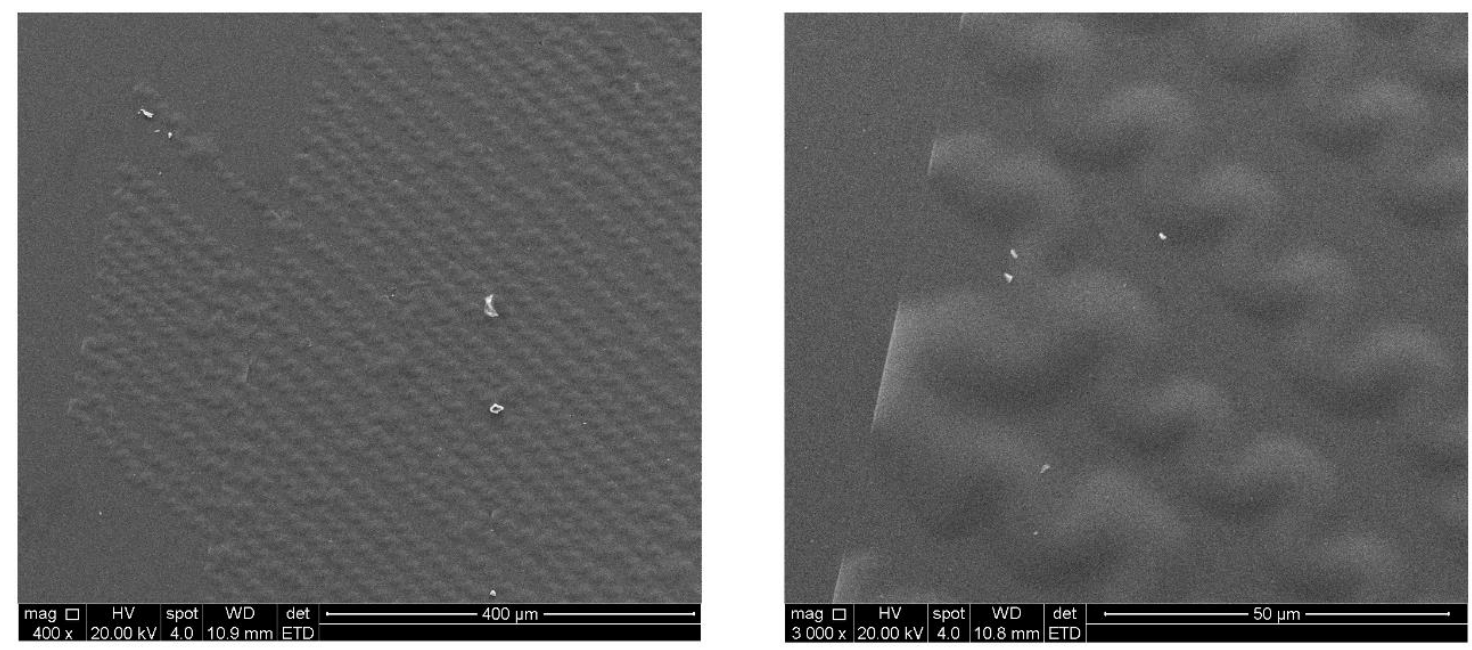

Figure 8-8 2D periodic wrinkles on our $\mathrm{AZO}$ on $\mathrm{CaF}_{2}$ samples in the form of buckle-delamination developed within a week after deposition. 
In the long term, some of the $\mathrm{AZO}$ on $\mathrm{CaF}_{2}$ samples developed cracks and delaminated, which was visible on a larger scale, as shown in the SEM images of Figure 8-9.
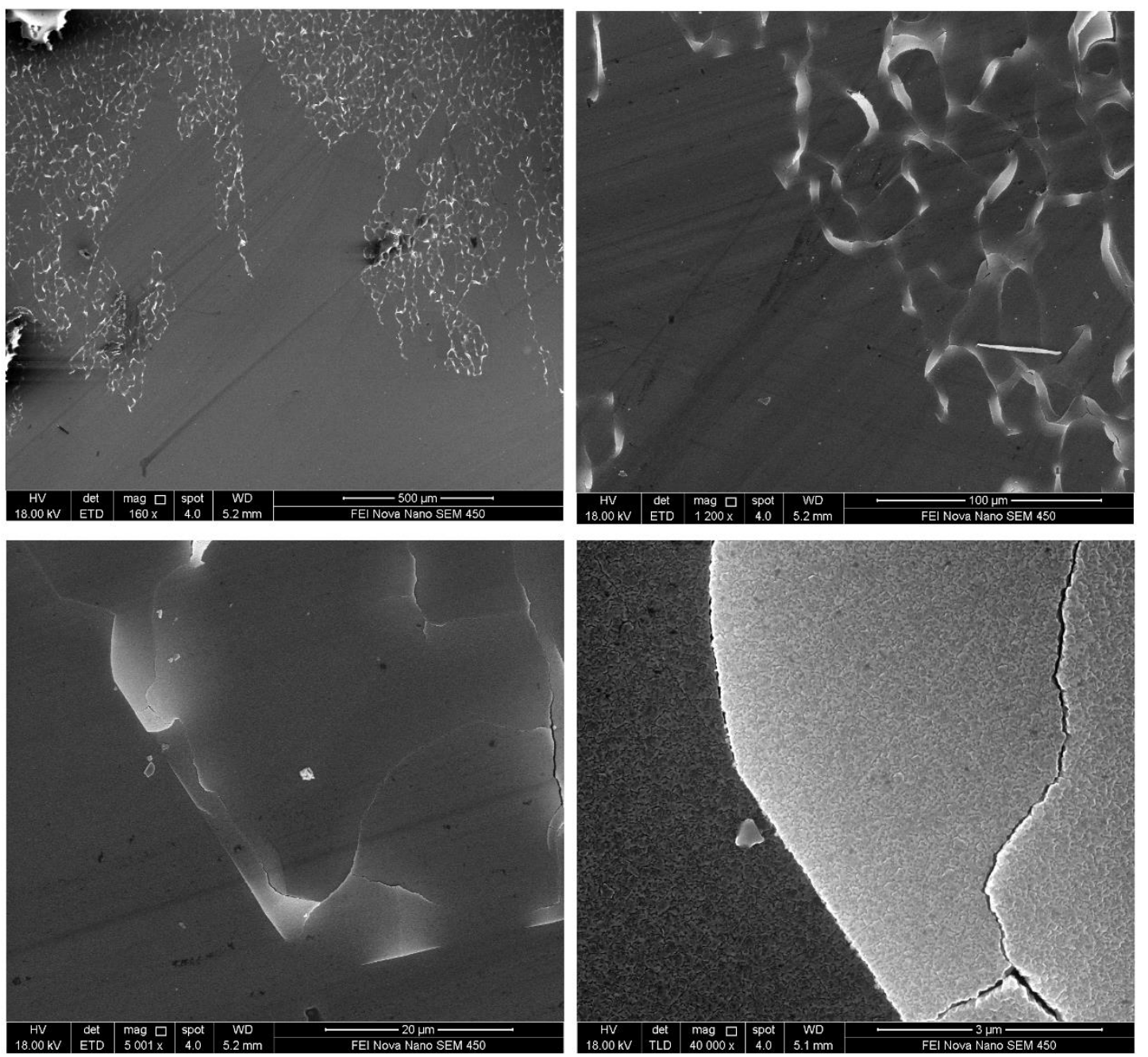

Figure 8-9 Cracks covering a large portion of the surface in $\mathrm{AZO}$ on $\mathrm{CaF}_{2}$ samples. This effect became more prominent over time.

Furthermore, some SEM images of the peeled $\mathrm{AZO}$ on $\mathrm{CaF}_{2}$ samples revealed numerous orthogonal micron-scaled "scratches", which probably was one of the 
failure modes of the thin film due to the inherent stress. A sample is shown in Figure 8-10.

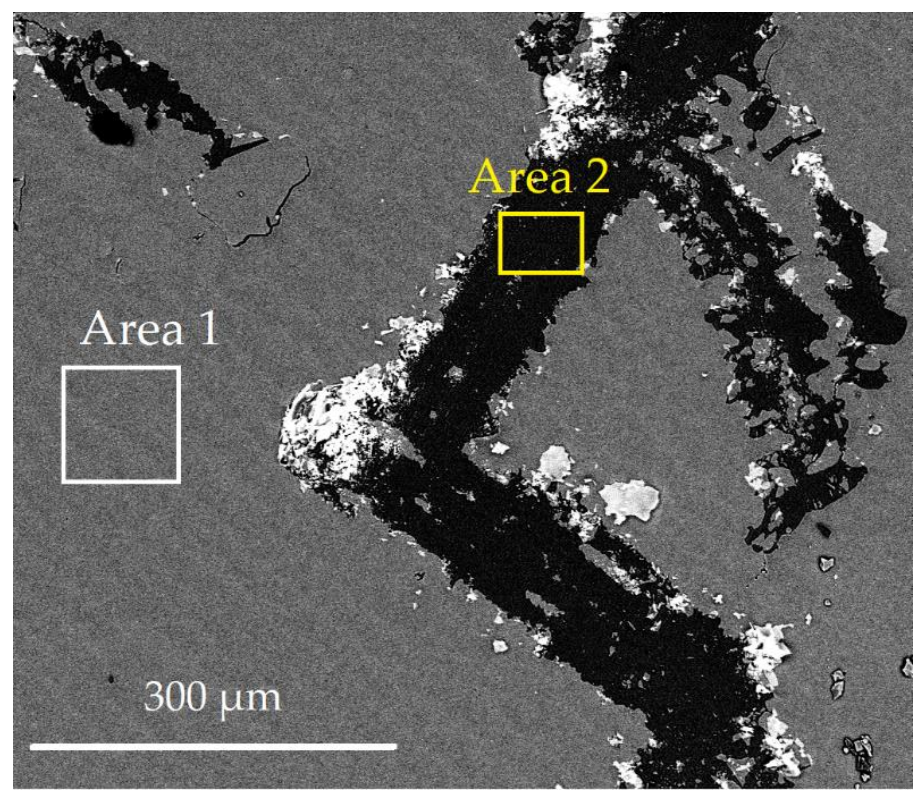

Figure 8-10 SEM image of $\mathrm{AZO}$ on $\mathrm{CaF}_{2}$ showing scratch-type damage. Areas 1 and 2 were selected for EDS analysis.

To confirm the observations of the SEM images, we performed EDS measurements for the two areas shown in Figure 8-10. 


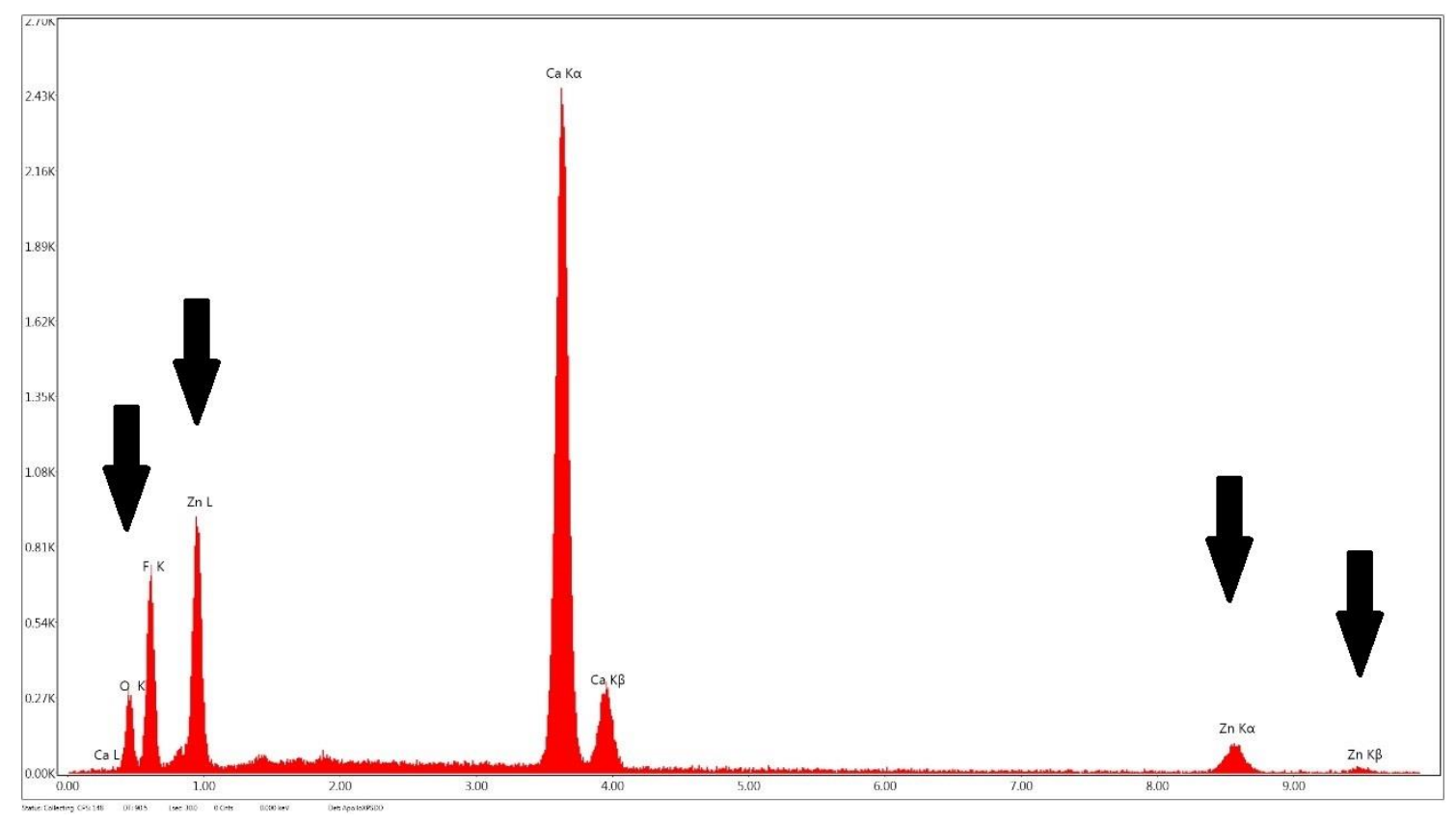

Figure 8-11 EDS analysis of AZO on $\mathrm{CaF}_{2}$ over Area 1 in Figure 8-10, arrows indicate the $\mathrm{Zn}$ and $\mathrm{O}$ peaks

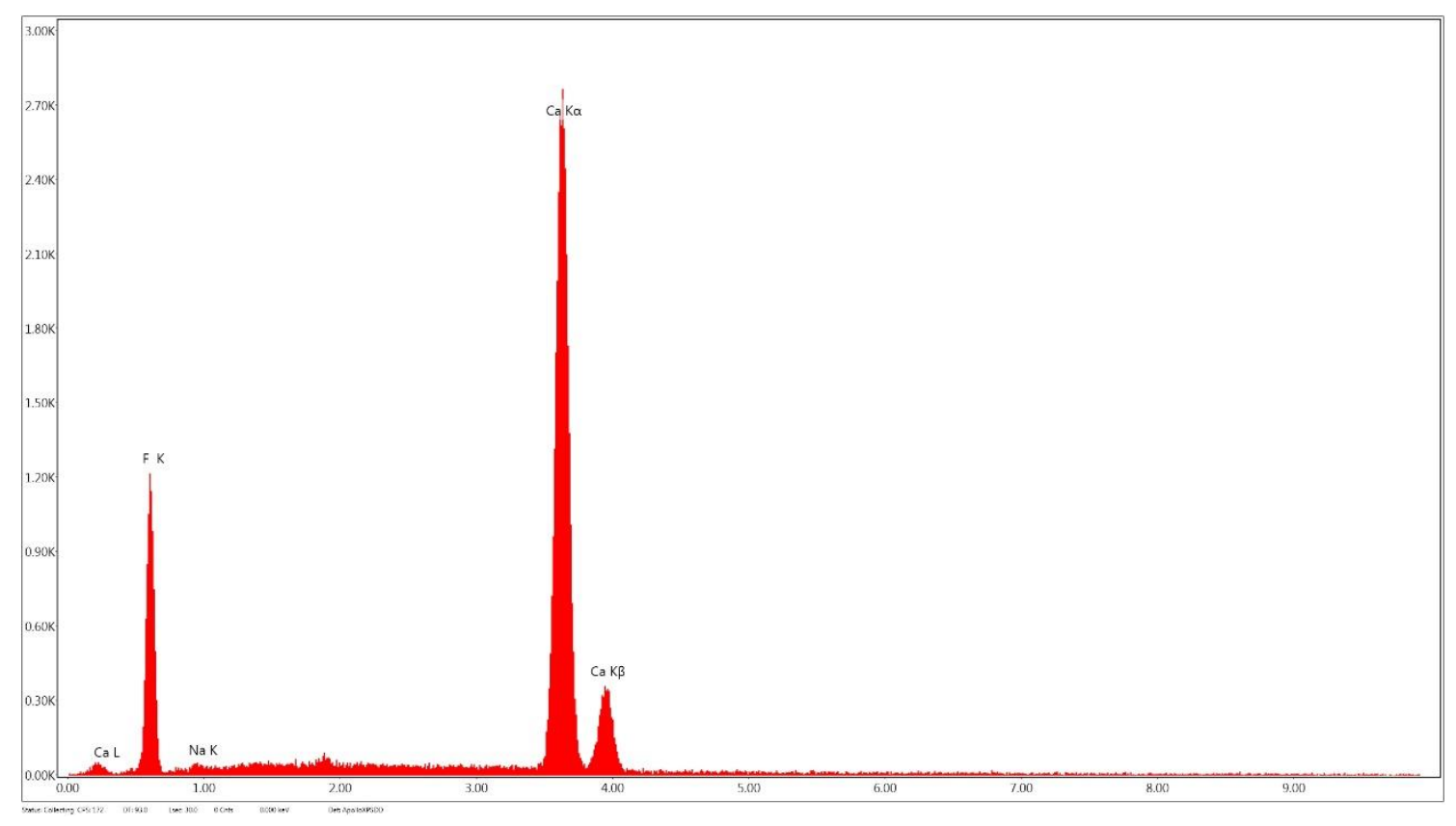

Figure 8-12 EDS analysis of AZO on $\mathrm{CaF}_{2}$ over Area 2 in Figure 8-10 
As can be seen, components of the thin film elements are not detected in the EDS analysis of Area 2, i.e., the scratched area.

Based on the results presented in this section, AZO thin films deposited on $\mathrm{CaF}_{2}$ had high residual stress levels due to the mismatch in the coefficient of thermal expansion between both materials. All other substrates showed no cracking or delamination signs, and remained stable over time, which confirmed their thermal suitability. Accordingly, despite being a promising substrate from an optical standpoint [438], $\mathrm{CaF}_{2}$ was found to be the least favourable candidate for depositing AZO thin films using our established process.

\subsection{Hall effect parameters}

The Hall effect is one of the primary characterization methods that were used to judge the suitability of our thin films for plasmonic applications. In this section, we present the Hall effect measurement results of our AZO samples on sapphire, $\mathrm{CaF}_{2}$ and $\mathrm{Si}$.

\subsubsection{Carrier concentration measurements}

The carrier concentration and resistivity for the AZO on sapphire samples from Set1 in section 8.2.1 are measured using our Hall effect setup. The results are shown in Table 8-13. 
Table 8-13 Hall effect parameters measured the for AZO thin films on sapphire from Set1. The carriers are n-type.

\begin{tabular}{|r|c|c|}
\hline Name & Ave carrier concentration $\left.\mathbf{( c m}^{-3}\right)$ & Av. $\boldsymbol{\rho}(\mathbf{\Omega . c m})$ \\
\hline Sap1set1 & $(1.60 \pm 0.06) \mathrm{E}+20$ & $(6.25 \pm 0.05) \mathrm{E}-03$ \\
\hline Sap2 set1 & $(1.57 \pm 0.09) \mathrm{E}+20$ & $(5.64 \pm 0.07) \mathrm{E}-03$ \\
\hline Sap3 set1 & $(1.40 \pm 0.05) \mathrm{E}+20$ & $(4.53 \pm 0.02) \mathrm{E}-03$ \\
\hline Sap4 set1 & $(1.85 \pm 0.08) \mathrm{E}+20$ & $(4.05 \pm 0.01) \mathrm{E}-03$ \\
\hline Sap5 set1 & $(1.60 \pm 0.09) \mathrm{E}+20$ & $(4.64 \pm 0.03) \mathrm{E}-03$ \\
\hline
\end{tabular}

Based on the results of chapter 7 , we defined $1 \mathrm{E}+20 \mathrm{~cm}^{-3}$ as the carrier concentration threshold for evaluating the suitability of our deposited AZO thin films for plasmonic applications. In addition, these films should have a resistivity of the order of 1E-3 ( $\Omega . \mathrm{cm})$. Accordingly, all reported carrier concentration and resistivity values in Table 8-13 satisfy these constraints, which suggested that all these samples are suitable for our plasmonic application. Table 8-14 shows the Hall effect values for the two AZO on sapphire samples from Set2 in section 8.2.1.

Table 8-14 Hall effect parameters measured for the AZO thin films on sapphire from Set2. The carriers are n-type.

\begin{tabular}{|r|c|c|}
\hline Name & Carrier concentration $\mathbf{( \mathbf { c m } ^ { - } \mathbf { 3 }}$ & $\boldsymbol{\rho}(\boldsymbol{\Omega . c m})$ \\
\hline Sap1 $1_{\text {set2 }}$ & $(4.02 \pm 0.03) \mathrm{E}+20$ & $(2.35 \pm 0.01) \mathrm{E}-03$ \\
\hline Sap2set2 & $(3.15 \pm 0.04) \mathrm{E}+20$ & $(2.52 \pm 0.01) \mathrm{E}-03$ \\
\hline
\end{tabular}


The carrier concentrations for the Set2 samples maintained the same order of magnitude $\left(1 \mathrm{E}+20 \mathrm{~cm}^{-3}\right)$, with their values being slightly better that those in Set1.

Next, Table 8-15 shows the carrier concentration and resistivity values for the $\mathrm{AZO}$ on $\mathrm{CaF}_{2}$ samples from section 8.2.2.

Table 8-15 Hall effect parameters measured for the AZO thin films on $\mathrm{CaF}_{2}$ substrate. The carriers are n-type.

\begin{tabular}{|c|c|c|}
\hline Name & Carrier concentration $\left(\mathbf{c m}^{-3}\right)$ & $\rho(\Omega . c m)$ \\
\hline non-transparent & $(1.04 \pm 0.07) \mathrm{E}+20$ & $(1.53 \pm 0.02) \mathrm{E}-02$ \\
\hline damaged & $(1.04 \pm 0.01) \mathrm{E}+21$ & $(3.01 \pm 0.04) \mathrm{E}-03$ \\
\hline thickest & $(2.64 \pm 0.03) \mathrm{E}+20$ & $(9.79 \pm 0.05) \mathrm{E}-03$ \\
\hline thinnest & $(1.89 \pm 0.06) \mathrm{E}+20$ & $(3.52 \pm 0.03) \mathrm{E}-03$ \\
\hline
\end{tabular}

Regardless of the differences between these samples, the carrier concentration values remain of the order of $1 \mathrm{E}+20 \mathrm{~cm}^{-3}$ or higher for all of them. The resistivity of the non-transparent sample, however, is significantly higher than other $\mathrm{CaF}_{2}$ samples.

Finally, Table 8-16 shows the measured Hall effect parameters for the four AZO on Si samples from section 8.2.3. 
Table 8-16 Hall effect parameters measured for the AZO thin films on silicon substrate. The carriers are n-type.

\begin{tabular}{|c|c|c|}
\hline Name & Carrier concentration $\mathbf{( \mathbf { c m } ^ { - 3 } )}$ & $\rho(\boldsymbol{\Omega} . \mathbf{c m})$ \\
\hline Si1 ${ }_{\text {Set } 1}$ & $(1.81 \pm 0.08) \mathrm{E}+20$ & $(4.58 \pm 0.03) \mathrm{E}-03$ \\
\hline Si2 set1 & $(1.58 \pm 0.02) \mathrm{E}+20$ & $(5.01 \pm 0.03) \mathrm{E}-03$ \\
\hline Si3 set1 & $(1.79 \pm 0.04) \mathrm{E}+20$ & $(4.55 \pm 0.01) \mathrm{E}-03$ \\
\hline Si1 ${ }_{\text {set2 }}$ & $(3.08 \pm 0.02) \mathrm{E}+20$ & $(2.31 \pm 0.01) \mathrm{E}-03$ \\
\hline
\end{tabular}

Samples from Set1 had comparable carrier concentration values, which indicated the uniformity of deposition across the studied area. The Si1set2 sample had a higher carrier concentration and almost half resistivity compared with Set1's samples.

In summary, all measured carrier concentration values reported in this chapter were of the order of $1 \mathrm{E}+20 \mathrm{~cm}^{-3}$ with no evident dependency on the substrate material. In addition, all samples had a low resistivity in the order of 1E-3 $(\Omega . \mathrm{cm})$. This indicates a consistently high number of free electrons in our deposited AZO thin films and suggests their potential suitability to create plasmonic devices in the near-infrared region of the EM spectrum.

All Hall effect measurements were performed at ambient temperatures. The next section presents the PPM results for our samples, providing an insight to their properties over a broader temperature range. 


\subsubsection{PPMS measurements}

Temperature dependent resistance of one AZO sample on each substrate from this chapter were measured using the PPMS. This choice was made given that most samples had comparable Hall effect and resistivity properties on a given substrate (except for the damaged sample on $\mathrm{CaF}_{2}$ ). The temperature dependant conductivity (inverse of resistivity) plots for these AZO samples are shown in Figure 8-13.

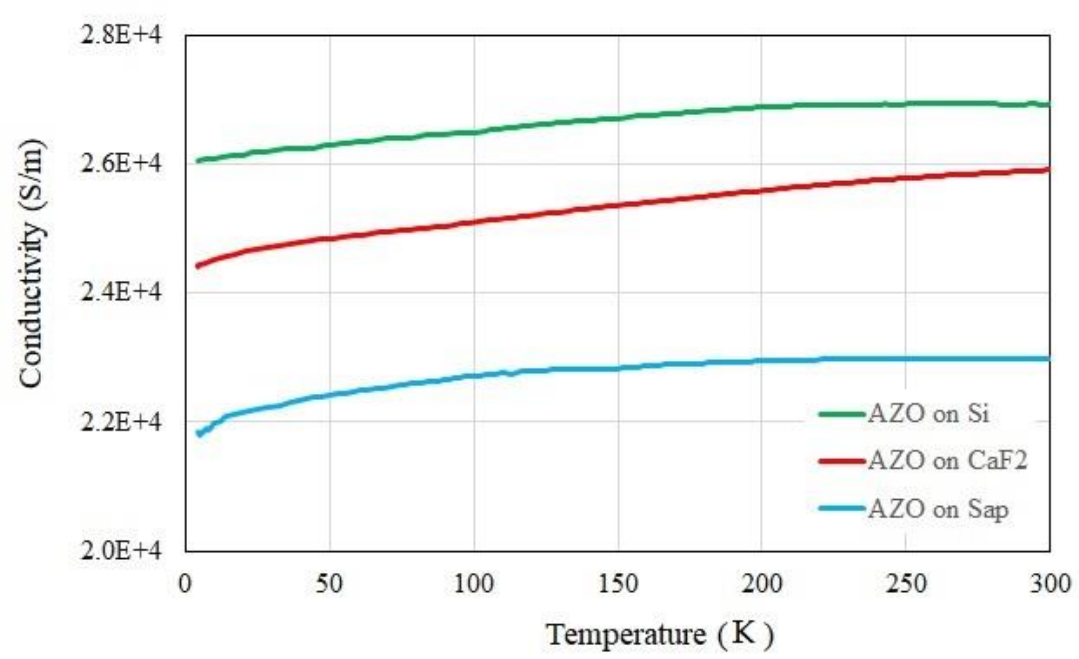

Figure 8-13 Temperature dependence conductivity of AZO

Given that our AZO thin films were highly doped beyond the degenerate point, we expected them to exhibit a behaviour that is closer to metals than semiconductors. Whereby metals have increased conductivity with temperature decrease due to decreased electron-phonon interactions [444], the conductivity of semiconductors drops exponentially with temperature decrease as electrons fall to lower energy states [445]. 
It can be seen from Figure 8-13 that our films had a slight linear decrease in conductivity as temperature dropped from $300 \mathrm{~K}$ to near $5 \mathrm{~K}$ during the measurements. The small decrease in conductivity confirmed our expectation regarding the film's electrical behaviour, which could be attributed to the high doping level as charged carriers do not experience a dramatic freeze out [446].

\subsection{FTIR measurements}

Based on the AZO spectral results in Chapter 7, we solely relied on the Vertex V80v Bruker FTIR spectrometer for transmission measurements in this chapter.

As mentioned in section 6.6.2, the FTIR measurements are carried under vacuum, where the pressure is about $2 \mathrm{hPa}$ in the Bruker spectrometer. This reduced pressure is expected to reduce scattering and absorption effects that arise from atmospheric conditions, where humidity in particular can affect the measured spectral properties of our samples. Thus, to get consistently accurate results, the system was left under vacuum for 15-30 mins after a new sample was placed or a detector was changed. As a result, all optical components had enough time to reach a steady state before measurements were started.

As reported in the previous chapter, the region below $2000 \mathrm{~cm}^{-1}$ is not transparent for the Borofloat33 substrate. This prevented the assessment of the optical properties of our AZO thin films in the near-infrared region. In this section, we present FTIR transmission spectra for AZO thin film samples on calcium fluoride, silicon and sapphire substrates, which are transparent in the NIR region and have low absorption bands in their transmission spectra, as explained in section 5.2.2 and section 8.1. 
Figure 8-14 shows the transmission plots for silicon, sapphire, and calcium fluoride blank substrates.

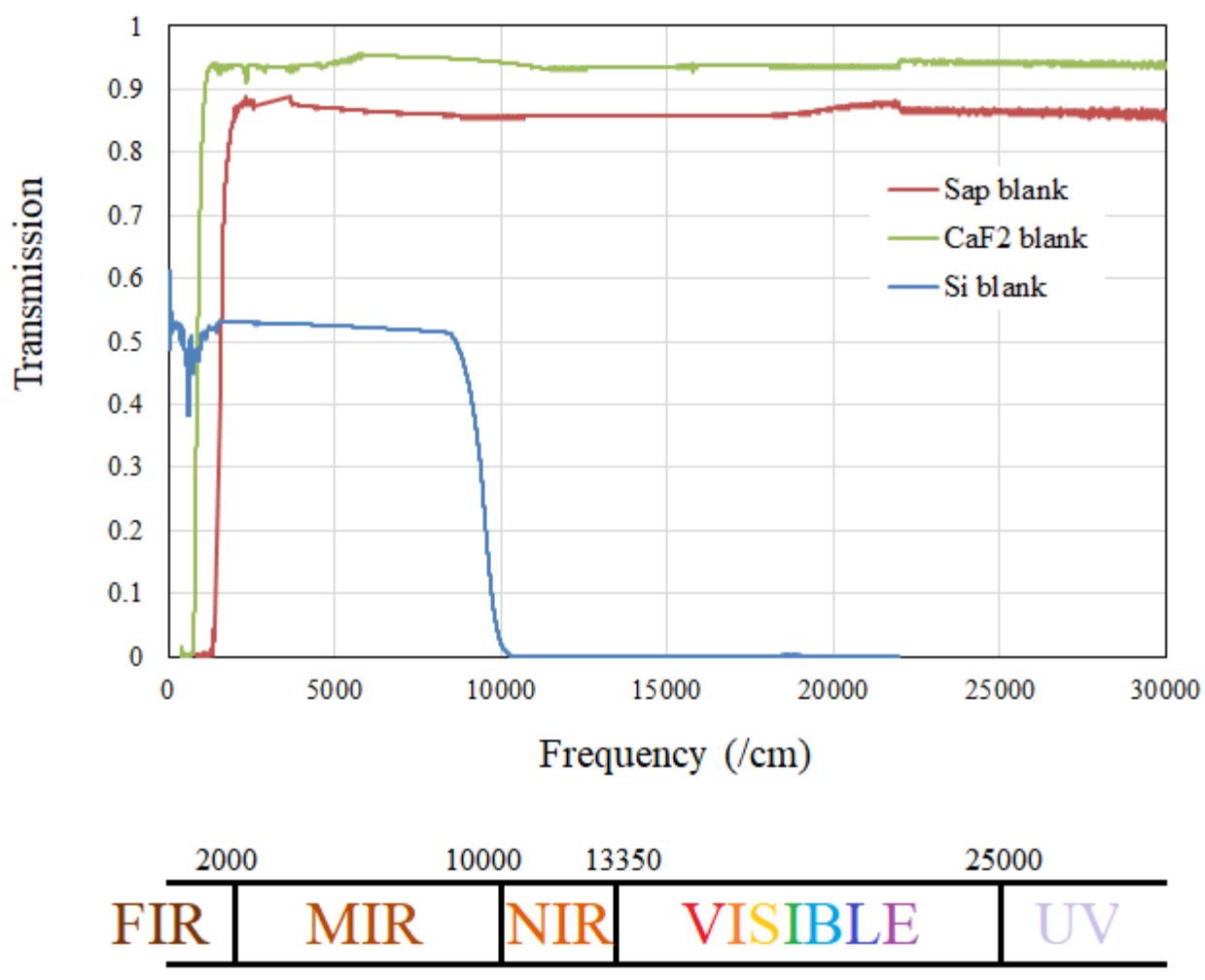

Figure 8-14 Transmission spectra for $\mathrm{CaF}_{2}$, Sapphire and Si substrates measured using the Bruker FTIR spectrometer.

It can be seen that the sapphire and calcium fluoride substrates were confirmed to be transparent over a broad spectral range. Silicon on the other hand had a relatively low transparency in the region of interest, nonetheless, it had about $50 \%$ transparency at the lowest frequency range $<1000 \mathrm{~cm}^{-1}$, while other substrates were completely opaque. This gives the opportunity to observe the optical properties of our deposited thin films in this range. 


\subsubsection{AZO on sapphire}

FTIR transmission spectra for AZO thin films on sapphire substrate from Set1 and Set 2 are presented in this section. Figure 8-15 shows the transmission plots of five AZO on sapphire samples from Set1.

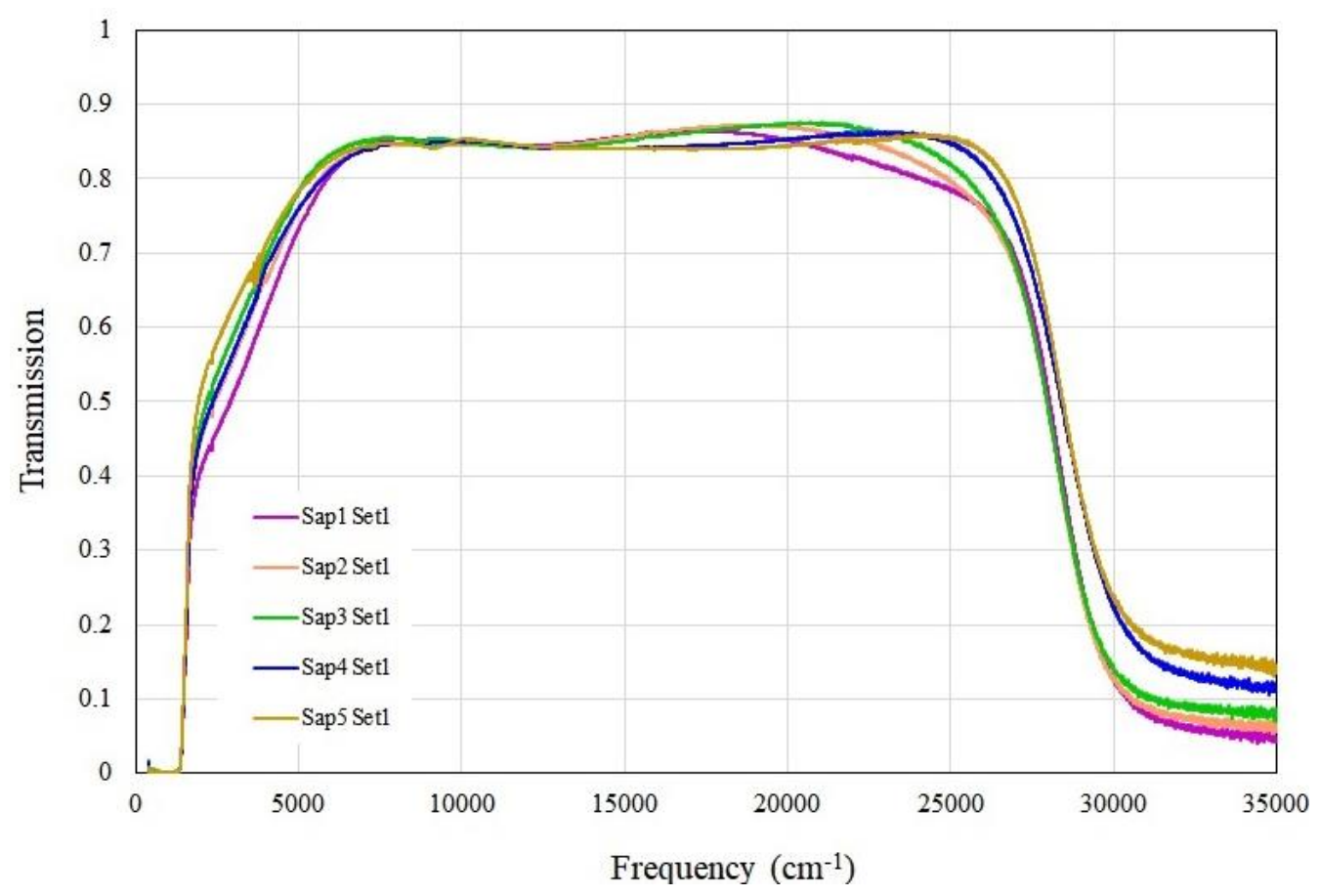

Figure 8-15 Transmission spectra for AZO thin films on sapphire substrate from Set1.

Given that these samples were prepared in a single sputtering process, they were expected to have comparable optical properties, were any differences would be attributed to their placement with respect to the centre of the plasma during deposition [447]. During deposition, Sap1set1-Sap3set1 were in the first row closest to the plasma centre while Sap4set1-Sap5set1 were further away in the second row. 
Comparing the transmission spectra of the AZO thin films deposited on sapphire to that of the blank sapphire substrate indicates that all samples maintained an optical transparency larger than $80 \%$ from the near UV to the beginning of the NIR range. However, the sample's sputtering position seemed to correlate with the differences in transmission spectra, as the Sap4set1 and Sap5set1 had higher UV and lower visible transmission than the Sap1set1-Sap3set1 samples.

Table 8-17 shows the RBS thickness measurements for the Sap1set-Sap5set1 samples. Accordingly, these samples are found to have some differences in thickness, where the first-row samples Sap1set-Sap3set1 were found to be $\sim 30 \mathrm{~nm}$ (or 25\%) thicker than the second-row samples Sap4set1 and Sap5set1.

Table 8-17 Set1 AZO on sapphire thickness measurement using RBS.

\begin{tabular}{|c|c|}
\hline Name & Thin film thickness (nm) \\
\hline Sap1 Set1 & $130 \pm 13$ \\
\hline Sap2 Set1 & $125 \pm 12$ \\
\hline Sap3 set1 & $115 \pm 11$ \\
\hline Sap4 set1 & $100 \pm 10$ \\
\hline Sap5 Set1 & $85 \pm 8$ \\
\hline
\end{tabular}

Considering the effects of thin film thickness on the transmission spectra of a given Set1 sample, it was found that thicker samples resulted in slightly lower transmission in the UV range. 
Next, we used the transmission spectra of these five AZO on sapphire samples to estimate their optical bandgaps using Tauc's plots [280]. Thus, the samples' spectra in the UV region were used for this estimation. For the Tauc plots presented in Figure 8-16, the vertical axis was $(\alpha E)^{2}$ while the horizontal axis was E, where $\alpha$ is the absorption coefficient and $\mathrm{E}$ is the energy given by $h f, h$ being Planck's constant and $f$ is the frequency.

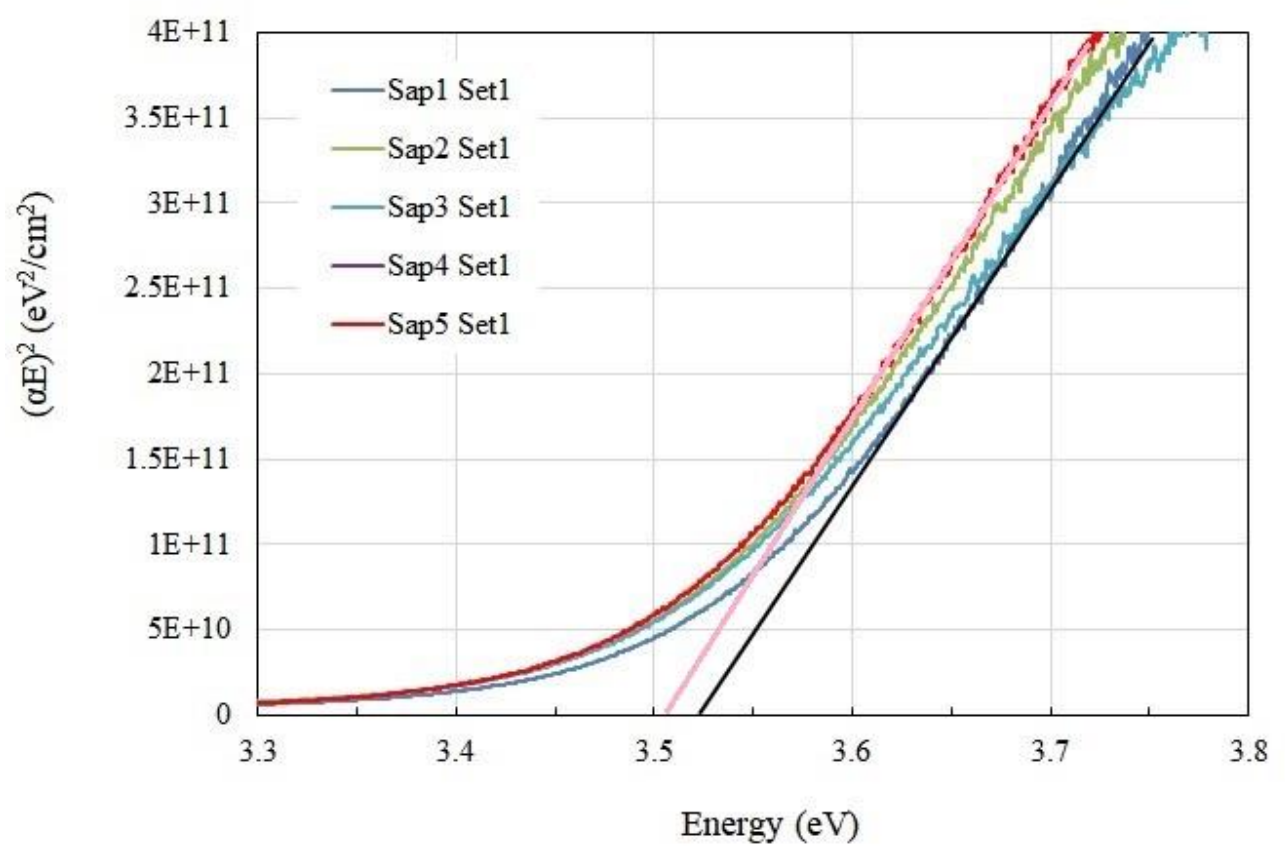

Figure 8-16 Tauc plots for the AZO on sapphire samples from Set1.

In all five plots of Figure 8-16, the asymptotic lines indicate a relatively wide optical bandgap around $\sim 3.5 \mathrm{eV}$, which could be attributed to the degenerate doping of $\mathrm{AZO}$, as described by the BM model [431]. Plotting the measured carrier concentration data in section 8.4.1 against the estimated optical bandgap energies for the different samples of Figure 8-16 is in a good fit with the BM model [428]. 
Figure 8-17 shows the FTIR spectra of the two AZO samples on sapphire that were selected from Set2, i.e., Sap1set2 and Sap2set2. For the sake of comparison with the selected samples from Set1, Sap1set2 and Sap2set2 were sputtered at the same locations of Sap4set1 and Sap2set1, respectively. Given this location mapping, we expected that Sap2set2 should be about 25\% thicker than Sap1set2.

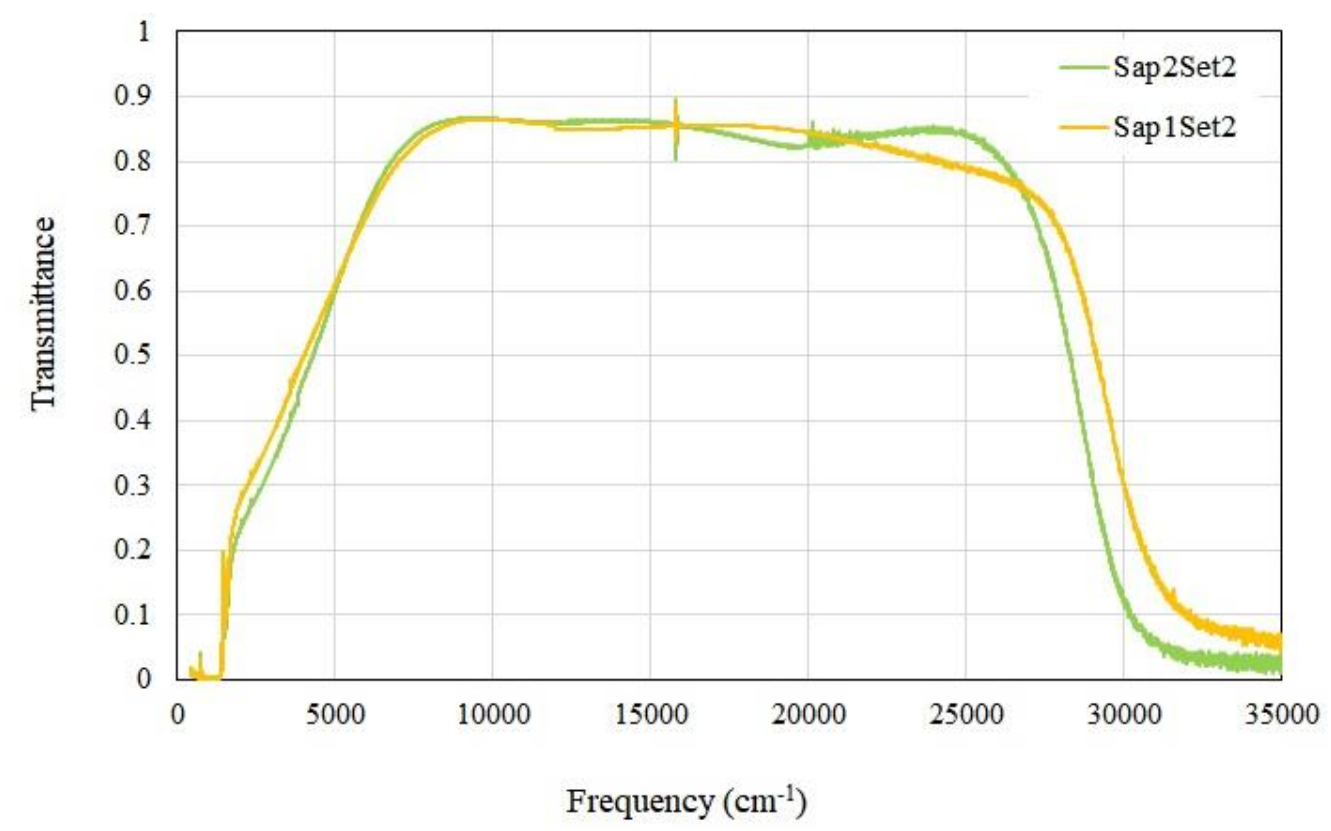

Figure 8-17 Transmission spectra for AZO thin films on sapphire substrate from Set2.

This was soon confirmed by the RBS measurements of Set2 samples, which are shown in Table 8-18. These RBS measurements also indicated that Set2's samples were significantly thicker (about 50\% thicker) than Set1's samples. 
Table 8-18 Set2 AZO on sapphire thickness measurement using RBS.

\begin{tabular}{|l|c|}
\hline Name & Thin film thickness (nm) \\
\hline Sap1 set2 & $145 \pm 14$ \\
\hline Sap2 set2 & $190 \pm 19$ \\
\hline
\end{tabular}

It can be seen from Figure 8-17 that the Set2 samples had comparable transmission properties to Set1's samples, where the thinner sample Sap1set2 had higher transmission in the UV range and lower transmission in the visible range. We note the slight difference in the mid-infrared transmission between Set2's samples and Set1's samples. This difference indicates a dissimilarity in the plasma frequency value between the two sets, as suggested by the findings of section 8.4.1, where samples from Set2 were found to have relatively higher carrier concentrations. This will be investigated in section 8.6.

Similar to our approach for Set1's samples, we used the transmission spectra for the AZO on sapphire samples from set2 to estimate the bandgap. The Tauc plots for Sap1set2 and Sap2set2 are shown in Figure 8-18. 


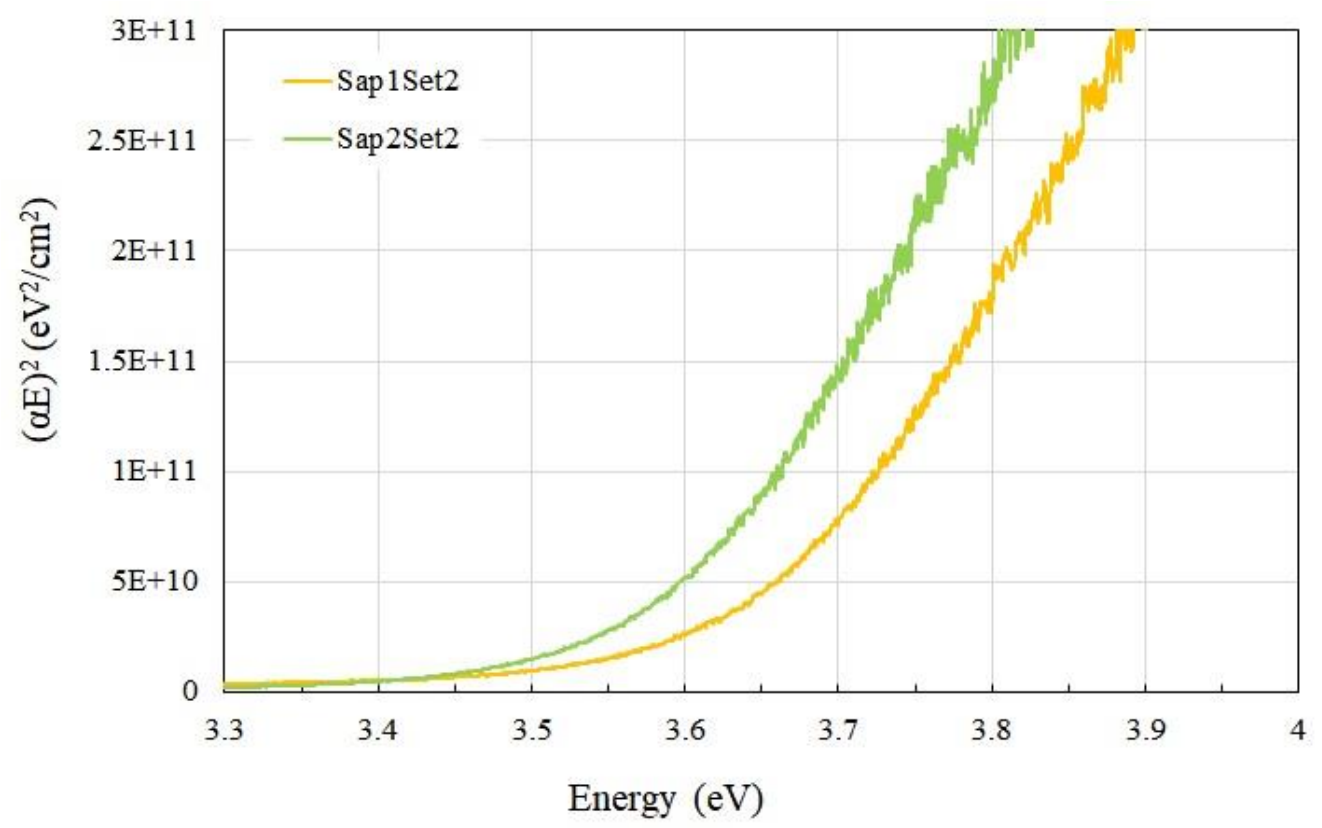

Figure 8-18 Tauc plots for AZO on sapphire samples from Set2.

Figure 8-18 shows bandgap energies of $3.65 \mathrm{eV}$ and $3.59 \mathrm{eV}$ for Sap1set2 and Sap2set2, respectively. The bandgap energies of Set2 samples are slightly higher than those of Set1, which were around $3.5 \mathrm{eV}$. This can be explained by the difference in the doping level between the two sets, which was confirmed in the Hall effect measurements of section 8.4.1. This extra doping level is expected to result in a larger overlap between the donor band and the conduction band, which results optical bandgap widening $[448,449]$.

In summary, transmission through AZO on sapphire samples was found to be higher than $80 \%$ in the region between the beginning of the near-IR to the near UV region. Also, there was no sharp absorption peaks in the samples' spectra due to the smooth transmission spectrum of the sapphire substrate. 


\subsubsection{AZO on $\mathrm{CaF}_{2}$}

In this section, transmission plots for the $\mathrm{AZO}$ on $\mathrm{CaF}_{2}$ samples are presented. Referring to Figure 8-14, the bare $\mathrm{CaF}_{2}$ substrate is highly transparent with a transmission above $90 \%$ over a broad range of frequencies. Therefore, in the transmission plots of $\mathrm{AZO}$ on $\mathrm{CaF}_{2}$ samples, the properties of the $\mathrm{AZO}$ thin film should dominate the behaviour of the transmission curve.

Figure 8-19 shows the transmission spectra for the four nominal $\mathrm{AZO}$ on $\mathrm{CaF}_{2}$ samples from different sputtering rounds, as defined in section 8.2.2. This covers the non-transparent sample, the damaged sample, the thickest and the thinnest samples. With regards to sample placement during the sputtering process, the thickest sample was the closest to the sputtering plasma while the thinnest sample had the farthest location from the sputtering plasma.

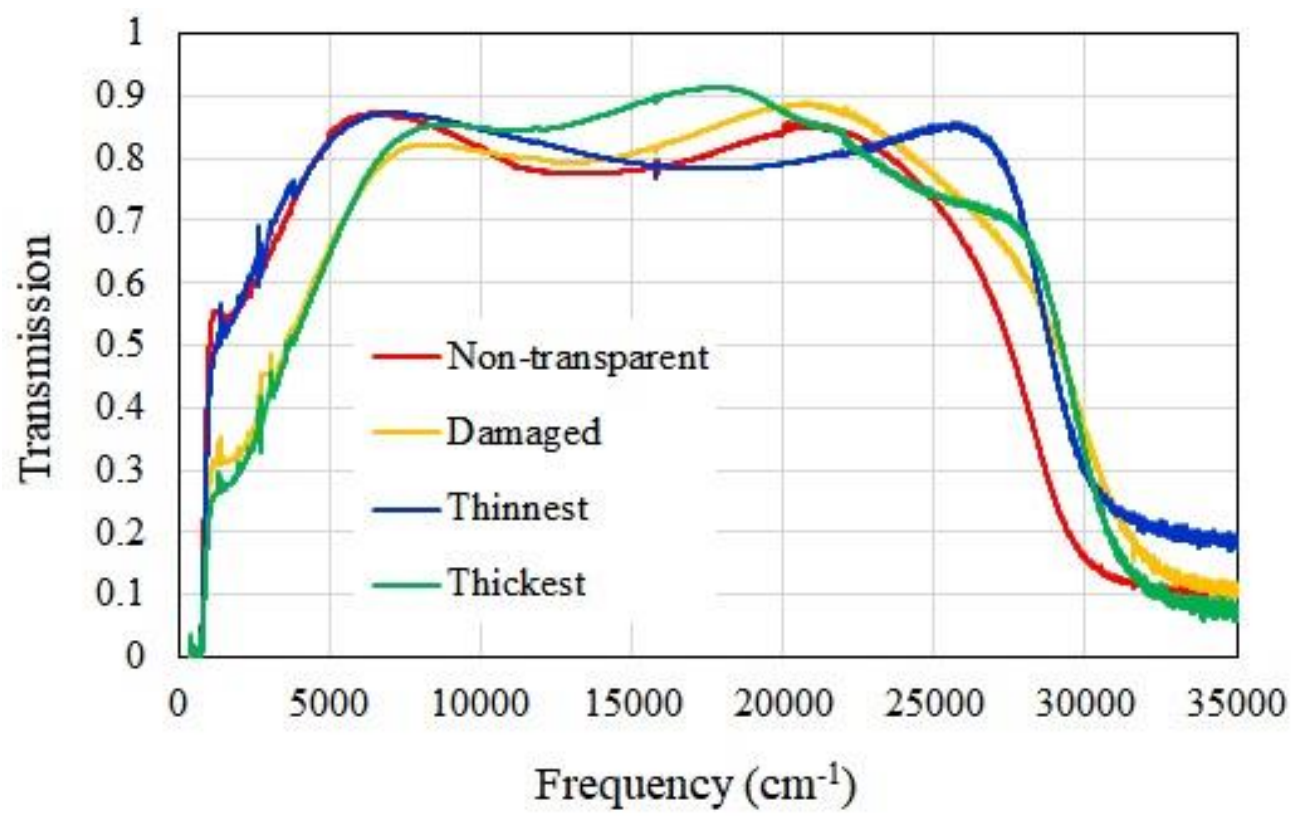

Figure 8-19 Transmission spectra for the four $\mathrm{AZO}_{\text {on }} \mathrm{CaF}_{2}$ samples. 
Despite our aim to maintain the resulting thin film properties from one sputtering round to the other via controlling the sputtering parameters, the quality of the deposited films was found to slightly vary. For the sake of simplifying the discussions in this section, we will describe the measured properties for each sample separately.

Non-transparent: this sample was found to have a film thickness of $\sim 110 \mathrm{~nm}$, as indicated by the RBS measurements. It had a grey tint when inspected under normal light, which indicated that the sample was not highly transparent in the visible region. The FTIR transmission spectrum of this sample is shown with a red solid curve in Figure 8-19. The visible tint of this sample was in agreement with the FTIR spectrum, where lower transmission was observed in the visible range. This sample showed a high MIR transmission.

Thinnest: RBS measurements indicated that this sample had a $\sim 80 \mathrm{~nm}$ thick AZO film. The FTIR transmission spectrum for this sample is shown with a blue solid curve in Figure 8-19. This relatively thin sample had one of the highest UV, NIR, and MIR transmission values amongst all $\mathrm{AZO}$ on $\mathrm{CaF}_{2}$ samples. Visually, this sample had a greenish yellow colour as light was reflected off it, a property which correlated well with the measured transmission spectrum, where transmission was lower in the corresponding visible range, starting from the end of green to orange wavelengths [450].

Damaged: this sample had a thickness of $\sim 120 \mathrm{~nm}$ as measured by the RBS technique. It notably had the highest carrier concentration amongst all our deposited $\mathrm{AZO}$ on $\mathrm{CaF}_{2}$ samples. During the FTIR measurements of this sample, 
extra care was taken to ensure aligning the light source with the non-damaged part of this sample, i.e., where there were no apparent cracks or peeling. Results indicated that this sample had one of the highest UV to visible transmission values, yet one of the lowest IR to MIR transmission values amongst the samples discussed in this section.

Thickest: RBS indicated that this sample had a thickness of $\sim 210 \mathrm{~nm}$. Optically, this sample showed a low transmission in the near UV and beginning of the visible region, as well as a low transmission at the end of the NIR towards the MIR region. Nonetheless, despite this sample being one the thickest samples in all our study, it had the highest transmission in a big portion of the visible region.

Figure 8-20 shows $(\alpha E)^{2}$ versus energy plots used to estimate the optical bandgap for these four selected samples.

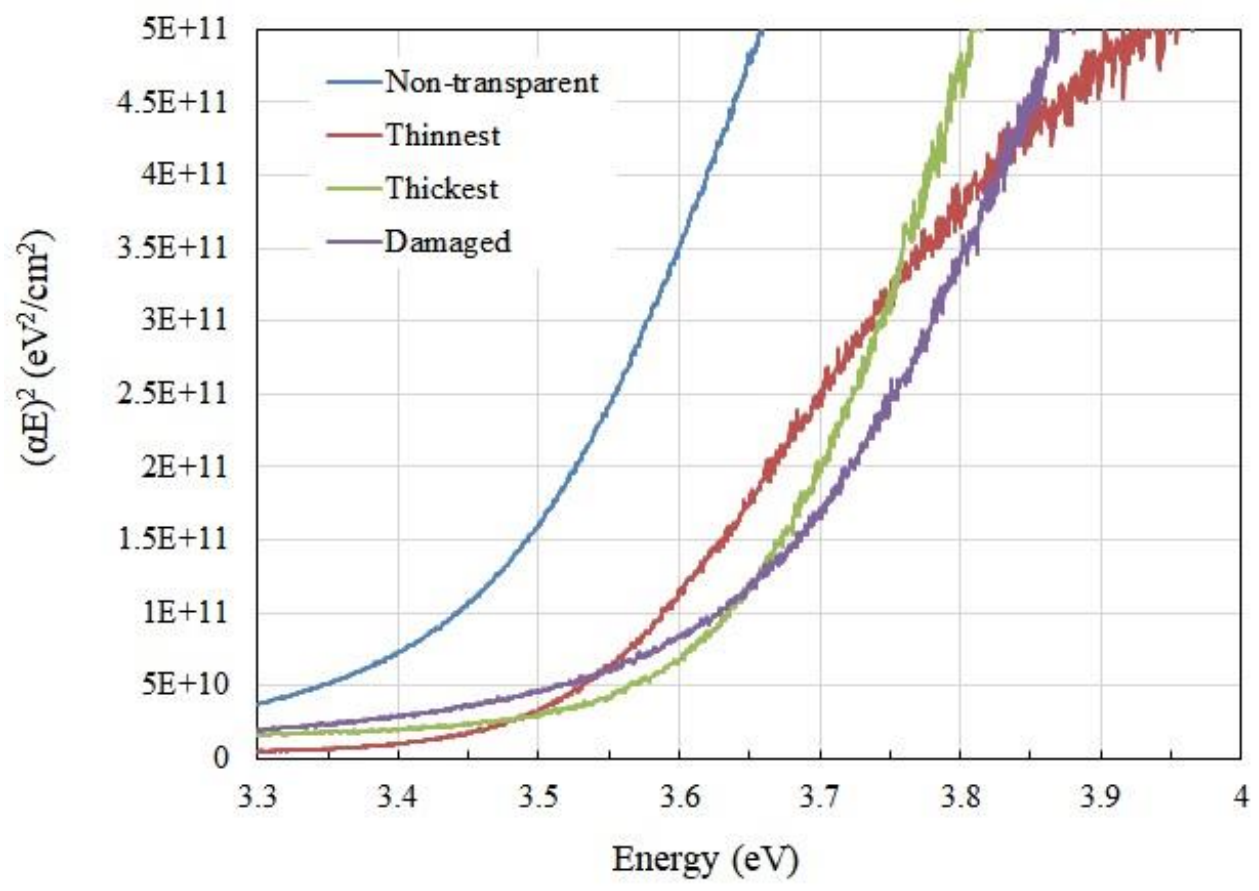

Figure 8-20 Tauc plots for the four AZO on $\mathrm{CaF}_{2}$ samples. 
Accordingly, the bandgap of each sample was estimated, where the values are summarized in Table 8-19.

Table 8-19 Estimated bandgap for the $\mathrm{AZO}$ on $\mathrm{CaF}_{2}$ samples. The carrier concentration values are supplied from section 8.4 .1 for comparison.

\begin{tabular}{|l|c|c|}
\hline Name & Bandgap (eV) & Carrier concentration $\mathbf{( \mathbf { c m } ^ { - 3 } )}$ \\
\hline Damaged & 3.63 & $(1.04 \pm 0.01) \mathrm{E}+21$ \\
\hline Thickest & 3.65 & $(2.64 \pm 0.03) \mathrm{E}+20$ \\
\hline Thinnest & 3.52 & $(1.89 \pm 0.06) \mathrm{E}+20$ \\
\hline Non-transparent & 3.44 & $(1.04 \pm 0.07) \mathrm{E}+20$ \\
\hline
\end{tabular}

These values confirmed that the carrier concertation correlated with the estimated optical bandgap for each sample, where having a higher carrier concentration resulted in a wider bandgap broadening according to the BursteinMoss (BM) effect. 


\subsubsection{AZO on Si}

Silicon is the third substrate studied in this chapter and is the only candidate that was partly transparent in the far-infrared region with a transmission of $\sim 50 \%$.

Thickness measurements using RBS for all selected AZO on Si samples are presented in Table 8-20. It is noted that during deposition, Si1set1-Si2set1 were in the first row closest to the plasma centre while Si3set1 was farther away in the second row. Si1set2 was placed at the same location of Si2set1 but in a different sputtering set. The higher thickness for Si1set2 samples was intentional as sputtering time was longer for Set2.

Table 8-20 Thickness measurements for the AZO on Si samples from Set1 and Set2 using RBS.

\begin{tabular}{|l|c|}
\hline Name & Thickness (nm) \\
\hline Si1 set1 & $110 \pm 11$ \\
\hline Si2 set1 & $130 \pm 13$ \\
\hline Si3 set1 & $110 \pm 11$ \\
\hline Si1 $1_{\text {set2 }}$ & $220 \pm 22$ \\
\hline
\end{tabular}

It is seen that samples from Set1 had comparable thin film thicknesses, with a variation of $20 \mathrm{~nm}$. The selected sample from Set2 had almost twice the thickness of Set1's samples, as expected from the longer deposition time. 
We recall from the previous sections that all our AZO on Si samples showed similar properties under different characterization techniques. Measuring the FTIR spectra for the AZO on Si samples from Set1 showed very similar transmission spectra for all three samples. Thus, Figure 8-21 only shows the transmission plot of a single sample from Set1, i.e., Si2set1, along with the spectrum of Si1set2. These two samples had the lowest and highest carrier concentration amongst the AZO on Si samples as indicated section 8.4.1, respectively. Thickness wise, AZO on Si1set2 was $\sim 90 \mathrm{~nm}$ thicker than Si2set1.

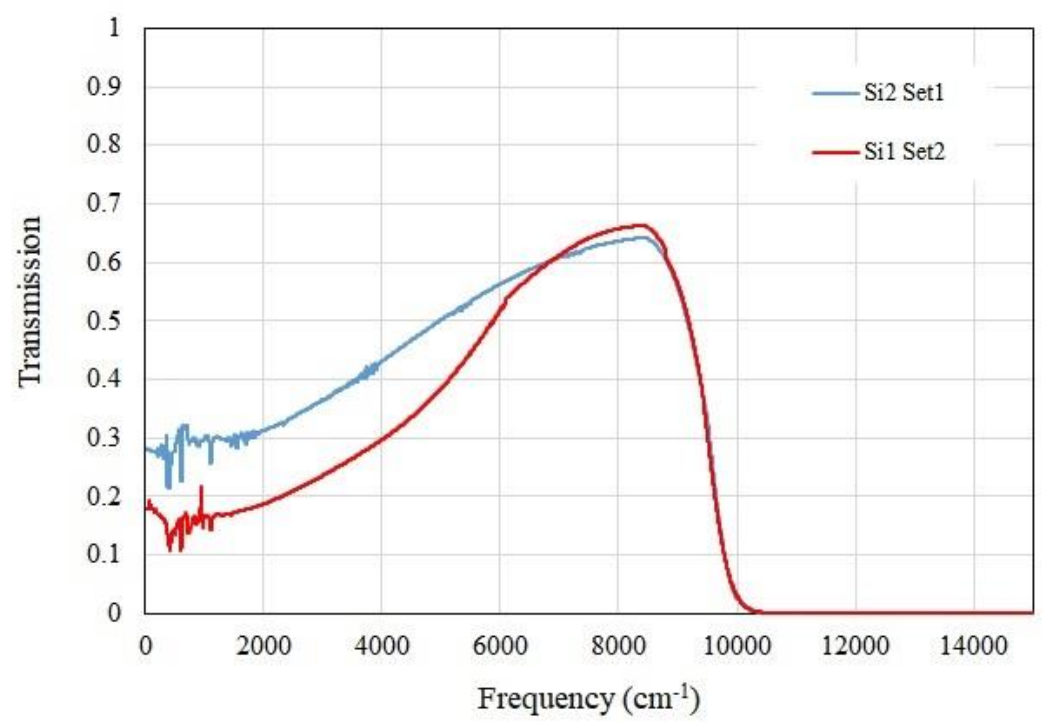

Figure 8-21 Transmission plots for the AZO thin films on Si.

These FTIR transmission spectra indicate that our AZO films are reasonably transparent in the far infrared region, where the increased thin film thickness resulted in a less transparent structure.

Bandgap calculations were not possible for the AZO samples deposited on $\mathrm{Si}$ as $\mathrm{Si}$ is not transparent for frequencies higher than $10000 \mathrm{~cm}^{-1}$. 


\subsection{Plasma frequency and dielectric permittivity of AZO}

Our plasma frequency estimation process became more accurate as we went through the fitting procedure for the AZO thin films on different substrates. As part of this process, we aimed to keep the scattering rate in the Reffit model close to the value suggested by the PMMS study, thereby making sure that all relevant measurement results were correctly represented in the fitting model.

Using the same process mentioned in Chapter 7, fitting the transmission spectra of our samples required fitting the blank substrates' transmission spectra first. Thus, for each substrate, the single layer model was used to estimate the relevant Lorentzian terms. Thereafter, these Lorentzian terms were applied in the multilayer model of the thin film-on-substrate, which results in an estimate for the thin film's plasma frequency. Similar to section 7.8, fitting the transmission data of a thin film-on-substrate was possible by using a simple Drude term for the thin film layer. Finally, adding more Lorentzian terms provided a well conforming fit to the measurements, which contributed to more accurate estimation of the dielectric permittivity.

\subsubsection{AZO on sapphire substrate}

Based on our previous fitting experience, it was found that plasma frequency values are dominantly affected by the samples' MIR transmission properties, where the absorption edge of our AZO exists. For the AZO on sapphire samples from Set1, the samples had a comparable MIR transmission response as shown 
in Figure 8-15, however, some variance in the transmission level was observed in that region. Thereby, we opted to select the most MIR transparent and least MIR transparent samples for plasma frequency estimation, as the rest of the samples from this set would have their plasma frequency values in between. These two selected samples were Sap1set1 and Sap5set1.

Figure 8-22 shows the FTIR measured transmission spectra for Sap1set1 and Sap5set1 in solid lines, while the dashed lines represent the corresponding modelled curves using the FTIR-Reffit method.

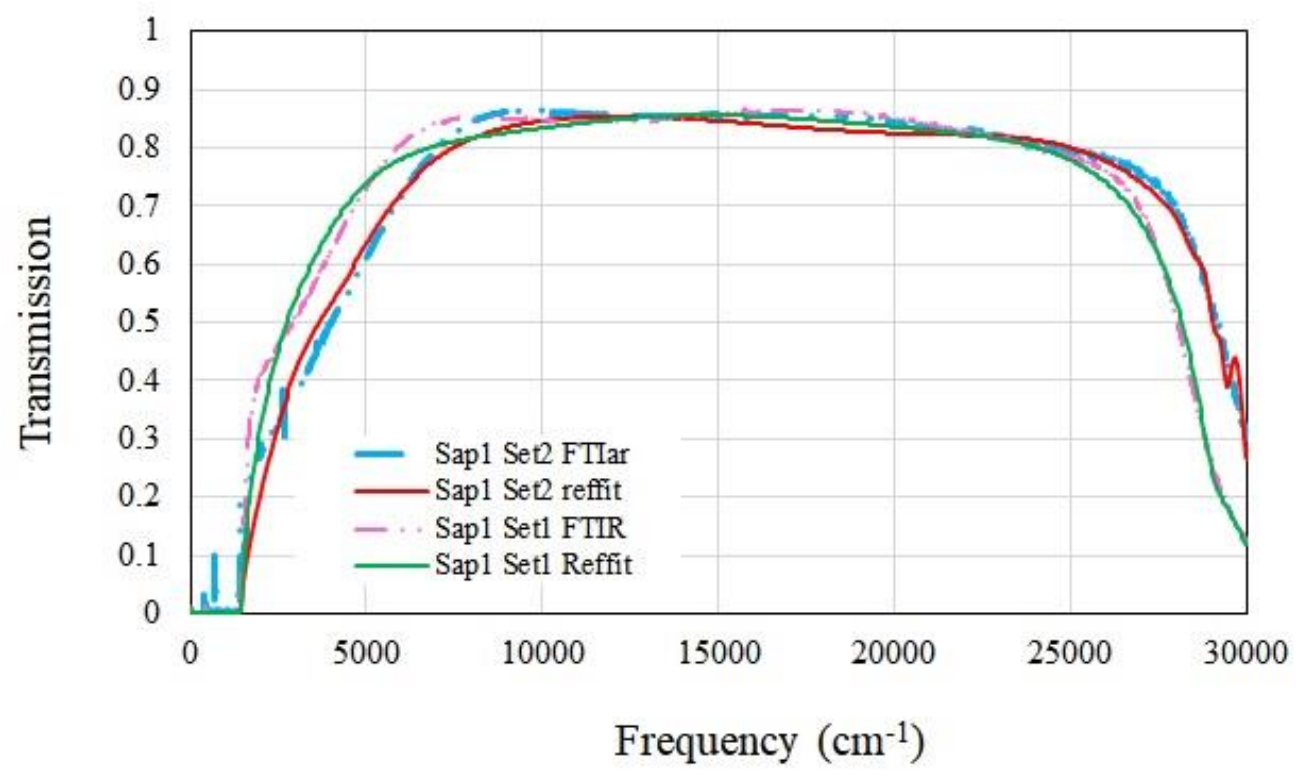

Figure 8-22 FTIR measured transmission spectra and Reffit models for two AZO on sapphire samples.

Based on the fitting results shown in Figure 8-22, the plasma frequency values were estimated. Table 8-21 shows these values for both samples. 
Table 8-21 Plasma frequency values calculated using two different methods for the AZO on sapphire samples from Set1.

\begin{tabular}{|l|c|c|c|c|}
\hline \multirow{2}{*}{ Name } & \multicolumn{3}{|c|}{ FTIR-Reffit method } & Hall effect-Drude method \\
\cline { 2 - 5 } & $G$ & $\boldsymbol{\varepsilon}_{\infty}$ & $\begin{array}{c}\text { Plasma frequency } \\
\left(\mathbf{c m}^{-1}\right)\end{array}$ & $\begin{array}{r}\text { Plasma frequency } \\
\left(\mathbf{c m}^{-\mathbf{1}}\right)\end{array}$ \\
\hline Sap1Set1 & 2000 & 1.3 & 4685 & 4686 \\
\hline Sap5Set1 & 2000 & 1.1 & 4710 & 4711 \\
\hline
\end{tabular}

These samples had the same scattering rate and had their plasma frequencies in the same infrared region. Using an effective mass of $m_{e}^{*}=0.65 m_{e}$ and the carrier concentration values from the Hall effect measurement reported in section 8.4.1, we could calculate the samples' plasma frequency via the Hall effect-Drude method. These results were found to be similar to those of the FTIR-Reffit method, as presented in Table 8-21.

Similarly, the plasma frequency values for the Sap1set2 and Sap2set2 samples are shown in Table 8-22.

Table 8-22 Plasma frequency values calculated using two different methods for the AZO on sapphire samples from Set2.

\begin{tabular}{|l|c|c|c|c|}
\hline \multirow{2}{*}{ Name } & \multicolumn{3}{|c|}{ FTIR-Reffit method } & Hall effect-Drude method \\
\cline { 2 - 5 } & $G$ & $\boldsymbol{\varepsilon}_{\infty}$ & $\begin{array}{c}\text { Plasma frequency } \\
\left(\mathbf{c m}^{-\mathbf{1}}\right)\end{array}$ & $\begin{array}{c}\text { Plasma frequency } \\
\left(\mathbf{c m}^{-\mathbf{1}}\right)\end{array}$ \\
\hline Sap15et2 & 1000 & 1.6 & 7445 & 7443 \\
\hline Sap2set2 & 1000 & 1.4 & 6590 & 6590 \\
\hline
\end{tabular}


As expected, the samples from Set 2 had a higher plasma frequency than those of Set1, which could be attributed to the higher carrier concentration and the different transmission response in the MIR region for the Set2 samples.

Using the transmission data and the plasma frequency values of our samples, we estimated the real and imaginary parts of the dielectric permittivity for Sap1set1, Sap5set1, Sap1set2 and Sap2set2. Figure 8-23 shows the modelled real and imaginary parts of the dielectric permittivity for Sap1set1 and Sap5set1.

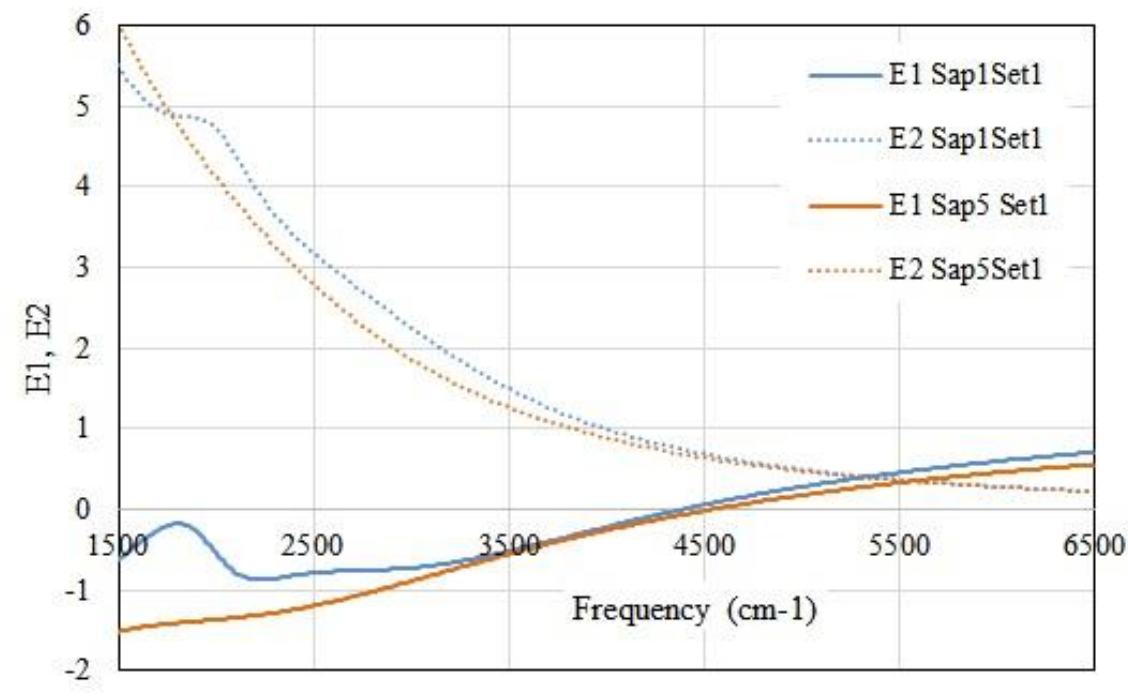

Figure 8-23 Real and imaginary parts of the dielectric permittivity for the AZO on sapphire samples from Set1.

Similarly, Figure 8-24 shows the modelled real and imaginary parts of the dielectric permittivity for Sap1set2 and Sap2set2 samples. 


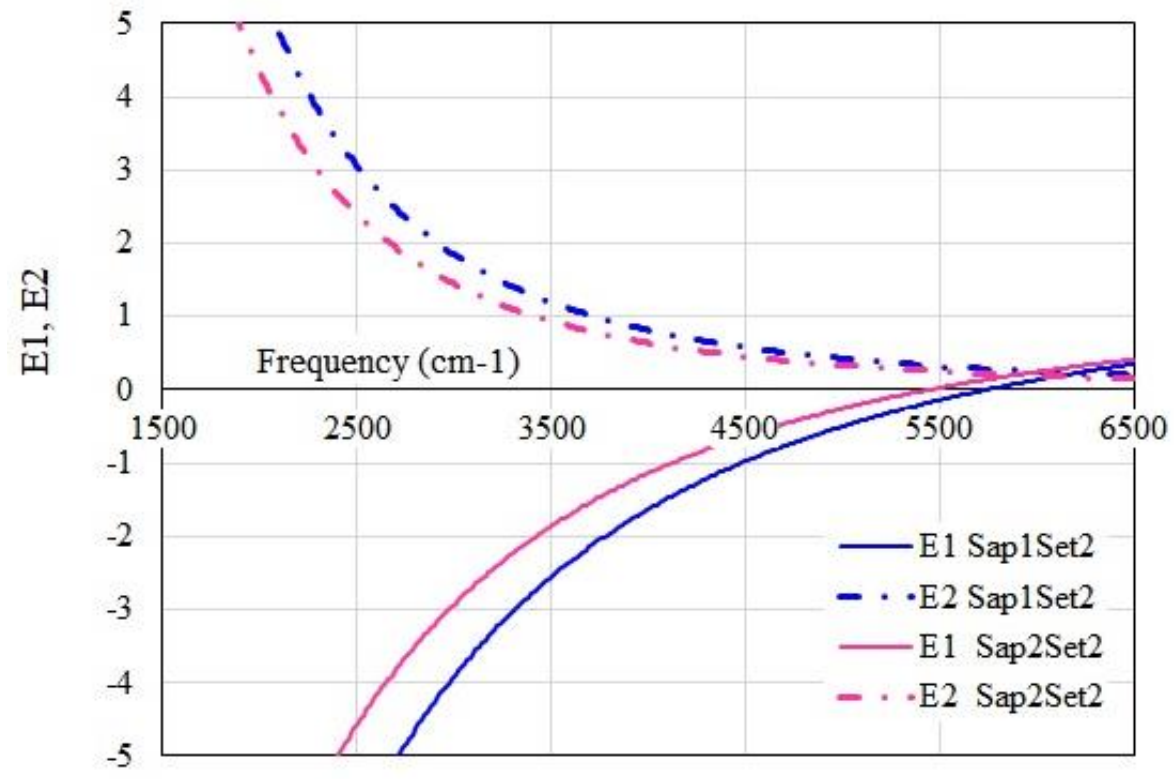

Figure 8-24 Real and imaginary parts of the dielectric permittivity for the AZO on sapphire samples from Set2.

It is seen that for both figures, the cross-over frequency where the real part of dielectric permittivity turns negative occurred in the region close to that of the estimated plasma frequency. Meanwhile, the imaginary part of dielectric permittivity was small at the cross-over frequency, which indicated low optical losses in our samples. Accordingly, these samples were found to have appropriate properties for our plasmonic application.

\subsection{2 $\mathrm{AZO}$ on $\mathrm{CaF}_{2}$ substrate}

The spectra presented for the $\mathrm{AZO}$ on $\mathrm{CaF}_{2}$ samples in Figure 8-19 shows that the damaged and the thickest samples had an almost identical response in the MIR region. Similarly, the thinnest and the non-transparent samples had identical transmission in the MIR region. Therefore, we only selected two 
samples for plasma frequency estimation, namely the thickest and thinnest samples, where the results are reported in Table 8-23.

Table 8-23 Plasma frequency values calculated using two different methods for the $\mathrm{AZO}$ on $\mathrm{CaF}_{2}$ samples.

\begin{tabular}{|c|c|c|c|c|}
\hline \multirow{2}{*}{ Name } & \multicolumn{3}{|c|}{ FTIR-Reffit method } & Hall effect-Drude method \\
\cline { 2 - 5 } & $G$ & $\boldsymbol{\varepsilon}_{\infty}$ & $\begin{array}{r}\text { Plasma } \\
\text { frequency } \\
\left(\mathbf{c m}^{-1}\right)\end{array}$ & $\begin{array}{r}\text { Plasma frequency } \\
\left(\mathbf{c m}^{-1}\right)\end{array}$ \\
\hline Thickest & 2500 & 1.6 & 6077 & 6030 \\
\hline Thinnest & 2000 & 1.3 & 5000 & 5064 \\
\hline
\end{tabular}

Plasma frequencies for both samples were estimated using the Hall effectDrude method and the FTIR-Reffit method, with both methods having similar outcomes.

Using these plasma frequency estimates, the real and imaginary parts of the dielectric permittivity for the $\mathrm{AZO}$ on $\mathrm{CaF}_{2}$ samples were modelled, as shown in Figure 8-25. 


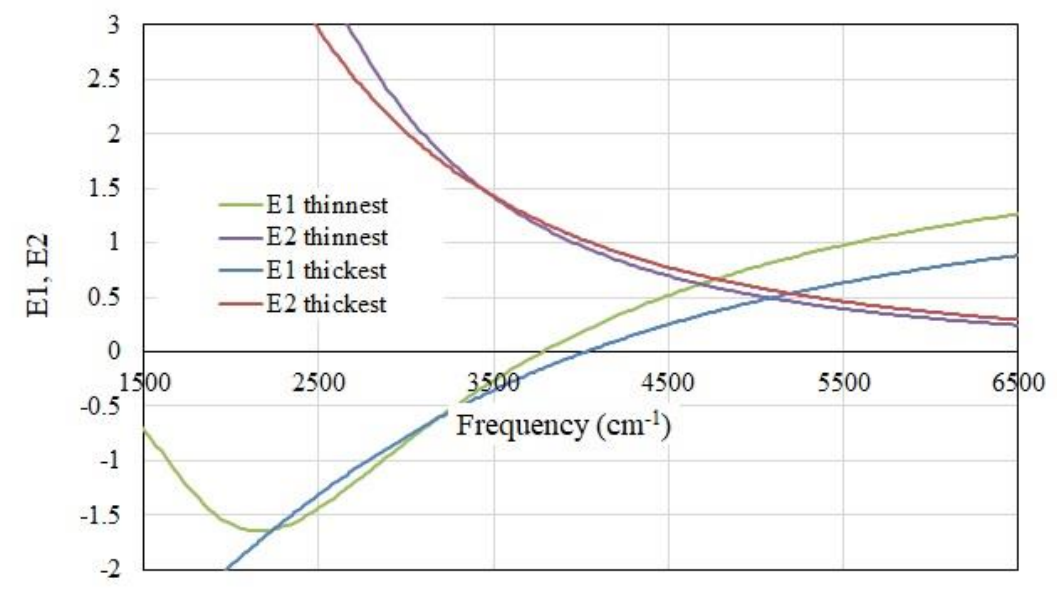

Figure 8-25 Real and imaginary parts of the dielectric permittivity for the thinnest and thickest $\mathrm{AZO}$ on $\mathrm{CaF}_{2}$ samples.

It can be seen that the crossover frequency the thickest sample was around $\sim 4000 \mathrm{~cm}^{-1}$, which was close to the plasma frequency, with the imaginary dielectric part being lower than 1 imaginary at the crossover frequency, indicating low optical losses. The thinnest sample had a crossover frequency of $\sim 3700 \mathrm{~cm}^{-1}$, with an imaginary part of the dielectric function being slightly larger than 1 at that frequency. Thus, both samples had comparable plasma frequency and dielectric properties that were suitable for MIR plasmonic applications.

\subsubsection{AZO on Si substrate}

In this section, selected $\mathrm{AZO}$ on $\mathrm{Si}$ samples had their plasma frequencies estimated using the Hall effect-Drude method and the FTIR-Reffit method. Table 8-24 shows these values, where both methods had similar outcomes. 
Table 8-24 Plasma frequency values calculated using two different methods for AZO on Si samples.

\begin{tabular}{|c|c|c|c|c|}
\hline \multirow{2}{*}{ Name } & \multicolumn{3}{|c|}{ FTIR-Reffit method } & Hall effect-Drude method \\
\cline { 2 - 5 } & $G$ & $\boldsymbol{\varepsilon}_{\infty}$ & $\begin{array}{c}\text { Plasma frequency } \\
\left(\mathbf{c m}^{-1}\right)\end{array}$ & $\begin{array}{r}\text { Plasma frequency } \\
\left(\mathbf{c m}^{-\mathbf{1}}\right)\end{array}$ \\
\hline Si2set1 & 1560 & 5.4 & 5107 & 4627 \\
\hline Si1set2 & 790 & 3.2 & 5976 & 6516 \\
\hline
\end{tabular}

These samples were found to have varying parameters, given than the Set2 sample was almost double in thickness.

Figure 8-26 shows the real and imaginary parts of the dielectric permittivity for Si2set1 and Si1set2, which were estimated using the plasma frequency values from Table 8-24. 


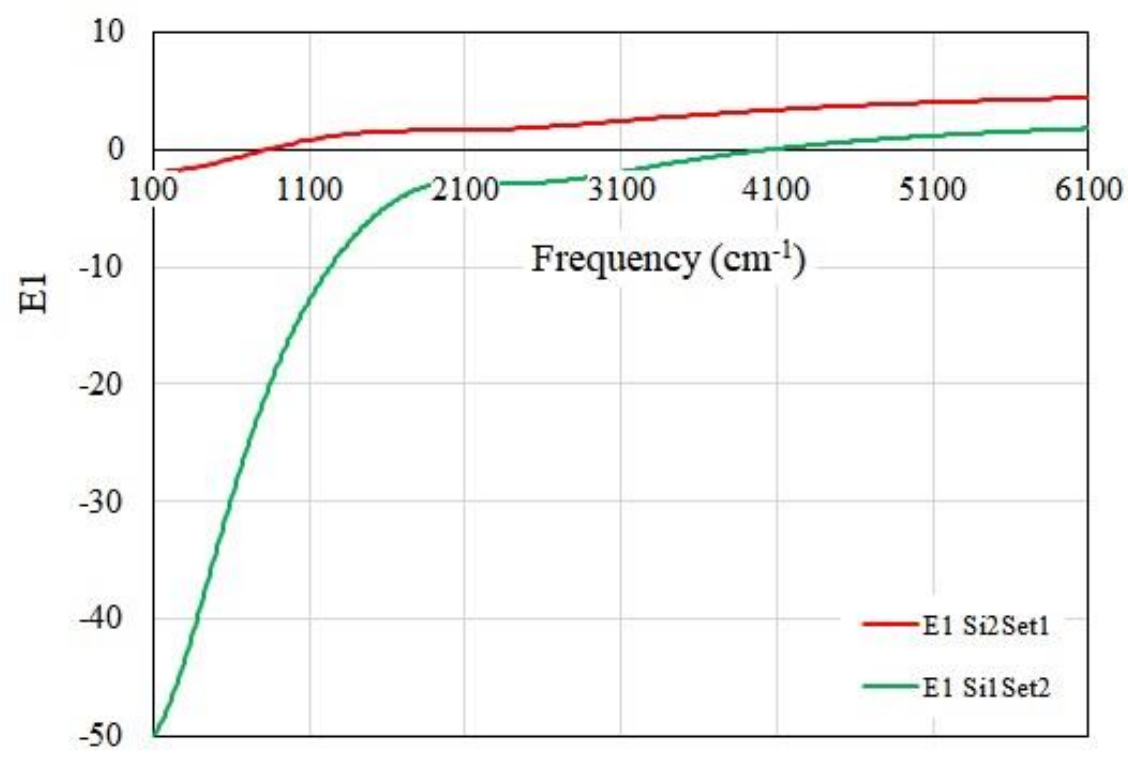

(a)

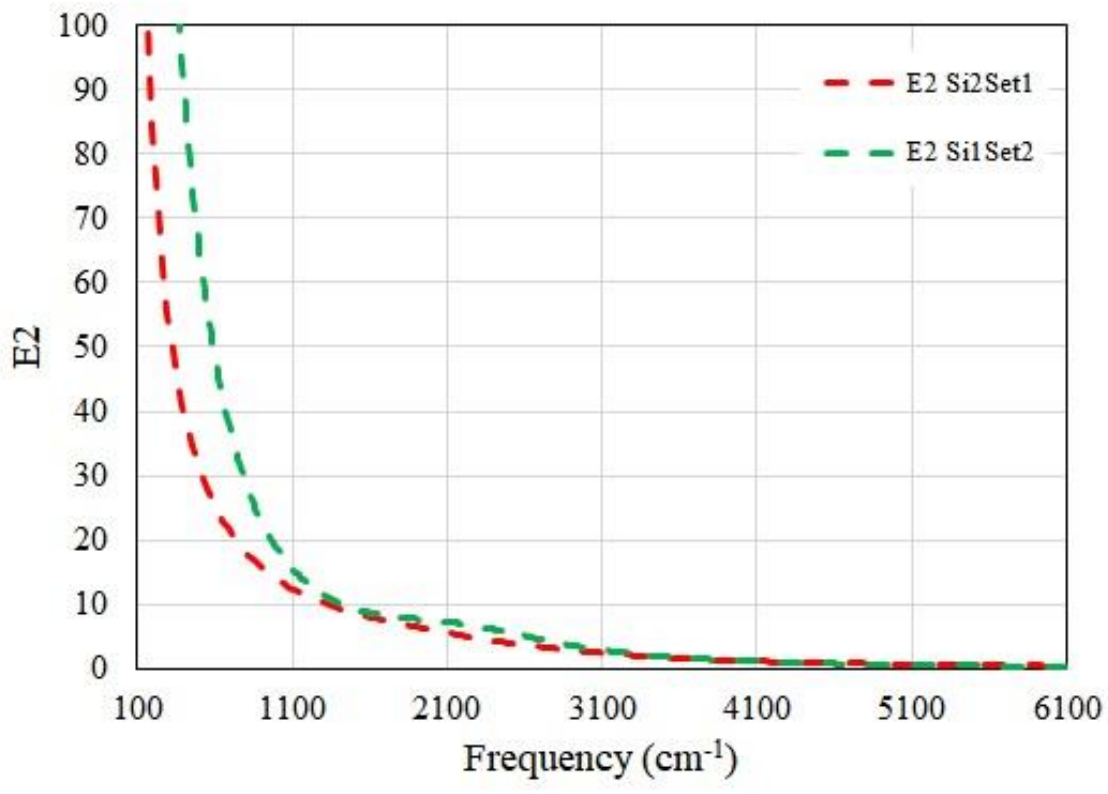

(b)

Figure 8-26 Real and imaginary parts of the dielectric permittivity for AZO on Si samples, namely, Si2set1 and Si1set2.

It can be seen that the crossover frequency for Si1set2 occurred at $\sim 4000 \mathrm{~cm}^{-1}$ and the imaginary part of the dielectric permittivity was low, indicating low losses 
near the plasma frequency. However, Si2set 1 had a crossover frequency around $1000 \mathrm{~cm}^{-1}$ (far from the plasma frequency) with a large imaginary part (high losses), both of which are not desirable for plasmonic applications.

Based on the results in this section, the FTIR transmission spectra showed that most of our films had reasonable transmission levels in the MIR of the EM spectrum, where the plasma frequency was often found. In addition, most samples had low imaginary dielectric part in that frequency range, which suggests low optical losses in the region of interest. This positive outcome confirmed that our sputtering process was applicable to all three substrates studied in this chapter, with the resulting AZO films having suitable properties for our plasmonic application in the MIR range.

\subsection{Chapter summary}

In this chapter, we investigated the optical and electrical properties of our AZO thin films deposited on three different substrates, namely (006) sapphire, (111) calcium fluoride and (100) silicon. Substrate selection was based on the desire to have a wider transparency range and a lower optical absorption. Transmission larger than $80 \%$ was observed for our $\mathrm{AZO}$ on sapphire and $\mathrm{CaF}_{2}$ samples, in the near-infrared, visible and UV regions of the electromagnetic spectrum.

The recorded SEM images showed the surfaces of the deposited AZO thin films on all three substrates. It is worth mentioning that the accurate implementation of our deposition recipe enabled us to create thin films with reproducible properties. This was particularly observed in the Hall effect 
properties of our samples, where a carrier concentration of the order of $1 \mathrm{E}+20$ and a low resistivity were achieved for most samples, regardless of the substrate.

Plasma frequency values for different samples were calculated using two independent methods. These values had a direct relation with the samples' spectral data, especially in the mid-infrared region of the spectrum. Values from the two methods were in good agreement, which validated the results.

The only limiting variable in our sputtering process was related to the sample's position with respect to the plasma centre, a shortcoming of our small RF-magnetron sputtering system, which resulted in slight variations in the properties of the deposited thin films. Our observations confirmed that thin films fabricated under the same sputtering conditions, and located close to each other during the sputtering process, tend to have a similar spectral response. A good example is the AZO on sapphire samples from section 8.5.1.

Based on the characterisation results of this chapter, our AZO samples deposited on all three substrates had reasonably similar properties that were suitable for the proposed plasmonic application. 



\section{Results C: thermal annealing effect on AZO thin films}



In this chapter, we present the characterization results for thermally annealed AZO thin films on all four substrates that were included in this study. This annealing experiment was carried out due to our interest in evaluating the impact of post-growth thermal annealing on the structural, electrical and optical properties of our thin films. In particular, we were interested in investigating the possibility of optimizing the carrier concentration and the plasma frequency of these films by such means. It is worth noting that such comprehensive annealing study on the deposited AZO thin film on four different substrates using an offcentre magnetron sputtering setup has never been reported to the best of author's knowledge and provides useful insight into the change in the properties of this class of deposited AZO thin films after annealing.

A key drive to investigate the effects of annealing on our films was based on the findings of the previous VUW study that recommended the use of a high substrate temperature during the $\mathrm{AZO}$ sputtering process to increase the transparency and the conductivity of the AZO films [253]. However, our sputtering chamber was not able to accommodate higher temperatures, as our recipe was already pushing the system to its limits. For instance, our most conductive films were deposited while the substrate temperature was held at 345 ${ }^{\circ} \mathrm{C}$. Therefore, post-growth thermal annealing in an inert environment was the next logical step for improving our fabrication process. 


\subsection{Chapter objectives}

In this chapter, the effects of thermal annealing on selected AZO samples deposited on the four different substrates are studied.

Accordingly, samples were characterized before and after each annealing step, where considered properties included resistivity, carrier concentration, optical transmission and crystal structure, as well as the dependant parameters like plasma frequency and dielectric permittivity.

Our deposited samples were stored at ambient conditions and were found to have stable properties over time. The only observed instability related to some AZO on CaF2 samples, which suffered from thin-film cracking and delamination that degraded with time.

During this study, we had access to two furnaces, both of which could thermally anneal the samples under inert conditions. The first furnace ran at normal atmospheric pressure with a pure Ar gas backfill, while the second was a vacuum furnace. Consequently, the results of three different sample groups are presented in this chapter:

- Samples annealed in Ar gas, which are provided in section 9.4.

- Samples annealed in vacuum, which are provided in section 9.4.2.

- Samples annealed in Ar first, then annealed in vacuum, denoted as "(Ar + Vac)" or "double annealed", which are provided in section 9.4.3.

Before elaborating the annealing results, it is important to note that some new samples will be introduced in this chapter for each substrate material. This was 
largely dictated by the realities of carrying the experimental work; for instance, samples had to be characterized in the four-point probe Hall effect setup then were diced in half to fit into the FTIR sample holder. This meant that once samples were characterized in the FTIR system, there was no way to conduct future Hall effect measurements on them. Furthermore, when embarking on this study, we did not anticipate the need to carry two different post-growth thermal annealing processes; however, this final outcome -of three sample groups- was driven by the developing results. In short, as our annealing runs increased, we had to introduce new samples and characterize them before and after annealing. Nonetheless, the "newly" presented samples were from the same fabrication runs as the previously presented samples, with properties on par with what was measured in the previous chapters.

\subsubsection{Annealing specifications}

Ar annealing: a high purity Ar gas flow with a constant rate of $0.5 \mathrm{~cm}^{3} / \mathrm{sec}$ was continuously present in the annealing furnace, i.e., the pre-heating stage, the main annealing process, and the cooling stage. We selected Ar as the annealing gas as it is inert and nontoxic [451]. Given that Ar is almost 1.3 times denser than air $[452,453]$, we set up the exhaust of our furnace in the upward direction, so that Ar would fill the entire chamber and no air gets trapped inside; a caution that had to be taken given that no pumps were used in this process and the pressure was maintained at $1 \mathrm{~atm}$. This annealing process lasted for a total of 6 hours, where temperature was elevated at a constant rate of $20^{\circ} \mathrm{C}$ per min from ambient to $600^{\circ} \mathrm{C}$, then held for one hour at $600^{\circ} \mathrm{C}$ and finally left to naturally cool down to ambient temperature. 
Vacuum annealing: in this process, samples were placed inside the furnace and pumped down over night to reach a pressure level less than $6 \mathrm{E}-6$ mbar. Temperature was then increased by steps of $100^{\circ} \mathrm{C}$ until the final temperature of $600{ }^{\circ} \mathrm{C}$ was reached. This annealing lasted for 30 minutes, where the process was similar to that reported in [454], which employed the same setup. Similar to the Ar annealing scenario, the samples were left to slowly cool down in vacuum, to prevent thermal shocks and to minimize introducing uncontrolled variables.

(Ar + Vac) double annealing: as the name implies, the double annealed samples were first annealed in the Ar furnace, then annealed in the vacuum furnace.

Concerning characterization, we first present the XRD and SEM analyses of samples from all three annealed groups, which are presented in sections 9.2 and 9.3, respectively.

\subsection{XRD results of annealed samples}

In this section, the measured XRD diffractograms are presented and analysed to highlight observed changes in our AZO thin film samples, which were deposited on all four considered substrates: sapphire, calcium fluoride, silicon and Borofloat33.

Based on the measured diffractograms of the post-growth annealed samples, we confirmed that the favourable $\mathrm{ZnO}(002)$ orientation remained the dominant crystal orientation for all annealed AZO thin films.

Next, we measured the FWHM for the dominant (002) peak to estimate the crystallite domain size using Scherrer's formula. In each annealing process, four 
AZO samples deposited on four different substrates were selected. Samples from chapters 7 and 8 can be identified by their names.

Table 9-1 to Table 9-4 show the FWHM and the mean crystallite domain size for samples at the original state and after vacuum annealing.

Table 9-1 Original and vacuum annealed AZO on Borofloat33 sample, namely $\mathrm{S} 7$ from Chapter 8.

\begin{tabular}{|c|c|c|}
\hline S7 & Original & Vac annealed \\
\hline FWHM $\left(^{\circ}\right)$ & 0.32 & 0.31 \\
\hline CDS (nm) & 25.9 & 26.3 \\
\hline
\end{tabular}

Table 9-2 Original and vacuum annealed AZO on sapphire sample, namely Sap2set2 from Chapter 8.

\begin{tabular}{|c|c|c|}
\hline Sap2 Set2 & Original & Vac annealed \\
\hline FWHM $\left(^{\circ}\right)$ & 0.37 & 0.36 \\
\hline CDS $(\mathrm{nm})$ & 22.4 & 23.1 \\
\hline
\end{tabular}


Table 9-3 Original and vacuum annealed $\mathrm{AZO}$ on $\mathrm{CaF}_{2}$ sample, namely thickest sample from Chapter 8.

\begin{tabular}{|c|c|c|}
\hline $\mathrm{AZO}$ on $\mathrm{CaF}_{2}$ (Thickest) & Original & Vac annealed \\
\hline $\mathrm{FWHM}\left(^{\circ}\right)$ & 0.45 & 0.42 \\
\hline $\mathrm{CDS}(\mathrm{nm})$ & 18.3 & 20.1 \\
\hline
\end{tabular}

Table 9-4 Original and vacuum annealed AZO on Si sample, namely Si2set1 from Chapter 8.

\begin{tabular}{|c|c|c|}
\hline Si2set1 & Original & Vac annealed \\
\hline FWHM $\left(^{\circ}\right)$ & 0.32 & 0.31 \\
\hline CDS (nm) & 26.0 & 26.7 \\
\hline
\end{tabular}

Thus, it can be seen that a very minor increase in the mean crystallite domain size was observed for all four substrates after vacuum annealing at $600^{\circ} \mathrm{C}$. This outcome was slightly underwhelming given that literature suggests a bigger increase in the mean crystallite domain size of $\mathrm{ZnO}$ due to thermal annealing at comparable temperatures $[455,456]$.

Next, using the technique described in section 6.2.2, the lattice constant (d) and the residual in-plane stress $(\zeta)$ were determined for our AZO thin films, before and after annealing, as shown in Table 9-5. Note that only films deposited on crystalline substrates could be included in this analysis as the technique relies on diffractogram adjustment with respect to the substrate's crystal peaks. 
Table 9-5 Variation in the lattice constant and residual in-plane stress for the vacuum annealed samples.

\begin{tabular}{|c|c|c|c|c|}
\cline { 2 - 5 } \multicolumn{1}{c|}{} & \multicolumn{2}{c|}{ Original } & \multicolumn{2}{c|}{ Vacuum Annealed } \\
\hline Name & $\mathbf{D}(\AA)$ & $\zeta(\mathbf{A P a})$ & $\mathbf{D}(\AA)$ & $\zeta$ (GPa) \\
\hline Sap25et2 & 2.611 & -0.85 & 2.602 & -0.04 \\
\hline Si2set1 & 2.606 & -0.40 & 2.601 & 0.04 \\
\hline
\end{tabular}

It can be seen that on both substrates, our AZO thin films were relieved from the residual stress by means of annealing, as was expected based on the reported literature $[456,455,253]$.

Next, the Ar annealed and the double annealed $(\mathrm{Ar}+\mathrm{Vac})$ samples on all four substrates were characterized. All samples except for that with a calcium fluoride substrate were already featured in chapters 7 and 8 . This sample was prepared in the same set as the "thickest" $\mathrm{AZO}$ on $\mathrm{CaF}_{2}$ sample, presented in chapter 8, with the main difference being its lower thickness measured at $145 \mathrm{~nm}$. This sample is referred to as $\mathrm{CaF}_{2}-\mathrm{B}$.

Table 9-6 to Table 9-9 show the calculated FWHM and mean crystallite domain size for these samples, before annealing and after each annealing step. 
Table 9-6 Ar annealed and (Ar + Vac) double annealed AZO on Borofloat33 sample, namely 59 from Chapter 7.

\begin{tabular}{|c|c|c|c|}
\hline S9 & Original & Ar annealed & Double annealed \\
\hline FWHM $\left(^{\circ}\right)$ & 0.30 & 0.28 & 0.28 \\
\hline CDS (nm) & 27.5 & 30.0 & 30.3 \\
\hline
\end{tabular}

Table 9-7 Original, Ar annealed and (Ar + Vac) double annealed AZO on sapphire sample, namely Sap1set2 from Chapter 8.

\begin{tabular}{|c|c|c|c|}
\hline Sap1 $1_{\text {set2 }}$ & Original & Ar annealed & Double annealed \\
\hline FWHM $\left(^{\circ}\right)$ & 0.39 & 0.34 & 0.33 \\
\hline CDS (nm) & 21.4 & 24.2 & 25.1 \\
\hline
\end{tabular}

Table 9-8 Original, Ar annealed and (Ar + Vac) double annealed AZO on $\mathrm{CaF}_{2}$ sample, namely $\mathrm{CaF}_{2}-\mathrm{B}$.

\begin{tabular}{|c|c|c|c|}
\hline $\mathrm{CaF}_{2}-B$ & Original & Ar annealed & Double annealed \\
\hline FWHM $\left(^{\circ}\right)$ & 0.39 & 0.39 & 0.38 \\
\hline CDS $(\mathrm{nm})$ & 21.1 & 21.1 & 21.9 \\
\hline
\end{tabular}


Table 9-9 Original, Ar annealed and (Ar + Vac) double annealed AZO on Si sample, namely Si1set2 from Chapter 8.

\begin{tabular}{|c|c|c|c|}
\hline Si1set2 & Original & Ar annealed & Double annealed \\
\hline FWHM $\left(^{\circ}\right)$ & 0.35 & 0.33 & 0.32 \\
\hline CDS (nm) & 23.9 & 25.3 & 26.0 \\
\hline
\end{tabular}

Comparing the FWHM of the original samples with their annealed versions shows that annealing results in reduced FWHM and increased mean crystallite size for all samples. Differences in the AZO thin film properties between the Ar annealing and the double annealing steps were not significant, with a minor improvement in the crystallite domain size.

Annealing induced changes in the lattice constant (d) and the in-plane residual stress for these AZO thin films are presented in Table 9-10.

Table 9-10 Lattice constant and residual in-plane stress variation for the Ar annealed and the double annealed samples

\begin{tabular}{|c|c|c|c|c|c|c|}
\hline & \multicolumn{2}{|c|}{ Original } & \multicolumn{2}{|c|}{ Ar annealed } & \multicolumn{2}{|c|}{$(\mathrm{Ar}+\mathrm{Vac})$ annealed } \\
\hline Name & d $(\AA)$ & $\zeta(\mathrm{GPa})$ & d $(\AA)$ & $\zeta(\mathrm{GPa})$ & d (Å) & $\zeta(\mathrm{GPa})$ \\
\hline Sap1set2 & 2.608 & -0.58 & 2.602 & -0.04 & 2.602 & -0.04 \\
\hline Si1 ${ }_{\text {Set2 }}$ & 2.610 & -0.76 & 2.601 & 0.04 & 2.601 & 0.04 \\
\hline
\end{tabular}


Once again, it was confirmed that either thermal annealing steps (Ar or vacuum) resulted in stress-free AZO films on both substrates.

\subsection{SEM results of annealed samples}

\subsubsection{Ar annealed samples}

Figure 9-1 shows the SEM image of one of our Ar annealed AZO on a sapphire samples. As can be seen, the image is not sharp due to charging effects. Charging was observed in all SEM images of our Ar annealed samples, which caused drifting in while imaging due to relatively high resistive path to electrical ground. To decrease this effect a faster acquisition rate was used at the expense of having less sharp images.

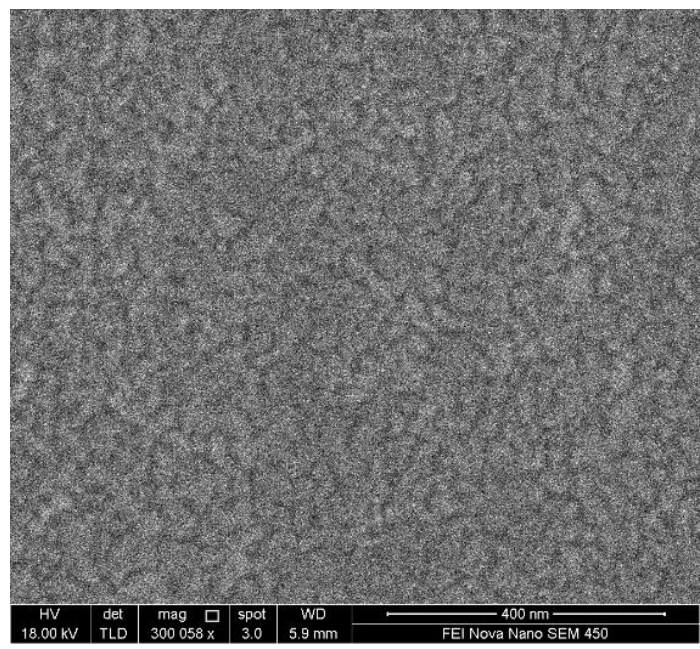

Figure 9-1 SEM image of an Ar annealed AZO on sapphire sample.

To judge the quality of the image against a reference, Figure 9-2 shows an identical SEM image of the original sample before Ar annealing was applied. 


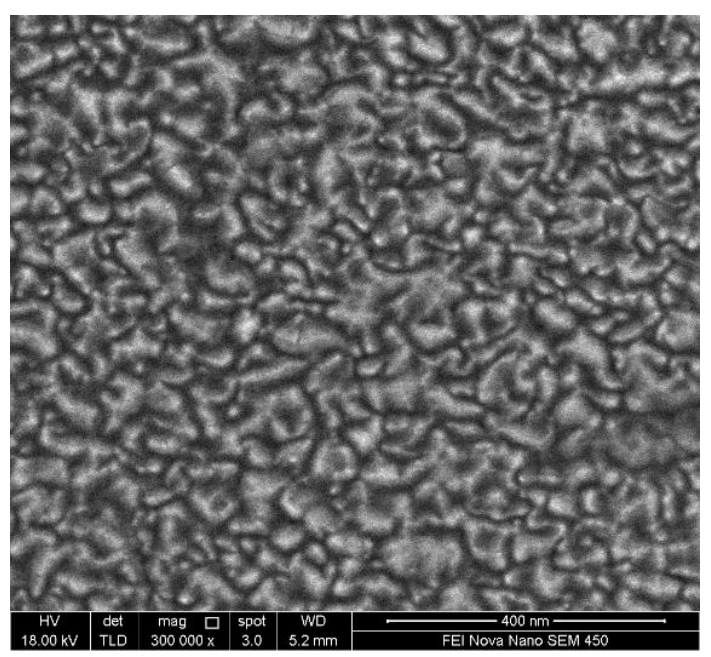

Figure 9-2 SEM image of an AZO on sapphire sample before annealing was applied.

It is evident that the SEM image quality degraded after Ar annealing, which can indicate a higher thin film resistivity. This was later confirmed in our electrical characterization results, which will be discussed in later sections.

\subsubsection{Vacuum annealed samples}

The SEM image of an AZO on Si sample (Si2set1) after vacuum annealing is shown in Figure 9-3. Figure 8-6 shows the before annealing image of the same sample. Contrary to the case of $\mathrm{Ar}$ annealing, samples that were vacuum annealed did not seem to suffer from charging effects during SEM imaging. 


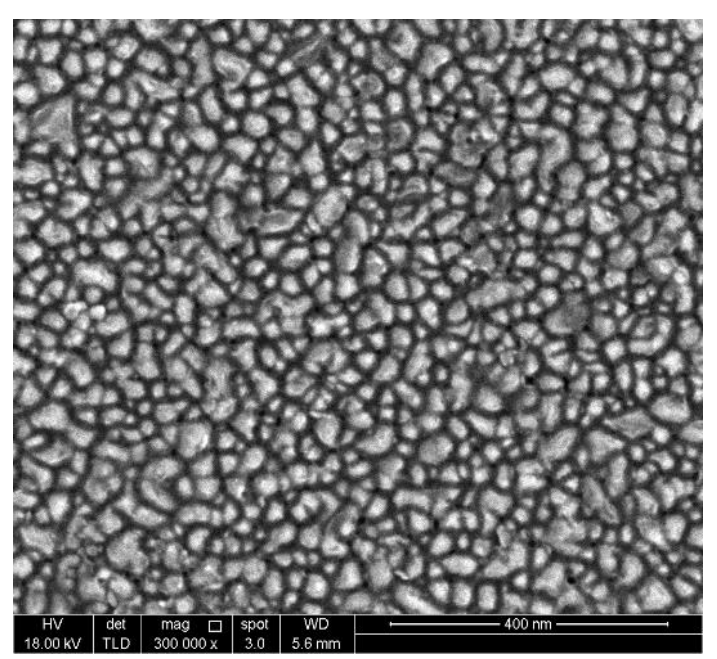

Figure 9-3 SEM image of the vacuum annealed AZO on Si sample, namely Si2set1.

It is noted that the grain shape and size before and after annealing did not seem to change significantly. Image processing using ImageJ confirmed this outcome as the mean grain diameter was found to be $44.75 \pm 4.47 \mathrm{~nm}$ for the original Si2set1 sample, while the vacuum annealed sample had a mean grain diameter of $46.79 \pm 4.67 \mathrm{~nm}$. Similar results were obtained for samples having other substrates.

\subsubsection{Double annealed samples}

Figure 9-4 shows the SEM image of an AZO on sapphire sample after the double annealing process. This was the same sample presented in Figure 9-2 (original state) and Figure 9-1 (Ar annealed). The scale was identical in all three images. 


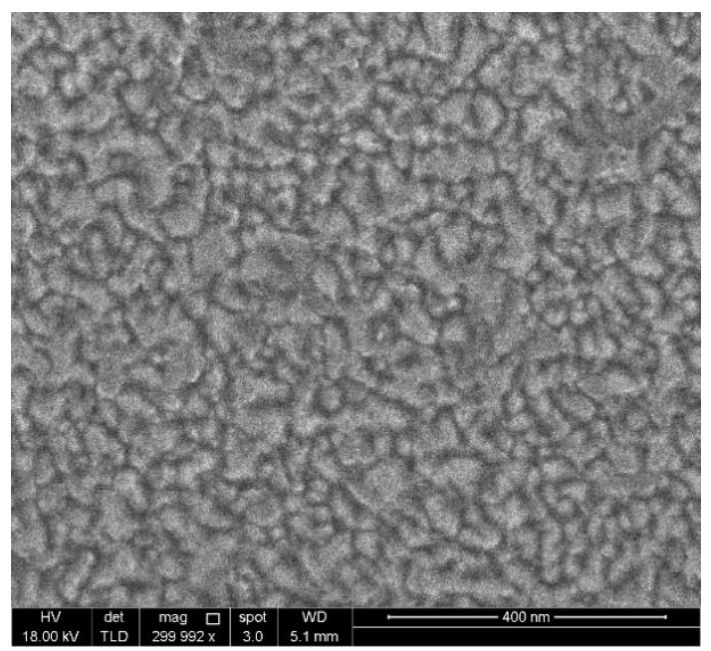

Figure 9-4 AZO on sapphire sample after the double annealing step.

Compared to the Ar annealed state, this sample seemed to be causing less charging while imaging under the same conditions in the SEM; thus, Figure 9-4 appears to be relatively sharper. This suggested that the AZO thin film regained some of its conductivity after the vacuum annealing step.

Using ImageJ, image processing was used to estimate the mean grain size for this sample at each state, as shown in Figure 9-2, Figure 9-1 and Figure 9-4. These results are presented in Table 9-11 that shows an increase in the mean grain diameter due to thermal annealing.

Table 9-11 Changes in the mean grain diameter for the AZO on sapphire sample due to thermal annealing.

\begin{tabular}{|l|c|}
\hline Name & Mean grain diameter $(\mathbf{n m})$ \\
\hline Original & $39.34 \pm 3.93$ \\
\hline Ar anneal & $45.29 \pm 4.52$ \\
\hline Double anneal & $47.26 \pm 4.72$ \\
\hline
\end{tabular}


This section provided a visual comparison between the as-sputtered (original) samples and the thermally annealed samples. These SEM images showed uniform film morphology with consistent grain shapes. A slight grain growth was observed in the annealed samples, which is consistent with our XRD results.

Literature suggests conflicting outcomes with regards to the effects of thermal annealing on the carrier concentration of AZO thin films, i.e., possible increase $[457,458]$ or decrease [459], possibly depending on the annealing conditions. In sections 9.4 to 9.4 .3 , we present the results regarding the optical and electrical properties of our annealed AZO samples using FTIR spectroscopy and Hall effect measurements.

\subsection{Optical and electrical properties}

\subsubsection{Ar annealed samples}

The effects of Ar annealing on the optical and electrical properties of selected AZO samples deposited on different substrates are presented in this section.

\section{AZO on Borofloat33}

The FTIR spectra for an AZO on Borofloat33 sample, namely S9 from Chapter 7, before and after Ar annealing are represented in Figure 9-5. 


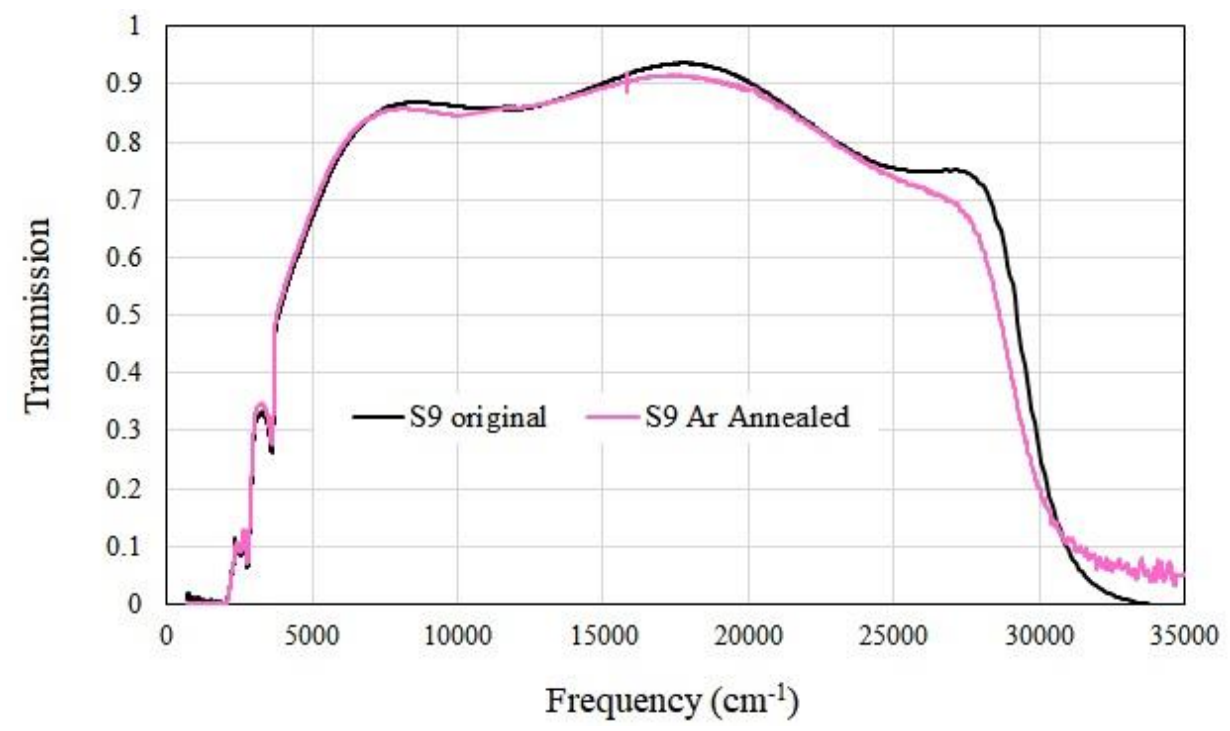

Figure 9-5 Transmission spectra for S9 before and after Ar annealing.

In Figure 9-5, the black curve shows the original S9 transmission in the assputtered state, while the pink curve shows the transmission spectrum after Ar annealing. Both transmission spectra almost overlap in the regions below $\sim 15000$ $\mathrm{cm}^{-1}$. For our AZO thin material, an identical transmission curve in MIR region might indicate that the two sample states had similar plasma frequency values. However, in this case, this assumption is not valid given that the high absorption in Borofloat33 substrate is a limiting factor for such estimation. With that being said, the modelled plasma frequency values for both states are presented in Table 9-12. 
Table 9-12 Plasma frequency before and after annealing using the FTIRReffit method.

\begin{tabular}{|c|c|c|c|c|}
\cline { 2 - 5 } \multicolumn{1}{c|}{} & \multicolumn{2}{c|}{ Original } & \multicolumn{2}{c|}{ Ar annealed } \\
\hline Name & $\mathrm{G}\left(\mathrm{cm}^{-1}\right)$ & $\omega_{p}\left(\mathrm{~cm}^{-1}\right)$ & $\mathrm{G}\left(\mathrm{cm}^{-1}\right)$ & $\omega_{p}\left(\mathrm{~cm}^{-1}\right)$ \\
\hline S9 & 3000 & 5980 & 3000 & 5441 \\
\hline
\end{tabular}

The after Ar annealing plasma frequency had a lower value, which indicated a red shift in the plasmonic region and a decrease in the carrier concentration.

We also noted that the spectra in Figure 9-5 showed a different response in the UV region, with the original sample having a higher transmission that extended further to higher frequencies. This indicated a decrease in the sample's optical bandgap and the carrier concentration after annealing. Table 9-13 shows the measured resistivity and carrier concentration for $\mathrm{S} 9$ before and after annealing.

Table 9-13 Carrier concentration (n) and resistivity $(\rho)$ from Hall measurements for original and after Ar annealing S9.

\begin{tabular}{|l|l|l|l|l|}
\cline { 2 - 5 } \multicolumn{1}{c|}{} & \multicolumn{2}{c|}{ Original } & \multicolumn{2}{c|}{ Ar Annealed } \\
\hline Name & $\rho(\Omega . \mathrm{cm})$ & $n\left(\mathrm{~cm}^{-3}\right)$ & $\rho(\Omega . \mathrm{cm})$ & $n\left(\mathrm{~cm}^{-3}\right)$ \\
\hline S9 & $2.75 \mathrm{E}-03$ & $2.60 \mathrm{E}+20$ & $4.52 \mathrm{E}-02$ & $5.02 \mathrm{E}+18$ \\
\hline
\end{tabular}


These measurements confirmed our FTIR transmission observations, as the Ar annealing step resulted in the sample becoming more resistive (an order of magnitude) with a lower carrier concentration (two orders of magnitude).

Furthermore, bandgap changes were calculated using Tauc plots, where these changes were in agreement with the decreased carrier concentration (the Burstein-Moss effect) as the sample's bandgap dropped from $3.75 \mathrm{eV}$ to $3.60 \mathrm{eV}$.

Obviously, this Ar annealing step resulted in degraded sample properties with respect to our target application.

\section{$\mathrm{AZO}$ on $\mathrm{CaF}_{2}$}

The FTIR measured spectra for two AZO on $\mathrm{CaF}_{2}$ samples before and after $\mathrm{Ar}$ annealing are presented in Figure 9-6.

$\mathrm{CaF}_{2}-\mathrm{A}$ and $\mathrm{B}$ were deposited in the same sputtering run as that of the "thickest" AZO on $\mathrm{CaF}_{2}$. They had a lower thickness than the thickest sample but their original optical transmission properties and carrier concentration levels were similar. 


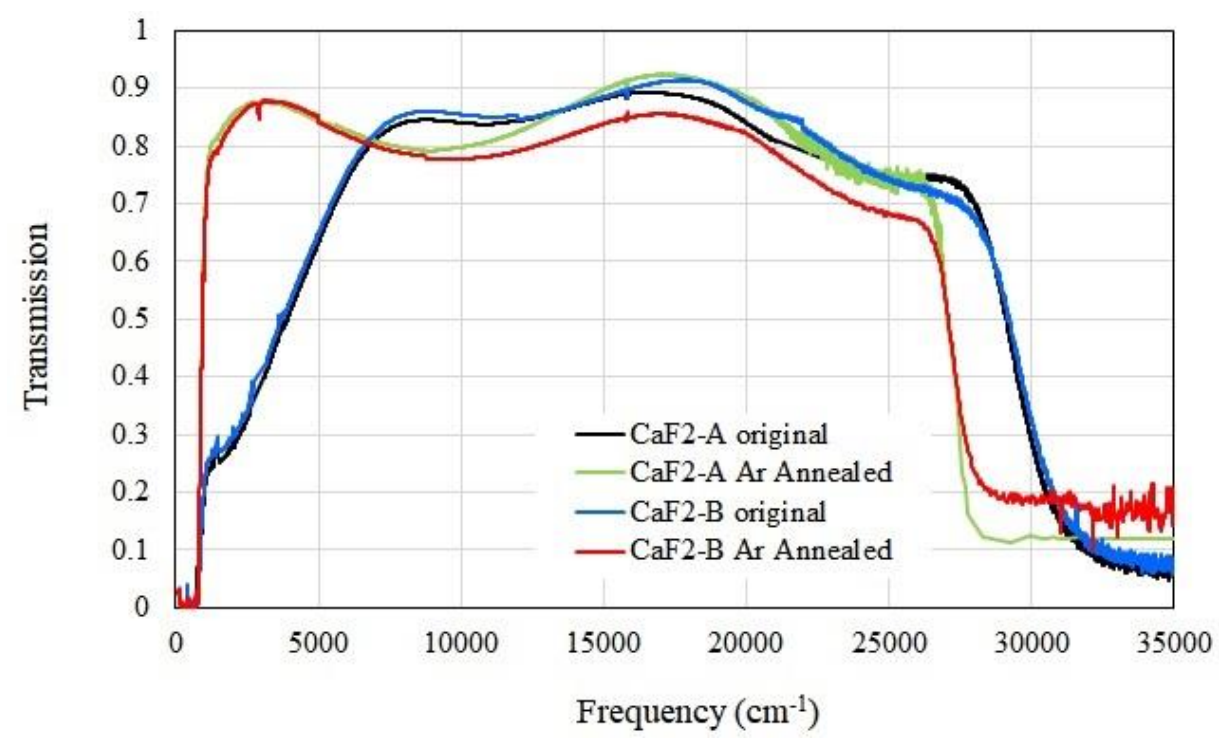

Figure 9-6 Transmission spectra for $\mathrm{CaF}_{2}-\mathrm{A}$ and $\mathrm{CaF}_{2}-\mathrm{B}$ before and after $\mathrm{Ar}$ annealing.

Figure 9-6 shows that the transmission response of both samples was chiefly affected in the IR region below $10000 \mathrm{~cm}^{-1}$ and in the UV region above $27000 \mathrm{~cm}^{-1}$ due to annealing. Thus, transmission increased from $\sim 0.3$ to $\sim 0.9$ in the mid-tofar IR region, while the samples became less transparent in the near UV region. These results resonated with what was reported for the Borofloat33 samples in the previous subsection, i.e., indicated a lower carrier concentration and a higher plasma frequency. The original and after annealing resistivity and carrier concentration for these two samples are shown in Table 9-14. 
Table 9-14 Original and after Ar annealing resistivity $(\rho)$ and carrier concentration (n) for $\mathrm{CaF}_{2}-\mathrm{A}$ and $\mathrm{B}$.

\begin{tabular}{|c|l|l|l|l|}
\hline \multirow{2}{*}{ Name } & \multicolumn{2}{|c|}{ Original } & \multicolumn{2}{c|}{ Ar Annealed } \\
\cline { 2 - 5 } & $\rho(\Omega . \mathrm{cm})$ & $n\left(\mathrm{~cm}^{-3}\right)$ & $\rho(\Omega . \mathrm{cm})$ & $n\left(\mathrm{~cm}^{-3}\right)$ \\
\hline$C a F 2-A$ & $2.45 \mathrm{E}-03$ & $4.36 \mathrm{E}+20$ & $2.70 \mathrm{E}-01$ & $2.49 \mathrm{E}+19$ \\
\hline$C a F 2-B$ & $2.62 \mathrm{E}-03$ & $2.76 \mathrm{E}+20$ & $6.75 \mathrm{E}-01$ & $6.24 \mathrm{E}+17$ \\
\hline
\end{tabular}

It is seen that despite both samples having a similar original carrier concentration of the order of $1 \mathrm{E}+20$, annealing resulted in significantly more decreased carrier concentration for the $\mathrm{CaF}_{2}-\mathrm{B}$ sample with a drop of 3 orders of magnitude, whereas the $\mathrm{CaF}_{2}-\mathrm{A}$ sample had a drop of one order of magnitude. Resistivity for both samples on the other hand, decreased by two orders of magnitude due to Ar annealing, with the B sample turning slightly more resistive (worse).

The calculated plasma frequency for the original and Ar annealed AZO on $\mathrm{CaF}_{2}$ samples are provided in Table 9-15.

Table 9-15 Plasma frequency before and after Ar annealing using the FTIR-Reffit method.

\begin{tabular}{|c|c|c|c|c|c|c|}
\hline \multirow{2}{*}{ Name } & \multicolumn{3}{|c|}{ Original } & \multicolumn{3}{c|}{ Ar annealed } \\
\cline { 2 - 7 } & $\omega_{p}\left(\mathrm{~cm}^{-1}\right)$ & $G\left(\mathrm{~cm}^{-1}\right)$ & $\varepsilon_{\infty}$ & $\omega_{p}\left(\mathrm{~cm}^{-1}\right)$ & $\mathrm{G}\left(\mathrm{cm}^{-1}\right)$ & $\varepsilon_{\infty}$ \\
\hline $\mathrm{CaF} F_{2}-A$ & 7751 & 1500 & 1.7 & 1851 & 1500 & 1.7 \\
\hline $\mathrm{CaF}_{2}-B$ & 6162 & 1800 & 1.2 & 293 & 1800 & 1.2 \\
\hline
\end{tabular}


As expected, thermal annealing in Ar resulted in decreased plasma frequency for both samples with a shift to the FIR region. The $\mathrm{CaF}_{2}-\mathrm{B}$ sample had a bigger shift in the plasma frequency, which agrees with the larger drop in its carrier concentration.

Using Tauc plots, the bandgap for both samples were estimated. Table 9-16 shows these results, where the bandgap dropped for both samples due to the Ar annealing, which also agreed with the carrier concentration change.

Table 9-16 Estimated optical bandgap for original and after Ar annealing $\mathrm{AZO}$ on $\mathrm{CaF}_{2}$.

\begin{tabular}{|c|c|}
\hline Name & Bandgap (eV) \\
\hline$C a F_{2}-A$ original & 3.65 \\
\hline$C a F_{2}-A$ Ar annealed & 3.38 \\
\hline$C a F_{2}-B$ original & 3.67 \\
\hline$C a F_{2}-B$ Ar annealed & 3.32 \\
\hline
\end{tabular}

\section{AZO on Si}

The before and after annealing FTIR spectra for the Si1set2 sample from Chapter 8 are shown in Figure 9-7. 


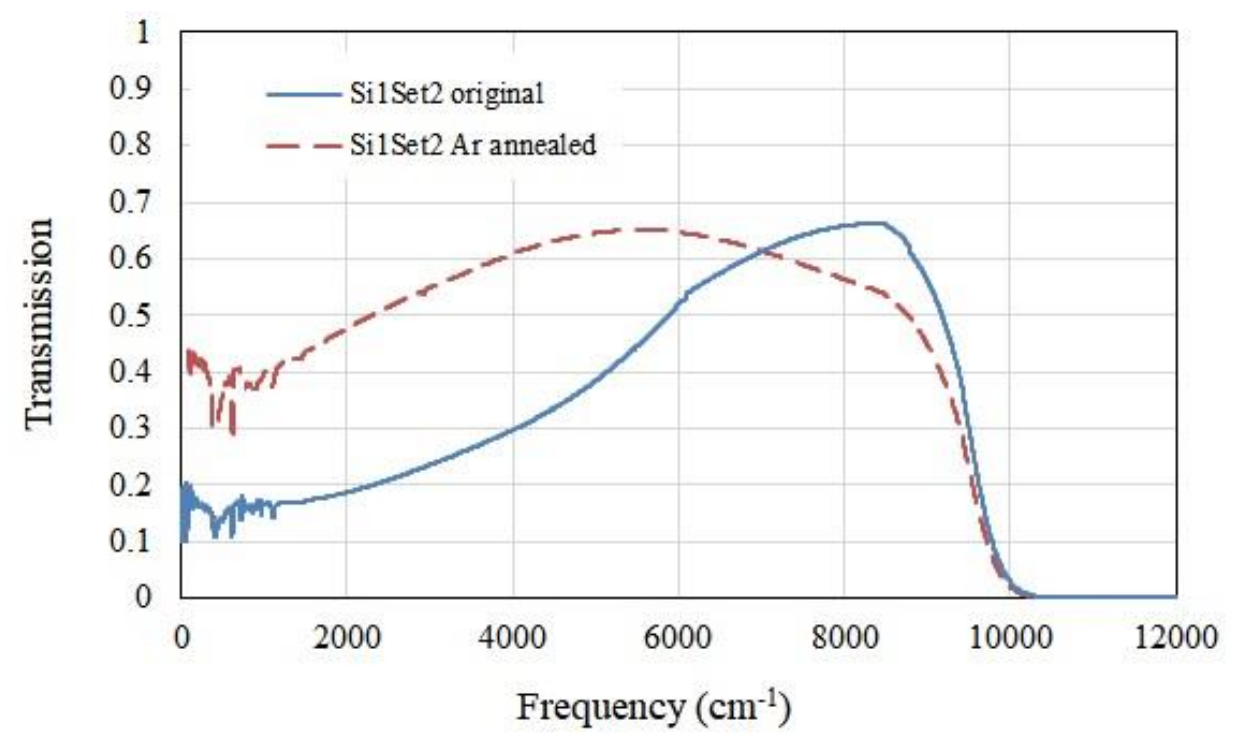

Figure 9-7 Transmission spectra for Si1set2 before and after Ar annealing.

Similar to observations on the other substrates, thermal annealing resulted in a red shift in the transmission spectrum of the sample, which can indicate a longer plasma wavelength and a lower carrier concentration. In addition, the sample became twice as transparent in the FIR region.

Table 9-18 presents the original and after annealing resistivity and carrier concentration for this sample.

Table 9-17 Plasma frequency before and after Ar annealing using the FTIR-Reffit method for Si1set2.

\begin{tabular}{|c|c|c|c|c|c|c|}
\hline \multirow{2}{*}{ Name } & \multicolumn{3}{|c|}{ Original } & \multicolumn{3}{c|}{ Ar annealed } \\
\cline { 2 - 7 } & $\omega_{\mathrm{p}}\left(\mathrm{cm}^{-1}\right)$ & $\mathrm{G}\left(\mathrm{cm}^{-1}\right)$ & $\varepsilon_{\infty}$ & $\omega_{\mathrm{p}}\left(\mathrm{cm}^{-1}\right)$ & $\mathrm{G}\left(\mathrm{cm}^{-1}\right)$ & $\varepsilon_{\infty}$ \\
\hline Si1 Set2 & 5976 & 790 & 3.2 & 2993 & 1969 & 4.5 \\
\hline
\end{tabular}


Accordingly, the plasma frequency decreased due to Ar annealing, a similar result to what was observed on other substrates. Finally, changes in the sample's carrier concentration and resistivity are summarized in Table 9-18, which indicated that resistivity increased by 3 orders of magnitude while carrier concentration decreased by two orders of magnitude.

Table 9-18 Original and after Ar annealing resistivity ( $\rho)$ and carrier concentration (n) for the AZO on Si sample.

\begin{tabular}{|c|c|c|c|c|}
\hline \multirow{3}{*}{ Name } & \multicolumn{2}{|c|}{ Original } & \multicolumn{2}{c|}{ Ar Annealed } \\
\cline { 2 - 5 } & $\rho(\Omega . \mathrm{cm})$ & $n\left(\mathrm{~cm}^{-3}\right)$ & $\rho(\Omega . \mathrm{cm})$ & $n\left(\mathrm{~cm}^{-3}\right)$ \\
\hline Si1set2 & $2.31 \mathrm{E}-3$ & $3.08 \mathrm{E}+20$ & $1.04 \mathrm{E}+00$ & $3.67 \mathrm{E}+18$ \\
\hline
\end{tabular}

It is worth noting that upon Ar annealing, we were not able to measure the samples' Hall properties using the common settings as the Hall effect setup indicated an open-circuit for all Ar annealed samples. Also, the I-V characteristics showed a non-ohmic (non-linear) behaviour, which confirmed the low carrier concentration of these samples. In an attempt to circumvent this issue, Hall measurements were performed under a lower applied current, and silver paint was applied on the corners of the Ar annealed samples to ensure a good electrical contact with the measurement probes. However, silver paint did not result in any improvement in the measured Hall parameters of the samples.

For all Ar annealed samples presented in this section, the decrease in the carrier concentration and estimated plasma frequency values were substantial, 
hence, we did not provide dielectric permittivity models for these samples as the dielectric permittivity showed negative values at longer wavelengths.

To conclude, Ar annealing resulted in a lower carrier concentration and optical bandgap, and a higher resistivity and plasma frequency for our deposited AZO thin films, regardless of the substrate material. This indicated that Ar annealing was not a desirable process for our intended application.

\subsubsection{Discussion}

Crystalline zinc oxide films are known to lose oxygen [262], which means that these films tend to be n-doped due to extra electrons in zinc. Once $\mathrm{Al}$ is used to dope the $\mathrm{ZnO}$ lattice, they replace zinc ions and the number of electrons in the lattice increases, hence conductivity increases [261, 264]. Therefore, Al doped $\mathrm{ZnO}$ can potentially have a decreased conductivity through two pathways. The first is by losing electrons due to losses in donor atoms, i.e., Zn and Al. The second pathway is by gaining oxygen atoms to fill available oxygen vacancies, which results in a depletion of the free electrons in the lattice.

The latter scenario provides a more probable explanation for the results achieved after our Ar annealing process, given the high temperature needed to sublimate $\mathrm{Al}$ or $\mathrm{Zn}$ in significant amounts, where the sublimation rate exponentially increases beyond $600{ }^{\circ} \mathrm{C}$ [460]. One possible source of oxygen during the Ar annealing process is air leakage due to faulty seals in the furnace setup.

If that was the case, then, the carrier concentration for Ar annealed AZO samples is decreased while the resistivity increased. This can explain the more pronounced charging effect that was observed while imaging these samples in the SEM system as compared to the original unannealed AZO films. 
In the next section, we perform a vacuum annealing on our AZO samples and check its impact on the samples properties, in particular the carrier concentration.

\subsubsection{Vacuum annealed sample}

In this section, we first report the FTIR transmission spectra for original and vacuum annealed AZO samples on different substrates. Figure 9-8 shows the transmission spectrum of an AZO on Boroflaot33 sample, namely S7 from Chapter 7 , before and after vacuum annealing.

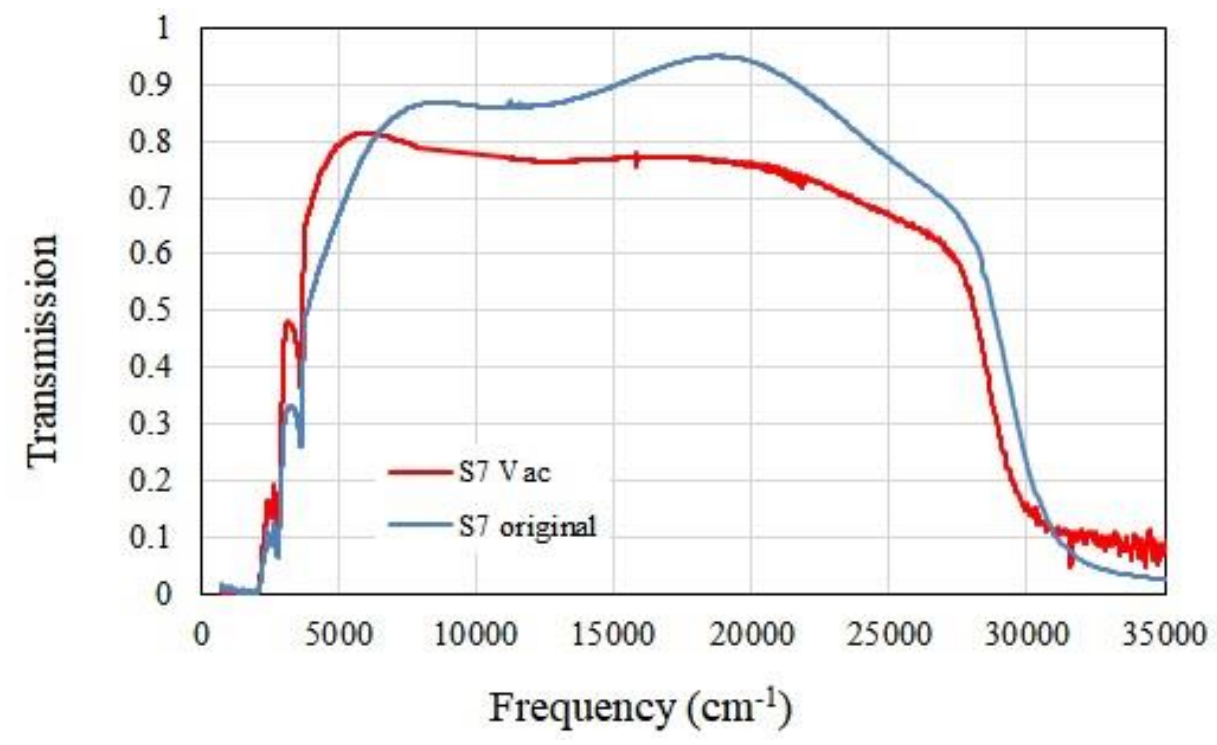

Figure 9-8 Transmission spectra for $\mathrm{S} 7$ before and after vacuum annealing.

The main observations in this plot were a red shift in the transmission response, and that with the exception of the region below $6000 \mathrm{~cm}^{-1}$, vacuum annealing resulted in a lower transparency for S7. 
Similarly, Figure 9-9 shows the transmission spectra for an AZO on sapphire sample, namely Sap2set2 from Chapter 8, before and after vacuum annealing.

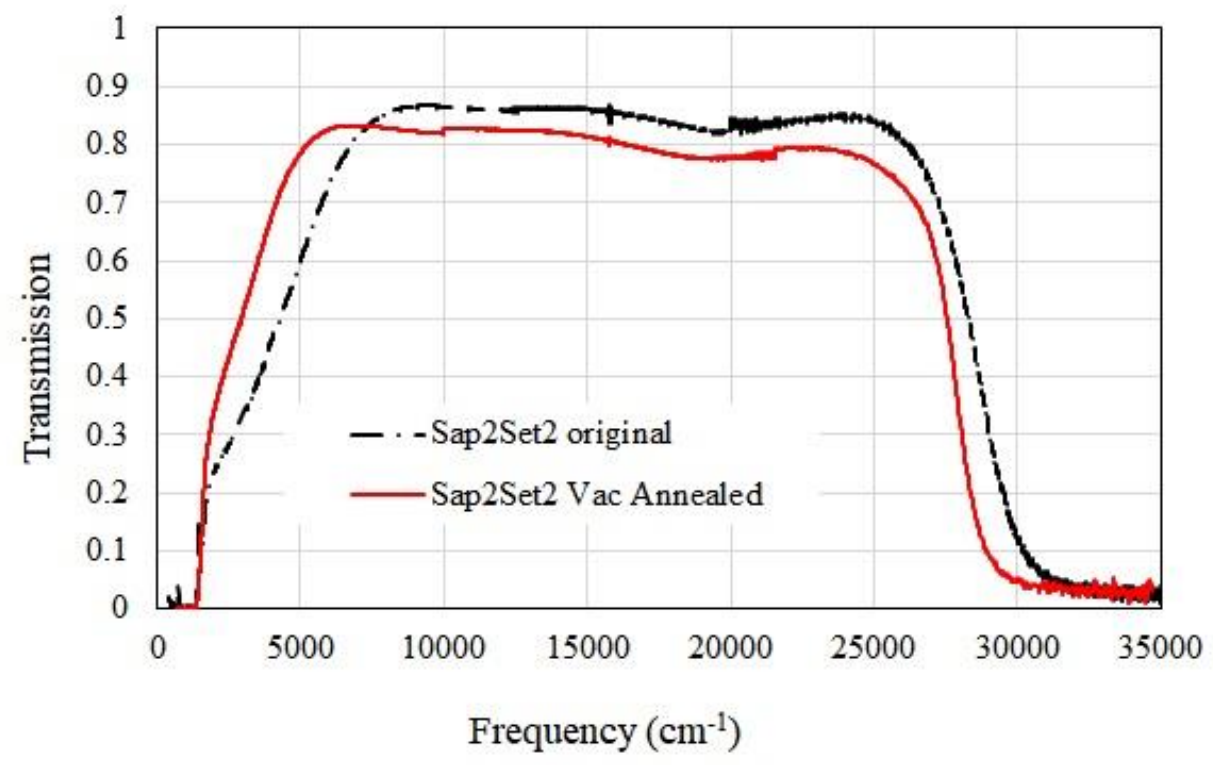

Figure 9-9 Transmission spectra for Sap2set2 before and after vacuum annealing.

Once again, the sample's transparency seemed to be decreased due to vacuum annealing, except for the spectral region below $6000 \mathrm{~cm}^{-1}$, and the whole transmission response seemed to be red shifted, as indicated by the higher transmission near the FIR range and the lack of transmission above $28000 \mathrm{~cm}^{-1}$.

Figure 9-10 shows the original and vacuum annealed FTIR spectra for thickest $\mathrm{AZO}$ on $\mathrm{CaF}_{2}$ sample from Chapter 8. After vacuum annealing, the transmission curve seems shifted towards lower frequencies (red shifted), as observed for the sapphire and Borofloat33 samples. 


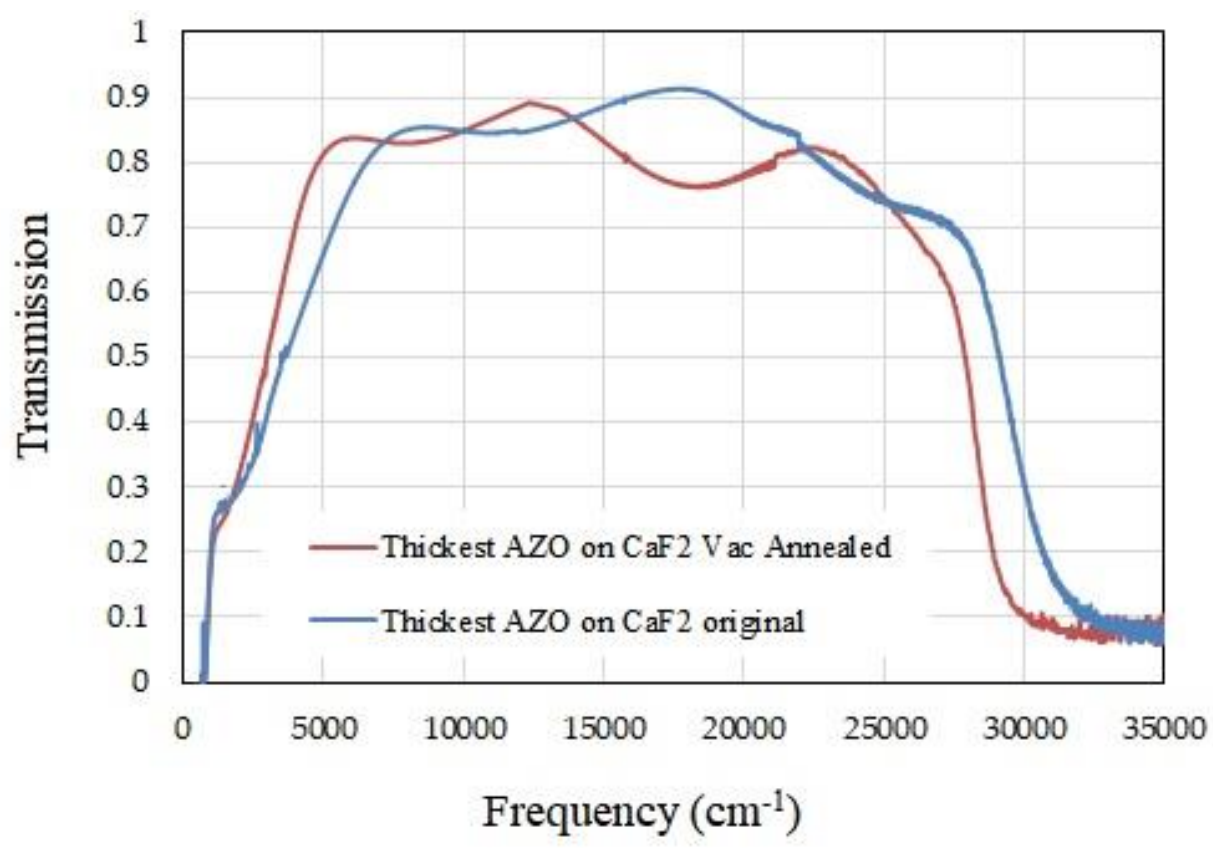

Figure 9-10 Transmission spectra for $\mathrm{AZO}$ on $\mathrm{CaF}_{2}$ (Thickest) before and after vacuum annealing.

It is noted that the change in the transparency of this sample due to vacuum annealing was more complex than the cases of sapphire and Borofloat33, as transparency became higher in some regions and lower in others, when compared with the original state. Nonetheless, a consistent observation was that this sample was also more transparent than its original state in the spectral region below $6000 \mathrm{~cm}^{-1}$.

Finally, Figure 9-11 shows the transmission spectra for an original and vacuum annealed AZO on Si sample, namely Si2set1. While $\mathrm{Si}$ is not transparent in the UV and visible regions, it is the only considered sample that was transparent in the FIR region. 


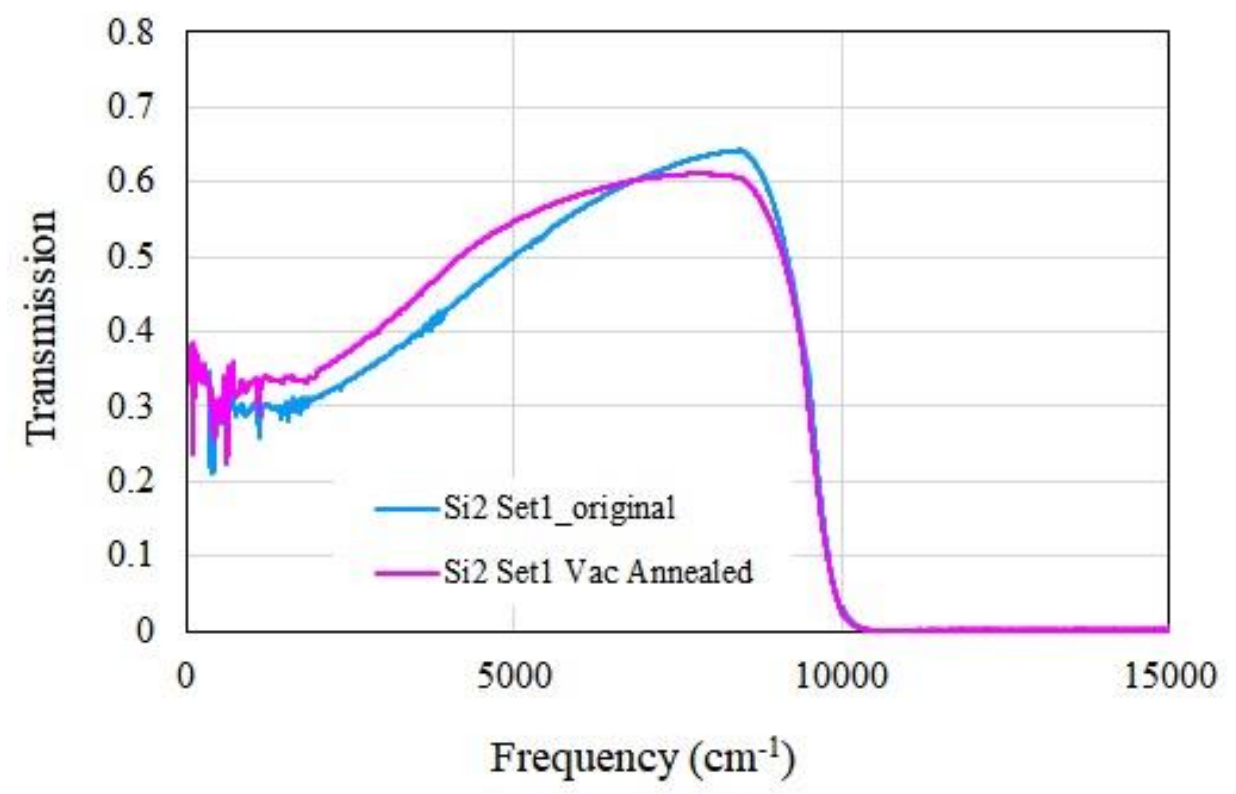

Figure 9-11 Transmission spectra for Si2set1 before and after vacuum annealing.

A red shift in the transmission response was also observed for Si2set1, where the sample became more transparent in region below $6000 \mathrm{~cm}-1$ after vacuum annealing.

Accordingly, AZO thin films on all substrates had a similar FTIR transmission outcome due to vacuum annealing, where the transmission response had a red shift towards longer wavelengths, and the samples became more transparent in the IR region below $6000 \mathrm{~cm}^{-1}$.

Table 9-19 shows a summary of the measured carrier concentration and resistivity before and after vacuum annealing for all four samples of this section. 
Table 9-19 Carrier concentration and resistivity before and after vacuum annealing for four AZO samples.

\begin{tabular}{|c|l|l|l|l|}
\hline \multirow{2}{*}{ Name } & \multicolumn{2}{|c|}{ Original } & \multicolumn{2}{c|}{ Vacuum Annealed } \\
\cline { 2 - 5 } & $\rho(\Omega . \mathrm{cm})$ & $n\left(\mathrm{~cm}^{-3}\right)$ & $\rho(\Omega . \mathrm{cm})$ & $n\left(\mathrm{~cm}^{-3}\right)$ \\
\hline Thickest & $9.79 \mathrm{E}-3$ & $2.64 \mathrm{E}+20$ & $10.64 \mathrm{E}-3$ & $1.22 \mathrm{E}+20$ \\
\hline S7 & $3.60 \mathrm{E}-03$ & $2.32 \mathrm{E}+20$ & $2.63 \mathrm{E}-03$ & $1.49 \mathrm{E}+20$ \\
\hline Sap2set2 & $2.52 \mathrm{E}-3$ & $3.15 \mathrm{E}+20$ & $2.99 \mathrm{E}-03$ & $1.45 \mathrm{E}+20$ \\
\hline Si2set1 & $5.01 \mathrm{E}-03$ & $1.58 \mathrm{E}+20$ & $5.17 \mathrm{E}-03$ & $1.13 \mathrm{E}+20$ \\
\hline
\end{tabular}

As can be seen in this table, vacuum annealing reduced the overall carrier concentration for all samples, however, this reduction was minor, as the carrier concentration remained higher than $1 \mathrm{E}+20$. This minor decrease could be due to the combination of two opposing mechanism that can occur while heating in vacuum; the first being the sublimation of $\mathrm{Zn}$ or $\mathrm{Al}$ atoms at such temperatures (lower carrier concentration effect), while the second is the loss of oxygen at raised temperature (higher carrier concentration effect). This outcome was in large contrast with that of the Ar annealing step, which caused a drop of $\sim 3$ orders of magnitude in the samples' carrier concentration.

Finally, the estimated plasma frequency for all vacuum annealed samples is provided in Table 9-20, where the FTIR-Reffit method was used. The table also shows the Drude terms for each sample as modelled in Reffit. 
Table 9-20 Plasma frequency values for all considered samples, before and after vacuum annealing.

\begin{tabular}{|c|c|c|c|c|c|c|}
\hline \multirow{2}{*}{ Name } & \multicolumn{3}{|c|}{ Original } & \multicolumn{3}{c|}{ Vac annealed } \\
\cline { 2 - 7 } & $\omega_{p}\left(\mathrm{~cm}^{-1}\right)$ & $\mathrm{G}\left(\mathrm{cm}^{-1}\right)$ & $\varepsilon_{\infty}$ & $\omega_{p}\left(\mathrm{~cm}^{-1}\right)$ & $\mathrm{G}\left(\mathrm{cm}^{-1}\right)$ & $\varepsilon_{\infty}$ \\
\hline Thickest & 6077 & 2500 & 1.6 & 4100 & 2500 & 1.6 \\
\hline S7 & 5650 & 3000 & 2.5 & 4523 & 3000 & 2.5 \\
\hline Sap2set2 & 6590 & 1000 & 1.4 & 4464 & 1000 & 1.4 \\
\hline Si2set1 & 5107 & 1560 & 5.4 & 5000 & 2181 & 6 \\
\hline
\end{tabular}

As can be seen, vacuum annealing caused a reduction in the plasma frequency for all considered samples, with the least reduction observed for the Si sample, while the largest reduction was observed for the sapphire sample.

In summary, the vacuum annealing process that we conducted did not improve the carrier concentration of our samples, where a slight reduction within the same order of magnitude was observed.

In the next section, we present the results of the last sample group included in this annealing study, which involves samples that were annealed in Ar first, then had a second vacuum annealing step. 


\subsubsection{Double annealed samples}

Hall effect measurements from section 9.4 indicated that the AZO samples had lower carrier concentrations due to the Ar annealing step. This outcome was not desirable for our intended application, with both the plasma wavelength and electrical resistivity significantly increasing. At that stage, we were not sure about the mechanism that caused such an outcome, whether it was driven by a sublimation of donor atoms or gained oxygen atoms (due to possible improper Ar furnace sealing). Consequently, we attempted the vacuum annealing process, which should eliminate the possibility of oxygen gain during the heating process, thereby isolating the two mechanisms. The main outcome of the vacuum annealing study was a slight reduction in the AZO films' carrier concentration, which confirmed our suspicion regarding an oxygen leak in the Ar annealing setup. Accordingly, the main focus in this section is to observe the impact of a second vacuum annealing process on the already Ar annealed samples.

Although all samples in this section underwent two annealing steps, we were only able to provide transmission spectra for the original and the double annealed sample states, i.e., we were not able to provide spectra for the Ar annealed state. This limitation was due to the size of the FTIR sample holder, where our samples needed to be cut in half to fit into the holder. Meanwhile, as soon as a sample was cut in half, it could not be measured in the Hall effect system anymore due to the associated sample holder. Therefore, the preference was to sacrifice the FTIR measurement after the Ar annealing stage, to monitor changes in the carrier concentration after each annealing step.

Figure 9-12 shows the FTIR transmission spectra for two AZO on sapphire samples, measured at the original and double annealed $(\mathrm{Ar}+\mathrm{Vac})$ states. These samples are denoted by Sap1set2 and Sap4set1. 


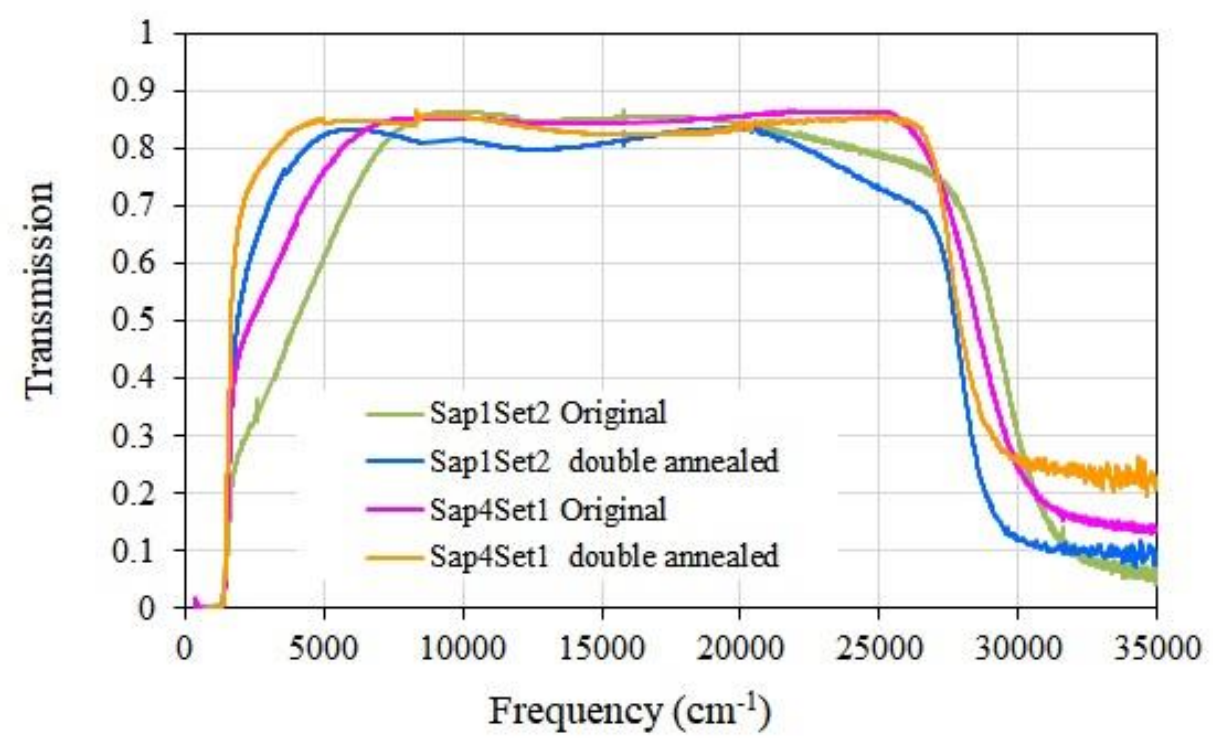

Figure 9-12 Transmission spectra for two Ar and vacuum annealed (double annealed) samples before and after annealing.

For both samples, the double annealing process increased transmission at frequencies below $\sim 7000 \mathrm{~cm}^{-1}$. Between $7000 \mathrm{~cm}^{-1}$ to $\sim 30000 \mathrm{~cm}^{-1}$, transmission levels of the double annealed samples were below those of the original spectra. Finally, the transmission levels of both double annealed samples exceeded those of the original states at frequencies above $31000 \mathrm{~cm}^{-1}$.

Figure 9-13 shows the Tauc plots for all four transmission curves from Figure 9-12, where a reduction in the optical bandgap was observed for both double annealed samples. 


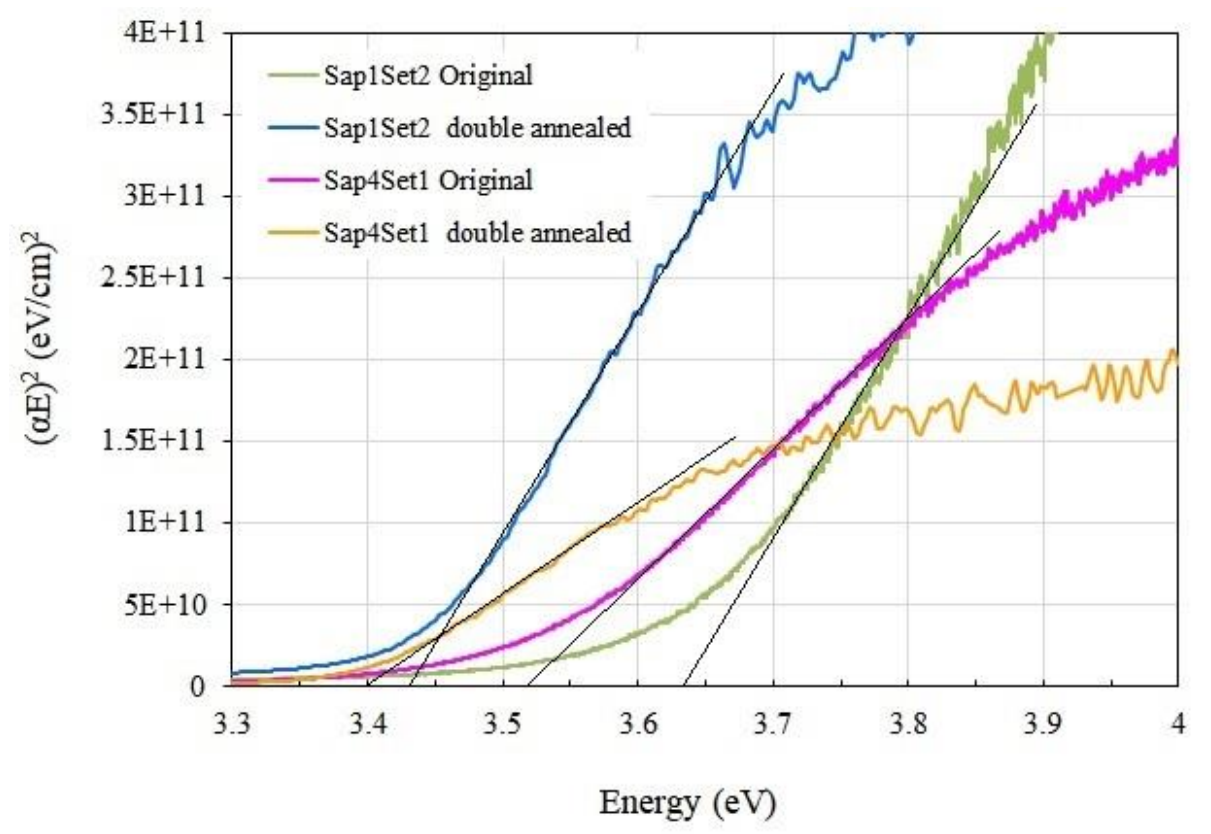

Figure 9-13 Tauc plots to estimate the optical bandgap for AZO on sapphire samples before and after the double annealing process.

Carrier concentration and resistivity were measured for our samples at the original state, after the first annealing (Ar annealing) and after the second annealing (Ar + Vac). Table 9-21 provides a summary of these results.

Table 9-21 Carrier concentration (n) and resistivity $(\rho)$ for double annealed samples compared to that of the original and Ar annealed samples.

\begin{tabular}{|c|c|c|c|c|c|c|}
\hline \multirow{2}{*}{ Name } & \multicolumn{2}{|c|}{ Original } & \multicolumn{2}{c|}{ Ar Annealed } & \multicolumn{2}{c|}{ Double Annealed } \\
\cline { 2 - 7 } & $\begin{array}{c}\rho \\
(\Omega . \mathrm{cm})\end{array}$ & $\begin{array}{c}n \\
\left(\mathrm{~cm}^{-3}\right)\end{array}$ & $\begin{array}{c}\rho \\
(\Omega . \mathrm{cm})\end{array}$ & $\begin{array}{c}n \\
\left(\mathrm{~cm}^{-3}\right)\end{array}$ & $\begin{array}{c}\rho \\
(\Omega . \mathrm{cm})\end{array}$ & $\begin{array}{c}n \\
\left(\mathrm{~cm}^{-3}\right)\end{array}$ \\
\hline Sap1set2 & $2 \mathrm{E}-3$ & $4.02 \mathrm{E}+20$ & $7 \mathrm{E}-2$ & $8.23 \mathrm{E}+18$ & $6 \mathrm{E}-3$ & $1.11 \mathrm{E}+20$ \\
\hline Sap4set1 & $4 \mathrm{E}-3$ & $1.85 \mathrm{E}+20$ & $2 \mathrm{E}-1$ & $6.93 \mathrm{E}+18$ & $4 \mathrm{E}-3$ & $8.72 \mathrm{E}+19$ \\
\hline
\end{tabular}


As can be seen, Ar annealing reduced the carrier concentration and increased the resistivity of our films, both of which are not desirable in our study. However, double annealing these samples at the same temperature in the vacuum furnace improved both parameters. This was another significant finding in the experimental part of this thesis, as thermal annealing was proven to be effective for tuning the key parameters of our plasmonic material.

With regards to the mechanism of this carrier concentration improvement, we attributed this to the possible outgassing of oxygen atoms in the vacuum furnace. Oxygen outgassing from AZO films during annealing processes has been reported in several studies [461, 462].

Figure 9-14 shows the original and double annealed transmission plots of an $\mathrm{AZO}$ on $\mathrm{CaF}_{2}$ sample from the same set as the "non-transparent" $\mathrm{AZO}$ on $\mathrm{CaF}_{2}$ in Chapter 8. We refer to this sample as non-transparent (B).

Being a part of this visually non-transparent sputtering set, this sample had a lower transmission in the visible region in the as-sputtered state.

After double annealing, the transparency of the sample was reduced by 20$30 \%$ through most of the characterized spectrum, from the near-infrared to the near UV region. This sample had a thickness of $90 \mathrm{~nm}$, as indicated by the RBS measurements. 


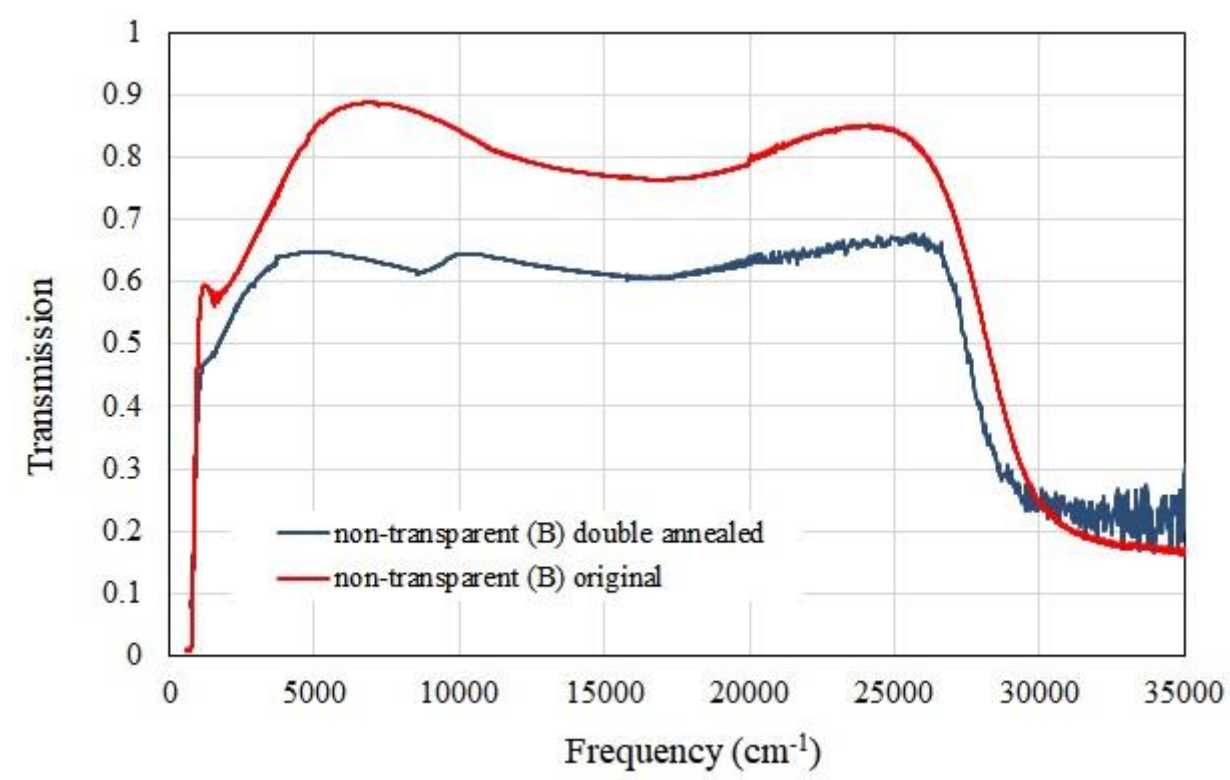

Figure 9-14 Transmission plots for the non-transparent (B) sample before and after double annealing.

Hall effect measurements showed that this sample had a lower carrier concentration of the order of $1 \mathrm{E}+19$ in the as-sputtered state. Table 9-22 shows the measured resistivity and carrier concentration for this sample in the original, Ar annealed and double annealed states.

Table 9-22 Carrier concentration $(n)$ and resistivity $(\rho)$ for double annealed $\mathrm{AZO}$ on $\mathrm{CaF}_{2}$ compared to the original and Ar annealed results.

\begin{tabular}{|c|c|c|c|c|c|c|}
\hline \multirow[b]{2}{*}{ Name } & \multicolumn{2}{|c|}{ Original } & \multicolumn{2}{|c|}{ Ar Annealed } & \multicolumn{2}{|c|}{ Double Annealed } \\
\hline & $\begin{array}{c}\rho \\
(\Omega . c m)\end{array}$ & $\begin{array}{c}n \\
\left(\mathrm{~cm}^{-3}\right)\end{array}$ & $\begin{array}{c}\rho \\
(\Omega . \mathrm{cm})\end{array}$ & $\begin{array}{c}n \\
\left(\mathrm{~cm}^{-3}\right)\end{array}$ & $\begin{array}{c}\rho \\
(\Omega . c m)\end{array}$ & $\begin{array}{c}n \\
\left(\mathrm{~cm}^{-3}\right)\end{array}$ \\
\hline Non-transparent (B) & 19E-3 & $8.45 \mathrm{E}+19$ & $2.7 \mathrm{E}-1$ & $4.24 \mathrm{E}+17$ & $22 \mathrm{E}-3$ & $5.01 E+19$ \\
\hline
\end{tabular}


Although, the carrier concentration and electrical conductivity of the original sample were substantially reduced after Ar annealing, these parameters were almost restored to the same original levels after vacuum annealing.

Estimated plasma frequency values using the FTIR-Reffit method for this subsection's samples are shown in Table 9-23.

Table 9-23 Plasma frequency using the FTIR-Reffit method before and after double annealing for all three samples included in this sub-section.

\begin{tabular}{|c|c|c|c|c|c|c|}
\hline \multirow{2}{*}{ Name } & \multicolumn{4}{|c|}{ Original } & \multicolumn{2}{c|}{ (Ar + Vac) double annealed } \\
\cline { 2 - 7 } & $G\left(\mathrm{~cm}^{-1}\right)$ & $\varepsilon_{\infty}$ & $\omega_{p}\left(\mathrm{~cm}^{-1}\right)$ & $\mathrm{G}\left(\mathrm{cm}^{-1}\right)$ & $\varepsilon_{\infty}$ & $\omega_{p}\left(\mathrm{~cm}^{-1}\right)$ \\
\hline Non-transparent (B) & 2000 & 2 & 3387 & 2000 & 2 & 2606 \\
\hline Sap1set2 & 1000 & 1.6 & 7445 & 1000 & 1.6 & 3920 \\
\hline Sap4set1 & 2000 & 1.4 & 4700 & 2000 & 1.4 & 3440 \\
\hline
\end{tabular}

It is observed that the plasma frequency values decreased for all studied samples after the double annealing process, which was in agreement with our expectations based on the reduced carrier concentration after double annealing.

Although the vacuum annealing and $(\mathrm{Ar}+\mathrm{Vac})$ double annealing processes did not improve the carrier concentration and plasma frequency values of our AZO thin films, the real and imaginary dielectric permittivity plots for one sample from each annealing method are shown below in Figure 9-15. These 
samples are Sap1set2 after $(\mathrm{Ar}+\mathrm{Vac})$ double annealing and Sap2set2 after vacuum annealing.

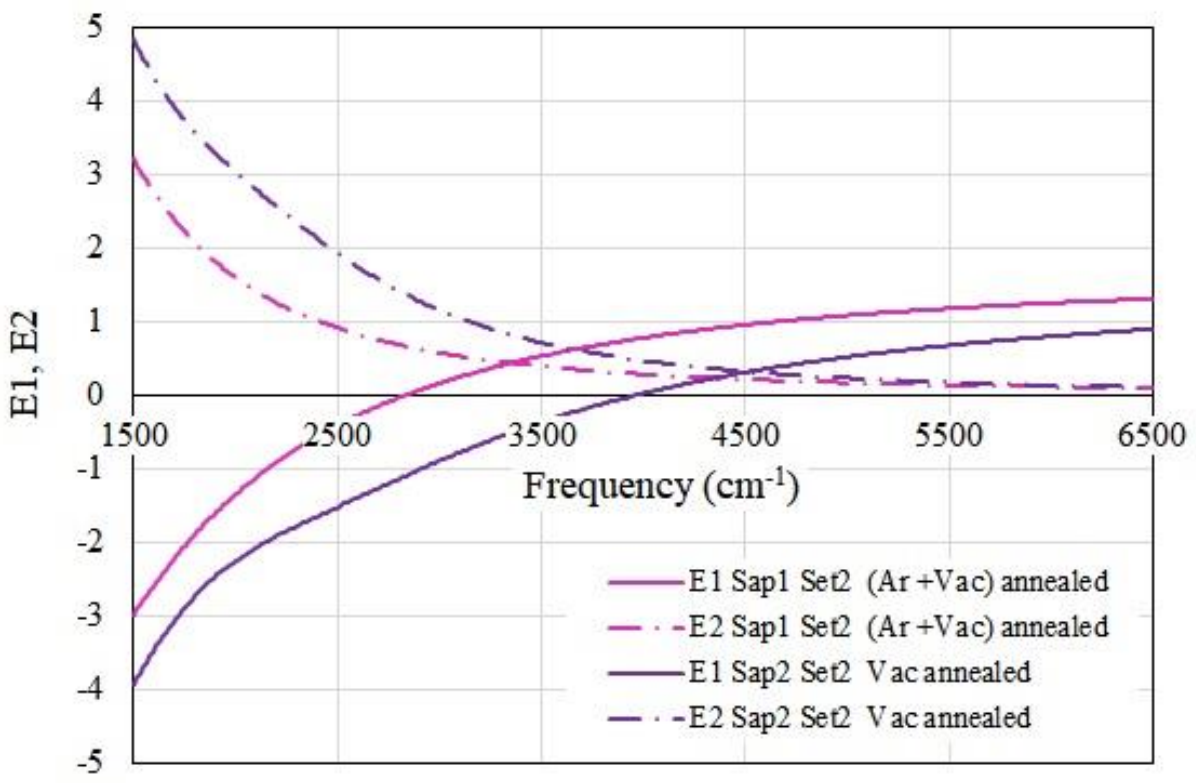

Figure 9-15 Real (E1) and imaginary (E2) dielectric permittivity for (Ar + Vac) double annealed Sap1set2 and vacuum annealed Sap2set2.

The curves of E1 and E2 in Figure 9-15 can be compared to those of the original state, which were presented in Figure 8-24. As can be seen, the cross-over frequency was shifted to larger wavelengths due to the double annealing process, which was not a desirable outcome for our thin films.

\subsection{Chapter summary}

In this chapter, we evaluated the effects of post-growth annealing on the properties of the deposited AZO samples. 
Firstly, we decided to Ar anneal our highly conductive AZO samples, aiming to improve the properties of the films. However, this resulted in reduced carrier concentration compared to that of the original samples. On average, the Hall effect measurements of $\mathrm{Ar}$ annealed samples showed at least two orders of magnitude of lower carrier concentration compared to that of the original state. A lower carrier concentration meant that our samples became less conductive. In short, this was a step in the wrong direction.

Next, we performed vacuum annealing on a different set of highly conductive AZO samples to examine the possibility of a different outcome than that of Ar annealing. The main observation was a slight reduction in the carrier concentration, which remained within the same order of magnitude of the assputtered films.

Finally, given the dissimilar outcomes to the two supposedly inert annealing processes, we were interested to check whether a second vacuum annealing could vary the properties of the already Ar annealed samples. Hall effect measurements of these double annealed samples confirmed that their carrier concentration increased to nearly the level achieved in the vacuum annealed samples. This indicated that the carrier loss in our Ar annealed samples could be reversed, and that the carrier concentration of our samples could be tuned by means of post-growth annealing. 



\title{
10 Summary of the experimental
}

\author{
results
}





\subsection{Substrate comparison}

Silicon was one of the substrates studied in this thesis. Given that the maximum optical transparency of silicon is almost half of what was measured for the other three substrate materials, and is not transparent in the UV, visible and NIR regions, it was not an ideal choice for our far-field superlens design. Nonetheless, we deliberately experimented using this material as it was the only substrate with transparency in the FIR region.

Optically, Borofloat33 was an acceptable substrate for our application, but only if the plasma frequency of the plasmonic thin film material was higher than $4000 \mathrm{~cm}^{-1}$, as the substrate is optically absorbent at lower frequencies. This substrate offers an advantage over other substrates given that it is made of an amorphous material, i.e., birefringence is not an issue.

Calcium fluoride was the best substrate from an optical response standpoint. However, this substrate resulted in large residual stress in our AZO thin films due to its relatively large coefficient of thermal expansion, causing significant film cracking and delamination. In addition, our sourced calcium fluoride substrates were defective as they were cut and polished at a tilted angle with respect to the proper $\left(\begin{array}{lll}1 & 1 & 1\end{array}\right)$ crystal orientation.

Finally, sapphire was the optimal candidate substrate in this study, given its optical and thermal characteristics, which resulted in consistent sample properties. Sapphire was slightly less transparent than calcium fluoride and was only opaque in FIR region. A disadvantage to sapphire was its crystalline nature, 
which results in birefringence in plasmonic application, such as the proposed FSL device.

As a general note, the transmission and reflection spectra hold information about the refractive index and dielectric permittivity $(\varepsilon)$, e.g. $T\left(\varepsilon_{1}, \varepsilon_{2}\right)$ and $R\left(\varepsilon_{1}, \varepsilon_{2}\right)$ of our AZO thin film samples. These transmission or reflection spectra could vary from a sample to the other due to numerous factors. For instance, thin films deposited on different substrates would possess different optical properties, regardless of our ability to isolate the optical transmission and reflection spectra of the thin films from those of the corresponding substrates in post-processing using Reffit. In addition, within our studied spectral range, optical transmission and reflection properties of each substrate material dictate our ability to infer the optical properties of our AZO thin films using the aforementioned postprocessing method; for example, a non-transparent substrate would prevent the measurement of the optical transmission property of a thin film deposited on that substrate. The effects of this dependency were observed at an earlier stage of our study, where AZO samples deposited on Borofloat33 were assumed to possess better dielectric permittivity values than what was more accurately determined later. This error was due to having a broad range of fittings that would conform with the measured transmission spectra of these samples, given the nontransparent nature of Borofloat33 in parts of the measured spectrum. This error was only realized when our study was expanded to include AZO samples deposited on $\mathrm{CaF}_{2}$, a substrate that was highly transparent across most parts of the characterized spectrum, which allowed us to understand the typical optical properties of our deposited AZO thin films.

On another note, variations in the properties of samples deposited on the same substrate and in the same sputtering run were always observed, which were due to the geometry of our sputtering system and the associated substrate heating 
constraints. In this case, our samples indicated compositional differences based on their location with respect to the sputtering target. These variations could be better understood by examining parameters such as resistivity and carrier concentration.

In this study, our knowledge evolved as we moved on from one substrate to the other, as each substrate has its unique optical and crystal properties. Generally, having a substrate with high level of transparency in the low frequency region allowed us to investigate the intraband effects of our AZO samples, which is directly related to their estimated plasma frequency values. On the other hand, substrates with high levels of transparency in the high frequency region enabled us to evaluate the interband effects of our AZO samples, where differences in the optical bandgap could arise.

This has shone much needed light on the properties of our deposited AZO thin films and ultimately enabled us to refine existing models that describe the properties of AZO. For instance, contrary to the value that exist in literature, we were able to establish a new effective mass value for AZO (0.65) that brought our fittings into good agreement with measurements, regardless of the used substrate.

Another sanity check of this effective mass value is presented in Figure 10-1, which shows a scatter plot of the bandgap versus carrier concentration for all reported AZO thin films deposited on Borofloat33 (from chapter 7), sapphire and calcium fluoride substrates (from chapter 8). The BM model (as described in section 4.4) is included in this figure to provide a reference to our results, noting that our effective mass value was employed in this model. 


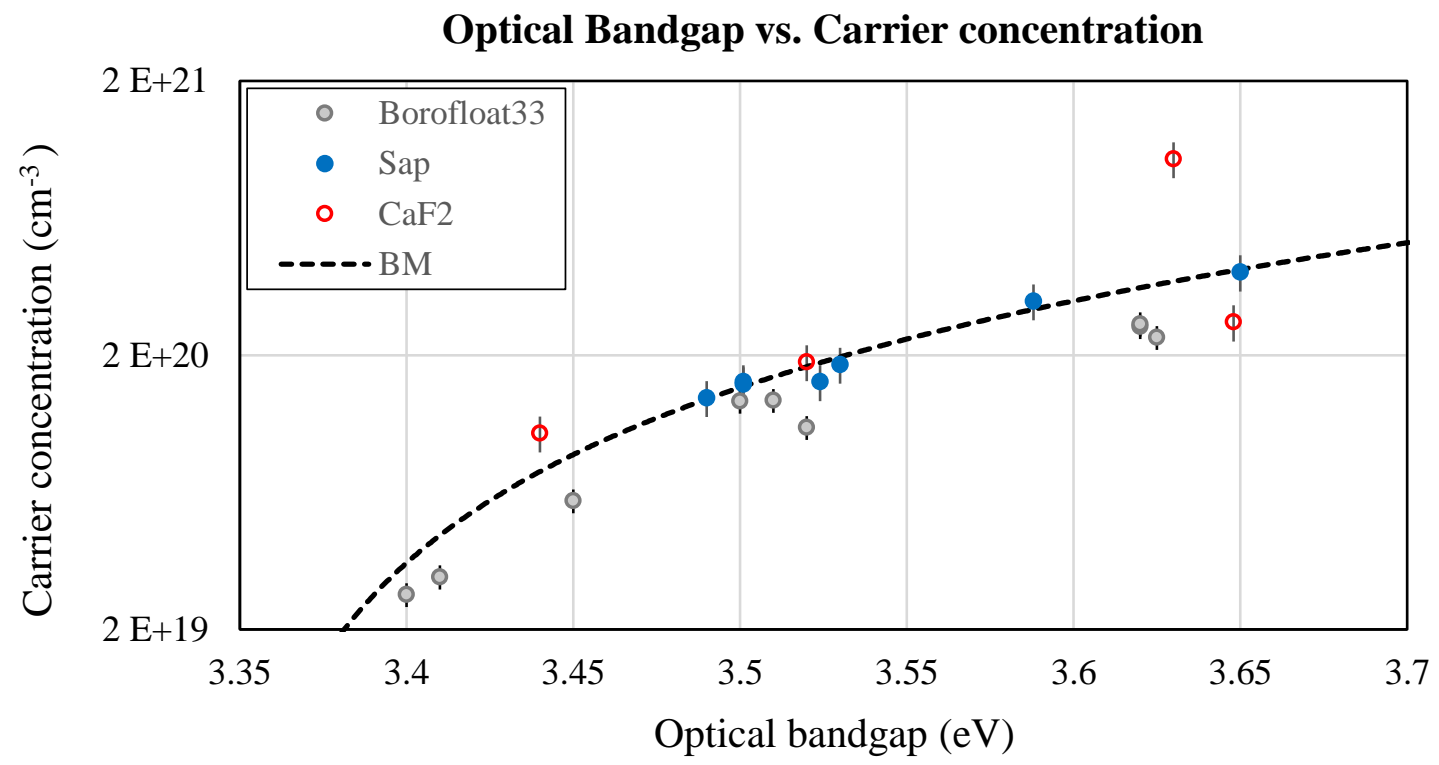

Figure 10-1 Plot of the measured carrier concentration versus the estimated optical bandgap for all AZO thin films on Borofloat33, sapphire and CaF2 substrates. The Burstein-Moss model (BM) is provided as a reference.

AZO samples on $\mathrm{Si}$ are excluded from this plot as $\mathrm{Si}$ is not transparent in the high frequency range, which highlights the limitations imposed by silicon's opacity in this spectral region.

The summary provided in this figure highlights a significant result in our study, where carrier concentration measurements and bandgap estimation for our AZO thin films on Borofloat33, sapphire and $\mathrm{CaF}_{2}$ were in a very good agreement with the Burstein-Moss model.

On a similar note, Figure 10-2 shows a scatter plot of the plasma frequency versus the carrier concentration for all our reported AZO thin films deposited on Borofloat33 (from chapter 7), sapphire, calcium fluoride and silicon substrates (from chapter 8) in this thesis. The dotted plot represents the fit of the Drude model equation as it passes through all represented points. 


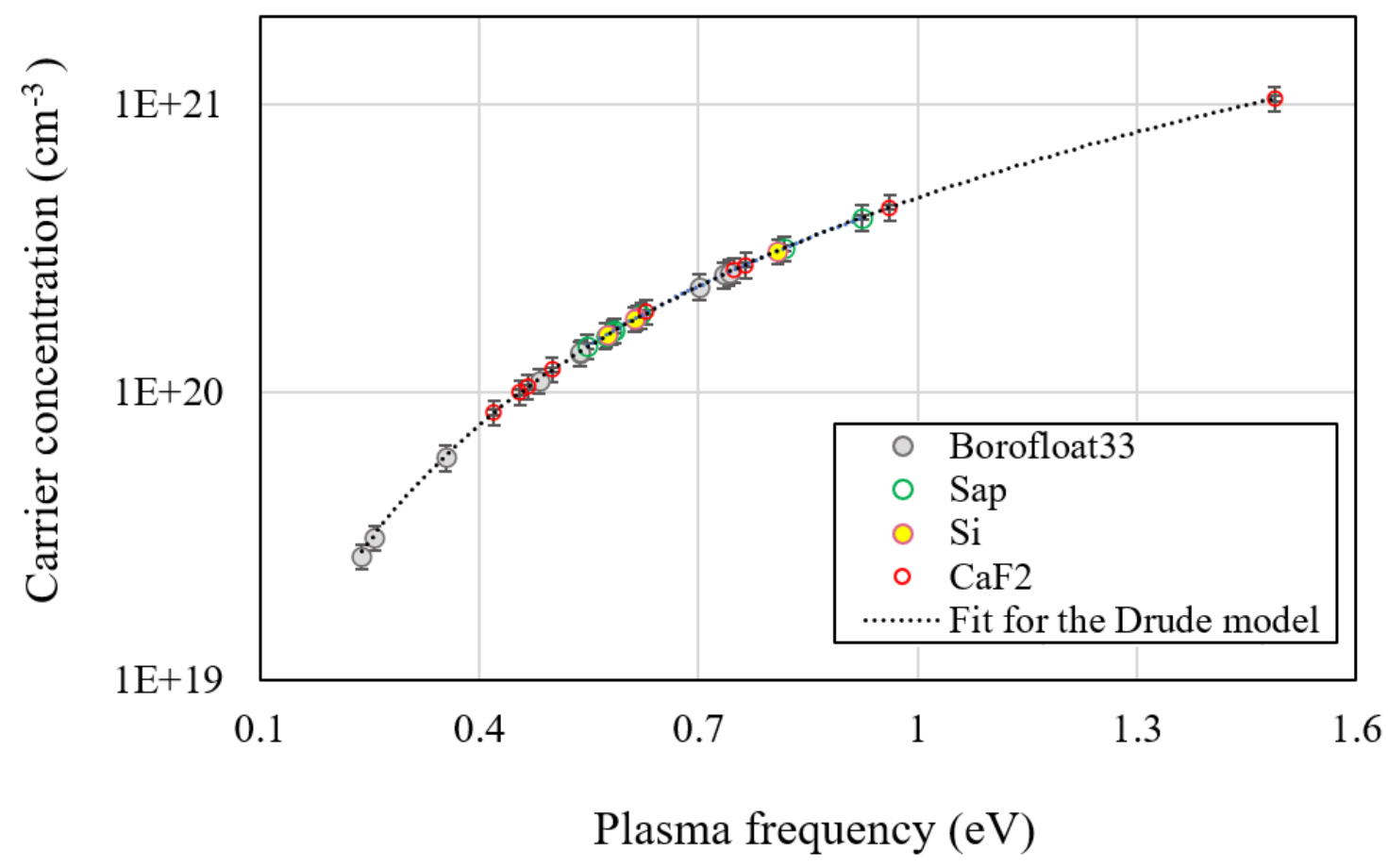

Figure 10-2 Plot of the calculated carrier concentration versus the estimated plasma frequency for all AZO thin films on Borofloat33, sapphire, $\mathrm{CaF}_{2}$ and silicon substrates.

This plot highlights the spread in plasma frequency values for our samples, which were produced using the same sputtering recipe inside the same sputtering system in different runs. The differences in carrier concentration values have been mainly attributed to variations in our sputtering conditions, in particular the target to sample placement. Future work relating to sputtering process optimization should seek to clarify and quantify this dependency to establish more consistent plasma frequency values for the produced thin films.

\subsection{Thin film properties}

It was observed from our samples that a good thickness control could be achieved during the sputtering process by controlling the sputtering rate and time and location with respect to the centre of the sputtering target. This was 
independent of the substrate material. Later thickness measurement, using the RBS method confirmed our estimations of the intended thickness based on the selected sputtering parameters. We observed that most of our deposited thin films had long-term structural and compositional stability as confirmed by SEM and EDX characterization methods, where selected samples showed no difference at all immediately after fabrication when compared to 24 months later.

We often re-measured our samples in the FTIR spectrometer to confirm stability of both our samples and the spectrometer. We could confirm that the optical properties of our deposited thin films remained constant even 24 months after the sputtering process took place.

Electrical DC resistivity measurements also were consistent over time.

Furthermore, our samples were measured in a Hall effect system, which showed that the majority of our samples had a carrier concentration of the order of $1 \mathrm{E}+20 \mathrm{~cm}^{-3}$.

Two independent calculation methods were employed to estimate the plasma frequency for our thin film samples; namely the FTIR-Reffit method and the HallDrude model. Based on the assumptions that we made for the effective mass of electrons in $\mathrm{AZO}$, plasma frequency values produced from these two independent methods were in full agreement. Moreover, the frequencydependent real and imaginary parts of the dielectric permittivity were determined for nominal samples.

Generally, an increase in the carrier concentration of our deposited thin films resulted in a shift towards shorter wavelengths, where a carrier concentration of $1 \mathrm{E}+20 \mathrm{~cm}^{-3}$ corresponded with a plasma frequency in the $\sim 6000 \mathrm{~cm}^{-1}$ range. For a given sample, the crossover frequency for the real part of the dielectric permittivity often occurred in the same spectral region of the plasma frequency. 
All our thin films were deposited in an off-centre arrangement. This allowed for eliminating damage on the thin film surface caused by energetic plasma species and also allowed for highly conductive samples with high carrier concentration. Summarizing the thickness, carrier concentration and resistivity measurement for all our samples from different runs of sputtering reveals a trend for samples of each set. Assuming a virtual line that diagonally connects the far edge of the sample holder to the edge that is closer to the target results in a gradient of thickness always measured the least for the sample placed at the far corner. This gradient has a reverse trend when comparing carrier concentration on the sample holder diagonally.

Thermal annealing of our samples was considered to improve the properties of the AZO thin films, in terms of achieving a higher carrier concentration, conductivity, plasma frequency and dielectric crossover frequency, to enable their plasmonic activity near the visible range. Although XRD measurement confirmed a consistent decrease in FWHM and increase in CDS values of the measured samples as shown in Figure 10-3, both considered annealing processes, resulted in degraded sample qualities as all four parameters were decreased. Nonetheless, an interesting outcome was achieved by this annealing study, as we proved the ability to tune the thin films' active plasmonic spectral range by means of controlling the parameters of the thermal annealing process. 

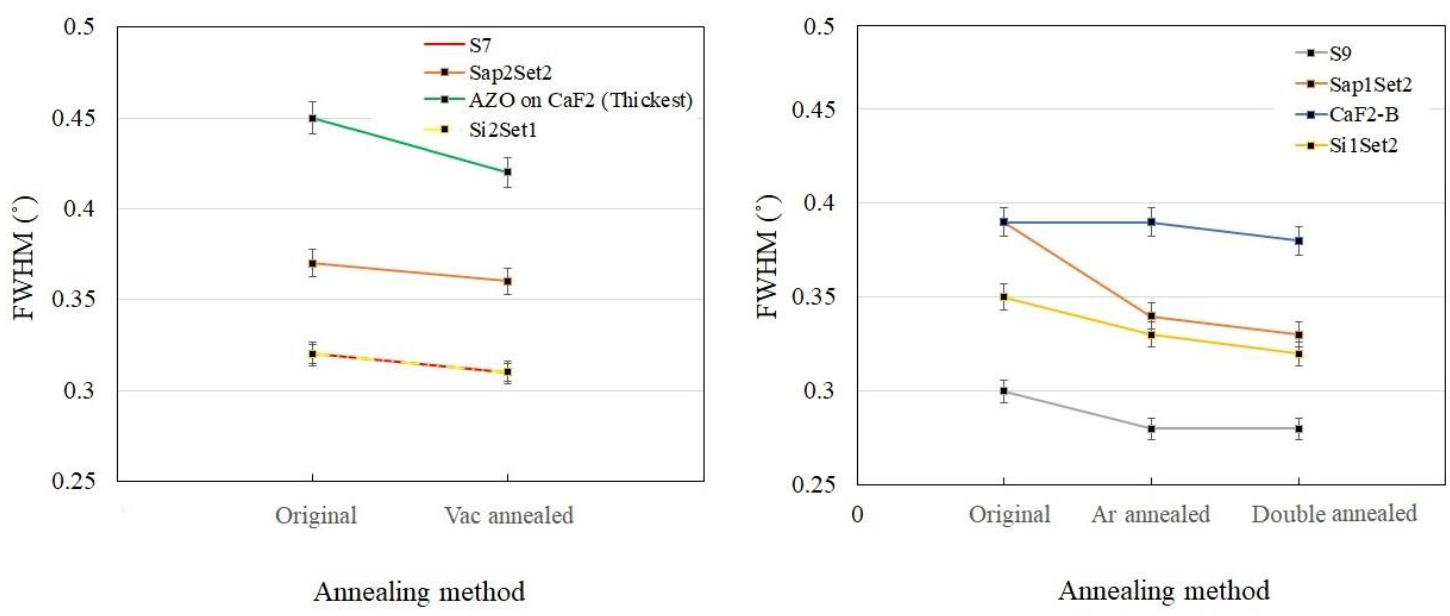

Figure 10-3 A comparison of FWHM $\left(^{\circ}\right)$ between the reported thermal annealing processes for AZO thin film samples deposited on different substrates.

In summary, over the course of this research, we characterized optical and electrical properties of our deposited AZO thin films intended for imaging applications. Results confirmed successful fabrication of uniform AZO thin films with high carrier concentrations, and the ability to control the plasma frequency values through optimization of the sputtering and annealing conditions. Contrary to most reported literature, our characterization results covered a wide range of the electromagnetic spectrum, which is useful for understanding the properties of ohmic AZO thin films over this spectral range. Therefore, this study is critical for the design and development of plasmonic devices that operate in the near infrared range of the EM spectrum, such as our proposed FSL concept. 


\section{Conclusion}



This chapter summarizes the work presented in this thesis. Chapters 1 to 3 form the first part, which includes a background study that describes the far-field superlens concept and provides the COMSOL Multiphysics ${ }^{\circledR}$ modelling results that relate to the design of our far-field superlens.

In Chapter 1, diffraction limit, which dictates the resolution limit of conventional lenses is discussed, and subwavelength imaging techniques, i.e., the near-field superlens and the far-field superlens are introduced. Furthermore, optical effects, i.e., Moiré and Talbot effects, relevant to the performance of a farfield superlens are described.

Chapter 2 introduces COMSOL Multiphysics ${ }^{\circledR}$ as a suitable modelling tool to design our far-field superlens.

In Chapter 3, the modelling results of our study, which covers two dimensional and three dimensional far-field superlens designs are presented. Such level of comprehensive 2D and 3D FSL simulation has never been reported in similar studies to the best of our knowledge. For instance, one of the main challenges in developing 3D models is resource management. In Chapter 3, utilization of proper unit cells was introduced. As part of this investigation, it was verified that by using suitable boundary conditions, minimum time and computation resources were required to study our far-field superlens designs; this simulation domain optimization is critical for enabling future modelling investigations. Furthermore, our study is unique in its examination of the relationship between the size of the object and the FSL nanogratings, and its effects on the far-field patterns of the FSL. While all previous studies limited their analysis of the performance of FSL to the generated Moiré pattern in the far-field, 
we did investigate the generation of a vertical periodicity, which we associated with the Talbot effect. The Talbot effect has thus never been investigated within the context of far-field superlenses, yet it has been consistently observed in all our simulation models. Finally, our 3D modelling provided a useful insight into how a FSL could resolve more details of the object by means of adjusting the provided illumination and using specific arrangements of the FSL nanogratings. This provides a unique benefit for lab based related research, whereby using only one laser setup and utilizing polarization properties of light, extra information could be resolved from an object's subwavelength features, which would have been impractical or impossible otherwise. Results and parameters presented in Chapter 3 are novel to this scientific field and not found in similar studies to the best of our knowledge. The main reason for focusing on these parameters was the lack of available information in literature, thus we sought to clarify the outcomes of different scenarios to understand more about the expected performance of the proposed far-field superlens designs.

Part two includes Chapter 4 to Chapter 10 and provides an overview of the experimental study, which include both theory and results. Part two introduces a novel material to build a superlens; a material that has never been characterized for this purpose and within the considered spectral range. We believe it was necessary to study the optical properties of this material within a wide spectral range to provide a thorough understanding of its expected performance if used to build a far-field superlens. To the best of our knowledge, this in-depth level of analysis has not been presented in the available literature, as most studies confine the characterization to the UV, visible, and NIR ranges.

In Chapter 4 we explain the reasoning behind selecting $\mathrm{Al}$ doped zinc oxide for the experimental study. This selection was based on a series of literature references, where transparent conductive oxides, such as Al doped zinc oxide 
have been suggested to have plasmonic properties. However, the lack of specific information related to the design and considerations of an AZO far-field superlens meant that we needed to adequately characterize this material before we understand what advantages it can offer.

In Chapter 5, we provide an introduction into relevant thin film deposition techniques. Then, we present a summary of our AZO deposition process, which was done using RF magnetron sputtering.

In Chapter 6, material characterization techniques used in the experimental part of this thesis are introduced. These techniques were used to generate the results presented in the following chapters.

In Chapter 7, AZO thin films deposited on Borofloat33 are studied. Characterization results and dielectric permittivity estimations are provided for these samples. However, our understanding and conclusions in this chapter were incomplete due to the limitations imposed by the optical properties of the selected substrate.

In Chapter 8, $\mathrm{AZO}$ thin films deposited on silicon, sapphire and $\mathrm{CaF}_{2}$ substrates are characterized. The transmission spectra of all three substrates were different from each other. This benefited our study in regard to having a broad spectral evaluation of the optical response of our deposited thin film samples. Given the comparative nature of this study, and by using several substrates, our confidence in the estimated values for the plasma frequency and the dielectric permittivity of our AZO thin films was increased.

In Chapter 9, we evaluated the effects of thermal annealing on the optical and electrical properties of nominal samples produced in our study.

It is worth mentioning that in each sputtering run for our $\mathrm{AZO}$ thin films samples, several samples had reasonably similar properties when characterized, 
thus, nominal samples in chapters 7 to 9 were selected to cover the breath of variation within our samples and highlight the achievable favourable properties for these samples. In Chapter 10 a summary highlighting the relationship between the carrier concentration-optical bandgap and carrier concentrationplasma frequency is represented. If the sputtering conditions could be replicated as described in this study, these plots could be considered as a reliable measure to estimate the optical bandgap and plasma frequency once the carrier concentration is measured accurately.

\subsection{Future work}

We suggest that future work relating to this thesis be focused on material characterization and microfabrication using $\mathrm{AZO}$ rather than modelling and simulation, to realize our proof of concept FSL design and confirm the expected performance based on our simulations using periodic objects. For instance, with regards to the sputtering process optimization, it is useful to clarify and quantify the dependency of plasma frequency values to carrier concentration to establish more consistent plasma frequency values for our deposited thin films. As part of this, identifying another substrate with high transparency that does not suffer from high thermal expansion would be favourable.

In the characterization part of our study, three different annealing processes have been shown to result in changes in the properties of our deposited AZO thin films. The main focus in our annealing section was on understanding variations in the carrier concentration due to thermal annealing, where the carrier concentration was measured after each step. In order to improve control over the carrier concentration and crystal properties of our AZO thin films, we suggest 
that future work should include a comprehensive vacuum annealing study with a broader range of considered temperatures to understand the dynamics of oxygen gain/loss and $\mathrm{Al}$ and Zn loss, as well as crystal and optical variations throughout this process. We also suggest that future studies consider thermal annealing in atmospheres with known oxygen concentrations, for example 1\% oxygen in nitrogen or argon, to establish control over the carrier concentration and plasmonic properties of the AZO thin films.

The FTIR transmission spectra of our annealed thin films indicated a general shift towards longer wavelengths compared to the transmission spectra of the same samples before annealing. Verifying whether this trend persists by including a larger group of samples in FTIR measurements would benefit this work.

We also believe it is a priority to vary annealing conditions and characterize the samples using Hall effect, FTIR spectroscopy-fitting method to establish clear correlation with the plasmonic properties of a given AZO sample.

Other utilized characterization techniques in our work included XRD, SEM, and FTIR spectroscopy. In the XRD section, regardless of the annealing technique or the type of substrate, the FWHM was found to reduce. We suggest that future work uses parallel beam XRD techniques to evaluate variations in samples' texture due to annealing.

We then suggest experimenting with lithography techniques to develop AZO gratings that can be used to construct a FSL structure. A starting point for the grating period is to be based on the measured plasma frequency for a given processing recipe, as presented in this thesis. The proposed test process to follow includes creating $1 \mathrm{D}$ and $2 \mathrm{D}$ grating structures using $\mathrm{AZO}$ and characterizing their electrical and optical properties. Thereafter, there is a need to verify the 
Moiré patterns of various AZO gratings on top of object gratings using a CCD camera placed at the far-field similar to available references. Consequently, it is necessary to incorporate AZO gratings in a far-field superlens design and evaluate its performance and subwavelength resolution using photoresist as shown in superlens studies. this work can proceed to characterizing the optical and plasmonic performance of such FSL prototypes to evaluate their functionality.

Following this proof of concept stage, one major piece of future work should focus on developing robust image processing algorithms that allow the reconstruction of various periodic object shapes using available information in the far-field of the FSL, such as the Moiré, Talbot and other identified patterns. We hope that progress in the development of such image processing algorithms for the far-field interactions of a far-field superlens and periodic object arrangements would provide some answers regarding resolving non-periodic objects.

Ultimately, future work should develop a complete setup that utilizes a FSL to image subwavelength objects reliably and in a robust manner. 


\section{References}

[1] J. B. Pendry, "Negative Refraction Makes a Perfect Lens," Phys. Rev. Lett., vol. 85, no. 18, pp. 3966-3969, 2000.

[2] N. Fang and e. al., "Sub-Diffraction-Limited Optical Imaging with a Silver Superlens," Science, vol. 308, p. 534-7, 2005.

[3] D. O. S. Melville and R. J. Blaikie, "Analysis and optimization of multilayer silver superlenses for near-field optical lithography," Physica B: Condensed Matter, vol. 394, no. 2, pp. 197-202, 2007.

[4] K. Aydin, I. Bulu and E. Ozbay, "Subwavelength resolution with a negative-index metamaterial superlens," Appl. Phys. Lett., vol. 90, no. 254102, 2007.

[5] D. O. S. Melville and R. J. Blaikie, "Super-resolution imaging through a planar silver layer," Optics Express, vol. 13, no. 6, p. 2127-2134, 2005.

[6] I. I. Smolyaninov, Y. J. Hung and C. C. Davis, "Magnifying Superlens in the Visible Frequency Range," Science, vol. 315, no. 5819, pp. 1699-1701, 2007. 
[7] A. Ourir, G. Lerosey, F. Lemoult, M. Fink and J. deRosny, "Far field subwavelength imaging of magnetic patterns," Applied Physics Letters, vol. 101, no. 11, 2012.

[8] V. Eckhouse, Z. Zalevsky, N. Konforti and D. Mendlovic, "Subwavelength structure imaging," Optical Engineering, vol. 43, no. 10, 2004.

[9] B. Zheng and e. al., "Broadband subwavelength imaging using non-resonant metamaterials," Applied physics letters, vol. 104, no. 7, p. 073502, 2014.

[10] M. Navarro-Cia and e. al., “Terahertz imaging of subwavelength particles with Zenneck surface waves," Applied physics letters, vol. 103, no. 22, 2013.

[11] M. Choi and A. Badano, "Planar small-angle x-ray scattering imaging of phantoms and biological samples," Appl. Phys. Lett., vol. 110, no. 15, 2017.

[12] S. A. Johnson, "Nanoscopy for nanoscience: how superresolution microscopy extends imaging for nanotechnology," Advanced Review, vol. 7, no. 3, pp. 266-281, 2015.

[13] M. Ting, Molecular Imaging in Nano MRI, John Wiley \& Sons, Inc, 2014.

[14] S. Diao and e. al, "Fluorescence Imaging In Vivo at Wavelengths beyond $1500 \mathrm{~nm}, "$ Communication, vol. 54, no. 49, pp. 1475814762, 2015. 
[15] G. Balasubramanian, A.Lazariev, S. R. Arumugam and D. Duan, “Nitrogen-Vacancy color center in diamond-emerging nanoscale applications in bioimaging and biosensing," Current Opinion in Chemical Biology, vol. 20, pp. 69-77, 2014.

[16] M. Khorasaninejad and e. al., "Metalenses at visible wavelengths: Diffraction-limited focusing and subwavelength resolution imaging," Science, vol. 352, no. 6290, pp. 1190-1194, 2016.

[17] A. Demming, “Scanning probe microscopy: a visionary development," Nanotechnology, vol. 24, no. 290201, 2013.

[18] P. Dvořák and e. al., "Control and near-field detection of surface plasmon interference patterns," Nano Letters, vol. 13, no. 6, pp. 2558-63, 2013.

[19] D. K. Gramotnev and M. W. Vogel, "Ultimate capabilities of sharp metal tips for plasmon nanofocusing, near-field trapping and sensing," Physics Letters A, vol. 375, no. 39, pp. 3464-3468, 2011.

[20] A. Demetriadou, "The impact of natural modes in plasmonic imaging," Scientific Reports, vol. 5, no. 18247, 2015.

[21] H. R. Colenso and e. al, "Comparison of seed layers for smooth, low loss silver films used in ultraviolet-visible plasmonic imaging devices," Thin Solid Films, vol. 656, pp. 68-74, 2018. 
[22] R. F. Gerton, Physical Principles of Electron Microscopy: An Introduction to TEM, SEM, and AEM, Springer Science \& Business Media, 2006.

[23] J. S. Silfies, S. A. Schwartz and M. W. Davidson, "The Diffraction Barrier in Optical Microscopy," [Online]. Available: https://www.microscopyu.com/techniques/super-resolution/thediffraction-barrier-in-optical-microscopy. [Accessed 2007 2018].

[24] L. Novotny, "The History of Near-field Optics," Progress in Optics, vol. 50, pp. 137-184, 2007.

[25] E. Abbe, "Beiträge zur Theorie des Mikroskops und der mikroskopischen Wahrnehmung," Archiv für mikroskopische Anatomie, vol. 9, no. 1, pp. 413-418, 1873.

[26] M. A. Lauterbach, "Finding, defining and breaking the diffraction barrier in microscopy - a historical perspective," Optical Nanoscopy, vol. 1, p. 8, 2012.

[27] M. Spencer, Fundamentals of Light Microscopy, CUP Archive, 1982.

[28] A. S. P. Khare, Engineering Physics: Fundamentals \& Modern Applications, Jones \& Bartlett Learning, 2009.

[29] G. deVilliers and E. R. Pike, The Limits of Resolution, CRC Press, 2016.

[30] B. El-Kareh, Fundamentals of Semiconductor Processing Technology, Springer Science \& Business Media, 2012. 
[31] M. J. Riedl, “Optical Design Fundamentals for Infrared Systems," 2001.

[32] G. Keiser, Biophotonics: Concepts to Applications, Springer, 2016.

[33] S. Ruzin, "Numerical Aperture," [Online]. Available: http://microscopy.berkeley.edu/courses/TLM/optics/na.html. [Accessed 3108 2017].

[34] K. R. Spring, M. J. Parry-Hill and M. W. Davidson, "Numerical Aperture Light Cones," 13112015. [Online]. Available: https://micro.magnet.fsu.edu/primer/java/nuaperture/index.html . [Accessed 2908 2017].

[35] A. Diaspro and M. A. M. J. vanZandvoort, Super-Resolution Imaging in Biomedicine, Series in Cellular and Clinical Imaging, CRC Press, 2016.

[36] U. J. Birk, Super-Resolution Microscopy: A Practical Guide, John Wiley \& Sons, 2017.

[37] Nature-Photonics-editorial, "Beyond the diffraction limit," Nature Photonics, vol. 3, no. 7, p. 361, 2009.

[38] D. Huang, "Optical coherence tomography," Science, vol. 254, no. 5035, pp. 1178-1181, 1991.

[39] D. Saeedkia, Handbook of Terahertz Technology for Imaging, Sensing and Communications, Elsevier, 2013. 
[40] Plunkett, J. W., Plunkett's Nanotechnology \& Mems Industry Almanac 2008: Nanotechnology \& Mems Industry Market Research, Statistics, Trends \& Leading Companies, Plunkett Research Ltd., 2008.

[41] M. J. Crocker, Handbook of Acoustics, John Wiley \& Sons, 1998.

[42] K. G. Foote, "Discriminating between the nearfield and the farfield of acoustic transducers," The Journal of the Acoustical Society of America , vol. 136, no. 1511, 2014.

[43] B. Zhao, B. Guizal, Z. M. Zhang, S. Fan and a. M. Antezza, "Near-field heat transfer between graphene/hBN multilayers," Phys. Rev. B , vol. 95, no. 245437, 2017.

[44] K. Joulain, Y. Ezzahri, J. Drevillon and P. Ben-Abdallah, "Modulation and amplification of radiative far field heat transfer: Towards a simple radiative thermal transistor," Appl. Phys. Lett., vol. 106, no. 133505, 2015.

[45] D. Courjon, Near-Field Microscopy and Near-Field Optics, World Scientific Publishing Company, 2003.

[46] L. Li, Manipulation of Near Field Propagation and Far Field Radiation of Surface Plasmon Polariton, Springer, 2017.

[47] D. R. Smith, "superlens brreaks optical barrier," Physics world, vol. 18, no. 8, pp. 23-24, 2005.

[48] X. C. Tong, Advanced Materials and Design for Electromagnetic Interference Shielding, CRC Press, 2008. 
[49] L. Novotny, "From near-field optics to optical antennas," physics Today, vol. 64, no. 7, pp. 47-52, 2011.

[50] J. Dong and e. al., "Image distance shift effect of the metal superlens and its applications to photolithography," EPL (Europhysics Letters), vol. 102, no. 2, 2013.

[51] N. Fang, "Imaging properties of a metamaterial superlens," Appl. Phys. Lett., vol. 82, no. 161, 2003.

[52] M. Haider, S. Uhlemann, E. Schwan, H. Rose, B. Kabius and K. Urban, Nature, no. 392, pp. 768-769, 1998.

[53] E. Betzig, M. Isaacson, H. Barshatzky, A. Lewis and K. Lin, "super-resolution imaging with near-field scanning optical microscopy (nsom)," Ultramicroscopy, vol. 25, no. 2, pp. 155-164, 1988.

[54] M. Kovar, M. A. Nohe, N. Petersen and P. Norton, "NSOM: Discovering New Worlds," EDU.photonics, University of Western Ontario.

[55] Z. Wang, "Microsphere Super-resolution Imaging," Nanoscience, vol. 3, pp. 193-210, 2016.

[56] A. García-Etxarri and e. al, "Influence of the tip in near-field imaging of nanoparticle plasmonic modes: Weak and strong coupling regimes," Phys. Rev. B, vol. 79, pp. 125439(1-5), 2009.

[57] S. W. Hell, "Toward Fluorescence nanoscopy," Nat. Biotechnol., vol. 21, pp. 1347-1355, 2003. 
[58] M. Troyon, D. Pastré, J. P. Jouart and J. L. Beaudoin, “Scanning near-field cathodoluminescence microscopy," Ultramicroscopy, vol. 75, no. 1, pp. 15-21, 1998.

[59] H. Lee, Z. Liu, Y. Xiong, C. Sun and X. Zhang, "Development of optical hyperlens for imaging below the diffraction limit," vol. 15, no. 24, pp. 15886-15891, 2007.

[60] X. Liu and Y. Zhang, "Metamaterials: a new frontier of science and technology," Chem. Soc. Rev., vol. 40, no. 5, pp. 2494-2507, 2011.

[61] Y. Huang, "Negative refraction in photonic crystals and metamaterials for transformation optics, Northeastern University," Boston (Mass.) : Northeastern University, 2011.

[62] V. G. Veselago, "The electrodynamics of substances with simultaneously negative values of $\varepsilon$ and $\mu$," Soviet Physics Uspekhi, vol. 10, pp. 509-514, 1968.

[63] J. B. Pendry, "Metamaterials and the Control of Electromagnetic Fields," Optical Society of America, p. CMB2, 2007.

[64] F. Capolino, Applications of Metamaterials, Taylor \& Francis, 2009.

[65] L. Venema, "A lensless ordinary," Nature Publishing Group, vol. 420, 2002. 
[66] G. V. Naik, V. M. Shalaev and A. Boltasseva, "Alternative plasmnoic materials: beyond gold and silver," Adv. Mater., vol. 25, no. 24, pp. 3264-3294, 2013.

[67] J. B. Pendry, "Pendry Replies.," Phys Rev Lett, vol. 87, no. 24, p. 249704, 2001.

[68] J. Pendry, "Pendry Replies," Phys Rev Lett, vol. 87, no. 24, p. 249702, 2001.

[69] J. B. Pendry, "Comment on "Left-Handed Materials Do Not Make a Perfect Lens"," Phys Rev Lett, vol. 91, no. 9, p. 099701, 2003.

[70] G. W. T. Hooft, "Comment on "Negative Refraction Makes a Perfect Lens," Phys Rev Lett, vol. 87, no. 24, p. 249701, 2001.

[71] J. M. Williams, "Some Problems with Negative Refraction," Phys Rev Lett, vol. 87, no. 24, p. 249703, 2001.

[72] M. Nieto-Vesperinas and N. Garcia, "Nieto-Vesperinas and Garcia Reply," Phys Rev Lett, vol. 91, no. 9, p. 099702, 2003.

[73] P. V. Parimi, W. T. Lu, P. Vodo and S. Sridhar, "Photonic crystals: Imaging by flat lens using negative refraction," Nature, vol. 426, p. 404, 2003.

[74] D. Melville and R. Blaikie, "Super-resolution imaging through a planar silver layer," Optics Express, vol. 13, no. 6, p. 2127-2134, 2005. 
[75] Z. Jacob and e. al., "Optical Hyperlens: Far-field imaging beyond the diffraction limit," Optics Express, vol. 14, no. 18, p. 8247-56, 2006.

[76] Z. Liu and e. al., "Far-Field Optical Hyperlens Magnifying SubDiffraction-Limited Objects," Science, vol. 315, p. 1686, 2007.

[77] A. Engheta and N. Salandrino, "Far-field subdiffraction optical microscopy using metamaterial crystals: Theory and simulations," Phys. Rev. B, vol. 74, pp. 075103 (1-5), 2006.

[78] D. Lu and Z. Liu, "Hyperlenses and metalenses for far-field super-resolution imaging," Nat. Commun., vol. 3, pp. 1205(1-9), 2012.

[79] K. Elsayad and K. G. Heinze, "Defining a Superlens Operating Regime for Imaging Fluorescent Molecules," Plos one, vol. 4, no. 12, pp. 1-7, 2009.

[80] W. Cai, D. A. Genov and V. Shalaev, "A Superlens Based on Metal-Dielectric Composites," PhysRevB., vol. 72, no. 193101, 2005.

[81] S. Durant, Z. Liu, N. Fang and X. Zhang, "Theory of optical imaging beyond the diffraction limit with a far-field superlens," in SPIE Optics + Photonics, San Diego, 2006.

[82] N. V. Selina and E. N. Tumayev, "Propagation of Electromagnetic Wave in Pendry Lens," Nanotechnologies in Russia, vol. 11, no. 5-6, pp. 349-354, 2016. 
[83] J. D. Jackson, Classical Electromagnetics, New York: John Wiley \& Sons, 1999.

[84] B. Klein, "Maxwell's Equations and the Helmholtz Wave Equation," [Online]. Available: http://bklein.ece.gatech.edu/laser-photonics/maxwells-equationsand-the-helmholtz-wave-equation/. [Accessed 151 2018].

[85] M. Schwartz, "Lecture 13: Electromagnetic waves," [Online]. Available:

http://users.physics.harvard.edu/ schwartz/15cFiles/Lecture13Light.pdf. [Accessed 2007 2018].

[86] ETH-Zurich-edu, "Chapter 2, The Wave Equation," [Online]. Available: https://www.photonics.ethz.ch/fileadmin/user_upload/Courses/ EM_FieldsAndWaves/WaveEquation.pdf. [Accessed 2007 2018].

[87] R. W. Ziolkowski and E. Heyman, "Wave propagation in media having negative permittivity and permeability," Physical Review E, vol. 64, pp. 056625(1-15), 2001.

[88] E. C. LeRu and P. G. Etchegoin, Principles of Surface-Enhanced Raman Spectroscopy : And Related Plasmonic Effects, Elsevier Science \& Technology, 2008.

[89] X. Wei and P. Mulvaney, "Modern Plasmonics," in andbook of Surface Science, Elsevier B.V., 2014, pp. 75-108.

[90] K. Liu, “The Optical Society Blog," 155 2014. [Online]. Available: http://www.osa.org/en- 
us/the_optical_society_blog/2014/may_2014/fundamental_and_p hysical_limitations_of_opto-elect/. [Accessed 2510 2016].

[91] M. C. Roco, C. A. Mirkin and M. C. Hersam, Nanotechnology Research Directions for Societal Needs in 2020: Retrospective and Outlook, Springer Science \& Business Media, 2011.

[92] P. R. West, S. Ishii, G. V. Naik, N. K. Emani and V. M. Shalaev, "Searching for better plasmonic materials," Laser Photonics Rev., vol. 4, no. 6, pp. 795-808, 2010.

[93] P. K. Jain, X. Huang, I. H. El-Sayed and M. A. El-Sayed, "Review of Some Interesting Surface Plasmon Resonance-enhanced Properties of Noble Metal Nanoparticles and Their Applications to Biosystems," Plasmonics, vol. 2, no. 3, pp. 107-118, 2007.

[94] R. P. H. Kooyman, "Chapter 2, Physics of Surface Plasmon Resonance," in Handbook of Surface Plasmon Resonance, The Royal Society of Chemistry, 2008, pp. 15-34.

[95] J. Friedel, "The Absorption of Light by Noble Metals and its Relation to the van der Waals Contribution to the Cohesive Energy," Proceedings of the Physical Society. Section B, vol. 65, no. 10, pp. 769-774, 1952.

[96] The-British-Museum, "The Lycurgus Cup," [Online]. Available: http://www.britishmuseum.org/research/collection_online/collec tion_object_details.aspx?objectId=61219\&partId=1\&searchText=1 ycurgus+cup\&page $=1$. [Accessed 2607 2018]. 
[97] S. Maier, "Plasmonics: Fundamentals and Applications," in Surface Plasmon Polaritons at Metal / Insulator Interfaces, New York, NY, Springer, 2007, pp. 21-37.

[98] J. M. Sanz and e. al., "UV Plasmonic Behavior of Various Metal Nanoparticles in the Near- and Far-Field Regimes: Geometry and Substrate Effects," J. Phys. Chem. C, vol. 38, no. 117, p. 1960619615, 2013.

[99] G. Gu, X. Lu, T. Kemsri and Y. Zhang, "Surface Plasmonics and Its Applications in Infrared Sensing," in Nanoplasmonics, Fundamentals and Applications, IntechOpen, 2017, pp. 195-215.

[100] Y. Gutiérrez, R. A. Osa, D. Ortiz, J. M. Saiz, F. González and F. Moreno, "Plasmonics in the Ultraviolet with Aluminum, Gallium, Magnesium and Rhodium," Appl. Sci., vol. 8, no. 64, pp. $1-14,2018$.

[101] G. Shvets and I. Tsukerman, Plasmonics and Plasmonic Metamaterials: Analysis and Applications, World Scientific, 2012.

[102] J. R. Reitz, F. J. Milford and R. W. Christy, Foundations of Electromagnetic Theory, Addison-Wesley, 1993.

[103] A. Stalmashonak, G. Seifert and A. Abdolvand, "Ultra-Short Pulsed Laser Engineered Metal-Glass Nanocomposites," in SpringerBriefs in Physics, Springer International Publishing, 2013, pp. 5-15. 
[104] H. Horvath, “Journal of Quantitative Spectroscopy \& Radiative Transfer, vol. 110," p. 787-799, 2009.

[105] G. Mie, "Beitra“ge zur Optik tru"ber Medien speziell kolloidaler Goldlo“ sungen," Ann Phys, vol. 25, pp. 377-445, 1908.

[106] A. Boltasseva and H. A. Atwater, "Low-Loss Plasmonic Metamaterials," Science, vol. 331, no. 6015, pp. 290-291, 2011.

[107] J. Harrington, Infrared Fibers and Their Applications, Bellingham, WA: SPIE Press, 2003.

[108] R. Singh, "Low and High Dielectric Constant Materials: Materials Science, Processing, and Reliability Issues : Proceedings of the Fourth International Symposium : And, Thin Film Materials for Advanced Packaging Technologies," Electrochemical Society, vol. 99, no. 7, 2000.

[109] X. Wang and e. al., "Excitation and propagation of surface plasmon polaritons on a non-structured surface with a permittivity gradient," Light: Science $\mathcal{E}$ Applications, vol. 5, pp. e16179(1-6), 2016.

[110] A. P. Knight and G. T. Reed, Silicon photonics: an introduction, John Wiley and Sons, SBN 0-470-87034-6., 2004.

[111] X. Luo, Y. Lv, C. Du, J. Ma, H. Wang, H. Li, G. Yang, X. Lin and H. Yao, "Nanoscopy of near-field distribution on plasmonic nanostructures," J. Vac. Sci. Technol. B, vol. 24, no. 1, pp. L1-L5, 2006. 
[112] A. Castanie, D. Felbacq and B. Guizal, "Surface Plasmon Guided Mode strong coupling," Advanced Electromagnetics, vol. 1, no. 2, pp. 85-88, 2012.

[113] A. V. Zayats, I. I. Smolyaninov and A. A. Maradudin, "Nanooptics of surface plasmon polaritons," Physics Reports, vol. 408, pp. 131-314, 2005.

[114] L. Novotny and B. Hecht, Principles of nano-optics, New York: Cambridge University press, 2006.

[115] O. Benson, “Chapter 7, Plasmonics," [Online]. Available: https://www.physik.huberlin.de/de/nano/lehre/Gastvorlesung\%20Wien/plasmonics. [Accessed 0808 2018].

[116] E. G. Lewen and E. Popov, Diffraction Gratings and Applications (Optical Science and Engineering), CRC Press, 1997.

[117] T. K. Gaylord and M. G. Moharam, "Analysis and Applications of Optical Diffraction by Gratings," PROCEEDINGS OF THE IEEE, vol. 73, no. 5, pp. 894-937, 1985.

[118] E. Popov, Gratings: Theory and Numeric Applications, Institut Fresnel, CNRS, AMU, 2012.

[119] Shimzo-Corp., "The Grating Equations," [Online]. Available: https://www.shimadzu.com/opt/guide/diffraction/03.html.

[Accessed 3107 2018]. 
[120] M. Zourob and A. Lakhtakia, Optical Guided-wave Chemical and Biosensors II, Springer Science \& Business Media, 2010.

[121] C. Ye and T. K. Wei, Tunable External Cavity Diode Lasers, World Scientific, 2004.

[122] O. K. Ersoy, Diffraction, Fourier Optics and Imaging, John Wiley \& Sons, 2006.

[123] E. L. C. Palmer, Diffraction Grating Handbook- Chapter 1, New York: Newpor Corporation, 2005.

[124] M. Born, E. Wolf and A. B. Bhatia, Principles of Optics: Electromagnetic Theory of Propagation, Interference and Diffraction of Light, Cambridge University Press, 1999.

[125] M. A. Ahmed, M. Haefner, M. Vogel, C. Pruss, A. Voss, W. Osten and T. Graf, "High-power radially polarized Yb:YAG thin-disk laser with high efficiency," Opt. Exp., vol. 19, no. 6, pp. 50935103, 2011.

[126] R. C. Rumpf, “Lecture 9, Diffraction Gratings," 2282018. [Online]. Available: https://empossible.net/wpcontent/uploads/2019/08/Lecture-9-Diffraction-gratings.pdf. [Accessed 210 2018].

[127] J. B. Pendry and D. R. Smith, "The quest for the superlens," Scientific American, pp. 60-67, 2006.

[128] J. B. Pendry, A. J. Holden, D. J. Robbins and W. J. Stewart, "Magnetism from Conductors and Enhanced Nonlinear 
Phenomena," IEEE Transactions on Microwave Theory and Techniques, vol. 47, no. 11, pp. 2075-2084, 1999.

[129] N. Engheta and R. W. Ziolkowski, Metamaterials: Physics and Engineering Explorations, John Wiley \& Sons, 2006.

[130] J. B. Pendry, "Negative refraction," Contemporary Physics, vol. 45, no. 3, pp. 191-202, 2004.

[131] G. V. Eleftheriades, "Enabling RF/microwave devices using negative-refractive-index transmission-line

(NRI-TL) metamaterials," IEEE Antennas and Propagation Magazine, vol. 49, no. 2, pp. 34 - 51, 2007.

[132] Y. S. Zhou, H. Y. Wang and H. Wang, "Zero refraction in natural materials and the mechanism of metal superlens," $E P L$ (Europhysics Letters), vol. 98, no. 6, p. 67005 (4pp), 2012.

[133] W. Adams, M. Sadatgol and D. Ö. Güney, "Review of near-field optics and superlenses for sub-diffraction-limited nanoimaging," AIP Advances, vol. 6, pp. 100701(1-17), 2016.

[134] J. Pendry, "Superlenses to overcome the diffraction limit," Nature Materials, vol. 7, pp. 435-441, 2008.

[135] H. Lee, Y. Xiong, N. Fang, W. Srituravanich, S. Durant, M. Ambati and X. Z. C. Sun, "Realization of Optical superlens imaging below the diffraction limit," New Journal of Physics, vol. 7, no. 255, pp. 1-16, 2005. 
[136] X. Zhang and Z. Liu, "Superlenses to overcome the diffraction limit," Nature Materials , vol. 7, pp. 435-441, 2008.

[137] L. Feng and e. al., “Metamaterials for Enhanced Polarization Conversion in Plasmonic Excitation," ACS Nano, vol. 5, no. 6, pp. 5100-5106, 2011.

[138] H. A. Atwater and A. Polman, "Plasmonics for improved photovoltaic devices," Nature Materials, vol. 9, pp. 205-213, 2010.

[139] A. Grbic. and G. V. Eleftheriades, "Overcoming the Diffraction Limit with a Planar Left-Handed Transmission-Line Lens," Phys. Rev. Lett., vol. 92, p. 117403, 2004.

[140] T. Taubner, D. Korobkin, Y. Urzhumov, G. Shvets and R. Hillenbrand, "Near-field microscopy through a SiC superlens," Science, vol. 313, p. 1595, 2006.

[141] A. N. Kissel and V. N. Lagarkov, "Near-Perfect Imaging in a Focusing System Based on a Left-Handed-Material Plate," Phys. Rev. Lett., vol. 92, no. 077401, 2004.

[142] Z. Liu and e. al., "Far-Field Optical Superlens," Nano Letters, vol. 7, no. 2, pp. 403-408, 2007.

[143] B. Foster, "Superresolution: Reality or a "STORM" in a Teacup?," Photonics media, Jan 2011. [Online]. Available: http://www.photonics.com/Article.aspx?AID=45696. [Accessed Oct 2016]. 
[144] Y. Nakano and K. Murata, "Talbot interferometry for measuring the focal length of a lens," Applied optics, vol. 24, no. 19, pp. 31623166, 1985.

[145] B. A. Parkinsona, F. S. Ohuchi, K. Ueno and A. Koma, "Periodic lattice distortions as a result of lattice mismatch in epitaxial films of two-dimensional materials," Appl. Phys. Lett. , vol. 58, no. 5, 1991.

[146] M. S. Kim, T. Scharf, C. Menzel, C. Rockstuhl and H. P. Herzig, "Talbot images of wavelength-scale amplitude gratings," Opt. Exp., vol. 20, no. 5, pp. 4903-4920, 2012.

[147] M. Berry, I. Marzoli and W. Schleich, "Quantum carpets, carpets of light," Phys. World, vol. 14, no. 6, pp. 39-46, 2001.

[148] A. W. Lohmann and D. E. Silva, "An interferometer based on the Talbot effect," Opt. Commun, vol. 2, no. 9, pp. 413-415, 1971.

[149] J. Wen, Y. Zhang and M. Xiao, "The Talbot effect: recent advances in classical optics, nonlinear optics, and quantum optics," Advances in Optics and Photonics, vol. 5, no. 1, pp. 83-130, 2013.

[150] E. L. Hu, M. Brongersma and A. Baca, "Applications: Nanophotonics and Plasmonics," in Nanotechnology Research Directions for Societal Needs in 2020: Retrospective and Outlook, Dordrecht, Springer Netherlands, 2011, pp. 417-444.

[151] H.-S. C. Er-Ping Li, Plasmonic Nanoelectronics and Sensing, Cambridge University Press, 2014. 
[152] Comsol, "The Finite Element Method (FEM)," COMSOL, 153 2016.

[Online].

Available:

https://www.comsol.com/multiphysics/finite-element-method.

[Accessed 137 2018].

[153] J. M. Jin, Theory and Computation of Electromagnetic Fields, John Wiley \& Sons, 2010.

[154] J. Leng, Handbook of Research on Computational Science and Engineering: Theory and Practice: Theory and Practice, Volume 2, IGI Global, 2011.

[155] Comsol, "Building the Mesh for a Model Geometry in COMSOL Multiphysics®," [Online]. Available: https://www.comsol.com/video/building-the-mesh-for-a-modelgeometry-in-comsol-multiphysics. [Accessed 0408 2018].

[156] Y. C. Liu, K. Chang, J. Fwu and L. Shih, "Performance comparison between FDTD and FEM for the simulation of plasmonic waveguide operating at optical communication frequency," in 2013 USNC-URSI Radio Science Meeting (Joint with AP-S Symposium), Lake Buena Vista, FL, 2013.

[157] T. Grosges, A. Vial and D. Barchiesi, "Models of near-field spectroscopic studies: comparison between Finite-Element and Finite-Difference methods," Opt. Express, vol. 13, no. 21, pp. 8483-8497, 2005.

[158] E. Süli and D. F. Mayers, An Introduction to Numerical Analysis, Cambridge University Press, 2003. 
[159] P. J. S. Cruz, Structures and Architecture: New concepts, applications and challenges, CRC Press, 2013.

[160] M. Tshilidzi, Finite Element Model Updating Using Computational Intelligence Techniques, London: SpringerVerlag, 2010.

[161] Y. Yan, H. Wang and M. Wan, "FEM modelling for press bend forming of doubly curved integrally stiffened aircraft panel," Transactions of Nonferrous Metals Society of China, vol. 22, no. 1, pp. S39-S47, 2012.

[162] T. Subramani and D. S. Kumar, "Fem Modelling and Analysis of Reinforced Concrete Section with Light Weight Blocks Infill," International Journal of Engineering Research and Applications, vol. 4, no. 6, pp. 142-149, 2014.

[163] S. Klink, D. Hoeche, F. L. Mantia, W. Schuhmann and S. Klink, "FEM modelling of a coaxial three-electrode test cell for electrochemical impedance spectroscopy in lithium ion batteries," Journal of Power Sources, vol. 240, pp. 273-280, 2013.

[164] H. Gothall, "How to Inspect Your Mesh in COMSOL Multiphysics®," $12052017 . \quad$ [Online]. Available: https://www.comsol.com/blogs/how-to-inspect-your-mesh-incomsol-multiphysics/. [Accessed 0608 2018].

[165] D. Roylance, "Finite Element Analysis," 28 Feb 2001. [Online]. Available: $\quad$ https://ocw.mit.edu/courses/materials-science-andengineering/3-11-mechanics-of-materials-fall- 
1999/modules/MIT3_11F99_fea.pdf.

[Accessed

MIT

OpenCourseWare].

[166] W. Frei, "Computational Electromagnetics Modeling, Which Module to Use?," Comsol, 109 2013. [Online]. Available: https://www.comsol.com/blogs/computational-electromagneticsmodeling-which-module-to-use/. [Accessed 137 2018].

[167] W. Frei, "Guide to Frequency Domain Wave Electromagnetics Modeling," comsol, $3062015 . \quad$ [Online]. Available: https://www.comsol.com/blogs/guide-to-frequency-domainwave-electromagnetics-modeling/. [Accessed 137 2018].

[168] B. Nine, "How to Implement a Mesh Refinement Study," 0608 2013. [Online]. Available: https://www.comsol.com/blogs/howto-implement-mesh-refinement-study/. [Accessed 0608 2018].

[169] Comsol, "Mesh and Geometry," [Online]. Available: https://www.comsol.com/release/5.2/mesh-and-geometry. [Accessed 0608 2018].

[170] J. Y. Wu and e. al., "The Advantages of Triangular and Tetrahedral Edge Elements for Electromagnetic Modeling with the Finite-Element Method," IEEE Transactions on Antennas and Propagation, vol. 45, no. 9, pp. 1431-1437, 1997.

[171] Y. Zhang and C. Bajaj, "Adaptive and Quality Quadrilateral/Hexahedral Meshing from Volumetric Data," Comput Methods Appl Mech Eng., vol. 195, no. 9, p. 942-960, 2006. 
[172] Comsol, “Comsol Multiphysics, User's Guide 4.3," 2012. [Online]. Available: http://people.ee.ethz.ch/ fieldcom/ppscomsol/documents/User\%20Guide/COMSOLMultiphysicsUsers Guide.pdf. [Accessed 201610 30].

[173] B. Bretz, "Improved Capabilities for Meshing with Tetrahedral Elements," $27 \quad 92016 . \quad$ [Online]. Available: https://www.comsol.com/blogs/improved-capabilities-formeshing-with-tetrahedral-elements/. [Accessed 228 2017].

[174] I. Kjelberg, " Unstructured and structured mesh in comsol," 30 08 2015. [Online]. Available: https://www.comsol.com/community/forums/general/thread/891 73/. [Accessed 2208 2017].

[175] C. Liu, "Discretizing the Weak Form Equations," 09022015. [Online]. Available: https://www.comsol.com/blogs/discretizingthe-weak-form-equations/. [Accessed 2208 2017].

[176] A. Datta and V. Rakesh, An Introduction to Modeling of Transport Processes: Applications to Biomedical Systems, New York: Cambridge University Press, 2010.

[177] M. Bern and P. Plassmann, "Mesh Generation," in Handbook of Computational Geometry, Elsevier, 2000, pp. 291-332.

[178] Comsol, "COMSOL multiphysics 5.2 release highlights, Mesh and Geometry," [Online]. Available: https://www.comsol.com/release/5.2/mesh-and-geometry. [Accessed 2208 2017]. 
[179] E. J. Kaiser, "Two simultaneous COMSOL instances on one machine can save time," $15 \quad 8$ 2014. [Online]. Available: https:/www.comsol.com/forum/thread/46779/twosimultaneous-comsol-instances-on-one-machine-can-save-time. [Accessed 137 2018].

[180] ECS-EResearch, "Science Faculty HPC Facility," $28 \quad 102016$. [Online]. Available: https://ecs.victoria.ac.nz/EResearch/ScienceFacultyHPCFacility. [Accessed 3110 2016].

[181] Comsol, "computing the Radar Cross Section of a Perfectly Conducting Sphere, COMSOL Multiphysics 5.2a," [Online]. Available: https://www.comsol.com/model/download/340371/models.rf.rcs _sphere.pdf. [Accessed 1907 2018].

[182] D. Darmofal, "Lecture 12, The Finite Element Method for OneDimensionla Diffusion," $2005 . \quad$ [Online]. Available: https://ocw.mit.edu/courses/aeronautics-and-astronautics/16901-computational-methods-in-aerospace-engineering-spring2005/lecture-notes/lect12.pdf. [Accessed 2016 Oct 30].

[183] W. Frei, "How Much Memory Is Needed to Solve Large COMSOL Models?," $24 \quad 10 \quad 2014 . \quad$ [Online]. Available: https://www.comsol.com/blogs/much-memory-needed-solvelarge-comsol-models/. [Accessed 111 2016].

[184] Comsol, "What does degrees of freedom (DOFs) mean in COMSOL Multiphysics?," [Online]. Available: 
https://www.comsol.com/support/knowledgebase/875/.

[Accessed 1208 2018].

[185] Comsol, “Augment Your COMSOL Multiphysics® Models with Material Properties from the Material Library," [Online]. Available: https://www.comsol.com/material-library. [Accessed 0608 2018].

[186] Comsol, "Comsol Products," [Online]. Available: https://www.comsol.com/products. [Accessed 0608 2018].

[187] Comsol, "Dedicated Multiphysics Node Introduced in COMSOL 4.4," $292013 . \quad$ [Online]. Available: https://www.comsol.com/blogs/dedicated-multiphysics-nodeintroduced-in-comsol-4-4/. [Accessed 0608 2018].

[188] Comsol, "Essentials of Postprocessing and Visualization in Comsol Multiphysics," $\quad$ [Online]. Available: https://www.comsol.com/zmags/essentials-of-postprocessingand-visualization. [Accessed 0608 2018].

[189] H. Lee, Z. Liu, Y. Xiong, C. Sun and X. Zhang, "Design, fabrication and characterization of a Far-field Superlens," Solid States Communications, vol. 146, pp. 202-207, 2008.

[190] Comsol, "RF Module user's guide, Introduction," Comsol Multyphysics, 2018.

[191] AllresistGmbH, "UV-patterning of PMMA resists," [Online]. Available: $\quad$ http://www.allresist.com/photorestist-other-resistsuv-patterning-of-pmma-resist/. [Accessed 0108 2018]. 
[192] Microchem, "Nano PMMA and Copolymer Data Sheet," [Online].

Available:

http://microchem.com/pdf/PMMA_Data_Sheet.pdf. [Accessed 01 08 2018].

[193] P. B. Johnson and R. W. Christy, "Optical Constants of the Noble Metals," Phys. Rev. B, vol. 6, no. 12, pp. 4370-4379, 1972.

[194] K-Patent-Process-Instruments, "Refractive Index, measurement principle," [Online]. Available: http://www.kpatents.com/assets/files/downloads/refractive_inde x_principle.pdf. [Accessed 310 2018].

[195] C. Zhao, Y. S. Zhou, Y. Zhang and H. Y. Wang, "The imaging properties of the metal superlens," Opt. Commun., vol. 368, pp. 180-184, 2016.

[196] P. Li and T. Taubner, "Multi-wavelength polar-dielectric superlens and graphene superlens," Opt. Exp. , vol. 20, no. 11, pp. 11787-95, 2012.

[197] C. P. Moore, “Optical Superlenses: Quality and Fidelity in SilverDielectric Near-Field Imaging Systems," University of Canterbury, Christchurch, 2011.

[198] F. F. Masouleh, P. Teal and C. Moore, "Spatial frequency characterisation of a far-field superlens to facilitate general purpose imaging," in Proceedings Volume 9889, Optical Modelling and Design IV; 98890I, Brussels, 2016. 
[199] "Multiphysics, Introduction to COMSOL, Version 5.0," 2014. [Online]. Available: http://www.comsol.com/.

[200] Nikon, “What is moiré?," 132 2017. [Online]. Available: https://www.nikonimgsupport.com/ni/NI_article?articleNo=0000 01760\&configured=1\&lang=en_US. [Accessed 2508 2018].

[201] J. Vangindertael, R. Camacho, W. Sempels, H. Mizuno, P. Dedecker and K. P. F. Janssen, "An introduction to optical superresolution microscopy for the adventurous biologist," Methods and Applications in Fluorescence, vol. 6, no. 2, pp. 022003(1-54), 2018.

[202] M. Kim, T. Scharf, C. Menzel, C. Rockstuhl and a. H. P. Herzig, “ Talbot images of wavelength-scale amplitude gratings," Opt. Express, vol. 20, no. 5, 2012.

[203] M. S. Kim, T. Scharf, C. Menzel, C. Rockstuhl and H. P. Herzig, "Phase anomalies in Talbot light carpets of self-images," vol. 21, no. 1, pp. 1287-1300, 2013.

[204] A. Macleod, "'Surface Plasmons: Fundamentals Part 1," Bulletin," Bulletin, pp. 24-29, 2011.

[205] T. Simonite, "Superlens' has its reach extended," 1222007. [Online]. Available: https://www.newscientist.com/article/dn11082-superlens-has-itsreach-extended/. [Accessed 1711 2018].

[206] C. Jeppesen, R. B. Nielsen, A. Boltasseva, S. Xiao, N. A. Mortensen and A. Kristensen, "Thin film Ag superlens towards 
lab-on-a-chip integration," Opt. Express, vol. 17, no. 25, pp. 22543-22552, 2009.

[207] T. Scharf, M. S. Kim and H. P. Herzig, "Measuring amplitude and phase of light emerging from microstructures with HRIM," in Optical Measurement Systems for Industrial Inspection VII, Vol. 8082, 80821O, 2011.

[208] W. Frei, “Using Perfectly Matched Layers and Scattering Boundary Conditions for Wave Electromagnetics Problems," 28 $12015 . \quad$ [Online]. Available:

https://www.comsol.com/blogs/using-perfectly-matched-layersand-scattering-boundary-conditions-for-wave-electromagneticsproblems/. [Accessed 292 2019].

[209] C. B. Wiebel, M. Singh and M. Maertens, "Testing the role of Michelson contrast for the perception of surface lightness Christiane B. Wiebel," Journal of Vision, vol. 16, no. 11, pp. 1-19, 2016.

[210] Comsol, “Wave Optics Module, User's guide, version 5.3," Comsol, 2018.

[211] C. Antonio, Epioptics-9 - Proceedings Of The 39th Course Of The International School Of Solid State Physics, World Scientific, 2008.

[212] E. Y. Tsymbal, "Section3: Electromagnetic Waves 1," [Online]. Available:

https://unlcms.unl.edu/cas/physics/tsymbal/teaching/EM- 
914/section3-Electromagnetic_Waves_1.pdf. [Accessed 1309 2018].

[213] S. Landis, Nano Lithography, John Wiley \& Sons, 2013.

[214] A. J. Wood, B. Chen, S. Pathan, S. Bok, C. J. Mathai, K. Gangopadhyay, S. A. Grant and S. Gangopadhyay, "Influence of silver grain size, roughness, and profile on the extraordinary fluorescence enhancement capabilities of grating coupled surface plasmon resonance," RSC Adv., vol. 5, pp. 78534-78544, 2015.

[215] S. Roh, T. Chung and B. Lee, "Overview of the Characteristics of Micro- and Nano-Structured Surface Plasmon Resonance Sensors," Sensors, vol. 11, no. 2, pp. 1565-1588, 2011.

[216] M. Jarrell, “Plane Waves and Wave Propagation," 2001. [Online]. Available:

http://www.phys.lsu.edu/ jarrell/COURSES/ELECTRODYNAMI CS/Chap7/chap7.pdf. [Accessed 1309 2018].

[217] W. Feri, "Modeling Electromagnetic Waves and Periodic $\begin{array}{lllll}\text { Structures," } & 17 & 1 & 2014 . & \text { [Online]. Available: }\end{array}$ https://www.comsol.com/blogs/modeling-electromagneticwaves-periodic-structures/. [Accessed 1309 2018].

[218] C. D. Geddes, Reviews in Plasmonics 2016, Springer, 2017.

[219] I. Pastoriza-Santos, C. Kinnear, J. Perez-Juste, P. Mulvaney and L. M. Liz-Marzan, "Plasmonic polymer nanocomposites," Nature Reviews Materials , 2018. 
[220] Z. Liu, S. Durant, H. Lee, Y. Pikus, Y. Xiong, C. Sun and X. Zhang, "Experimental studies of far-field superlens for subdiffractional optical imaging," Opt. Express, vol. 15, no. 11, pp. 6947-54, 2007.

[221] M. Jiang, C. L. Wyatt and G. Wang, "X-Ray Phase-Contrast Imaging with Three 2D Gratings," International Journal of Biomedical Imaging, Hindawi Publishing Corporation, vol. doi:10.1155/2008/827152, p. 8 pages, 2008.

[222] M. L. Grilli, A. Sytchkova, S. Boycheva and A. Piegari, “Transparent and conductive Al-doped $\mathrm{ZnO}$ films for solar cells applications," Phys. Status Solidi A, vol. 210, no. 4, pp. 748-754, 2013.

[223] Nature-Editorial, "Beyond the diffraction limit," Nature Photonics, vol. 3, p. 361, 2009.

[224] P. N. Kumar, M. Deepa and A. K. Srivastava, "Ag plasmonic nanostructures and a novel gel electrolyte in a high efficiency TiO2/CdS solar cell," Phys. Chem. Chem. Phys., vol. 17, pp. 1004010052, 2015.

[225] C. A. DiMarzio, "Optics for Engineers," in CRC Press, 2011.

[226] W. Cai and V. Shalaev, Optical Metamaterials: Fundamentals and Applications, Springer Science \& Business Media, 2009.

[227] G. Manickam, R. Gandhiraman, R. K. Vijayaraghavan, L. Kerr, C. Doyle, D. E. Williams and S. Daniels, "Protection and functionalisation of silver as an optical sensing platform for 
highly sensitive SPR based analysis," Analyst, vol. 137, no. 22, pp. 5265-5271, 2012.

[228] W. S. Chang, B. A. Willingham, L. S. Slaughter, B. P. Khanal, L. Vigderman, E. R. Zubarev and S. Link, "Low absorption losses of strongly coupled surface plasmons in nanoparticle assemblies," Proc Natl Acad Sci U S A, vol. 108, no. 50, pp. 19879-19884, 2011.

[229] T. Stefaniuk, P. Wróbel, E. Górecka and T. Szoplik, “Optimum deposition conditions of ultrasmooth silver nanolayers," Nanoscale Res Lett., vol. 9, no. 1, pp. 1-9(153), 2014.

[230] W. J. Lee, J. E. Kim, H. Y. Park and M. H. Lee, "Silver superlens using antisymmetric surface plasmon modes," Opt. Exp., vol. 18, no. 6, pp. 5459-5465, 2010.

[231] D. Jing, B. Unal, F. L. Qin, C. Yuen, J. W. Evans, C. J. Jenks, D. J. Sordelet and P. A. Thiel, "Stranski-Krastanov-like growth of an Ag film on a metallic glass," Thin Solid Films, vol. 24, no. 517, p. 6486-6492, 2009.

[232] Z. Liu, S. Durant, H. Lee, Y. Pikus, N. Fang, Y. Xiong, C. Sun and X. Zhang, "Far-Field Optical Superlens," Nano Let., vol. 7, no. 2, pp. 403-408, 2007.

[233] D. Nga, N. Lan and N. Viet, "Second Quantization Model of Surface Plasmon Polariton at Metal Planar Surface," Journal of Physics: Conference Series, vol. 627, no. 012018, 2015.

[234] C. Wang, W. Zhang, Z. Zhao, Y. Wang, P. Gao, Y. Luo and X. Luo, "Plasmonic Structures, Materials and Lenses for Optical 
Lithography beyond the Diffraction Limit: A Review," Micromachines, vol. 7, no. 7, pp. 118(1-33), 2016.

[235] G. V. Naik and A. Boltasseva, "A comparative study of semiconductor-based plasmonic metamaterials," Metamaterials, vol. 5, no. 1, pp. 1-7, 2011.

[236] Photonics-Media, "Simplified Superlens Captures IR Light," 31 March 2011. [Online]. Available: http://www.photonics.com/Article.aspx?AID=46533.

[237] M. Fehrenbacher and e. al., "Plasmonic Superlensing in Doped GaAs," Nano Lett., vol. 15, no. 2, p. 1057-1061, 2015.

[238] G. V. Naik and A. Boltasseva, "Semiconductors for plasmonics and metamaterials," vol. 4, no. 10, pp. 295-297, 2010.

[239] G. V. Naik, J. Kim and A. Boltasseva, “Oxides and nitrides as alternative plasmonic materials in the optical range," Opt. Mater. Express, vol. 1, pp. 1090-1099, 2011.

[240] F. Ren, Compound Semiconductor Power Transistors and: Stateof-the-Art Program on Compound Semiconductors, The Electrochemical Society, 1998.

[241] H. Hosono and K. Ueda, Transparent Conductive Oxides, Springer Handbook of Electronic and Photonic Materials, Springer, 2017.

[242] S. Szunerits and R. Boukherroub, Introduction to Plasmonics: Advances and Applications, CRC Press, 2015. 
[243] P. R. West, S. Ishii, G. Naik, N. Emani, I. M. Shalaev and A. Boltasseva, "Searching for Better Plasmonic Materials," Laser and Photonics Review, vol. 4, no. 6, pp. 795-808, 2010.

[244] H. Hogan, "Putting more than glass in the toolbox," Photonics Spectra, Laurin Publishing , 2012.

[245] G. Naik, J. Liu, A. V. Kildishev, V. M. Shalaev and A. Boltasseva, “Demonstration of $\mathrm{Al}: \mathrm{ZnO}$ as a plasmonic component for nearinfrared metamaterials," Proceddings of the Nationla Academy of Science of the United States of America, vol. 109, no. 23, p. 88348838, 2012.

[246] A. Sytchkova, M. L. Grilli, A. Rinaldi, S. Vedraine, P. Torchio, A. Piegari and F. Flory, "Radio frequency sputtered Al:ZnO-Ag transparent conductor: A plasmonic nanostructure with enhanced optical and electrical properties," physica status solidi (a), vol. 210, pp. 748-754, 2013.

[247] k. Omae, M. Nakano, A. Tanaka, M. Hirata, T. Hamaguchi and T. Chonan, "Indium lung-case reports and epidemiology," International Archives of Occupational and Environmental Health, vol. 84, no. 5, pp. 471-477, 2011.

[248] K. J. Cummings and e. al., "Indium Lung Disease," vol. 141, no. 6, pp. 1512-1521, 2012.

[249] R. Buonsanti, A. Llordes, S. Aloni, B. A. Helms and D. J. Milliron, “Tunable Infrared Absorption and Visible Transparency of 
Colloidal Aluminum-Doped Zinc Oxide Nanocrystals," Nano Letters, vol. 11, no. 11, p. 4706-4710, 2011.

[250] M. Chen, Z. L. Pei, C. Sun, J. Gong, R. F. Huang and L. S. Wen, "ZAO: an attractive potential substitute for ITO in flat display panels," Mat. Sci. and Eng. B, vol. 85, no. 2-3, pp. 212-217, 2001.

[251] M. C. Jun, S. U. Park and J. H. Koh, "Comparative studies of Aldoped $\mathrm{ZnO}$ and Ga-doped $\mathrm{ZnO}$ transparent conducting oxide thin films," Nanoscale Res. Lett., vol. 7, no. 10, pp. 1064-1076, 2012.

[252] X. Li, L. Jiao, H. Xu, Y. Lu, C. Zhu, J. Duan, X. Zhang, N. Dai and W. Song, “Near-infrared subwavelength imaging using Al:ZnObased near-field superlens," Opt. Mater. Express, vol. 6, no. 12, pp. 3892-3898, 2016.

[253] I. Sinno, "ZnO thin-film optimization towards the fabrication of high-frequency ultrasound transducers," Victoria University of Wellington, 2017.

[254] E. Venere, "New materials may bring advanced optical technologies, cloaking," Purdue University, 21 Jan 2011. [Online]. Available: http://www.purdue.edu/newsroom/research/2011/110121Boltass vaMetamats.html. [Accessed 91 2017].

[255] V. E. Babicheva, A. Boltasseva and A. V. Lavrinenko, “Transparent conducting oxides for electro-optical plasmonic modulators," Nanophotonics, vol. 4, no. 1, pp. 165-185, 2015. 
[256] F. Wang, "Plasmonics," [Online]. Available: http://web.stanford.edu/group/melosh/plasmonics.html. [Accessed 211 2017].

[257] M. M. Masis, A. Dabirian, F. Dauzou, B. Delaup, L. Fanni and T. Thosophorn, "Transparent Conductive Oxides (TCOs)," EPFL, [Online]. Available: http://pvlab.epfl.ch/tco. [Accessed 175 2017].

[258] J. Y. Lin, K. D. Zhong and P. T. Lee, "Plasmonic behaviors of metallic AZO thin film and AZO nanodisk array," Opt. Express, vol. 24, no. 5, pp. 5125-5135, 2016.

[259] T. Coutts, T. Mason, J. Perkins and D. Ginley, "Transparent Conducting Oxides: Status and Opportunities in Basic Research," in 195th Meeting of the Electrochemical Society, Seattle, Washington, 1999.

[260] A. Frölich and M. Wegener, "Spectroscopic characterization of highly doped $\mathrm{ZnO}$ films grown by atomic-layer deposition for three-dimensional infrared metamaterials," Optical Materials Express, vol. 1, no. 5, pp. 883-889, 2011.

[261] A. Janotti and C. G. V. deWalle, "Fundamentals of zinc oxide as a semiconductor," Reports on Progress in Physics, vol. 72, no. 126501, pp. 1-29, 2009.

[262] Ü. Özgür, Y. I. Alivov, C. Liu, A. Teke, M. A. Reshchikov, S. Doğan, V. Avrutin, S. Cho and H. Morkoç, "A comprehensive review of $\mathrm{ZnO}$ materials and devices," Journal of Applied Physics, vol. 98, no. 041301, 2005. 
[263] H. Morkoç and Ü. Özgür, Zinc Oxide: Fundamentals, Materials and Device Technology, Wiley, 2009.

[264] D. C. Look, "Recent advances in $\mathrm{ZnO}$ materials and devices," Materials Science and Engineering: B, vol. 80, no. 1-3, pp. 383-387, 2001.

[265] C. Jagadish and S. Pearton, Zinc Oxide Bulk, Thin Films and Nanostructures, New York: Elsevier, 2006.

[266] Q. A. Acton, Zinc Compounds-Advances in Research and Application: 2013 Edition: ScholarlyBrief, ScholarlyEditions, 2013.

[267] N. P. Dasgupta, S. Neubert, W. Lee, O. Trejo, J. R. Lee and F. B. Prinz, "Atomic Layer Deposition of Al-doped ZnO Films: Effect of Grain Orientation on Conductivity," Chem. Mater., vol. 22, no. 16, pp. 4769-4775, 2010.

[268] A. J. Walle and C. G. Vande, "Native point defects in ZnO," Physical Review B, vol. 76, no. 16, pp. 165202(1-22), 2007.

[269] K. Ellmer, A. Klein and B. Rech, Transparent Conductive Zinc Oxide, Basics and Applications in Thin Film Solar Cells, Berlin: Springer, 2008.

[270] A. Akdag, H. F. Budak, M. Yilmaz, A. Efe, M. Buyukaydin, M. Can, G. Turgut and E. Sonmez, "Structural and Morphological Properties of Al doped ZnO Nanoparticles," J. Phys., Conference series, vol. 707, 2016. 
[271] J. P. Colinge and C. Colinge, Physics of Semiconductor Devices, Springer Science \& Business Media, 2005.

[272] J. Y. Noh, H. Kim, Y. S. Kim and C. H. Park, "Electron doping limit in Al-doped $\mathrm{ZnO}$ by donor-acceptor interactions," Journal of Applied Physics, vol. 113, no. 153703, 2013.

[273] F. J. Haug, "Essential semiconductor physics, Degenerate semiconductors," [Online]. Available: http://www.superstrate.net/pv/physics/. [Accessed 201711 28].

[274] B. V. Zeghbroeck, "Principles of semiconductor devices," in Semiconductor fundamentals, Colorado.edu, 2011.

[275] E. Burstein, "Anomalous Optical Absorption Limit in InSb," Phys. Rev., vol. 93, no. 3, p. 632, 1954.

[276] T. Moss, Proc. Phys. Soc. London, no. B67, p. 775, 1964.

[277] Z. Zhao, D. L. Morel and C. S. Ferekides, "Electrical and optical properties of tin-doped $\mathrm{CdO}$ films deposited by atmospheric metalorganic chemical vapor deposition," Thin Solid Films, vol. 413, no. 1, pp. 203-211, 2002.

[278] H. H. C. Lai and e. al., "Dopant-induced bandgap shift in Aldoped $\mathrm{ZnO}$ thin films prepared by spray pyrolysis," Journal of Applied Physics, vol. 112, no. 083708, pp. 1-6, 2012.

[279] F. K. Shan and Y. S. Yu, "Band gap energy of pure and Al-doped $\mathrm{ZnO}$ thin films," Journal of the European Ceramic Society, vol. 24, no. 6, pp. 1869-1872, 2004. 
[280] J. Tauc, R. Grigorovici and A. Vancu, "Optical Properties and Electronic Structure of Amorphous Germanium," Phys. Stat. Sol. (b), vol. 15, no. 2, pp. 627-637, 1966.

[281] W. Miao, X. Li, Q. Zhang, L. Huang, Z. Zhang, L. Zhang and X. Yan, "Transparent conductive In2O3:Mo thin films prepared by reactive direct current magnetron sputtering at room temperature," Thin Solid Films, vol. 500, no. 1-2, pp. 70-73, 2006.

[282] M. Kumar, N. Umezawa, S. Ishii and T. Nagao, "Examining the performance of refractory conductive ceramics as plasmonic materials: a theoretical approach," ACS Photonics, vol. 3, no. 1, p. 43-50, 2016.

[283] G. V. Naik, J. Kim, P. R. West, N. K. Emani and A. Boltasseva, "The Road Ahead for Metamaterials and Plasmonics: Improved Material Building Blocks," in Integrated Photonics Research, Silicon and Nanophotonics, Colorado Springs, 2012.

[284] P. West, S. Ishii, G. Naik, N. Emani and A. Boltasseva, "Identifying low-loss plasmonic materials," 10 Nov 2010. [Online]. Available: http://spie.org/newsroom/3167-identifyinglow-loss-plasmonic-materials?ArticleID $=x 42239 . \quad$ [Accessed $\quad 9 \quad 1$ 2017].

[285] D. B. Murphy, K. R. Spring, T. J. Fellers and M. W. Davidson, "Principles of Birefringence page navigation, Introduction to Optical Birefringence," $\quad$ [Online]. Available: 
https://www.microscopyu.com/techniques/polarizedlight/principles-of-birefringence. [Accessed 2908 2017].

[286] S. Ruzin, "Anisotropy and Birefringence," [Online]. Available: http://microscopy.berkeley.edu/courses/TLM/plm/birefr.html. [Accessed 2808 2017].

[287] Photonics-EDU., “Optically isotropic crystal," [Online]. Available: https://www.photonics.com/EDU/Term.aspx?TermID=5874. [Accessed 2110 2018].

[288] M. C. Gupta and J. Ballato, The Handbook of Photonics, CRC Press, 2006.

[289] Schott-LTD, "Schott Borofloat, The versatile floated borosilicate glass - with an infinite number of applications," [Online]. Available: $\quad$ https://psec.uchicago.edu/glass/borofloat_33_e.pdf. [Accessed 1007 2017].

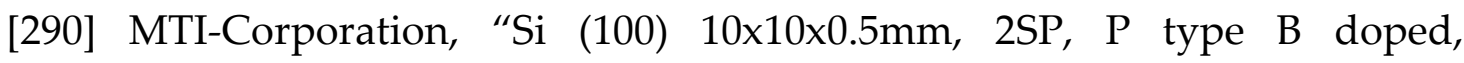
R: 8-32 ohm-cm," [Online]. Available: http://www.mtixtl.com/SiB-a-101005S2.aspx. [Accessed 112 2018].

[291] University-Wafer-Inc., "Sapphire Wafers in volume if you don't have to!," [Online]. Available: http://www.universitywafer.com/Wafers_Services/Sapphire/sap phire.html. [Accessed 112 2018].

[292] Eksma-Optics, "CALCIUM FLUORIDE (CAF2) WINDOWS," [Online]. Available: http://eksmaoptics.com/optical- 
components/uv-and-ir-optics/calcium-fluoride-caf2-windows/. [Accessed 112 2018].

[293] Crystran-Ltd., "Calcium Fluoride (CaF2)," [Online]. Available: https://www.crystran.co.uk/optical-materials/calcium-fluoridecaf2. [Accessed 0405 2018].

[294] ACEPT-W3-Group, "Silicon - a Material Transparent to Infrared," [Online]. Available: https://www.asu.edu/courses/phs208/patternsbb/PiN/rdg/silicon /index.htm. [Accessed 282 2018].

[295] M. J. Weber, Handbook of Optical Materials, Press, 2003.

[296] C. Kruger, D. Heinert, A. Khalaidovski, J. Steinlechner, R. Nawrodt, R. Schnabel and H. Luck, "Birefringence Measurements on Crystalline Silicon," 2015.

[297] J. F. Lamond and J. H. Pielert, Significance of Tests and Properties of Concrete and Concrete-making Materials, 169, Part 4, ASTM International, 2006.

[298] F. Cverna, ASM Ready Reference: Thermal properties of metals, ASM Ready Reference Series, ASM International, 2002.

[299] D. E. Aspnes and A. A. Studna, "Dielectric functions and optical parameters of $\mathrm{Si}, \mathrm{Ge}, \mathrm{GaP}, \mathrm{GaAs}, \mathrm{GaSb}$, InP, InAs, and InSb from 1.5 to 6.0 eV," Phys. Rev. B, vol. 27, no. 2, pp. 985-1009, 1983. 
[300] I. H. Malitson and M. Dodge, "Refractive index and Birefringence of Synthetic Sapphire," J. Opt. Soc. Am. , vol. 62, no. 1405, 1972.

[301] I. H. Malitson, "A Redetermination of Some Optical Properties of Calcium Fluoride," Appl. Opt. , vol. 2, no. 11, pp. 1103-107, 1963.

[302] Ioffe-Inst., "Si- Silicon Thermal Properties," [Online]. Available: http://www.ioffe.ru/SVA/NSM/Semicond/Si/thermal.html. [Accessed 0405 2018].

[303] MolTech-GmbH, "Sapphire (Al2O3)," [Online]. Available: http://www.mtberlin.com/frames_cryst/descriptions/sapphire.htm. [Accessed 0405 2018].

[304] M. Ohring, Materials Science of Thin Films, San Diego: Academic Press, 2002.

[305] K. Ellmer, "Magnetron sputtering of transparent conductive zinc oxide: relation between the sputtering parameters and the electronic properties," J. Phys. D: Appl. Phys., vol. 33, p. R17-R32, 2000.

[306] S. Schiller, U. Heisig, K. Goedicke, K. Schade, G. Teschner and J. Henneberger, "Advances in high rate sputtering with magnetron-plasmatron processing and instrumentation," Thin Solid Films, vol. 64, no. 3, pp. 455-467, 1979.

[307] I. MacMillan, "OPTICAL FABRICATION: Advances in sputtering benefit coating costs," 41 2009. [Online]. Available: 
http://www.laserfocusworld.com/articles/2009/04/opticalfabrication-advances-in-sputtering-benefit-coating-costs.html. [Accessed 2408 2017].

[308] R. Hong, H. Qi, J. Huang, Z. Fan and J. Shao, "Influence of oxygen partial pressure on the structure and photoluminescence of direct current reactive magnetron sputtering $\mathrm{ZnO}$ thin films," Thin Solid Films, vol. 473, no. 1, pp. 58-62, 2005.

[309] B. G. Choi and e. al., "Electrical, optical and structural properties of transparent and conducting $\mathrm{ZnO}$ thin films doped with $\mathrm{Al}$ and F by rf magnetron sputter," Journal of the European Ceramic Society, vol. 25, no. 12, pp. 2161-2165, 2005.

[310] D. L. Raimondi and E. Kay, "High Resistivity Transparent ZnO Thin Films," Journal of Vacuum Science and Technology, vol. 96, no. 7, 1970.

[311] S. Elhamali, W. M. Cranton, N. Kalfagiannis, X. Hou, R. Ranson and D. C. Koutsogeorgis, "Enhanced electrical and optical properties of room temperature deposited Aluminium doped Zinc Oxide (AZO) thin films by excimer laser annealing," Optics and Lasers in Engineering, vol. 80, pp. 45-51, 2016.

[312] Magnetron-Sputtering-Technology, MicroMagnetics, [Online]. Available:

http://www.directvacuum.com/pdf/what_is_sputtering.pdf. [Accessed 101 2017]. 
[313] Aultimut-International-LLC, “Advances in RF Sputtering," AUltimut International LLC., [Online]. Available: http://www.aultimut.com/technologies-2/plasmaprocesses/advances-in-rf-sputtering/. [Accessed 91 2017].

[314] M. M. Waite, S. I. Shah and D. A. Glocker, "Sputtering Sources," in 50 Years of Vacuum Coating Technology and the growth of the Society of Vacuum Coaters, vol. 50, Society of Vacuum Coaters, 2007, pp. 41-50.

[315] B. M. Smirnov, Physics of Ionized Gases, John Wiley \& Sons, 2008.

[316] M. Hughes, “What is RF Sputtering?,” 2710 2016. [Online]. Available: $\quad$ http://www.semicore.com/news/92-what-is-rfsputtering. [Accessed 1311 2017].

[317] D. Hoffman, B. Singh and J. H. Thomas, Handbook of Vacuum Science and Technology, Elsevier, 1997.

[318] R. G. Driggers, Encyclopedia of Optical Engineering: Pho-Z, Dekker Encyclopedias Series, CRC Press, 2003.

[319] Micro-Magnetics-Inc., "Magnetron Sputtering Technology," [Online]. Available: http://www.directvacuum.com/PDF/what_is_sputtering.pdf. [Accessed 2210 2018].

[320] J. I. Owen, Growth, Etching, and Stability of Sputtered ZnO:AI for Thin-film Silicon Solar Cells, Forschungszentrum Jülich, 2011. 
[321] D. L. Smith, Thin-Film Deposition: Principles Practice, McGrawHill, 1995.

[322] H. Majeed, "Sputtering of Thin Films," 2503 2013. [Online]. Available: http://physlab.org/wpcontent/uploads/2016/04/Hassanpres_sputtering.pdf.

[Accessed 2504 2018].

[323] M. M. Waite, S. I. Shah and D. A. Glocker, "Sputtering Sources, In 50 Years of Vacuum Coating Technology and the growth of the Society of Vacuum Coaters," Springer Bulletin, pp. 42-50, 2010.

[324] Y. Nishi and R. Doering, Handbook of Semiconductor Manufacturing Technology, CRC Press, 2007.

[325] D. M. Mattox, Handbook of Physical Vapor Deposition (PVD) Processing, Cambridge University Press, 2014.

[326] F. F. Masouleh, I. Sinno, R. G. Buckley, G. Gouws and C. P. Moore, "Characterization of conductive Al-doped $\mathrm{ZnO}$ thin films for plasmonic applications," Appl. Phys. A, vol. 124, no. 174, 2018.

[327] A. Barranco, A. Borras, A. Gonzalez Elipe and A. Palmero, "Perspectives on oblique angle deposition of thin films: From fundamentals to devices," Progress in Mateials Science, vol. 76, pp. 59-153, 2016. 
[328] K. Tominaga, K. Kuroda and O. Tada, "Radiation Effect due to Energetic Oxygen Atoms on Conductive Al-Doped ZnO Films," Jpn. J. Appl. Phys., vol. 27, no. 7, pp. 1176-1180, 1988.

[329] K. Tominaga, N. Veshiba, Y. Shintani and O. Tada, "Hi qhEnergy Neutral Atoms in the Sputterinq of $\mathrm{ZnO}$," Jpn. J. Appl. Phys., vol. 20, pp. 519-526, 1981.

[330] K. Tominaga, S. Iwamura., I. Fujita, Y. Shintani and O. Tada, "Influence of Bombardment by Energetic Atoms on C-axis Orientation of ZnO Films," Jpn. J. Appl. Phys., vol. 21, pp. 999$1002,1982$.

[331] T. Welzel, R. Kleinhempel, T. Dunger and F. Richter, "Ion Energy Distributions in Magnetron Sputtering of Zinc Aluminum Oxide," in PSE Proceedings, 2009.

[332] M. K. Jayaraj, A. Antony and M. Ramachandran, “Transparent conducting zinc oxide thin film prepared by off-axis $\mathrm{rf}$ magnetron sputtering," B. Mater. Sci., vol. 25, no. 3, pp. 227-230, 2002.

[333] W. Roentgen, “Uber Eine Neue Art Von Strahlen," Wurzburg Stahel, 1896.

[334] P. P. Ewald, "Fifty Years of X-ray Diffraction. Utrecht: NVA Oosthoek's Uitgeversmaatschappij, 1962.," [Online]. Available: http://www.iucr.org/publ/50yearsofxraydiffraction. [Accessed 23 $12018]$. 
[335] M. R. Kundu, S. M. White, N. Gopalswamy and J. Lim, “Millimeter, Microwave, Hard X-ray and Soft X-ray Observations of Energetic Electron Populations in Solar Flares," Astrophysical Journal Supplement Series, vol. 90, no. 2, pp. 599-610, 1994.

[336] D. Attwood, Soft X-Rays and Extreme Ultraviolet Radiation: Principles and Applications, Cambridge University Press, 1999.

[337] S. Suckewer and C. H. Skinner, "Soft X-ray Lasers and Their Applications," Science, vol. 247, no. 4950, pp. 1553-1557, 1990.

[338] P. Frame, "Coolidge X-ray Tubes," Oak Ridge Associated Universities, $24 \quad 6 \quad 2009 . \quad$ [Online]. Available: https://www.orau.org/ptp/collection/xraytubescoolidge/coolidge information.htm. [Accessed 231 2018].

[339] C. Flynn, "Lecture 3 : Accelerated charges and bremsstrahlung," Tuorla Observatory, University of Turku, 1510 2006. [Online]. Available: $\quad$ http://www.astro.utu.fi/ cflynn/astroII/l3.html. [Accessed 231 2018].

[340] B. Cullity and S. Stock, "Properties of X-Rays," in Elements of XRay Diffraction, 3rd edition, Pearson Education Limited, 2014, pp. $1-30$.

[341] T. Kuwana, Physical Methods in Modern Chemical Analysis, Volume 2, Elsevier, 2012. 
[342] P. F. Fewster, X-Ray Scattering from Semiconductors and Other Materials, Frontiers in nanobiomedical research, World Scientific, 2015.

[343] N. D. Spencer and J. H. Moore, Encyclopedia of Chemical Physics and Physical Chemistry: Fundamentals, Volume 1 of Encyclopedia of Chemical Physics and Physical Chemistry, Taylor \& Francis, 2001.

[344] M. Eckert, "Disputed discovery: the beginnings of X-ray diffraction in crystals in 1912 and its repercussions," Acta Crystallographica: Foundations of Crystallography, vol. 68, pp. 30-39, 2012.

[345] C. Suryanarayana and M. G. Norton, X-Ray Diffraction: A Practical Approach, Springer Science \& Business Media, 2013.

[346] R. J. D. Tilley, Understanding Solids: The Science of Materials, John Wiley \& Sons, 2013.

[347] R. J. D. Tilley, Crystals and Crystal Structures, John Wiley \& Sons, 2006.

[348] R. I. Masel, Principles of Adsorption and Reaction on Solid Surfaces, John Wiley \& Sons, 1996.

[349] D. Brabazon, E. Pellicer, F. Zivic, J. Sort, M. D. Baró, N. Grujovic and K. L. Choy, Commercialization of Nanotechnologies-A Case Study Approach, Springer, 2017. 
[350] B. D. Cullity and S. R. Stock, Elements of X-ray Diffraction, Pearson Education, Limited, 2013.

[351] ZnO Powder, Calculated from ICSD using POWD-12++, Reference code:01-070-8072, 2001.

[352] PANalytical, "PANalytical-X'Pert Powder," [Online]. Available: http://www.panalytical.com/en/XPert3-Powder.htm.

[353] W. D. JrCallister, Materials Science and Engineering, An Introduction, John Wiley \& Sons, Inc., 2007.

[354] A. L. Patterson, "The Scherrer Formula for X-Ray Particle Size Determination," Phys. Rev. , vol. 56, no. 978, 1939.

[355] V. V. Kharton, Solid State Electrochemistry I: Fundamentals, Materials and their Applications, John Wiley \& Sons, 2009.

[356] J. Wilson and A. Langford, "Sherrer after Sixty Years: A Survey and Some New Results in the Determination of Crystallite Size," J. Appl. Cryst., vol. 11, pp. 102-113, 1978.

[357] Y. Wang and M. A. Nastasi, Handbook of modern ion beam materials analysis, Warrendale, Pa. : Materials Research Society, 1950-c2009 2nd ed..

[358] A. W. Czanderna and D. M. Hercules, "Ion Spectroscopies for Surface Analysis," in Part of the Methods of Surface Characterization book series (MOSC, volume 2), Boston, MA, Springer, 1991.

[359] A. Saha and A. R. Barron, "Physical Methods in Chemistry and Nano Science - Rutherford Backscattering of Thin Films," 
[Online].

Available:

https://cnx.org/contents/uieDnVBC@25.2:2jLvz-

IO@3/Rutherford-Backscattering-of-Thin-Films. [Accessed $18 \quad 18$ 2018].

[360] M. Mayer, "Rutherford Backscattering Spectrometry (RBS), MaxPlanck-Institut fur Plasmaphysik, EURATOM Association,"” 195 $2003 . \quad$ [Online]. Available:

http://users.ictp.it/ pub_off/lectures/Ins022/Mayer_1/Mayer_1.p

df. [Accessed 2110 2018].

[361] M. O. Thompson, "RUMP: RBS Analysis and Plotting," 2111 1996. [Online]. Available: http://www.genplot.com/doc/rump.htm. [Accessed 2110 2018].

[362] L. Reimer, Scanning Electron Microscopy: Physics of Image Formation and Microanalysis, Volume 45 of Springer Series in Optical Sciences, 2013: Springer.

[363] J. Goldstein, D. E. Newbury, D. C. Joy, C. E. Lyman, P. Echlin, E. Lifshin, L. Sawyer and J. R. Michael, Scanning Electron Microscopy and X-ray Microanalysis, third Ed., Springer US, 2003.

[364] A. D. Gree, "The History and Working Principle of the Scanning Electron Microscope (SEM)," 2003 2015. [Online]. Available: https://www.azonano.com/article.aspx?ArticleID=3995.

[Accessed 2904 2018]. 
[365] L. C. Sawyer, Polymer Microscopy, Springer Science \& Business , 2012.

[366] J. Manifacier, J. Gasiot and J. Fillard, "A simple method for the determination of the optical constants $\mathrm{n}$ and $\mathrm{k}$ and the thickness of a weakly absorbing film," Journal of Physics E: Scientific Instruments, vol. 9, no. 11, p. 1002, 1976.

[367] G. S. Leadstone, "The discovery of the Hall effect," Phys. Educ., vol. 14, pp. 374-379, 1979.

[368] R. Green, "Design How-To, Hall effect measurements in materials characterization," 178 2011. [Online]. Available: https://www.eetimes.com/document.asp?doc_id=1279015. [Accessed 2504 2018].

[369] E. D. Sisson, "The Hall Effect Devices and Applications," Analytical Chemistry, vol. 43, no. 7, pp. (67-71) A, 1971.

[370] R. Green, "Hall Effect Measurements in Materials Characterization, White Paper," Keithley Instruments, Inc., 261 2011. [Online]. Available: file:///C:/Users/fadakarfar/Downloads/HallEffect_WhtPapr.pdf. [Accessed 101 2017].

[371] R. S. Poppovic, Hall Effect Devices: Magnetic Sensors and Characterization of Semiconductors, Series in Sensors, CRC Press, 1991.

[372] R. S. Popovic, Hall Effect Devices, Second Edition, CRC Press, 2003. 
[373] L. J. VanderPauw, "A method of measuring specific resistivity and Hall effect of discs of arbitrary shape," Philips Research Reports, vol. 13, pp. 1-9, 1958.

[374] PML-Webmaster, "Resistivity and Hall Measurements," $16 \quad 4$ 2010.

[Online].

Available:

https://www.nist.gov/pml/engineering-physics-

division/popular-links/hall-effect/resistivity-and-hall-

measurements. [Accessed 262 2018].

[375] NIST, “Hall Effect Measurements Algorithm," 15042010. [Online]. Available: https://www.nist.gov/pml/engineeringphysics-division/popular-links/hall-effect/hall-effectmeasurements-algorithm. [Accessed 138 2018].

[376] E. Ramsden, Hall-Effect Sensors: Theory and Application, Elsevier, 2011.

[377] D. P. Joshi and K. Sen, "Effect of grain size on the resistivity of polycrystalline material," Solar Cells, vol. 9, no. 4, pp. 261-267, 1983.

[378] A-level-physics-tutor, "Thermal Physics, Thermometers," [Online]. Available: http://www.a-levelphysicstutor.com/thermthermometers.php. [Accessed 20179 20].

[379] LOT-Quantum-Design-Europe, "PPMS® Physical Property measurement system, Integrated measurement applications," [Online]. Available: http://www.lao.cz/data/ke- 
stazeni/Bro\%C5\%BEura\%20PPMS-d573.pdf. [Accessed $23 \quad 10$ 2018].

[380] A. M. Helmenstine, "Spectroscopy Introduction, Introduction to Spectroscopy and Types of Spectroscopy," 63 2017. [Online]. Available: $\quad$ https://www.thoughtco.com/introduction-tospectroscopy-603741. [Accessed 1412 2017].

[381] T. Creighton, "Gravitational wave spectrum," [Online]. Available:

http://www.tapir.caltech.edu/ teviet/Waves/gwave_spectrum.ht ml. [Accessed 1010 2019].

[382] G. Bonacucina, "Acoustic spectroscopy: A powerful analytical method for the pharmaceutical field?," International Journal of Pharmaceutics, vol. 503, no. 1-2, pp. 174-195, 2016.

[383] H. Yang, V. Paschalidis, K. Yagi, L. Lehner, F. Pretorius and N. Yunes, "Gravitational wave spectroscopy of binary neutron star merger remnants with mode stacking," PHYSICAL REVIEW D, vol. 97, p. 024049, 2018.

[384] I. Newton, "A letter of Mr. Isaac Newton, Professor of the Mathematicks in the University of Cambridge; containing his new theory about light and colors: sent by the author to the publisher from Cambridge, in order to be communicated to the R. Society," Phil. Trans., vol. 6, no. 80, pp. 3075-3087, 1671. 
[385] J. J. Workman, Concise Handbook Of Analytical Spectroscopy, The: Theory, Applications, And Reference Materials (In 5 Volumes), World Scientific, 2016.

[386] S. A. Dyer, Wiley Survey of Instrumentation and Measurement, John Wiley \& Sons, 2004.

[387] https://commons.wikimedia.org/wiki/User:Inductiveload, "The Electromagnetic Spectrum," [Online]. Available: https://en.wikipedia.org/wiki/Electromagnetic_spectrum\#/media /File:EM_Spectrum_Properties_edit.svg. [Accessed 0109 2019].

[388] R. G. Compton, New Techniques for the Study of Electrodes and Their Reactions, Volume 29 of Comprehensive Chemical Kinetics, Elsevier, 1989.

[389] Bruker, “VERTEX 80/80v user manual," Bruker Optics Inc.

[390] Nayuki, "How to implement the discrete Fourier transform," 20 6 2018. [Online]. Available: https://www.nayuki.io/page/how-toimplement-the-discrete-fourier-transform. [Accessed 2310 2018].

[391] S. R. J. Brueck, “Optical and Interferometric Lithography Nanotechnology Enablers," in Proceedings of the IEEE, New Mexico Univ., Albuquerque, NM, USA, 2005.

[392] AZoOptics-editorial, "What is a Globar?," 216 2013. [Online]. Available: https://www.azooptics.com/Article.aspx?ArticleID=566. [Accessed 89 2017]. 
[393] M. Berciu, "Part VI: Properties of metals and insulators," [Online].

Available:

https://www.phas.ubc.ca/ berciu/TEACHING/PHYS315/LECTU

RES/FILES/properties.pdf. [Accessed 103 2018].

[394]

D. L. Reger, S. R. Goode and D. W. Ball, Chemistry: Principles and Practice, Third Edition, Belmont; USA: Brooks/Cole, Cengage Learning, 2010.

[395] J. Yvon, “Drude Dispersion Model," [Online]. Available: http://www.horiba.com/fileadmin/uploads/Scientific/Downloads /OpticalSchool_CN/TN/ellipsometer/Drude_Dispersion_Model.p df. [Accessed 173 2017].

[396] M. A. Ordal, L. L. Long, R. J. Bell, S. E. Bell, R. R. Bell, R. W. Alexander and C. A. Ward, "Optical properties of the metals Al, $\mathrm{Co}, \mathrm{Cu}, \mathrm{Au}, \mathrm{Fe}, \mathrm{Pb}, \mathrm{Ni}, \mathrm{Pd}, \mathrm{Pt}, \mathrm{Ag}, \mathrm{Ti}$, and $\mathrm{W}$ in the infrared and far infrared," Applied Optics, vol. 22, no. 7, pp. 1099-1120, 1983.

[397] M. I. Marković and A. D. Rakić, "Determination of the reflection coefficients of laser light of wavelengths $\lambda \in(0.22 \mu \mathrm{m}, 200 \mu \mathrm{m})$ from the surface of aluminum using the Lorentz-Drude model," Appl. Opt., vol. 29, no. 24, pp. 3479-3483, 1990.

[398] R. P. Lucht, "Drude Model for dielectric constant of metals," [Online]. Available: http://optics.hanyang.ac.kr/ shsong/27Metals.pdf. [Accessed 199 2019].

[399] R. Aachen, “Electrical conductivity of metals, semiconductors, and superconductors," $21 \quad 8$ 2008. [Online]. Available: 
https://institut2a.physik.rwthaachen.de/de/teaching/praktikum/Anleitungen/FK09Leitfaehigkeit-englisch.PDF. [Accessed 288 2019].

[400] A. Calzolari, A. Ruini and A. Catellani, "Transparent Conductive Oxides as Near-IR Plasmonic Materials: The Case of Al-Doped ZnO Derivatives," ACS Photonics, vol. 1, no. 8, pp. 703-709, 2014.

[401] I. F. Almog, M. S. Bradley and V. Bulovi'c, "The Lorentz Oscillator and its Applications," [Online]. Available: https://ocw.mit.edu/courses/electrical-engineering-andcomputer-science/6-007-electromagnetic-energy-from-motors-tolasers-spring-2011/readings/MIT6_007S11_lorentz.pdf.

[Accessed 29 2017].

[402] R. E. Smallman and R. J. Bishop, Modern Physical Metallurgy and Materials Engineering: Science, Process, Applications, Butterworth-Heinemann, 1999.

[403] M. S. Dresselhaus, Solid State Physics, Part II, Optical Properties of Solids, 2001.

[404] G. Chen, "Chapter 6 OPTICAL PROPERTIES OF SOLIDS," 2006. [Online]. Available: https://ocw.mit.edu/courses/mechanicalengineering/2-58j-radiative-transfer-spring2006/readings/chap6_solid_prop.pdf. [Accessed 1703 2018].

[405] A. Kogar, "Plasma Frequency, Screening Response Time and the Independent Electron Approximation," 1511 2015. [Online]. Available: 
https:/thiscondensedlife.wordpress.com/2015/11/15/plasmafrequency-screening-response-time-and-the-independentelectron-approximation/. [Accessed 153 2017].

[406] R. P. Feynman, R. B. Leighton and M. Sands, "The Feynman Lectures on Physics, Vol. 2, Reading, Chap. 32.," MA: AddisonWesley, 1964.

[407] R. Noriega and e. al., "Probing the electrical properties of highlydoped Al:ZnO nanowire ensembles," J. Appl. Phys., vol. 107, no. 074312, pp. 1-7, 2010.

[408] F. J. Himpsel, "The dielectric function of a Metal ("Jellium")," [Online]. Available: http://uw.physics.wisc.edu/ himpsel/551/Lectures/Plasmonics.p df. [Accessed 1810 2018].

[409] A. F. Levi, "The Lorentz oscillator model," in Essential Classical Mechanics for Device Physics, Morgan \& Claypool Publishers, 2016.

[410] J. Yvon, "Classical Dispersion Model," Horiba, 11 2006. [Online]. [Accessed 1903 2018].

[411] B. Ung, "The dielectric function of dispersive media," 2007. [Online].

Available:

http://theses.ulaval.ca/archimede/fichiers/24879/ch03.html\#d0e20

35. [Accessed 1703 2018].

[412] M. I. Marković and A. D. Rakić, "Determination of optical properties of aluminum including electron reradiation in the 
Lorentz-Drude model," Opt. Laser Technol., vol. 22, no. 6, pp. 394-398, 1990.

[413] R. Umeda, C. Totsuji, K. Tsuruta and H. Totsuji, "An FDTD analysis of nanostructured electromagnetic metamaterials using parallel computer," Materials Transactions, vol. 50, no. 5, p. 994998, 2009.

[414] B. Harbecke, "Coherent and incoherent Reflection and Transmission of Multilayer Structures," Appl. Phys. B., vol. 39, no. 3, pp. 165-170, 1986.

[415] A. Kuzmenk, “Reffit,” University of Geneva, Genève, 2004.

[416] M. Billardon, M. E. Couprie, J. M. Ortega and M. Velghe, "FabryPerot effects in the exponential decay and phase shift reflectivity measurement methods," Applied Optics, vol. 30, no. 3, pp. 344$351,1991$.

[417] S. G. Lipson, H. Lipson and D. S. Tannhauser, Optical Physics 3rd Edition, London: Cambridge University Press, 1995.

[418] R. G. Buckley and D. Beaglehole, "Absorptance of thin films," Applied optics, vol. 16, no. 9, pp. 2495-2499, 1977.

[419] W. M. Kim, H. Kim, J. H. Ko, B. Cheong, T. S. Lee, K. S. Lee, D. Kim and T. Y. Seong, "Density-of-state effective mass and nonparabolicity parameter of impurity doped $\mathrm{ZnO}$ thin films," J. Phys. D: Appl. Phys., vol. 41, p. 195409 (5pp), 2008. 
[420] V. Romanyuk and e. al., "Optical and Electrical Properties of Highly Doped ZnO:Al Films Deposited by Atomic Layer Deposition on $\mathrm{Si}$ Substrates in Visible and Near Infrared Region," ACTA Physica Polonica A, vol. 129, 2016.

[421] E. Muchuweni, T. S. Sathiaraj and H. Nyakotyo, "Synthesis and characterization of zinc oxide thin films for optoelectronic applications," Heliyon, vol. 3, no. 4, 2017.

[422] Y. Li and e. al., "Micro/Nano hierarchical peony-like Al doped $\mathrm{ZnO}$ superhydrophobic film: The guiding effect of (100) preferred seed layer," Sci. Rep., vol. 6, no. 19187, pp. 1-9, 2016.

[423] P. Singh, A. Kumar and D. Kaur, "Growth and characterization of $\mathrm{ZnO}$ nanocrystalline thin films and nanopowder via low-cost ultrasonic spray pyrolysis," Journal of Crystal Growth, vol. 306, pp. 303-310, 2007.

[424] M. H. Aslan, A. Y. Oral, E. Mensur, A. Gul and E. Basaran, "Preparation of c-axis-oriented zinc-oxide thin films and the study of their microstructure and optical properties," Solar Energy Materials and Solar Cells, vol. 82, no. 4, pp. 543-552, 2004.

[425] P. Scherrer, "Bestimmung der Groesse und der inneren Struktur von Kolloidteilchen mittels Roentgenstrahlen," Nachr. Ges. Wiss. Goettingen, no. 26, pp. 98-100, 1918.

[426] V. Srikant and D. R. Clarke, "On the optical band gap of zinc oxide," Journal of Applied Physics, vol. 83, no. 5447, 1998. 
[427] A. H. Ramelan, S. Wahyuningsih, H. Munawaroh and R. Narayan, "ZnO wide bandgap semiconductors preparation for optoelectronic devices," in IOP Conf. Series: Materials Science and Engineering , Indonesia, 2016.

[428] B. E. Sernelius, K. F. Berggren, Z. C. Jin, I. Hamberg and C. G. Granqvist, "Band-gap tailoring of $\mathrm{ZnO}$ by means of heavy $\mathrm{Al}$ doping," Phys. Rev. B , vol. 37, no. $10244,1988$.

[429] B. Benhaoua, A. Rahal and S. Benramache, "The Structural, optical and electrical properties of nanocrystalline $\mathrm{ZnO}: \mathrm{Al}$ thin films," Superlattices and Microstructures, vol. 68, pp. 38-47, 2014.

[430] P. Uprety, M. M. Junda, K. Ghimire, D. Adhikari, C. R. Grice and N. J. Podraza, "Spectroscopic ellipsometry determination of optical and electrical properties of aluminum doped zinc oxide," Appl. Surf. Sci., vol. 421 (Part B), pp. 852-858, 2017.

[431] A. Walsh, J. L. F. D. Silva and S. H. Wei, "Origins of Band-Gap Renormalization in Degenerately Doped Semiconductors," Physical Review. B, Condensed Matter and Materials Physics, vol. 78, no. 7, pp. 075211(1-5), 2008.

[432] H. Zheng, R. J. Zhang, D. H. Li, X. Chen, S. Y. Wang, Y. X. Zheng, M. J. Li, Z. G. Hu, N. Dai and L. Y. Chen, "Optical Properties of Al-Doped $\mathrm{ZnO}$ Films in the Infrared Region and Their Absorption Applications," Nanoscale Research Letters, vol. 13, pp. 149 (1-7), 2018. 
[433] A. Sharmin, S. Tabassum, M. S. Bashar and Z. H. Mahmood, “Depositions and characterization of sol-gel processed Al-doped $\mathrm{ZnO}(\mathrm{AZO})$ as transparent conducting oxide (TCO) for solar cell application," Journal of Theoretical and Applied Physics, vol. 13, pp. 123-132, 2019.

[434] A. K. Pradhan, R. M. Mundle, K. Santiago, J. R. Skuza, B. Xiao, K. D. Song, M. Bahoura, R. Cheaito and P. E. Hopkins, "Extreme tunability in aluminum doped Zinc Oxide plasmonic materials for near-infrared applications," Scientific Reports, vol. 4, pp. 6415 (1-6), 2015.

[435] Y. Wang, A. Capretti and L. D. Negro, "Wide tuning of the optical and structural properties of alternative plasmonic materials," Optical Materials Express, vol. 5, no. 11, 2015.

[436] F. K. Shan and e. al., "Blueshift of near band edge emission in $\mathrm{Mg}$ doped $\mathrm{ZnO}$ thin films and aging," J. Appl. Phys., vol. 95, no. 9, pp. 4772-4776, 2004.

[437] R. H. Fowler and L. Nordheim, "Electron emission in intense electric fields," The Royal Society Publishing, vol. 119, no. 781, 1928.

[438] EksmaOptics, "Calcium Fluoride (CaF2) $\quad$ Components," $30 \quad 7$ 2017. [Online].

Available:

http://eksmaoptics.com/out/media/Calcium_Fluoride_Optical_C omponents_EKSMA_Optics.pdf. 
[439] nih.gov, "Download (ImageJ)," NIH US, [Online]. Available: https://imagej.nih.gov/ij/download.html. [Accessed 16 2019].

[440] The-Gale-Group-Inc., "Thermal Expansion, Science of Everyday Things," [Online]. Available: http://www.encyclopedia.com/science-andtechnology/physics/physics/thermal-expansion. [Accessed $21 \quad 11$ 2017].

[441] A. A. Khan, "X-ray determination of thermal expansion of zinc oxide," Acta Crystallographica Section A, vol. A24, p. 403, 1968.

[442] H. Mei, C. M. Landis and R. Huang, "Concomitant wrinkling and buckle-delamination of elastic thin films on compliant substrates," Mechanics of Materials, vol. 43, pp. 627-642, 2011.

[443] M. W. Moon, J. E. Chung, K. R. Lee, K. H. Oh, R. Wang and A. G. Evans, "An experimental study of the influence of imperfections on the buckling of compressed thin films," Acta Materialia, vol. 50, no. 1219-1227, p. 5, 2002.

[444] G. Grosso and G. P. Parravicini, Solid State Physics, Elsevier, 2000.

[445] J. Halpern, "Chapter 12.6: Metals and Semiconductors," 128 2016. [Online]. Available: https://chem.libretexts.org/LibreTexts/Howard_University/Gene ral_Chemistry\%3A_An_Atoms_First_Approach/Unit_5\%3A_Stat es_of_Matter/Chapter_12\%3A_Solids/Chapter_12.06\%3A_Metals _and_Semiconductors. [Accessed 14 2018]. 
[446] S. M. Sze, Semiconductor Devices, Physics and Technology, Wiley, 2001.

[447] A. Goswami, Thin Film Fundamentals, New Age International, 1996.

[448] S. Salam, M. Islam and M. Akram, "Sol-gel synthesis of intrinsic and aluminum-doped zinc oxide thin films as transparent conducting oxides for thin film solar cells," Thin Solid Films, vol. 529, p. 242-247, 2013.

[449] J. G. Lu and S. Fujita, "Carrier Concentration Dependence of Band Gap Shift in n-Type ZnO: Al Films," Journal of Applied Physics, vol. 101(8), no. 083705, 2007.

[450] I. Thorn and C. O. Au, Applications of Wet-End Paper Chemistry, SpringerLink: Springer e-Books, Springer Science \& Business Media, 2009.

[451] P. M. S. Monk , Physical Chemistry: Understanding our Chemical World, John Wiley and Sons, 2008.

[452] N. R. Mandal, Aluminum Welding, Woodhead Publishing Series in Welding and Other Joining Technologies Series, Woodhead Publishing, 2002.

[453] A. C. Davies, The Science and Practice of Welding: Volume 2 of The Science and Practice of Welding, Arthur Cyril Davies, Cambridge University Press, 1992. 
[454] J. Kennedy, P. P. Murmu, J. Leveneur, A. Markwitz and J. Futter, "Controlling preferred orientation and electrical conductivity of zinc oxide thin films by post growth annealing treatment," Applied Surface Science, vol. 367, pp. 52-58, 2016.

[455] R. Hong, J. Huang, H. He and Z. S. J. Fan, "Influence of different post treatments on the structure and optical properties of zinc oxide films," Applied Surface Science, vol. 242, p. 346, 2005.

[456] T. Miyoshi, "Evaluation of $\mathrm{Pb}(\mathrm{Zr}, \mathrm{Ti}) \mathrm{O} 3$ Ceramics Prepared by Aerosol Deposition," Jpn J Appl Phys, vol. 46, p. 7023, 2007.

[457] H. Rotella and e. al., "Role of vacancy defects in $\mathrm{Al}$ doped $\mathrm{ZnO}$ thin films for optoelectronic devices," Journal of Physics D: Applied Physics, vol. 50, pp. 485106(1-7), 2017.

[458] H. Wang and e. al., "Materials Science; Research on materials science described," Electronics Business Journal, p. 253, 2010.

[459] A. Wang and e. al., "Effects of doping and annealing on properties of $\mathrm{ZnO}$ films grown by atomic layer deposition," Nanoscale Res Lett., vol. 10, no. 75, 2015.

[460] D. Kohl, M. Henzler and G. Heiland, “Low temperature sublimation processes from clean cleaved polar surfaces of zinc oxide crystals during first heating," Surface Science, vol. 41, no. 2, pp. 403-411, 1974.

[461] G. J. Fang, D. Li and B. L. Yao, "Influence of post-deposition annealing on the properties of transparent conductive nanocrystalline ZAO thin films prepared by RF magnetron 
sputtering with highly conductive ceramic target," Thin Solid Films, vol. 418, pp. 156-162, 2002.

[462] G. Fang, D. Li and B. L. Yao, "Fabrication and vacuum annealing of transparent conductive AZOthin films prepared by DC magnetron sputtering," Vacuum, vol. 68, pp. 363-372, 2003.

[463] Schott, "Data Sheet, Schott," 112 2014. [Online]. Available: http://www.sydor.com/wp-content/uploads/SCHOTT-KG1Shortpass-Filter.pdf. [Accessed 2005 2018].

[464] A. Serpenguzel, Y. O. Yyilmaz, U. K. Ayaz and A. Kurt, "PART III Integrated Photonic Technologies: Microring Structures, Silicone Microspheres for VLSI Photonics," in VLSI Micro- and Nanophotonics: Science, Technology, and Applications,, CRC Press, 2010.

[465] University-of-Reading, "infrared Multilayer laboratory, Silicon (Si)," [Online]. Available: http://www.reading.ac.uk/irinfraredmaterials-si.aspx. [Accessed 1505 2018].

[466] N. Neves, "AZO - The Replacement for ITO?," Ceramic Applications, vol. 3, pp. 62-66, 2015.

[467] S. O. Elhamali, W. M. Cranton, N. Kalfagiannis, X. Hou, R. Ranson and D. C. Koutsogeorgis, "Enhanced electrical and optical properties of room temperature deposited Aluminium doped Zinc Oxide (AZO) thin films by excimer laser annealing," Optics and Lasers in Engineering, vol. 80, pp. 45-51, 2016. 
[468] Sigma-Aldrich, "Indium tin oxide coated PET," [Online]. Available:

https://www.sigmaaldrich.com/catalog/product/aldrich/639303?1 ang=en\&region=NZ. [Accessed 12 2018]. 



\section{Appendix A}

\subsection{Ethics}

No foreseeable ethics approvals were required for this study.

\subsection{Budget and Resources}

All direct project costs were organized and provided by external project income. This covered lab materials and equipment operating costs. Useful textbooks and online literature materials were provided by the university library. A scholarship was provided by Victoria University of Wellington. 



\section{Appendix B}

\subsection{Challenges of FTIR measurements in our study}

A well-adjusted spectrometer produces linear and smooth spectral response despite variations in the included detectors and subsystems throughout a given measurement. However, during our measurements with the Bruker FTIR spectrometer, we observed a discontinuity in the acquired spectrum, in the region where the visible and UV ranges join. This anomaly is seen in Figure 13-1.

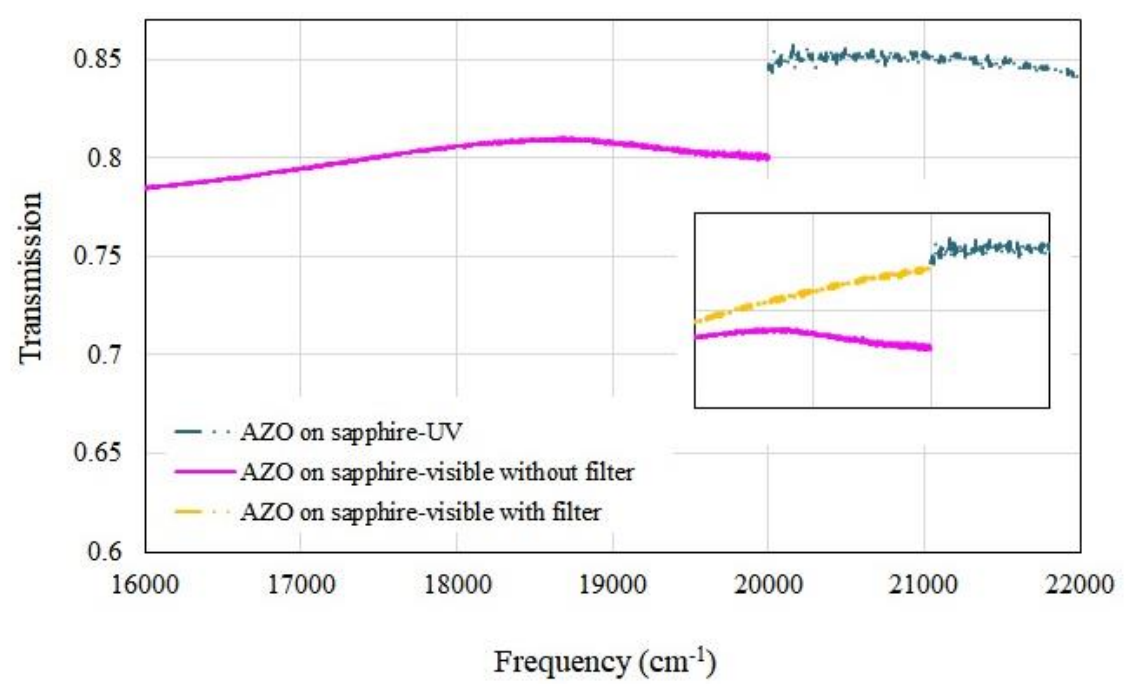

Figure 13-1 Transmission discontinuity at the end of the visible range in the Bruker system. The inset shows the corrected spectrum using our filter. 
To understand the reasons behind this discontinuity, we checked the recorded reference spectrum that was used to normalize the measured sample spectrum. This reference spectrum is shown in Figure 13-2(a), where a high-intensity peak at $\sim 16000 \mathrm{~cm}^{-1}$ can be observed.

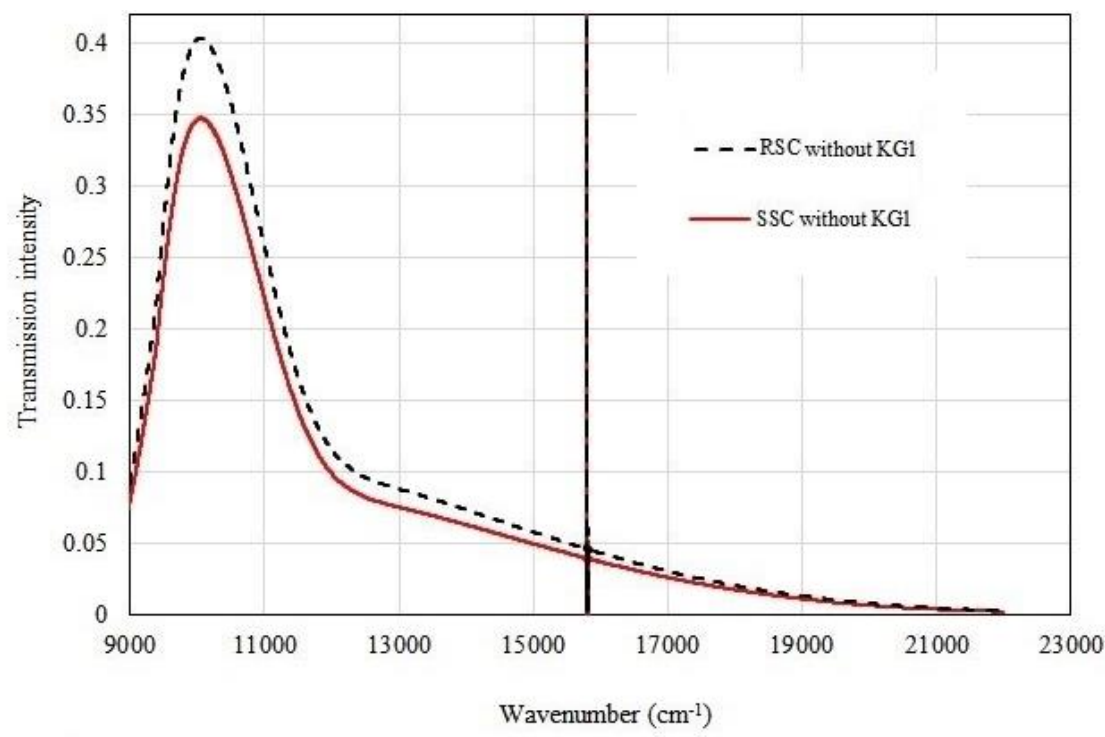

(a)

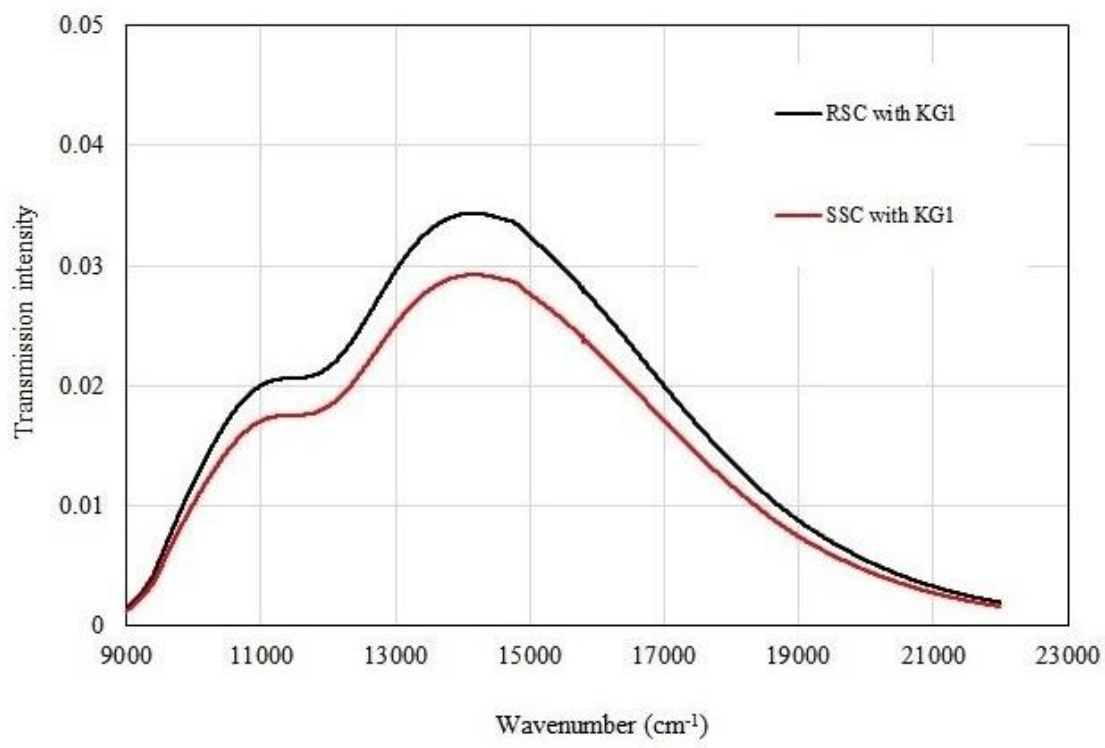

(b)

Figure 13-2 Reference spectrum (RSC) and sample spectrum (SSC) (a) before and (b) after placing the filter. 
This high-intensity peak was attributed to the alignment laser. Suspecting this to be the reason for the discontinuity in the spectral response, we decided to remove this peak by means of introducing a-band filter (KG1 [463]). The before and after spectra of the reference (RSC) and sample (SSC) compartments are shown in Figure 13-2(a) and Figure 13-2 (b), respectively.

It can be seen that the KG1 filter significantly blocked the infrared signal, which resulted in a lower signal to noise ratio over the measured spectrum. However, this filter successfully removed the anomaly of the alignment laser.

By dividing the sample spectrum to the reference spectrum in each part of Figure 13-2, the normalized sample spectra are obtained for both scenarios (with and without the filter). These spectra are shown below in Figure 13-3.

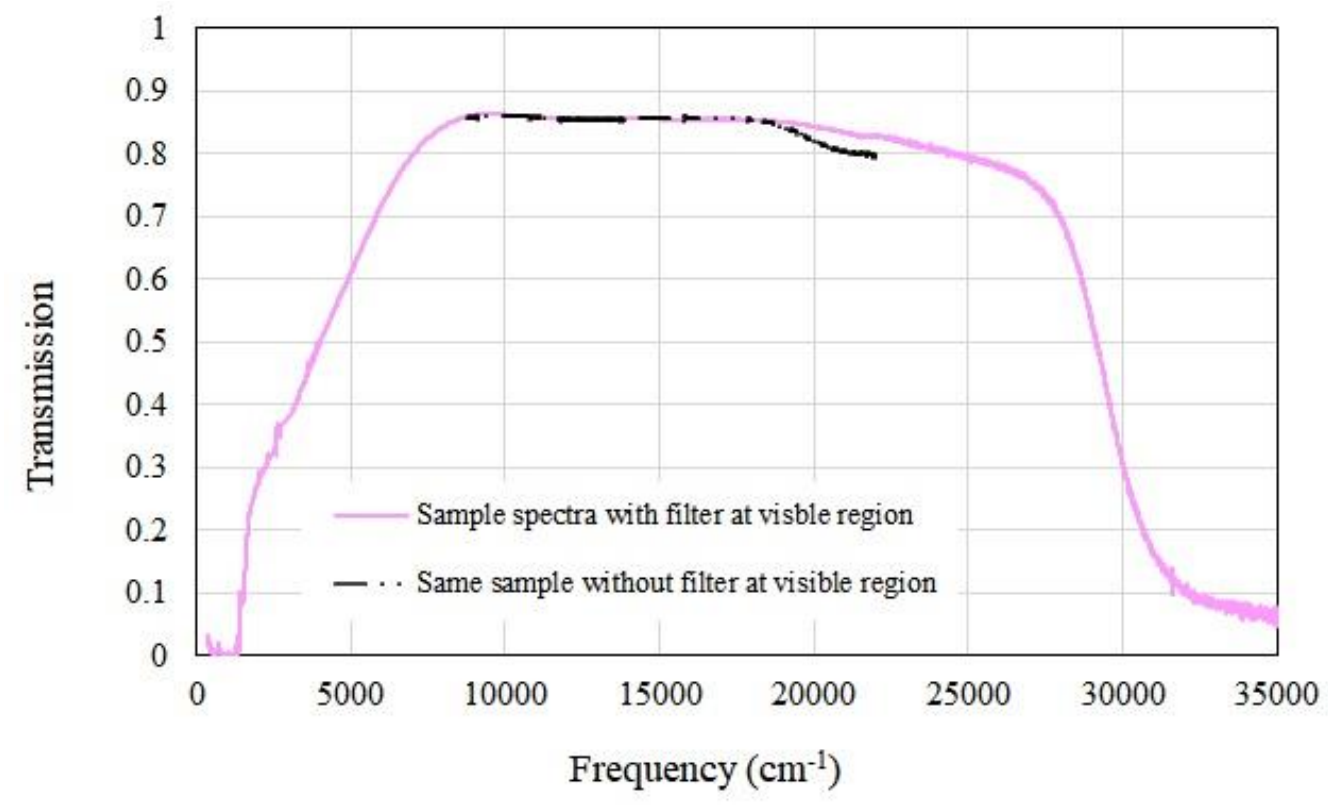

Figure 13-3 FTIR spectrum for AZO sample with (pink curve) and without filter (black curve) in the visible region. 
As can be seen, the filter absorbed specific wavelengths where the undesired artefacts were, with a trade-off of reduced RSC and SSC intensities by an order of magnitude. This was an acceptable outcome given that the signal to noise ratio ample.

\section{Silicon substrate's anomalies}

Generally, in a spectroscopic measurement, it is important to have a fixed sample compartment to ensure an exact $90^{\circ}$ angle between the sample surface and the incoming light beam.

During our measurements, it became evident that the final spectrum of a $\mathrm{Si}$ sample was sensitive to the angle between the surface of the sample and the light beam. As a result, the spectra of silicon samples were not stable in the infrared region. This can be attributed to the high reflectivity of the surface of silicon in that region. This condition was not observed for samples that had other substrates.

Therefore, we designed a manual goniometer to check the reflected intensity of the light beam as a function of the angle of incidence. Our consequent measurements confirmed that rotation of silicon blank substrate from the normal position resulted in an unrealistic transmission signal. To capture the effect of this anomaly on the resulting transmission curve, we first rotated the silicon blank substrate to establish the angle at which the highest intensity of the light beam was detected. The maximum intensity happened at an angle less than $5^{\circ}$. Next, measurement was done while the sample was kept at $90^{\circ}$ with respect to the light beam, which is the ideal scenario. Under each condition, a spectrum was recorded; the results are shown in Figure 13-4. 


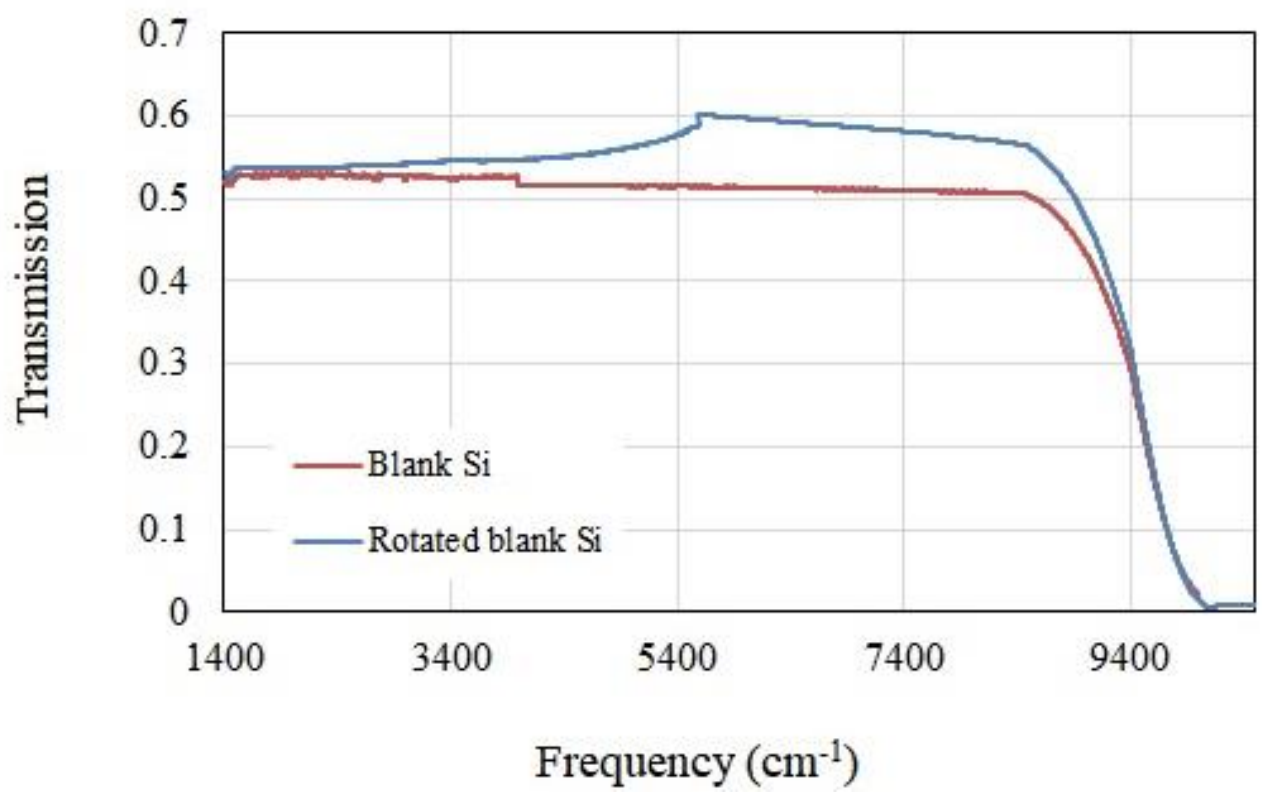

Figure 13-4 Transmission anomaly from the Si surface.

As can be seen, the rotated blank $\mathrm{Si}$ substrate presented a maximum transmission up to $60 \%$ which did not conform with the expected transmittance properties of $\mathrm{Si}$ as reported in literature $[464,465]$. Therefore, it was important to ensure that $\mathrm{Si}$ samples were mounted at an exact $90^{\circ}$ angle with respect to the light beam. Finally, to minimize the error involved with manual operation, a fully automated process was used to measure spectra for these samples and the repeatability of the measured data was confirmed. 



\section{Appendix C}

\subsection{Comparing ITO with AZO}

Indium tin oxide (ITO) is one the most established transparent conductive oxides with numerous applications in the relevant industries [466]. AZO however, offers environmental advantageous over ITO, as well as lower optical losses at a large part of the EM spectrum, with comparable electrical and optical properties; hence, AZO has been becoming a popular material for research and industry [467].

Here, we report our measured FTIR transmission results for an ITO thin film and compare it with nominal AZO samples from our reported studies. Thus, we measured the transmission spectral response for an ITO thin film on a flexible polyethylene terephthalate (PET) substrate. The sample was purchased from Sigma-Aldrich [468], where the ITO thin film thickness was $130 \mathrm{~nm}$ and the PET substrate had a thickness of $127 \mu \mathrm{m}$.

To compare the FTIR transmission of AZO with ITO, we selected AZO on $\mathrm{CaF}_{2}$ samples because $\mathrm{CaF}_{2}$ had the broadest transparency response between our four candidate substrates. Figure 14-1 shows the transmission spectra of a blank PET substrate, an ITO on PET sample, a blank $\mathrm{CaF}_{2}$ substrate and an $\mathrm{AZO}$ on $\mathrm{CaF}_{2}$ sample, where the thickness of AZO thin films was $120 \mathrm{~nm}$. The thickness of the $\mathrm{CaF}_{2}$ blank substrate was $1022 \mu \mathrm{m}$. 


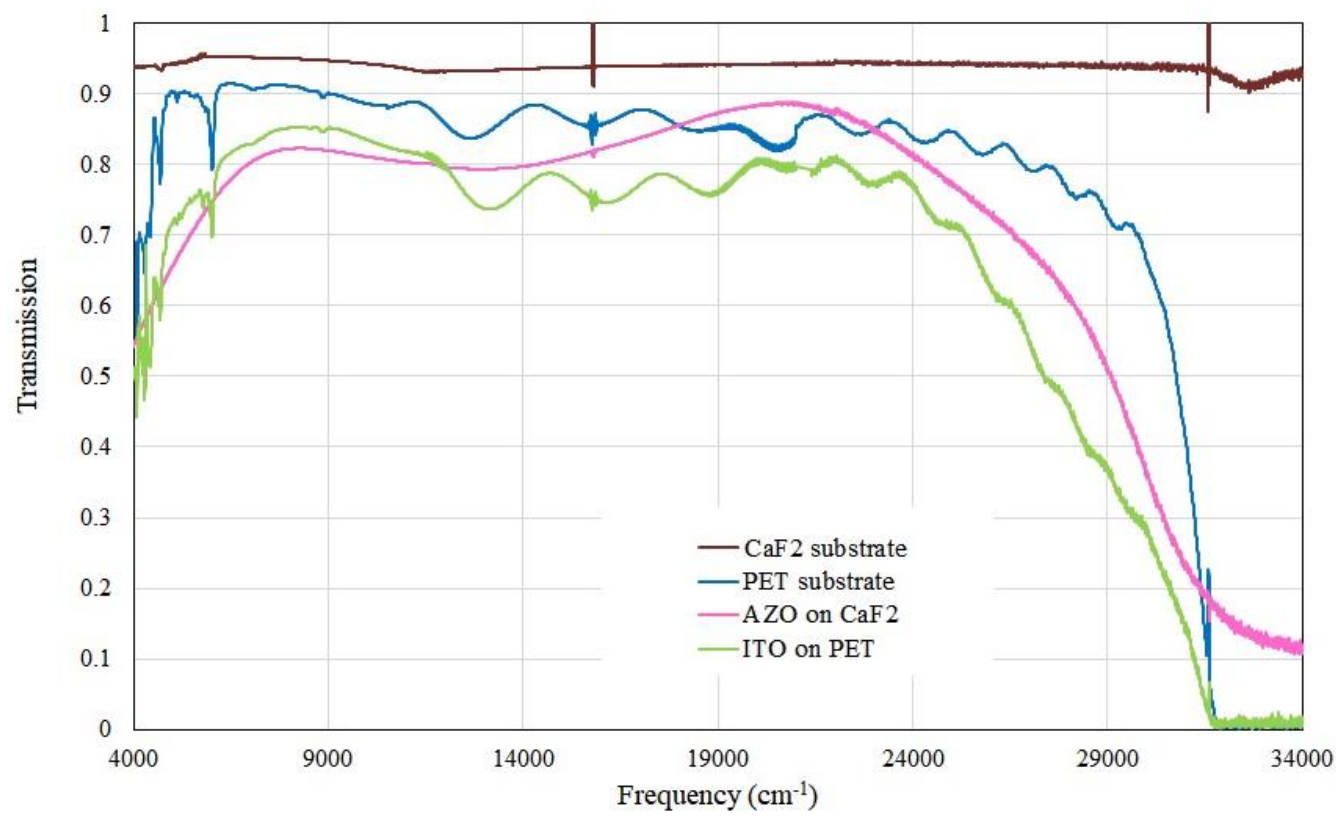

Figure 14-1 Transmission spectra included in our AZO - ITO comparison.

The PET substrate had a transmission of over $80 \%$ starting from the infrared to the UV, where a sharp cut-off is observed. The ITO thin film on PET had a transmission of over $70 \%$ from the infrared towards the end of the visible range. In comparison, $\mathrm{CaF}_{2}$ was highly transparent with a transmission over $90 \%$ in the whole presented range. Our $\mathrm{AZO}$ on $\mathrm{CaF}_{2}$ sample had a comparable transmission response to the ITO thin film, with a slightly higher transparency. Therefore, our AZO films were proven to be a suitable alternative for ITO films where transparency is important.

We also performed Hall effect measurements on our ITO sample. Considering that this sample was commercially established product, it had a high carrier concentration of the order of $1 \mathrm{E}+20 \mathrm{~cm}^{-3}$ and a resistivity of the order of $1 \mathrm{E}-4$ $\Omega . c m$, both of which are comparable with our nominal AZO samples reported in this thesis. 
